\title{
Die Iromycine und das Collinolacton: Synthese mikrobieller Naturstoffe aus Streptomyces sp.
}

\author{
Dissertation \\ zur Erlangung des Doktorgrades \\ der Mathematisch-Naturwissenschaftlichen Fakultäten \\ der Georg-August-Universität zu Göttingen
}

vorgelegt von

Heydar Shojaei

aus Teheran/Iran

Göttingen 2007 
D7

Referent:

Prof. Dr. A. de Meijere

Korreferent:

Prof. Dr. H. Laatsch

Tag der mündlichen Prüfung: 01./02.05.2007 
Die vorliegende Arbeit wurde in der Zeit von August 2003 bis Januar 2007 am Institut für Organische Chemie der Georg-August-Universität Göttingen angefertigt.

Ich möchte mich bei Herrn Prof. Dr. A. de Meijere für die interessante Themenstellung und die Bereitstellung der Möglichkeit zur Durchführung dieser Arbeit bedanken. Herrn Dr. E. P. von Zeschwitz danke ich für die hilfreichen Diskussionen und Anregungen bei der Realisierung der gesetzten Ziele im Rahmen der Naturstoffsynthese.

Ich bedanke mich weiterhin für die Unterstützung des Sonderforschungsbereiches, ohne dessen Hilfe die Durchführung dieser Arbeit für mich nicht möglich gewesen wäre. 



\section{Inhaltsverzeichnis}

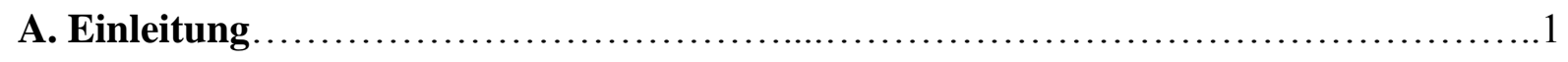

I. Naturstoffe und die Bedeutung ihrer Totalsynthese.................................

II. Die Naturstoffe Iromycin und Collinolacton aus Streptomyces sp.......................6

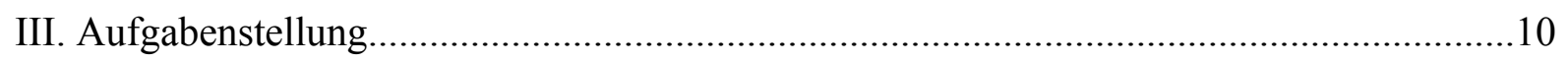

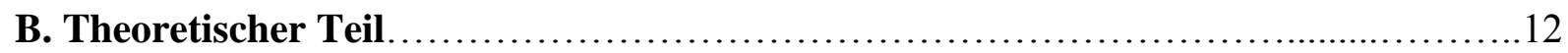

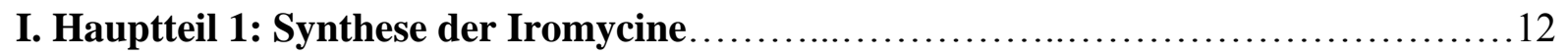

1. Retrosynthetische Aspekte zu einer diversitäts-orientierten Synthese der Iromycine........12

2. Synthese der Iromycine A und R ausgehend von der Kreuzkupplung von Brommethyl-

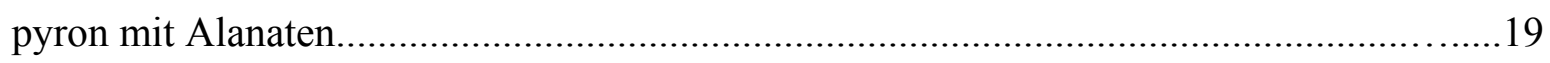

2.1. Synthese des Ringfragments als 6-Brommethylpyron...........................20

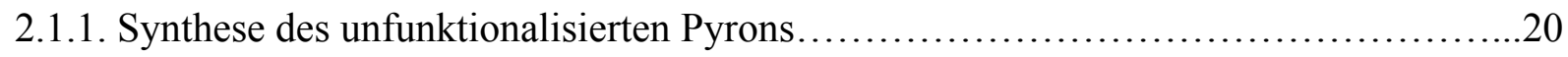

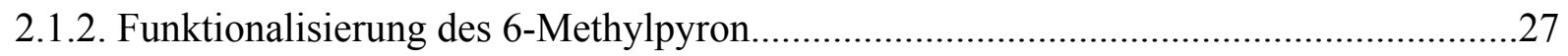

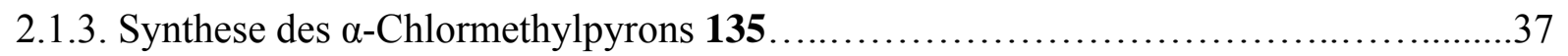

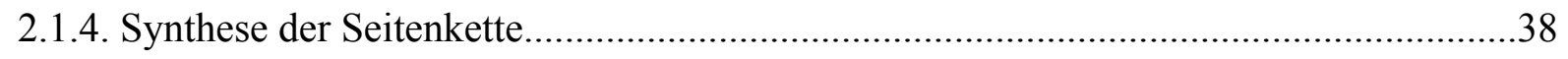

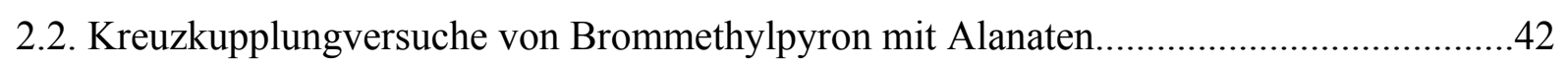

2.2.1. Zirconocen-katalysierte Darstellung der Alane aus Alkinen.........................................43

2.2.2. Versuche zur Darstellung der Iromycinvorläufer aus Bromiden mit Alanaten...............45

2.2.2.1. Vorversuche zu Kreuzkupplungsreaktionen von Benzylbromiden mit Alanaten........47

2.2.2.2. Darstellung der Iromycinvorläufer aus Pyronhalogeniden mit Alanaten.....................50

2.3. Synthese der Iromycine A und R sowie deren Analogons durch O-N-Austausch............59

3. Kreuzkupplungsreaktionen des Brommethylpyridons mit Alanaten..................................66

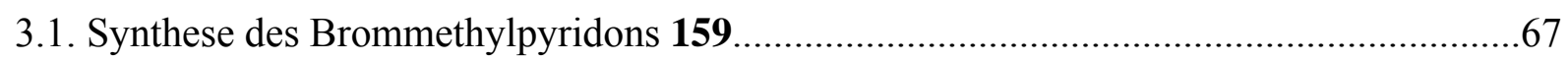

3.1.1. O-N-Austausch des Pyrons 63 zu dem Pyridon 62 ....................................................67

3.1.2. Synthese des Pyridonacetates 156 aus dem Pyridon 62 .............................................73

3.1.3. Funktionalisierung des Pyridonacetates $156 \mathrm{zu}$ dem Brommethylpyridon 159............75

3.2. Kreuzkupplungsreaktionen des Brommethylpyridons 159 mit Alanaten.........................80

3.2.1. Kupplungversuche des Brommethylpyridons 159 mit dem Alanat 124.......................80

3.2.2. Kreuzkupplungversuche des Brommethylpyridons 159 mit Alanaten durch in situ

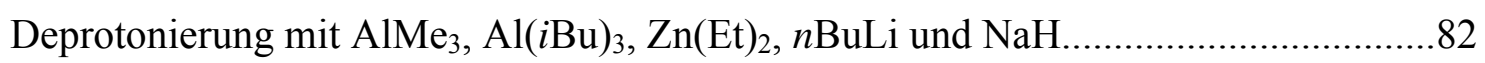

3.2.3. Kreuzkupplungsversuche des $N$-geschützten Brommethylpyridons 159 mit dem

Alanat 124. 
3.2.3.1. Versuche zur Schützung des Brommethylpyridons 159 an Stickstoff.

3.2.3.1.1. Versuche zur Schützung des Brommethylpyridons 159 mit Anhydriden,

Silanen, Chlormethylmethylether und Acetylchlorid..... .86

3.2.3.1.2. Versuche zur Schützung des Brommethylpyridons 159 durch Methylierung .93

3.2.3.2. Kreuzkupplungversuche der $N$-geschützten Brommethylpyridone 169 und 174 mit dem Alanat 124. .98

4. Kreuzkupplungversuche des Brommethylpyridins 181 mit Alanat 124. 100

5. Metallkatalysierte Kreuzkupplungsversuche. 102

5.1. Pd-katalysierte Kreuzkupplung mit Alanen. 102

5.1.1. Pd-katalysierte Kreuzkupplung der Bromide mit in situ generierten Alanen. 103

5.1.2. Pd-katalysierte Stille-Kupplung des Brommethylpyrons 183 mit Vinylstannen.

5.1.3. Versuche zur Pd-katalysierten Kreuzkupplung von Zinkorganylen mit Alkenylhalogeniden.

5.1.4. Versuche zu Pd-katalysierten Kreuzkupplungsreaktionen von $\gamma$-Stannylorganylund $\gamma$-Zinkorganyl-maskierten Trienolaten mit Alkenyliodid.

5.2. Ni-katalysierte Kreuzkupplung mit Alanen.

5.2.1. Ni-katalysierte Kreuzkupplung der Benzylhalogenide 130 und 222 sowie der Pyronhalogenide 96 und 135 mit dem Alan 123.

5.2.2. Ni-katalysierte Kreuzkupplung der Chlormethylpyridine 223 und 179 mit dem Alan 123.

5.3. Cu-katalysierte Kreuzkupplungsreaktionen.

5.3.1. Cu-katalysierte Kreuzkupplung des Brommethylpyrons 96 mit Alanen.

5.3.2. Cu-katalysierte Kreuzkupplung von Lithium-Alkenylen mit Benzylbromid bzw. Brommethylpyron.

5.3.3. Cu-katalysierte Kreuzkupplung von Grignard-Reagentien mit Bromiden. 134

5.3.4. Versuche zur Cu-katalysierten Alkinylierung des Brommethylpyrons 96. 140

\section{Hauptteil 2: Stand der Arbeiten zur Synthese von Collinolacton.}

1. Retrosynthese und Reaktionsmechanismen der Collinolactonsynthese. 143

2. Darstellung der Ausgangssubstanzen. 148

2.1. Synthese der Methylenkomponenten 273, 274 und 275. 149

2.2. Synthese des (E,E)-6-(tert-Butyldimethylsilanoxy)hexa-3,5-dienals (281). 154

3. C-C-Kupplungsversuche zur Darstellung des Aldoladditionsproduktes.

3.1. Versuche zur Aldoladdition unter basischen Bedingungen. 164 


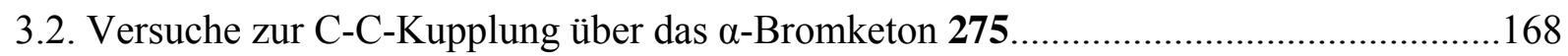

3.3. Versuche zur Mukaiyama-Aldoladdition unter Verwendung von Lewissäuren..............170

3.3.1. Versuch zur Mukaiyama-Aldoladdition mit $\mathrm{TiCl}_{4}$ als Lewissäure...............................170

3.3.2. Versuch zur Mukaiyama-Aldoladdition mit $\mathrm{ZnCl}_{2}$ als Lewissäure.............................171

3.3.3. Versuch zur Mukaiyama-Aldoladdition mit $\mathrm{Yb}(\mathrm{OTf})_{3}$ als Lewissäure.......................172

3.3.4. Versuch zur Mukaiyama-Aldoladdition mit TBAF als Lewissäure.............................173

3.3.5. Versuche zur Mukaiyama-Aldoladdition mit BINOL/Ti(O-iPr $)_{4}$ als Lewissäure-

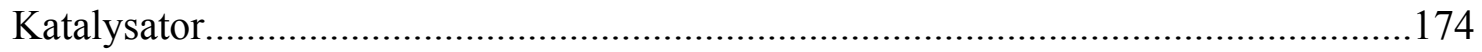

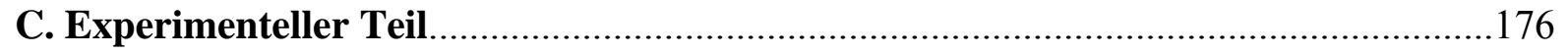

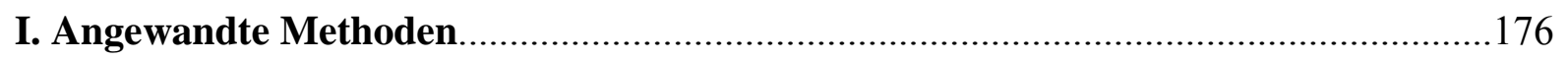

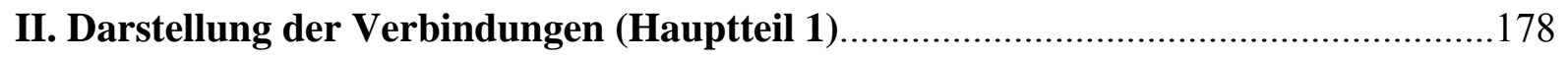

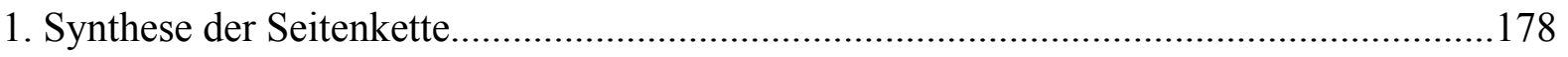

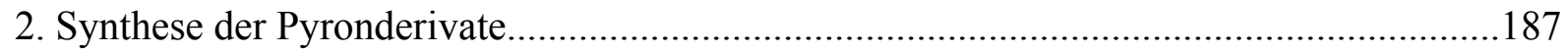

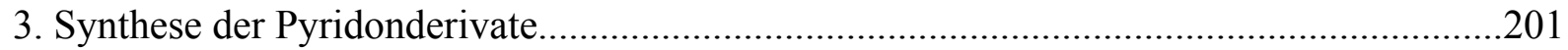

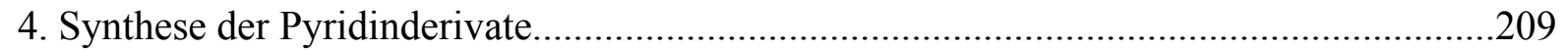

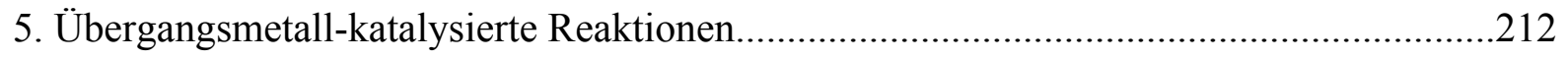

III. Darstellung der Ausgangssubstanzen zur Collinolactonteilsynthese (Hauptteil 2)..217

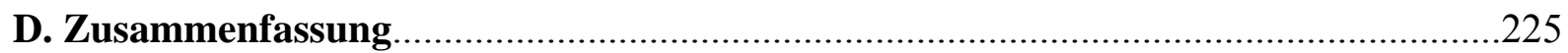

\section{E. Abkürzungen}

\section{F. Literaturverzeichnis}

G. Spektrenanhang 



\section{A. Einleitung}

\section{Naturstoffe und die Bedeutung ihrer Totalsynthese}

Seit den Tagen der Frühgeschichte stellt die Natur den Menschen eine unerschöpfliche Quelle von Heilmitteln und anderen biologisch aktiven Substanzen zur Verfügung. Schon die Medizinmänner und Schamanen der Naturvölker kannten den Nutzen von Pflanzen und Pilzen bei der Behandlung Kranker. Auch in den frühen Hochkulturen der Ägypter, Maya, Perser oder Chinesen wurden aus Pflanzen gewonnene Heiltränke zur Behandlung zahlreicher Krankheiten eingesetzt. Allein in China gibt es auch heute noch ca. 11.000 aus Pflanzen hergestellte traditionelle Heilmittel. ${ }^{[1]}$ Die Erkenntnis, dass er von der Natur lernen könne, brachte den Menschen im Verlaufe der Geschichte auch dazu, die biologischen Systeme eingehend zu studieren, zu imitieren und zu versuchen, ihre Zusammenhänge aufzuklären. Diesem Ansatz entsprang auch die Naturstoffchemie, deren Entwicklung durch die bedeutenden Fortschritte der Chemie bei der Isolierung und Synthese organischer Verbindungen im 19. Jahrhundert ihren Anfang nahm. Bereits im Jahr 1805 war es gelungen, das Morphin als erstes Alkaloid zu isolieren, welches als Opiumbestandteil schon seit vorchristlicher Zeit zur Schmerzbehandlung eingesetzt worden war. ${ }^{[2]}$ Im Jahr 1828 schaffte es schließlich Friedrich Wöhler erstmalig, die organische Verbindung Harnstoffsäure aus einer anorganischen Substanz herzustellen, womit er der Totalsynthese den Weg bereitete.

Seit jener Zeit gewann die Naturstoffchemie zum Zwecke der Isolierung und Reproduktion biologisch aktiver Verbindungen immer mehr an Bedeutung, vor allem auf dem Gebiet der Arzneimittel. So beruhen etwa 40\% der heute weltweit eingesetzten Arzneimittel auf Naturstoffen oder Naturstoffderivaten. ${ }^{[3]}$ Die Bedeutung der Naturstoffe bei der Arzneimittelherstellung wird auch dadurch deutlich, dass mehr als ein Drittel aller Arzneimittelumsätze in der Zeit von 1981-2004 auf Naturstoffen oder deren "intellectual DNA" beruhten, während Naturstoffe im Vergleich zu Synthetika lediglich 1\% aller bekannten organischen Verbindungen ausmachen. $^{[4]}$

Hatte man die nützlichen biologischen Aktivitäten eines Naturstoffes erkannt, bereitete es aber oftmals Schwierigkeiten, diesen größeren Kreisen der Weltbevölkerung zugänglich zu machen, da Naturstoffe auf biologischem Weg nur begrenzt verfügbar und reproduzierbar sind ${ }^{[5]}$ Die natürlichen Vorkommen sind für eine kommerzielle Nutzung nicht immer ausrei- 
chend und beschränken sich zudem teilweise auf schwer zugängliche Gebiete, wie den tropischen Regenwald. Auch eine künstliche Nachzüchtung der Pflanzen lieferte nicht immer die Lösung, da für eine Gewinnung der begehrten Naturstoffe in relevanter Menge oftmals eine unverhältnismäßig große Menge an Pflanzenmaterial erforderlich war und es zudem auch vorkam, dass Pflanzen außerhalb ihres natürlichen Ökosystems die gesuchten Inhaltsstoffe überhaupt nicht mehr produzierten. ${ }^{[6]}$ Den Ausweg brachte allein die Totalsynthese der Naturstoffe, die deren synthetische, industrielle Herstellung erst ermöglichte und die Naturstoffe damit unbegrenzt verfügbar machte. Eine weitere wichtige Funktion der Totalsynthese besteht in der über die Strukturaufklärung des Moleküls ermöglichten Synthese von Naturstoffanaloga und - derivaten, da häufig erst diesen die gewünschten biologisch aktiven Eigenschaften zukommen.

Eines der historisch bedeutendsten Beispiele für die gelungene Totalsynthese eines Naturstoffes stellt die Synthese des Penicillins dar (vgl. Schema 1). Penicillin ist eines der ältesten und wichtigsten Antibiotika und gehört zu der Gruppe der $\beta$-Lactam-Antibiotika. ${ }^{[7]}$ Antibiotika, vor allem das Penicillin, haben Millionen Menschenleben gerettet und das Leiden vieler Patienten gelindert. ${ }^{[5]}$ Die $\beta$-Lactam-Antibiotika wurden als erste Naturstoffklasse zur Therapie bakterieller Infektionen verwendet und noch heute gehört diese Substanzgruppe zu den klinisch meistverwendeten Antibiotika. ${ }^{[5]}$ Entdeckt wurde das Penicillin 1928 von dem schottischen Bakteriologen Alexander Fleming als Stoffwechselprodukt des Schimmelpilzes Penicillium notatum. 1939 gelang es dann Boris Chain und Walter Florey, das Penicillin zu isolieren und dessen bemerkenswerte biologische Aktivität gegen verschiedene pathogene Keime zu ermitteln, wofür sie gemeinsam mit Fleming 1945 den Nobelpreis für Medizin erhielten.

Schema 1. Penicillin V 1 bzw. Penicillin G 3 als Naturstoffe.

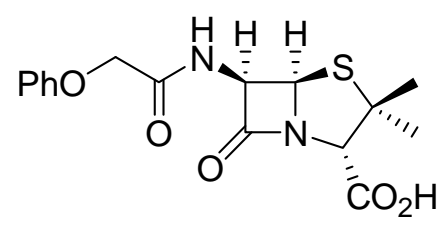

Penicillin V 1

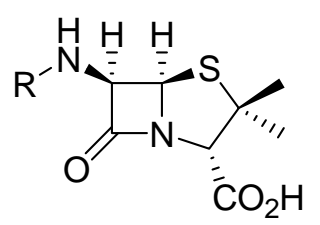

Penicillin-Grundstruktur 2<smiles>CC1(C)S[C@@H]2[C@H](NC(=O)Cc3ccccc3)C(=O)N2[C@H]1C(=O)O</smiles>

Penicillin G 3 
Nach der Entdeckung des großen Potentials des Penicillins im Kampf gegen die bis dahin oft tödlich verlaufenden Infektionskrankheiten bestand die große Schwierigkeit darin, das Penicillin in einer für den weltweiten Bedarf ausreichenden Menge herzustellen, da der Schimmelpilz selbst nur winzige Dosen produzierte. Nur kurze Zeit nach den Veröffentlichungen von Chain und Florey wurden daher große Anstrengungen zur Erforschung eines chemischen Syntheseweges für das Penicillin unternommen und annähernd 1.000 Chemiker arbeiteten hieran allein in den USA und Großbritannien gleichzeitig, ohne dass ihnen jedoch ein Durchbruch gelang. ${ }^{[8]}$ Selbst über die Struktur des Penicillins konnten die Wissenschaftler anfangs keine Einigkeit erzielen, bis es D. Crowfoot-Hodgkin 1945 gelang, die Struktur des Penicillins G 3 durch Röntgen-Kristallographie zu entschlüsseln. Doch auch nach dieser Entdeckung scheiterten sämtliche Syntheseversuche, so dass die Synthese des Penicillin-Moleküls schon als unmögliche Aufgabe bezeichnet wurde. ${ }^{[8]}$ Die Probleme bei der Totalsynthese des Penicillins wurden durch dessen 4-gliedrigen $\beta$-Lactam-Ring hervorgerufen, der für die instabile und reaktive Natur des Moleküls unter sauren wie basischen Reaktionsbedingungen, aber auch für dessen gewünschte antibakterielle Eigenschaften verantwortlich ist. Erst 1957 gelang es J. Sheehan und seinen Mitarbeitern die erste rationale Totalsyntheseroute für Penicillin zu entwickeln, indem sie neue effektive und selektive synthetische Methoden anwandten, die zugleich mild genug waren, um einen Zerfall des Penicillins zu verhindern. ${ }^{[8]}$

Sheehan plante als ersten Schritt zur Totalsynthese des Penicillins V 1, ausgehend von racemischen ( \pm )-Valin (4) als Startverbindung, die Darstellung des enantiomerenreinen Penicillaminhydrochlorids 15 (vgl. Schema 2). ${ }^{[9]}$ Hierzu wurde das Valin (4) in einer $N$-Acylierungsreaktion mit Chloracetylchlorid zunächst in das Amid 5 überführt, dessen Cyclisierung in heißem Essigsäureanhydrid zur Bildung des Lactonimins 7 führte, welches zu dem Oxazolon 8 isomerisiert wurde. Die Behandlung des als Michael-Akzeptor prädestinierten Oxazolons 8 mit Schwefelwasserstoffsäure und Methanolat initialisierte die Ringöffnungsreaktion, die zur chemoselektiven Bildung des Thiols 12 führte. Die Behandlung des Amids 12 mit wässrigem $\mathrm{HCl}$ führte in einer simultanen Ester- sowie $N$-Acetylspaltung zur Bildung eines Carbonsäureamins, das in einer Kondensations- und Cyclisierungsreaktion mit Aceton den Thiazolidinring 13 lieferte. Die Formylierungsreaktion des Thiazolidins 13 mit Ameisensäure führte zur Bildung des racemischen $N$-Formyl-isopropyliden-DL-penicillamins 14, dessen Behandlung mit einer wässrigen Brucin-Lösung eine diasteromere Salzmischung ergab. Da selektiv nur ein Diastereomer in Form von Kristallen ausfiel, konnte das Gemisch durch Filtration getrennt 
werden. Eine Behandlung der Kristalle mit 2 N HCl führte nach einer Ringöffnungsreaktion zum Erhalt des gewünschten reinen D-Penicillinhydrochlorids $\mathbf{1 5}$ als Intermediat.

Schema 2. Synthese des D-Penicillaminhydrochlorids 15 als Intermediat der Synthese des Kalium-Salzes von Penicillin V 1.

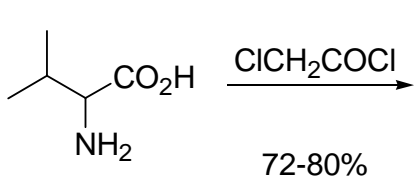

( \pm -Valin 4<smiles>CC(C)C(NC(=O)CCl)C(=O)O</smiles>

5<smiles>CC#CCC(=O)OC(=O)C(NC(=O)CCl)C(C)C</smiles>

6<smiles>C=C1N=C(C(C)C)C(=O)O1</smiles>

7

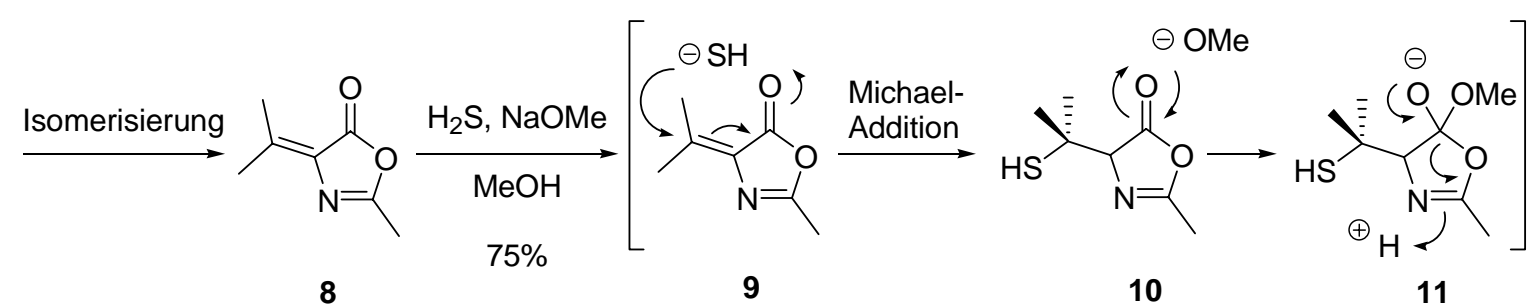

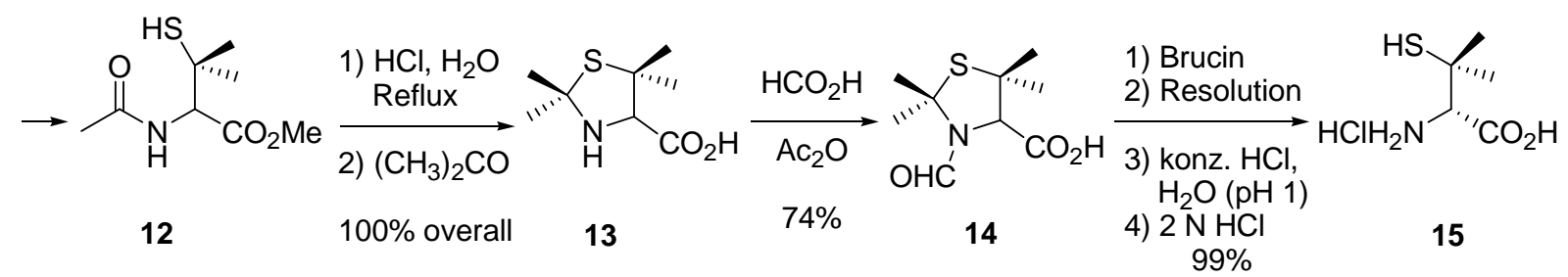

Sheehan et al. ${ }^{[9]}$ verwendeten als weiteres Intermediat zur Synthese des Penicillins V 1 den tert-Butylphthalimidmalonataldehyd 16 (vgl. Schema 3), der aus entsprechendem tertButylphthalimidacetat in Gegenwart von tert-BuONa und tert-BuOCHO dargestellt wurde. Die Verknüpfung der Intermediate D-Penicillinhydrochlorid 15 und Aldehyd 16 verlief unter Einsatz von wässrigem Natriumacetat in Ethanol, welches unter nucleophilen Angriffen der Amin- bzw. Thiolgruppe an die elektrophile Carbaldehydfunktion zur Cyclisierung und Bildung eines diasteromeren Gemisches aus dem unerwünschten D- $\gamma$-Thiazolidin 17 und dem zur Darstellung des Penicillins V 1 erforderlichen D- $\alpha$-Thiazolidin-Isomers 18 führte. Die Hydrazinolyse des D- $\alpha$-Thiazolidin-Isomers 18 und dessen anschließende Behandlung mit wässriger HCl-Lösung ergab das freie D- $\alpha$-Hydrochlorid 19, das unter Verwendung von $\mathrm{NEt}_{3}$ 
und Phenoxyacetylchlorid in das $\alpha$-tButyl-D-phenoxymethylpenicilloat 20 als charakteristische Vorstufe des Penicillins V 1 überführt werden konnte.

Schema 3. Synthese des Kaliumsalzes von Penicillin V 1.

16

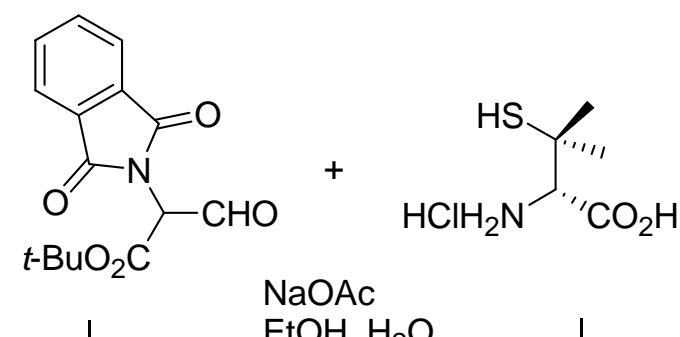

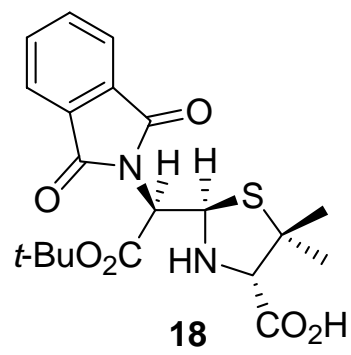
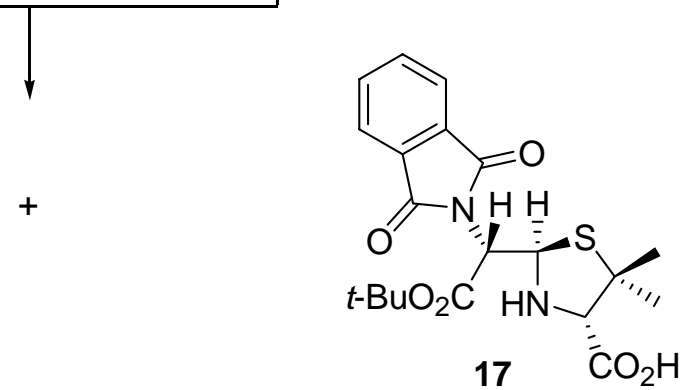

overall $82 \%$

1. $\mathrm{N}_{2} \mathrm{H}_{4}, 13^{\circ} \mathrm{C}$

2. $\mathrm{HCl}, \mathrm{H}_{2} \mathrm{O}$

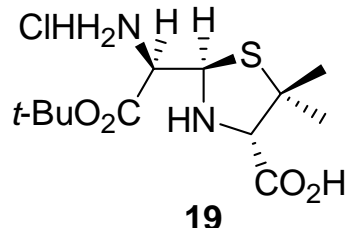

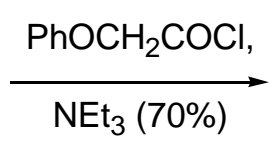

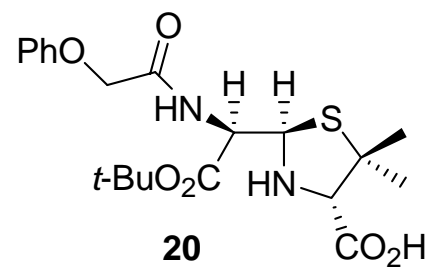

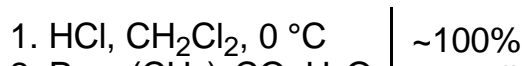

2. Pyr., $\left(\mathrm{CH}_{3}\right)_{2} \mathrm{CO}, \mathrm{H}_{2} \mathrm{O} \downarrow$ overall<smiles>[X]OC(=O)[C@H]1S[C@@H]2[C@H](NC(=O)COc3ccccc3)C(=O)N12</smiles>

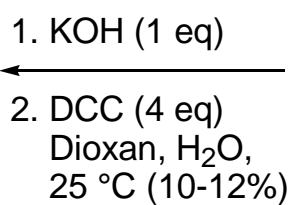

K-Salz von (+)-Penicillin V 1

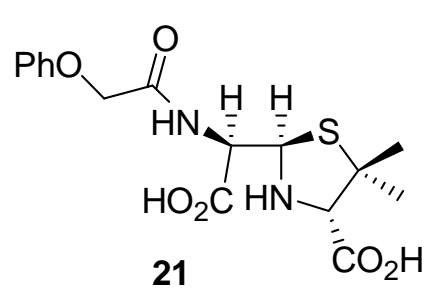<smiles>CCC</smiles> 
Die Verseifung des tert-Butylesters 20 gelang Sheehan unter Behandlung des Esters mit wasserfreier HCl-Lösung in Dichlormethan, gefolgt von der Kristallisation der resultierenden Carbonsäure in wässriger Acetonlösung unter Einsatz von 1.0 eq Pyridin. Die intramolekulare Lactamisierung des aus Amin 21 und KOH dargestellten Carboxylates erfolgte über den Säureaktivierungsschritt 22 des Salzes mit $N$-N'-Dicyclohexylcarbodiimid (DCC), welches zur Vollendung der ersten Totalsynthese des Kaliumsalzes des Penicillins V 1 führte.

Die dargestellte Syntheseroute des Penicillins V nach Sheehan stellt einen Meilenstein in der organischen Synthese dar. Sheehan et al. entwickelten einige synthetische Methoden von allgemeinem Nutzen. Bemerkenswert sind dabei vor allem der Einsatz von Schutzgruppen aus sofort spaltbaren Phthalimiden und tert-Butylestern sowie die Verwendung von aliphatischen Carbodiimiden zum Ringschluss des $\beta$-Lactam-Rings.

\section{Die Naturstoffe Iromycin und Collinolacton aus Streptomyces sp.}

Die Iromycine sind Vertreter einer neuartigen Substanzklasse und bisher als Naturstoffe oder deren Abbauprodukte nicht literaturbekannt. Die Naturstoffe Iromycin A und B (vgl. Schema 4) sind Sekundär-Metabolite des Bakterienstammes Streptomyces sp. Gö Dra 17, der aus einer aus der Nähe von Göttingen stammenden Bodenprobe eines Kalkmagerrasens isoliert werden konnte. ${ }^{[10]}$

Schema 4. Die Naturstoffe Iromycin A (23A), Iromycin B (23B) und Iromycin C (23C) sowie deren synthetisch darzustellende Analoga 23 und Iromycin R (23R).<smiles>CCCc1c(C/C=C(\C)C/C=C/C(C)C)[nH]c(=O)c(C)c1O</smiles><smiles>CCCc1c(C/C=C(\C)C/C=C/C(C)(C)O)[nH]c(=O)c(C)c1O</smiles><smiles>CC/C=C/C/C(C)=C/Cc1[nH]c(=O)c(C)c(O)c1CCC</smiles><smiles>CCCCC/C(C)=C/Cc1[nH]c(=O)c(C)c(O)c1CCC</smiles><smiles>[R16][R16]([H])=CCCc1c(C/C=C(\C)C/C=C/C(C)(C)C)[nH]c(=O)c(C)c1O</smiles> 
Das als ölige, gelb-grünliche Flüssigkeit isolierte Iromycin A zeigte eine UV-Löschung bei $254 \mathrm{~nm}$. Massenspektroskopische Untersuchungen ergaben einen Molekülpeak bei $m / z=303$, was einer Summenformel von $\mathrm{C}_{19} \mathrm{H}_{29} \mathrm{NO}_{2}$ entspricht. Die Struktur der Iromycine konnte durch NMR- und röntgenstrukturanalytische Daten aufgeklärt werden, die einen hochsubstituierten Pyridonring mit einer ungesättigten $\mathrm{C}_{10}$-Seitenkette als Strukturfragmente belegten (vgl. Schema 4).

Dem Iromycin A konnte durch Untersuchungen ein interessantes biologisch-aktives Wirkprofil zugewiesen werden. Sukenaga et al. konnten eine antibakterielle Wirkung des Iromycins A gegen Bacillus subtilis und Staphylococcus aureus nachweisen und ließen es als Inhibitor der NO-Bildung und Hemmer der NO-Synthase patentieren. ${ }^{[11]}$ Untersuchungen zur Einsetzbarkeit gegen weitere Krankheiten wie Schlaganfall, Parkinsonsche Krankheit, Arthritis, Diabetes, Schmerz u.a. sind noch nicht abgeschlossen. ${ }^{[11]}$ Das Interesse an einer pharmazeutischen Nutzbarkeit des Iromycins A liegt einerseits darin, dass aufgrund der Verbreitung von bakteriellen Resistenzen sowie Multiresistenzen gegen bekannte Antibiotika der Bedarf an neuartigen Wirkstoffen stetig zunimmt und andererseits gerade niedermolekulare Wirkstoffstrukturen wie Iromycin A für die industrielle Synthese und medizinische Einsetzbarkeit vorteilhaft sind.

Für die unterschiedliche Bioaktivität der Iromycine werden hauptsächlich die Variationen der Seitenkettenenden verantwortlich gemacht. Im Gegensatz zum Iromycin A konnte beim Iromycin B in Untersuchungen keinerlei antibakterielle Wirkung nachgewiesen werden. Da der einzige strukturelle Unterschied zwischen Iromycin A und Iromycin B darin besteht, dass bei Iromycin B das Proton der isoPropylgruppe des Iromycins A an der C-7'-Position durch eine OH-Gruppe ersetzt ist, wird die Bioaktivität des Iromycins A auf die unsubstituierte isoPropylgruppe zurückgeführt. Es wurde daraufhin auch angenommen, dass das Iromycin B ein durch Oxidation entstandenes metabolisches Abbauprodukt des Iromycins A darstellt. Das Iromycin C ist ein Derivat des Iromycins A, das am Ende der Seitenkette anstatt der isoPropylgruppe eine Ethylgruppe trägt. Seine biologische Aktivität liegt zwischen derjenigen von Iromycin A und B.

Das Collinolacton (24) (vgl. Schema 5) ist ebenso wie die Iromycine ein neuer Naturstoff und zeichnet sich besonders durch ein cytostatisches Bioaktivitätsprofil aus. ${ }^{[12]}$ Das Collinolacton ist ein von Streptomyceten-Stämmen produziertes Stoffwechselprodukt. Es konnte bisher aus 
dem aus einer bolivianischen Bodenprobe resultierenden Stamm Streptomyces sp. Gö 40/10 und dem aus einer japanischen Bodenprobe herrührenden Stamm Streptomyces collinus sp. Tü 1892 isoliert werden. ${ }^{[13]}$ Das Collinolacton lag nach der Isolierung als farblose, amorphkristalline Verbindung vor, die auf dem Dünnschichtchromatogramm UV-Licht von $254 \mathrm{~nm}$ löschte. Die hochauflösende ESI-Massenspektroskopie führte $\mathrm{zu}$ der Summenformel $\mathrm{C}_{21} \mathrm{H}_{28} \mathrm{O}_{5}{ }^{[14]}$ Die Struktur des Collinolactons 24 ist bislang noch nicht abschließend geklärt. Gesichert ist, dass das Molekül aus einem Cyclodecatriengerüst, einem annelierten Siebenring und einem sechsgliedrigen Lactonring besteht (vgl. Schema 5). Damit weist das Collinolacton ein für Naturstoffe einzigartiges Grundgerüst auf. Während HPLC-Analysen des Collinolactons das Vorliegen einer einheitlichen Verbindung bestätigten, wiesen sowohl das ${ }^{1} \mathrm{H}$ - als auch das ${ }^{13}$ C-NMR-Spektrum einen doppelten Satz an Signalen mit einem Intensitätsverhältnis von 4:1 auf. Aufgrund dessen wurde zunächst angenommen, dass das Collinolacton nach der Isolierung als für einen Naturstoff ungewöhnliches E/Z-Isomerengemisch 24 an der Doppelbindung C-14/C-15 vorliegt. ${ }^{[12]}$

Schema 5. Mögliche E,Z-Isomere des Naturstoffes Collinolacton (24).

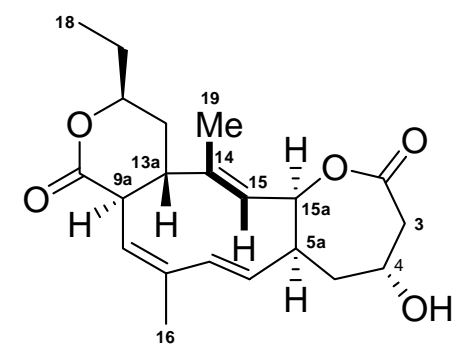

Collinolacton 24

E-Isomer

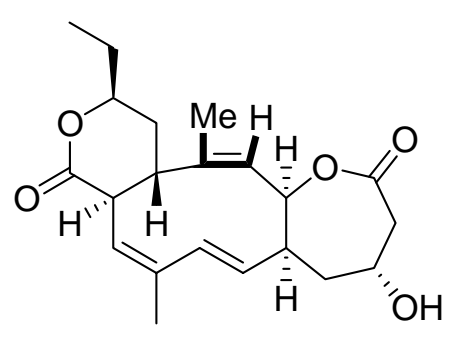

Collinolacton 24 Z-Isomer

Der zunächst gemachte Strukturvorschlag konnte jedoch durch weiterführende Untersuchungen lediglich hinsichtlich des Vorliegens der E-Konfiguration verifiziert werden, während sich das Vorliegen der Z-Konfiguration nicht bestätigen ließ. ${ }^{[14]}$ Gegen das Vorliegen eines stabilen E/Z-Isomerengemisches sprach zunächst, dass sich die Komponenten mittels chromatographischer Verfahren als nicht trennbar erwiesen. Um NMR-Spektren mit verbesserter Separierung der Signalmuster zu erhalten, wurden die ${ }^{1} \mathrm{H}-\mathrm{NMR}-\mathrm{Spektren}$ sowohl in $\mathrm{CD}_{2} \mathrm{Cl}_{2}$ als auch in $\mathrm{C}_{6} \mathrm{D}_{6}$ gemessen. Die Messungen bestätigten zunächst das Vorliegen eines Integral- 
verhältnisses von 4:1 für die beiden Komponenten. Die Auswertung weiterführender HMBCund NOESY-Experimente ergab für die Hauptkomponente eine sich mit dem vorgeschlagenen E-Isomer deckende Molekülstruktur, während die Minderkomponente als Rotamer der Hauptkomponente identifiziert wurde, eine Verbindung gleicher Konfiguration, die durch Rotation des Molekülfragmentes C14/C15/C19 um die Bindungen 13a/14 und 15a/15 mit der Hauptkomponente im dynamischen Gleichgewicht steht. Die Doppelbindung C-14/C-15 liegt damit um 180 Grad gekippt, aber weiterhin in E-Konfiguration vor. Bezogen auf die Methylgruppen C-16 und C-19 und ihre relative Stellung zueinander wurde die Hauptkomponente als anti-Collinolacton und die Minderkomponente als syn-Collinolacton charakterisiert (vgl. Schema 6). ${ }^{[14]}$ Eine abschließende Strukturaufklärung des Collinolactons steht aber noch aus, da es bislang nicht möglich war, röntgentaugliche Kristalle zu erhalten.

Schema 6: Hauptkomponente (anti) bzw. Minderkomponente (syn) des Collinolactons (24).

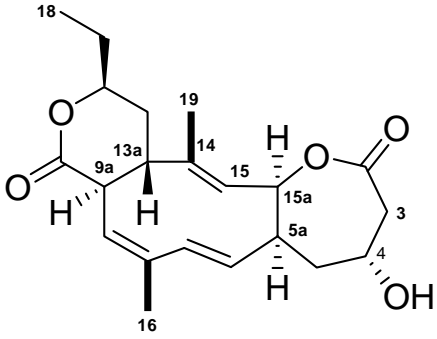

24 anti
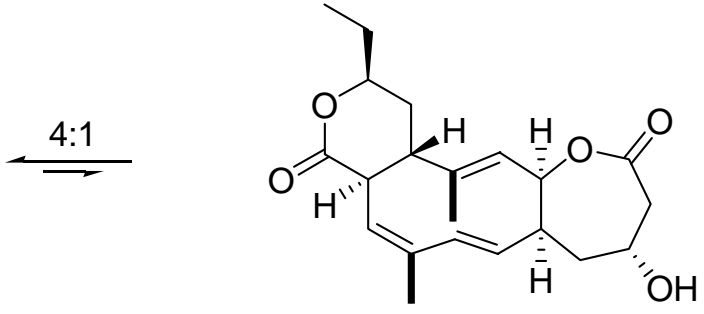

$\mathbf{2 4}_{\text {syn }}$

Aufgrund der cytostatischen Aktivität von Collinolacton wird auch in möglichen Derivaten des Collinolactons erhebliches biologisch-aktives Potential vermutet, weshalb der Darstellung von Derivaten und deren Untersuchung großes Interesse zukommt. Bisherige Untersuchungen von möglichen Derivaten (vgl. Schema 7) erbrachten für die Acetyl- 28, Benzoyl- 29 sowie $p$ Brombenzoylderivate 30 vielversprechende Ergebnisse für cytotoxische Aktivitäten. Diese Derivate zeigten bei in vitro-Tests eine Hemmung des Zellwachstums von Magencarcinom-, Lebercarcinom- und Mammacarcinom-Tumorzellen. ${ }^{[14]}$ 
Schema 7. Derivatisierung des Collinolactons (24) an der Doppelbindung bzw. an der Alkoholfunktion.

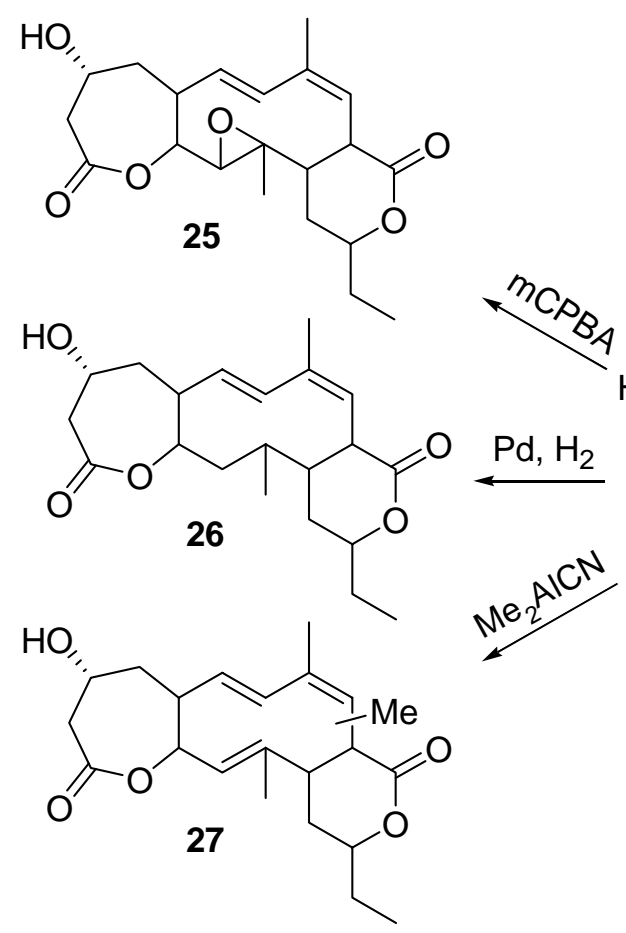<smiles>CC(=O)O[C@H]1CC(=O)OC2C=CC=CC2C1</smiles>

28

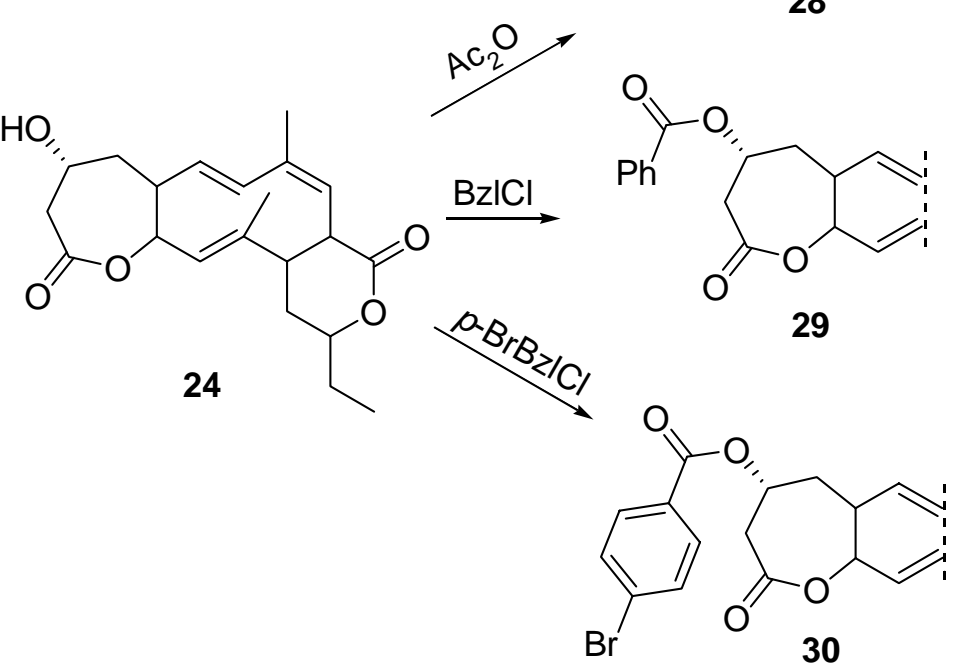

\section{Aufgabenstellung}

Im Rahmen dieser Doktorarbeit sollte eine Synthesestrategie zur Darstellung der Naturstoffe Iromycin sowie Collinolacton erarbeitet werden. Die vorgenannten, in Göttingen isolierten Naturstoffe werden derzeit im Rahmen einer Zusammenarbeit des Arbeitskreises von Dr. v. Zezschwitz mit dem biochemisch-organischen Arbeitskreis von Dr. Stephanie Grond chemisch und biochemisch untersucht.

Im Hinblick auf den Naturstoff Iromycin wurde im Rahmen der o.g. Zusammenarbeit angestrebt, weitere Derivate des Iromycins A (23A) auf biologische Aktivitäten hin zu untersuchen, da für die differenten Bioaktivitäten der Iromycine hauptsächlich die Variationen der Seitenkettenenden verantwortlich gemacht werden. Die synthetischen Arbeiten dieser Dissertation dienten dem Zweck, einen Zugang zu ausgewählten Derivaten der Iromycine zu ermöglichen. Als Hauptziel der Arbeit war daher ausgegeben worden, einen effizienten Syntheseweg für das Iromycin A zu entwickeln, der zugleich auf die Synthese anderer Derivate übertragbar sein sollte. Um eine rasche Synthese von Strukturanaloga (vgl. Schema 4, 23 und 
23R) zu gewährleisten, sollte ein Zugang in hochkonvergenter Form durch getrennte Darstellung der Seitenkette und des entsprechenden Pyronfragmentes gefunden werden, die dann durch Kreuzkupplungen miteinander verbunden werden sollten.

Die Totalsynthese des Collinolactons 24 sollte in erster Linie durch röntgenstrukturanalytische Untersuchungen von synthetisch hergestellten Derivaten eine vollständige und abschließende Strukturaufklärung des Naturstoffes ermöglichen. Die Aufstellung der Syntheseroute sollte auf der Basis von bereits durchgeführten Untersuchungen zur Biosynthese ${ }^{[15]}$ vorgenommen werden. Desweiteren sollten durch die gefundene Syntheseroute auch Derivate des Collinolactons zur weiteren Aufklärung des biologischen Wirkungsprofils zugänglich gemacht werden.

Die Synthesearbeiten zu den Iromycinen werden im Hauptteil 1 behandelt. Der Hauptteil 2 befasst sich mit dem Stand der Synthesearbeiten von Collinolacton. 


\section{B. Theoretischer Teil}

\section{Hauptteil 1: Synthese der Iromycine}

\section{Retrosynthetische Aspekte zu einer diversitäts-orientierten Synthese der Iromycine}

Der strukturelle Aufbau der Iromycine besteht aus zwei zentralen Strukturfragmenten, einem Pyridonring und einem ungesättigten $\mathrm{C}_{10}$-Seitenkettengerüst, die zur Erzielung einer größtmöglichen Diversität zunächst getrennt synthetisiert und erst in einem späteren Schritt gekuppelt werden sollten.

Die in der Literatur beschriebenen Syntheseverfahren von Naturstoffen mit ähnlichem grundstrukturellem Molekülaufbau, bei denen die Synthese ausgehend von einem einzigen Strukturfragment angelegt worden war, schienen für die Iromycinsynthese nicht in gleichem Maße geeignet. Unter anderem Oppolzer et al. ${ }^{[16]}$ wandten eine derartige Vorgehensweise bei der Synthese des aus Geflügelnebennieren, Tuberkel Bazilli sowie Meeresmollusken isolierten Naturstoffes Deoxypolypropionat 40 als Pyronderivat an (vgl. Schema 8).

Schema 8. Synthese des Naturstoffes Deoxypolypropionat 40 nach Oppolzer.

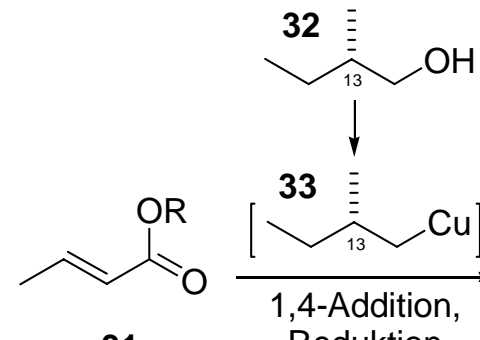

31
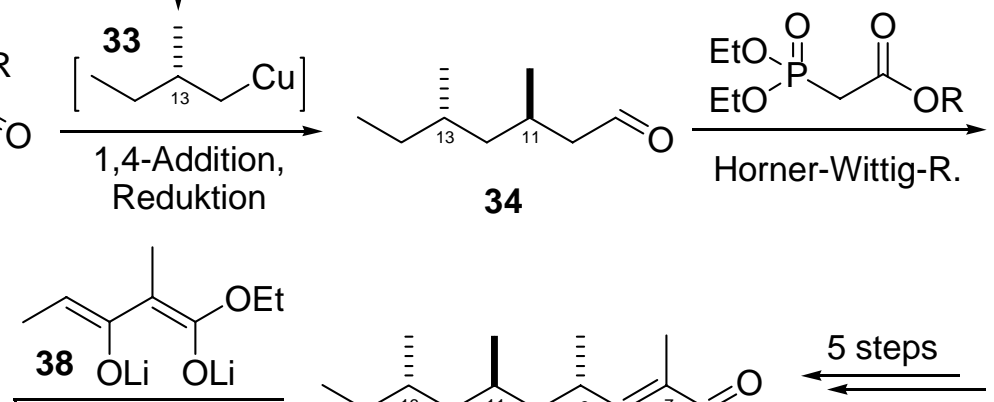

34<smiles>[R7]C(=O)/C=C/C[C@H](C)C[C@H](C)CC</smiles>
35<smiles>[R2]OC(=O)C[C@@H](C)C[C@H](C)C[C@H](C)CCC#CC[AsH3-]</smiles>
37 36

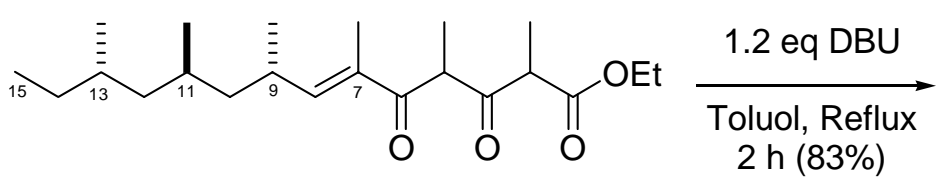

39
40<smiles>CC[C@H](C)C[C@@H](C)C[C@H](C)/C=C(\C)c1oc(=O)c(C)c(O)c1C</smiles> 
Die Verbindung 40 wurde von Oppolzer et al. im Rahmen einer 18-stufigen Synthese aus dem acyclischen Intermediat 39 dargestellt (vgl. Schema 8). Zur Darstellung des acyclischen Schlüsselintermediates 39 sowie zur Generierung der gewünschten Topizität der methylsubstituierten Stereozentren (absolute sowie relative Konfiguration) machte man sich unter Einsatz von chiralen Auxilarien, ausgehend von Crotonat 31 und dem (s)-2-Methyl-1-butanol 32, die kombinatorische Anwendung von Horner-Wittig- sowie 1,4-Additionsreaktionen zu Nutze. Hierbei stellten Oppolzer et al. zunächst das Intermediat 39 dar, das in einer Cyclisierungsreaktion mit DBU in einem letzten Syntheseschritt in das enantiomerenreine Pyronderivat 40 überführt wurde.

Im Gegensatz zu der Vorgehensweise von Oppolzer et al. erschien bei der Synthese des Iromycins A (23A) die frühe Einführung der Seitenkette wegen der bei Iromycin erhöhten Anzahl an Doppelbindungsfunktionen ungünstig, da hierdurch die möglichen Synthesemethoden limitiert worden wären. Zudem ermöglicht eine zunächst getrennte Teilsynthese der Seitenkette deren Variation in wenigen Syntheseschritten. Gerade der Ermöglichung der Diversität der Seitenkette kommt bei der Iromycinsynthese besondere Bedeutung zu, da die Seitenkettenenden aufgrund von NO-Synthase-Untersuchungen für die Biaktivität der Iromycine verantwortlich gemacht werden.

$\mathrm{Zu}$ den Naturstoffen, die eine grundstrukturelle Ähnlichkeit zu Iromycin A aufweisen und deren Synthese bereits Gegenstand der Untersuchungen zahlreicher Arbeitsgruppen ${ }^{[17]}$ waren, zählen auch die Piericidine 45, 49 und 52 (vgl. Schemata 9, 10 und 11). Piericidine sind prototypische Mitglieder einer der wichtigsten Klassen von biologisch aktiven Naturstoffen, die aus Streptomyces mobaraensis und S. Pactam isoliert wurden. ${ }^{[17 \mathrm{c}]}$ Ihnen wird unter anderem Cytotoxizität, anti-mikrobielle Wirkung sowie insektizidale Aktivität zugewiesen. ${ }^{[17 a]}$ Unter den Piericidinen ist insbesondere Piericidin $A_{1}$ als effektiver Inhibitor der mitochondriellen Elektronen-Transportkette des Proteins NADH-Ubichinon-Reduktase und Antagonist des als Co-Enzym $Q_{n}$ bezeichneten Ubichinons 53 bekannt (vgl. Schema 12). ${ }^{[17 b]}$ Die Piericidine riefen daher großes synthetisches Interesse hervor. Den bislang veröffentlichten Arbeiten zur Piericidinsynthese ${ }^{[17]}$ ist gemeinsam, dass zunächst zwei Molekülfragmente getrennt aufgebaut und erst in einem anschließenden Schritt zum Piercidin kreuzgekuppelt wurden.

Philips et al. ${ }^{[17 \mathrm{a}]}$ berichteten von der Synthese des 7-Demethylpiericidins $\mathrm{A}_{1}$ (45) (vgl. Schema 9). Hierbei gingen sie bei der retrosynthetischen Syntheseplanung im Gegensatz zu Oppolzer 
von der Darstellung zweier Kettenfragmente $\mathbf{4 2}$ und $\mathbf{4 4}$ aus und nahmen einen Bindungsbruch an den mit C-6' und C-1 bezeichneten Zentren des Piericidins an. Zur Darstellung des Hauptgrundgerüstes 42 als eines der Molekülfragmente ausgehend von dem Bromid 41 nutzte man die sich für eine Funktionalisierung besonders eignende freie ortho-Position des Pyridinringes aus. Die geplante Pd-katalysierte Kreuzkupplung der Stannylverbindung 42 sowie der Seitenkette 44 führte man im Wege einer Stille-Kupplung durch und erhielt nach anschließender Entschützung das gewünschte Piericidin 45.

Schema 9. Synthese des Naturstoffes 7-Demethylpiericidin $A_{1}$ (45) nach Philips.<smiles>COc1nc(Br)c(C)c(OC)c1OC</smiles>

41 $\mid \begin{aligned} & \text { tert-BuLi } \\ & \mathrm{Bu}_{3} \mathrm{SnCl}\end{aligned}$<smiles>CCCCCCCCCCCCCCCCCC</smiles>
42<smiles>C=CC(O[Si](C#CC=CCC(C)=CCOS(=O)(=O)[O-])(C(C)C)C(C)C)C(C)=CC</smiles>
$\underset{\mathrm{Ti}\left(\mathrm{OPr}^{\prime}\right)_{3} \mathrm{Cl}}{i \mathrm{PrgCl}} \downarrow$<smiles>C/C=C(\C)C1O[Si](PCC)(P(C)C)/C(=C\C=C\CC(C)=CCC(=O)OC)[C@@H]1C</smiles>

1) $\mathrm{Pd}_{2}(\mathrm{dba})_{3}$, $\mathrm{LiCl}$,

44 DMF $(55 \%)$

2) TBAF, DMF (70\%)<smiles>C/C=C(\C)C(O)[C@H](C)/C=C/C=C/C/C(C)=C/Cc1nc(OC)c(OC)c(O)c1C</smiles>

Rapoport et al. versuchten bei der Synthese der Piericidine 49 (vgl. Schema 10) deren starke Toxizität, die die Anwendbarkeit des Naturstoffes limitierte, unter Beibehaltung der antagonistischen Eigenschaften der Piericidine gegenüber dem Ubichinon $\mathrm{CoQ}_{\mathrm{n}} \mathbf{5 3}$ herabzusetzen. ${ }^{[17 b]}$ Auch dieser Arbeitskreis nahm den retrosynthetischen Bindungsbruch zwischen der C-6' und der C-1-Position der Piericidinverbindung vor. Als Seitenkettenfragmente für die 
Kreuzkupplung wurden Prenylbromide 48 aus Prenol, Geraniol, Farnesol, Phytol bzw. Solanesol dargestellt. Desweiteren setzten Rapoport et al. genauso wie Philips et al. Brommethylpyridin 46 als Hauptgrundgerüst ein. Die Synthese des Piericidins 49 wurde über eine Transmetallierung des Brommethylpyridins 46 und die anschließende Kupplung des hieraus resultierenden Lithiopyridins 47 mit dem Prenylbromid 48 unter Einsatz von Buthyllithium vorgenommen.

Schema 10. Synthese der Piericidine 49 nach Rapoport.<smiles>COc1nc(Br)c(C)c(OCc2ccccc2)c1OC</smiles>

46<smiles>COc1nc(Cl)c(C)c(OCc2ccccc2)c1OC</smiles>

47

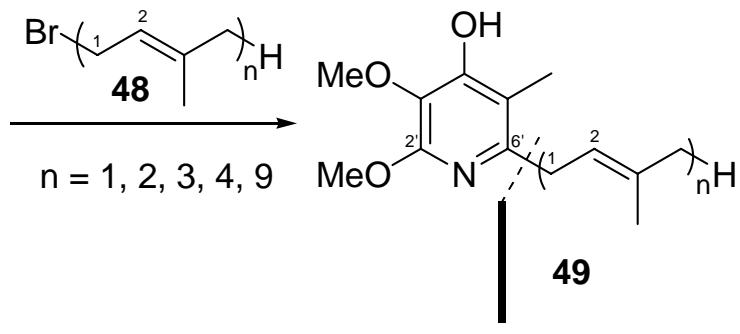

Transmetallierung

Als weitere Piericidin-Analoga wurden die Piericidine $\mathrm{A}_{1}$ und $\mathrm{B}_{1}(\mathbf{5 2})$ von Boger et al. ${ }^{[18]}$ über 26 Stufen synthetisiert (vgl. Schema 11).

Schema 11. Synthese der Piericidine $A_{1}$ und $B_{1}(\mathbf{5 2})$ nach Boger.

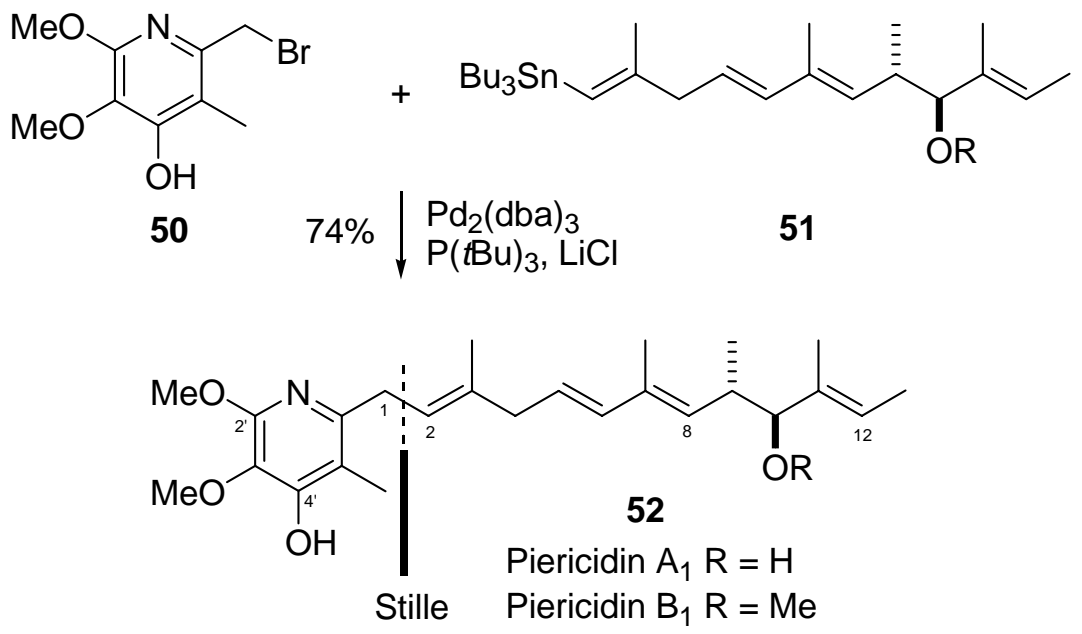


Anders als Philips et al. und Rapoport et al. nahmen Boger et al. im Rahmen der retrosynthetischen Überlegungen einen Bindungsbruch an der C1- und C2- $\sigma$-Bindung zwischen dem Heterocyclus und dem Seitenkettenfragment an, wodurch das Piericidin in zwei Segmente, das Ringsystem 50 und die Seitenkette 51, aufgespalten wurde. Zur Synthese der Piericidine verwendeten Boger et al. als Molekülfragmente das heterocyclische Pyridinylbromid $\mathbf{5 0}$ und das Tetraenstannat der Seitenkette 51, die über eine Pd-katalysierte Stille-Kupplung verknüpft und nach abschließenden Entschützungsschritten in die gewünschten Piericidine (52) als Kreuzkupplungsprodukte überführt wurden.

Als weiterer wichtiger Naturstoff, der eine strukturelle Ähnlichkeit mit den Iromycinen aufweist, ist das bereits oben genannte und als Ubichinon 53 bekannte Co-Enzym $Q_{n}(n=1-12)$ zu nennen, das als essentielle Komponente der zellularen Humanbiologie als reduktiver Carrier fungiert und als Antagonist der o.g. Piericidine gilt, woraus die Wichtigkeit der synthetischen Darstellung der Verbindung 53 resultiert (vgl. Schema 12).

Schema 12. Synthese des Ubichinons $53\left(\mathrm{CoQ}_{\mathrm{n}}\right)$ nach Lipshutz.<smiles>CCC(C)=CC1=C(C)C(=O)C(OC)=C(OC)C1=O</smiles>

53 Ubichinon $\left(\mathrm{CoQ}_{n}\right)$<smiles>CCC(C)=CCc1c(C)c(OC)c(OC)c(OC)c1OC</smiles>

54<smiles>[X]Cc1c(C)c(OC)c(OC)c(OC)c1C[CH+]</smiles>

55<smiles>COc1c(C)c(CCl)c(OC)c(OC)c1OC</smiles>

58

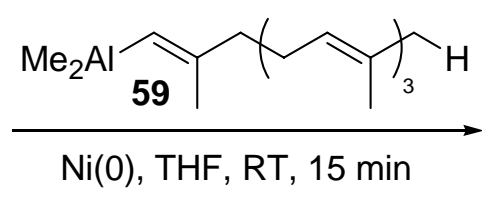

$87 \%$<smiles>C#CCCCC(C)=CCCC</smiles>

57<smiles>COc1c(C)c(CC=C(C)C)c(OC)c(OC)c1OC</smiles>

60 
Die Synthese der Ubichinone stand daher im Fokus der Arbeiten von Lipshutz et al. ${ }^{[19]}$, die die Ubichinone in einer Übergangsmetall-katalysierten Kreuzkupplungsreaktion ebenfalls ausgehend von zwei Molekülfragmenten darstellten. Im Rahmen der Retrosynthese nahmen Lipshutz et al. analog der Vorgehensweise von Boger et al. den Bindungsbruch zwischen der C-1' und der C-2'-Position der Seitenkette vor (vgl. Schema 12), wodurch sich das Halogenid 55 und das Alkin 57 ergaben. Entsprechend des retrosynthetischen Aufbaus gelang ihnen die Ni-katalysierte Kreuzkupplung des Chlorids 58 mit dem aus dem Alkin 57 dargestellten Alan 59 zu dem Ubichinon-Vorläufer 60.

Der Vorzug der Synthesemethode nach Lipschutz gegenüber den Vorgehensweisen von Philips, Rapoport und Boger besteht darin, dass durch die Annahme eines Bindungsbruches an der C-1'- und C-2'-Position der Seitenkette und eine hieraus folgende Fragmentierung des Zielmoleküls in das Allylhalogenid 55 und das Alkin 57, eine Methylierung und Funktionalisierung der Seitenkette in einem einzigen Syntheseschritt ermöglicht wird. Die Carboaluminierung des Alkins 57 führte auf diese Weise sowohl zu einer selektiven Methylierung der Dreifachbindung als auch zur Generierung einer Doppelbindungsbildung mit einfacher Geometrie.

Für das retrosynthetische Konzept der Iromycinsynthese wurde ebenso wie Lipschutz ein Bindungsbruch an der C-1'- und C-2'-Position der Seitenkette des Iromycins 23 geplant (vgl. Schema 13). Neben den bereits oben erwähnten Vorzügen der Synthesemethode über eine Carboaluminierung des Alkins, erschien dieses Retrosynthesekonzept im Fall des Iromycins auch deshalb vorteilhaft, da sich die aus dem Bindungsbruch resultierende Methylgruppe an der C-6-Position des Pyridonrings aufgrund ihrer chemischen Eigenschaften für eine Funktionalisierung in besonderem Maße eignet. Demzufolge bietet eine Analyse des Molekülaufbaus für die geplante Kreuzkupplung der beiden Strukturfragmente drei potentielle Ringsysteme, nämlich $\alpha$-Pyridon 62, $\alpha$-Pyron 63 und $\alpha$-Pyridin 71 (vgl. Schema 13), an. Die Grundstruktur der genannten Ringsysteme ist bereits aufgrund der wissenschaftlichen und synthetischen Erforschung ähnlicher Verbindungen Gegenstand zahlreicher Publikationen. ${ }^{[20]}$

Alkylpyridine wie die Verbindung 71 sind unter anderem befähigt, Reaktionen wie Seitenketten-Halogenierungen und oxidative Funktionalisierungen einzugehen und weisen eine Acidität der direkt zum Heterocyclus benachbarten C-H-Bindungen auf, die in 2-, 4- und 6-Stellung stärker ausgeprägt ist als in 3- bzw. 5-Stellung (vgl. Schema 13). Die leichte Deprotonierbar- 
keit an der 2-, 4- und 6-Position wird durch die Mesomerie-Stabilisierung des entsprechenden Carbanions unter Beteiligung des Ringstickstoffs hervorgerufen. ${ }^{[21]}$ Dementsprechend dürfte das Alkylpyridin $\mathbf{7 1}$ trotz des Vorhandenseins mehrerer Alkylgruppen am Ring bevorzugt an der Methylgruppe der C-6-Position basenvermittelte Reaktionen eingehen ${ }^{[21]}$ und wird daher als eines der potentiellen Ringsysteme für die Darstellung des Iromycins in Betracht gezogen. Das Alkylpyridin $71(\mathrm{R}=\mathrm{Ac})$ ist durch zweifache Acylierung aus dem Pyridon 62 darstellbar.

Schema 13. Retrosynthetische Analyse des Iromycins.<smiles>[R9]c1nc(CN=C(C)CC=CC([R])(C)C)c(CCC)c([R])c1C</smiles><smiles>[R]C(C)(C)/C=C/C/C(C)=C/Cc1[nH]c(=O)c(C)c(O)c1CCCCCCCCCC</smiles><smiles>[X]Cc1nc([R20])c(C)c([R])c1CCC</smiles><smiles>[X]Cc1[nH]c(=O)c(C)c(O)c1CCC</smiles><smiles>[M]C=C(C)CC=CC([R])(C)C</smiles><smiles>[Y]CCCc1c(C)nc([R20])c(C)c1O[R]</smiles><smiles>C1=CCC=C1</smiles><smiles>CCCc1c(C)[nH]c(=O)c(C)c1O</smiles><smiles>[R]C(C)(C)/C=C/CC#C</smiles>

乃<smiles>CCCc1c(C)oc(=O)c(C)c1O</smiles><smiles>[X]CC=CC([R])(C)C</smiles>

67

$\sqrt{2}$

$\mathrm{M}=\mathrm{Al}, \mathrm{Mg}$

$\mathrm{X}=\mathrm{Br}, \mathrm{Cl}$<smiles>CCCC(C(C)=O)C(=O)C(C)C(=O)OCC</smiles><smiles>[M]C=C</smiles><smiles>[R]C(C)(C)C=O</smiles>

68 
Durch ihre spezielle 4-Hydroxy-Gruppe sind Pyridone $\left(p K_{s}=11\right)$ und Pyrone $\left(p K_{s}=14\right)$ schwache Säuren. Die physikalischen und chemischen Eigenschaften des Pyridons und des Pyrons entsprechen denen eines ungesättigten Lactams bzw. Lactons. ${ }^{[22]}$ Sie besitzen eine ähnliche elektronische Struktur und zeigen eine Seitenketten-Reaktivität. Die Carbonylgruppe übt durch den -M-Induktiven Effekt einen Einfluß auf die Substituenten am Ring aus. Die Methylgruppen in 6-Position sind daher für eine Funktionalisierung aktiviert und somit leicht für eine Derivatisierung zugänglich. Die Pyridonstruktur und deren O-Analoga sind bereits aus vielen Publikationen bekannt und wurden in zahlreichen Arbeitskreisen ${ }^{[20]}$ in verschiedenen Varianten als Gegenstand der Synthese untersucht und funktionalisiert. Ebenso wurden des öfteren Pyrone aus den entsprechenden Tricarbonylen synthetisiert.

Bei dem Aufbau des Seitenkettenfragmentes wurde die Methylierung des Alkins 36 zum Alken 35 und dessen anschließende metallkatalysierte Kreuzkupplung mit dem Ringsystem unter Bildung einer C-C-Bindung anvisiert (vgl. Schema 13). Die Auswahl eines aus einem Aldehyd und dem Vinylgrignard-Reagenz 68 darstellbaren Allylhalogenids 67 bestimmt bereits zu Beginn der Synthese die Struktur des Seitenkettenfragmentes und gewährleistet die gewünschte Variabilität des Syntheseweges auch für Naturstoffderivate.

\section{Synthese der Iromycine $A$ und $R$ ausgehend von der Kreuzkupplung von Brom- methylpyron mit Alanaten}

Aufgrund der retrosynthetischen Überlegungen sollte das Iromycin als Zielmolekül über die Kreuzkupplung eines Halogenidderivates des Pyrons 63 und eines Organometallalkenyls als Alkenylalan 65 unter Bildung einer C-C-Bindung synthetisiert werden (vgl. Schema 8). Die Bildung des Alkenylalans $\mathbf{6 5}$ sollte über eine selektive Carboaluminierung des Alkins $\mathbf{6 6}$ herbeigeführt werden. Die Behandlung des Alkenylalans mit $n$ BuLi ergibt das Alkenylalanat, das in einen nucleophilen Angriff unter Verdrängung des Halogenatoms der Seitenkette unter CC-Bindungsbildung an das Hauptgrundgerüst kuppeln sollte. Die Durchführung der Kreuzkupplungsreaktion mit Alanaten erschien gegenüber dem Einsatz von Alanen vorteilhaft, da diese im Vergleich zu den Alanen aufgrund ihres ionischen Charakters eine erhöhte Nucleophilie aufweisen. 


\subsection{Synthese des Ringfragments als 6-Brommethylpyron}

\subsubsection{Synthese des unfunktionalisierten Pyrons}

Zur geplanten Synthese des gewünschten Heptansäureethylesters 84 wurden als Ausgangssubstanzen die kommerziell erhältlichen Verbindungen 2-Methyl-3-oxo-butansäureethylester (83) und Propylbromid bzw. -iodid als Alkylierungsmittel eingesetzt (vgl. Schema 14). Die nucleophile Substitution von $\beta$-Dicarbonylverbindungen in $\gamma$-Position ist eine Reaktion, die von Weiler et al. ${ }^{[23]}$ schon im Jahr 1974 untersucht wurde. Hierzu generierten sie durch zweifache Deprotonierung mit starken Basen das entsprechende Dianion, das bei Zugabe eines Äquivalents eines Alkylierungsmittels mit hoher Selektivität eine Reaktion in der $\gamma$-Position eingeht, da diese aufgrund der schlechteren Stabilisierung der negativen Ladung wesentlich reaktiver ist. Dabei kamen als Basen entweder 2 Äquivalente LDA oder 1 Äquivalent NaH und 1 Äquivalent $n$ BuLi in den Lösungsmitteln THF, DME, Et $t_{2} \mathrm{O}$ oder HMPA zum Einsatz. Entsprechend dieser Arbeiten sollte hier durch nucleophile Substitution das C-4Kohlenstoffgerüst des eingesetzten $\beta$-Ketoesters 83 um drei Kohlenstoffatome verlängert werden. Als Propylierungsmittel wurde zunächst das preisgünstige Propylbromid eingesetzt.

Schema 14. Kettenverlängerung durch Alkylierung des $\beta$-Ketoesters 83 zum $\beta$-Ketoester 84.<smiles>CCOC(=O)C(C)C(C)=O</smiles>

83
1) $2.2 \mathrm{eq}$ LDA, THF $0{ }^{\circ} \mathrm{C}, 30 \mathrm{~min}$

3) $83,0^{\circ} \mathrm{C}, 1 \mathrm{~h}$

2) $1.1 \mathrm{eq} n \mathrm{Prl}, \mathrm{THF}$ $0{ }^{\circ} \mathrm{C}, 2.5 \mathrm{~h}$

$56 \%$

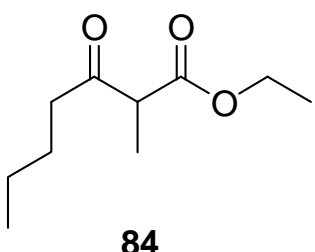

84

Tabelle 1. Alkylierung des $\beta$-Ketoesters 83 zum $\beta$-Ketoester 84.

\begin{tabular}{cccc}
\hline Eintrag & Alkylhalogenid & $\mathrm{t}[\mathrm{h}]$ & Ausbeute 84 $(\%)^{\mathrm{a})}$ \\
\hline 1 & Propylbromid & 0.5 & 25 \\
2 & Propyliodid & 1.5 & 44 \\
3 & Propyliodid & 2.5 & 56 \\
4 & Propyliodid & 2.0 & $84^{\mathrm{b})}$ \\
\hline
\end{tabular}

${ }^{\text {a) }}$ Ausbeuten nach destillativer Reinigung. ${ }^{\text {b) }}$ Rohprodukt. 
Die Generierung von LDA wurde aufgrund der geringeren Polarität des $\mathrm{Et}_{2} \mathrm{O}$, der extremen Toxizität des HMPA und des nachteiligen höheren Siedepunktes von DME in THF vorgenommen. Die Umsetzungen wurden aufgrund der hohen Löslichkeit der Enolverbindungen in $\mathrm{H}_{2} \mathrm{O}$ grundsätzlich mit konz. $\mathrm{HCl}$ hydrolysiert, um eine größere Verdünnung und hierdurch bedingte Verluste bei der Aufarbeitung zu vermeiden. Als Extraktionsmittel wurde stets $\mathrm{Et}_{2} \mathrm{O}$ verwendet, die erhaltenen Rohprodukte wurden fraktionierend destilliert (s. Exp. Teil, Allg. Arbeitsvorschrift A6). Die Umsetzung mit Propylbromid lieferte nach der Aufreinigung das Zielprodukt in einer Ausbeute von 25\% (vgl. Schema 14, Tabelle 1, Eintrag 1). Der Einsatz des Iodids erwies sich gegenüber dem Einsatz des entsprechenden Bromids aufgrund besserer Qualität der Abgangsgruppe als effektiver, da hierdurch die Ausbeute erheblich erhöht werden konnte (vgl. Tabelle 1, Einträge 2-4). Es ergaben sich nach 1.5 h Reaktionszeit 44\% des Zielprodukts und nach $2.5 \mathrm{~h}$ sogar 56\%. Vor der Destillation entsprach die Menge an Rohprodukt in der Regel noch den theoretischen Ausbeuten. Erst bei der destillativen Reinigung wurden größere Massenverluste beobachtet. Da das Rohprodukt praktisch vollständig aus dem gewünschten $\beta$-Ketoester ohne sonstige Nebenprodukte bestand, war für die geringe Ausbeute an Ketoester $\mathbf{8 4}$ die Zersetzung des Produkts während der Destillation verantwortlich. Es wird vermutet, dass entweder das Produkt selbst eine geringe thermische Stabilität aufweist oder dass aufgrund des Einsatzes von konz. $\mathrm{HCl}$ noch Säurespuren im Rohprodukt enthalten waren, was zur Erhöhung der Siedetemperatur oder des Salzcharakters des Rohprodukts geführt haben könnte. Bei langsamer Zugabe von konz. HCl und der Einstellung des pH-Wertes (5-6) konnte dennoch keine signifikante Ausbeuteerhöhung erzielt werden. Daher ist für künftige Arbeiten eine Aufreinigung durch Säulenfiltration empfehlenswert.

Im nächsten Schritt der Synthese sollte 2-Methyl-3-oxo-heptansäureethylester (84) an der C4-Position zu einem $\beta, \delta$-Diketoester (Tricarbonyl) acyliert werden. Müller et al. ${ }^{[24]}$, Harris et al. ${ }^{[25]}$, Weinreb et al. ${ }^{[26]}$ und Hiyama et al. ${ }^{[27]}$ berichteten von exzellenten Ausbeuten beim Einsatz von Weinrebamid als Acylierungsmittel in ähnlichen Umsetzungen. In Anlehnung hieran wurde die Reaktion des Ketoesters 84 mit 2.2 eq LDA als Base und 1.5 eq Weinrebamid in THF bei $-10{ }^{\circ} \mathrm{C}$ durchgeführt (vgl. Tabelle 2, Eintrag 1). Hierbei konnte allerdings auch bei Erhöhung der Temperatur auf Raumtemperatur nach 5 h kein Ester 64 detektiert werden. Hagiwara et al. ${ }^{[28]}$ berichteten von der Anwendung sowohl von Essigsäuremethylester als auch von $\mathrm{N}$-Acetylimidazol als Acylierungsmittel. Die Umsetzung von Dicarbonyl 84 mit Essigsäuremethylester unter Verwendung von LDA erbrachte jedoch nicht das gewünschte Substitutionsprodukt 64 (vgl. Tabelle 2, Eintrag 2). 
Die Acylierung gelang schließlich beim Einsatz von $N$-Acetylimidazol, das in quantitativen Ausbeuten aus Acetylchlorid und Imidazol in THF dargestellt wurde. ${ }^{[29]}$ Hierzu wurde erst das Bisenolat aus LDA und dem $\beta$-Ketoester $\mathbf{8 4}$ generiert und anschließend mit einer Lösung von $N$-Acetylimidazol in THF umgesetzt (vgl. Schema 15, Tabelle 2).

Schema 15. Acylierung des $\beta$-Ketoesters 84 mit $N$-Acetylimidazol zu dem $\beta, \delta$-Diketoester 64.

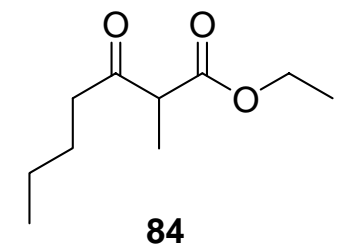
1) $\mathrm{HN}(\mathrm{iPr})_{2}, n \mathrm{BuLi}$ THF, $0^{\circ} \mathrm{C}, 20 \mathrm{~min}$
2) $84, \mathrm{THF}, 0^{\circ} \mathrm{C}, 1 \mathrm{~h}$
3) N-Acetylimidazol THF, $-78^{\circ} \mathrm{C}, 2 \mathrm{~h}$ 64:84 (4.76:1)<smiles>CCCC(C(C)=O)C(=O)C(C)C(=O)OCC</smiles>

Tabelle 2. Versuche zur Darstellung des Heptansäureethylesters 64.

\begin{tabular}{cccccc}
\hline Eintrag & Acylierungsmittel [eq] ${ }^{\mathrm{a})}$ & LDA [eq] & $\mathrm{T}\left[{ }^{\circ} \mathrm{C}\right]$ & $\mathrm{t}[\mathrm{h}]$ & {$[\mathbf{6 4 : 8 4}]^{\mathrm{b})}$} \\
\hline 1 & $\mathrm{~A}(1.5)$ & 2.2 & $-10 \rightarrow \mathrm{RT}$ & 5 & $1: 0$ \\
2 & $\mathrm{~B}(1.05)$ & 2.2 & $-10 \rightarrow \mathrm{RT}$ & 2 & $1: 0$ \\
3 & $\mathrm{C}(1.05)$ & 2.6 & -78 & 2 & $3: 1$ \\
4 & $\mathrm{C}(1.20)$ & 2.6 & -78 & 1 & $2.3: 1$ \\
& & & -40 & 4 & $2: 1$ \\
5 & $\mathrm{C}(1.25)$ & 2.6 & -78 & 2 & $3.2: 1$ \\
6 & $\mathrm{C}(1.50)$ & 2.6 & -78 & 2 & $4.76: 1$ \\
7 & $\mathrm{C}(2.00)$ & 2.6 & -78 & 2 & $1: 1.25$ \\
8 & $\mathrm{C}(1.20)$ & 2.1 & -78 & 2 & $1: 1.4$ \\
9 & $\mathrm{C}(1.50)$ & 2.2 & -78 & 2.5 & $1: 1.6$ \\
10 & $\mathrm{C}(1.2)$ & $\mathrm{c})$ & -78 & 3 & $4.3: 1$ \\
11 & $\mathrm{C}(1.2)$ & $\mathrm{c})$ & -78 & 1 & $4: 1$ \\
\hline
\end{tabular}

a) A: $\mathrm{CH}_{3} \mathrm{CON}\left(\mathrm{CH}_{3}\right) \mathrm{OCH}_{3}, \mathrm{~B}: \mathrm{CH}_{3} \mathrm{CO}_{2} \mathrm{CH}_{3}, \mathrm{C}: N$-Acetylimidazol. b) Verhältnis 1t. ${ }^{1} \mathrm{H}-\mathrm{NMR}$ des Rohprodukts. ${ }^{\mathrm{c})} 1.2$ eq $\mathrm{NaH}$ u. 1.0 eq $n$ BuLi als Basen.

Die Acylierungsreaktion wurde im Rahmen dieser Arbeit bezüglich der Menge an Acylierungsmittel und Base sowie hinsichtlich der Reaktionstemperatur optimiert (s. Exp. Teil, Allg. Arbeitsvorschrift A7). Da sich das Produkt bei allen Aufreinigungsversuchen als sehr zersetzlich erwies, wurde als Maß für die Ausbeute das Verhältnis zwischen dem Edukt 84 und dem Produkt 64 aus den ${ }^{1}$ H-NMR-Spektren des Rohprodukts bestimmt. Die Menge an $N$-Acetylimidazol wurde bei den Versuchen von 1.05 eq bis 2.0 eq variiert (vgl. Tabelle 2). Das beste 
Ergebnis wurde bei dem Versuch mit 1.5 eq Acylierungsmittel erzielt, bei dem nach der Aufarbeitung der Reaktionslösung ein Gemisch aus dem Produkt 64 und dem Edukt 84 im Verhältnis von 4.8:1 isoliert werden konnte (vgl. Tabelle 2, Eintrag 6). Eine Erhöhung der Menge an Acylierungsmittel ergab dagegen einen schlechteren Umsatz. Bei den Versuchen wurden überwiegend 2.6 eq LDA verwendet, da geringere Mengen an Base zu schlechteren Ergebnissen geführt hatten (vgl. Tabelle 2, Einträge 8 u. 9). Als optimal erwies sich eine Reaktionstemperatur von $-78{ }^{\circ} \mathrm{C}$, da durch eine Erhöhung der Temperatur von $-78{ }^{\circ} \mathrm{C}$ auf $-40{ }^{\circ} \mathrm{C}$ kein höherer Umsatz erzielt werden konnte (vgl. Tabelle 2, Eintrag 4).

Sowohl Hagiwara et al. ${ }^{[28]}$ als auch Weiler et al. ${ }^{[23]}$ berichteten von der Anwendung von $\mathrm{NaH}$ und $n \mathrm{BuLi}$ in THF zur Bildung des Dianions. Dieses Basengemisch wurde deshalb verwendet, da die Basizität von NaH nicht für eine zweifache Deprotonierung ausreicht und zudem die Verwendung von $n \mathrm{BuLi}$ im ersten Deprotonierungsschritt nicht möglich ist, weil die Deprotonierung mit der nucleophilen Addition an die Carbonylfunktionen konkurriert. Der Einsatz dieses Basensystems wurde daher bei den Versuchen 10 und 11 untersucht (vgl. Tabelle 2, Einträge 10 u. 11). Die Acylierung mit jeweils 1.2 eq NaH und 1.0 eq $n \mathrm{BuLi}$ in THF in Gegenwart von 1.2 eq $N$-Acetylimidazol bei $-78^{\circ} \mathrm{C}$ führte zunächst zu einem $4.3: 1$ und 4:1-Edukt-Produkt-Verhältnis zugunsten des Tricarbonyls 64. Bei einer Temperaturerhöhung auf $-40{ }^{\circ} \mathrm{C}$ ergab sich jedoch ein vermindertes Verhältnis von 2.5:1 und bei einer Reaktionstemperatur von $0{ }^{\circ} \mathrm{C}$ sogar nur noch ein Verhältnis von 2:1, was auf die Zersetzung des Produktes 64 bei erhöhter Temperatur hindeutet. Daher wurde die Hydrolyse des Reaktionsgemisches mit ges. $\mathrm{NH}_{4} \mathrm{Cl}$-Lösung bei $-78{ }^{\circ} \mathrm{C}$ durchgeführt. Insgesamt ergaben die Versuche mit diesem Basensystem einen hohen Umsatz. Allerdings zeigten größere aliphatische Signale im ${ }^{1}$ H-NMR-Spektrum die Bildung von Nebenprodukten, vermutlich durch Polymerisation, und damit eine geringe Reinheit an, weshalb sich statt $\mathrm{NaH} / n \mathrm{BuLi}$ die Verwendung von LDA als Base empfiehlt. Die erhaltenen Rohprodukte wurde ohne weitere Reinigung im nächsten Syntheseschritt eingesetzt, da die Versuche der säulenchromatographischen wie auch destillativen Reinigung zu erheblichen Verlusten an Rohprodukt führten.

Als nächster Schritt der Synthese sollte das Tricarbonyl 64 in einer Cyclisierungsreaktion unter Verwendung von sterisch anspruchsvollen Basen wie DBU in das gewünschte Pyron 63 überführt werden. Pyrone kommen aufgrund ihres molekularen Aufbaus als Teilstruktur und Baustein der Polyketidderivate in vielen Naturstoffen vor und sind seit längerer Zeit bekannt. Die Darstellung von Pyronverbindungen ist bereits Gegenstand vieler Synthesen gewesen. ${ }^{[30]}$ 
Unter anderem wurde die Cyclisierung zu Pyronen ausgehend von $\beta$-Diketoestern durchgeführt, die durch die Acylierung des Dianions von 1,3-Diketonen dargestellt wurden. ${ }^{[22]} \mathrm{Me}-$ chanistisch verläuft die Pyrondarstellung über eine Deprotonierung von $\beta, \delta$-DiketoesterVerbindungen unter Verwendung von DBU an dem methylenaktiven Kohlenstoffzentrum (C4-Position) unter kinetischen Bedingungen, hier anhand der Tricarbonylverbindung 64 beispielhaft dargestellt (vgl. Schema 16). Dabei wird die "ambidente Nucleophilie" des EnolatIons 85 ausgenutzt. Der Einsatz eines polar aprotischen oder unpolaren Lösungsmittels verstärkt den nucleophilen Angriff des O-Atoms, da das Enolat "nackt" -also völlig unsolvatisiert- vorliegt. ${ }^{[31]}$ Desweiteren wird die Cyclisierung über den Sauerstoff sowohl über das sog. Konzept der harten und weichen Säuren und Basen $(\mathrm{HSAB})^{[31]}$ als auch über die Bildung eines stabilen 6er-Rings begünstigt. Die Hydrolyse des Anions 87 nach Abspaltung eines Protons an der C-3-Position der Spezies 86 durch das Ethanolat liefert dann das gewünschte Pyron 63 .

Schema 16. Mechanismus der Cyclisierung des $\beta, \delta$-Diketoesters 64 zu dem Pyron 63.

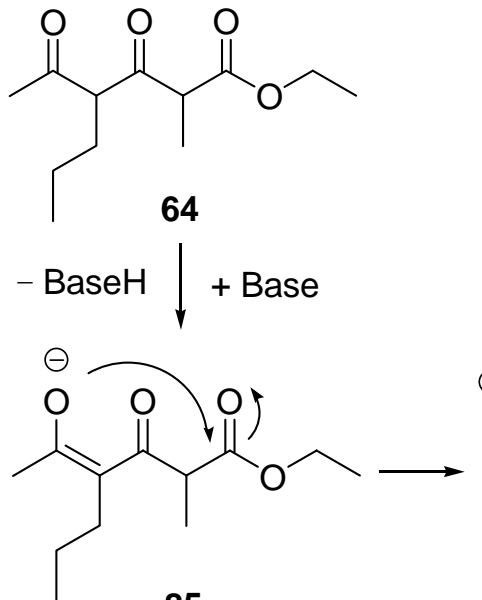

85
$\mathrm{DBU}, \Delta$

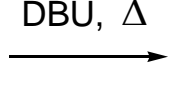<smiles>CCCc1c(C)oc(=O)c(C)c1O</smiles>

63<smiles>[PH3+][IH+]</smiles>

87

Enders et al. ${ }^{[32]}$ und Oppolzer et al. ${ }^{[33]}$ beschrieben die Darstellung entsprechender Pyronderivate. Hierzu verwendeten sie DBU als Base in Toluol unter Rückflussbedingungen bei einer Reaktionszeit von $3 \mathrm{~h}$ bzw. $2 \mathrm{~h}$. Anlehnend an diese Literaturquellen wurde die Umwandlung des $\beta$, $\delta$-Diketoesters 64 zu dem Pyron 63 zunächst in Toluol unter identischen Bedingungen untersucht (vgl. Schema 17, Tabelle 3). 
Schema 17. Cyclisierungsreaktion des $\beta, \delta$-Diketoesters 64 zu dem Pyron 63 in Toluol.

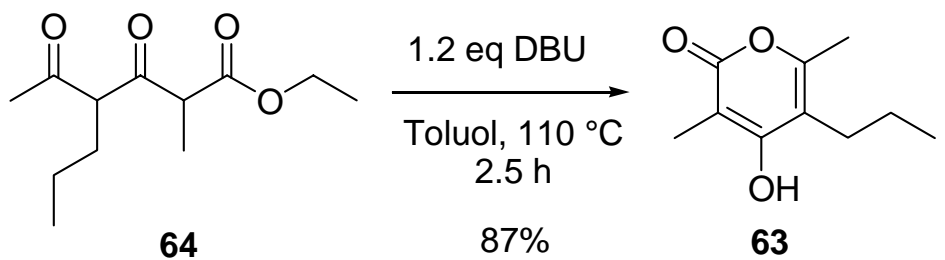

Tabelle 3. Cyclisierungsreaktion des $\beta, \delta$-Diketoesters 64 zum Pyron 63 in Toluol.

\begin{tabular}{ccc}
\hline Eintrag & $\mathbf{6 3}^{\text {a) }}: \mathbf{6 4}^{\text {b) }}$-Verhältnis & Ausbeute $(\%)$ \\
\hline 1 & $0.69: 1$ & 87 \\
2 & $0.65: 1$ & 22 \\
3 & $0.66: 1$ & 39 \\
\hline
\end{tabular}

a) Rohprodukt an Pyron 63. ${ }^{\text {b) }}$ Edukt 64.

Die Reaktionslösungen der ersten beiden Cyclisierungsversuche wurden mit $\mathrm{NH}_{4} \mathrm{Cl}$-Lösung und $\mathrm{Et}_{2} \mathrm{O}$ aufgearbeitet und säulenchromatographisch gereinigt (vgl. Tabelle 3, Einträge $1 \mathrm{u}$. 2). Die Reaktionslösung des Versuchs 3 (vgl. Tabelle 3, Eintrag 3) wurde hingegen erst mit einer ges. $\mathrm{NaHCO}_{3}$-Lösung versetzt $(\mathrm{pH}=9)$ und dann mit $\mathrm{Et}_{2} \mathrm{O}$ extrahiert. Auf diese Weise konnte das Pyron als Salz in die wässrige Phase überführt und hierdurch der $\beta$-Ketoester 84 aus der Reaktionslösung entfernt werden. Der $\beta$-Ketoester 84 stammte sowohl aus der als Gemisch eingesetzten Ausgangssubstanz (diese wurde stets ohne Isolierung als ein Gemisch der Verbindungen 64 und 84 eingesetzt, da Reinigungsversuche bei der Darstellung von Tricarbonyl $64 \mathrm{zu}$ erheblichen Verlusten geführt hatten), als auch aus der eventuellen Zersetzung des $\beta, \delta$-Diketoesters 64 während des Cyclisierungsprozesses. Die verbliebene wässrige Phase (aus der $\mathrm{NaHCO}_{3}$-Phase) wurde mit $1.0 \mathrm{M} \mathrm{HCl}$ auf $\mathrm{pH}=5$ eingestellt und aufgearbeitet. Das erhaltene Rohprodukt wurde säulenchromatographisch gereinigt. Aus dem Vergleich der in der Tabelle 3 aufgeführten Ausbeuten wird ersichtlich, dass die erste Reaktion (Eintrag 1) nicht in vergleichbar hoher Ausbeute reproduziert werden konnte. In Anbetracht der Durchführung der Umsetzungen unter identischen Bedingungen und der Analyse der errechneten Massenverhältnisse des Rohproduktes 63 und der eingesetzten Ausgangsverbindung 64 kann die auffallende Divergenz der isolierten Ausbeuten damit erklärt werden, dass die Umsetzung 3 (8.8 g Edukt) im Gegensatz zu der Reaktion 1 (61 mg Edukt) in viel größerem Maßstab durchgeführt wurde. Desweiteren könnte für die geringe Ausbeute bei den Versuchen 2 und 3 
der geringere tatsächliche Anteil an Tricarbonyl 64 in der Ausgangssubstanz verantwortlich sein, da dieser über das entsprechende Spektrum nur theoretisch errechnet wurde.

Hagiwara et al. ${ }^{[28]}$ berichteten bei der Totalsynthese des Phytotoxins Solanapyron D und E über eine Cyclisierung des $\beta, \delta$-Diketoesters zu Pyron unter Einsatz von DBU in Benzol. Yamamura et al. ${ }^{[34]}$ führten die Cyclisierung eines $\beta, \delta$-Diketoesters bei der Totalsynthese von Phenoxan ebenfalls unter Verwendung von DBU in Benzol durch. Durch den entsprechenden Einsatz des niedrig siedenderen Benzols bei den Cyclisierungsreaktionen des Diketoesters 64 zu dem Pyron 63 konnten die Umsetzungen bei einer gegenüber den Reaktionen mit Toluol $\left(110{ }^{\circ} \mathrm{C}\right)$ schonenderen Temperatur von $90{ }^{\circ} \mathrm{C}$ durchgeführt werden (vgl. Schema 18, Tabelle 4). Die Reaktionszeiten für die vollständigen Umsetzungen betrugen 2-6 h. Das gewünschte Pyron 63 konnte in einer Ausbeute von 80\% dargestellt werden (s. Exp. Teil, Allg. Arbeitsvorschrift A8).

Schema 18. Cyclisierungsreaktion des $\beta, \delta$-Diketoesters 64 zu dem Pyron 63 in Benzol.<smiles>CCCc1c(C)oc(=O)c(C)c1O</smiles>

Tabelle 4. Cyclisierungsreaktion des $\beta, \delta$-Diketoesters 64 zu dem Pyron 63 in Benzol.

\begin{tabular}{|c|c|c|c|c|}
\hline Eintrag & Zeit [h] & Reinigung & Ausbeute $(\%)^{a)}$ & Ausbeute $(\%)^{b)}$ \\
\hline 4 & 2 & $\mathrm{KRD}^{\mathrm{c})}$ & 67 & 47 \\
\hline 5 & 6 & $\mathrm{KRD}^{\mathrm{c})}$ & 78 & 61 \\
\hline 6 & 2 & $\mathrm{KRD}^{\mathrm{c})}$ & 43 & 63 \\
\hline 7 & 6 & $C C^{d)}$ & 80 & 64 \\
\hline 8 & 6 & $\mathrm{CC}^{\mathrm{d})}$ & 71 & 61 \\
\hline 9 & 6 & $\mathrm{CC}^{\mathrm{d})}$ & 70 & 49 \\
\hline 10 & 6 & $\mathrm{CC}^{\mathrm{d})}$ & 75 & 56 \\
\hline
\end{tabular}


Die Versuche zur Reinigung des stark viskosen Pyrons 63 durch Kugelrohrdestillation erwiesen sich als ineffektiv. Es wurden stets Gemische aus dem Pyron 63 und dem Ketoester 84 erhalten. Nach der Aufarbeitung der Reaktion in zwei Testproben mit $\mathrm{NH}_{4} \mathrm{Cl}$ (s. Tabelle 4, Eintrag 4) bzw. $1 \mathrm{M} \mathrm{HCl}$ und ges. $\mathrm{NaHCO}_{3}$-Lösung wurden fast identische Produktmassen von 37.8 und 38.1 mg Pyron als Rohprodukt erhalten. Die auf pH-Einstellung beruhende Aufarbeitungsmethode mit $1 \mathrm{M} \mathrm{HCl}$ und $\mathrm{NaHCO}_{3}$-Lösung erwies sich somit als vorteilhaft (vgl. Tabelle 4, Einträge 5-10). So konnten sowohl das Pyron 63 als auch der in Reaktionslösung verbliebene Diketoester 84 in sehr effizienter Weise isoliert und vorgereinigt werden. Die erhaltenen Rohprodukte des Pyrons 63 konnten so ohne weitere Reinigung umgesetzt werden. Diese effiziente Trennung ist darauf zurückzuführen, dass die 4-OH-Gruppe des Pyrons 63 eine starke Acidität aufweist, was experimentell durch die vollständige Deprotonierung der $\mathrm{OH}-$ Gruppe mit $\mathrm{NaHCO}_{3}$ bestätigt wurde. Die nicht zufriedenstellenden Ausbeuten könnten auf den unterschiedlichen Verunreinigungsgrad des $\beta, \delta$-Diketoesters 64 zurückzuführen sein. Bei Durchführung des Cyclisierungsprozesses in geringerer Lösungskonzentration wurde eine deutliche Ausbeutesteigerung beobachtet, allerdings musste die Reaktionszeit erheblich verlängert werden. Das Pyron fiel bei der Aufbewahrung in hoch konzentrierter Lösung aus Diethylether bei tiefer Temperatur $\left(-32{ }^{\circ} \mathrm{C}\right)$ als weißer Feststoff aus.

\subsubsection{Funktionalisierung des 6-Methylpyrons}

Zur Funktionalisierung des 6-Methylpyrons 63 sollte zunächst die OH-Gruppe aufgrund ihrer hohen Acidität geschützt werden, um für die weiteren Funktionalisierungsschritte eine Umsetzung im basischen Medium zu ermöglichen. Hoffmann et al. ${ }^{[35]}$ berichteten über eine Acetylierung der Pyrone mit Acetylchlorid in Gegenwart von Pyridin in einer Reaktionszeit von 2 h. Die Acylierung des Pyrons 63 wurde im Rahmen dieser Arbeit unter Verwendung von 1.0 eq Acetylchlorid und 1.0 eq Pyridin durchgeführt (vgl. Schema 19, Tabelle 5, Exp. Teil, Allg. Arbeitsvorschrift A9, Variante 2). Eine vollständige Umsetzung konnte im Gegensatz zur Darstellung in der Literatur erst nach einer deutlich längeren Reaktionszeit von $20 \mathrm{~h}$ detektiert werden. Dies könnte auf die geringere Nucleophilie des Pyronium-Ions als Folge der sterischen Hinderung durch die Propylgruppe zurückzuführen sein. Eine Destillation des öligen Rohproduktes war aufgrund des niedrigen Schmelzpunktes erfolglos. Die Acetylierungsprodukte wurden daher säulenchromatographisch gereinigt (vgl. Tabelle 5, Einträge 1 u. 2). Es konnte zwar bei der Reaktion 1 eine Ausbeute von 63\% erzielt werden, jedoch traten bei den 
Reinigungsversuchen zumeist Voll- bzw. Teilentschützungen auf. Darüberhinaus erwies sich das Acetat bei späteren Versuchen zur Reduktion des Acetatcarbaldehyds 92 mit $\mathrm{NaBH}_{4}$ (vgl. Schema 22 u. Tabelle 7) ebenfalls als instabil und damit für die Fortführung des Syntheseweges als ungeeignet. Aufgrunddessen musste das Acetat durch eine stabilere Benzoylschutzgruppe ersetzt werden.

Schema 19. Acylierung des Pyrons 63 zu den Estern 88, 89 und 90.

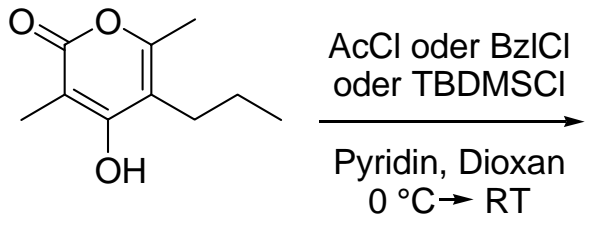

63<smiles>[R]Oc1c(CCC)c(C)oc(=O)c1C</smiles>

$88(R=A c, 63 \%)$

$89(\mathrm{R}=\mathrm{Bzl}, 89 \%)$

$90(\mathrm{R}=\mathrm{TBDMS}, 0 \%)$

Tabelle 5. Acylierung des Pyrons 63 zum Essigsäure- 88 bzw. Benzoesäureester 89.

\begin{tabular}{|c|c|c|c|c|}
\hline Eintrag & Acylierungsmittel & Base & $\mathrm{t}[\mathrm{h}]$ & Ausbeute (\%) \\
\hline $1^{\text {a) }}$ & Acetylchlorid & Pyridin & 20 & 63 \\
\hline $2^{\text {a) }}$ & Acetylchlorid & Pyridin & 18 & 47 \\
\hline $3^{\mathrm{b})}$ & Benzoylchlorid & $\mathrm{NEt}_{3}$ & 5 & 83 \\
\hline $4^{\text {c) }}$ & Benzoylchlorid & Pyridin & 20 & 45 \\
\hline $5^{c)}$ & Benzoylchlorid & Pyridin & 44 & 89 \\
\hline $6^{\mathrm{c}, \mathrm{f})}$ & Benzoylchlorid & Pyridin & 19 & - \\
\hline $7^{\mathrm{d})}$ & TBDMSCl & Pyridin & 126 & 0 \\
\hline $8^{e)}$ & TBDMSCl & Imidazol & 19 & 0 \\
\hline \multicolumn{5}{|c|}{$\begin{array}{l}\text { a) } 1.0 \text { eq } \mathrm{AcCl}, 1.0 \text { eq Pyridin, Dioxan, } 0{ }^{\circ} \mathrm{C} \rightarrow \mathrm{RT} .{ }^{\text {b) }} 1.2 \text { eq Benzoylchlorid, } 1.0 \text { eq } \\
\mathrm{NEt}_{3} \text {, Toluol, } \Delta .{ }^{\text {c) }} 1.1 \text { eq Benzoylchlorid, Pyridin im Überschuß, } 0{ }^{\circ} \mathrm{C} \rightarrow \mathrm{RT} \text {. }{ }^{\mathrm{d})} 2.0 \\
\text { eq TBDMSCl, Pyridin im Überschuß, } 40{ }^{\circ} \mathrm{C}, 15 \mathrm{~h} \text {. }{ }^{\mathrm{e})} 5.0 \text { eq TBDMSCl, } 10 \text { eq Imi- } \\
\text { dazol, RT, } 19 \mathrm{~h} .{ }^{\mathrm{f})} \text { Als Ausgangssubstanz wurde das Diol } 91 \text { eingesetzt. }\end{array}$} \\
\hline
\end{tabular}

Hoffmann et al. ${ }^{[35]}$ und T. Kappe et al. ${ }^{[36]}$ berichteten bei dem Benzoylierungsversuch eines Pyrons (Synthese von Hydroxychinolinen) bzw. eines Pyridons (Synthese von Hydroxycoumarinen) vom Einsatz von Benzoylchlorid in Toluol und $\mathrm{NEt}_{3}$ als Base (Reaktionszeit 5 h, Ausbeute 23-91\%). C. O. Kappe et al. ${ }^{[37]}$ beschrieben hingegen die Synthese des Pyridincarbonitrils und die Schützung der entsprechenden Pyridine unter Zugabe von Pyridin als Base. Sie führten die Reaktion zwar bei einer längeren Reaktionszeit von $12 \mathrm{~h}$ durch, verwendeten 
aber schonendere Reaktionsbedingungen (Raumtemperatur) und erzielten eine Ausbeute von $86 \%$.

Das Pyron 63 wurde unter den in der Tabelle 5 angegebenen Bedingungen mit Benzoylchlorid in $\mathrm{NEt}_{3}$ (Eintrag 3) bzw. Pyridin (Einträge 4-6) acyliert und untersucht. Die Acylierungsreaktion mit $\mathrm{NEt}_{3}$ wurde mit $\mathrm{CH}_{2} \mathrm{Cl}_{2}$ und ges. NaCl-Lösung aufgearbeitet. Trotz der zufriedenstellenden Ausbeute von 83\% nach der säulenchromatographischen Reinigung konnte die zu große Anzahl von Signalen im ${ }^{13} \mathrm{C}$-NMR-Spektrum nicht erklärt werden. Jedes Kohlenstoffatom wies eine Signalverdopplung mit sehr geringem Intensitätsunterschied und chemischer Verschiebung auf. Dies legte aufgrund der Struktur des Moleküls die Vermutung nahe, dass es sich um Keto-Enol-Tautomere handeln könnte. Eine Monoacylierung der CO-Gruppe an der C-2-Position des Ringes konnte über das ${ }^{1} \mathrm{H}-\mathrm{NMR}$-Spektrum nicht bestätigt werden und wird auch aufgrund der Durchführung der Reaktion bei Raumtemperatur für sehr unwahrscheinlich gehalten. Zudem spricht gegen eine Monoacylierung an der CO-Gruppe der Umstand, dass das $\mathrm{sp}^{2}$-O-Atom der Carbonylgruppe eine geringere Nucleophilie als die des $\mathrm{sp}^{3}$-O-Atoms an der C-4-Position des Pyronrings 63 aufweist. Dieser Sachverhalt wurde jedoch nicht näher untersucht. Der Optimierungsversuch mit ges. NaCl-, $1.0 \mathrm{M} \mathrm{KHSO}_{4}$ - und ges. $\mathrm{NaHCO}_{3}-\mathrm{Lö}-$ sung erbrachte nicht den erhofften Effekt (vgl. Tabelle 5, Eintrag 4). Diese Reaktion ergab nur eine Ausbeute von 45\%. Die Verdopplung der Protonenzahl im aromatischen Bereich deutete auf die Bildung eines Nebenproduktes hin. Das durch säulenchromatographische Reinigung isolierte Fraktionsgemisch wurde mit $\mathrm{NaHCO}_{3}$-Lösung und EtOAc aufgearbeitet. Hierdurch konnte das Nebenprodukt als Benzoesäure, das Hydrolyseprodukt des Benzoylchlorids, identifiziert werden. Zudem wurde eine geringe Menge des entschützten Produktes beobachtet, dessen Bildung auf die Aufarbeitung mit $\mathrm{NaHCO}_{3}$-Lösung zurückzuführen sein könnte. Ein Vergleich des ${ }^{13} \mathrm{C}$-NMR-Spektrums des Versuchs 4 (vgl. Tabelle 5, Eintrag 4) mit demjenigen des Versuchs 3 (vgl. Tabelle 5, Eintrag 3) wies ein in hohem Maße ähnliches Strukturmuster auf, was auf einen gleichen Reaktionsverlauf hindeutet. Bei der Reaktion 5 (vgl. Tabelle 5, Eintrag 5) konnten nach Optimierung der Aufarbeitung $\left(\mathrm{NaHCO}_{3} / 1.0 \mathrm{M} \mathrm{HCl}\right)$ das überschüssige Pyridin und das Hydrolyseprodukt Benzoesäure leicht aus dem Reaktionsgemisch entfernt und eine Ausbeute von 89\% erzielt werden (s. Exp. Teil, Allg. Arbeitsvorschrift A9, Variante 1). Aufgrund der geringen Stabilität des Acetatesters 88 (s.o.) wurde der Benzoesäureester 89 vorab auf die Stabilität während des geplanten Reduktionsprozesses untersucht. Der Stabilitätstest mit $\mathrm{NaBH}_{4}$ als Reduktionsmittel ergab eine höhere Stabilität der Benzoylfunktion im Vergleich zur Acetylgruppe. Bei der säulenchromatographischen Reinigung wie auch 
bei der basischen Aufarbeitung wurde allerdings eine partielle Entschützung beobachtet, was zu geringen Ausbeuten führte. Abschließend durchgeführte Schützungsversuche mit TBDMSCl und Imidazol unter den in der Tabelle 5 angegebenen Bedingungen schlugen insgesamt fehl (Einträge 7 u. 8).

Der nächste Schritt der Funktionalisierung war die Oxidation des Benzoesäureesters 89 und des Essigsäureesters 88 an der C-1'-Position (vgl. Schemata 20 u. 21). Hierzu wurde die sog. "Seitenketten-Reaktivität" des Pyrongrundgerüstes ausgenutzt. Der Mesomerie-Effekt der C2-Oxo-Gruppe des Moleküls übt einen Einfluß (-M-Effekt) auf die Substituenten am Ring aus, der die Seitenkette aktiviert und somit eine oxidative Funktionalisierung der Methylgruppe an der 6-Stellung des Rings ermöglicht. Der Oxidationsprozeß liefert den Aldehyd, dem eine Schlüsselfunktion für die weiteren Umfunktionalisierungsvorhaben zukommt. Für die Funktionalisierung der Seitenkette kann Selendioxid $\mathrm{SeO}_{2}$ eingesetzt werden, das gewöhnlich sowohl zur Oxidation von Ketonen und Aldehyden zu $\alpha$-Dicarbonylverbindungen als auch zur Oxidation von Allylkohlenwasserstoffen bzw. Alkylaromaten verwendet wird. Suzuki et al. ${ }^{[38]}$, Hoffmann et al. ${ }^{[35]}$ und Moreno-Maňas et al. ${ }^{[39]}$ verwendeten für ähnliche Oxidationsreaktionen ebenfalls Selenoxid als Oxidationsmittel. Suzuki et al. setzten die Umsetzung in einem Bombenrohr an, erhitzten die Reaktionsmischung $1 \mathrm{~h}$ auf $165{ }^{\circ} \mathrm{C}$ und erhielten ein Gemisch aus einem Aldehyd und dem entsprechenden Alkohol in einem Verhältnis von 65:25\% und einer Ausbeute von 52\%. Hoffmann et al. gelang die oxidative Funktionalisierung nach einer Reaktionszeit von $18 \mathrm{~h}$ bei $170{ }^{\circ} \mathrm{C}$ unter Rückflußbedingungen mit einer Ausbeute von 96\% des Alkohols als Rohprodukt.

Im Rahmen der hier durchgeführten Funktionalisierung wurde die basische Oxidation des Acetatesters 88 zum Aldehyd 92 (vgl. Tabelle 6, Einträge 1-3) unter Einsatz von je 3.0 eq aktiviertem $\mathrm{SeO}_{2}{ }^{[40]}$ bei einer im Vergleich zur Literatur schonenderen Reaktionstemperatur von $110^{\circ} \mathrm{C}$ durchgeführt (s. Exp. Teil, Allg. Arbeitsvorschrift A10). Eine vollständige Umsetzung des Eduktes konnte nach 27 h (Einträge 1 u. 3) bzw. 31 h (Eintrag 2) erreicht werden. Als Produkt des Oxidationsprozesses wurde stets ein Gemisch aus dem Aldehyd 92 und dem Alkohol 93 in quantitativer Menge erhalten. Bei den Umsetzungen 1 und 3 entstand nach jeweils 27 h ein Aldehyd-Alkohol-Gemisch von 2.74:1 bzw. 2.6:1. Der höhere Anteil des Aldehyds 92 im Falle der Reaktion 2 (5.11:1) im Vergleich zu den Versuchen 1 und 3 ist wahrscheinlich auf die längere Reaktionszeit zurückzuführen, in der der gebildete Alkohol 93 als primäres Oxidationsprodukt zum Aldehyd 92 "hochoxidiert" worden sein dürfte. 
Schema 20. Oxidative Funktionalisierung der Seitenkette des Acetats $\mathbf{8 8}$ zu dem Aldehyd $\mathbf{9 2 .}$<smiles>CCCc1c(I)oc(=O)c(C)c1OC(C)=O</smiles>

88<smiles>CCCc1c(C=O)oc(=O)c(C)c1OC(C)=O</smiles>

92<smiles>CCCc1c(CO)oc(=O)c(C)c1OC(C)=O</smiles>

93

Tabelle 6. Oxidation des Essigsäureesters $\mathbf{8 8}$ und des Benzoesäureesters 89 zu den entsprechenden Aldehyden 92 und 94.

\begin{tabular}{cccccc}
\hline Eintrag & Edukt & $\mathrm{SeO}_{2}[\mathrm{eq}]$ & $\mathrm{T}\left[{ }^{\circ} \mathrm{C}\right]$ & $\mathrm{t}[\mathrm{h}]$ & $\mathbf{9 2 : 9 3}$ \\
\hline $1^{\text {a) }}$ & $\mathbf{8 8}$ & 3.0 & 110 & 27 & $2.74: 1$ \\
$2^{\text {a) }}$ & $\mathbf{8 8}$ & 3.0 & 110 & 31 & $5.11: 1$ \\
$3^{\text {a) }}$ & $\mathbf{8 8}$ & 3.0 & 110 & 27 & $2.6: 1$ \\
\hdashline $4^{\text {a) }}$ & $\mathbf{8 9}$ & 8.0 & 110 & 77 & $1: 0$ \\
$5^{\text {b) }}$ & $\mathbf{8 9}$ & 5.0 & 110 & 42 & $1: 0$ \\
$6^{\text {b) }}$ & $\mathbf{8 9}$ & 7.3 & 110 & 4 & $1: 0$ \\
$7^{\text {b) }}$ & $\mathbf{8 9}$ & 5.3 & 130 & 18 & \\
${ }^{\text {a) }}$ Rückfluß. & & 130 & 16 & $1: 0$ \\
\end{tabular}

Die entsprechende Funktionalisierung des Benzoats 89 an der C-1'-Position zum Aldehyd lieferte ausschließlich den Aldehyd 94 als Hauptprodukt (vgl. Schema 21, Tabelle 6, Einträge 4-7; Exp. Teil, Allg. Arbeitsvorschrift A10). Trotz erhöhten Einsatzes von 8.0 bzw. 5.0 eq $\mathrm{SeO}_{2}$ bei den bei $110{ }^{\circ} \mathrm{C}$ durchgeführten Versuchen konnte der vollständige Umsatz zum Aldehyd erst nach erheblich längerer Reaktionszeit von $77 \mathrm{~h}$ und $42 \mathrm{~h}$ erzielt werden (Einträge 4 u. 5). Die Erhöhung der Temperatur auf $130{ }^{\circ} \mathrm{C}$ bei den Umsetzung 6 und 7 führte erwartungsgemäß zu einer Verkürzung der Reaktionszeit auf 18 h bzw. 16 h (Einträge 6 u. 7).

Schema 21. Oxidative Funktionalisierung des Benzoats 89 zu dem Aldehyd 94.<smiles>CCCOc1c(CCC)c(I)oc(=O)c1C</smiles>

89

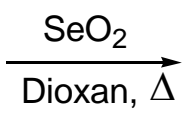

99\%<smiles>CCCc1c(C=O)oc(=O)c(C)c1OCC</smiles>

94 
Ein Vergleich der oxidativen Umsetzungen der Acetate und Benzoate ließ eindeutig auf eine geringere Seitenketten-Reaktivität der Benzoate schließen. Dies wird sowohl durch den erhöhten Einsatz von $\mathrm{SeO}_{2}$ als auch durch die erheblich längere Reaktionszeit bei den Umsetzungen mit dem Benzoat 89 gestützt. Ausschlaggebend für die Reaktionszeit dürfte hauptsächlich die Aktivierungsenergie des Umfunktionalisierungsprozesses sein. Dies wird durch den Vergleich der Einträge 4 und 5 mit 6 bzw. 7 ersichtlich (vgl. Tabelle 6). Die Temperaturerhöhung um $20^{\circ} \mathrm{C}$ bewirkte somit eine um 2.5-4.5-fache Erhöhung der Umsatzgeschwindigkeit. Für die langsamere Umsetzung des Benzoates 89 zum Aldehyd 94 im Vergleich zu der Oxidation der Acetate dürfte die Benzoylschutzfunktion verantwortlich sein. Die Verlängerung der Reaktionszeit könnte durch den $\pi$-mesomeren Substitutionseffekt der Benzoylfunktion, die Koordination der elektrophilen Se-Verbindung am Phenylring mit der Folge einer Destabilisierung des Übergangszustandes sowie durch die sterische Hinderung des Phenylringes hervorgerufen werden. Für das Ausbleiben der Alkohole (vgl. Tabelle 6, Einträge 1-3) bei der Umfunktionalisierung der Benzoate (Einträge 4-7) kann vermutlich der erhöhte Einsatz von Selendioxid verantwortlich gemacht werden, da der primär gebildete Alkohol als das reaktivere Oxidationsprodukt erst in einem zweiten elektrophilen Angriff des Selenoxids in die höher oxidierte Stufe zum Aldehyd umgewandelt wird.

Die Reduktion des aus der Oxidation mit $\mathrm{SeO}_{2}$ erhaltenen Aldehydgemisches (s.o.) sollte durch Umsetzung mit $\mathrm{NaBH}_{4}$ erreicht werden, um im anschließenden Funktionalisierungsschritt ausschließlich den Alkohol 93 als Edukt einsetzen zu können. McGarry et al. ${ }^{[41]}$ berichteten von der Reduktion eines allylischen Formylesters mit $\mathrm{NaBH}_{4}$. Moreno-Maňas et al. ${ }^{[39]}$ reduzierten ebenfalls ein 4-Methoxy-6-methyl-2-pyron erfolgreich mit $\mathrm{NaBH}_{4}$. Die Reduktion des Aldehyd-Alkohol-Gemisches 92 und 93 zum Erhalt des Alkohols 93 (vgl. Tabelle 6, Einträge 1-3) wurde mit $\mathrm{NaBH}_{4}$ durchgeführt (s. Experimenteller Teil, Allgemeine Arbeitsvorschrift A11). Bei allen Umsetzungen wurde der unerwünschte entschützte Pyronalkohol 91 (vgl. Schema 22, Tabelle 7, Einträge 1-3) erhalten. Die Aufarbeitung der reduktiven Umsetzung sowohl mit einem konz. $\mathrm{HCl} / \mathrm{EtOH}-G e m i s c h$ im 1:1-Verhältnis als auch mit ges. $\mathrm{NH}_{4} \mathrm{Cl}-$ Lösung lieferte das entschützte Diol 91 als Hauptprodukt sowie in geringem Anteil die dehydroxylierte Verbindung 63 als Nebenprodukt. Testreaktionen der Acetate sowohl in basischem $\left(\mathrm{NH}_{4} \mathrm{OH}\right.$-Lösung und $\left.\mathrm{NaBH}_{4}\right)$ wie auch in saurem Medium zeigten die besondere Instabilität der Schutzgruppe an. 
Schema 22. Versuche zur Reduktion des Aldehyd-Alkohol-Gemisches 92 u. 93.

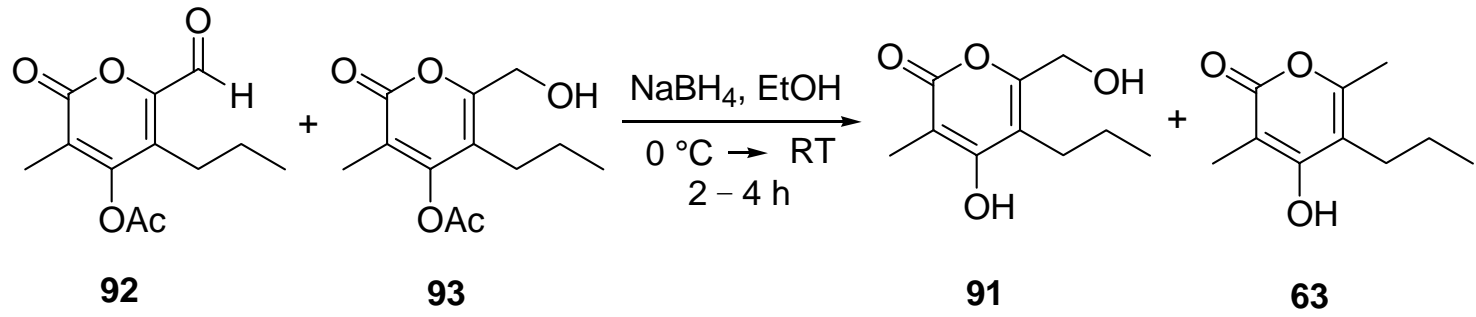

Tabelle 7. Versuche zur Darstellung der Pyronalkohole 93 u. 95.

\begin{tabular}{cccc}
\hline Eintrag & Lösungsmittel. & $\mathrm{t}[\mathrm{h}]$ & Massenbilanz 95 (\%) \\
\hline 1 & EtOH & 2.5 & - \\
2 & EtOH & 2.5 & - \\
3 & EtOH & 4.5 & - \\
4 & EtOH & 4.0 & 91 \\
5 & EtOH & 2.5 & 82 \\
6 & EtOH & 2.0 & 70 \\
$7^{\text {a) }}$ & $\mathrm{MeOH}$ & 0.5 & 41 \\
\hline a) 1.2 eq $\mathrm{NaBH}_{4}, 2.4$ eq $\mathrm{KH}_{2} \mathrm{PO}_{4}, \mathrm{MeOH}, 0^{\circ} \mathrm{C}$.
\end{tabular}

Die entsprechenden Benzoate 94 erwiesen sich in den Reduktionsversuchen unter den angegebenen Bedingungen als stabiler (vgl. Schema 23). Es konnte der Alkohol 95 erfolgreich in einer Ausbeute von 91\% isoliert werden (vgl. Tabelle 7, Einträge 4-7, s. Exp. Teil, Allg. Arbeitsvorschrift A11).

Schema 23. Reduktion des Aldehyds 94 zu dem Alkohol 95.<smiles>CCCc1c(C=O)oc(=O)c(C)c1OCC</smiles>

94<smiles>CCOC(=O)ON(CC)c1ccccc1</smiles>

$91 \%$<smiles>CCCc1c(CO)oc(=O)c(C)c1OCC</smiles>

95 
Die höhere Stabilität der Benzoylfunktion im Vergleich zur Acetylfunktion konnte bei einer Umsetzung des unfunktionalisierten Benzoats 89 in einer Testreaktion mit $\mathrm{NaBH}_{4}$ bestätigt werden. Die Säurestabilität belegte auch die Aufarbeitung einer Probe mit konz. HCl/EtOHGemisch im 1:1-Verhältnis, bei der keine Entschützung detektiert wurde. Der aus den Reduktionsversuchen erhaltene Alkohol 95 (Einträge 4-6) konnte aufgrund seiner sehr sauberen Qualität und der geringen Verunreinigung des Rohproduktes grundsätzlich ohne weitere Aufreinigung umgesetzt werden.

Eine weitere nach Bhattacharya et al. ${ }^{[42]}$ durchgeführte reduktive Umsetzung des Aldehyds 94 mit $\mathrm{NaBH}_{4}$ in Gegenwart von $\mathrm{KH}_{2} \mathrm{PO}_{4}$ ergab ein Gemisch aus dem Alkohol 95 und dem unerwünschten Diol 91 im Verhältnis von 2:1 (vgl. Tabelle 7, Eintrag 7). Nach säulenchromatographischer Reinigung wurde die Verbindung 95 in einer Ausbeute von 41\% isoliert. Die moderate Ausbeute an dem Alkohol 95 wird neben dem Massenverlust, der aus der Bildung des Diols 91 herrührt, unter anderem auch der stetigen Entschützung des Alkohols zum Diol 91 während der sauren Aufreinigung (Kieselgel) angelastet. Umkristallisationsversuche mit heißem Essigsäureester führten zu einer Vergrößerung des Anteils an dem Diol 91 bis hin zur vollständigen Debenzoylierung. Da die reduktiven Umsetzungen qualitativ wie auch quantitativ exzellent verliefen, wurde auf eine Aufreinigung verzichtet.

Als letzter Schritt der Funktionalisierung sollte der Alkohol 95 in das Brommethylpyron 96 umgewandelt werden. Das Bromid 96 stellt die Schlüsselkomponente der nucleophilen Substitutionsreaktion der C-C-Kreuzkupplung mit Alkenylalanaten dar (vgl. Schema 24). Hoffmann et al. ${ }^{[35]}$ berichteten von der Bromierung eines ähnlichen Pyronalkohols mit 1.1 eq $\mathrm{PBr}_{3}$ in $\mathrm{Et}_{2} \mathrm{O}$ bei $40{ }^{\circ} \mathrm{C}$ und einer Ausbeute von $84 \%$. In Abweichung der Vorgaben von Hoffmann wurden die entsprechenden Umwandlungen des Hydroxybenzoates 95 aufgrund dessen geringer Löslichkeit in $\mathrm{Et}_{2} \mathrm{O}$ in polar aprotischem Dioxan durchgeführt (s. Exp. Teil, Allg. Arbeitsvorschrift A12, Variante 1).

Schema 24. Nucleophile Substitution des Benzoatalkohols 95 zu dem Brommethylpyron 96.

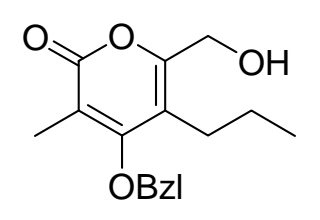

95

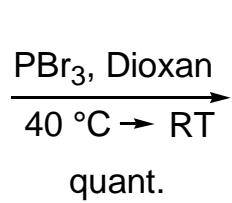

quant.<smiles>[Z17]Oc1c(CCC)c(CBr)oc(=O)c1C</smiles>

96 
Durch die Umfunktionalisierungsversuche wurde das Bromid 96 quantitiv als Rohprodukt in sehr hohem Reinheitsgrad erhalten (vgl. Tabelle 8). Bei dem Versuch der säulenchromatographischen Reinigung wurden stets Spuren des Entschützungsprodukts 97 (Schema 25) und geringe Mengen des Alkohols 95 beobachtet, welches auf Feuchtigkeitsspuren im Kieselgel zurückzuführen ist. Die Umsetzungen verliefen im Allgemeinen komplikationslos und in exzellenter Ausbeute und Qualität, so dass eine weitere Reinigung nicht notwendig war. Umkristallisationsversuche aus EtOAc waren nicht erfolgreich.

Tabelle 8. Bromierung der Alkohole 95 u. 91 zu den Bromiden 96 u. 97.

\begin{tabular}{cccc}
\hline Eintrag & $\mathrm{PBr}_{3}[\mathrm{eq}]$ & $\mathrm{t}[\mathrm{h}]$ & Ausbeute $(\%)$ \\
\hline 1 & 1.1 & 20 & quant. \\
2 & 1.1 & 15 & quant. \\
3 & 1.1 & 15 & 70 \\
4 & 1.1 & 22 & 37 \\
5 & 1.9 & 18 & 50 \\
\hline
\end{tabular}

Schema 25. Nucleophile Substitution des Diols 91 zu dem Bromid 97.<smiles>CCCc1c(CO)oc(=O)c(C)c1O</smiles>

91

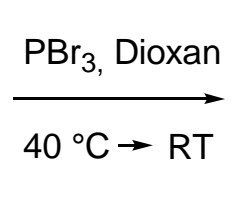
.<smiles>CCCc1c(CBr)oc(=O)c(C)c1O</smiles>

97

Der bei den reduktiven Hydrierungsreaktionen mit $\mathrm{NaBH}_{4}$ entschützte Pyronalkohol 91 (vgl. Tabelle 7, Einträge 1-3) wurde unter identischen Bedingungen mit 1.1 eq bzw. 1.9 eq $\mathrm{PBr}_{3}$ bromiert (vgl. Tabelle 8, Einträge 4 u. 5). Das ${ }^{1}$ H-NMR des Rohproduktes des Bromids 97 (Eintrag 5) zeigte zwei Verbindungen. Die auffällige Parallelstellung der chemischen Verschiebung der entsprechenden Signalmuster im Spektrum weiter ins Tieffeld wies auf unterschiedlich polare Verbindungen hin. Das Singulettsignal bei 4.45 bestätigte die Bildung des Bromids 97. Das Spektrum der mit 1.1 eq $\mathrm{PBr}_{3}$ durchgeführten Reaktion (Eintrag 4) enthielt ebenfalls die gleichen Signalmuster, war jedoch aufgrund erhöhter Verunreinigung schlecht auswertbar. Nach säulenchromatographischer Reinigung wurde das Bromid 97 in einer Ausbeute von bis zu 50\% (Eintrag 5) erhalten. 
Nachfolgend soll versucht werden, die geringe Ausbeute des Bromids 97 über eine Betrachtung der Reaktionsmechanismus zu klären. Der Alkohol 91 reagiert zunächst mit Phosphortribromid unter Ausbildung einer protonierten anorganischen Esterverbindung, die als Derivat der phosphorigen Säure aufgefaßt werden kann. Die $\mathrm{OPBr}_{2}{ }^{-}$-Gruppe ist eine gute Abgangsgruppe und wird durch einen elektrophilen Angriff des freien Bromids verdrängt. Die gesteigerte Elektrophilie des $\mathrm{HOPBr}_{2}$ erhöht die statistische Wahrscheinlichkeit eines nucleophilen Angriffs des freien Bromidanions auf die vinylische Hydroxyfunktion der Bromidzwischenstufe, obwohl an sich die vinylische Hydroxyfunktion (C-4-Position) gegenüber der allylischen Hydroxylfunktion (C-1'-Position) weniger reaktiv ist. Die OH-Funktion des Pyrongrundgerüstes wird dadurch an der C-4-Position in einer Konkurrenzreaktion phosphoryliert. Die nun gebildete Sauerstoffbindung des Dihalogenphosphinsäurederivats (-OPOBr am Ring ist kürzer und stabiler als die der analogen vinylischen $\mathrm{OH}-\mathrm{Gruppen}$. Durch die Aufarbeitung der Reaktionen mit ges. $\mathrm{NaHCO}_{3}$-Lösungen wurden daher sowohl das Bromid 97 (Schema 25, Tabelle 8, Einträge 4 u. 5) als auch das an der C-4-Position phosphorylierte Bromid erhalten, wodurch die oben erwähnte parallele chemische Verschiebung der Protonensignale erklärt werden kann. Die Aufarbeitung einer Probe der Umsetzung 5 lieferte den Nachweis dieser Hypothese. Durch die Aufarbeitung mit konz. HCl wurde die Phosphorsäureesterfunktion als sehr gute Abgangsgruppe abgespalten und durch eine OH-Gruppe ersetzt. Das entsprechende Spektrum zeigte nun lediglich die erwarteten Protonensignale. Der Einsatz von 1.9 eq $\mathrm{PBr}_{3}$ im Vergleich zu 1.1 eq bei dem Versuch 4 erklärt die erhöhte Ausbeute des Bromids bei dem Versuch 5. Die isolierten Bromide wurden benzoyliert und weiter umgesetzt.

Die Benzoylierung des Diols 91, welches aus der reduktiven Umsetzung eines Gemisches von Aldehyd 92 und Alkohol 93 mit $\mathrm{NaBH}_{4}$ resultierte (vgl. Schema 22, Tabelle 5, Eintrag 8), führte aufgrund der starken Säureempfindlichkeit des Diols 91 zur Abspaltung der Hydroxylfunktion. Nach der Aufarbeitung mit ges. $\mathrm{NH}_{4} \mathrm{Cl}$-Lösung wurde nur das dehydroxylierte Edukt 89 erhalten (Schema 26). Das Rohprodukt 89 wurde anschließend ohne weitere Aufreinigung in einer Oxidationsreaktion zu dem Aldehyd 94 (vgl. Schema 21) umfunktionalisiert.

Schema 26. Benzoylierung des durch Reduktion mit $\mathrm{NaBH}_{4}$ gebildeten Diols 91.

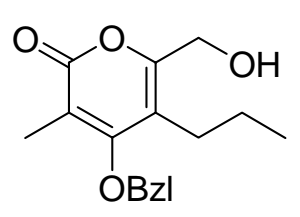

95

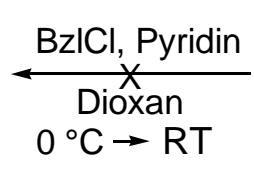

$0^{\circ} \mathrm{C} \rightarrow \mathrm{RT}$

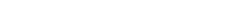

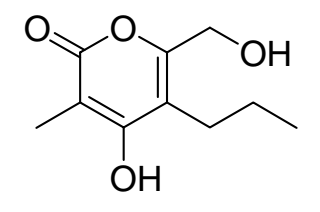

91

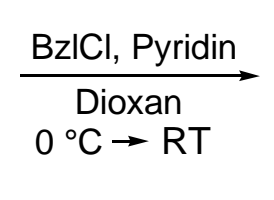

36 


\subsubsection{Synthese des $\alpha$-Chlormethylpyrons 135}

Da für spätere Kupplungen mit der Seitenkette auch die entsprechenden Chloride verwendet werden sollten, wurde die Konvertierung des Brommethylpyrons 96 zu dem korrespondierenden Chlorid 135 (vgl. Schema 27) nach Oae et al. ${ }^{[43]}$ mit wasserfreiem Tetraethylammoniumchlorid $\mathrm{NEt}_{4} \mathrm{Cl}$ durchgeführt. Das gewünschte Chlormethylpyron 135 konnte nach Aufarbeitung mit einer sehr guten Ausbeute von 91\% (vgl. Tabelle 9, Eintrag 1) erhalten werden. Der Chlorierungsversuch des Bromids 96 bei Raumtemperatur lieferte eine exzellente Ausbeute des Chlorids 135 ((Eintrag 2: $>99 \%)$. Aufgrund der ausgezeichneten Qualität $\left({ }^{1} \mathrm{H}-\right.$ und ${ }^{13} \mathrm{C}$ NMR) des Chlorids 135 aus den Versuchen 1 und 2 wurden die isolierten Rohprodukte ohne weitere Reinigung umgesetzt (s. Exp. Teil, Allg. Arbeitsvorschrift A12, Variante 3).

Schema 27. Umfunktionalisierung des Brommethylpyrons 96 zu dem Chlorid 135.

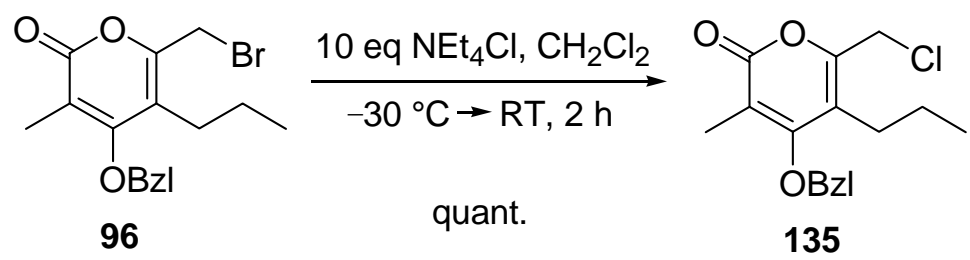

Tabelle 9. Umfunktionalisierung des Brommethylpyrons 96 zu dem Chlorid 135.

\begin{tabular}{cccccc}
\hline Eintrag & Chlorierungsmittel & {$[\mathrm{eq}]$} & $\mathrm{T}\left[{ }^{\circ} \mathrm{C}\right]$ & $\mathrm{t}[\mathrm{h}]$ & Ausbeute $\mathbf{1 3 5}(\%)$ \\
\hline 1 & $\mathrm{NEt}_{4} \mathrm{Cl}$ & 10 & $-30 \rightarrow \mathrm{RT}$ & 2 & 91 \\
2 & $\mathrm{NEt}_{4} \mathrm{Cl}$ & 10 & $\mathrm{RT}$ & 1 & $>99$ \\
3 & $\mathrm{SnCl}_{4}$ & 3 & $-30 \rightarrow \mathrm{RT}$ & 2 & 0 \\
\hline
\end{tabular}

Desweiteren wurde ein Chlorierungsversuch mit $\mathrm{SnCl}_{4}$ nach Ashby et al. ${ }^{[44]}$ durchgeführt, der aber nicht erfolgreich verlief (vgl. Tabelle 9, Eintrag 3). Entsprechend den von Ashby angegebenen Reaktionsbedingungen wurde im Rahmen der Chloridsynthese eine Lösung des Brommethylpyrons 96 in trockenem $\mathrm{CH}_{2} \mathrm{CH}_{2}$ bei $-30{ }^{\circ} \mathrm{C}$ mit wasserfreiem $\mathrm{SnCl}_{4}$ umgesetzt. Hierbei wurde das eingesetzte Brommethylpyron 96 unumgesetzt in vollem Umfang zurückerhalten. 


\subsubsection{Synthese der Seitenkette}

Für die Darstellung der zur Seitenkettensynthese der Iromycine benötigten Alkine 103A, 103R und 116 wurden im Einklang zu dem eingangs dargestellten retrosynthetischen Aufbauschema (vgl. Schema 13) die kommerziell erhältlichen Substanzen isoButyraldehyd für Iromycin A, Pivalinaldehyd für Iromycin R, Cinnamylbromid 114 (Schema 29) sowie das von J. Siewert aus dem hiesigen Arbeitskreis dargestellte 4-(E)-Methyl-hepta-1,4-dien-3-ol (119) (Schema 30) eingesetzt.

Die Darstellung der Allylalkohole 99A und 99R nach Kaga et al. ${ }^{[45]}$ gelang bei Verwendung von 1.05 eq Ethenylmagnesiumbromid in einer exzellenten Ausbeute von 99\% für 99A und in einer zufriedenstellenden Ausbeute von 65\% für 99R (vgl. Schema 28, Exp. Teil, Allg. Arbeitsvorschrift A1). Die trotz der grundsätzlich gut verlaufenden Umsetzungen entstandene Ausbeuteminderung im Falle des Alkohols 99R wurde einer schlechten Qualität der Vinylgrignardlösung zugeschrieben.

Schema 28. Syntheseschritte der Seitenketten 103A und 103R.

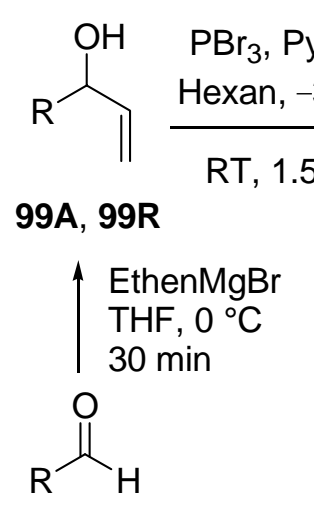

98A, 98R

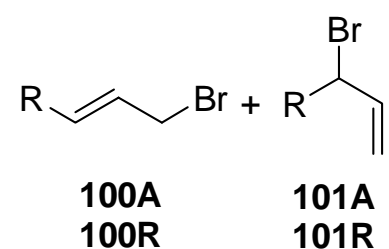

98A R $=\mathrm{CH}\left(\mathrm{CH}_{3}\right)_{2}$ für Iromycin $\mathrm{A}$ 98R $\mathrm{R}=\left(\mathrm{CH}_{3}\right)_{3}$ für Iromycin $\mathrm{R}$ $2.5 \mathrm{~h}$
1) $\mathrm{TMS}=$ EthylMgBr THF, RT, $3 \mathrm{~h} R$

2) 100,101 , CuCN, THF, 102A, 102R $-10^{\circ} \mathrm{C}, \mathrm{RT}$, $\mathrm{NaOH}$ $\mathrm{MeOH}$ $\mathrm{RT}, 4 \mathrm{~h}$<smiles>[R]C=CCC#C</smiles>
103A, 103R

Die Bildung der Allylbromid-Gemische 100A bzw. 101A (32\%) und 100R bzw. 101R (55\%) gelang mit 0.5 eq $\mathrm{PBr}_{3}$ und 0.4 eq Pyridin in Hexan bei $-30{ }^{\circ} \mathrm{C}$ nach Vorgaben von Bloodworth et al. ${ }^{[46]}$ und Hoffmann et al. ${ }^{[47]}$ (vgl. Schema 28, Exp. Teil, Allg. Arbeitsvorschrift A2). Es wurde ein Gemisch der Bromide 100A und 101A (3.7:1) bzw. 100R (fast ausschließlich) und 101R (Spuren) als Produkte von $\mathrm{S}_{N^{\prime}}$ - und $\mathrm{S}_{N}$-Reaktionen erhalten. Trotz einer fraktionie- 
renden Destillation konnte keine Trennung der Strukturisomere erreicht werden. Die Bildung eines höheren Produktanteils aus der langsamer verlaufenden $S_{N}$-Substitutionsreaktion 100A gegenüber der deutlich schneller verlaufenden $\mathrm{S}_{N^{-}}$-Reaktion 101A ist zwar verwunderlich, angesichts der Lage der Protonen im ${ }^{1} \mathrm{H}-\mathrm{NMR}-$ Spektrum jedoch sicher. Die Auswertung des Spektrums ergab für das 4-H-Proton des Verbindungsgemisches (4-H der Isobutylgruppe) je ein Multiplett, das bei dem Bromid 100A aufgrund der allylischen Position und der Nachbarschaft zur Doppelbindung um 0.4 ppm weiter ins Tieffeld verschoben war. In der Literatur ${ }^{[48][49]}$ wurde ebenfalls von der Darstellung des Bromids in einem Verhältnis von 4:1 zugunsten des bekannten Produktes 100A berichtet.

Das Gemisch der Bromide wurde zum Zweck der Alkinylierung in einem Cu-katalysierten Verfahren zu den Alkinen 102A und 102R umgesetzt. Hierbei wird in einem Ummetallierungsprozeß das terminale Alkin von $\mathrm{Mg}$ auf $\mathrm{Cu}$ übertragen. Es bildet sich das Kupferacetylid, das dann mit den Bromiden eine allylische Substitution eingeht. Sato et al. ${ }^{[50]}$ setzten bei $10{ }^{\circ} \mathrm{C}$ eine Lösung von Trimethylsilylethinylmagnesiumbromid in THF zu einer Suspension aus $\mathrm{CuBr}$ und Allylbromid in THF ein, rührten die Reaktion $1 \mathrm{~h}$ bei $0{ }^{\circ} \mathrm{C}$ und anschließend 1 $\mathrm{h}$ bei Raumtemperatur (Ausbeute 82\%). Analog hierzu wurde im Rahmen der Seitenkettensynthese Trimethylsilylacetylen in THF bei Raumtemperatur mit Alkylmagnesiumbromid als Base deprotoniert. Eine Lösung von vorgeneriertem Trimethylsilylethinylmagnesiumbromid wurde jeweils zu entsprechenden Suspensionen aus den Allylbromidgemischen 100A und 101A, 100R und 101R bzw. Cinnamylbromid 114 (vgl. Schema 29) und CuCN zugegeben (s. Exp. Teil, Allg. Arbeitsvorschrift A3). Die Reaktionen verliefen ohne nennenswerte Probleme und wurden nach der Aufarbeitung mittels einer Kieselgelfiltration gereinigt. Die Umsetzungen lieferten trans-selektiv Trimethylsilyl-geschützte Enine in Ausbeuten von 95\% (102A), 53\% (102R) und 92\% (115).

Normant et al. ${ }^{[51]}$ und Grimaldi et al. ${ }^{[52]}$ führten TMS-Entschützungsversuche strukturverwandter Verbindungen unter basischer Behandlung der Alkinylsilane mit einer NaOH-Lösung durch. Die Lösungen der TMS-Enine 102A, 102R und 115 wurden nach Vorgaben von Normant mit NaOH-Lösung desilyliert (s. Exp. Teil, Allg. Arbeitsvorschrift A4, Variante 1). Nach Destillation über eine Vigreuxkolonne konnten die gewünschten Enine 103A (99\%) und 103R (59\%) isoliert werden (vgl. Schema 28). Die Desilylierung des phenylischen Enins 115 (vgl. Tabelle 10, Eintrag 1) ergab nach destillativer Abtrennung des Lösungsmittels ein intensiv orangefarbenes, viskoses Rohproduktgemisch aus Feststoff und Flüssigkeit. Die Umkon- 
densation zur Trennung der Strukturisomere war erfolglos. Die analytische Auswertung zeigte ein Gemisch des gewünschten Enins 116 (39\%) und des unerwünschten Allens (E)-Penta1,3,4-trienylbenzol (118, 61\%) im Verhältnis 1:1.55 (vgl. Schema 29, Tabelle 10). Durch Charakterisierung einer Testprobe nach säulenchromatographischer Reinigung konnte die Bildung des Allens bestätigt werden. Auch Grimaldi et al. ${ }^{[52]}$ hatten die Bildung der Allene ausgehend von alkylsubstituierten Eninen bestätigt. Die Bildung des Allens kann durch die basenvermittelte Deprotonierung des aciden Protons an der allylischen Position der Spezies 117 erklärt werden (vgl. Schema 29).

Schema 29. Synthese des arylsubstituierten Alkins 116 ausgehend von Cinnamylbromid 114.<smiles>BrC/C=C/c1ccccc1</smiles>

114

$$
\begin{aligned}
& \text { 1) } \mathrm{TMS}-\overline{\overline{\bar{N}}}, \mathrm{EtMgBr} \\
& \mathrm{THF}, \mathrm{RT}, 3 \mathrm{~h} \\
& \hline \text { 2) } \begin{array}{c}
114, \mathrm{CuCN}, \mathrm{THF}, \\
-10^{\circ} \mathrm{C}, \mathrm{RT}, 2.5 \mathrm{~h}
\end{array}
\end{aligned}
$$

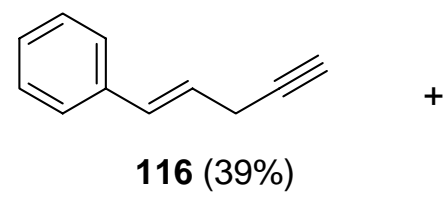<smiles>[AsH3][14C]#C/C=C/c1ccccc1</smiles>

115<smiles>C=C=C/C=C/c1ccccc1</smiles>

$118(61 \%)$

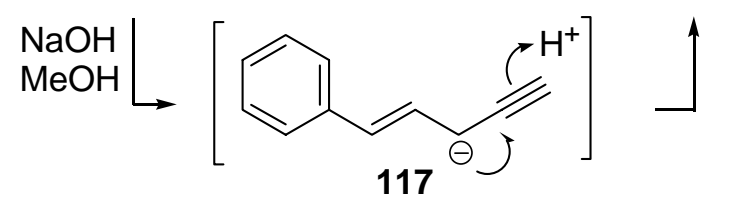

$115 \underset{\mathrm{THF}, 0{ }^{\circ} \mathrm{C}, 4 \mathrm{~h}}{\stackrel{2.0 \text { eq TBAF }}{\longrightarrow}} \quad 116(16 \%)+\quad 118(84 \%)$

$115 \stackrel{2.0 \text { eq KF }}{\stackrel{\mathrm{DMF}, \mathrm{RT}, 5 \mathrm{~h}}{\mathrm{~N}}} \quad 116(77 \%)$

Tabelle 10. Desilylierung des 1-Trimethylsilyl-(4E)-5-phenylpent-4-en1-ins 115 zu (4E)-5-Phenylpent-4-en-1-in 116 und dem Allen 118.

\begin{tabular}{ccccccc}
\hline Eintrag & Base & {$[\mathrm{eq}]$} & $\mathrm{Lsm}$. & $\mathrm{T}\left[{ }^{\circ} \mathrm{C}\right]$ & $\mathrm{t}[\mathrm{h}]$ & $\mathbf{1 1 6 : 1 1 8}$ \\
\hline 1 & $\mathrm{NaOH}$ & 1.6 & $\mathrm{MeOH}$ & 25 & 5 & $1: 1.55$ \\
2 & $\mathrm{TBAF}$ & 2.0 & $\mathrm{THF}$ & 0 & 4 & $1: 3.33$ \\
3 & $\mathrm{TBAF}$ & 2.0 & $\mathrm{THF}$ & 25 & 2 & $1: 5.4$ \\
4 & $\mathrm{KF}$ & 2.0 & $\mathrm{DMF}$ & 25 & 5 & $7.2: 1$ \\
\hline
\end{tabular}


Bergeron et al. ${ }^{[53]}$ und Chemin et al. ${ }^{[54]}$ berichteten von erfolgreichen Desilylierungsversuchen mit TBAF in THF bzw. Kaliumfluorid in DMF. Die entsprechend durchgeführten Umsetzungen des Silans 115 mit TBAF führten ebenfalls zur Bildung eines Gemisches aus dem Enin 116 und dem Allen 118 als Hauptprodukt (vgl. Tabelle 10, Einträge 2 u. 3). Die Entschützung des TMS-Phenylenins 115 gelang schließlich nach Bergeron bei der Behandlung des Eduktes mit KF in DMF bei Raumtemperatur (vgl. Schema 29, Tabelle 10, Eintrag 4) im Verhältnis 7.2:1 zugunsten des Enins 116 in einer Ausbeute von 77\% (Exp. Teil, Allg. Arbeitsvorschrift A4, Variante 3).

Als weitere Seitenkette sollte das Dienin 121 ausgehend von dem Alkohol 119 hergestellt werden. Enders et al. ${ }^{[55]}$ berichteten von der Bromierung eines terminalen Dienylalkohols mit ähnlicher Molekülstruktur wie 4(E)-Methyl-hepta-1,4-dien-3-ol (119) (vgl. Schema 30) unter Verwendung von $\mathrm{PBr}_{3}$ in $\mathrm{Et}_{2} \mathrm{O}$ (Ausbeute 93\%). Sie empfahlen die unverzügliche Umsetzung der bromierten Komponenten aufgrund der schnellen Zersetzung.

Schema 30. Bromierung und Alkinylierung des 4(E)-Methylhepta-1,4-dien-3-ols (119).

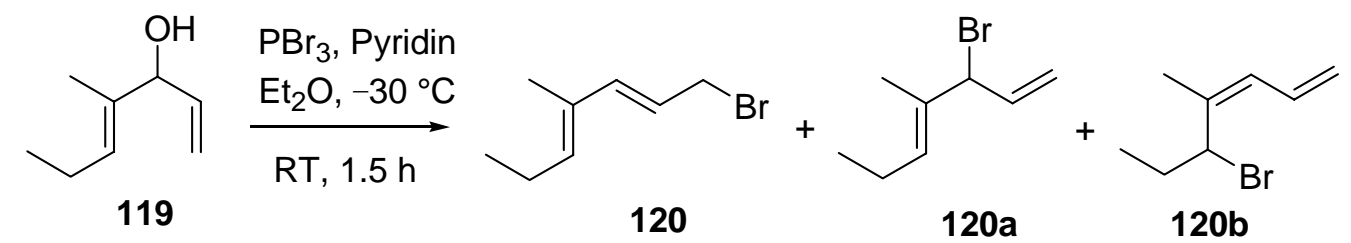

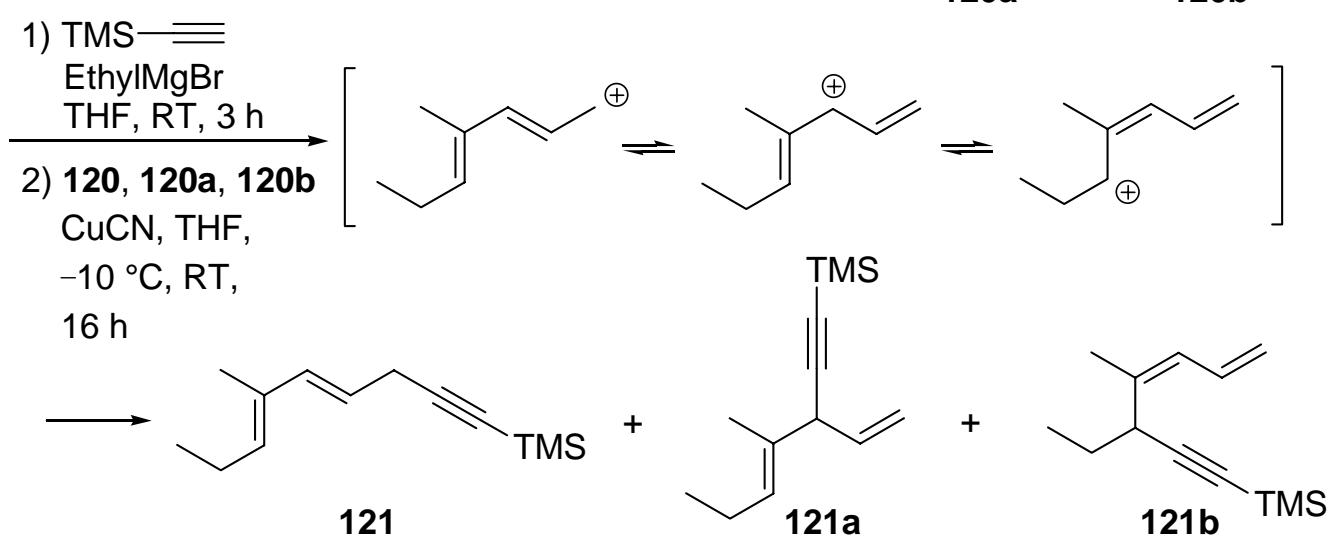

In Anbetracht der gewonnenen Erkenntnisse bezogen auf die Chemoselektivität des Bromierungsverfahrens bei den Alkoholen 99A und 99R (vgl. Schema 28), welche stets zur Bildung eines Gemisches aus den Bromiden 100 und 101 geführt hatte, wurde angenommen, dass die 
Chemoselektivität bei den Dienen der Struktur 119 eher noch geringer ausfällt. Die Halogenierung des Dienalkohols 119 wurde aufgrunddessen unter veränderten Temperaturbedingungen bei $-30{ }^{\circ} \mathrm{C}$ in $\mathrm{Et}_{2} \mathrm{O}$ durchgeführt. Nach $1.5 \mathrm{~h}$ Reaktionszeit und Aufarbeitung der Reaktion mit eiskalter ges. $\mathrm{K}_{2} \mathrm{CO}_{3}$-Lösung und Pentan konnte ein Gemisch aus den Bromierungsprodukten 120a und 120 (2.49:1) sowie des Bromids 120b in Spuren erhalten werden (Gesamtausbeute 95\%). Dieses Ergebnis stimmte mit schon zuvor gewonnenen experimentellen Ergebnissen (100A, 101A bzw. 100R und 101R, vgl. Schema 28) überein. Es lag aufgrund der zweifachen Doppelbindungsstruktur sowie der Lage der Alkoholfunktion an der C3-Position eine doppelte statistische Wahrscheinlichkeit der Regioselektivität der $\mathrm{S}_{N}$ 'Reaktion sowie eine einfache Regioselektivität der schneller verlaufenden $\mathrm{S}_{N}$-Reaktion vor. Die geringere Bildung des über eine langsamer verlaufende Reaktion entstandenen terminalen $\mathrm{S}_{N^{\prime}}$-Produkts 120 dürfte mit einem energetisch ungünstigeren und weniger stabilen primären Kation im Übergangszustand erklärt werden. Die Bildung des $S_{N}$-Produkts 120a verläuft hingegen über einen Übergangszustand, in dem ein stabileres sekundäres Kation vorliegt, da beide Doppelbindungen konjungiert zum Reaktionszentrum stehen. Das Bromid 120b ist aufgrund sterischer Hinderung nur in Spuren entstanden.

Die Alkinylierung des Bromidgemisches 120 zu den Alkinen 121, 121a und 121b (Schema 30) wurde unverzüglich nach dessen Herstellung gemäß den im Schema 28 angegebenen Reaktionsbedingungen durchgeführt. Die Auswertung des erhaltenen Spektrums der Umsetzung erwies sich wegen starker Verunreinigungen als schwierig und ließ keine zuverlässige Aussage über die Bildungsanteile der entstandenen Produkte 121, 121a und 121b zu. Bei dem Versuch der NMR-Analytik wurde in $\mathrm{CDCl}_{3}$ sowie $\mathrm{CD}_{3} \mathrm{OD}$ vollständige Zersetzung beobachtet.

\subsection{Kreuzkupplungversuche von Brommethylpyron mit Alanaten}

Die geplante Synthese der Iromycine A und R sollte über eine C-C-Bindungsbildung in einer Kreuzkupplungsreaktion des Brommethylpyrons 96 und der Seitenkettenfragmente 122, 103A und 103R ablaufen und die Iromycin-Vorläufer 125, 125A und 125R liefern (vgl. Schema 31). Das Iromycin-Analogon 125 wurde im Rahmen von Untersuchungs- sowie Optimierungsreaktionen als Modellsubstrat dargestellt. Der anschließende O-N-Austausch des Kreuzkupplungsproduktes sollte die gewünschten Iromycine 23, 23A und 23R ergeben. Die Iromycin-Vorläufer, nämlich der Benzoesäureester 125 als Modellsubstrat (vgl. Schema 31), der 
Benzoesäureester 125A als Iromycin A-Vorläufer sowie der Benzoesäureester 125R als Iromycin R-Vorläufer, sollten ausgehend von Brommethylpyron 96 und den entsprechenden Alkinderivaten 103A und 103R bzw. 1-Heptin (122) aufgebaut werden (vgl. Schema 31).

Schema 31. Darstellung der Kreuzkupplungsprodukte 125, 125A und 125R aus dem Brommethylpyron 96 und den Alanaten 124, 124A und 124R.

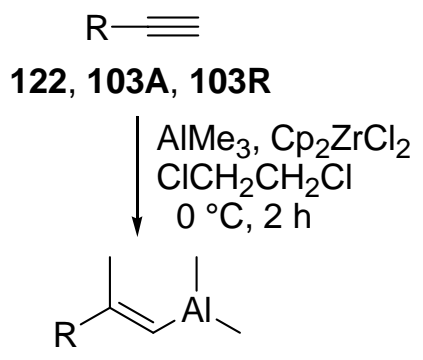

\begin{tabular}{l|l|l} 
Alkin & Alan & \multicolumn{1}{|c}{$\mathrm{R}$} \\
\hline 122 & 123 & $\mathrm{CH}_{3}\left(\mathrm{CH}_{2}\right)_{4}$ \\
103A & 123A & $\left(\mathrm{CH}_{3}\right)_{2} \mathrm{CHCH}=\mathrm{CHCH}_{2}$ \\
103R & 123R & $\left(\mathrm{CH}_{3}\right)_{3} \mathrm{CCH}=\mathrm{CHCH}_{2}$
\end{tabular}

123, 123A, 123R

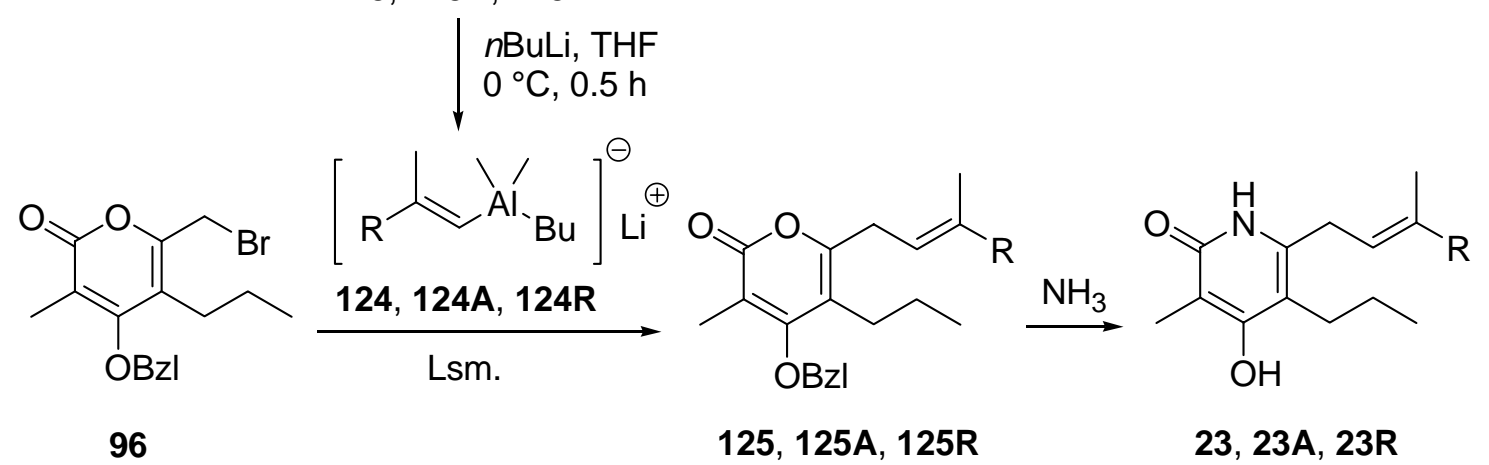

\subsubsection{Zirconocen-katalysierte Darstellung der Alane aus Alkinen}

Zur Durchführung der Kreuzkupplungsversuche von Brommethylpyron 96 mit Alanaten sollten zunächst die Alkine 122, 103A und 103R in einer Carboaluminierungsreaktion zu einer metallorganischen Verbindung hochselektiv am C-2 der Alkinylfunktion methyliert werden, um hierdurch über eine $c i s$-selektive Addition die trans-Alkenyldimethylalane 124, 124A und 124R zu erhalten. Die Umsetzungen der Alane mit Brommethylpyron 96 sollten dann die gewünschten Vorläufermoleküle 125, 125A und 125R liefern.

Die Carboaluminierungsreaktion der Alkine wurde zunächst nach Negishi et al ${ }^{[56]}$ untersucht. Negishi entwickelte die Carboaluminierungsmethode und wandte diese zur Darstellung von Geraniol, Farnesol, Monocyclofarnesol, $\alpha$-Farnesol, Vitamin A und weiteren diversen Natur- 
stoffen an. Nach dieser neuartigen Methode gelang es Negishi, terminale Alkine in einer $\mathrm{Cp}_{2} \mathrm{ZrCl}_{2}$-katalysierten Reaktion unter Verwendung von $\mathrm{AlMe}_{3}$ in die gewünschten $(E)$-2Methyl-1-alkenylalane zu überführen, die in den entsprechenden Kreuzkupplungsreaktionen eingesetzt wurden. Im Rahmen der hier durchgeführten Iromycinsynthese wurde nach Negishi 1.0 eq Alkin bei Raumtemperatur mit einer Suspension von 0.2 eq $\mathrm{Cp}_{2} \mathrm{ZrCl}_{2}$ und 2.0 eq Al$\mathrm{Me}_{3}$ in Dichlorethan umgesetzt. Bei diesen mit in situ generierten Alanlösungen durchgeführten Kreuzkupplungsreaktionen zeigten sich jedoch Probleme, da die Reaktionen entweder überhaupt nicht verliefen oder die Übertragung der Methylgruppe auf das Brommethylpyron 96 beobachtet wurde, welches dem Überschuss an $\mathrm{AlMe}_{3}$ zugeschrieben wurde (zur Bildung des methylierten Produktes vgl. auch Abschnitte 2.2.2.2 u. 5.3.1).

Lipshutz et al. ${ }^{[57]}$ und Mori et al. ${ }^{[58]}$ beschrieben die Zirconocen-katalysierte Darstellung von $\mathrm{AlMe}_{3}$-freien Alanen (vgl. Schema 31). Lipshutz et al. führten die Carboaluminierung durch Zugabe einer $\mathrm{AlMe}_{3}$-Lösung zu Zirconocendichlorid $\left(\mathrm{CpZrCl}_{2}\right)$ unter Argonatmosphäre bei $0^{\circ} \mathrm{C}$ durch und entfernten dann unter vermindertem Druck bei $0^{\circ} \mathrm{C}$ das Lösungsmittel (Hexan). Anschließend wurde zunächst Dichlorethan (DCE) als Lösungsmittel und dann eine Lösung des Alkins in DCE hinzugegeben. Nach Beendigung der Reaktion (Dauer 12-17 h) entfernten sie das Dichlorethan und versetzten die Reaktionsmischung mit Hexan, um die Zirconocen-Salze auszufällen. Die anschließende Isolierung über eine Umkehrfritte lieferte dann das entsprechende Alan. Mori et al. gaben eine $\mathrm{AlMe}_{3}-\mathrm{Lösung} \mathrm{zu} \mathrm{CpZrCl}_{2}$ in Dichlormethan (DCM) und rührten die Reaktionslösung 30 min bei Raumtemperatur. Nach Zugabe von Alkin wurde die Reaktion $2 \mathrm{~h}$ bis zur vollständigen Umsetzung bei eben dieser Temperatur gerührt. Anschließend erhöhten sie die Temperatur auf $30^{\circ} \mathrm{C}$ und entfernten das überschüssige $\mathrm{AlMe}_{3}$ sowie das Lösungsmittel unter vermindertem Druck. Aufgrund der Probleme bei den Kreuzkupplungsreaktionen nach Negishi mit in situ dargestellten Alanen wurden entsprechend der Vorgehensweise von Lipshutz und Mori bei der Iromycinsynthese ebenfalls $\mathrm{AlMe}_{3}$-freie Alane eingesetzt.

Die Darstellung der extrem luft- und hydrolyseempfindlichen Alane wurden entsprechend der vorstehend angeführten Literaturangaben Zirconocen-katalysiert unter Einsatz von $\mathrm{AlMe}_{3}(2.0$ M Lösungen in Hexan) in DCE, jedoch unter den in der Tabelle 11 dargestellten veränderten experimentellen Bedingungen durchgeführt und optimiert (s. Exp. Teil, Allg. Arbeitsvorschrift A5, Varianten 1 und 2). Die Umsetzungen zur Darstellung von (E)-2-Methylhepta-1enyldimethylalan (123) verliefen insgesamt relativ glatt und sauber. Die Ausbeuten der iso- 
lierten Alane 123, 123A und 123R betrugen 62-100\% bezogen auf die eingesetzte Eduktmenge (vgl. Tabelle 11). Die erzielte höhere Reinheit des Alans 123 im Vergleich zu derjenigen der Alane 123A und 123R wurde mit der Qualität des eingesetzten Alkins erklärt. Im Allgemeinen wird die Darstellung der Alane aber vor allem von der Qualität der $\mathrm{AlMe}_{3}$-Lösungen sowie dem Einsatz von wasserfreien Lösungsmitteln beeinflusst.

Tabelle 11. Versuche zur Darstellung der Alane 123, 123A und 123R.

\begin{tabular}{ccccccc}
\hline Eintrag & Alkin & $\mathrm{Cp}_{2} \mathrm{ZrCl}_{2}[\mathrm{eq}]$ & $\mathrm{AlMe}_{3}[\mathrm{eq}]$ & $\mathrm{T}\left[0{ }^{\circ} \mathrm{C}\right]$ & $\mathrm{t}[\mathrm{h}]$ & Ausbeute $(\%)^{\mathrm{c})}$ \\
\hline 1 & $\mathbf{1 2 2}$ & 0.25 & 1.5 & $0 \rightarrow \mathrm{RT}$ & 15 & 62 \\
2 & $\mathbf{1 2 2}$ & 0.25 & 1.5 & $0 \rightarrow \mathrm{RT}$ & 15 & 85 \\
3 & $\mathbf{1 2 2}$ & 0.25 & 1.5 & $0 \rightarrow \mathrm{RT}$ & 15 & quant. \\
4 & $\mathbf{1 2 2}$ & 0.20 & 2.0 & $0 \rightarrow \mathrm{RT}$ & 15 & quant. \\
5 & $\mathbf{1 2 2}$ & 0.20 & 2.0 & $0 \rightarrow \mathrm{RT}$ & 15 & 88 \\
\hline 6 & $\mathbf{1 0 3 A}$ & 0.25 & 1.5 & $0 \rightarrow \mathrm{RT}$ & 16 & quant. \\
7 & $\mathbf{1 0 3 A}$ & 0.25 & 1.5 & $0 \rightarrow \mathrm{RT}$ & 16 & 54 \\
8 & $\mathbf{1 0 3 A}$ & 0.20 & 2.0 & $0 \rightarrow \mathrm{RT}$ & 17 & $70^{\text {a) }}$ \\
9 & $\mathbf{1 0 3 R}$ & 0.20 & 2.0 & $0 \rightarrow \mathrm{RT}$ & 16 & $70^{\mathrm{b})}$ \\
10 & $\mathbf{1 0 3 R}$ & 0.20 & 2.0 & 0 & 2 & 89 \\
\hline a) ${ }^{1} \mathrm{H}-\mathrm{NMR}$ des Alans zeigte das Hydrolyseprodukt an. ${ }^{\text {b) }}$ Keine Methylübertragung auf \\
das Alkin. ${ }^{\text {c) }}$ Ausbeute der isolierten Alane.
\end{tabular}

\subsubsection{Versuche zur Darstellung der Iromycinvorläufer aus Bromiden mit Alanaten}

Entsprechend dem im Schema 31 dargestellten Syntheseweg (vgl. Abschnitt 2.2) wurde als nächster Syntheseschritt die Kreuzkupplung von Brommethylpyron 96 mit Alanaten zur Darstellung der Iromycinvorläufer untersucht. Ähnliche C-C-Bindungsbildungsreaktionen von Halogeniden und Alanaten wurden bereits in der Literatur beschrieben. Negishi et. al. ${ }^{[59]}$ berichteten in zahlreichen Publikationen unter anderem über die selektive Synthese von disubstituierten trans-Alkenen (Ausbeute $>98 \%$ ) in einer Eintopf-Reaktion von Alkylhalogeniden mit trans-Alkenylalanaten, die über die Behandlung des Alkenyldimethylalans mit Alkyllithiumorganylen wie $n \mathrm{BuLi}$ oder MeLi generiert wurden. Die entsprechenden Alkenyldialkylalane konnten unter Umsetzung der Alkine mit DIBAH bzw. AlMe 3 dargestellt werden. Desweiteren beschrieben Utimoto et al. ${ }^{[60]}$ die in situ Alkenylierung von Allylhalogeniden, wie z.B. Allylchlorid, Methylallylchlorid und 2,3-Dichlorpropen, zu den entsprechenden Allylprodukten (Ausbeuten 77-84\%). Die benötigten Alkenylalanate wurden ausgehend von einer Hydroaluminierung terminal substituierter Trimethylsilylalkine mit DIBAH und anschließen- 
der Behandlung mit MeLi in situ dargestellt. Frejd et al. ${ }^{[61]}$ nutzten die Alkinyl- bzw. Alkenylalanate aufgrund ihrer erhöhten Nucleophilie im Vergleich zu den entsprechenden Alanen zur Epoxidöffnung von Zuckerderivaten, und erreichten die Übertragung der Alkenyl- und Alkinylreste vorwiegend in guten Ausbeuten von 30-95\%. Takeuchi et al. ${ }^{[62]}$ gelang unter anderem bei der Synthese von Steronen bzw. Castasteronen und Brassinoliden sogar die Vinylübertragung (Ausbeute 55\%) via eines Vinylalanates in einem nucleophilen Angriff des Alanats auf den Aldehyd. Aus den vorgenannten Arbeiten, vor allem derjenigen von Takeuchi et al., wird als charakteristische Eigenschaft der Alanate deren effektive Nucleophilie deutlich, die auch bei der anvisierten Iromycinsynthese ausgenutzt werden sollte. Der Einsatz von Alanaten ist insbesondere auch gegenüber der Verwendung von Alanen vorteilhaft, weil bei den Alanaten aufgrund ihres ionischen Charakters die in unpolaren Lösungsmitteln (Hexan, Toluol, etc.) vorherrschende starke Neigung der Alane zur Bildung dimerer Spezies völlig aufgehoben ist, wodurch ein nucleophiler Angriff der Seitenkettenverbindungen (Alanat) auf das Bromid begünstigt ist. Auch wenn Alane in koordinierenden Lösungsmitteln wie THF vollständig als Monomere vorliegen, ist ein nucleophiler Angriff im Vergleich entweder nicht gegeben oder nur sehr schwach ausgeprägt.

Aufgrund der starken Nucleophilie der Alanate und der Struktur des Brommethylpyrons ist der Ablauf der geplanten Kreuzkupplung über eine nucleophile $\mathrm{S}_{N}$ 2-Substitutionsreaktion anzunehmen. Die Analyse der elektronischen Struktur des Brommethylpyrons 96 (vgl. Schema 31) lässt die Konjugation der Doppelbindungen entlang des ungesättigten Lactonrings (cyclischer Ester) erkennen, welche mit der Carbonylgruppe an der C-2-Position ein ausgedehntes $\pi$-System bildet. Man spricht von einem doppelt vinylogen Ester. Diese Struktur erlaubt eine ausgedehnte Delokalisierung des Doppelbindungssystems der konjugierten Dienverbindung. Zudem ist durch die Funktionalisierung der C-1'-Position des Pyrongrundgerüstes als Halogenid die Voraussetzung für ein als Allylhalogenid dargestelltes System gewährleistet. Diese Delokalisierung im Allylsystem ist die Ursache für die ungewöhnliche Reaktivität von Bindungen in der Allylstellung. Daher gehen Allylhalogenide relativ schnelle $\mathrm{S}_{N} 1$ - und $\mathrm{S}_{N}$ 2-Reaktionen ein. Diese elektronischen Eigenschaften des Moleküls prädestinieren damit das Allybromid 96 im besonderen Maße für einen nucleophilen Angriff der Alanate an der vinylogen Position. Die starke Nucleophilie der Alanate als Aluminiumorganyle begünstigt zudem den Ablauf von $\mathrm{S}_{N} 2$-Substitutionsreaktionen. Daher sollten die Kreuzkupplungsreaktionen unter den Bedingungen einer $\mathrm{S}_{N} 2$-Reaktion, nämlich Verwendung polar aprotischer Lö- 
sungsmittel, hoher Konzentration des Nucleophils, Einsatz eines starken Nucleophils sowie Einsatz einer geeigneten Abgangsgruppe wie Bromid, untersucht werden.

\subsubsection{Vorversuche zu Kreuzkupplungsreaktionen von Benzylbromiden mit Alanaten}

Vorversuche zur Kreuzkupplung von Bromiden mit Alanaten wurden zunächst mit Benzylbromiden durchgeführt, da diese ein mit Brommethylpyron 96 vergleichbares Allylsystem aufweisen. Die Vorversuche wurden über eine Generierung des Alanats unter Zugabe entsprechender Äquivalente an $n$ BuLi zu einer Lösung des Alans in THF bzw. Acetonitril bei 0 ${ }^{\circ} \mathrm{C}$ und anschließender Umsetzung des Alanats mit Benzylbromid durchgeführt und untersucht (vgl. Schema 32, Tabelle 12, Exp. Teil, Allg. Arbeitsvorschrift A13).

Schema 32. Vorversuche zu Kreuzkupplungsreaktionen des Benzylbromids 130 mit Alanaten.

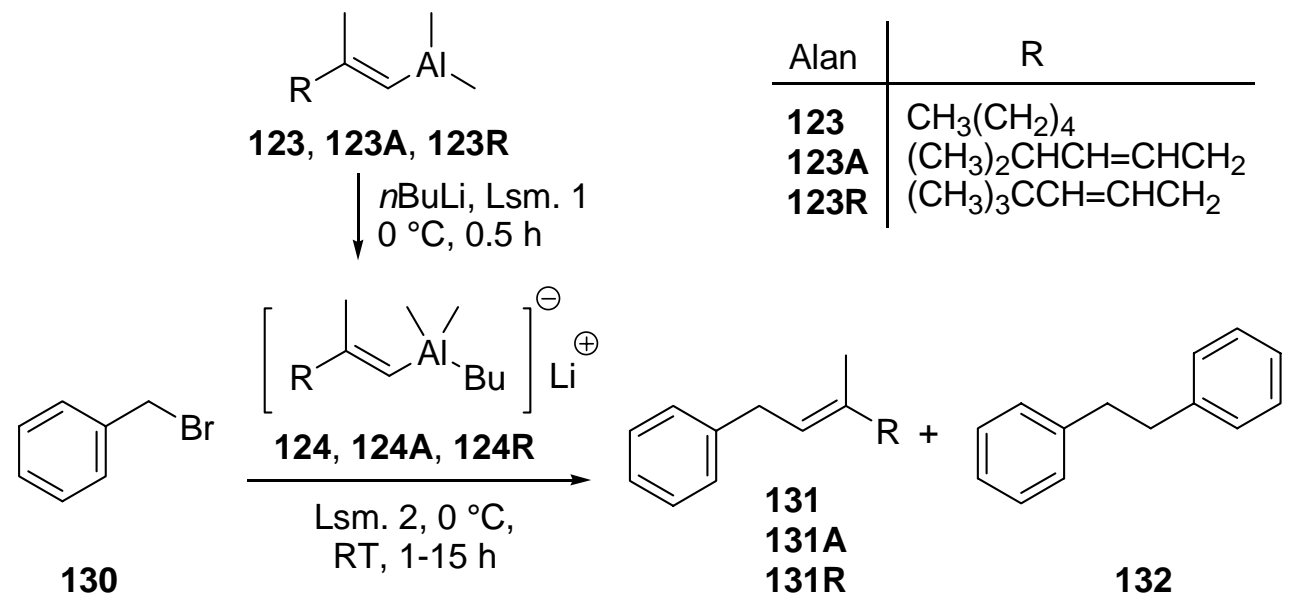

Ziel der Vorversuche war unter anderem die Beobachtung des Einflusses der polar aprotischen Lösungsmittel THF und MeCN auf das Reaktionsgeschehen, deren Einsatz wegen eines angenommenen Reaktionsverlaufes über eine nucleophile Substitution nach einem $\mathrm{S}_{N} 2$ Mechanismus (s. Abschnitt 2.2.2.) untersucht wurde. Während die Reaktion in THF (vgl. Tabelle 12, Eintrag 1) in einer sauberen und vollständigen Umsetzung das gewünschte Kreuzkupplungsprodukt 131 lieferte, blieb dessen Entstehung bei dem unter Verwendung des polareren $\mathrm{MeCN}$ als Lösungsmittel durchgeführten Versuch trotz einer vollständigen Umsetzung völlig aus (vgl. Tabelle 12, Eintrag 2). Bei dem in THF durchgeführten Versuch (Eintrag 1) 
wurde nach der Aufarbeitung neben dem gewünschten Kreuzkupplungsprodukt 131 als Hauptprodukt auch das Homokupplungsprodukt 132 als Nebenprodukt in einem Verhältnis von $94 \%$ zu $6 \%$ erhalten.

Tabelle 12. Kreuzkupplungsreaktionen des Benzylbromids 130 mit Alanaten.

\begin{tabular}{|c|c|c|c|c|c|c|c|}
\hline Eintrag & $\begin{array}{l}\text { Alan } \\
\text { [eq] }\end{array}$ & $\begin{array}{c}n \mathrm{BuLi} \\
{[\mathrm{eq}]}\end{array}$ & Lsm.1 & Lsm.2 & $\begin{array}{c}\mathrm{t} \\
{[\mathrm{h}]}\end{array}$ & $\begin{array}{l}\text { Verhältnis } \\
\text { in }(\%)\end{array}$ & $\begin{array}{c}\text { Massenverhältnis } \\
\text { in }(\%)^{\mathrm{a})}\end{array}$ \\
\hline & 123 & & & & & 130:131:132 & $131: 130$ \\
\hline 1 & 3.0 & 3.0 & $\mathrm{THF}$ & $\mathrm{THF}$ & 15 & $0: 94: 6$ & 74 \\
\hline 2 & 3.0 & 3.0 & $\mathrm{MeCN}$ & $\mathrm{MeCN}$ & 15 & $0: 0: 0$ & 100 \\
\hline 3 & 1.1 & 1.1 & THF & THF & 3 & $0: 56: 44$ & 48 \\
\hline \multirow[t]{2}{*}{4} & 2.0 & 2.0 & THF & THF & 22 & $12: 75: 13$ & 42 \\
\hline & $123 \mathrm{~A}$ & & & & & 130:131A:132 & 131A:130 \\
\hline 5 & 1.1 & 1.1 & THF & THF & 3 & 100:0:0 & 33 \\
\hline 6 & 2.0 & 2.0 & THF & THF & 24 & $15: 85: 0$ & 27 \\
\hline 7 & 2.0 & 2.0 & THF & THF & 22 & $0: 90: 10$ & 30 \\
\hline \multirow[t]{2}{*}{8} & 2.0 & 2.0 & THF & THF & 15 & $35: 47: 18$ & 43 \\
\hline & 123R & & & & & 130:131R:132 & 131R:130 \\
\hline 9 & 2.5 & 2.5 & THF & THF & 2 & 0:Spur:Spur & 48 \\
\hline
\end{tabular}

Die Identifizierung der unerwünschten Produkte durch das ${ }^{1} \mathrm{H}-\mathrm{NMR}-$ Spektrum erwies sich aufgrund zahlreicher Singulett-Signale als sehr schwierig. Das Ausbleiben des gewünschten Produktes 131 bei Verwendung des im Vergleich zu THF stärker polaren MeCN (vgl. Tabelle 12, Eintrag 2) kann mit einem nucleophilen Angriff des $n \mathrm{BuLi}$ auf das Acetonitrilkohlenstoffzentrum und einem nucleophilen oder elektrophilen Angriff des $n \mathrm{BuLi}$ auf das reaktive Benzylbromid erklärt werden. Der nucleophile Angriff des Li-Organyls auf das Acetonitrilkohlenstoffzentrum führte zur Bildung des Adduktes Imin als Zwischenprodukt. Hierdurch wurde das zur Generierung des Alanats notwendige $n \mathrm{BuLi}$ teilweise verbraucht. Die anschließende Hydrolyse bei der Aufarbeitung lieferte dann das korrespondierende Keton. Desweiteren könnte eine Solvatisierung des $n$ Butyl-Anions durch das elektronenarme und stark polarisierbare Acetonitril zur Abschwächung der Nucleophilie führen. Dies wird vor allem durch die starke Tendenz der freien Li-Ionen zur Salzbildung gestützt. Der Ausfall von LiBr hätte allerdings die Bildung eines instabilen Benzylkations zur Folge, dessen Hydrolyse folglich zu Benzylalkohol geführt hätte (vgl. Schema 33). Die Entstehung des bei den Versuchen vorwiegend erhaltenen Toluols schließlich setzt die Bildung des Benzylanions voraus, dessen saure 
Hydrolyse Toluol liefert (vgl. Schema 33). Die Erklärung für die Bildung des Toluols aus Benzylbromid 130 könnte wiederum ein in der Reaktionslösung befindlicher Überschuß an $n$ BuLi erbringen (vgl. Schema 33). Die Bildung von Toluol (2.38 ppm), Benzylalkohol (4.4 ppm) und 1-Phenyl-propan-2-on (2.2 und $3.4 \mathrm{ppm}$ ) wurde von den beobachteten Signalen gestützt und daher theoretisch für möglich gehalten. Die Überlegung, dass $n$ Buli bei dem Reaktionsgeschehen, wie oben beschrieben, verbraucht worden und hierdurch eine Alanatbildung ausgeblieben sein könnte, führte desweiteren zu der Annahme, dass die Nucleophilie des Alans alleine als solche für einen nucleophilen Angriff auf das Bromid und die Bildung des Kreuzkupplungsproduktes nicht ausreicht. Ein radikalischer Mechanismus würde die divers gebildeten Produkte zwar eher erklären, ist aufgrund der Reaktionsbedingungen aber eher unwahrscheinlich.

Schema 33. Bildung des Toluols aus dem Benzylbromid 130.

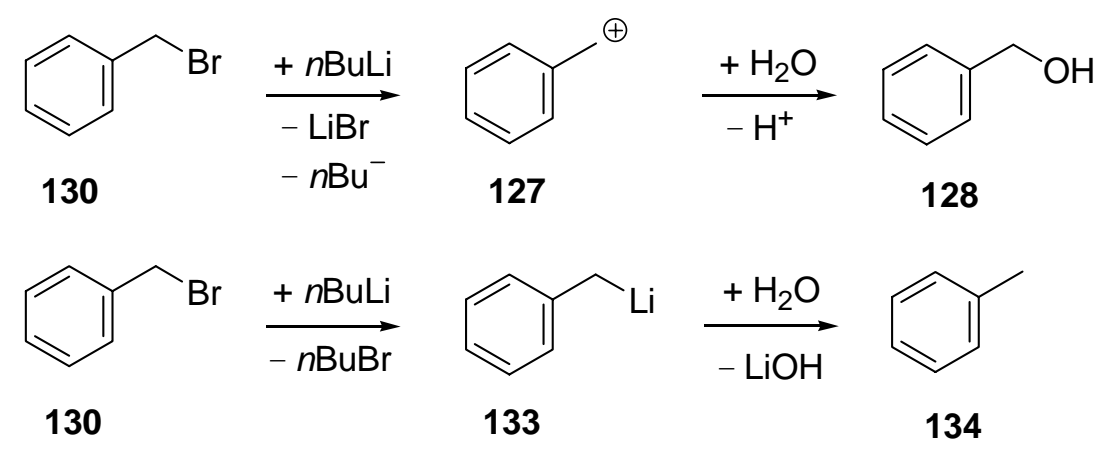

Im einem weiteren Versuch ergab der Einsatz von je 1.1 eq Alan $\mathbf{1 2 3}$ und $n \mathrm{BuLi}$ in THF nach $3 \mathrm{~h}$ ein Gemisch aus dem gewünschten Produkt 131 als Hauptprodukt und der Homokupplungsverbindung 132 als Nebenprodukt im Verhältnis 1.27:1 (vgl. Tabelle 12, Eintrag 3). Die großen aliphatischen Signale wiesen trotz vollständiger Umsetzung des Benzylbromids auf einen geringen Anteil an erhaltenem Kreuzkupplungsproduktes 131 und Nebenprodukt 132 im Rohprodukt hin. Die geringe Bildung der Verbindung 131 ist auf einen geringen Anteil an eingesetztem Alan und Lithiumorganyl von jeweils 1.1 eq zurückzuführen. Bei Verwendung von je 2.0 eq Alan und $n \mathrm{BuLi}$ (vgl. Tabelle 12, Eintrag 4) konnte das Ergebnis des Versuches mit jeweils 3.0 eq (Eintrag 1) zwar angenähert, aber nicht reproduziert werden. Die nicht vollständige Umsetzung des Bromids wurde wiederum auf die Qualität des Alans zurückgeführt. 
Die zu den Umsetzungen des als Modellsubstrat verwendeten Alans 123 analog durchgeführten Kreuzkupplungsreaktionen mit den Alanen 123A und 123R ergaben ähnlich divergente Ergebnisse (vgl. Tabelle 12, Einträge 5-9). Während bei dem Versuch 5 (Eintrag 5) keine Umsetzung beobachtet wurde, konnte das Kreuzkupplungsprodukt bei den Umsetzungen 6 und 7 (Einträge 6 u. 7) als Hauptanteil des Rohprodukts erhalten werden. Hingegen halbierte sich bei dem Versuch 8 (Eintrag 8) der Anteil des gewünschten Kreuzkupplungsprodukt 131A auf 47\%. Die Umsetzung des Benzylbromids mit dem Alanat 124R (Eintrag 9) lieferte trotz vollständiger Umsetzung nur Spuren des Kreuzkupplungsproduktes 131R. Auffällig waren besonders kleine Rohproduktmassen bei den mit den Alanen 123A und 123R durchgeführten Reaktionen (vgl. Tabelle 12, Einträge 5-9).

Aufgrund des Ergebnisses der Vorversuche ist die Verwendung von 3.0 eq Alanat (vgl. Tabelle 12, Eintrag 1) anscheinend als stöchiometrisches Idealmaß für die Kreuzkupplungsreaktion zu betrachten. Die zum Teil sehr guten Bildungsanteile der Kreuzkupplungsprodukte bestätigten die Effektivität der Methodik für die C-C-Bindungsbildung. Als weiteres Ziel zur Verbesserung der Methodik blieb jedoch, die Bildung des Homokupplungsproduktes 132 zu minimieren und parallel die schlechte Massebilanz der Rohprodukte zu erhöhen. Auf diesem Weg erschien die Optimierung der Verhältnisse zwischen der $n \mathrm{BuLi}$ - und der Alankonzentration wichtig zu sein. Der gravierende Verlust an Rohmasse wurde hauptsächlich auf die Bildung des flüchtigen Toluols zurückgeführt.

\subsubsection{Darstellung der Iromycinvorläufer aus Pyronhalogeniden mit Alanaten}

Die C-C-Bindungsbildung des Brommethylpyrons 96 und der Seitenkettenalanate 124, 124A bzw. 124R zu den Iromycinvorläufern 125, 125A bzw. 125R wurde entsprechend der mit Benzylbromid und Alanat untersuchten Methode unter $\mathrm{S}_{N}$ 2-Bedingungen (vgl. Abschnitte 2.2.2. u. 2.2.2.1) durchgeführt (vgl. Schema 34). Die Alanate 124, 124A und 124R wurden ausgehend von den zuvor hergestellten Lösungen der entsprechenden Alane 123, 123A und 123R (vgl. Abschnitt 2.2.1.) generiert. Die Zugabe von $n \mathrm{BuLi}$ zu den Alanen (1.0 M Lösungen in Hexan) bei $0{ }^{\circ} \mathrm{C}$ führte nach 30 min zur Bildung der Alanate 124, 124A und 124R als eigentliche nucleophile Spezies. 
Schema 34. Kreuzkupplungsreaktionen des Brommethylpyrons 96 mit den Alanaten 124, 124A und 124R.

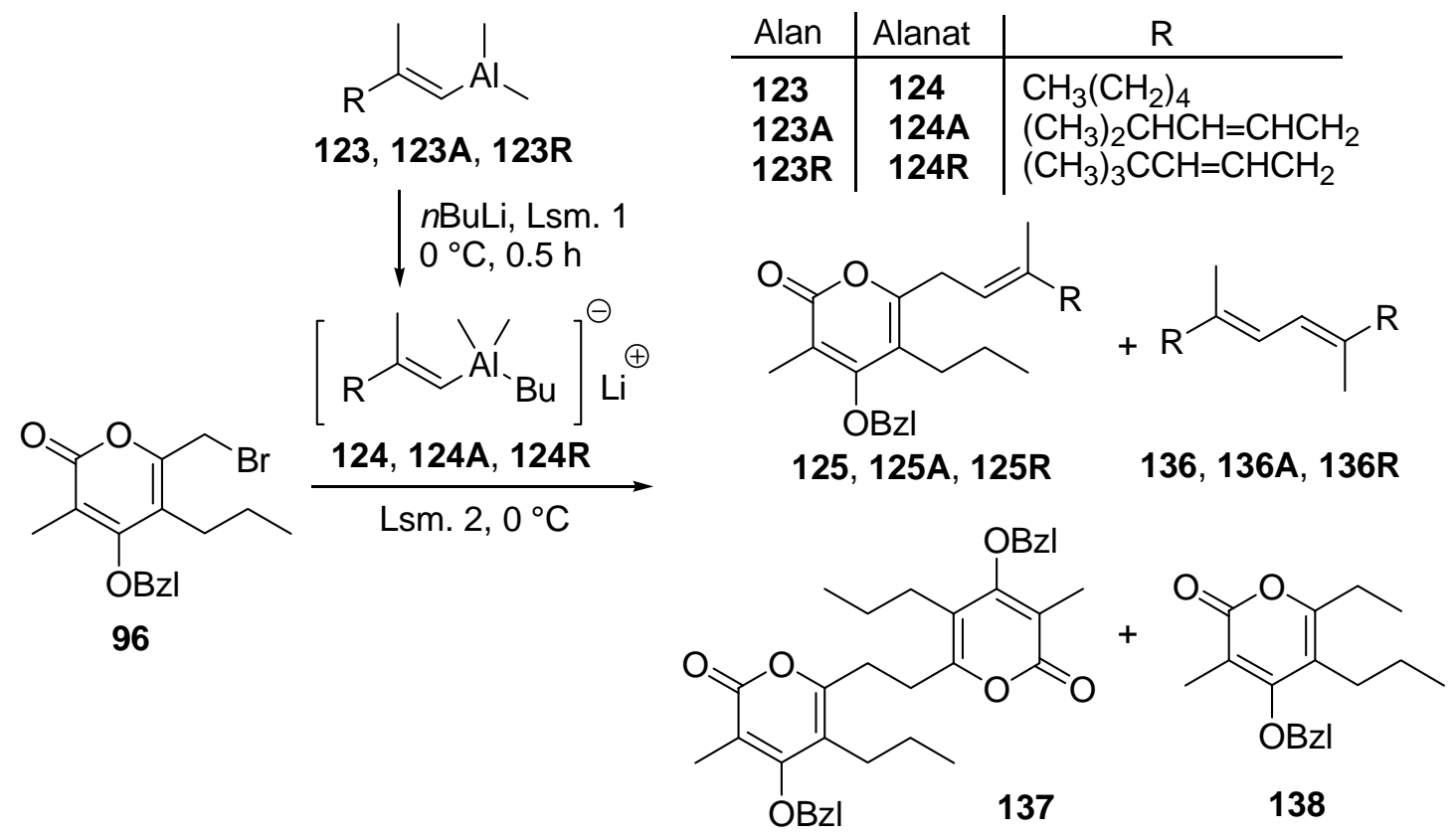

Die Kreuzkupplungen des Brommethylpyrons 96 mit dem Heptenylalanat 124 sowie den Alkenylalanaten 124A und 124R (vgl. Tabellen 13-15, Exp. Teil, Allg. Arbeitsvorschrift A13) lieferten, wie die zuvor mit Benzylbromid durchgeführten Vorversuche (vgl. Tabelle 12), die gewünschten Kreuzkupplungsprodukte 125, 125A und 125R. Daneben konnte allerdings die Bildung von drei weiteren Verbindungen als Neben- bzw. Hauptprodukte beobachtet werden. Bei einer dieser Verbindungen handelte es sich um das Dipyronethan (DPE) 137, das Homokupplungsprodukt des Hauptgrundgerüstes 96 (vgl. Schema 34), das als Analogon zu der bei den Kreuzkupplungsreaktionen der Benzylbromide beobachteten Homokupplungsverbindung Diphenylethan 132 angesehen werden kann. Als weitere Verbindungen wurden die Homokupplungsprodukte 136, 136A und 136R (vgl. Schema 34) der entsprechenden Seitenkettenfragmente als Strukturdimere isoliert und nachgewiesen. Zudem wurde das Methylierungsprodukt des Brommethylpyrons 96 als Nebenprodukt identifiziert. Die Umsetzung des Chlormethylpyrons 135 mit dem Heptenylalanat 124 (vgl. Tabelle 13, Eintrag 5) führte bei Ausbleiben des Kreuzkupplungsproduktes zur vollständigen Zersetzung des Eduktes 135, weshalb Chlormethylpyron als Ausgangssubstanz nicht weiter eingesetzt wurde. Die Darstellung des gewünschten Kreuzkupplungsproduktes 125 wurde daher ausschließlich ausgehend von Brommethylpyron 96 durchgeführt und optimiert. 
Die Betrachtung der in der Tabelle 13 aufgeführten Kupplungsversuche mit dem Brommethylpyron 96 und dem Alanat 124 zeigt, dass die Massenbilanz der Rohprodukte der Einträge 2 und 9 (48-50\%) trotz Massenzunahme des gewünschten Kreuzkupplungsproduktes und der vollständigen Umsetzung des Bromides 96 erheblich unter den erhofften theoretischen Rohproduktausbeuten geblieben war. Dies dürfte daher herrühren, dass ein Teil der umgesetzten Masse als Salz bei der wässrigen Aufarbeitung ausgeschieden wurde. Eventuell kann dies auch mit einem erhöhten Anteil von $n \mathrm{BuLi}$ zusammenhängen, der durch den geringeren tatsächlichen Gehalt des reaktiven Alans zustandegekommen sein könnte. Während bei den Umsetzungen 2 und 5 (Tabelle 13, Einträge 2 u. 5) hauptsächlich eine Zersetzung eintrat und nur Spuren von Produkt und Nebenprodukten entstanden, bildete sich bei dem Versuch 9 (Tabelle 13, Eintrag 9) lediglich das Homokupplungsprodukt 137 als Hauptprodukt.

Tabelle 13. Kreuzkupplungsreaktionen des Brommethylpyrons 96 bzw. Chlormethylpyrons 135 mit dem Heptenylalanat 124 zu dem Kreuzkupplungsprodukt 125.

\begin{tabular}{|c|c|c|c|c|c|c|c|c|}
\hline Eintrag & $\begin{array}{l}123 \\
{[\mathrm{eq}]}\end{array}$ & $\begin{array}{c}n \mathrm{BuLi} \\
{[\mathrm{eq}]}\end{array}$ & Lsm.1/Lsm.2 & $\begin{array}{c}\mathrm{T} \\
{\left[{ }^{\circ} \mathrm{C}\right]}\end{array}$ & $\begin{array}{c}\mathrm{t} \\
{[\mathrm{h}]}\end{array}$ & $\begin{array}{c}\text { 96:125:136:137:138 } \\
(\%)\end{array}$ & $\begin{array}{l}\text { AR } \\
(\%)^{\text {a) }}\end{array}$ & $\begin{array}{l}\text { KKP } \\
(\%)^{b)}\end{array}$ \\
\hline 1 & 1.0 & 1.5 & THF/THF & $0 \rightarrow \mathrm{RT}$ & 4.5 & $0: 79: 5: 15: 1$ & 90 & - \\
\hline \multirow[t]{3}{*}{2} & 1.5 & 1.5 & Toluol/Toluol & -30 & 8 & & & \\
\hline & 0.7 & 0.7 & & 0 & 17 & & & \\
\hline & & & & RT & 5 & $0: S^{d)}: S: S: 0$ & 48 & - \\
\hline 3 & 1.5 & 1.5 & $\mathrm{Et}_{2} \mathrm{O} / \mathrm{Et}_{2} \mathrm{O}$ & -30 & 2 & $0: 52: 0: 37: 11$ & 69 & - \\
\hline 4 & 1.5 & 1.5 & THF/THF & -30 & 2 & $35: 0: 0: 64.9: 0$ & 65 & - \\
\hline \multirow[t]{2}{*}{$5^{c)}$} & 2.0 & 2.0 & $\mathrm{THF} / \mathrm{THF}$ & 0 & 6 & & & \\
\hline & & & & $0 \rightarrow \mathrm{RT}$ & 15 & Zersetzung & 48 & - \\
\hline \multirow[t]{2}{*}{6} & 2.0 & 2.0 & $\mathrm{THF} / \mathrm{MeCN}$ & 0 & 6 & & & \\
\hline & & & & $0 \rightarrow \mathrm{RT}$ & 16 & 0:60:0:40:0 & 100 & 70 \\
\hline 7 & 2.0 & 2.0 & THF/THF & 0 & 1.5 & $0: 66: 18: 16: 0$ & 95 & 46 \\
\hline 8 & 2.0 & 2.0 & THF/THF & 0 & 2.5 & $0: 96: 0: 4: 0$ & 100 & 83 \\
\hline 9 & 2.0 & 3.0 & THF/THF & 0 & 3 & 0:0:0:100:0 & 50 & - \\
\hline 10 & 3.0 & 3.0 & THF/THF & $0 \rightarrow \mathrm{RT}$ & 20 & 30:61:0:9:0 & 71 & - \\
\hline
\end{tabular}

Bei den Umsetzungen 1 und 3 konnte als weiteres Nebenprodukt neben dem Dien 136 und dem Dipyronethan 137 das methylierte Pyron 138 charakterisiert werden. Die häufig beobachtete Bildung des Methylierungsproduktes 138 wurde auf die Unselektivität der Substituenten-Übertragung von den Alanaten 124, 124A bzw. 124R auf das Brommethylpyron 96 im Verlauf der Kreuzkupplungsreaktion zurückgeführt, da neben der Übertragung der Alke- 
nylgruppe auch teilweise die Methylgruppe übertragen wurde. Der im Vergleich zur Alkenylübertragung geringere Anteil der Methylübertragung ist mit dem Umstand zu erklären, dass die Alkenylgruppen aufgrund ihrer besseren Stabilisierung sowie ihres größeren sterischen Anspruchs leichter übertragen werden. Auch wenn im Allgemeinen die Synthese des Kreuzkupplungsproduktes mit 83\%-iger Ausbeute nach säulenchromatographischer Reinigung gelang, wurde stets die Bildung der genannten Nebenprodukte in unterschiedlichen Produktanteilen beobachtet. Bemerkenswert war das Ergebnis der Umsetzungen 4 und 9 (vgl. Tabelle 13), bei denen als einzige gebildete Produkte ausschließlich die unerwünschten Verbindungen Dien 136 und Dipyronethan 137 in 65\%- und 100\%-igem Anteil gebildet wurden.

Auch bei der Synthese des Iromycin A-Vorläufers 125A mit Brommethylpyron 96 und dem Alanat 124A wurde erwartungsgemäß die Bildung des Diens als Strukturdimer der Seitenkette 136A, des Dipyronethans 137 als Homokupplungsprodukt und des 6-Ethylpyrons 138 als Methylierungsprodukt des Eduktes 96, das durch die Substitution der Bromidfunktion mit einer Methylgruppe entstand, beobachtet (vgl. Tabelle 14). Bei dem Versuch 1 wurde das methylierte Produkt 138 als Hauptprodukt (Tabelle 14, Eintrag 1: Ausbeute 56\%) gebildet und bei der Umsetzung 2 (Tabelle 14, Eintrag 2) in einem etwa gleichhohen Bildungsanteil von 38\% neben dem Dipyronethan (42\%). Die Verbindungen 137 und 138 wurden isoliert und analytisch nachgewiesen. Die Synthese des Kreuzkupplungsproduktes 125A konnte schließlich in erfreulichen Ausbeuten bei Einsatz von je 3.0 eq Alan 123A und $n$ BuLi (Tabelle 14, Eintrag 5: Ausbeute 90\%) bzw. 3.0 eq Alan 123A und 2.0 eq $n$ BuLi im Unterschuß (Tabelle 14, Eintrag 7: Ausbeute 95\%) bei einer Temperatur von $0{ }^{\circ} \mathrm{C}$ realisiert werden.

Tabelle 14. Kreuzkupplungsreaktionen des Brommethylpyrons 96 mit dem Alanat 124A zu dem Iromycin A-Vorläufer 125A.

\begin{tabular}{cccccccc}
\hline Eintrag & $\begin{array}{c}\text { 123A } \\
{[\mathrm{eq}]}\end{array}$ & $\begin{array}{c}n \mathrm{BuLi} \\
{[\mathrm{eq}]}\end{array}$ & $\begin{array}{c}\mathrm{T} \\
{\left[{ }^{\circ} \mathrm{C}\right]}\end{array}$ & $\begin{array}{c}\mathrm{t} \\
{[\mathrm{h}]}\end{array}$ & $\begin{array}{c}\mathbf{9 6 : 1 2 5 A : 1 3 6 A : 1 3 7 : 1 3 8} \\
(\%)\end{array}$ & $\begin{array}{c}\text { MB } \\
(\%)^{\mathrm{a})}\end{array}$ & $\begin{array}{c}\text { Ausbeute } \\
(\%)^{\mathrm{b})}\end{array}$ \\
\hline $1^{\mathrm{c})}$ & 2.0 & 2.0 & $0, \mathrm{RT}$ & 3,3 & $0: 4: 7: 33: 56$ & 63 & - \\
$2^{\mathrm{d})}$ & 2.0 & 2.0 & $0, \mathrm{RT}$ & 3,1 & $0: 16: 4: 42: 38$ & 99 & 43 \\
3 & 2.0 & 3.0 & $0 \rightarrow \mathrm{RT}$ & 15 & $20: 52: 1: 27: 0$ & 72 & - \\
4 & 3.0 & 3.0 & $0 \rightarrow \mathrm{RT}$ & 20 & $7: 84: 0: 9: 0$ & 93 & 33 \\
5 & 3.0 & 3.0 & $0, \mathrm{RT}$ & 2,2 & $0: 96: 0: 4: 0$ & 94 & 90 \\
6 & 3.0 & 3.0 & 0 & 2 & $0: 95: 0: 5: 0$ & 100 & 56 \\
7 & 3.0 & 2.0 & 0 & 6 & $0: 100: 0: 0: 0$ & 100 & 95 \\
8 & 4.0 & 4.0 & $0, \mathrm{RT}$ & 2,4 & $9: 79: 0: 12: 0$ & 94 & - \\
\hline a)
\end{tabular}

${ }^{a}$ Massenbilanz: Verhältnis der erhaltenen Rohproduktmasse und eingesetzten Eduktmasse. b) Ausbeute nach säulenchromatographischer Reinigung. ${ }^{\text {c) }}$ Methylierungsprodukt $(60 \%) .{ }^{d)}$ Methylierungsanteil (38\%). 
Die Versuche zur Darstellung des Vorläuferproduktes 125R des Iromycins R wurden ausschließlich unter Unterschußbedingungen an $n$ BuLi durchgeführt (vgl. Tabelle 15, Einträge 1, 2 und 3), um das Ausbleiben eventueller Zersetzungserscheinungen und Debromierungen zu gewährleisten. Hierbei konnte das Kreuzkupplungsprodukt 125R in Ausbeuten von 38-45\% isoliert werden. Die erhöhten Reaktionszeiten der Umsetzungen 2 und 3 waren auf eine unbekannte Verunreinigung zurückzuführen, die über DC detektiert wurde. Da diese eine identische Polarität mit dem eingesetzten Bromid 96 aufwies, war zunächst angenommen worden, dass die Umsetzung noch nicht vollständig erfolgt war. Die Tatsache jedoch, dass nach Zugabe weiterer Alanatlösung 124R keine weitere Reaktionsentwicklung beobachtet werden konnte, ließ die Vermutung zu, dass die Reaktion schon nach 2 h, wie erwartet, vollständig abgelaufen war.

Tabelle 15. Kreuzkupplungsreaktionen des Brommethylpyrons 96 mit dem Alanat 124R zu dem Iromycin R-Vorläufer 125R.

\begin{tabular}{cccccc}
\hline Eintrag & $\begin{array}{c}\text { Alan 123R } \\
{[\mathrm{eq}]}\end{array}$ & $\begin{array}{c}n \mathrm{BuLi} \\
{[\mathrm{eq}]}\end{array}$ & $\begin{array}{c}\mathrm{t} \\
{[\mathrm{h}]}\end{array}$ & $\begin{array}{c}\text { 96:125R:136R:137 } \\
(\%)\end{array}$ & $\begin{array}{c}\text { Ausbeute } \\
(\%)^{\mathrm{a})}\end{array}$ \\
\hline 1 & 3.0 & 2.0 & 2 & $0: 75: 5: 20: 0$ & - \\
2 & 6.0 & 4.0 & 6 & $14: 69: 10: 7: 0$ & 45 \\
3 & 4.5 & 3.0 & 6 & $0: 100: 0: 0: 0$ & 38 \\
\hline
\end{tabular}

Bei den durchgeführten Kreuzkupplungsreaktionen mit Pyronhalogeniden und Alanaten wurden zum Zwecke der Optimierung des Syntheseverfahrens der Einfluss des Lösungsmittels, der Alanatmenge sowie der Reaktionstemperatur auf das Reaktionsgeschehen systematisch untersucht. Im Rahmen der Untersuchung des Lösungsmitteleinsatzes wurde neben der Verwendung von THF sowohl der Einsatz von polaren $\left(\mathrm{Et}_{2} \mathrm{O}, \mathrm{MeCN}\right)$ wie unpolaren Lösungsmitteln (Toluol) überprüft. Die Variation des Lösungsmittels wurde hauptsächlich in den Umsetzungen des Bromids 96 bzw. des Chlorids 135 mit dem Alanat 124 zu dem Kreuzkupplungsprodukt 125 untersucht. Insgesamt konnte bei dem Einsatz von Toluol, $\mathrm{Et}_{2} \mathrm{O}$ und $\mathrm{MeCN}$ keine merkliche Verbesserung des Bildungsanteils des gewünschten Kreuzkupplungsproduktes beobachtet werden, weshalb diese Lösungsmittel bei den in den Tabellen 14 und 15 aufgeführten Umsetzungen nicht mehr verwendet wurden. Die in der Tabelle 13 aufgeführten Umsetzungen in THF führten überwiegend zu zufriedenstellenden, im Einzelfall sogar zu sehr guten Bildungsanteilen des Kreuzkupplungproduktes 125 (vgl. Eintrag 8, Anteil 96\%, Ausbeute 
83\%). Sowohl bei Verwendung von im Vergleich zu THF polareren als auch unpolareren Lösungsmitteln wurde tendenziell eher eine nachteilige Auswirkung auf das Gesamtbild des Reaktionsgeschehens festgestellt (vgl. Tabelle 13). Während die Umsetzung in Toluol (vgl. Tabelle 13, Eintrag 2) bei vollständigem Umsatz des Bromids 96 nur Spuren des Kreuzkupplungsproduktes 125 ergab, konnte bei dem in $\mathrm{Et}_{2} \mathrm{O}$ durchgeführten Versuch bei $-30{ }^{\circ} \mathrm{C}(\mathrm{vgl}$. Tabelle 13, Eintrag 3) ein akzeptabler Anteil von 52\% an dem Iromycinvorläufer 125 erreicht werden. Alternativ wurde auch die kombinatorische Umsetzung von THF und MeCN untersucht (vgl. Tabelle 13, Eintrag 6). Hierzu wurde das Alanat lediglich in THF generiert, um die Bildung der bei den Versuchen mit Benzylbromid beobachteten unerwünschten Nebenprodukte (vgl. Abschnitt 2.2.2.1 u. Tabelle 12, Eintrag 2) zu vermeiden, und anschließend eine Lösung des Bromids 96 in MeCN zu dem vorgenerierten Alanat gegeben. Es entstand ein Gemisch aus dem Kreuzkupplungsprodukt 125 als Hauptprodukt und dem Dipyronethan 137 als Nebenprodukt im Verhältnis 60:40 zugunsten des Vorläuferproduktes des Iromycinanalogons 125. Insgesamt konnte festgestellt werden, dass die Bildungsanteile des Kreuzkupplungsproduktes 125 in dem polar koordinierenden Lösungsmittel THF am Besten reproduziert werden konnten, da es zu einer effektiveren Stabilisierung des Alans während des Alanatbildung beizutragen imstande ist.

Die Generierung der Alanate wurde sowohl unter Verwendung von stöchiometrischen als auch von überschüssigen Mengen an Alanen und $n \mathrm{BuLi}$ durchgeführt und untersucht. Insgesamt wurde ein breites Spektrum von Äquivalenzen an Alanen und $n$ BuLi von 1.0-6.0 eq in den Versuchen eingesetzt (vgl. Tabellen 13-15). Die Versuchsergebnisse lassen eine Tendenz zu höheren Produktanteilen sowie exzellenten Ausbeuten bei Verwendung überschüssiger Äquivalenzmengen von 3.0-4.5 eq an Reagenzien erkennen (vgl. Tabelle 14, Eintrag 7: 100\% Produktanteil u. 95\% Ausbeute, Eintrag 5: 96\% Produktanteil u. 90\% Ausbeute sowie Eintrag 8: 79\% Produktanteil u. 94\% Ausbeute an Rohprodukt; vgl. Tabelle 15, Eintrag 3: 100\% Umsatz u. 38\% Ausbeute). Die erzielten hohen Produktanteile bzw. Ausbeuten sind zwar auf die hohe Alan-Konzentration zurückzuführen, jedoch ist ein hoher Alan-Einsatz keine zwingend notwendige Voraussetzung für das Erlangen von hohen Umsätzen und Ausbeuten. Dies belegen einerseits die von 1.5-2.0 eq ausgehenden Umsetzungen, bei denen recht akzeptable Produktanteile erzielt wurden (vgl. Tabelle 13, Eintrag 1: 79\% Produktanteil u. 90\% Rohprodukt, Eintrag 8: 96\% Produktanteil u. 83\% Ausbeute), und andererseits die unzufriedenstellenden Produktanteile mit mäßigen Ausbeuten unter Einsatz eines Überschusses von 4-6 eq an Reagenzien (vgl. Tabelle 15, Eintrag 2: 69\% Produktanteil u. 45\% Ausbeute). Diese scheinbar 
widersprüchlichen Ergebnisse werden wohl auf die fehlende Konzentrationsabstimmung (reales Stoffmengenverhältnis) zwischen der Alan- und $n$ BuLi-Konzentration zurückzuführen sein, deren Abstimmmung sich als schwierig erwies. Da ein hoher Anteil an $n$ BuLi gegenüber dem Alan nachteilig zu einer Debromierung bzw. zur Bildung von Nebenprodukten führte, wie in den analogen Umsetzungen des Benzylbromids kurz beschrieben wurde (vgl. Abschnitt 2.2.2.1), sollte ein anteiliger Überschuß an $n$ BuLi vermieden werden. Eines der Probleme bei der massenbezogenen Herstellung der Alanlösungen (zur Alandarstellung vgl. Abschnitt 2.2.1 u. Exp. Teil, Allg. Arbeitsvorschrift A5, Varianten 1 und 2) besteht zudem darin, dass die hergestellte Lösung nicht die tatsächliche Konzentration an reaktivem Alan wiedergibt, sondern allenfalls annäherungsweise, denn die in dem eingewogenen Alan vorhandenen Verunreinigungen, wie Zirconiumsalze oder evtl. geringfügige $\mathrm{AlMe}_{3}$-Reste, verfälschen die isolierte Masse. Darüber hinaus ist eine mehrmalige Abfiltration der Alane zur Entfernung der Verunreinigungen vor allem wegen der bestehenden besonders großen Hydrolyseempfindlichkeit der Alane und etwaiger Massenverluste nicht empfehlenswert. Daher bietet sich als Konsequenz entweder eine besonders genaue Bestimmung der Alankonzentration durch die Verwendung von Referenzsubstanzen bei der GC-analytischen Konzentrationsbestimmung oder der Einsatz von $n$ BuLi im Unterschuß im Verhältnis zum eingesetzten Alan an. Durch Letzteres bleibt zwar der Unterschuß an Base in der Reaktionslösung gewährleistet, jedoch ist diese Vorgehensweise mit einem Verlust an Alan und damit auch einer verminderten Alanatbildung verbunden.

Die Temperaturveränderung als weiterer maßgeblicher Einflußfaktor auf das Reaktionsgeschehen wurde vorwiegend in den in der Tabelle 13 aufgeführten Versuchen mit Brommethylpyron 96 und dem Alanat 124 untersucht. Der Vergleich der Reaktionsbedingungen der Versuche in Toluol, $\mathrm{Et}_{2} \mathrm{O}$ und THF bei $-30{ }^{\circ} \mathrm{C}$ (vgl. Tabelle 13, Einträge 2-4) führte unter Einsatz von je 1.5 eq Alanat 124 im Falle der Umsetzungen 2 und 4 zu Spuren bzw. zum Ausbleiben des Kupplungsproduktes, während bei dem Versuch 3 ein Anteil von 52\% des Kreuzkupplungsproduktes gebildet wurde. Unter Berücksichtigung des Lösungsmitteleinflusses (Toluol, $\mathrm{Et}_{2} \mathrm{O}$ und $\mathrm{MeCN}$ ) könnte für das Ausbleiben des Kupplungsprodukts in THF bei $-30^{\circ} \mathrm{C}$ angenommen werden, dass tiefere Temperaturen bei der Durchführung der Kreuzkupplungsreaktionen anscheinend nicht als ideale Reaktionsbedingungen zu betrachten sind. Angesichts der erzielten exzellenten Produktanteile und Ausbeuten bei einer Reaktionstemperatur von $0{ }^{\circ} \mathrm{C}$ und einer Reaktionszeit von $2 \mathrm{~h}$ (vgl. Tabelle 13, Eintrag 8: 96\% Produktanteil u. 83\% Ausbeute, vgl. Tabelle 14, Eintrag 5: 96\% Produktanteil u. 90\% Ausbeute, Eintrag 6: 
95\% Produktanteil u. 56\% Ausbeute) und der Tatsache, dass eine weitere Temperaturerhöhung auf Raumtemperatur weder zu erhöhten Ausbeuten noch zu einer merklich sichtbaren Beschleunigung der Reaktionsgeschwindigkeit geführt hatte, kann die Durchführung der Umsetzungen bei $0{ }^{\circ} \mathrm{C}$ als bevorzugte Temperaturbedingung angesehen werden. Als Gesamtergebnis der Optimierungsversuche konnte festgestellt werden, dass eine Generierung des Alanats in THF unter Einsatz von 3.0 eq Alan und 2.0 eq $n \mathrm{BuLi}$ bei $0{ }^{\circ} \mathrm{C}$ und die Durchführung der Reaktion nach Zugabe einer Lösung von Pyronhalogeniden in THF bei dieser Temperatur angestrebt werden sollte.

Auffällig bei den Kreuzkupplungsreaktionen war die Bildung der Nebenprodukte 136, 136A, 136R, 137 und 138 (vgl. Schema 34), die maßgeblich zu den erzielten geringen Kreuzkupplungsproduktausbeuten beitrug. Die Nebenprodukte entstanden teilweise sogar als Hauptprodukte der Umsetzungen. Insbesondere die Bildung des durch Seitenkettenhomokupplungen enstandenen Diens 136 bzw. des aus dem eingesetzten Hauptgrundgerüst Brommethylpyron 96 stammenden Dipyronethans 137 wurde fast bei allen Kreuzkupplungsreaktionen beobachtet. Die erhaltenen Verbindungen Tetradecadien 136 und Dipyronethan 137 wurden spektroskopisch sowie massenanalytisch untersucht und nachgewiesen. Das Dien 136 und das Dipyronethan 137 zeigten jeweils ein charakteristisches Singulett, nämlich im Falle des Diens 136 bei 6.0 ppm für zwei olefinische Protonen und im Falle des Dipyronethans 137 bei 2.98 ppm für vier aliphatische Protonen. Bei 6-Ethylpyron 138 wurden ein Triplett sowie ein Quartett bei $1.27 \mathrm{ppm}$ und $2.6 \mathrm{ppm}$ den Protonen der beiden an der C-2'- und C-1'-Position vorhandenen Kohlenstoffzentren zugeordnet. Aufgrund fehlender geeigneter Reaktionsbedingungen dürften sowohl eine radikalische Reaktion als auch eine auf einem getrennten Ionen-Paar basierende nucleophile Substitutionsreaktion $\left(\mathrm{S}_{N} 1\right)$ als Ursachen für die Nebenproduktbildung mit höchster Wahrscheinlichkeit ausgeschlossen werden können. Gegen die Annahme einer radikalischen Reaktion sprechen das Fehlen von hohen Temperaturen bzw. Licht sowie die Verwendung eines unpolaren Lösungsmittels. Für das Vorliegen einer $\mathrm{S}_{N} 1$-Reaktion fehlen die Verwendung eines polar protisches Lösungsmittels, die Qualität der Abgangsgruppe sowie die Bildung eines instabilen primären Kations. Da die vorherrschenden Reaktionsbedingungen hingegen für eine nucleophile Substitution $\mathrm{S}_{N} 2$ prädestiniert sind, könnte die Bildung des Dipyronethans 137 auf den im nachfolgenden Schema 35 dargestellten zwei Wegen erfolgt sein. 
Schema 35. Mechanismus der Bildung des Nebenproduktes 137.

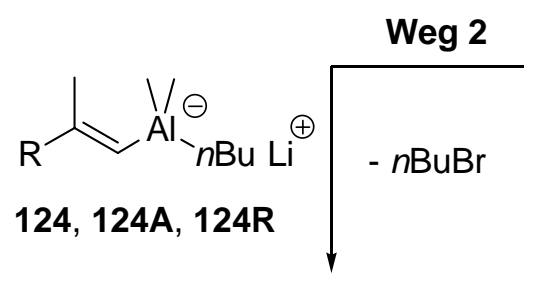<smiles>CCCc1c(CBr)oc(=O)c(C)c1OC(=O)OC</smiles>
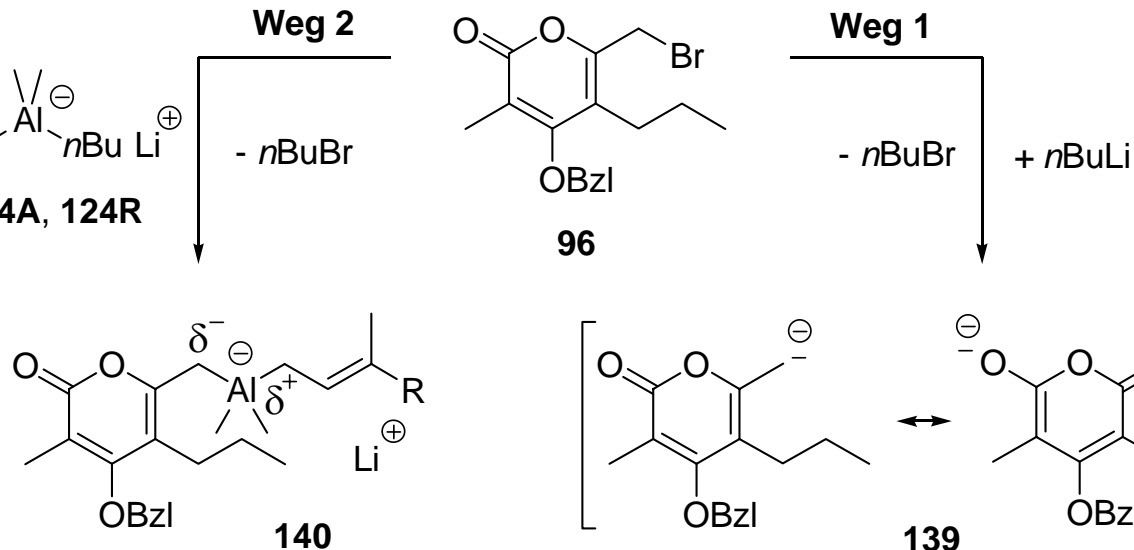<smiles>C=C(O)c1c(CCC)c([O-])oc(=O)c1C</smiles>

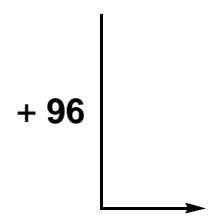<smiles>[R17]c1c(CCC)c(CCc2oc(=O)c(C)c([R8])c2CCC)oc(=O)c1C</smiles>

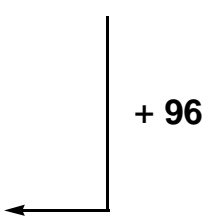

Eine auf einem getrennten Ionenpaar basierende nucleophile Substitution $\mathrm{S}_{N} 1$ ist aufgrund der elektronischen Struktur des Moleküls unwahrscheinlich. Bei einer theoretisch möglichen Abspaltung eines Bromid-Anions im Brommethylpyron 96 würde das gebildete allylische, ohnehin strukturell instabile primäre Kation - auch wenn bei Allylkationen die Elektronenlücke durch Konjugation der Doppelbindung vermindert ist und diese daher stabiler sind als die nicht allylischen primären Kationen - zusätzlich durch den starken Elektronenzug der Carbonylgruppe entlang der doppelt vinylogen Doppelbindungsstruktur des Ringes in Richtung der Carbonylgruppe aufgrund des $-M$-Mesomeren Effekts destabilisiert. Daher wird ein anionischer Reaktionsmechanismus bei der Bildung des Homokupplungsproduktes 137 favorisiert. Dieser Reaktionsweg wird durch einen anfänglichen Angriff von überschüssigem $n \mathrm{BuLi}$ an das Bromidatom aufgrund der Polarisierbarkeit des Halogenids unter Lithium-HalogenAustausch und die Bildung des $n$ Butylbromids initialisiert (vgl. Schema 35, Weg 1). Im Gegensatz zu der kationischen Spezies ( $\mathrm{S}_{N} 1$-Substitution) dürfte das Anion 139 aufgrund der Durchkonjugation der Doppelbindungen wegen des $-M$-Mesomeren Effekts der Carbonylgruppe, begründet auf mehreren mesomeren Grenzstrukturen, einen erheblichen Stabilitätszuwachs aufweisen, die dem Anion 139 eine Langlebigkeit im Vergleich zu seinem analogen Kation sichert. Daher ist ein durch den Überschuß an $n$ BuLi induziertes Anion 139 nun 
selbst in der Lage, als Nucleophil zu fungieren und direkt das Brommethylpyron 96 nucleophil anzugreifen. Der elektrophile Angriff des noch im Reaktionsmedium befindlichen, unverbrauchten Brommethylpyrons 96 auf das Anion 139 könnte dann zu der Bildung des Homokupplungsproduktes 137 führen. Das Ausmaß der Bildung des Dipyronethans 137 wird durch die überschüssige Menge an $n \mathrm{BuLi}$ in der Reaktionslösung bestimmt. Eine weitere Entstehungsvariante des Homokupplungsproduktes 137, die gegenüber dem vorstehend dargestellten Bildungsweg allerdings weniger wahrscheinlich erscheint, könnte in der Carboaluminierung des Hauptgrundgerüstes 96 zu der Spezies 140, die auf dem im Schema 35 dargestellten Weg 2 initialisiert werden kann, liegen. Die Bildung des Alanats 140 könnte hiernach durch einen versuchten nucleophilen Angriff der Alkenylalanate 124, 124A und 124R herbeigeführt werden, der auf die Polarisierbarkeit des Bromidatoms zurückzuführen wäre (vgl. Schema 35, Weg 2).

Die Ergebnisse der Versuche zur Kreuzkupplung des Brommethylpyrons 96 mit dem Heptenylalanat 124 sowie den Alkenylalanaten 124A und 124R zeigten das Gelingen der C-CBindungsbildung und die hohe Effektivität dieser Methode auf dem Weg zur Darstellung der gewünschten Iromycine bzw. Iromycin-Analoga. Die Kreuzkupplungsprodukte 125 und 125A konnten in guten bis exzellenten Ausbeuten von $83 \%$ bzw. $95 \%$ dargestellt werden, das Kreuzkupplungsprodukt 125R in akzeptabler Ausbeute von $45 \%$. Im Allgemeinen lieferte die Darstellung der Iromycin-Vorläufer über die Generierung des Alanats in THF unter Einsatz von 3.0 eq Alan und 2.0 eq $n$ BuLi bei $0{ }^{\circ} \mathrm{C}$ und die Durchführung der Reaktion nach Zugabe einer Lösung des Brommethylpyrons 96 in THF bei dieser Temperatur die besten Ergebnisse.

\subsection{Synthese der Iromycine A und R sowie deren Analogons durch O-N-Austausch}

Als nächster Schritt zur synthetischen Darstellung der Iromycine 23A und 23R und des Iromycin-Analogons 23 war die Transformation der O-Analoga 125A, 125R und 125 zu den NAnaloga geplant (vgl. Schema 36). Die O-N-Austausch-Versuche wurden parallel unter Ver-

wendung von $\mathrm{NH}_{3}$ und $\mathrm{NH}_{2} \mathrm{NH}_{2}$ untersucht. Moreno-Maňas et al. ${ }^{[63]}$ berichteten von der Umwandlung der Pyrone zu den entsprechenden $N$-Analoga unter Einsatz eines Überschusses an wässriger $\mathrm{NH}_{3}$-Lösung im Bombenrohr bei $100-110{ }^{\circ} \mathrm{C}$ (Reaktionszeit $26 \mathrm{~h}$, Ausbeute 86\%). Diese Methode wurde für den durchgeführten O-N-Austausch bei den hier verwendeten Pyronen entsprechend angewandt. Mechanistisch verläuft der O-N-Austausch bei den 
Kreuzkupplungsprodukten 125, 125A und 125R über einen nucleophilen Angriff von $\mathrm{NH}_{3}$ unter Debenzoylierung und Bildung des Benzamids zu den entschützten Pyronen 150, 150A und 150R (vgl. Schema 36). Ein weiterer Angriff des Ammoniaks initialisiert die Ringöffnung unter Umfunktionalisierung der Molekülkette zu dem Amid 151, dessen intramolekularer Angriff an die Ketofunktion unter Ausscheidung des Wassers die Triebkraft der Recyclisierung zu dem Kation 152 darstellt. Die anschließende Protonenwanderung unter Wiederherstellung der Doppelbindung liefert die gewünschte Iromycin-Grundstruktur 23.

Schema 36. O-N-Austausch des Kreuzkupplungsprodukts $125 \mathrm{zu}$ den Iromycinen 23A und 23R und dem Iromycin-Analogon 23.<smiles>[R17]Oc1c(CCC)c(CC=C([R])C)oc(=O)c1C</smiles>

$125,125 A, 125 R$

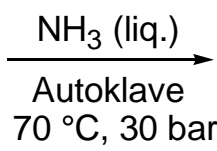

44-48 h<smiles>[R]C(C)=CCc1[nH]c(=O)c(C)c(O)c1CCC</smiles>

23, 23A, 23R $-\mathrm{PhCONH}_{2} /+\mathrm{NH}_{3}$<smiles>[R]/C(C)=C/Cc1oc(=O)c(C)c(O)c1CCC</smiles>

150, 150A, 150R<smiles>[R]C(=O)/C(C)=C(/O)C(CCC)C(=N)CC=C([R])C</smiles>

151<smiles>[R]C(C)=CCC1=NC(=O)C(C)=C(O)C1(C)CCC</smiles>

152

\begin{tabular}{l|c|c} 
Edukt & Produkt & \multicolumn{1}{|c}{$\mathrm{R}$} \\
\hline 125 & 23 & $\mathrm{CH}_{3}\left(\mathrm{CH}_{2}\right)_{4}$ \\
125A & $23 \mathrm{~A}$ & $\left(\mathrm{CH}_{3}\right)_{2} \mathrm{CHCH}=\mathrm{CHCH}_{2}$ \\
$125 \mathrm{R}$ & $23 \mathrm{R}$ & $\left(\mathrm{CH}_{3}\right)_{3} \mathrm{CCH}=\mathrm{CHCH}_{2}$
\end{tabular}

Die Vorversuche wurden zunächst mit dem Kreuzkupplungsprodukt 125 und einer übersättigten methanolischen $\mathrm{NH}_{3}$-Lösung nach Moreno-Maňas durchgeführt (vgl. Schema 37). Zur Durchführung des O-N-Austausches des Kreuzkupplungsproduktes 125 wurden die Transformationsversuche jeweils entsprechend den in der Tabelle 16 angegebenen milden Reaktionsbedingungen durchgeführt. Die erhaltenen Spektren der bei Raumtemperatur durchgeführten Versuche (vgl. Tabelle 16, Einträge 1 und 2) bestätigten die über Dünnschichtchroma- 
tographie gewonnenen Schlussfolgerungen. So zeigte eine Referenzauftragung (DC) die Bildung des Benzamids an, die die erwartete Entschützung im basischen Medium belegte (Tabelle 16, Eintrag 1). Die Auswertung der Spektren ergab als Hauptprodukt das debenzoylierte Entschützungsprodukt 150 (vgl. Schema 37). Es konnte keine entsprechende Umsetzung zu dem N-Analogon des Eduktes 125 festgestellt werden. Eine nach säulenchromatographischer Reinigung durchgeführte, hochauflösende massen- sowie IR-spektroskopische Analyse der Umsetzungen (fehlende Schlüsselbande für Amide bei $1640-1530 \mathrm{~cm}^{-1}$, lit. $1660 \mathrm{~cm}^{-1}$ ) wies den Erhalt des Entschützungsproduktes 150 (Schlüsselbande bei $1734 \mathrm{~cm}^{-1}$ ) als O-Analogon nach.

Schema 37. O-N-Austausch des Kupplungsprodukts $125 \mathrm{zu}$ dem Iromycin-Analogon 23.<smiles>CCCc1c(C/C=C(\C)[Po])oc(=O)c(C)c1OCC</smiles>

125

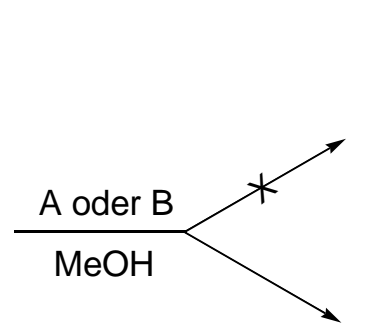

A: $\mathrm{NH}_{4} \mathrm{OH}$ (Überschuß) B: $\mathrm{NH}_{4} \mathrm{OAc}(1.0 \mathrm{eq})$<smiles>CCCPC(C)=CCc1[nH]c(=O)c(C)c(O)c1CCC</smiles>

150

Tabelle 16. O-N-Austausch des Kreuzkupplungsprodukts 125 zu dem IromycinAnalogon 23 mit $\mathrm{NH}_{4} \mathrm{OH}$-Lösung in $\mathrm{MeOH}$.

\begin{tabular}{|c|c|c|c|c|c|}
\hline Eintrag & N-Träger & Umsetzung in & $\mathrm{T}\left[{ }^{\circ} \mathrm{C}\right]$ & $\mathrm{t}[\mathrm{h}]$ & 150:23 (\%) \\
\hline 1 & $\mathrm{NH}_{4} \mathrm{OH}^{\mathrm{a})}$ & Autoklave & RT & 18 & $1: 0$ \\
\hline 2 & $\mathrm{NH}_{4} \mathrm{OH}^{\mathrm{a})}$ & Standard $^{\mathrm{b})}$ & RT & 72 & $1: 0$ \\
\hline 3 & $\mathrm{NH}_{4} \mathrm{OH}^{\mathrm{a})}$ & Bombenrohr & 60 & 72 & \\
\hline & & & 80 & 72 & $1: 0$ \\
\hline 4 & $\mathrm{NH}_{4} \mathrm{OAc}$ & Standard $^{\text {b) }}$ & RT & 96 & $1: 0$ \\
\hline
\end{tabular}

Die im Bombenrohr durchgeführte Umsetzung bei $60-80{ }^{\circ} \mathrm{C}$ (Tabelle 16, Eintrag 3) sowie der Versuch des O-N-Austausches unter Einsatz von $\mathrm{NH}_{4} \mathrm{OAc}$ in $\mathrm{MeOH}^{[64]}$ (Tabelle 16, Eintrag 4) führten ausschließlich zur Bildung des Entschützungsprodukts 150 (s. Exp. Teil, Allg. Ar- 
beitsvorschrift A14, Variante 3). Dies konnte über Detektion mit einer Referenzprobe 150, die durch Entschützung des Benzoates 125 in 10\%-iger NaOH-Lösung in THF bei RT nach $26 \mathrm{~h}$ hergestellt wurde, bestätigt werden. Die möglichen Ursachen für das Misslingen der Vorversuche dürften hauptsächlich in der für die O-N-Austauschsreaktion benötigten hohen Aktivierungsenergie sowie daneben in geringem Maße in der Verschiebung des Gleichgewichtes zum Nachteil der Bildung des Iromycin-Analogons durch die Ausscheidung von Wasser liegen. Daher erschienen die Erhöhung der Temperatur und die Durchführung der Reaktion bei drastischeren Bedingungen erforderlich.

Aufgrund der vorstehenden Erwägungen wurde die Durchführung der Umsetzungen in reinem Ammoniak unter Ausschluß von Wasser untersucht. Hierzu wurden jeweils Lösungen der entsprechenden Iromycin-Vorläufer 125, 125A und 125R in einem Autoklave gegeben und das Lösungsmittel unter vermindertem Druck entfernt. Anschließend wurde bei $-78{ }^{\circ} \mathrm{C}$ wasserfreier Ammoniak in dem Autoklave einkondensiert (s. Exp. Teil, Allg. Arbeitsvorschrift A14, Variante 1). Die O-N-Transformationsreaktionen wurden entsprechend der in der Tabelle 17 angegebenen Reaktionsbedingungen durchgeführt. Die Umsetzung der Reaktion unter milden Bedingungen bei Raumtemperatur (vgl. Tabelle 17, Eintrag 1) - die milden Bedingungen waren im Hinblick auf die Doppelbindungsfunktionen der Iromycine sowie im Falle späterer Synthesen des Iromycins B für dessen sensible OH-Funktionen an der Seitenkette von besonderer Relevanz - ergab nach Aufarbeitung und anschließender säulenchromatographischer Reinigung ausschließlich das Entschützungsprodukt 150. Der Umstand, dass der erhöhte Druck alleine nicht als ausreichende Bedingung für die O-N-Transformation ausreicht, konnte in weiteren Versuchen durch Erhöhung der Temperatur belegt werden (vgl. Tabelle 17, Einträge 2 u. 3). Die bei $50{ }^{\circ} \mathrm{C}$ durchgeführte Reaktion ergab lediglich das debenzoylierte Produkt 150. Die Erhöhung der Temperatur auf $65{ }^{\circ} \mathrm{C}$ bei einem Druck von 20 bar führte nach $18 \mathrm{~h}$ zum Erhalt eines Gemisches aus dem Entschützungsprodukt 150 (Anteil 74\%) und dem gewünschten N-Analogon 23 (Anteil 26\%).

Der vollständige O-N-Austausch zu dem Iromycin-Analogon 23 gelang schließlich erst bei Durchführung des Umfunktionalisierungprozesses unter Erhöhung der Reaktionstemperatur auf $70{ }^{\circ} \mathrm{C}$ bei 30 bar nach $48 \mathrm{~h}$ (Tabelle 17, Eintrag 4). Die analytischen Untersuchungen $\left({ }^{1} \mathrm{H}-\right.$ NMR, ${ }^{13}$ C-NMR sowie HMRS) belegten die Bildung des Iromycin-Analogons 23. Der Vergleich des ${ }^{13} \mathrm{C}-\mathrm{NMR}-$ Spektrums des synthetisierten Stickstoffderivats $\mathbf{2 3}$ mit dem des isolierten Naturstoffs Iromycin A zeigte eine identische chemische Verschiebung für die charakte- 
ristischen C-6- und C-4-Kohlenstoffatome des Rings an $\left[{ }^{13} \mathrm{C} \mathrm{NMR},\left(75.5 \mathrm{MHz}, \mathrm{CD}_{3} \mathrm{OD}\right)\right.$ : 143.08 ( $\left.\mathrm{C}_{\text {quart }}, \mathrm{C}-6\right), 166.01$ ( $\left.\mathrm{C}_{\text {quart }}, \mathrm{C}-4\right)$, für Iromycin A (Lit.-Wert): 142.98 ( $\left.\mathrm{C}_{\text {quart }}, \mathrm{C}-6\right)$, $\left.166.01\left(\mathrm{C}_{\text {quart }}, \mathrm{C}-4\right)\right]$.

Schema 38. Aufarbeitung des 4-Aminopyridons $153 \mathrm{zu}$ dem Iromycin-Derivat 23 sowie ${ }^{1} \mathrm{H}-$ NMR-Spektrum des Iromycins A (23A).<smiles>[R10]Oc1c(CCC)c(CC=C([R])C)oc(=O)c1C</smiles>

$125,125 A, 125 R$ $70{ }^{\circ} \mathrm{C}, 30$ bar 44-48 h<smiles>CCCc1c(C/C=C(\C)[Pb])[nH]c(=O)c(C)c1N</smiles>

153, 153A, 153R
$1 \mathrm{M} \mathrm{KHSO}_{4}$<smiles>C1CCCCC1</smiles>

23, 23A, 23R

\begin{tabular}{l|c|c} 
Edukt & Produkt & $\mathrm{R}$ \\
\hline 125 & 23 & $\mathrm{CH}_{3}\left(\mathrm{CH}_{2}\right)_{4}$ \\
125A & 23A & $\left(\mathrm{CH}_{3}\right)_{2} \mathrm{CHCH}=\mathrm{CHCH}_{2}$ \\
125R & 23R & $\left(\mathrm{CH}_{3}\right)_{3} \mathrm{CCH}=\mathrm{CHCH}_{2}$
\end{tabular}

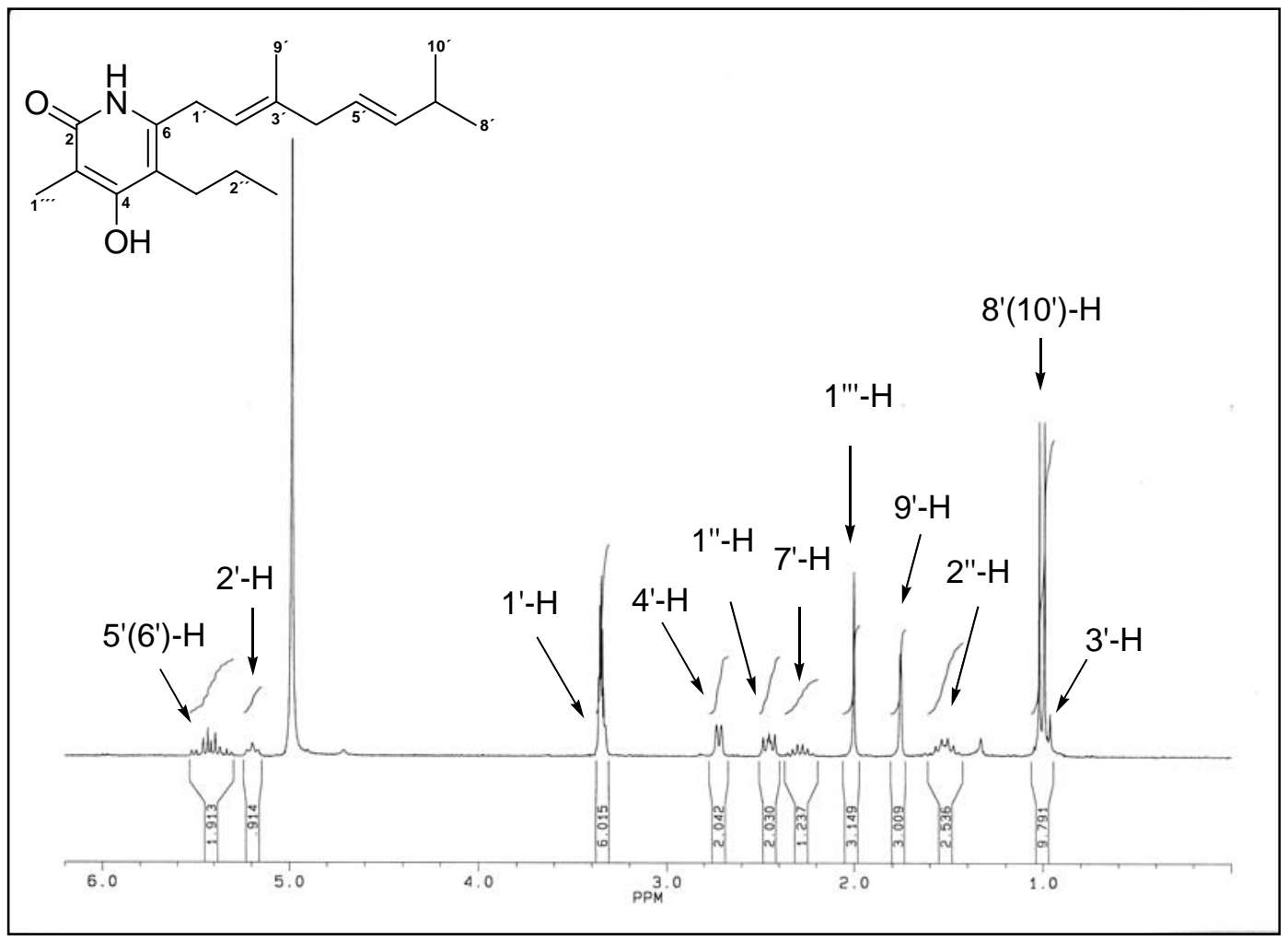


Hérault et al. ${ }^{[65]}$ beobachteten bei der Umwandlung der Pyrone zu den Pyridon-Analoga durch O-N-Austausch darüber hinaus die Substitution der 4-Hydroxygruppe zu einer 4Aminfunktion. Dies ließ die Annahme zu, dass im Rahmen der hier durchgeführten O-NAustausch-Reaktionen ein Angriff des $\mathrm{NH}_{3}$ auf das Michaelsystem des Ringes an der C-4Position stattgefunden und zur Substitution der funktionellen OH-Gruppe und der Bildung des Amins 153 geführt haben könnte (vgl. Schema 38). Die Reaktivität des Michael-Systems und die parallel zu den Versuchen mit $\mathrm{NH}_{3}$ durchgeführten O-N-Austauschreaktionen mit $\mathrm{NH}_{2} \mathrm{NH}_{2}$ (siehe unten, Schema 43), bei denen die entsprechende Aminierung der 4-Position des Pyrons 63 zu dem Hydrazin- 160 und dem Hydrazonderivat 161 beobachtet wurde, ließen sowohl die Bildung des 4-Aminopyrons 153 als auch die Resubstitution des Amins $153 \mathrm{zu}$ dem 4-Hydroxypyron 23 als wahrscheinlich erscheinen. Um eine eventuelle Bildung des 4Aminoprodukts 153 als Endprodukt auszuschließen, wurde eine Probe der zuvor mit PufferLösung und EtOAc aufgearbeiteten Reaktion (vgl. Tabelle 17, Eintrag 4) zusätzlich mit 1.0 M $\mathrm{KHSO}_{4}$-Lösung und EtOAc aufgearbeitet und die Stabilität des Iromycin-Analogons 23 geprüft. Durch weitere dünnschichtchromatographische und analytische Untersuchung der Probe konnte die Bildung des 4-Aminoprodukts 153 als Endprodukt definitiv ausgeschlossen werden (Bildung des 4-Aminoprodukts 153 als Zwischenprodukt ist jedoch möglich). Entsprechende Umsetzungen der Iromycin-Vorläufer 125A und 125R (Tabelle 17, Einträge 5 u. 6) ergaben unter identischen Reaktionsbedingungen und Aufarbeitung mit $1.0 \mathrm{M} \mathrm{KHSO}_{4^{-}}$ Lösung nach Reaktionszeiten von 48 h bzw. 44 h schließlich die gewünschten Naturstoffe Iromycin A (23A) und Iromycin R (23R) in einer Ausbeute von 64\% bzw. 63\%.

Tabelle 17. Transformation der Iromycin-Vorläufer 125A, 125R u. 125 in die Iromycine 23A u. 23R sowie das Iromycin-Analogon 23 mit wasserfreiem $\mathrm{NH}_{3}$.

\begin{tabular}{ccccccc}
\hline Eintrag & Edukt & $\mathrm{T}\left[{ }^{\circ} \mathrm{C}\right]$ & $\mathrm{P}[\mathrm{bar}]$ & $\mathrm{t}[\mathrm{h}]$ & $\mathbf{1 5 0 : 2 3}(\%)$ & Ausbeute $(\%)^{\mathrm{b})}$ \\
\hline 1 & $\mathbf{1 2 5}$ & $\mathrm{RT}$ & 10 & 60 & $100^{\mathrm{a})}: 0$ & - \\
2 & $\mathbf{1 2 5}$ & 50 & 20 & 6 & $100^{\mathrm{a})}: 0$ & - \\
3 & $\mathbf{1 2 5}$ & 65 & 20 & 18 & $74^{\mathrm{a})}: 26$ & - \\
4 & $\mathbf{1 2 5}$ & 70 & 30 & 48 & $0: 100$ & 58 \\
5 & $\mathbf{1 2 5 A}$ & 70 & 30 & 48 & $0: 100$ & 64 \\
6 & $\mathbf{1 2 5 R}$ & 70 & 30 & 44 & $0: 100$ & 63 \\
\hline
\end{tabular}

a) Es wurde stets nur das entschützte Kreuzkupplungsprodukt 150 erhalten. ${ }^{\text {b) }}$ Ausbeute nach säulenchromatographischer Reinigung. 
Da bei den Vorversuchen zum O-N-Austausch mit $\mathrm{NH}_{3}$ Probleme aufgetreten waren, wurde parallel der Einsatz von Hydrazin als nucleophilerer Stickstoffträger zur Überführung des Kreuzkupplungsprodukts 125 in das entsprechende $N$-Analogon untersucht (vgl. Schema 39). Pednekar et al. ${ }^{[66]}$ berichteten von der Überführung der Pyrone in die entsprechenden $\mathrm{N}$ Aminopyridone unter Verwendung von Hydrazin in Dioxan unter Rückflußbedingungen. Diese Methode war zuvor bereits erfolgreich bei der Darstellung des Pyridons 62 aus dem Pyron 63 ausprobiert worden (s. Abschnitt 3.1.1).

Schema 39. Überführung des Kreuzkupplungsproduktes 125 in das Iromycin-Analogon 23 unter Verwendung von $\mathrm{N}_{2} \mathrm{H}_{4}$.<smiles>CCCc1c(C/C=C(\C)[Po])oc(=O)c(C)c1OCC</smiles>

125<smiles>CCCc1c(NN)c(C)c(=O)n(N)c1C/C=C(\C)[Po](C)N</smiles>

154
1) $\mathrm{N}_{2} \mathrm{H}_{4}$, Dioxan

2) $1 \mathrm{M} \mathrm{KHSO}_{4}$-Lsg

3) $\mathrm{NaNO}_{2}, \mathrm{~d}-\mathrm{HOAc}$<smiles>CCCc1c(C/C=C(\C)[Po])[nH]c(=O)c(C)c1O</smiles>

23

1) $1 \mathrm{M} \mathrm{KHSO}_{4}$-Lsg

2) $\mathrm{NaNO}_{2}, \mathrm{~d}-\mathrm{HOAc}$<smiles>CCCC1=C(C/C=C(\C)PO)N(N)C(=O)C(C)/C1=N/N</smiles>

155

Entsprechend der Vorgaben von Pednekar wurde eine Lösung des Kreuzkupplungsproduktes 125 und $\mathrm{N}_{2} \mathrm{H}_{4}$ in Dioxan (je 25 eq, 1:1) eingesetzt und unter den in der Tabelle 18 angeführten Reaktionsbedingungen gerührt. Nach den in dem Schema 39 dargestellten Produktstrukturen sollten zunächst die gebildeten $N$-Aminopyridon-4-hydrazone 154 und 155 durch die Behandlung mit 1.0 $\mathrm{M} \mathrm{KHSO}_{4}$-Lösung in die entsprechenden $N$-Aminopyridone überführt werden. Die anschließende Reduktion mit $\mathrm{NaNO}_{2}$ in Essigsäure sollte dann zum gewünschten Iromycin-Analogon führen, was jedoch mißlang. Bei allen drei unternommenen Versuchen (vgl. Tabelle 18, Einträge 1-3) trat eine vollständige Zersetzung ein, so daß eine Auswertung der Spektren nicht erfolgen konnte. 
Tabelle 18. Überführung des KK-Produkts 125 in das Iromycin-Analogon 23 unter Verwendung von $\mathrm{N}_{2} \mathrm{H}_{4}$.

\begin{tabular}{ccccc}
\hline Eintag & $\mathrm{T}\left[{ }^{\circ} \mathrm{C}\right]$ & $\mathrm{t}[\mathrm{h}]$ & Auswertung & $\mathbf{2 3}$ \\
\hline 1 & $\mathrm{RT}$ & 72 & Zersetzung & - \\
2 & 60 & 5 & Zersetzung & - \\
3 & 60 & 5 & Zersetzung & - \\
\hline
\end{tabular}

\section{Kreuzkupplungsreaktionen des Brommethylpyridons mit Alanaten}

Da die Iromycinsynthese über den zuvor dargestellten Weg der Kreuzkupplung des Brommethylpyridons 159 mit Alanaten bei dem Syntheseschritt des O-N-Austausches zunächst Probleme bereitete (vgl. Abschnitt 2.3), wurden parallel Kreuzkupplungsvorversuche des Brommethylpyridons 159 mit dem Alkenylalanat 124 untersucht. Hierbei wurde im Gegensatz zu dem eingeschlagenen Syntheseweg bei dem Pyron 63 die Einführung des Stickstoffs in den Ringcyclus erst vor dem geplanten und entscheidenden Kreuzkupplungsreaktionsschritt durchgeführt (vgl. Schema 40). Als Ausgangssubstanzen sollten entsprechend dem in Abschnitt 2.2.2.2 (vgl. Schema 34) dargestellten Syntheseweg das Brommethylpyridon 159 als Analogon des Brommethylpyrons 96 mit den Alkenylalanaten 124, 124A und 124R eingesetzt werden (vgl. Schema 40), um zu einem direkten Zugang zu den Iromycinen zu gelangen.

Schema 40. Darstellung des Iromycin-Analogons 23 aus dem Brommethylpyridon 159 und dem Alanat 124.<smiles>CCCc1c(CBr)[nH]c(=O)c(C)c1OC(C)=O</smiles>

159

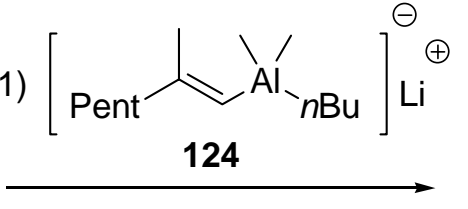

2) basische Entschützung<smiles>CCCc1c(CC=C(C)PC)[nH]c(=O)c(C)c1O</smiles>

23

Bei der Synthese des Bromids 159 wurde zunächst der in dem Abschnitt 2 beschriebene retrosynthetische Syntheseweg ausgehend von Pyron 63 beschritten (vgl. Schema 41). Hierbei werden nach Transformation des Pyrons 63 zu dem Pyridon 62 zunächst die Umfunktionalisierung zum entsprechenden Aldehyd bzw. Alkohol und die anschließenden Halogenierungs- 
versuche erläutert. Abschließend werden die Kreuzkupplungsversuche des Pyridonhalogenids mit dem Alanat 124 als Modellsubstrat (vgl. Schema 40) zur Synthese des IromycinAnalogons 23 behandelt.

Schema 41. Retrosynthetische Darstellung des Brommethylpyridons 159 aus dem Pyron 63.<smiles>C=CCc1c(C)oc(=O)c(C)c1O</smiles>

63<smiles>C=CCc1c(C=O)[nH]c(=O)c(C)c1OC(C)=O</smiles>

157<smiles>C=CCc1c(C)[nH]c(=O)c(C)c1O</smiles>

62<smiles>CCCc1c(CO)[nH]c(=O)c(C)c1OC(C)=O</smiles>

158<smiles>C=CCc1c(C)[nH]c(=O)c(C)c1OC(C)=O</smiles>

156<smiles>CCCc1c(CBr)[nH]c(=O)c(C)c1OC(C)=O</smiles>

159

\subsection{Synthese des Brommethylpyridons 159}

\subsubsection{O-N-Austausch des Pyrons 63 zu dem Pyridon 62}

Der O-N-Austausch des Pyrons 63 zu dem Pyridon 62 als erster Schritt zur Darstellung des Brommethylpyridons 159 wurde zunächst mit Ammoniaklösung untersucht. Es ist bereits durch die Literatur bekannt, daß Ammoniak Pyrone in korrespondierende Pyridone transformiert. Wang et al. ${ }^{[67]}$ führten die Umwandlung in $\mathrm{NH}_{4} \mathrm{OH}$ bei $100{ }^{\circ} \mathrm{C}(6 \mathrm{~h})$ durch und erhielten nach Einkonzentration der Reaktionslösung und Umkristallisation des Feststoffes aus wässrigem EtOH das von ihnen angestrebte Pyridon (Ausbeute 83\%). Manas et al. ${ }^{[63]}$ setzten eine Pyronlösung in einem $\mathrm{NH}_{4} \mathrm{OH}$ und EtOH-Gemisch bei $100-110^{\circ} \mathrm{C} 26 \mathrm{~h}$ in einem Bombenrohr ein und erhielten nach Aufarbeitung mit $\mathrm{HCl}$ das korrespondierende Pyridon (Ausbeute $86 \%$ ). Groutas et al. ${ }^{[68]}$ berichteten von der Umwandlung eines Pyrons mit $\mathrm{NH}_{4} \mathrm{OH}$ in Dioxan bei $110{ }^{\circ} \mathrm{C}$ (Reaktionszeit $1 \mathrm{~h}$ ) und kristallisierten das entsprechende Pyridon aus wässrigem EtOH um. Die im Rahmen dieser Arbeit durchgeführten Versuche wurden gemäß den in der Tabelle 19 angegebenen Reaktionsbedingungen mit konz. $\mathrm{NH}_{3}$ durchgeführt (vgl. Schema 42, Exp. Teil, Allg. Arbeitsvorschrift A15). Die Behandlung des Pyrons 63 mit Am- 
moniak bei hohen Temperaturen von $110-140{ }^{\circ} \mathrm{C}$ führte sowohl mit als auch ohne Verwendung von Lösungsmitteln in den überwiegenden Fällen zur vollständigen Umsetzung und Bildung des Pyridons 62 (vgl. Tabelle 19). Lediglich die bei $110{ }^{\circ} \mathrm{C}$ durchgeführte Reaktion (Eintrag 2), bei der kein Umsatz beobachtet wurde, blieb erfolglos. Da der Umwandlungsversuch 1 bei milder Raumtemperatur nach einer Reaktionszeit von $68 \mathrm{~h}$ unter vollständiger Umsetzung erfolgreich verlief (Eintrag 1), blieb das Ausbleiben des Pyridons 62 bei der Umsetzung unter der verhältnismäßig hohen Temperatur von $110{ }^{\circ} \mathrm{C}$ (Eintrag 2) erstaunlich. Die Ursache hierfür dürfte in der nach Groutas et al. festgelegten kurzen Reaktionszeit von $1 \mathrm{~h} z u$ sehen sein.

Schema 42. Darstellung des 6-Methylpyridons 62 ausgehend von dem Pyron 63 mit $\mathrm{NH}_{3}$.<smiles>CCCc1c(C)oc(=O)c(C)c1O</smiles>

63

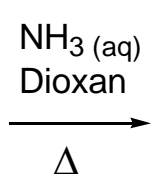

$75 \%$<smiles>CCCc1c(C)[nH]c(=O)c(C)c1O</smiles>

62

Tabelle 19. Darstellung des Pyridons 62 ausgehend von dem Pyron 63 mit $\mathrm{NH}_{3}$.

\begin{tabular}{ccccccc}
\hline Eintrag & Lsm. & Vorrichtung & $\mathrm{T}\left[{ }^{\circ} \mathrm{C}\right]$ & $\mathrm{t}[\mathrm{h}]$ & Umsatz $(\%)$ & Massenbilanz (\%) $^{\mathrm{d})}$ \\
\hline $1^{\text {c) }}$ & - & Bombenrohr & $\mathrm{RT}$ & 68 & 100 & 39 \\
$2^{\text {a) }}$ & Dioxan & Rückfluß & 110 & 1 & 0 & 83 \\
$3^{\text {a) }}$ & Dioxan & Rückfluß & 120 & 5.5 & 100 & 93 \\
$4^{\text {a) }}$ & Dioxan & Bombenrohr & 120 & 54 & 100 & 96 \\
$5^{\text {c) }}$ & - & Bombenrohr & 120 & 3 & 100 & 88 \\
$6^{\text {b) }}$ & EtOH & Autoklave & 120 & 5 & 100 & 99 \\
$7^{\text {c) }}$ & - & Autoklave & 138 & 24 & 100 & 74 \\
$8^{\text {a) }}$ & Dioxan & Bombenrohr & 140 & 2 & 100 & 75 \\
\hline
\end{tabular}

${ }^{\text {a) }}$ Einsatz von je 25 eq $\mathrm{NH}_{4} \mathrm{OH}\left(28-30 \mathrm{Gew} \%\right.$-Lösung von $\mathrm{NH}_{3}$ in $\left.\mathrm{H}_{2} \mathrm{O}\right) /$ Dioxan (1:1). ${ }^{\text {b) }}$ Einsatz von reinem flüssigen $\mathrm{NH}_{3}$ (liq) bei $\mathrm{P}=16 \mathrm{kP} / \mathrm{cm}$. ${ }^{\mathrm{c})} \mathrm{NH}_{4} \mathrm{OH}$ im Überschuß (25 eq). ${ }^{\text {d) }}$ Verhältnis des erhaltenen Rohprodukts zum eingesetzten Edukt.

Der Vergleich der in der Tabelle 19 dargestellten Ergebnisse zeigt, dass die Umfunktionalisierung des Pyrons 63 sowohl bei milden wie auch hohen Temperaturen realisiert werden kann, wobei die Durchführung der Reaktion bei Raumtemperatur mit einer längeren Reaktionszeit verbunden ist. Von Relevanz für den Erfolg der Umsetzungen scheint zudem sicherlich der 
hohe Überschuß an $\mathrm{NH}_{3}$ gewesen zu sein. Die Bilanz der erhaltenen Rohproduktausbeuten könnte desweiteren dafür sprechen, dass die Transformationsreaktionen in Lösungsmittel besser verliefen als diejenigen ohne Lösungsmitteleinsatz. Aufgrund der nicht erfolgten systematischen Reinigung der Rohprodukte kann jedoch keine positive Aussage darüber getroffen werden, ob auch die Qualität und Quantität des gewünschten Produktes durch den Lösungsmitteleinsatz begünstigt wird.

Die Reaktionslösungen wurden im Allgemeinen aufgrund der stark polaren Eigenschaft des Pyridons 62 und seiner hohen Löslichkeit in alkoholischen Lösungen sowie in Wasser bevorzugt stark eingeengt und anschließend mit $\mathrm{KHSO}_{4}$-Lösung hydrolysiert. Die saure Aufarbeitung der Reaktionsmischung wurde wegen des Verdachts der Aminbildung an der C-4Position des Ringes, die allerdings während der Untersuchungen nicht beobachtet wurde, durchgeführt. Am Vorteilhaftesten erwies sich die in dem Autoklave durchgeführte Transformation des Pyrons 63 zu dem Pyridon 62 in reinem Ammoniak nach 5 h (vgl. Tabelle 19, Eintrag 6). Für das Pyridon 62 wurden IR-Banden bei $1602 \mathrm{~cm}^{-1}$ für NH (Lit. $\left.1630 \mathrm{~cm}^{-1}\right)^{[63]}$ sowie spektroskopische Daten $\left[{ }^{13} \mathrm{C}\right.$ NMR (62.9 MHz, $\mathrm{CD}_{3} \mathrm{OD}, 140.56$ ( $\left.\mathrm{C}_{\text {quart }}, \mathrm{C}-6\right), 166.08$ $\left(\mathrm{C}_{\text {quart }}, \mathrm{C}-4\right), 166.19$ ( $\left.\mathrm{C}_{\text {quart }}, \mathrm{C}-2\right)$ ] analytisch nachgewiesen. Die in $\mathrm{CDCl}_{3}$ aufgenommenen ${ }^{1}$ H-NMR-Spektren waren sehr unscharf und machten eine Auswertung der Umsetzungen teilweise schwierig. Daher wurde das Pyridon 62 bevorzugt in $\mathrm{CD}_{3} \mathrm{OD}$ oder DMSO aufgenommen.

Als weitere Möglichkeit zur Einführung des Stickstoffatoms in den Ringzyclus des Pyrons 63 und damit zur Darstellung des Pyridons 62 wurde parallel der Einsatz des im Vergleich zu $\mathrm{NH}_{3}$ stärker nucleophilen Hydrazins untersucht, dessen Reaktionsmechanismus ähnlich dem Mechanismus der Pyrontransformation mit Ammoniak verläuft (vgl. Schema 36). Eine anschließende Entschützung sollte zur Darstellung des Pyridons 62 führen (vgl. Schema 43). Baddar et al. ${ }^{[69]}$, Pednekar et al. ${ }^{[66]}$ und El-Kholy et al. ${ }^{[70]}$ führten den O-N-Austausch bei vergleichbaren Pyronen unter Rückflußbedingungen mit Hydrazinhydrat in EtOH durch. ElKholy et al. reduzierten das Amin anschließend unter Behandlung des Aminopyridons mit einer wässrigen $\mathrm{NaNO}_{2}$-Lösung in Essigsäure. Die Versuche zum O-N-Austausch des Pyrons 63 zu dem Pyridon 62 mit Hydrazin im Rahmen der vorliegenden Arbeit wurden nur in Anlehnung an die genannten Literaturangaben, aber unter modifizierten Reaktionsbedingungen durchgeführt. Die Umfunktionalisierung gelang bei $60{ }^{\circ} \mathrm{C}$ in sehr glatter und sauberer Umsetzung und ergab ein Gemisch aus dem Hydrazin 160 und dem Hydrazon 161, das analytisch 
nachgewiesen wurde (s. Exp. Teil, Allg. Arbeitsvorschrift A15, Variante 1). Die Reduktion des $N$-Aminopyridons 162 (Phenol-Verkochung) zu dem gewünschten Pyridon 62 verlief unter Einsatz von $\mathrm{NaNO}_{2}$ und $\mathrm{d}^{4}-\mathrm{HOAc}$ bei Raumtemperatur ebenfall sehr glatt und sauber (s. Exp. Teil, Allg. Arbeitsvorschrift A15, Variante 2). Die analytischen Daten $\left({ }^{1} \mathrm{H}-\mathrm{NMR}\right.$, IRund Massenspektroskopie) entsprachen den aus der Darstellung des Pyrons 63 mit Ammoniak gewonnenen analytischen Werten des Pyridons 62 (s. Exp. Teil, Allg. Arbeitsvorschrift A15, Variante 2).

Schema 43. Umfunktionalisierung des Pyrons 63 zu dem Pyridon 62 mit Hydrazin.<smiles>CCCc1c(C)oc(=O)c(C)c1O</smiles>

$\mid \begin{aligned} & \mathrm{N}_{2} \mathrm{H}_{4} \\ & \text { Dioxan, } \Delta\end{aligned}$<smiles>CCCc1c(NN)c(I)c(=O)n(N)c1I</smiles>

160
1) $\mathrm{N}_{2} \mathrm{H}_{4}$, Dioxan, $\Delta$

2) $1 \mathrm{M} \mathrm{KHSO}_{4}$-Lsg

3) $\mathrm{NaNO}_{2}, \mathrm{~d}-\mathrm{HOAC}$<smiles>CCCC1=C(C)N(N)C(=O)C(C)/C1=N/N</smiles>

161<smiles>CCCc1c(C)[nH]c(=O)c(C)c1O</smiles>

$\uparrow \begin{aligned} & \mathrm{NaNO}_{2}(\mathrm{aq}) \\ & \mathrm{d}-\mathrm{HOAc}\end{aligned}$<smiles>CCCc1c(O)c(C)c(=O)n(N)c1C</smiles>

162

Da bei der Darstellung des Pyridons 62 aus dem Pyron 63 durch O-N-Austausch mit $\mathrm{NH}_{3}$ zunächst nicht der erwünschte Erfolg erzielt werden konnte, wurde parallel hierzu die Einführung des Stickstoffes in den Pyronring mit Benzylamin und $p$-Methoxybenzylamin untersucht, welches zur Bildung der $N$-Benzylpyridone und nach deren Entschützung zum gewünschten Pyridon 62 führen sollte. Kiang et al. ${ }^{[71]}$ führten die Reaktion mit einem ähnlichen Pyron mit Benzylamin in Essigsäure durch. Hérault et al. ${ }^{[65]}$ führten die Umfunktionalisierung der Pyrone bei hoher Temperatur in Benzylamin durch. Wiley et al. ${ }^{[72]}$ fügten eine Lösung von Methylkumulat in Methanol zu einer Lösung von $p$-Methoxybenzylamin in $\mathrm{MeOH}$ und ließen die Lösung 8 Tage stehen. Nach Zugabe von konz. NaOH-Lösung wurde die Reaktionslösung 20 min unter Rückflussbedingungen gerührt. Die Behandlung mit $\mathrm{HCl}$ ergab ein $N-(p$ Methoxybenzyl)pyridon als Feststoff (Ausbeute 81\%). Der geplante O-N-Austausch des Py- 
rons 63 zu dem $N$-Benzylpyridon 163 und dem $N$-(4-methoxybenzyl)pyridon 164 wurde in einer Untersuchungsreihe in Lösungsmitteln (Dioxan, $\mathrm{C}_{6} \mathrm{D}_{6}$ und $d$-DMSO) bzw. ohne Lösungsmittel sowie unter variierenden Temperaturbedingungen durchgeführt und untersucht (vgl. Tabelle 20).

Schema 44. O-N-Austausch des Pyrons 63 zu dem N-Benzylpyridon 163 bzw. 164 mit anschließender Reduktion zu dem Pyridon 62.

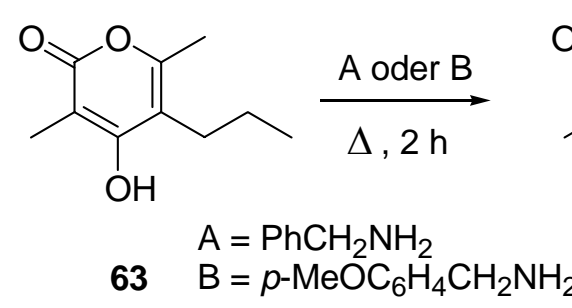

$63 \mathrm{~B}=p-\mathrm{MeOC}_{6} \mathrm{H}_{4} \mathrm{CH}_{2} \mathrm{NH}_{2}$

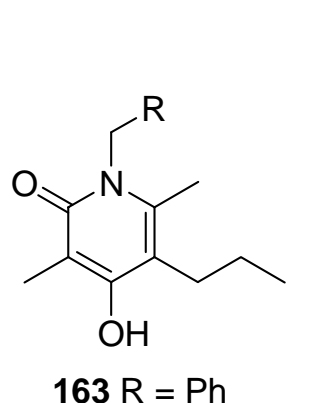

$164 \mathrm{R}=p-\mathrm{MeOC}_{6} \mathrm{H}_{4}$
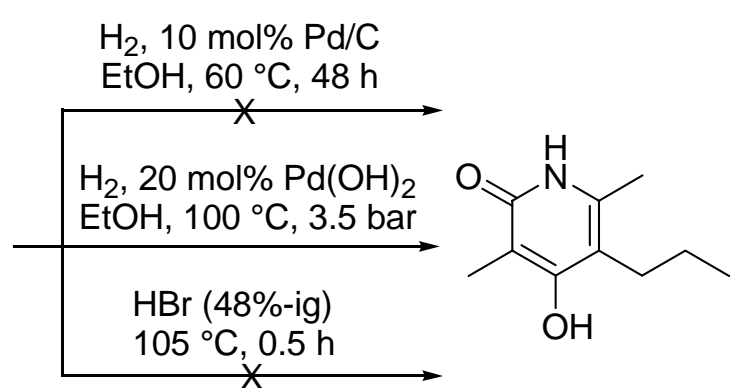

62

Tabelle 20. O-N-Austausch des Pyrons 63 zu dem N-Benzylpyridon 163.

\begin{tabular}{|c|c|c|c|c|}
\hline Eintrag & $\mathrm{PhCH}_{2} \mathrm{NH}_{2}$ [eq] & Lsm. & $\mathrm{T}\left[{ }^{\circ} \mathrm{C}\right], \mathrm{t}[\mathrm{h}]$ & 63:163 (\%) \\
\hline 1 & 1.0 & - & $120(2)$ & $27: 73$ \\
\hline 2 & 1.0 & $\mathrm{H}_{2} \mathrm{O}$ & $120(2)$ & $30: 70$ \\
\hline 3 & 2.0 & - & $120(1.5)$ & $0: 100$ \\
\hline 4 & 2.0 & - & RT (3.5) & 100:0 \\
\hline 5 & 2.0 & Dioxan & $\mathrm{RT}(3)$ & 100:0 \\
\hline & $p-\mathrm{MeOC}_{6} \mathrm{H}_{4} \mathrm{NH}_{2}[\mathrm{e}$ & & & 63:164(\%) \\
\hline 6 & 5.4 & - & $\begin{array}{l}\mathrm{RT}(15), 50(5) \\
80(4), 100(4)\end{array}$ & Zersetzung \\
\hline 7 & 4.4 & Dioxan & $\begin{array}{l}\text { RT (15), } 50(5), \\
80(4), 100(4)\end{array}$ & Zersetzung \\
\hline 8 & 2.2 & $\mathrm{C}_{6} \mathrm{D}_{6}$ & $\begin{array}{l}\text { RT (2), } 50(5) \\
80(4), 100(4)\end{array}$ & Zersetzung \\
\hline 9 & 2.2 & $d$-DMSO & $\begin{array}{l}\text { RT (2), } 50(5), \\
80(4), 100(4)\end{array}$ & $100: 0$ \\
\hline
\end{tabular}

Während bei den unter milden Bedingungen (Raumtemperatur) durchgeführten Reaktionen (Tabelle 20, Einträge 4 u. 5) keine Umsetzung festgestellt wurde, ergaben die Versuche bei $120^{\circ} \mathrm{C}$ (Tabelle 20, Einträge 1 u. 2) ein Gemisch aus dem Edukt 63 und dem gewünschten $N$ Benzylpyridon 163. Eine vollständige Umsetzung zum Produkt 163 gelang letztlich bei der 
lösungsmittelfreien Reaktion (Tabelle 20, Eintrag 3). Dabei wurden 58\% der eingesetzten Menge als Rohprodukt erhalten, was einer Ausbeute von 58 mol\% des eingesetzten Eduktes entspricht. Die Durchführung des Umwandlungsprozesses unter Verwendung des nucleophileren $p-\mathrm{MeOC}_{6} \mathrm{H}_{4} \mathrm{NH}_{2}$ mißlang gänzlich (vgl. Tabelle 20). Auch bei Vornahme einer Temperaturerhöhung $\left(50{ }^{\circ} \mathrm{C}, 80^{\circ} \mathrm{C}, 100{ }^{\circ} \mathrm{C}\right)$ und Zugabe weiterer Äquivalente des Amins konnte das gewünschte $N$-(4-Methoxybenzyl)pyridon 164 nicht nachgewiesen werden. Während der Versuch ohne Lösungsmitteleinsatz (Tabelle 20, Eintrag 6) sowie Versuche in den Lösungsmitteln Dioxan (Tabelle 20, Eintrag 7) und $\mathrm{C}_{6} \mathrm{D}_{6}$ (Tabelle 20, Eintrag 8) zur vollständigen Zersetzung führten, wurde bei der Reaktion mit $d$-DMSO (Tabelle 20, Eintrag 9) das Pyron 63 unzersetzt zurückerhalten.

Bei der Debenzylierung des $N$-Benzylpyridons 163 zu dem Pyridon 62 (vgl. Schema 44) wurde eine Reihe von Methoden nach Angaben aus der Literatur untersucht. Kappe et al. ${ }^{[73]}$ berichteten von der Debenzylierung von Pyridonen mit Wasserstoff als Reduktionsmittel unter Verwendung von Pd auf Aktivkohle. Die Hydrogenolyse des $N$-Benzylpyridons 163 wurde zunächst nach Kappe et al. entsprechend den in der Tabelle 21 angegebenen Reaktionsbedingungen durchgeführt (vgl. Tabelle 21, Einträge 1 u. 2). Hierbei wurden die eingesetzten Edukte quantitativ zurückerhalten, woraufhin diese Methode nicht weiter verfolgt wurde. Kurihara et al. ${ }^{[74]}$ führten die Debenzylierung der entsprechenden $N$-Benzylpyridone unter Einsatz von 48\%-iger Bromwasserstoffsäure durch. Die analog hierzu durchgeführte Debenzylierungsreaktion mit 48\%-iger $\mathrm{HBr}$ (Tabelle 21, Eintrag 5) blieb ebenfalls erfolglos.

Tabelle 21. Reduktion des N-Benzylpyridons 163 zu dem Pyridon 62.

\begin{tabular}{cccccccc}
\hline Eintrag & $\begin{array}{c}\text { Entschützungsmittel } \\
{[\mathrm{eq}]}\end{array}$ & $\begin{array}{c}\text { Kat. } \\
{[\mathrm{mol} \%]}\end{array}$ & Lsm. & $\begin{array}{c}\mathrm{T} \\
{\left[{ }^{\circ} \mathrm{C}\right]}\end{array}$ & $\begin{array}{c}\mathrm{P} \\
{[\mathrm{bar}]}\end{array}$ & $\begin{array}{c}\mathrm{t} \\
{[\mathrm{h}]}\end{array}$ & $\mathbf{1 6 3 : 6 2}$ \\
\hline 1 & $\mathrm{H}_{2}$ & $\mathrm{~A}, 13$ & $\mathrm{EtOH}$ & 60 & $\mathrm{P}^{\circ}$ & 4 & $1: 0$ \\
2 & $\mathrm{H}_{2}$ & $\mathrm{~A}, 10$ & $\mathrm{EtOH}$ & 60 & $\mathrm{P}^{\circ}$ & 48 & $1: 0$ \\
3 & $\mathrm{H}_{2}$ & $\mathrm{~B}, 20$ & $\mathrm{EtOH}$ & 100 & 3.5 & 34 & $1: 5$ \\
4 & $\mathrm{H}_{2}$ & $\mathrm{~B}, 20$ & $\mathrm{EtOH}$ & 100 & 3.4 & 44 & $0: 1$ \\
\hdashline 5 & $\mathrm{HBr}$ & - & - & 105 & - & 0.5 & $1: 1$ \\
\hline
\end{tabular}

A: $\mathrm{Pd} / \mathrm{C} . \mathrm{B}: \mathrm{Pd}(\mathrm{OH})_{2} . \mathrm{P}^{\circ}:$ Normaldruck.

Takahashi et al. ${ }^{[75]}$ gelang die katalytische Hydrierung von $N$-Benzylpyridonen unter Verwendung eines Pearlman-Katalysators an einer Parr-Apparatur. Zur Reduktion des $\mathrm{N}$ Benzylpyridons 163 wurde entsprechend der Arbeitsvorschrift nach Takahashi eine Lösung 
von $N$-Benzylpyridon 163 und einem Pearlman-Katalysator $\left[\mathrm{Pd}(\mathrm{OH})_{2} /\right.$ Aktivkohle, $\left.20 \% \mathrm{Pd}\right]$ in EtOH bei $100{ }^{\circ} \mathrm{C}$ an der Parr-Apparatur hydriert (s. Exp. Teil, Allg. Arbeitsvorschrift A15, Variante 3). Nach 34 h konnte ein Gemisch aus dem Edukt 163 und dem Pyridon 62 als Hauptprodukt im Verhältnis 1:5 erhalten werden (Tabelle 21, Eintrag 3). Eine vollständige Umsetzung der Hydrogenolysereaktion gelang schließlich nach $44 \mathrm{~h}$ in quantitativer Ausbeute (Tabelle 21, Eintrag 4). Der erhaltene weiße Feststoff wurde sowohl massenspektroskopisch als auch IR-spektroskopisch untersucht und als Pyridon 62 nachgewiesen. Für das IR wurde eine Schlüsselbande bei $1601 \mathrm{~cm}^{-1}$ (nach Lit. bei $1615 \mathrm{~cm}^{-1}$ ) ${ }^{[73]}$ gefunden.

\subsubsection{Synthese des Pyridonacetates 156 aus dem Pyridon 62}

Die Veresterung der 4-Hydroxygruppe des Pyridons 62 zu dem Pyridonacetat 156 (vgl. Schema 45) wurde nach Hoffmann et al. ${ }^{[35]}$ zunächst mit jeweils 1.0 eq an Acetylchlorid und Pyridin als Aktivierungsreagenz in $\mathrm{Et}_{2} \mathrm{O}$ bzw. Dioxan durchgeführt (Tabelle 22, Einträge 1 u. 2). Die Umsetzungen verliefen glatt und sehr sauber, sodaß eine Reinigung an sich nicht erforderlich war $\left({ }^{1} \mathrm{H}\right.$ - sowie ${ }^{13} \mathrm{C}-\mathrm{NMR}$ zeigten keine Verunreinigungen an). Nach säulenchromatographischer Reinigung konnte das Acetat 156 mit einer Ausbeute von 59\% (Tabelle 22, Eintrag 1) und einer Ausbeute von 69\% (Tabelle 22, Eintrag 2) isoliert werden. Die Ausbeutesteigerung auf 69\% könnte durch die erhöhte Polarität des Dioxans als Lösungsmittel bedingt sein. Die verlängerte Reaktionszeit bei dem Versuch 2 im Vergleich zu dem Versuch 1 wurde auf die Verwendung von Dioxan als Lösungsmittel und eine hierdurch herrührende Verdünnung des Reaktionsmediums zurückgeführt, da dies die Bildung der aktivierten $N$ Acetylpyridiniumchlorid-Zwischenstufe verlangsamt haben dürfte.

Schema 45. Synthese des Pyridonacetats 156 aus dem Pyridon 62.

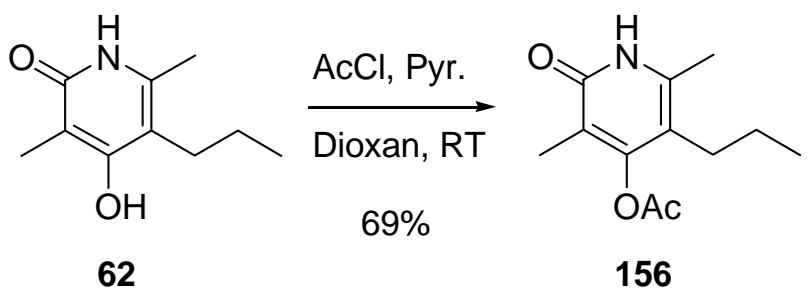


Tabelle 22. Synthese des Pyridonacetats 156 aus dem Pyridon 62.

\begin{tabular}{cccccccc}
\hline Eintrag & $\begin{array}{c}\text { AcCl } \\
{[\mathrm{eq}]}\end{array}$ & $\begin{array}{c}\text { Pyridin } \\
{[\mathrm{eq}]}\end{array}$ & Lsm. & $\begin{array}{c}\mathrm{T} \\
{\left[{ }^{\circ} \mathrm{C}\right]}\end{array}$ & $\begin{array}{c}\mathrm{t} \\
{[\mathrm{h}]}\end{array}$ & $\begin{array}{c}\text { Massenbilanz } \\
(\%)^{\mathrm{a})}\end{array}$ & $\begin{array}{c}\text { Ausbeute } \\
(\%)^{\mathrm{b})}\end{array}$ \\
\hline 1 & 1.0 & 1.0 & $\mathrm{Et}_{2} \mathrm{O}$ & $0 \rightarrow \mathrm{RT}$ & 41 & quant. & $59^{\mathrm{c})}$ \\
2 & 1.0 & 1.0 & Dioxan & $0 \rightarrow \mathrm{RT}$ & 63 & quant. & $69^{\mathrm{c})}$ \\
3 & 1.1 & 25 & - & $\mathrm{RT}$ & 17 & 93 & $67^{\mathrm{d})}$ \\
4 & 1.1 & 25 & - & $\mathrm{RT}$ & 38 & quant. & $60^{\mathrm{c})}$ \\
\hline a) Massenverhältnis des Rohprodukts und des eingesetzten Eduktes. & ${ }^{\text {b) }}$ Ausbeute \\
nach entsprechender & Reinigung. & c) Säulenchromatographische Reinigung. d) \\
Umkristallisation.
\end{tabular}

Zum Zwecke der Ausbeuteerhöhung wurden die Acylierungsversuche des Pyridons 62 zudem im Gegensatz zu den Versuchen 1 und 2 (vgl. Tabelle 22, Einträge 1 u. 2) in Pyridin als Lösungsmittel durchgeführt. Kappe et al. ${ }^{[37]}$ berichteten von der Acylierung der Pyridone unter dem Einsatz von Pyridin als Lösungsmittel (Ausbeute 86\%). Jonas und Horeni et al. ${ }^{[76]}$ acylierten Cumarin-Derivate ebenfalls mit Pyridin im Überschuß (Ausbeute 70\%). Die Schützung des Pyridons 62 wurde im Rahmen der Iromycin-Synthese analog der oben angegebenen Literaturvorgaben unter lösungsmittelfreien Reaktionsbedingungen durchgeführt. Die lösungsmittelfreie Durchführung sowie die Verwendung des Pyridins im Überschuß bei den Acylierungsversuchen (vgl. Tabelle 22, Einträge 3 u. 4) erbrachte keine merkliche Ausbeutesteigerung (60-67\%). Auch wenn diese Acylierungsversuche im Vergleich zu den Acylierungsversuchen ihrer O-Analoga (Pyron 63) in Gegenwart von Pyridin im Überschuß relativ glatt und sehr sauber abliefen, konnten geringfügig erhöhte Verunreinigungsanteile gegenüber den in Lösungsmittel durchgeführten Versuchen (vgl. Tabelle 22, Einträge 1 u. 2) aufgrund des Vergleichs der entsprechenden Spektren beobachtet werden. Vorteilhaft erwiesen sich deutlich kürzere Reaktionszeiten, was zweifellos auf das stärker konzentrierte Medium (erhöhte Reaktionsgeschwindigkeit des Aktivierungsschrittes bei der Bildung der $N$-Acetylpyridiniumchlorid-Zwischenstufe) durch den Überschuß an Pyridin zurückzuführen ist. Ein Vergleich der Massenbilanzen mit den Produktausbeuten des Esters 156 (vgl. Tabelle 22) zeigte einen Verlust des Rohproduktes von bis zu 41\% bei den Aufreinigungsversuchen an. Diese Entwicklung setzte sich sowohl bei späteren chromatographischen Aufreinigungen wie auch bei Umkristallisationsversuchen fort. Eine Umsetzung des Rohproduktes ohne weitere Reinigung ist daher empfehlenswert, zumal die Qualität des erhaltenen Rohproduktes 156 als sehr sauber eingestuft werden konnte. 


\subsubsection{Funktionalisierung des Pyridonacetates 156 zu dem Brommethylpyridon 159}

Die Funktionalisierung der Methylgruppe an der C-6-Position des Pyridonacetats 156 zum entsprechenden Pyridoncarbaldehyd 157 wurde, wie bei der Synthese des Pyroncarbaldehyds 92 bzw. 94 (vgl. Abschnitt 2.1.2, Tabelle 6), mit basischem $\mathrm{SeO}_{2}$ nach den Arbeitsvorschriften von Suzuki et al. ${ }^{[38]}$, Hoffmann et al. ${ }^{[35]}$ und Moreno-Maňas et al. ${ }^{[39]}$ systematisch durchgeführt. Das Oxidationsmittel $\left(\mathrm{SeO}_{2}\right)$ wurde nach bekannter Arbeitsvorschrift ${ }^{[77]}$ aktiviert und eingesetzt. Hierbei musste hohe Sorgfalt ausgeübt werden, denn $\mathrm{SeO}_{2}$ weist eine 20-25-fach höhere Toxizität als Cyanidsalze auf. Bei der oxidativen Funktionalisierung nach Hoffmann unter Rückfluß konnte nach 44 h keine vollständige Umsetzung erzielt werden (Tabelle 23, Eintrag 3). Die Durchführung der Oxidation im geschlossenen System (Bombenrohr) erwies sich aus experimenteller Sicht als vorteilhaft, da sich die Reaktionszeit auf 4-17 h verringerte (vgl. Tabelle 23).

Schema 46. Oxidation des Pyridonesters 156 zum Pyridoncarbaldehyd 157.

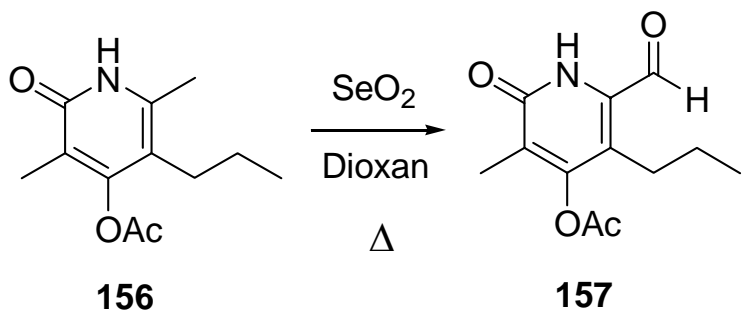

Aus dem Vergleich der Versuchsergebnisse ist auf einen direkten Zusammenhang zwischen der Reaktionszeit und dem Temperatureinfluß einerseits sowie der eingesetzten Menge an Selenoxid andererseits zu schließen (vgl. Tabelle 23). Während bei den Versuchen bei $170{ }^{\circ} \mathrm{C}$ (Einträge 6 u. 7) ein vollständiger Umsatz nach 4 h erreicht wurde, ergaben die Umsetzungen bei $120^{\circ} \mathrm{C}$ (Einträge 2, 4 u. 5) nach 7-10 h und die Umsetzung bei $110^{\circ} \mathrm{C}$ (Eintrag 1) nach 17 h den gewünschten Aldehyd 157. Durch die erhöhte Temperatur ist eine gesteigerte Reaktivität der C-H-Bindungen der Methylgruppe gegeben (vgl. Abschnitt 2.1.2). Ersichtlich wirkt sich ebenso die erhöhte Menge an Selenoxid positiv auf die Reaktionsgeschwindigkeit der Funktionalisierung aus. Die erzielte vollständige Umsetzung bei dem Versuch mit einer verlängerten Reaktionszeit von 17 h (Tabelle 23, Eintrag 1) wurde auf die niedrige Temperatur von $110^{\circ} \mathrm{C}$ und den Einsatz von 4.0 eq $\mathrm{SeO}_{2}$ zurückgeführt. 
Tabelle 23. Oxidation des Pyridonesters 156 zu dem Pyridoncarbaldehyd 157.

\begin{tabular}{cccccccc}
\hline Eintrag & $\begin{array}{c}\mathrm{SeO}_{2} \\
{[\mathrm{eq}]}\end{array}$ & Vorrichtung & $\begin{array}{c}\mathrm{T} \\
{\left[{ }^{\circ} \mathrm{C}\right]}\end{array}$ & $\begin{array}{c}\mathrm{t} \\
{[\mathrm{h}]}\end{array}$ & $\begin{array}{c}\mathbf{1 5 7 : 1 5 6} \\
(\%)\end{array}$ & $\begin{array}{c}\text { Massenbilanz } \\
(\%)^{\mathrm{a})}\end{array}$ & $\begin{array}{c}\text { Ausbeute } \\
(\%)\end{array}$ \\
\hline 1 & 4.0 & Bombenrohr & 110 & 17 & $1: 0$ & quant. & - \\
2 & 6.0 & Bombenrohr & 120 & 7 & $1: 0$ & quant. & - \\
3 & 6.8 & Rückfluß & 120 & 44 & $1: 2$ & 65 & - \\
4 & 8.0 & Bombenrohr & 120 & 8 & $1: 0$ & 97 & 30 \\
5 & 10 & Bombenrohr & 120 & 10 & $1: 0$ & 82 & 35 \\
6 & 3.0 & Bombenrohr & 170 & 4 & $1: 0$ & quant. & - \\
7 & 3.4 & Bombenrohr & 170 & 4 & $1: 0$ & quant. & - \\
\hline
\end{tabular}

Alle Reaktionen wurden in Dioxan als Lösungsmittel durchgeführt. ${ }^{\text {a) }}$ Massenverhältnis des Rohprodukts und des eingesetzten Edukts.

Analog der Acetylierungsversuche (vgl. Tabelle 22) verliefen auch die Oxidationen des Pyridonesters 156 im Vergleich zu den Acetylierungs- und Oxidationsreaktionen der Pyronanaloga (vgl. Abschnitt 2.1.2, Tabelle 6) qualitativ relativ glatt und sauber. Im Gegensatz zu den Oxidationsreaktionen der Pyronester 88 und 89 mit Selenoxid (vgl. Tabelle 6), bei denen häufig ein Gemisch aus dem Pyroncarbaldehyd 92 und dem Pyronalkohol 93 beobachtet wurde, konnte bei der Funktionalisierung des Pyridonesters $156 \mathrm{zu}$ dem Aldehyd 157 die Bildung entsprechender Alkohole nicht detektiert und nachgewiesen werden (vgl. Tabelle 23). Die Umsetzungen der Pyronester 88 und 89 zu den Aldehyden 92 und 94 verliefen erheblich langsamer, sodaß eine vollständige Umsetzung erst nach deutlich längeren Reaktionszeiten erreicht werden konnte. Diese Feststellungen belegen eine stärkere Reaktivität des Pyridons 62 im Vergleich zum Pyron 63. Zur Erklärung der Reaktivitätsunterschiede des Pyrons 63 und des Pyridons 62 in Bezug auf den Oxidationsprozess kann ein Vergleich der elektronischen Struktur sowie der Elektronendichteverhältnisse des Hauptgrundgerüstes beitragen. Da es sich hier um eine Oxidationsreaktion handelt, dürfte die Tendenz zur Elektronenabgabe an dem C1'-Zentrum als relevantes Kriterium für die Reaktivität der Umsetzung angesehen werden. Aufgrund des Umstandes, dass die Variationen der Schutzgruppen scheinbar keinen ersichtlichen Einfluß auf die Reaktivität der Oxidationsreaktionen ausüben (vgl. Tabellen 6 u. 23), dürften die Reaktivitätsunterschiede nur bei dem $\mathrm{sp}^{3}$-Hybrid-Orbital des O- und N-Atoms des Lacton- und Lactamrings zu suchen sein. Zudem ist die Tendenz des $M$-mesomeren Effekts und somit die Bereitschaft zu einer Beteiligung des freien Elektronenpaars im Falle des Stickstoffatoms im Vergleich zu dem des Sauerstoffatoms (dem Sauerstoffatom wird nur eine sehr schwach formale Fähigkeit zum $M$-mesomeren Effekt zugeordnet) erheblich stärker ausgeprägt, so dass dem Pyridonester 156 eine erleichterte Oxidationstendenz zugeschrieben werden kann. 
Die reduktive Umfunktionalisierung des Pyridoncarbaldehyds 157 zum Pyridonalkohol 158 wurde nach Moreno-Maňas ${ }^{[39]}$ mit 1.2 eq $\mathrm{NaBH}_{4}$ in EtOH bei $0{ }^{\circ} \mathrm{C}$ durchgeführt (vgl. Schema 47, s. Exp. Teil). Die Reduktionsversuche wurden mit einem Gemisch aus konz. HCl und EtOH (Einträge 1, 3, 4 u. 6) bzw. mit $1.0 \mathrm{M} \mathrm{HCl}$ (Einträge 2 u. 5) hydrolysiert. Während bei den Versuchen eine teilweise (Eintrag 1) sowie sogar eine vollständige Abspaltung der Acetylgruppe (Eintrag 3) auftrat, konnte neben dieser Entschützung auch der Verlust der Hydroxylgruppe beobachtet werden (Eintrag 6). Die Abspaltung der Schutzgruppe wurde auf den Einsatz konz $\mathrm{HCl}$ bei der Hydrolyse des Aldehyds 157 zurückgeführt. Zur Hydrolyse wurden bei der Umsetzung 1 (Eintrag 1) Säure und EtOH in einem Verhältnis von 1:1 eingesetzt, bei dem Versuch 4 (Eintrag 4) hingegen im Verhältnis 1:2. Dies dürfte vermutlich der Grund dafür sein, weshalb bei dem Versuch 4 keine Entschützung auftrat. Die Aufarbeitung der Umsetzungen 2 und 5 mit 5.0 eq $1.0 \mathrm{M} \mathrm{HCl}$ (Einträge 2 u. 5) verlief glatt und ohne Entschützungserscheinungen.

Schema 47. Reduktive Umfunktionalisierung des Aldehyds 157 zu dem Pyridonalkohol 158.<smiles>CCCc1c(C=O)[nH]c(=O)c(C)c1OC(C)=O</smiles>

157

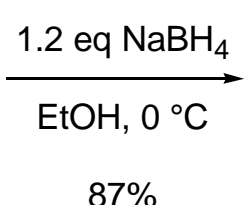

$87 \%$<smiles>CCCc1c(CO)[nH]c(=O)c(C)c1OC(C)=O</smiles>

158

Tabelle 24. Reduktion des Aldehyds 157 zu dem Pyridonalkohol 158.

\begin{tabular}{cccc}
\hline Eintrag & $\mathrm{t}[\mathrm{h}]$ & Massenbilanz $(\%)^{\mathrm{a})}$ & Ausbeute $(\%)$ \\
\hline 1 & 2 & quant. & $53^{\mathrm{b})}$ \\
2 & 2 & quant. & $-{ }^{\mathrm{c})}$ \\
3 & 4 & quant. & $-{ }^{\mathrm{c})}$ \\
4 & 4 & quant. & $29^{\mathrm{b})}$ \\
5 & 2.5 & quant. & $87^{\mathrm{d})}$ \\
6 & 2.5 & 81 & $13^{\mathrm{d})}$ \\
\hline
\end{tabular}

a) Massenverhältnis des erhaltenen Rohprodukts und des eingesetzten Edukts. ${ }^{\text {b) }}$ Säulenchromatographische Reinigung. ${ }^{\text {c) }}$ Alkohol wurde ohne Aufreinigung umgesetzt. ${ }^{\text {d) }}$ Umkristallisation. 
Die Pyridonalkohole zeigten sich auch bei der säulenchromatographischen Reinigung als instabil. Dies wird besonders durch den Vergleich mit den Massenbilanzen und Ausbeuten des isolierten Alkohols 158 (vgl. Tabelle 24) deutlich. Die chromatographische Reinigung bei dem Versuch 4 (Eintrag 4) führte zu einem gravierenden Massenverlust von $3 / 4$ des zu reinigenden Rohproduktes. Die Umkristallisation aus heißem EtOAc erwies sich nur als bedingt erfolgreich. Während der Umkristallisationsversuch 6 (Eintrag 6) gerade eine Ausbeute von 13\% ergab, konnte hingegen der Alkohol 158 bei dem Versuch 5 (Eintrag 5) in einer Ausbeute von $87 \%$ isoliert werden.

Die Bromierung der nach einem $\mathrm{S}_{N} 2$-Mechanismus verlaufenden Substitutionsreaktion des Alkohols $158 \mathrm{zu}$ dem Brommethylpyridon 159 wurde nach Hoffmann et al. ${ }^{[35]}$ mit $\mathrm{PBr}_{3}$ durchgeführt (vgl. Schema 48). Die Umsetzungen 3 und 4 (vgl. Tabelle 25, Einträge 3 und 4) wurden in Dioxan durchgeführt, da der Alkohol 158 nur eine geringe Löslichkeit in $\mathrm{Et}_{2} \mathrm{O}$ aufwies (vgl. Tabelle 25, Einträge 1 u. 2). Die säulenchromatographische Reinigung einer Probe des Bromids 159 führte zu einem großen Massenverlust sowie zur Bildung von Spuren des Alkohols 158. Die Rückbildung des Alkohols 158 aus dem Bromid 159 wird auf die Hydrolyse (Spuren von $\mathrm{H}_{2} \mathrm{O}$ auf Kieselgel) und die hierdurch bedingte Resubstitution des Bromids als bessere Abgangsgruppe gegenüber der OH-Funktion zurückgeführt. Die Bromierungsversuche verliefen stets sehr glatt und mit hoher Sauberkeit (vgl. Tabelle 25), sodaß eine entsprechende Aufreinigung an sich nicht erforderlich war. Bei den dennoch durchgeführten Reinigungsversuchen erwies sich die Umkristallisation aus EtOAc (Eintrag 3, Ausbeute 62\%) gegenüber der säulenchromatographischen Reinigung, die mit Zersetzungserscheinungen, Massenverlust sowie der Rückbildung des Alkohols aus dem Bromid verbunden war, als bessere Reinigungsmethode für das Brommethylpyridon 159.

Schema 48. Bromierung des Pyridonalkohols 158 zu dem Brommethylpyridon 159.

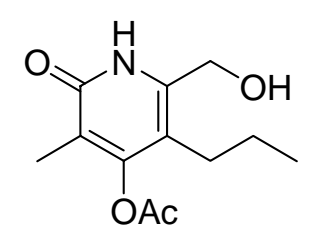

158

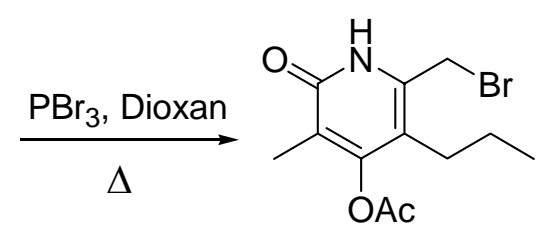

159 
Tabelle 25. Bromierung des Pyridonalkohols 158 zum Brommethylpyridon 159.

\begin{tabular}{cccccc}
\hline Eintrag & $\mathrm{PBr}_{3}[\mathrm{eq}]$ & $\mathrm{Lsm}$. & $\mathrm{T}\left[{ }^{\circ} \mathrm{C}\right]$ & $\mathrm{t}[\mathrm{h}]$ & Massenbilanz $(\%)^{\mathrm{a})}$ \\
\hline 1 & 1.1 & $\mathrm{Et}_{2} \mathrm{O}$ & $40 \rightarrow \mathrm{RT}$ & 20 & quant. \\
2 & 1.1 & $\mathrm{Et}_{2} \mathrm{O}$ & $40 \rightarrow \mathrm{RT}$ & 24 & 70 \\
3 & 1.1 & Dioxan & $40 \rightarrow \mathrm{RT}$ & 15 & $79^{\mathrm{b})}$ \\
4 & 1.1 & Dioxan & $40 \rightarrow \mathrm{RT}$ & 15 & 50 \\
a) Massenverhältnis des Rohprodukts und des eingesetzten Eduktes. ${ }^{\text {b) }}$ Umkristalli- \\
sation aus heißem EtOAc (62\%).
\end{tabular}

Um die Funktionalisierungsschritte des Pyridonacetats 156 zu dem Bromids 159 (s. oxidative Funktionalisierung zum Aldehyd 157 und anschließende Reduktion zum Alkohol 158, vgl. Schemata 46 u. 47) unter Umständen zu verkürzen, wurde die direkte radikalische Bromierung des Pyridonesters 156 nach Moreno-Maňas et al. ${ }^{[39]}$ in Gegenwart einer Mischung von $\mathrm{N}$-Bromsuccinimid (NBS) und Benzoylperoxid (DBPO) als Radikalstarter in $\mathrm{CCl}_{4}$ untersucht (Exp. Teil, Allg. Arbeitsvorschrift A12, Variante 2). Die angewandten radikalischen Bromierungsversuche erwiesen sich jedoch aufgrund der geringen Selektivität des Bromierungsverfahrens als ungeeignet (vgl. Schema 49). Die Auswertung der Spektren zeigte zusätzlich zu dem gewünschten Singulett des Methylenbromids 159 (s, 2 H, 4.40 ppm) weitere unerwünschte Signale bei 4.52 und 4.63 ppm, die der zweifach bromierten Verbindung 149 (vgl. Schema 49) sowie einem weiteren unidentifizierten Bromderivat zugeordnet wurden. Es konnten für die Mono- und Dibromid-Produkte der Versuche Anteile von 1:3 bzw. 1:10 zugunsten des Dibromierungsproduktes 149 berechnet werden. Das gewünschte Brommethylpyridon 159 wurde dementsprechend nur als Nebenprodukt gebildet. Moreno-Maňas et al. berichteten ebenfalls von einer zweifachen Bromierung an der C-1'-Position sowie einer Bromierung an dem C-3-Kohlenstoff des Hauptgrundgerüstes. Diese Methode wurde daher aufgrund geringer Selektivität des Bromierungsverfahrens nicht weiter untersucht.

Schema 49. Radikalische Bromierung des Pyridonesters 156 zum Brommethylpyridon 159.

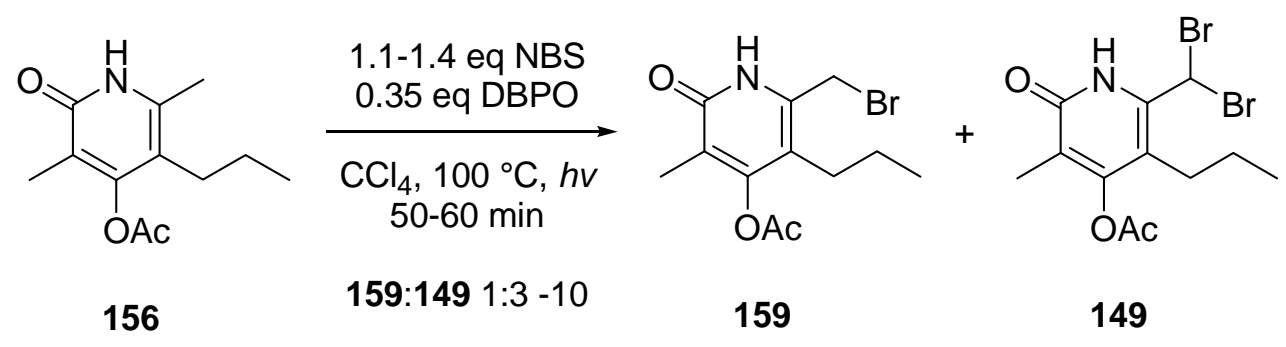




\subsection{Kreuzkupplungsreaktionen des Brommethylpyridons 159 mit Alanaten}

\subsubsection{Kupplungversuche des Brommethylpyridons 159 mit dem Alanat 124}

Analog der im Abschnitt 2.2.2.2 (vgl. Schema 34) schon beschriebenen Kreuzkupplungversuche des Brommethylpyrons 96 mit dem Alanat 124 (als Modellsubstrat), 124A und 124R wurden entsprechende C-C-Bindungsbildungversuche des Brommethylpyridons 159 mit dem Alanat 124 nach Negishi et al. ${ }^{[5]}$, Utimoto et al. ${ }^{[60]}$, Frejd et al. ${ }^{[61]}$ und Takeuchi et al. ${ }^{[62]}$ untersucht. Zur Generierung des Alanats 124 wurde nach der im Abschnitt 2.2.2.2 (vgl. Schema 34) angewandten Methode und entsprechend der in der Tabelle 26 angegebenen Bedingungen verfahren. Hierzu wurde eine $n \mathrm{BuLi}$-Lösung in Hexan bei $0{ }^{\circ} \mathrm{C}$ einer Lösung des Alans 123 in THF zugegeben und 30 min gerührt. Die Reaktionslösung wurde anschließend mit einer Lösung von 1.0 eq Brommethylpyridon 159 in THF versetzt und untersucht (s. Exp. Teil, Allg. Arbeitsvorschrift A13). Da bei dem ersten durchgeführten Versuch bei $0{ }^{\circ} \mathrm{C}(\mathrm{vgl}$. Tabelle 26 , Eintrag 1) trotz Zugabe weiterer 2.0 eq des Alanats 124 (ingesamt 4.0 eq Alanat 124) nach 41 h kein Kreuzkupplungsprodukt detektiert werden konnte, wurden weitere 2.0 eq des Alanats 124 generiert und mit 1.0 eq des Brommethylpyrons 183 in einer Referenzreaktion $17 \mathrm{~h}$ beobachtet. Durch Zugabe des Brommethylpyrons 183 als O-Analogon in Gegenwart des Brommethylpyridons 159 als N-Analogon sollte untersucht werden, ob unter identischen Bedingungen eine entsprechende Kreuzkupplungsreaktion des O-Analogons stattfindet oder nicht.

Schema 50. Kreuzkupplungsversuche der Bromide 159 und 183 mit dem Alanat 124 zur Darstellung der Iromycin-Analoga 165 und 184.<smiles>CCCc1c(CBr)[nH]c(=O)c(C)c1OC(C)=O</smiles>

159<smiles>CCCc1c(CBr)oc(=O)c(C)c1OC(C)=O</smiles>

183
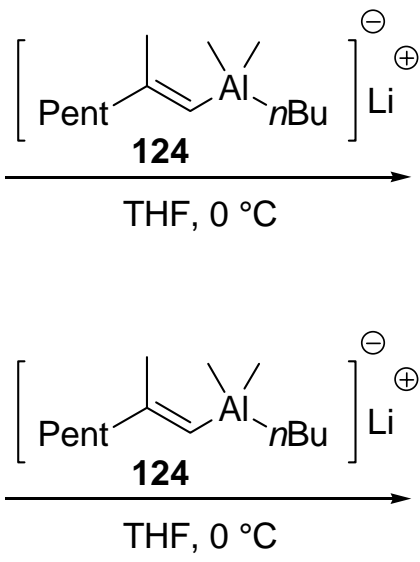

THF, $0{ }^{\circ} \mathrm{C}$<smiles>CCCc1c(C/C=C(\C)[Po])[nH]c(=O)c(C)c1OC(C)=O</smiles>

165<smiles>CCCc1c(C(C)=O)oc(=O)c(C)c1C/C=C(\C)P</smiles>

184 
Tabelle 26. Kreuzkupplungversuche der Bromide 159 und 183 mit dem Alanat 124.

\begin{tabular}{ccccccl}
\hline Eintrag & $\begin{array}{c}\text { Alan 124 } \\
{[\mathrm{eq}]}\end{array}$ & $\begin{array}{c}n \text { BuLi } \\
{[\mathrm{eq}]}\end{array}$ & Bromid & $\begin{array}{c}\text { Bromid } \\
{[\mathrm{eq}]}\end{array}$ & $\begin{array}{c}\mathrm{t} \\
{[\mathrm{h}]}\end{array}$ & Auswertung, Produkte \\
\hline 1 & 4.0 & 4.0 & $\mathbf{1 5 9}$ & 1.0 & 41 & Zersetzung, Dien 136 \\
dann & 2.0 & 2.0 & $\mathbf{1 8 3}$ & 1.0 & 17 & Zersetzung, Dien 136 \\
2 & 2.0 & 4.0 & $\mathbf{1 5 9}$ & 1.0 & 17 & Zersetzung, Entschützung \\
3 & 2.0 & 4.0 & $\mathbf{1 8 3}$ & 1.0 & 17 & $\begin{array}{l}\text { Kreuzkupplungsprodukt 184, } \\
\text { (15ien 136 u. DPE }\end{array}$ \\
& & & & & & 137 \\
4 & 2.0 & 2.0 & $\mathbf{1 5 9}$ & 1.0 & 2 & Zersetzung, Entschützung \\
\hline
\end{tabular}

a) Dipyronethan 137.

Die erste Variante der Umsetzung führte zu einer vollständigen Zersetzung der Bromide 159 und 183 (vgl. Tabelle 26, Eintrag 1). Die entsprechenden Spektren zeigten jedoch die Bildung des Diens 136 an. In zwei weiteren Versuchsvarianten (vgl. Tabelle 26, Einträge 2 u. 3) wurde das Alanat 124, im Gegensatz zu der Umsetzung, bei der das $\mathrm{AlMe}_{3}$-freie Alan 123 eingesetzt wurde (Eintrag 1), in situ generiert, so dass die Reaktionslösung noch 1.0 Äquivalent überschüssiges $\mathrm{AlMe}_{3}$ enthielt. Daher wurden hier 4.0 eq $n$ Buli eingesetzt, um das Alkenylalan und das überschüssige $\mathrm{AlMe}_{3}$ in das Alanat zu überführen. Die generierte Alanatlösung wurde dann in zwei Vergleichsreaktionen (Einträge 2 u. 3) mit jeweils einer Lösung der Bromide 159 und 183 versetzt. Während der Versuch 2 sowohl zur Zersetzung als auch zur Entschützung der Acetatfunktion führte (keine Spur des Kreuzkupplungsprodukt 165), zeigte die Auswertung des Versuchs 3 die Bildung eines Gemisches aus dem Kreuzkupplungsprodukt 184 (vgl. Schema 65), dem Dipyronethan 137 und dem Dien 136. Auch bei dem entsprechenden Versuch mit dem $\mathrm{AlMe}_{3}$-freien Alanat 124 und dem Bromid 159 konnte das gewünschte Kreuzkupplungsprodukt 165 (vgl. Schema 50) nicht nachgewiesen werden (vgl. Tabelle 26, Eintrag 4).

Das Scheitern der Kreuzkupplungsversuche des Brommethylpyridons 159 mit dem Alanat 124 und der Umstand, dass die Versuche mit dem Brommethylpyron 183, das O-Analogon des Brommethylpyridons 159, immerhin teilweise zur Bildung des Kreuzkupplungsprodukts führten, könnten möglicherweise auf die Acidität des Protons an Stickstoff (vgl. Schema 51) und auf die hohe Säureempfindlichkeit des Alanats 124 zurückzuführen sein. Andererseits stünden für eine mögliche Deprotonierung von 1.0 eq Brommethylpyridon 159 durch das Alanat 124 aber noch weitere 3.0 eq des Alanats 124 zur Verfügung (vgl. Tabelle 26, Eintrag 1), so dass das Proton des Stickstoffes allein an das Ausbleiben des Kreuzkupplungsprodukts noch nicht erklärt. Durch den Überschuß an Alanat 124 dürfte dennoch genügend Triebkraft 
für einen nucleophilen Angriff auf das Bromid gewährleistet sein, weshalb eine saure Zersetzung des Alans durch das acide Proton des Brommethylpyridons allein höchstens zu einer Ausbeuteminimierung führen könnte. Auch eine eventuelle Koordination des Alanats 124 als Lewissäure an die Carbonylfunktion der Amidgruppe dürfte zwar eine Ausbeuteminderung bewirken, andererseits aber auch zu einer erhöhten Reaktivität des Allylbromids und damit zu einer schnelleren Kreuzkupplungsreaktion führen. Diese Frage wurde im Rahmen dieser Arbeit jedoch nicht näher untersucht.

\subsubsection{Kreuzkupplungversuche des Brommethylpyridons 159 mit Alanaten durch in situ Deprotonierung mit $\mathrm{AlMe}_{3}, \mathrm{Al}(\mathrm{iBu})_{3}, \mathrm{Zn}(\mathrm{Et})_{2}, \mathrm{nBuLi}$ und $\mathrm{NaH}$}

Aufgrund der nicht erfolgreichen Kreuzkupplungsversuche des Brommethylpyridons 159 mit dem Alanat 124, für welche das Stickstoffproton des Bromids 159 als möglicher Hinderungsgrund angenommen wurde (vgl. Abschnitt 3.2.1), sollte in einer weiteren Versuchsreihe die Möglichkeit einer in situ Deprotonierung des Stickstoffprotons der Amidgruppe unter Einsatz von Metallorganylen als Basen untersucht werden (vgl. Schema 51). Die anschließende Zugabe von vorgeneriertem Alanat 124 zur Lösung der Spezies 166 sollte nach C-C-Bindungsbildung und entsprechender Hydrolyse zum gewünschten Kreuzkupplungsprodukt 165 führen.

Schema 51. Kreuzkupplungversuch durch in situ Deprotonierung des Brommethylpyridons 159 an Stickstoff mit den Metallorganylen $\mathrm{AlMe}_{3}, \mathrm{Al}(\mathrm{iBu})_{3}, \mathrm{Zn}(\mathrm{Et})_{2}, n \mathrm{BuLi}$ und $\mathrm{NaH}$.

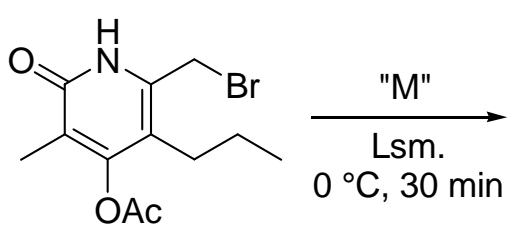

159<smiles>[M]n1c(CBr)c(CCC)c(OC(C)=O)c(C)c1=O</smiles>

166

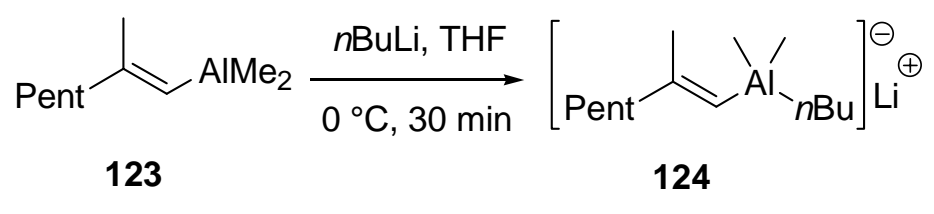

"M" = AlMe $3, \mathrm{Al}(\mathrm{iBu})_{3}, \mathrm{Zn}(\mathrm{Et})_{2}, n \mathrm{BuLi}, \mathrm{NaH}$

$\mathrm{M}=\mathrm{AlMe}_{2}, \mathrm{Al}(\mathrm{iBu})_{2}, \mathrm{ZnEt}, \mathrm{Li}, \mathrm{Na}$<smiles>CCCc1c(C/C=C(\C)[Pb])[nH]c(=O)c(C)c1OC(C)=O</smiles><smiles>CCCc1c(CCc2[nH]c(=O)c(C)c(OC(C)=O)c2CCC)[nH]c(=O)c(C)c1OC(C)=O</smiles> 
Tabelle 27. Kreuzkupplung des Brommethylpyridons 159 mit dem Alanat 124 unter Einsatz der Basen $\mathrm{AlMe}_{3}, \mathrm{Al}(\mathrm{iBu})_{3}, \mathrm{Zn}(\mathrm{Et})_{2}, n \mathrm{BuLi}$ und $\mathrm{NaH}$.

\begin{tabular}{|c|c|c|c|c|c|c|c|}
\hline $\begin{array}{l}\text { Ein- } \\
\text { trag }\end{array}$ & $\begin{array}{c}\text { Alanat } \\
\mathbf{1 2 4}[\mathrm{eq}]\end{array}$ & Base & $\begin{array}{l}\text { Base } \\
\text { [eq] }\end{array}$ & Lsm. 2 & $\begin{array}{c}\mathrm{T} \\
{\left[{ }^{\circ} \mathrm{C}\right]}\end{array}$ & $\begin{array}{c}\mathrm{t} \\
{[\mathrm{h}]}\end{array}$ & $\begin{array}{c}\text { 159:165:136:167:156 } \\
(\%)^{\mathrm{a})}\end{array}$ \\
\hline 1 & 2.0 & $\mathrm{Al}(\mathrm{Me})_{3}$ & 1.0 & THF & $0 \rightarrow \mathrm{RT}$ & 16 & $32: 6: 0: 2: 60$ \\
\hline 2 & 2.0 & $\mathrm{Al}(\mathrm{Me})_{3}$ & 1.2 & THF & $0 \rightarrow \mathrm{RT}$ & 70 & $5: 17: 1: 1: 76$ \\
\hline 3 & 2.0 & $\mathrm{Al}(\mathrm{Me})_{3}$ & 1.2 & $\mathrm{MeCN}$ & $0 \rightarrow \mathrm{RT}$ & 15 & $13: 10: 1: 1: 75$ \\
\hline 4 & 2.0 & $\mathrm{Al}(\mathrm{Me})_{3}$ & 1.2 & DMF & $0 \rightarrow \mathrm{RT}$ & 15 & 11:0:0:0:89 \\
\hline 5 & 3.0 & $\mathrm{Al}(\mathrm{Me})_{3}$ & 2.0 & $\mathrm{MeCN}$ & $0 \rightarrow \mathrm{RT}$ & 15 & 30:0:0:0:70 \\
\hline 6 & 3.0 & $\mathrm{Al}(\mathrm{Me})_{3}$ & 2.0 & $\mathrm{MeCN} / \mathrm{THF}$ & $0 \rightarrow \mathrm{RT}$ & 15 & $12: 12: 0: 0: 76$ \\
\hline 7 & 3.0 & $\mathrm{Al}(\mathrm{Me})_{3}$ & 2.0 & $\mathrm{MeCN} / \mathrm{THF}$ & 50 & 7 & $0: 23: 1: 0: 76$ \\
\hline 8 & 3.0 & $\mathrm{Al}(\mathrm{Me})_{3}$ & 2.0 & NMP & $0 \rightarrow \mathrm{RT}$ & 15 & -b) \\
\hline 9 & 2.0 & $\mathrm{Al}(i \mathrm{Bu})_{3}$ & 1.2 & THF & $0 \rightarrow \mathrm{RT}$ & 14 & $32: 3: 0: 1: 64$ \\
\hline 10 & 3.0 & $\mathrm{Zn}(\mathrm{Et})_{2}$ & 1.1 & THF & $0 \rightarrow \mathrm{RT}$ & 15 & $35: 3: 1: 1: 60$ \\
\hline 11 & 3.0 & $n \mathrm{BuLi}$ & 1.0 & THF & $-78 \rightarrow \mathrm{RT}$ & 18 & $0: 20: 3: 3: 74$ \\
\hline 12 & 3.0 & $n \mathrm{BuLi}$ & 1.0 & $\mathrm{MeCN} / \mathrm{THF}$ & -78 & 1 & \\
\hline & & & & $(3: 1)$ & -40 & 2 & \\
\hline & & & & & -30 & 22 & \\
\hline & & & & & -10 & 18 & \\
\hline & & & & & 0 & 6 & b) \\
\hline 13 & 3.0 & $\mathrm{NaH}$ & 1.0 & $\begin{array}{c}\text { 18-Krone-6 } \\
\text { THF }\end{array}$ & $-30 \rightarrow \mathrm{RT}$ & 15 & -b) \\
\hline
\end{tabular}

a) Prozentuale Anteile der Komponenten 159:165:136:137:156 zueinander. ${ }^{\text {b) }}$ Auswertung war nicht möglich.

Es sollten zunächst die Deprotonierungs- bzw. Protonierungsschritte mit $\mathrm{AlMe}_{3}$-Lösung und ges. $\mathrm{NH}_{4} \mathrm{Cl}$-Lösung geprüft werden. Ziel der Voruntersuchungen war die Feststellung, ob das Brommethylpyridon 159 unzersetzt wieder zurückgewonnen werden kann. Desweiteren sollte eine vollständige Deprotonierung durch Einsatz von Metallorganylen als Basen bestätigt werden. Da die Deprotonierungsreaktion des Bromids 159 mit $\mathrm{AlMe}_{3}$ unter Freisetzung von $\mathrm{Me}-$ than abläuft, wird eine Deprotonierung durch Gasentwicklung angezeigt. Bei den durchgeführten Deprotonierungs- bzw. Protonierungsuntersuchungen des Brommethylpyridons 159 mit $\mathrm{AlMe}_{3}$ bzw. mit $\mathrm{Zn}(\mathrm{Et})_{2}$ wurde keine Zersetzung des Eduktes beobachtet. Die durchgeführten Kreuzkupplungsversuche (vgl. Schema 51 und Tabelle 27) unter Einsatz der Lösungen von $\mathrm{AlMe}_{3}$, Triisobutylaluminium $\mathrm{Al}(\mathrm{iBu})_{3}$ bzw. Diethylzink $\mathrm{Zn}(\mathrm{Et})_{2}$ als Basen wurden unter den in der Tabelle 27 angegebenen Reaktionsbedingungen nach der Allgemeinen Arbeitsvorschrift A17 durchgeführt (s. Exp. Teil).

Ein Vergleich der Ergebnisse der Tabelle 27 verdeutlicht, dass das gewünschte Kreuzkupplungsprodukt 165 stets als Minderprodukt gebildet wurde. Die Auswertung des Spektrums der 
Umsetzung bei $50{ }^{\circ} \mathrm{C}$ (Eintrag 7) zeigte die Bildung des höchsten Anteils an Kreuzkupplungsprodukt 165 (Anteil 23\%). Desweiteren wurden neben dem Debromierungsprodukt 156 (vgl. Schema 45), das stets als Hauptprodukt ermittelt wurde, das Dien 136 bei 6.0 ppm (vgl. Schema 34), das Dipyridonethan 167 bei 2.85 ppm und der Pyridonalkohol 158 bei 4.65 ppm (vgl. Schema 47) als Hydrolyseprodukt des Brommethylpyridons 159 beobachtet und massenspektroskopisch $(156,158,167)$ nachgewiesen. Zusätzlich wurde ausnahmslos bei allen Umsetzungen ein Singulett bei 4.52 ppm beobachtet, das zum Teil in geringer Menge (Tabelle 27, Einträge 1, 2, 9 u. 10), zum Teil aber auch in erheblichem Anteil (Tabelle 27, Einträge 3-8) beobachtet wurde. Der Wert der chemischen Verschiebung des genannten Signals entsprach dem bei den radikalischen Bromierungsversuchen des Pyridonesters 156, bei dem die dibromierte Verbindung als Hauptprodukt gebildet wurde (3:1 bzw. 10:1, vgl. Abschnitt 3.1.3, Schema 49), beobachteten Zuordnungswert für die Dibromspezies. Jedoch wäre eine Bildung des Dibromids unter den gegebenen Reaktionsbedingungen nicht erklärlich. Die höchsten Bildungsanteile der unbekannten Verbindung, deren chemischer Verschiebungswert einem Dibromid entsprach, wurden bei den Versuchen unter Einsatz von die nucleophile Substitution fördernden, polar aprotischen Lösungsmitteln, wie Acetonitril (MeCN), Dimethylformamid (DMF) und N-Methyl-2-pyrrolidon (NMP), beobachtet (vgl. Tabelle 27, Einträge 3-8). Die in Gegenwart des geringer polaren THF durchgeführten Umsetzungen (vgl. Tabelle 27, Einträge 1, 2, 9 u. 10) ergaben hingegen nur geringe Mengen bis hin zu bloßen Spuren dieser Verbindung.

Als Grund für die Debromierung kann die Lewissäure-Eigenschaft des Aluminiums angenommen werden (vgl. Schema 51), wodurch auch die teilweise Bildung des Alkohols 158 begründet werden könnte. Durch die koordinative Wechselwirkung des Aluminiummetalls mit dem benachbarten Sauerstoff der Carbonylgruppe der Struktur 166 (vgl. Schema 51) bildet sich ein aktiviertes System, das nach hydrolytischer Aufarbeitung die Bildung des Alkohols 158 erklären könnte. Die Bildung diverser Nebenprodukte stützt die Existenz mehrerer, nebeneinander ablaufender mechanistischer Reaktionen. Das Gesamtbild der diversen Produktbildungen blieb auch bei Einsatz anderer Metallorganyle wie $\mathrm{Al}(i \mathrm{Bu})_{3}$ und $\mathrm{Zn}(\mathrm{Et})_{2}$ unverändert (vgl. Tabelle 27, Einträge 9 u. 10). Bei dem Einsatz von $\mathrm{Zn}(\mathrm{Et})_{2}$ dürfte aufgrund der schwächeren lewissauren Eigenschaft des Metalls die geringste Umsetzung stattgefunden haben. 
Wie oben schon erwähnt, bestand die theoretische Annahme, dass die Substitution des Stickstoffprotons der NH-Gruppe unter Bildung einer Metall-Stickstoffbindung zu einer Erhöhung der Elektronendichte des Brommethylpyridons 159 und damit zur Verstärkung des doppelt vinylogen Effekts des Ringes geführt haben könnte, was eine Steigerung der Reaktivität der nucleophilen Substitution zur Folge gehabt hätte. Diese Vermutung konnte jedoch durch die vorhergehenden Versuche mit Alan- und Zinkorganylen nicht bestätigt werden. Das Scheitern der Umsetzungen könnte vielmehr sogar einen Beleg für die erhöhte Reaktivität anderer Prozesse anstelle der gewünschten Begünstigung der nucleophilen Substitution liefern, wodurch die Bildung der diversen unerwünschten Produkte initialisiert worden sein könnte. Daher wurden weitergehende Versuche mit $n \mathrm{BuLi}$ und $\mathrm{NaH}$ als Deprotonierungsmittel untersucht. Die Kreuzkupplungsversuche unter Einsatz von $n \mathrm{BuLi}$ und $\mathrm{NaH}$ wurden unter den in Tabelle 27 angegebenen Reaktionsbedingungen nach der Allgemeinen Arbeitsvorschrift A17 (s. Exp. Teil) durchgeführt. Während bei dem Versuch 11 (vgl. Tabelle 27, Eintrag 11) das Kreuzkupplungsprodukt 165 (Anteil 20\%) und das Debromierungsprodukt 156 (Anteil 74\%) erhalten wurden, konnten die Versuche 12 und 13 (vgl. Tabelle 27, Eintrag 12 u. 13) nicht ausgewertet werden.

\subsubsection{Kreuzkupplungsversuche des $N$-geschützten Brommethylpyridons 159 mit dem} Alanat 124

\subsubsection{Versuche zur Schützung des Brommethylpyridons 159 an Stickstoff}

Wie im letzten Abschnitt 3.2.2 beschrieben, konnte das gewünschte Kreuzkupplungsprodukt 165 bei der in situ Deprotonierung des Brommethylpyridons 159 nur anteilig (23\%) gebildet werden. Daher wurde als weitere Möglichkeit zur Erzielung der C-C-Bindungsbildung die Schützung der Stickstoffgruppe untersucht (vgl. Schema 52). Die Untersuchung sollte zeigen, ob das an Stickstoff gebundene saure Proton tatsächlich eine Hinderung für die Kreuzkupplungsreaktion darstellt. Die Versuche zum Einbau einer geeigneten Schutzgruppe wurden mit Anhydriden (Boc $2 \mathrm{O}$, TFAA), Silanen (TMSCl, TMSI, TMSOTf, TBDMSCl), Chlormethylmethylether $(\mathrm{MOMCl})$ und Acetylchlorid $(\mathrm{AcCl})$ durchgeführt. Als Methylierungsmittel wurden Diazomethan $\left(\mathrm{CH}_{2} \mathrm{~N}_{2}\right)$ und Methyliodid (MeI) verwendet. 
Schema 52. Kreuzkupplungsversuche mit den $N$-geschützten Brommethylpyridonen 168-174 und dem Alanat 124.<smiles>[R]C(C)(C)c1c(CBr)[nH]c(=O)c(C)c1OC(C)=O</smiles>

159<smiles>[R]n1c(CBr)c([PH])c(OC(C)=O)c(C)c1=O</smiles>

168-174

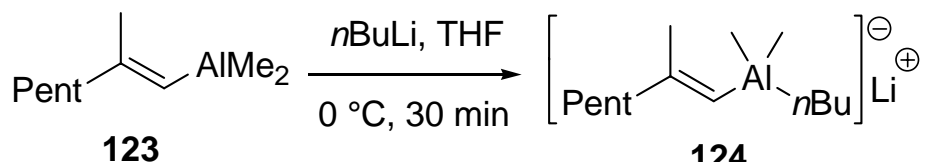<smiles>[R]n1c(CC=C(C)[R7][H])c(CCC)c(O[C+](=O)[O-])c(C)c1=O</smiles>

175

176

\begin{tabular}{c|l|c|l} 
Edukt & $\mathrm{R}=$ & Produkt & N-R $=$ \\
\hline $\mathbf{1 6 8}$ & Boc & - & N-Boc \\
$\mathbf{1 6 9}$ & TMS & $\mathbf{1 7 5}$ & N-TMS \\
$\mathbf{1 7 0}$ & TBDMS & - & N-TBDMS \\
$\mathbf{1 7 1}$ & MOM & - & N-MOM \\
$\mathbf{1 7 2}$ & $\mathrm{CF}_{3} \mathrm{CO}_{2}$ & - & N-COCF \\
$\mathbf{1 7 3}$ & Ac & - & N-Ac \\
$\mathbf{1 7 4}$ & $\mathrm{Me}$ & $\mathbf{1 7 6}$ & N-Me
\end{tabular}

3.2.3.1.1. Versuche zur Schützung des Brommethylpyridons 159 mit Anhydriden, Silanen, Chlormethylmethylether und Acetylchlorid

Nishiyama et al. ${ }^{[78]}$ schützten den Pyridonen vergleichbare Piperazine an der Amingruppe erfolgreich unter Verwendung von $\mathrm{Boc}_{2} \mathrm{O}$. Die Schützung des Brommethylpyridons 159 wurde daher nach der von Nishiyama et al. angegebenen Arbeitsvorschrift in Gegenwart von DMAP untersucht (vgl. Schema 53).

Schema 53. Darstellung des $N$-geschützten Brommethylpyridons 168 mit $\mathrm{Boc}_{2} \mathrm{O}$.

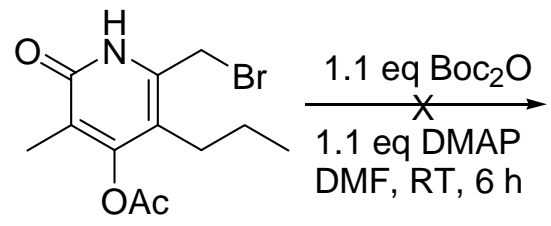

159<smiles>CCCc1c(OC(C)C)c(C)c(=O)n(C(=O)C(C)(C)C)c1CBr</smiles>

168 
Die Auswertung des Spektrums erwies sich als schwierig. Das bei $1.5 \mathrm{ppm}$ erhaltene Singulett deutete zwar auf die am Stickstoff gebundene $t$ Butyloxy-Funktion hin. Die Abwesenheit des Methylenbromid-Signals bei 4.4 ppm zeigte jedoch eine Abspaltung des Bromids an der C-1'Position und damit das Ausbleiben des gewünschten Produktes 168 an. Eine entsprechende Testreaktion wurde in Abwesenheit von $\mathrm{Boc}_{2} \mathrm{O}$ mit Brommethylpyridon 159 (2 mg) und DMAP in Dichlormethan bei Raumtemperatur durchgeführt und über Nacht gerührt. Die DCKontrolle zeigte eine vollständige Zersetzung des Eduktes 159 an. Die wässrige Aufarbeitung der Reaktionslösung mit $1 \mathrm{M} \mathrm{KHSO}_{4}$-Lösung belegte die schon zuvor beobachtete Zersetzungserscheinung. Die Boc-Gruppe erwies sich daher als nicht geeignet und es wurden keine weiteren Schützungsversuche hiermit untersucht.

Hua et al. ${ }^{[79]}$ überführten Pyrrolidinone in ihre silylierte Form durch Zugabe von TMSCl zu einer Lösung von Pyrrolidinon und $\mathrm{NEt}_{3}$ in Toluol. Die in Anlehnung an Hua im Rahmen dieser Arbeit durchgeführten Untersuchungsreihen (vgl. Tabelle 28) wurden zwecks spektroskopischer Beobachtung zunächst in einem NMR-Röhrchen (Einträge 1-5) und unter Argonatmosphäre durchgeführt. Die geplante Silylierungsreaktion des Brommethylpyridons 159 wurde in Gegenwart von $\mathrm{NEt}_{3}$ und TMSCl in $\mathrm{C}_{6} \mathrm{D}_{6}$ sowie $\mathrm{CDCl}_{3}$ untersucht (s. Exp. Teil, Allg. Arbeitsvorschrift A16). Die stöchiometrischen Angaben bzw. die weiteren variablen Reaktionsbedingungen der Versuche sind in der Tabelle 28 angegeben. Bei den Reaktionen wurde reines TMSCl (vgl. Tabelle 28, Einträge 1 u. 2), ein $0.5 \mathrm{M}$ Lösungsgemisch aus TMSCl/NEt $\mathrm{N}_{3}$ in $\mathrm{C}_{6} \mathrm{D}_{6}$ (vgl. Tabelle 28, Eintrag 3) und eine $0.5 \mathrm{M}$ TMSCl-Lösung in $\mathrm{C}_{6} \mathrm{D}_{6}$ (vgl. Tabelle 28, Einträge 4-8) verwendet.

Schema 54. $N$-Silylierung des Brommethylpyridons 159 mit TMSX (X = Cl, I, OTf).
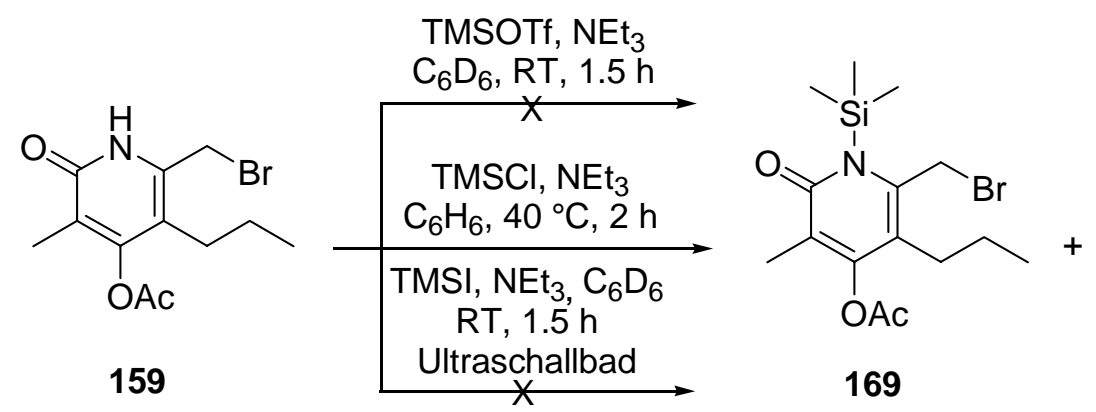<smiles>CCCc1c(OC(=O)[O-])c(C)c(=O)n([Si](C)(C)C)c1CCl</smiles> 
Tabelle 28. $N$-Silylierung des Brommethylpyridons 159 mit TMSX (X = Cl, I, OTf).

\begin{tabular}{cccccccc}
\hline Eintrag & Reagenz & [eq] & $\mathrm{NEt}_{3}$ [eq] & $\mathrm{LSM}$ & $\mathrm{T}\left[{ }^{\circ} \mathrm{C}\right]$ & $\mathrm{t}[\mathrm{h}]$ & $N$-TMS (\%) \\
\hline 1 & $\mathrm{TMSCl}$ & 1.1 & 1.25 & $\mathrm{C}_{6} \mathrm{D}_{6}$ & $\mathrm{RT}$ & 48 & 20 \\
2 & $\mathrm{TMSCl}$ & 4.0 & 5.0 & $\mathrm{C}_{6} \mathrm{D}_{6}$ & $\mathrm{RT}$ & 14.5 & - \\
3 & $\mathrm{TMSCl}$ & 3.0 & 3.0 & $\mathrm{C}_{6} \mathrm{D}_{6}$ & )$))^{\mathrm{a})}, \mathrm{RT}$ & 1.5 & - \\
4 & $\mathrm{TMSCl}$ & 2.0 & 4.0 & $\mathrm{CDCl}_{3}$ & )$))^{\mathrm{a})}, \mathrm{RT}$ & 19.5 & $\left.-{ }^{-}\right)$ \\
5 & $\mathrm{TMSCl}$ & 1.6 & 1.75 & $\mathrm{C}_{6} \mathrm{D}_{6}$ & $\mathrm{RT} \rightarrow 40$ & 3.5 & 88 \\
6 & $\mathrm{TMSCl}$ & 2.2 & 4.25 & $\mathrm{C}_{6} \mathrm{D}_{6}$ & $\mathrm{RT} \rightarrow 40$ & 3.5 & 100 \\
7 & $\mathrm{TMSCl}$ & 2.2 & 4.25 & $\mathrm{CDCl}_{3}$ & $\mathrm{RT}$ & 3.5 & 100 \\
8 & TMSCl & 1.5 & 3.0 & $\mathrm{C}_{6} \mathrm{H}_{6}$ & $\mathrm{RT} \rightarrow 40$ & 2 & 100 \\
9 & TMSI & 3.0 & 1.2 & $\mathrm{C}_{6} \mathrm{D}_{6}$ & )$))^{\mathrm{a})}, \mathrm{RT}$ & 1.5 & - \\
10 & TMSOTf & 2.0 & 2.5 & $\mathrm{C}_{6} \mathrm{D}_{6}$ & $0 \rightarrow \mathrm{RT}$ & 1.5 & - \\
\hline
\end{tabular}

a) Ultraschallbad. ${ }^{\text {b) }}$ Bildung von Derivaten.

Die Spektren der Silylierungsprobe des unter Verwendung von 1.1 eq TMSCl durchgeführten Versuchs (vgl. Tabelle 28, Eintrag 1) zeigten neben dem gewünschten Singulett bei 0.41 ppm für die $N$-TMS-Schutzgruppe und damit für die Bildung des gewünschten Silylamins 169 in einem Anteil von 20\% auch das Erscheinen weiterer unerwünschter Singuletts bei 4.2 ppm an, welche auf die Bildung des Chloridisomers 190 als Nebenprodukt hinwies. Die Auswertung der Reaktionsproben eines weiteren, zunächst unter Einsatz von 2.0 eq TMSCl und 2.5 eq $\mathrm{NEt}_{3}$ durchgeführten Versuchs (vgl. Tabelle 28, Eintrag 2) zeigte trotz Zugabe weiterer Äquivalente an TMSCl und $\mathrm{NEt}_{3}$ eine Silylierung des Edukts 159 zum Produkt 169 an, dessen Bildungsanteil allerdings bei längerer Reaktionszeit zurückging (Anteil 169: 17\% nach 1 h, 25\% nach 3 h, 14\% nach 4.5 h, 0\% nach 14.5 h). Das auch hier bei 4.2 ppm beobachtete ${ }^{1} \mathrm{H}-\mathrm{NMR}$ Signal zeigte ebenfalls die Bildung des Chloridisomers 190 an, allerdings nur in geringer Menge. Um eine stöchiometrisch genaue Dosierung der Reagenzien zu erreichen, wurde ein Lösungsgemisch aus TMSCl und $\mathrm{NEt}_{3}$-Lösung eingesetzt (vgl. Tabelle 28, Eintrag 3). Die Auswertung zeigte nicht das gewünschte Produkt 169 an. Dies wurde auf die fehlende Reaktivität der eingesetzten Verbindungen im Lösungsgemisch zurückgeführt. Aufgrund der starken Trübung der homogenen TMSCl-Lösung nach der Zugabe des Amins bei der Herstellung des Lösungsgemisches wurde auf die Adduktbildung der Lösungskomponenten geschlossen (Bildung quartärer Ammoniumsalze). Bei dem unter Einsatz einer 0.5 M TMSCl-Lösung in $\mathrm{CDCl}_{3}$ durchgeführten Versuch (vgl. Tabelle 28, Eintrag 4) wurde nach $1 \mathrm{~h}$ eine vollständige Umsetzung des Bromids 159 zu dem Silan 169 beobachtet. Während die Beobachtung des Reaktionsgeschehens nach 1.5 bzw. 2.0 h keine weitere Entwicklung aufzeigte, wurde nach 15 h eine Veränderung im ${ }^{1}$ H-NMR (Signale bei 4.1-4.25 ppm) festgestellt, die auf die Instabilität des Produktes 169 hinwies. Die Silylierungsreaktion wurde aufgrund der geringen Lös- 
lichkeit des Bromids 159 in $\mathrm{C}_{6} \mathrm{D}_{6}$ zudem bei einer erhöhten Reaktionstemperatur von $40{ }^{\circ} \mathrm{C}$ durchgeführt (vgl. Tabelle 28, Eintrag 5). Während die Auswertung der Spektren eine Umsetzung des silylierten Produkts 169 (Anteil 88\%) anzeigte, konnte das Produkt 169 massenspektroskopisch nicht nachgewiesen werden. Dies wurde jedoch auf die besondere Instabilität der Schutzgruppe und die Fragmentierung des Silylproduktes schon zu Beginn der Messung zurückgeführt. Die in größerem Maßstab durchgeführten Umsetzungen (vgl. Tabelle 28, Einträge 6-8) lieferten das gewünschte Produkt 169. Der Versuch 7 (Eintrag 7) wurde nach Beendigung der Reaktion zunächst i.V. einkonzentriert. Der Rückstand wurde in Benzol aufgenommen, über einen Spritzenfilter filtriert und mit Chloroform gewaschen. Die erhaltene organische Phase wurde i.V. konzentriert und spektroskopisch untersucht. Die bei $40{ }^{\circ} \mathrm{C}$ durchgeführten Versuche (Einträge 6 u. 8) wurden dagegen ausschließlich über einen Spritzenfilter filtriert und einkonzentriert (s. Exp. Teil, Allg. Arbeitsvorschrift A16). Die Durchführung der Reaktionen mit TMSCl führte unter optimierten Bedingungen zu sauberen Umsetzungen in quantitativen Ausbeuten (vgl. Tabelle 28). Scheinbar ist der Überschuß an Base notwendig, um Nebenreaktionen zu vermeiden. Die Versuche haben desweiteren gezeigt, dass die Silylierungsreaktion in $\mathrm{C}_{6} \mathrm{D}_{6}$ wegen der im Vergleich zu $\mathrm{CDCl}_{3}$ geringeren Löslichkeit des Brommethylpyridons 159, nicht bei Raumtemperatur, sondern zum Erzielen gleicher Ergebnisse wie bei den Versuchen mit $\mathrm{CDCl}_{3}$ bei höheren Temperaturen durchgeführt werden muß.

Bei der Umsetzung des Brommethylpyridons 159 mit TMSI in $\mathrm{CDCl}_{3}$ (vgl. Tabelle 28, Eintrag 9) blieb die gewünschte Schützung des Stickstoffs aufgrund von Löslichkeitsproblemen nach $1.5 \mathrm{~h}$ trotz Verwendung eines Ultraschallbades aus. Die Verwendung von TMSOTf in $\mathrm{C}_{6} \mathrm{D}_{6}$ (vgl. Tabelle 28, Eintrag 10) führte nach 90 min zur vollständigen Zersetzung des Eduktes 159.

Trotz der mit TMSCl erfolgreich durchgeführten Schützungsversuche wurde die Schützung der Amidgruppe desweiteren mit dem gegenüber TMSCl stabileren TBDMSCl als Schützungsreagenz untersucht, da sich das aus der Schützung mit TMSCl resultierende $N$ Trimethylsilylpyridon 169 bei den Kreuzkupplungsversuchen mit dem Alanat 124 als instabil erwies (vgl. Abschnitt 3.2.3.2). Hart et al. ${ }^{[80]}$ und Reider et al. ${ }^{[81]}$ verwendeten bei der Silylierung ähnlicher cyclischer Amide mit TBDMSCl ebenfalls $\mathrm{NEt}_{3}$ als Base. Die entsprechende Silylierung des Brommethylpyridons 159 (vgl. Schema 55) wurde mit einer TBDMSClLösung analog der oben beschriebenen Verfahrensweise der mit TMSCl gelungenen Silylierungsversuche (vgl. Tabelle 28, Einträge 6-8) durchgeführt. Das ${ }^{1}$ H-NMR-Spektrums des 
Versuchs wies eine Verdopplung der Protonensignale auf. Dies wurde mit der Bildung der $N$ Silylverbindung 170 und des an der C-2-Position O-silylierten Produktes 186 im Verhältnis von 50:50 interpretiert. Die Bildung dieser N- und O-silylierten Produkte als Gemisch kann mit der im Vergleich zu der TMS-Funktion sterisch anspruchsvolleren TBDMS-Schutzgruppe sowie der größeren Affinität der Si-O-Bindung erklärt werden. Deshalb wurde auf weitere Versuche mit TBDMSCl verzichtet.

Schema 55. $N$-Silylierung des Brommethylpyridons 159 mit tert-Butyldimethylsilylchlorid.

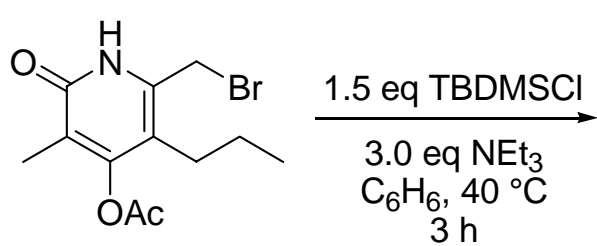

159<smiles>CCCc1c(OC(C)=O)c(C)c(=O)n(CC(C)=O)c1CBr</smiles>

170
TBDMSO<smiles>CCCc1c(CBr)nc(O)c(C)c1OC(C)=O</smiles>

186

Alternativ wurde zudem die Schützung des Brommethylpyridons 159 mit MOMCl untersucht (vgl. Schema 56). Entsprechend einer allgemeinen Arbeitsvorschrift nach Mori et al. ${ }^{[82]}$ wurden die Umsetzungen unter Einsatz von $\mathrm{NaH}$ bzw. $\mathrm{NEt}(i \operatorname{Pr})_{2}$ als Base durchgeführt (vgl. Tabelle 29, Einträge 1 u. 2). Die Auswertung des Spektrums des mit NaH durchgeführten Versuches (Eintrag 1) zeigte eine 26\%-ige Umsetzung zu dem gewünschten Produkt 171. Die Singuletts bei $3.41\left(\mathrm{CH}_{2} \mathrm{OCH}_{3}\right)$ und $4.61\left(\mathrm{NCH}_{2} \mathrm{O}\right)$ ppm wurden der Verbindung 171 zugeordnet.

Schema 56. Schützung der Stickstofffunktion des Brommethylpyridons 159 mit MOMCl.

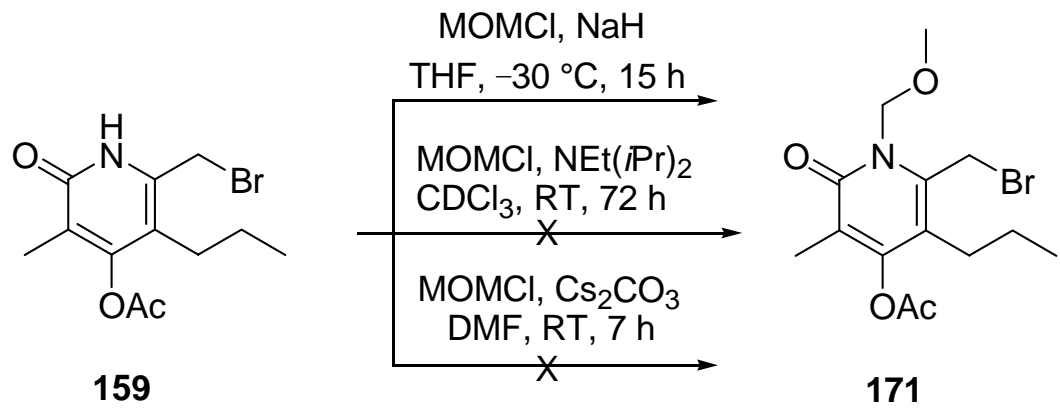


Tabelle 29. Schützung der funktionellen Stickstoffgruppe des Brommethylpyridons 159.

\begin{tabular}{ccccccccc}
\hline Eintrag & Reagenz & [eq] & Base & [eq] & LSM & $\mathrm{T}\left[{ }^{\circ} \mathrm{C}\right]$ & $\mathrm{t}[\mathrm{h}]$ & $N-\mathrm{CH}_{2} \mathrm{OMe}(\%)$ \\
\hline 1 & MOMCl & 1.1 & $\mathrm{NaH}$ & 1.1 & $\mathrm{THF}$ & $-30 \rightarrow \mathrm{RT}$ & 15 & 26 \\
2 & $\mathrm{MOMCl}$ & 5.0 & $\mathrm{NEt}(\mathrm{iPr})_{2}$ & 10 & $\mathrm{CDCl}_{3}$ & $0 \rightarrow \mathrm{RT}$ & 72 & - \\
3 & $\mathrm{MOMCl}$ & 1.0 & $\mathrm{Cs}_{2} \mathrm{CO}_{3}$ & 3.0 & $\mathrm{DMF}$ & $\mathrm{RT}$ & 7 & - \\
\hline
\end{tabular}

Die Schützung des Bromids 159 mit $\mathrm{MOMCl}$ unter Verwendung von $\operatorname{NEt}(i \operatorname{Pr})_{2}$ in $\mathrm{CDCl}_{3}$ wurde in einem NMR-Röhrchen im Mikromaßstab durchgeführt (vgl. Schema 56 u. Tabelle 29, Eintrag 2). Hierzu wurde MOMCl mit einer Lösung von Brommethylpyridon 159 (2 mg) und $\operatorname{NEt}(i \operatorname{Pr})_{2}$ in $\mathrm{CDCl}_{3}$ bei $0{ }^{\circ} \mathrm{C}$ umgesetzt. Die Auswertung der Umsetzung unter mehrmaliger spektroskopischer Untersuchung zeigte die Bildung diverser Nebenprodukte, die nicht eindeutig zu identifizieren waren. Der Versuch zur vollständigen Entfernung der Aminreste bei reduziertem Druck führte zu einem vollständigen Verlust der Schutzgruppe, was auf die relativ hohe Instabilität der funktionellen Gruppe zurückgeführt wurde. Eine weitere Umsetzung wurde analog nach Nadin et al. ${ }^{[83]}$ mit $\mathrm{Cs}_{2} \mathrm{CO}_{3}$ durchgeführt, wobei anstatt des von Nadin verwandten 4-Methoxybenzylchlorids $\mathrm{MOMCl}$ als Reagenz eingesetzt wurde (vgl. Tabelle 29, Eintrag 3). Dieser Versuch führte zur Bildung diverser unbekannter Nebenprodukte. Aufgrund des sehr komplexen Spektrums wurde diese Methode für nicht geeignet befunden.

Als weitere Variante zur Schützung des Brommethylpyridons 159 wurden Versuche mit TFAA $^{[84]}$ als Reagenz untersucht (vgl. Schema 57). Die Umsetzungen wurden im Mikromaß-

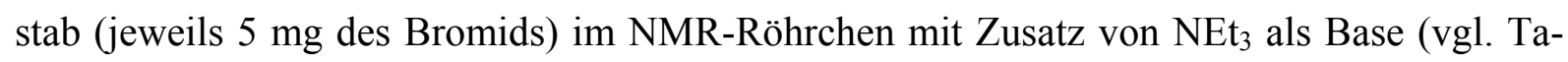
belle 30, Einträge 1 u. 2) und ohne Zusatz von $\mathrm{NEt}_{3}$ (vgl. Tabelle 30, Eintrag 3) untersucht

Schema 57. Schützung der Stickstofffunktion des Brommethylpyridons 159 mit TFAA.

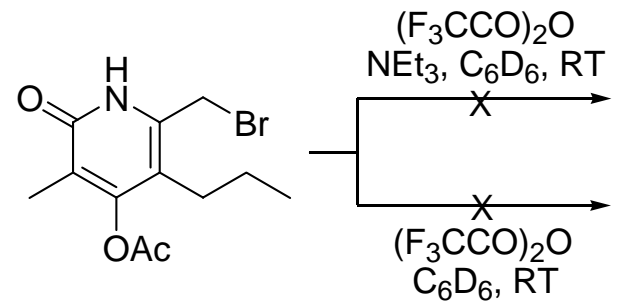

159<smiles>CCCc1c(OC(C)=O)c(C)c(=O)n(C(=O)C(F)(F)F)c1CBr</smiles>

172<smiles>CCCc1c(CBr)nc(OC(=O)C(F)(F)F)c(C)c1OC(C)=O</smiles>

188 
Tabelle 30. Schützung der Stickstoffgruppe des Brommethylpyridons 159 mit TFAA.

\begin{tabular}{ccccccccc}
\hline Eintrag & Reagenz & [eq] & Base & {$[\mathrm{eq}]$} & $\mathrm{LSM}$ & $\mathrm{T}\left[{ }^{\circ} \mathrm{C}\right]$ & $\mathrm{t}[\mathrm{h}]$ & $N-\mathrm{COCF}_{3}(\%)$ \\
\hline 1 & $\left(\mathrm{~F}_{3} \mathrm{CCO}\right)_{2} \mathrm{O}$ & 1.2 & $\mathrm{NEt}_{3}$ & 2.0 & $\mathrm{C}_{6} \mathrm{D}_{6}$ & $\mathrm{RT}$ & 3.5 & - \\
2 & $\left(\mathrm{~F}_{3} \mathrm{CCO}\right)_{2} \mathrm{O}$ & 1.2 & $\mathrm{NEt}_{3}$ & 3.0 & $\mathrm{CDCl}_{3}$ & $\mathrm{RT} \rightarrow 50$ & 3 & - \\
3 & $\left(\mathrm{~F}_{3} \mathrm{CCO}\right)_{2} \mathrm{O}$ & 8.7 & - & - & $\mathrm{C}_{6} \mathrm{D}_{6}$ & $\mathrm{RT}$ & 1.5 & - \\
\hline
\end{tabular}

Zunächst wurden das Brommethylpyridon 159 und $\mathrm{NEt}_{3}$ in $\mathrm{C}_{6} \mathrm{D}_{6}$ bzw. $\mathrm{CDCl}_{3}$ gelöst und anschließend eine Lösung von TFAA in den entsprechenden Lösungsmitteln zugegeben (vgl. Tabelle 30, Einträge 1 u. 2). Die Tatsache, dass alle erhaltenen ${ }^{1} \mathrm{H}-\mathrm{NMR}$-Spektren nach $1 \mathrm{~h}, 2$ h, $3 \mathrm{~h}$ und $3.5 \mathrm{~h}$ identische Protonenzahlen aufwiesen und keine weitere Veränderung mehr anzeigten, wurde als Anzeichen für eine vollständige Umsetzung nach $1 \mathrm{~h}$ gewertet. Es wurde im Vergleich zum Edukt 159 eine starke Verschiebung aller Protonensignale ins Hochfeld beobachtet. Bei der C-1"'- und der Acetatgruppe betrug die chemische Verschiebung 0.4 ppm. Aufgrund fehlender Protonensignale bei Trifluoracetat als Schutzgruppe konnte jedoch keine definitive Aussage darüber getroffen werden, ob es sich um das $\mathrm{N}$-Acylierungs- 172 oder OAcylierungsprodukt 188 handelte. Das entsprechende ${ }^{13} \mathrm{C}$-NMR-Spektrum (während der Messung fiel $\mathrm{NH}_{4} \mathrm{COOCF}_{3}$-Salz aus) deutete auf die O-Acylierung und Bildung des Pyridins 188 hin.

Das ${ }^{1} \mathrm{H}-\mathrm{NMR}-$ Spektrum des ohne Einsatz von $\mathrm{NEt}_{3}$ durchgeführten Versuchs (vgl. Tabelle 30, Eintrag 3) zeigte eine Verschiebung der C-1"'-Gruppe und der Acetatgruppe jeweils um 0.45 und 0.8 ppm ins Hochfeld an. Das Protonensignal des C-1'-Methylenbromids wurde um 0.4 ppm ins Hochfeld verschoben. Die Ursache dieser Verschiebung wurde nicht näher untersucht. Ein eventueller Austausch der Acetatgruppe mit einer Trifluoressigsäuregruppe wurde aufgrund der geringen Reaktivität des Esters jedenfalls für unwahrscheinlich gehalten. Längeres Aufbewahren (48 h) des Rohproduktes aus dem Versuch 3 (vgl. Tabelle 30, Eintrag 3) unter vermindertem Druck führte zur Rückverschiebung aller Protonensignale auf ihre ursprünglichen Positionen entsprechend denen des Brommethylpyridons 159, was eine Abspaltung der Schutzgruppe anzeigte. Die massenspektroskopische Analyse wies das eingesetzte Edukt 159 nach. Die Spaltung der funktionellen Gruppe unabhängig von dem Ort der Schützung zeigte zum wiederholten Male die Instabilität und den Schwierigkeitsgrad der Schützung des Brommethylpyridons 159 im Vergleich zur Schützung der entsprechenden Aminverbindungen auf. 
Als weitere Schützungsmöglichkeit wurde schließlich die Acylierung des Brommethylpyridons 159 mit $\mathrm{AcCl}$ nach Nishiyama et al. ${ }^{[78]}$ untersucht (vgl. Schema 58). Die Umsetzung wurde im Mikromaßstab in einem NMR-Röhrchen in $\mathrm{CDCl}_{3}$ als Lösungsmittel durchgeführt (s. Exp. Teil). Der Schützungsversuch führte in einer sauberen Reaktion nach $1.5 \mathrm{~h}$ zur vollständigen Umsetzung. Spektroskopische und massenanalytische Untersuchungen wiesen jedoch statt der Bildung des gewünschten Bromids 173 ausschließlich die Bildung des doppelt acetylierten Pyridins 181 nach.

Schema 58. Schützung der Stickstofffunktion des Brommethylpyridons 159 mit AcCl.<smiles>CCCc1c(CBr)[nH]c(=O)c(C)c1OC(C)=O</smiles>

159

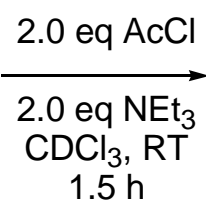

$1.5 \mathrm{~h}$

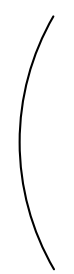<smiles>CCCc1c(CBr)c(C)c(=O)n(C(C)=O)c1C</smiles>

173<smiles>CCCc1c(CBr)nc(OC(C)=O)c(C)c1OC(C)=O</smiles>

181

\subsection{Versuche zur Schützung des Brommethylpyridons 159 durch Methylierung}

Die Versuche zur Methylierung des Brommethylpyridons 159 wurden zunächst nach Pattenden et. al. ${ }^{[85]}$ mit einer Diazomethan-Lösung untersucht (vgl. Schema 59). In Abweichung von den Vorgaben von Pattenden wurden die Reaktionen allerdings bei $0{ }^{\circ} \mathrm{C}$ durchgeführt. Hierbei wurde zu einer Lösung des Bromids $159\left(\mathrm{CHCl}_{3}\right.$ bzw. THF) eine etherische Lösung des Diazomethans zugetropft und gerührt. Die Reaktionen wurden im Allgemeinen nach Beendigung der Umsetzungen ausschließlich konzentriert.

Schema 59. Methylierung des Brommethylpyridons 159 an der Stickstofffunktion mit Diazomethan zu dem $N$-Methylbrommethylpyridon 174.<smiles>CCCc1c(CBr)[nH]c(=O)c(C)c1OC(C)=O</smiles>

159

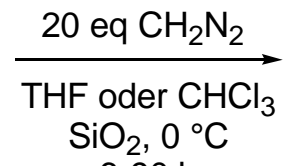
$6-90 \mathrm{~h}$<smiles>CCCc1c(OC(C)=O)c(C)c(=O)n(C)c1CBr</smiles>

174<smiles>CCCc1c(CBr)nc(OC)c(C)c1OC(C)=O</smiles>

177 
Zunächst wurden gemäß der Arbeitsvorschrift von Pattenden et al. 2.0 eq Diazomethan eingesetzt (vgl. Tabelle 31, Eintrag 1). Da die dünnschichtchromatographische Detektion keine Umsetzung anzeigte, wurden nach $2 \mathrm{~h}$ weitere 2.0 eq Diazomethan zugegeben und $6 \mathrm{~h}$ gerührt. Es wurde ein Gemisch aus dem N-Methyl- 174 und dem O-Methyl-Isomer 177 (vgl. Schema 59) bei einem Umsatz von lediglich 8\% erhalten (3.68 ppm für NMe und 3.92 ppm für OMe). Pattenden et al. ${ }^{[85]}$ berichteten ebenfalls von der Bildung eines Isomerengemisches (bei 3.69 ppm für NMe und bei 3.93 ppm für OMe). Als Konsequenz des geringen Umsetzungsanteils wurden daher die weiteren Methylierungsversuche unter Einsatz des Diazomethans im Überschuß (20 eq) durchgeführt (vgl. Tabelle 31, Einträge 2-8).

Tabelle 31. Methylierung des Brommethylpyridons 159 mit Diazomethan zu dem N-Methylbrommethylpyridon 174.

\begin{tabular}{cccccc}
\hline Eintrag & Diazomethan [eq] & $\mathrm{SiO}_{2}$ & $\mathrm{Lsm}$. & $\mathrm{t}[\mathrm{h}]$ & $\mathbf{1 5 9 : 1 7 4 : 1 7 7 ( \% )}$ \\
\hline 1 & 4.0 & - & $\mathrm{CHCl}_{3}$ & 6 & $92: 5: 3$ \\
2 & 20 & - & $\mathrm{CHCl}_{3}$ & 90 & $22: 18: 60$ \\
3 & 20 & - & $\mathrm{THF}$ & 44 & $0: 52: 48$ \\
4 & 20 & + & $\mathrm{THF}$ & 20 & $0: 53: 47$ \\
5 & 20 & + & $\mathrm{THF}$ & 20 & $0: 46: 54$ \\
6 & 20 & + & $\mathrm{THF}$ & 20 & $0: 44: 56$ \\
7 & 20 & + & $\mathrm{THF}$ & 20 & $0: 38: 62$ \\
8 & 20 & + & THF & 22 & $0: 36: 64$ \\
\hline
\end{tabular}

Eine vollständige Umwandlung des Bromids 159 konnte bei der katalysatorfreien Umsetzung (vgl. Tabelle 31, Eintrag 2) trotz Einsatzes von Diazomethan im Überschuß (20 eq) und einer längeren Reaktionszeit von $90 \mathrm{~h}$ nicht erreicht werden. Es wurde hauptsächlich das OMeIsomer 177 in einem Bildungsanteil von 60\% gebildet. Dagegen konnte bei dem katalysatorfreien Methylierungsversuch in THF (vgl. Tabelle 31, Eintrag 3) das gewünschte $N$-MethylIsomer 174 im Verhältnis 52:48 als Hauptprodukt erhalten werden. Wegen der höheren Löslichkeit des Bromids 159 wurde dieser Versuch im polaren Lösungsmittel THF durchgeführt. Der katalytische Einsatz von $\mathrm{SiO}_{2}$ führte zur Beschleunigung der Reaktionsgeschwindigkeit und zur Umkehrung der Isomerenanteile zugunsten des $N$-Methylierungsproduktes 174 (vgl. Tabelle 31, Einträge 4-8). In allen Fällen wurde weiterhin stets ein Gemisch aus beiden Isomeren erhalten. Die Auswertung der Tabelle 31 lässt hierbei erkennen, dass mit dem Einsatz von $\mathrm{SiO}_{2}$ im Vergleich zu den Versuchen ohne Anwendung des Katalysators keine effektive Erhöhung des Isomerenanteils bei dem Methylierungsprozeß mit Diazomethan zugunsten des 
$N$-Isomers 174 erreicht werden konnte. Die Isomeren wurden über säulenchromatographische Reinigung getrennt (s. Exp. Teil, Allg. Arbeitsvorschrift A18). Die Bildung des O-Isomers ist auf die Aromatisierung des Rings zurückzuführen. Bei den mit $\mathrm{SiO}_{2}$ durchgeführten Versuchen ist das Selektivitätsproblem daneben höchstwahrscheinlich auf die energetisch günstigere C-O- bzw. Si-O-Bindung im Vergleich zur energetisch benachteiligten C-N- bzw. Si-NBindung zurückzuführen, die wiederum in der stärkeren Affinität der Si-Verbindung zum Sauerstoffatom begründet liegt.

In einer weiteren Variante zur $N$-Methylierung des Brommethylpyridons 159 wurde Methyliodid (MeI) als Alkylierungsmittel eingesetzt (vgl. Schema 60). Als Basen wurden unter anderem $n \mathrm{BuLi}$, LDA und Alkalihydride (NaH oder $\mathrm{KH}$ ) verwendet. Im Hinblick auf den $p$ KsWert der Pyridone (15-17) dürften die oben genannten Basen eine ausreichende Basizität für eine vollständige Deprotonierung aufweisen.

Schema 60. Methylierung des Brommethylpyridons 159 mit Methyliodid unter Verwendung von $n \mathrm{BuLi}$, LDA, $\mathrm{NaH}$ und $\mathrm{KH}$.

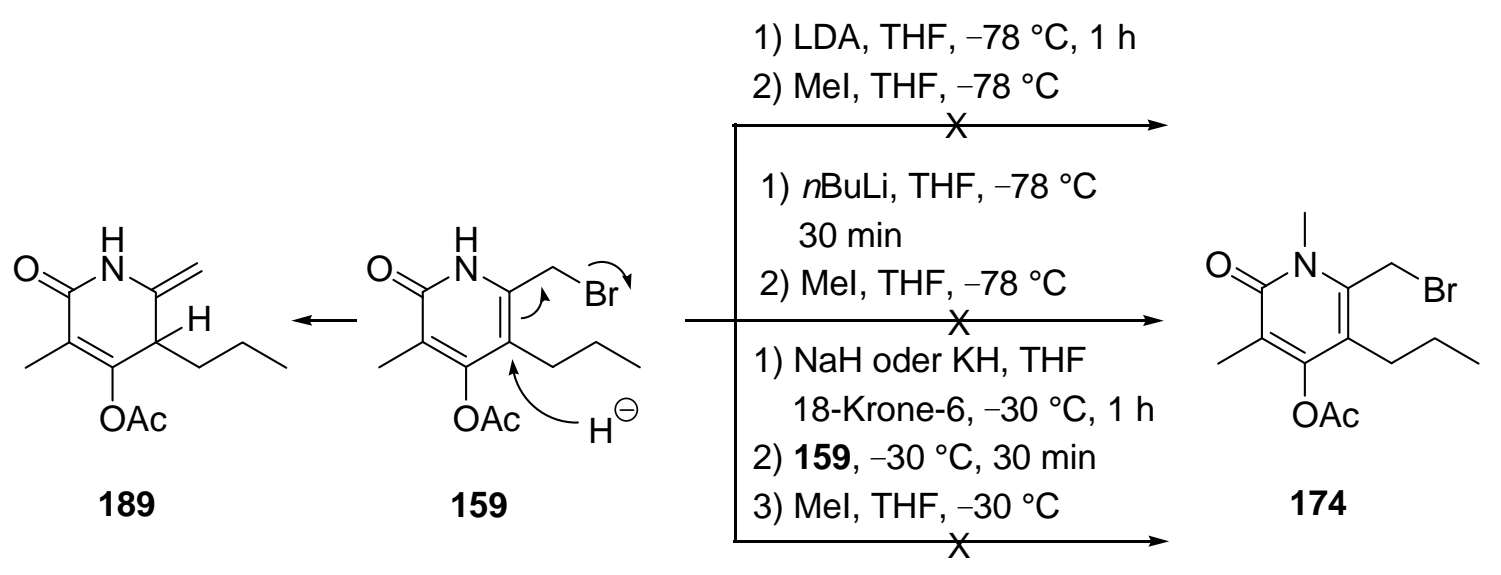

In der ersten Variante des Methylierungsversuches mit MeI wurde bei $-78^{\circ} \mathrm{C} n \mathrm{BuLi}$ zu einer Lösung des Bromids 159 in THF zugegeben und 30 min gerührt (vgl. Tabelle 32, Eintrag 1). Anschließend wurde langsam bei $-78^{\circ} \mathrm{C}$ das MeI zugegeben. Die Reaktionslösung wurde aufgrund fehlender Umsetzung bei $-78{ }^{\circ} \mathrm{C}$ bzw. $0{ }^{\circ} \mathrm{C}$ (DC-Detektion) auf Raumtemperatur erwärmt. Die Auswertung der Umsetzung nach wässriger Aufarbeitung belegte neben der Bildung diverser Nebenprodukte auch die Abspaltung des Bromids in erheblichem Anteil. 
Dies ist auf die Bildung des $n$ Butylbromids und die Lithierung der C-1'-Position zurückzuführen, die nach der Hydrolyse zu dem debromierten Produkt 156 führte (vgl. Schema 49). Zusätzlich tendieren Lithiumorganyle ( $n \mathrm{BuLi})$ im Vergleich zu Lithiumamiden (LDA) dazu, häufiger als Nucleophile und seltener als Basen zu fungieren. Das Erscheinen des Protonensignals bei 9.91 ppm wurde als geringfügige Bildung eines Oxidationsprodukts, wie z.B. Aldehyd, gewertet, obwohl an sich reduktive Reaktionsbedingungen vorlagen. Bei der zweiten Versuchsvariante wurden nicht-nucleophile sowie schwächer basische Lithiumamide (LDA) als Deprotonierungsmittel eingesetzt (vgl. Tabelle 32, Einträge 2-4). Das LDA wurde aus einer Lösung von $n \mathrm{BuLi}$ und Diisopropylamin entsprechend der in der Tabelle 32 angegebenen Reaktionsbedingungen vorgeneriert. Bei allen mit LDA durchgeführten Versuchen (Einträge 2-4) blieb das $N$-Methylierungsprodukt 174 trotz Verwendung von Methyliodid im Überschuß bzw. 1.6 eq LDA (Eintrag 4) gänzlich aus.

Tabelle 32. Basenvermittelte Methylierung des Bromids 159 mit Methyliodid.

\begin{tabular}{cccccccccc}
\hline Eintrag & Base & $\begin{array}{c}\text { Base } \\
{[\mathrm{eq}]}\end{array}$ & $\begin{array}{c}\mathrm{T}_{1} \\
{\left[{ }^{\circ} \mathrm{C}\right]}\end{array}$ & $\begin{array}{c}\mathrm{t}_{1} \\
{[\mathrm{~h}]}\end{array}$ & $\begin{array}{c}\mathrm{T}_{2} \\
{\left[{ }^{\circ} \mathrm{C}\right]}\end{array}$ & $\begin{array}{c}\mathrm{t}_{2} \\
{[\mathrm{~h}]}\end{array}$ & $\begin{array}{c}\mathrm{MeI} \\
{[\mathrm{eq}]}\end{array}$ & $\begin{array}{c}\mathrm{T}_{3} \\
{\left[{ }^{\circ} \mathrm{C}\right]}\end{array}$ & $\begin{array}{c}\mathrm{t}_{3} \\
{[\mathrm{~h}]}\end{array}$ \\
\hline 1 & $n$ BuLi & 1.0 & -78 & 0.5 & -78 & 0.5 & 1.0 & -78 & 2 \\
& & & & & & & & 0 & 1 \\
& & & & & & & & $\mathrm{RT}$ & 20 \\
2 & LDA & 1.1 & 0 & 1 & -78 & 0.5 & 1.0 & $-78 \rightarrow 0$ & 15 \\
& & & & & & & 1.0 & 0 & 4 \\
3 & LDA & 1.6 & -78 & 1 & -78 & 1 & 1.0 & -78 & 19 \\
& & & & & & & & -40 & 4 \\
4 & LDA & 1.6 & -78 & 1 & -78 & 1 & 3.0 & -78 & 3 \\
& & & & & & & & -60 & 15 \\
& & & & & & & 60 & -60 & 4 \\
\hline
\end{tabular}

Ebenso misslangen die geplanten Schützungsversuche mit Methyliodid in Anwesenheit der Alkalihydride $\mathrm{NaH}$ und $\mathrm{KH}$ beim Zusatz von 1.1 eq 18-Krone-6 als Chelatisierungsmittel (vgl. Schema 60, Tabelle 33, Einträge 1-3). Die Versuche ergaben zwar alle das gewünschte Methylierungsprodukt 174 in einem 68-, 71- bzw. 85\%-igen Anteil. Allerdings entstand auch eine große Menge weiterer Produkte, so daß die $N$-Methylierungsanteile tatsächlich geringer ausgefallen sein dürften. Das Erscheinen eines unsauberen Dubletts bei 5.25-5.28 ppm sowie der bereits aus vorhergehenden Versuchen bekannte Debromierungseffekt bei den mit $\mathrm{NaH}$ durchgeführten Versuchen (Einträge 1 u. 2) wurde als wahrscheinliche Folge der Bildung einer exo-cyclischen Doppelbindung und damit der Bildung der Verbindung 189 gewertet 
(vgl. Schema 60). Ebenso wurde das erhaltene ${ }^{13}$ C-NMR-Signal bei 98 ppm als ein Indiz für die Bildung einer exo-cyclischen Doppelbindung angesehen. Die Bildung einer exocyclischen Doppelbindung war überraschend, da in der Regel die exo-cyclischen Doppelbindungen instabiler als ihre entsprechenden endo-cyclischen Doppelbindungen sind. Auch die Beobachtung von Multiplett-Signalen im ${ }^{1} \mathrm{H}-\mathrm{NMR}-$ Spektrum bei 1.9-2.05 sowie 3.6-3.75 ppm könnte die Addition eines Hydrid-Nucleophils an der stark elektrophilen C-5-Position des Ringgerüstes und die Initialisierung eines neuen Stereozentrums begründen (vgl. Schema 60). Die durch einen nucleophilen Hydrid-Angriff an der C-5-Position des Rings initialisierte Umlagerung der endo-cyclischen zu einer exo-cyclischen Doppelbindung könnte, anders als bei dem durch $n \mathrm{BuLi}$ initialisierten Debromierungsprozeß (vgl. Tabelle 32, Eintrag 1), zur Verdrängung und Abspaltung des Bromidanions geführt haben. Die mögliche Abspaltung des Bromidanions trotz Einsatz von 18-Krone-6 als Kationsolvator dürfte neben dem kleineren Radius des Natriumkations wahrscheinlich auf die nicht optimale Qualität des Kroneethers zurückzuführen sein, denn das Ausbleiben des Debromierungseffektes bei dem unter Einsatz von $\mathrm{KH}$ als Base durchgeführten Versuch (Eintrag 3) spricht aufgrund des größeren Ionenradius für eine effektiver erfolgte Stabilisierung des Kaliumions durch den Kronenether. Die Rolle des Kronenethers bei dem Debromierungseffekt könnte darin gelegen haben, dass eine Stabilisierung des Kaliumions durch den Kronenether zu einer erhöhten Verfügbarkeit des Hydridanions als Base anstatt als Nucleophil geführt hat. Somit könnte statt einer langsamer verlaufenden nucleophilen Substitutionsreaktion eine schneller verlaufende Deprotonierungsreaktion abgelaufen sein, wodurch unter Umständen auch der erhöhte Bildungsanteil des $N$ Methylierungsproduktes entstanden sein könnte.

Während bei den Versuchen mit NaH (vgl. Tabelle 33, Einträge 1 u. 2) eine Abspaltung der funktionellen Acetatgruppe des Brommethylpyridons 159 aufgrund des komplexen Spektrums weder widerlegt noch bestätigt werden konnte, wurde bei der Reaktion mit KH (vgl. Tabelle 33, Eintrag 3) ein Entschützungsanteil von $72 \%$ gegenüber dem $N$-Methylierungsanteil von $28 \%$ ermittelt. Zusätzlich wurde bei diesem Versuch (Eintrag 3) ein nicht minder hoher Anteil des O-Methylierungsprodukts 177 (vgl. Schema 59) beobachtet. Insgesamt wurde trotz zufriedenstellender $N$-Methylierungsanteile aufgrund der Bildung diverser Nebenprodukte und des Auftretens von Entschützungs- bzw. Debromierungsproblemen sowie der Bildung einer exo-cyclischen Doppelbindung keine weitere Untersuchung durchgeführt. Das methylierte Derivat 174 wurde nach Isolierung bei den im folgenden Abschnitt behandelten Kreuzkupplungsversuchen eingesetzt. 
Tabelle 33. Methylierung des Bromids 159 mit MeI und NaH bzw. KaH als Basen.

\begin{tabular}{cccccccccc}
\hline Eintrag & $\begin{array}{c}\text { Base } \\
{[\mathrm{eq}]}\end{array}$ & $\begin{array}{c}\mathrm{T}_{1} \\
{\left[{ }^{\circ} \mathrm{C}\right]}\end{array}$ & $\begin{array}{c}\mathrm{t}_{1} \\
{[\mathrm{~h}]}\end{array}$ & $\begin{array}{c}\mathrm{T}_{2} \\
{\left[{ }^{\circ} \mathrm{C}\right]}\end{array}$ & $\begin{array}{c}\mathrm{t}_{2} \\
{[\mathrm{~h}]}\end{array}$ & $\begin{array}{c}\mathrm{T}_{3} \\
{\left[{ }^{\circ} \mathrm{C}\right]}\end{array}$ & $\begin{array}{c}\mathrm{t}_{3} \\
{[\mathrm{~h}]}\end{array}$ & $\begin{array}{c}\text { MeI } \\
{[\mathrm{eq}]}\end{array}$ & $\begin{array}{c}\text { 159:174:177:156 } \\
(\%)\end{array}$ \\
\hline $1^{\mathrm{a})}$ & $1.1 \mathrm{NaH}$ & -30 & 1 & -30 & 0.5 & $-30 \rightarrow 0$ & 15 & 1.0 & \\
& & & & & & 0 & 4 & & $0: 68: 0: 32$ \\
$2^{\text {a) }}$ & $1.1 \mathrm{NaH}$ & -78 & 1 & -78 & 1 & -78 & 1 & 1.0 & \\
& & & & & & -78 & 18 & & \\
& & & & & & -40 & 4 & & $0: 71: 0: 29$ \\
$3^{\text {a) }}$ & $1.1 \mathrm{KaH}$ & -78 & 1 & -78 & 1 & -78 & 3 & 3.0 & \\
& & & & & & -60 & 15 & & $0: 85: 15: 0$ \\
& & & & & & $\mathrm{RT}$ & 6 & & \\
\hline
\end{tabular}

${ }^{\text {a) }}$ Es wurde 1.1 eq 18-Krone-6 eingesetzt.

\subsubsection{Kreuzkupplungversuche der $N$-geschützten Brommethylpyridone 169 und 174 mit dem Alanat 124}

Die in den vorigen Abschnitten behandelten Schützungsversuche gelangen lediglich im Falle des Einsatzes von Trimethylsilylchlorid sowie Diazomethan, bei denen die Brommethylpyridone 169 und 174 erhalten wurden. Die Kreuzkupplungsversuche wurden entsprechend der in Schema 52 dargestellten Methode daher nur mit den $N$-geschützten Brommethylpyridonen 169 oder 174 und dem Alanat 124 durchgeführt (für stöchiometrische Angaben sowie Reaktionsbedingungen vgl. Tabelle 34). Hierzu wurde bei $0{ }^{\circ} \mathrm{C}$ eine Lösung der Bromide $169 \mathrm{bzw}$. 174 zum vorgenerierten Alanat zugegeben. Bei den mit dem Bromid 169 in Gegenwart von 2.0 eq Alanat 124 in THF bzw. MeCN bei Raumtemperatur durchgeführten Versuchen (vgl. Tabelle 34, Einträge 1 u. 2) konnte das gewünschte Kreuzkupplungsprodukt 175 nach einer Reaktionszeit von $60 \mathrm{~h}$ nicht nachgewiesen werden. Die DC-Detektion sowie die Auswertung des Spektrums der Umsetzungen belegten eine vollständige Abspaltung der TMS-Gruppe, was auf die geringe Stabilität der Schutzgruppe zurückzuführen ist. Desweiteren konnte in beiden Fällen neben Spuren des Diens 136 der schon mehrfach beschriebene Debromierungseffekt durch die Bildung des 6-Methylpyridons 156 beobachtet werden. Für den Versuch 1 (Eintrag 1) wurde ein Anteil von 47\% für das 6-Methylpyridon 156 berechnet. Bei der Umsetzung 2 (Eintrag 2) konnte dieser Anteil aufgrund von Verunreinigungen nicht genau berechnet werden.

Die Auswertung der entsprechenden Kreuzkupplungsversuche des $N$-Methylpyridons 174 mit 3.0 eq des Alanats 124 (vgl. Tabelle 34, Einträge 3 u. 4) erwies sich aufgrund erheblicher Verunreinigungen als schwierig. Es wurden als charakteristische Signale für das ungeschützte 
Kreuzkupplungsprodukt 165 ein Dublett für das allylische 1'-H bei 3.3 ppm und ein Triplett für das benachbarte olefinische 2'-H bei $5.2 \mathrm{ppm}$ erwartet, welche schon bei den Versuchen zuvor nachgewiesen werden konnten (vgl. Schema 51, Tabelle 27, Einträge 2 u. 7). Das erwartete Dublett bei 3.3 ppm konnte jedoch wegen der Unschärfe des Spektrums nicht eindeutig identifiziert werden. Die erhaltenen Protonensignale der Versuche 3 und 4 (vgl. Tabelle 34, Einträge 3 u. 4) bei 5.35 ppm (scharfes Triplett), 5.20 und $5.41 \mathrm{ppm}$ (unscharfes Triplett) könnten ein Indiz für die Bildung des Kreuzkupplungsproduktes 176 darstellen. Ob die unterschiedliche chemische Verschiebung der Protonensignale von $0.21 \mathrm{ppm}$ im Vergleich zu den für das Kreuzkupplungsprodukt erwarteten Signalen auf Wechselwirkungs- und Lösungsmitteleffekte im Rohprodukt zurückzuführen war, erschien zwar möglich. Eine sichere Bestätigung für die Bildung des gewünschten Kreuzkupplungsproduktes konnte hieraus jedoch nicht abgeleitet werden. Wegen der geringen Intensitäten der oben genannten Protonensignale sowie der erheblichen Nebenproduktbildung erschien eine weitere Untersuchung nicht sinnvoll.

Tabelle 34. Kreuzkupplungversuche der $N$-geschützten Brommethylpyridone 169 bzw. 174 mit dem vorgenerierten Alanat 124 zu den Kreuzkupplungsprodukten 175 bzw. 176.

\begin{tabular}{cccccccc}
\hline Eintrag & $\begin{array}{c}\text { Alan } \\
{[\mathrm{eq}]}\end{array}$ & $\begin{array}{c}n \text { BuLi } \\
{[\mathrm{eq}]}\end{array}$ & Lsm.1 & Bromid & Lsm.2 & $\begin{array}{c}\mathrm{t} \\
{[\mathrm{h}]}\end{array}$ & $\begin{array}{c}\mathbf{1 6 9 : 1 5 9 : 1 7 5 : 1 3 6 : 1 3 7 : 1 5 6} \\
(\%)\end{array}$ \\
\hline 1 & 2.0 & 2.0 & THF & $\mathbf{1 6 9}$ & THF & 60 & $0: 52: 0: 1: 0: 47$ \\
2 & 2.0 & 2.0 & MeCN & $\mathbf{1 6 9}$ & MeCN & 60 & $0: 95: 0: 5: 0:-{ }^{\text {b) }}$ \\
3 & 3.0 & 3.0 & THF & $\mathbf{1 7 4}$ & THF & 16 & $-^{\text {c) }}$ \\
4 & 3.0 & 3.0 & MeCN & $\mathbf{1 7 4}$ & MeCN & 16 & $-^{\text {c) }}$ \\
${ }^{\text {a) }}$ Debromiertes Edukt. ${ }^{\text {b) }}$ Der Debromierungsanteil konnte nicht berechnet werden. Der \\
prozentuale Anteil des Diens 136 wurde daher nur bezogen auf das Bromid 175 berechnet. \\
c) Keine Auswertung möglich.
\end{tabular}

Bei den Kreuzkupplungsversuchen des Brommethylpyridons 159 mit dem Alanat 124, die im Gegensatz zu den Kreuzkupplungsversuchen mit dem Brommethylpyron 96 schlechte Ausbeuten lieferten, ergab sich die Fragestellung, ob das Proton der Amidgruppe des Brommethylpyridons 159 einen Störfaktor für die gewünschte C-C-Bindungsbildung darstellte und die Schützung des Amids mit einer geeigneten Schutzgruppe die Kreuzkupplung ermöglichen würde. Die erhaltenen Ergebnisse aus den Kreuzkupplungsversuchen der $N$-geschützten Brommethylpyridone 169 und 174 mit dem Alanat 124 ließen jedoch keine eindeutige Aussage darüber zu, ob das Proton der Stickstoffgruppe der tatsächliche Hinderungsgrund für die gewünschte Kreuzkupplung darstellte, da, wie beschrieben, auch mit dem $N$-geschützten Py- 
ridon keine Produktbildung erfolgte. Der Syntheseweg der Iromycine über die Pyridone erschien auf dem hier untersuchten Weg aufgrund der erhaltenen Ergebnisse insgesamt nicht erfolgversprechend.

\section{Kreuzkupplungversuche des Brommethylpyridins 181 mit Alanat 124}

Auf dem Weg zur Synthese der Iromycine wurden entsprechende Pyridine als weiteres potentielles Ringsystem zur Darstellung des Kreuzkupplungsproduktes untersucht. Entsprechend der schon beschriebenen Vorgehensweise bei den Kreuzkupplungsreaktionen von Pyronhalogeniden (vgl. Abschnitt 2.2) bzw. Pyridonhalogeniden (vgl. Abschnitt 3.2) sollten die gewünschten Kreuzkupplungsprodukte ausgehend von Pyridinhalogeniden und Alanaten synthetisiert und nach anschließender Entschützung im basischen Medium in die gewünschten Iromycine überführt werden (vgl. Schema 61).

Schema 61. Kreuzkupplung des Brommethylpyridins 181 mit dem Alanat 124.

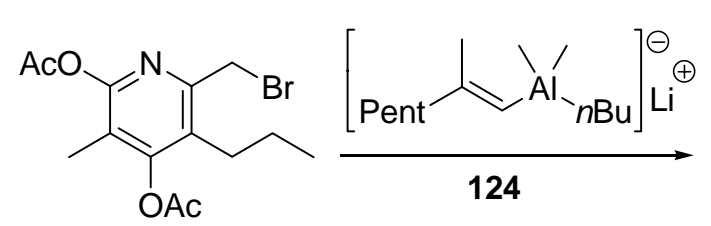

181<smiles>CCCc1c(CC=C(C)[OH2+])nc(OC(C)=O)c(C)c1OC(C)=O</smiles>

182

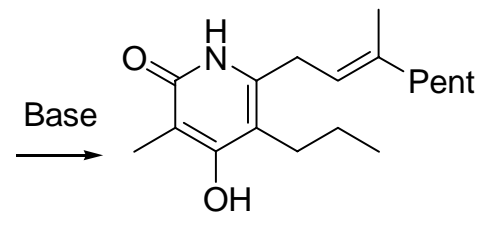

23

Zur Durchführung der geplanten Kreuzkupplungsreaktionen wurde zunächst die Synthese der benötigten Ausgangsverbindungen Diacetoxypyridin 178, Chlormethylpyridin 179, Pyridincarbaldehyd 180 und Brommethylpyridin 181 aus Pyridon 62 untersucht (vgl. Schema 62). Die Darstellung des Diacetoxypyridins 178 nach Mukkala et al. ${ }^{[86]}$ verliefen sehr sauber. Hierzu wurde eine Lösung des Pyridons 62 in Essigsäureanhydrid (Überschuß) 2-3 h unter Rückfluß bei $140{ }^{\circ} \mathrm{C}$ gerührt (s. Exp. Teil, Allg. Arbeitsvorschrift A19). Die Funktionalisierung des Diacetoxypyridins 178 zum Pyridincarbaldehyd 180 wurde nach Suzuki et al. ${ }^{[38]}$ geprüft. Hierzu wurde zunächst eine Lösung des Diacetoxypyridins 178 in Dioxan in Anwesenheit von 3.0 eq $\mathrm{SeO}_{2}$ bei $170{ }^{\circ} \mathrm{C}$ erhitzt und $1.5 \mathrm{~h}$ gerührt. Die Reaktion führte zwar zur vollständigen Funktionalisierung der Seitenkette zum Aldehyd, es fand jedoch eine Abspal- 
tung beider Acetatgruppen zum 4-Hydroxypyridonaldehyd statt. Die analoge Umsetzung bei $110{ }^{\circ} \mathrm{C}$ ergab nach $18.5 \mathrm{~h}$ ein Gemisch aus dem eingesetzten Diacetat 178 und dem gewünschten Pyridincarbaldehyd 180 im Verhältnis von 1.3:1 (s. Exp. Teil, Allg. Arbeitsvorschrift A10, Versuch 1).

Schema 62. Synthese der Pyridinderivate 178, 179, 180 und 181 aus dem Pyridon 62.

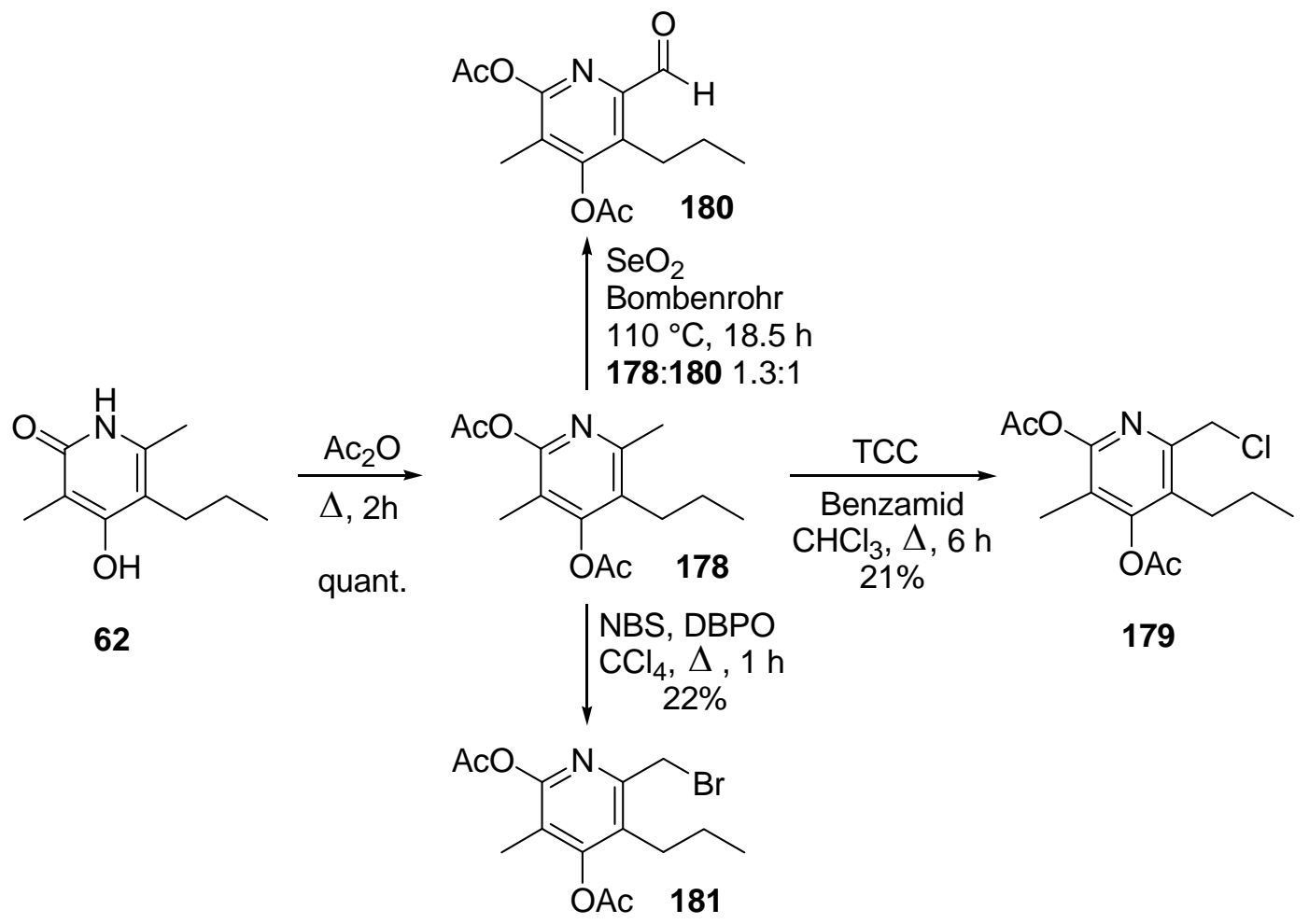

Die Direktsynthese des Chlorids 179 und des Bromids 181 aus Diacetoxypyridin 178 (vgl. Schema 62) wurde nach Jeromin et al. ${ }^{[87]}$ und Moreno-Maňas et al. ${ }^{[39]}$ durchgeführt. Für die Darstellung von 2-(Chlormethyl)pyridin 179 wurde eine Mischung aus dem Diacetat 178, Benzamid und Trichlorisocyansäure (TCC) als Chlorierungsmittel unter Rückfluß umgesetzt. Die nach wässriger Aufarbeitung durchgeführte säulenchromatographische Reinigung ergab ein Gemisch aus zweifach chloriertem Diacetat (9\%), monochloriertem Diacetat 179 (21\%) und zweifach chloriertem 4-Acetoxypyridon (34\%) (Exp. Teil, Allg. Arbeitsvorschrift A20). Die nach Moreno-Maňas et al. ${ }^{[39]}$ durchgeführte radikalische Funktionalisierung des Diacetoxypyridins 178 zum Bromid 181 verlief unter Einsatz von NBS und DBPO in $\mathrm{CCl}_{4}$ unter 
Rückfluß unsauber. Das gewünschte Bromid 181 konnte nach säulenchromatographischer Reinigung in einer Ausbeute von 22\% isoliert werden (s. Exp. Teil).

Die geplante Kreuzkupplungsreaktion (vgl. Schema 61) mit 2.0 eq Alanat 124 in THF wurde nach der allgemeinen Arbeitsvorschrift A13 (s. Exp. Teil) durchgeführt. Nach einer Reaktionszeit von 48 h konnten nur geringe Spuren des gewünschten Kreuzkupplungsproduktes 182 als Pyridinverbindung und des in Folge einer in situ Entschützung gebildeten IromycinAnalogons 23 (4-Acetoxypyridon) beobachtet werden. Aufgrund des nur geringen Anteils des Kreuzkupplungsproduktes sowie der Probleme bei der Darstellung der Ausgangsverbindungen, welche sich in geringeren Ausbeuten als bei der Pyron- und Pyridonsyntheseroute sowie in der schlechten Qualität der Rohprodukte widerspiegelte, wurde der hier untersuchte Weg für die synthetische Darstellung der Iromycine als nicht erfolgversprechend befunden und daher nicht weiter verfolgt.

\section{Metallkatalysierte Kreuzkupplungsversuche}

Als weitere Möglichkeit zur Synthese der Iromycine A und R sowie der entsprechenden Iromycinanaloga wurde die C-C-Bindungsknüpfung mittels Übergangsmetall-katalysierter Reaktionen untersucht. Die Anwendung der metallkatalysierten Kreuzkupplungsmethode erschien aufgrund des eingangs dargestellten retrosynthetischen Syntheseplans naheliegend, da die beiden Fragmente, nämlich das Halogenid als Hauptfragment sowie die aus Alkinen dargestellten Alkenyle, für eine Übergangsmetall-katalysierte Verküpfung prädestiniert schienen. Metallkatalysierte Reaktionen zeichnen sich nämlich dadurch aus, dass Alkenyl- oder VinylGruppen auf Aryl-, Vinyl- oder Alkenylhalogenide übertragen werden können. Zur Darstellung der Iromycine wurden Palladium-, Kupfer- sowie Nickel-katalysierte Kupplungsreaktionen der beiden Kettenfragmente untersucht.

\subsection{Pd-katalysierte Kreuzkupplung mit Alanen}

Negishi et al. ${ }^{[56]}$ berichteten von der hoch stereo- und regioselektiven Durchführung von Pdkatalysierten Kreuzkupplungen von Benzylhalogeniden mit Alkenylalanen. Hierzu setzte man das Benzylbromid 130 bzw. Benzylchlorid 222 unter Verwendung von $\left[\mathrm{Pd}\left(\mathrm{PPh}_{3}\right)_{4}\right]$ mit dem 
in situ generierten Octenylalan 142 um, das in einer Zirconocen-katalysierten Carboaluminierungsreaktion aus entsprechendem 1-Octin 141 dargestellt wurde (vgl. Schema 63). Negishi et al. erzielten auf diese Weise die gewünschten Kreuzkupplungsprodukte in Ausbeuten von 92\% bzw. 93\%. Lipshutz et al. ${ }^{[57]}$ beschrieben ebenfalls die Pd-katalysierte C-C-Kupplung von substituierten Benzylchloriden mit Alkenylalanen. Nach Lipshutz ergab die Umsetzung von Alkenylalan 145 und $p$-Fluorbenzylchlorid 144 in Anwesenheit von $5 \mathrm{~mol} \%\left[\operatorname{Pd}\left(\mathrm{PPh}_{3}\right)_{4}\right]$ nach $12 \mathrm{~h}$ bei Raumtemperatur das gewünschte Kreuzkupplungsprodukt 146 in einer Ausbeute von $67 \%$ (vgl. Schema 63). Aufgrund der Veröffentlichungen von Negishi und Lipshutz wurde im Rahmen dieser Arbeit die Untersuchung von Pd-katalysierten Kreuzkupplungen von Pyron- und Brommethylpyridonen mit Alanen geplant.

Schema 63. Pd-katalysierte Kreuzkupplungsreaktionen nach Negishi und Lipshutz.

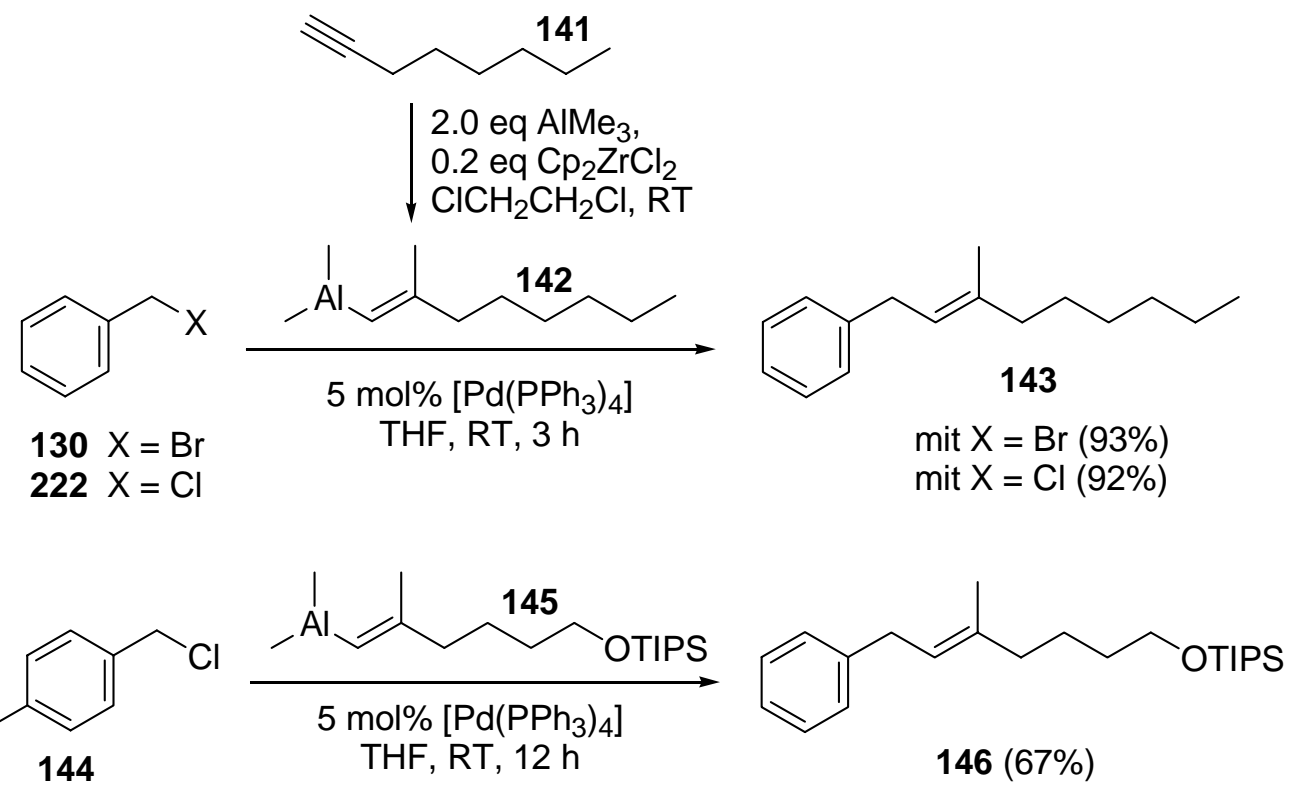

\subsubsection{Pd-katalysierte Kreuzkupplung der Bromide mit in situ generierten Alanen}

Die Vorversuche zur Untersuchung der geplanten Kupplung des Grund- und Seitenkettengerüstes durch Pd-katalysierte Kreuzkupplung wurden mit Benzylbromid 130 und den Alanen 123 und 123A nach Negishi et. al. ${ }^{[88]}$ und Lipshutz et al. ${ }^{[57]}$ durchgeführt (vgl. Schema 64). Im Gegensatz zu den Kreuzkupplungsreaktionen mit Alanaten, bei denen $\mathrm{AlMe}_{3}$-freie Alane eingesetzt wurden, wurden die Pd-katalysierten Kreuzkupplungsreaktionen mit in situ generier- 
ten, d.h. nicht $\mathrm{AlMe}_{3}$-freien Alanen durchgeführt. Die benötigten Alane 123 und 123A wurden in einer Zirconocen-katalysierten Umsetzung mit $\mathrm{AlMe}_{3}$ dargestellt (s. Exp. Teil, Allg. Arbeitsvorschrift A23). Die vorgenerierte Alan-Lösung wurde bei Raumtemperatur mit einer Suspension aus dem Benzylbromid 130 und dem Pd-Katalysator entsprechend der im Schema 64 und der Tabelle 35 angegebenen Reaktionsbedingungen umgesetzt.

Schema 64. Pd-katalysierte Kreuzkupplung des Benzylbromids 130 mit den in situ generierten Alanen 123 bzw. 123A.

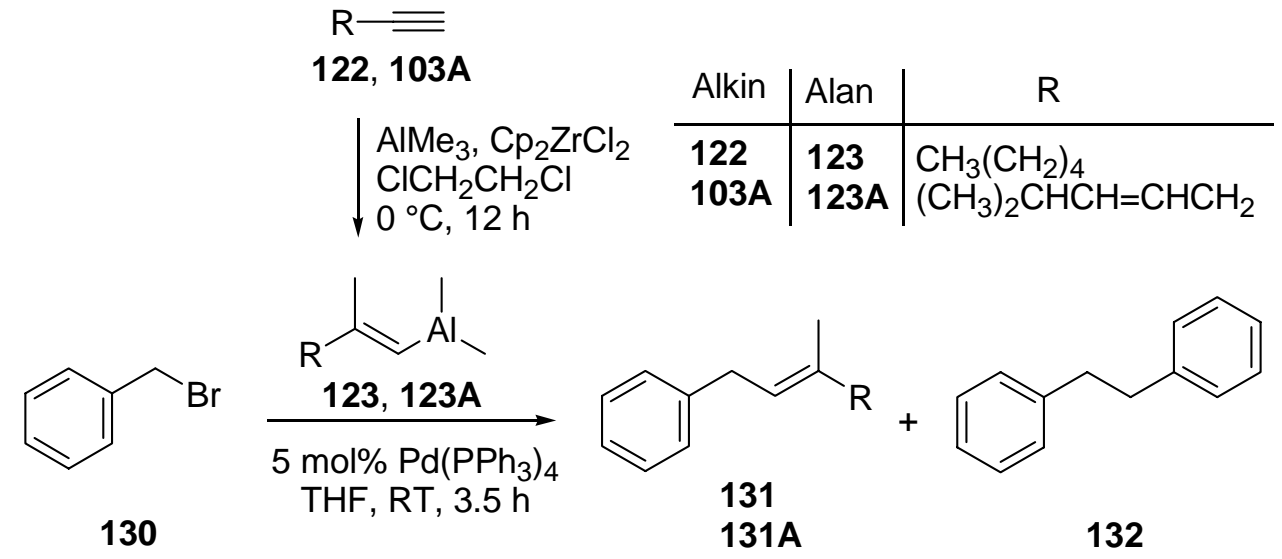

Tabelle 35. Pd-katalysierte Kreuzkupplung des Benzylbromids 130 mit den in situ generierten Alanen 123 bzw. 123A zu den Kreuzkupplungsprodukten 131 bzw. 131A.

\begin{tabular}{cccccccc}
\hline Eintrag & $\mathrm{AlMe}_{3}$ & Alan & $\begin{array}{c}\text { BnBr } \\
{[\mathrm{eq}]}\end{array}$ & $\begin{array}{c}\text { Lsm:DCE }^{\mathrm{a})} \\
{[50: 15]}\end{array}$ & $\begin{array}{c}\mathrm{t} \\
{[\mathrm{h}]}\end{array}$ & $\begin{array}{c}\mathbf{1 3 1 : 1 3 0} \\
(\%)\end{array}$ & $\begin{array}{c}\mathbf{1 3 0}: \mathbf{1 3 1}: \mathbf{1 3 2} \\
(\%)\end{array}$ \\
\hline 1 & 2.0 & $\mathbf{1 2 3}$ & 1.0 & THF:DCE & 3.5 & 100 & $6: 93: 1$ \\
2 & 2.0 & $\mathbf{1 2 3}$ & 1.0 & THF:DCE & 3.5 & 100 & $5: 94: 1$ \\
3 & 2.0 & $\mathbf{1 2 3}$ & 1.0 & THF:DCE & 15 & 11 & $2: 97: 1$ \\
4 & 2.0 & $\mathbf{1 2 3}$ & $1.0^{\mathrm{c})}$ & THF:DCE & 15 & 25 & $0: 98: 2$ \\
5 & 2.0 & $\mathbf{1 2 3}$ & $1.0^{\mathrm{d})}$ & THF:DCE & 15 & 21 & $21: 79: 2$ \\
\hline 6 & 2.0 & $\mathbf{1 2 3 A}$ & 0.75 & THF:DCE & 3.5 & 70 & $45: 55: 0$ \\
7 & 2.5 & $\mathbf{1 2 3 A}$ & 1.0 & THF:DCE & 4 & 100 & $20: 80: 0$ \\
8 & 1.0 & $\mathbf{1 2 3 A}$ & 1.0 & DMF:DCE & 4 & 50 & $100: 0: 0$ \\
\hline
\end{tabular}

a) 1,2-Dichlorethan. ${ }^{\text {b) }}$ Massenverhältnis der erhaltenen Rohprodukte 131 oder 131A zum Edukt 130. ${ }^{\text {c) }}$ Plus 1.0 eq Pyridonacetat 156. ${ }^{\text {d) }}$ Plus 1.0 eq Diacetoxypyridin 178.

Während die Kreuzkupplungsreaktionen des Benzylbromids 130 mit dem Alan 123 zu fast vollständigen Umsetzungen unter C-C-Bindungsbildung in Anteilen von 93\% und 94\% führ- 
ten (vgl. Tabelle 35, Einträge 1 u.2), konnten bei den analogen Umsetzungen des Bromids 130 mit dem Alan 123A in THF Bildungsanteile des Kreuzkupplungsproduktes von 55\% und $80 \%$ berechnet werden (vgl. Tabelle 35, Einträge 6 u. 7). Bei dem Pd-katalysierten Versuch in DMF blieb das gewünschte Produkt 131A vollständig aus (Eintrag 8). Da das Alan bei diesem Versuch ausgehend von nur 1.0 eq $\mathrm{AlMe}_{3}$ generiert wurde, kann das Ausbleiben des Kreuzkupplungsprodukts entweder am Lösungsmittel DMF oder an einer unterbliebenen Carboaluminierung liegen.

Im Hinblick auf die geplanten Pd-katalysierten Kreuzkupplungsreaktionen des Brommethylpyridons 159 mit Alanen sollte zudem im Vorfeld die Auswirkung der Stickstoffverbindungen auf das Reaktionsgeschehen des Kreuzkupplungversuchs untersucht werden. Hierzu wurde in drei Referenzversuchen analog zu den vorgenannten Versuchen 1 und 2 (vgl. Tabelle 35, Einträge 1 u. 2) jeweils eine Lösung des vorgenerierten Alans $123 \mathrm{zu}$ einer Lösung von $1.0 \mathrm{eq}$ Benzylbromid 130 und $\left[\mathrm{Pd}\left(\mathrm{PPh}_{3}\right)_{4}\right](5 \mathrm{~mol} \%)$ gegeben (vgl. Tabelle 35, Einträge 3-5). Anschließend wurde zu der Reaktionslösung des Versuches 4 (Eintrag 4) 1.0 eq nichtfunktionalisiertes Pyridonacetat 156 (vgl. Schema 45) und zu der Reaktionslösung des Versuches 5 (Eintrag 5) 1.0 eq nichtfunktionalisiertes Diacetoxypyridin 178 (vgl. Schema 62) zugegeben. Während das Kreuzkupplungsprodukt 131 in den Referenzversuchen 3 und 4 (Einträge 3 u. 4) jeweils in Anteilen von 97\% und 98\% gebildet wurde, führte der Referenzversuch 5 (Eintrag 5) zur Bildung des Produktes 131 in einem Anteil von 79\%. Die Rohproduktausbeuten blieben jedoch mit 11\% (Eintrag 3), $25 \%$ (Eintrag 4) und 21\% (Eintrag 5) weit hinter den eingesetzten Massen zurück. Insgesamt erbrachten die durchgeführten Vorversuche aber das Ergebnis, dass die Pd-katalysierte Kreuzkupplungsreaktion des Benzylbromids 130 mit Alanen komplikationslos zu realisieren war.

Die Pd-katalysierten Kreuzkupplungsversuche des Brommethylpyrons 183 bzw. des Brommethylpyridons 159 mit den Alanen 123A bzw. 123 (vgl. Schema 65) wurden entsprechend der bei den Vorversuchen beschriebenen Vorgehensweise durchgeführt. Die modifizierten Reaktionsbedingungen sind in der Tabelle 36 aufgeführt. Die in situ Carboaluminierung des Alkins 103A zu dem Alan 123A wurde mit AlMe 3 -Lösung (2.0 eq) und $\mathrm{Cp}_{2} \mathrm{ZrCl}_{2}$ (0.2 eq) in $\mathrm{ClCH}_{2} \mathrm{CH}_{2} \mathrm{Cl}$ bei $0{ }^{\circ} \mathrm{C}$ durchgeführt und $12 \mathrm{~h}$ bei Raumtemperatur gerührt. Sowohl bei Kreuzkupplungversuchen des Brommethylpyrons 183 als auch bei denen des Brommethylpyridons 159 mit den entsprechenden Alkenylalanen 123A bzw. 123 konnte keine Bildung der gewünschten Kreuzkupplungsprodukte 184 und 165 beobachtet werden. Während für die er- 
ste Umsetzung (vgl. Tabelle 36, Eintrag 1) ein Anteil des infolge der Debromierung gebildeten Produkts 88 von 29\% berechnet wurde, führten die Versuche 2 bei 5 mol\% (vgl. Tabelle 36, Eintrag 2) sowie 3 und 4 bei $10 \mathrm{~mol} \%$ des Katalysators (vgl. Tabelle 36, Einträge 3 u. 4) zur vollständigen Debromierung des Eduktes.

Schema 65. Pd-katalysierte Kreuzkupplungsreaktionen des Brommethylpyrons 183 mit dem Alan 123A und des Brommethylpyridons 159 mit dem Alan 123.<smiles>CCCc1[X]c(=O)c(C)[Y](C(C)=O)c1CBr</smiles>

$159 \mathrm{Y}=\mathrm{NH}$

$183 Y=O$

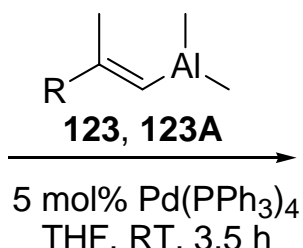

THF, RT, $3.5 \mathrm{~h}$<smiles>[X][Y]1c(C)c(OC(C)C)c(CCC)c(C)c1CC=C([R])C</smiles>

$165 \mathrm{Y}=\mathrm{NH}$

$184 Y=O$

\begin{tabular}{l|c|l} 
Alan & Produkt & \multicolumn{1}{|c}{$\mathrm{R}$} \\
\hline 123 & $\mathbf{1 6 5}$ & $\mathrm{CH}_{3}\left(\mathrm{CH}_{2}\right)_{4}$ \\
123A & $\mathbf{1 8 4}$ & $\left(\mathrm{CH}_{3}\right)_{2} \mathrm{CHCH}=\mathrm{CHCH}_{2}$
\end{tabular}

Tabelle 36. Pd-katalysierte Kreuzkupplungsreaktionen des Brommethylpyrons 183 mit dem Alan 123A und des Brommethylpyridons 159 mit dem Alan 123.

\begin{tabular}{|c|c|c|c|c|c|c|c|}
\hline Eintrag & Bromid & $\begin{array}{c}{\left[\mathrm{Pd}\left(\mathrm{PPh}_{3}\right)_{4}\right]} \\
{\left[\mathrm{mol}_{0}\right]}\end{array}$ & $\begin{array}{c}\text { Lsm.:DCE }^{\text {b) }} \\
{[50: 15]}\end{array}$ & $\begin{array}{c}\mathrm{T} \\
{\left[{ }^{\circ} \mathrm{C}\right]}\end{array}$ & $\begin{array}{c}\mathrm{t} \\
{[\mathrm{h}]}\end{array}$ & $\begin{array}{c}184: 183 \\
\left.(\%)^{c}\right)\end{array}$ & $\begin{array}{c}\text { 183:184:88 } \\
(\%)\end{array}$ \\
\hline 1 & 183 & 5 & THF:DCE & $\mathrm{RT}$ & 16 & 100 & $71: 0: 29$ \\
\hline 2 & 183 & 5 & THF:DCE & RT & 75 & 50 & $0: 0: 100$ \\
\hline 3 & 183 & 10 & THF:DCE & 40 & 19 & 100 & 0:0:100 \\
\hline 4 & 183 & 10 & DMF:DCE & RT & 19 & 50 & 0:0:100 \\
\hline $5^{\text {a) }}$ & 183 & 5 & THF:DCE & RT & 2 & 90 & - d) \\
\hline & & & & & & 165:159 & $159: 165: 156$ \\
\hline 6 & 159 & 5 & THF:DCE & $\mathrm{RT}$ & 8 & 64 & $36: 8: 56$ \\
\hline 7 & 159 & 5 & THF:DCE & RT & 21 & 14 & d) \\
\hline
\end{tabular}

Da Negishi et al. ${ }^{[88]}$ über positive Effekte in ähnlichen Umsetzungen durch Zugabe von $\mathrm{ZnCl}_{2}$ berichteten, wurde im Versuch 5 (vgl. Tabelle 36, Eintrag 5) zusätzlich 1.0 eq $\mathrm{ZnCl}_{2}$ eingesetzt. Jedoch führte dies hauptsächlich zur Bildung unbekannter Nebenprodukte. Die Kreuz- 
kupplungsversuche des Brommethylpyridons 159 blieben ebenfalls ohne Erfolg. Während bei dem Versuch 6 (vgl. Tabelle 36, Eintrag 6) das Produkt 165 in geringen Mengen (8\%) gebildet wurde, konnte der Versuch 7 (vgl. Tabelle 36, Eintrag 7) überhaupt nicht ausgewertet werden.

Bei der Ursachenermittlung für das Scheitern der Kreuzkupplung wurde zunächst eine eventuelle sterische Hinderung durch die Propylgruppe als Grund ausgeschlossen, da diese eine freie Drehbarkeit aufweist. Da die Pyridone schwache Säuren sind, könnte für das Ausbleiben des gewünschten Kreuzkupplungsproduktes bei der Umsetzung des Alkenylalans mit dem eingesetzten Brommethylpyridon 159 desweiteren die schwache Acidität der NH-Gruppe verantwortlich gemacht werden, welche zum Abfangen des Katalysators und damit zur Inaktivität des $\left[\mathrm{Pd}\left(\mathrm{PPh}_{3}\right)_{4}\right]$ geführt haben könnte. Schließlich könnte ein Ausbleiben des Kupplungsproduktes auch mit der Eigenschaft der Alane, sehr empfindlich auf Säureprotonen zu reagieren, begründet werden, wodurch ein saures Medium selbst bei schwacher Acidität der NHGruppe eine augenblickliche Zersetzung des Alans (sehr schnelle Säure-Base-Reaktion in Konkurrenz zu einer langsam katalytisch verlaufenden Kreuzkupplungsreaktion) zur Folge hätte. Die beiden vorstehenden Erwägungen stehen jedoch im Widerspruch zu den erhaltenen experimentellen Ergebnissen. Dies wird aus dem Vergleich der Versuche von Brommethylpyron 183 (vgl. Tabelle 36, Einträge 1-4) mit denen des Brommethylpyridons 159 (vgl. Tabelle 36, Einträge 6-7) ersichtlich. Während die Bildung des Kreuzkupplungsproduktes 184 ganz ausblieb, konnte das Stickstoff-Analogon 165 immerhin als Nebenprodukt in kleiner Menge (8\%) gebildet werden. Zur Begründung des Ausbleibens des Kreuzkupplungsproduktes wurde auch angenommen, dass die Koordination bzw. die Komplexierung des Katalysators als Lewissäure an der funktionellen Gruppe der Bromide (Carbonyl- bzw. Stickstoffgruppe) erfolgt. Im Rahmen dieser Hypothese hätte eine Erhöhung der Katalysatormenge durch die Erhöhung der Elektrophilie der allylischen Position zur gesteigerten Tendenz der oxidativen Addition und damit zur Verbesserung des Bildungsanteils an Kreuzkupplungsprodukt führen müssen. Dies blieb jedoch in den Versuchen 3 und 4 (vgl. Tabelle 36, Einträge 3 u. 4) trotz Zugabe von $10 \mathrm{~mol} \%$ des $\left[\mathrm{Pd}\left(\mathrm{PPh}_{3}\right)_{4}\right]$ aus.

Zum Nachweis bzw. zur Widerlegung der o.g. Hypothesen wurden Referenzversuche (vgl. Tabelle 35, Einträge 3, 4 u. 5) unter identischen Bedingungen durchgeführt, bei denen das Pyridonacetat 156 und das Diacetoxypyridin 178 als Nicht-Bromidderivate eingesetzt wurden (eine Kreuzkupplungsreaktion war somit nicht möglich). Das Versuchsergebnis ließ jedoch 
keine eindeutige Aussage zu. Man erhielt jeweils nur die Kupplungsprodukte $\mathbf{1 3 1}$ in geringen Ausbeuten. Ursprünglich wurde angenommen, dass das Alan die Amidgruppe des Pyridons deprotoniert und dass dies durch die Zersetzung des Alans der Grund für das Ausbleiben des Kupplungsproduktes darstellt. Dieser Annahme stand aber entgegen, dass das Produkt 131 bei der in Gegenwart von Pyridonacetat 156 durchgeführten Reaktion (vgl. Tabelle 35, Eintrag 4, Anteil 98\%) nicht ausblieb. Die entsprechend gute Ausbeute bei der Umsetzung 3 (vgl. Tabelle 35, Eintrag 3) könnte andererseits die These einer irreversiblen Koordination des verwendeten Katalysators an den Heterocyclen (freies Elektronenpaar des Stickstoffs bzw. des Sauerstoffs) als Grund für das Ausbleiben des Kreuzkupplungsproduktes stützen. Das freie Elektronenpaar des Stickstoffs in der Pyridonverbindung dürfte eine strukturell größerere Bereitschaft zur Koordination an dem Katalysator aufweisen als die freien sp $^{3}$-Elektronenpaare des Sauerstoff-Analogons. Dies wird jedoch durch den Versuch widerlegt, bei dem das Kreuzkupplungsprodukt in einem Anteil von 8\% gebildet wurde (vgl. Tabelle 36, Eintrag 6). Im Fall einer Anlagerung des Katalysators an das freie Elektronenpaar des Stickstoffes hätte jedoch im Gegensatz dazu das Kreuzkupplungsprodukt 184 als Sauerstoffderivat gebildet werden müssen. Desweiteren ergaben die Referenzversuche (vgl. Tabelle 35, Einträge 3-5), dass das Kreuzkupplungsprodukt 131 in hohen Anteilen gebildet werden konnte. Der Versuch 3 (Eintrag 3) wurde in Abwesenheit, der Versuch 4 (Eintrag 4) in Anwesenheit von Pyridonacetat 156 durchgeführt, wobei jeweils das Kreuzkupplungsprodukt 131 in gleichen Anteilen (97\% und 98\%) gebildet wurde. Im Vergleich zu den Pyridonen weisen die Pyridine bessere Ligandeneigenschaften als Lewisbase für das Palladium auf. Dies illustriert sich in dem Versuch 5 (Eintrag 5), bei dem das Diacetoxypyridin 178 als Beimenge zugesetzt wurde. Der Anteil des Kreuzkupplungsprodukts betrug in diesem Fall 79\% (geringer als bei Versuch 4). Dies belegt, dass scheinbar die Koordination des Katalysators am Heterocyclus zwar das Reaktionsgeschehen in geringem Maße beeinflusst, jedoch nicht der maßgebliche Grund für das Ausbleiben des Kreuzkupplungsprodukts darstellt.

Die erheblichen Anteile an debromierten Produkten sowohl im Falle des Pyron- 183 wie auch des Pyridonanalogons 159 (vgl. Tabelle 36) zeigen, dass in der Tat die oxidative Addition stattfindet. Dem schließt sich aber dann keine Transmetallierung mit dem Alan, sondern eine Reduktion an. Ähnliche Beobachtungen waren bereits in anderen Arbeiten zu Pd-katalysierten Reaktionen gemacht worden. ${ }^{[89]}$ 


\subsubsection{Pd-katalysierte Stille-Kupplung des Brommethylpyrons 183 mit Vinylstannen}

Als weitere Möglichkeit zur C-C-Bindungsbildung wurde die Stille-Kupplung untersucht, die ebenfalls zur Familie der Pd-katalysierten Kreuzkupplungsreaktionen gehört. Hierbei wird eine Organozinnverbindung mit einem Kohlenstoffelektrophil Pd-katalysiert verknüpft. Nach dem oxidativen Additionsschritt des Kohlenstoffelektrophils mit Pd (0) unter Bildung des Pd(II)-Komplexes findet ein Transmetallierungsprozeß statt, bei dem die Organylreste der Zinnverbindung auf die Pd-Spezies übertragen werden. Eine anschließende reduktive Eliminierung führt zur Freisetzung des Kupplungsproduktes und zur Generierung des Katalysators.

Zur Darstellung des Kreuzkupplungsproduktes 194 (vgl. Schema 66) als Vorversuch auf dem Weg zur Synthese der Iromycine wurde die Umsetzung des Bromids 183 und des Tributylvinylstannans mit $\left[\mathrm{Pd}\left(\mathrm{PPh}_{3}\right)_{4}\right]$ untersucht. Die Umsetzung ergab $14 \%$ des eingesetzten Eduktes 183 und $86 \%$ seines debromierten Analogons. Es konnte keine Bildung des Kreuzkupplungsprodukts 194 beobachtet werden, weshalb diese Methodik als gescheitert betrachtet wurde.

Schema 66. Pd-katalysierte Stille-Kupplung des Brommethylpyrons 183 mit Tributylvinylstannan.

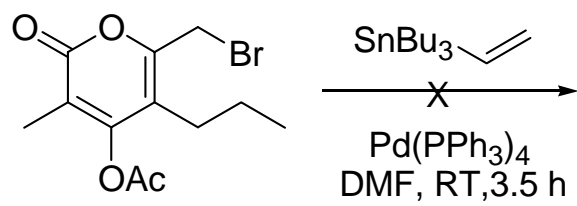

183<smiles>C=CCc1oc(=O)c(C)c(OC(C)=O)c1CCC</smiles>

194

\subsubsection{Versuche zur Pd-katalysierten Kreuzkupplung von Zinkorganylen mit Alkenyl- halogeniden}

Knochel et al. ${ }^{[90]}$ beschrieben die Pd-katalysierte C-C-Verknüpfung von Zinkorganylen und Alkenyliodiden. Nach der von Knochel angegebenen Arbeitsvorschrift wurden die geplanten Kreuzkupplungsversuche sowohl mit Benzylbromid 130 (vgl. Schema 68) als Modellsubstrat als auch mit Brommethylpyron 96 (vgl. Schema 69) durchgeführt und optimiert. Die Zinkorganyle 197 und 198 wurden zunächst jeweils ausgehend von Benzylbromid 130 und Brom- 
methylpyron 96 in zwei Varianten nach Knochel et al. ${ }^{[90]}$ dargestellt (vgl. Schema 67, Exp. Teil, Allg. Arbeitsvorschrift A23). Bei Einsatz der Zinkorganyle 197 und 198 wurde nach Knochel et al. ${ }^{[90]}$ von einer $80 \%$-igen Konzentration $(0.8 \mathrm{M}$ bezogen auf die eingesetzten Edukte) ausgegangen und dies anschließend optimiert. In den durchgeführten Kreuzkupplungsreaktionen wurden die kommerziell erhältlichen Katalysatoren Bis(dibenzylidenaceton)palladium $\left[\mathrm{Pd}(\mathrm{dba})_{2}\right]$ und Tetrakis(triphenylphosphin)palladium $\left[\mathrm{Pd}\left(\mathrm{PPh}_{3}\right)_{4}\right]$ verwendet. Das verwendete Tris(2-furyl)phosphin (TFP) wurde nach den Vorgaben von Santelli-Rouvier, Santelli et al. ${ }^{[91]}$ hergestellt. Das Heptenyliodid 195 wurde ausgehend von 1-Heptin (122) nach Negishi $^{\left[{ }^{[92]}\right.}$ gemäß der allgemeinen Arbeitsvorschrift A21 (s. Exp. Teil) dargestellt. Dazu wurde das Heptin carboaluminiert und dann das Alkenylalan mit $\mathrm{I}_{2}$ umgesetzt.

Schema 67. Darstellung der Zinkorganyle 197 bzw. 198 aus dem Benzylbromid 130 bzw. dem Brommethylpyron 96 nach den Varianten A oder B.
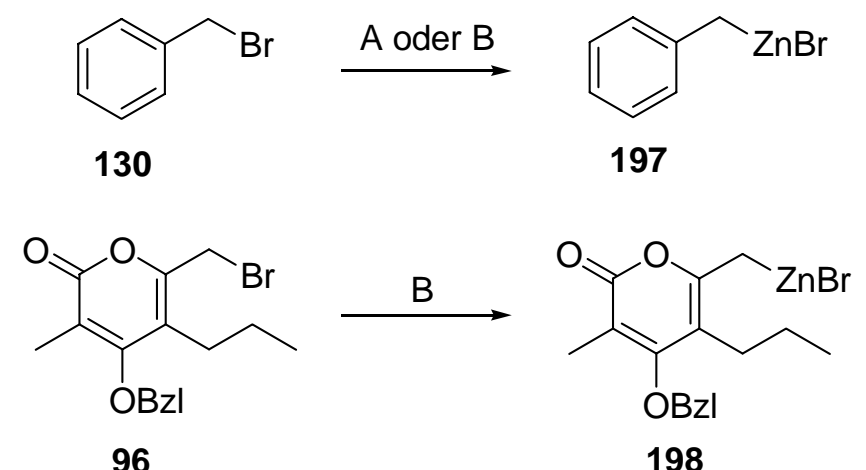

A: $\mathrm{Zn}, \mathrm{BrCH}_{2} \mathrm{CH}_{2} \mathrm{Br}, \mathrm{TMSCl}, \mathrm{THF}$, Reflux $\rightarrow \mathrm{RT}, 0^{\circ} \mathrm{C}, \mathrm{RT}, 30 \mathrm{~min}$

$\left.\left.\left.\mathrm{B}: \mathrm{Zn}, \mathrm{I}_{2}, \mathrm{THF},\right)\right)\right), 45 \mathrm{~min}$

Die Vorversuche zur Darstellung des Kreuzkupplungsprodukts 131 wurden zunächst in Anwesenheit von $\left[\mathrm{Pd}(\mathrm{dba})_{2}\right]$ und TFP durchgeführt (vgl. Schema 68, Variante C; Tabelle 37, Einträge 1-5). Hierzu wurde entsprechend den in der Tabelle 37 angegebenen Reaktionsbedingungen zu einer Suspension des Iodids 195 mit $\left[\mathrm{Pd}(\mathrm{dba})_{2}\right]$ und TFP in THF bzw. in einem THF/DMF-Gemisch eine Lösung des Zinkorganyls 197 zugegeben und gerührt. Das gewünschte Kreuzkupplungsprodukt 131 blieb bei den durchgeführten Versuchen vollständig aus. Als Nebenprodukt wurde das Homokupplungsprodukt 132 in Anteilen von 4-17\% beobachtet. Das Homokupplungsprodukt 132 stammt nach Knochel et al. ${ }^{[90]}$ aus der Zinkorganyllösung und wird bei der Darstellung der Organozink-Verbindung gebildet. 
Schema 68. Pd-katalysierte Kreuzkupplungsreaktionen des aus dem Benzylbromid 130 generierten Zinkorganyls 197 mit 1-Heptenyliodid 195 zu dem Kreuzkupplungsprodukt 131.

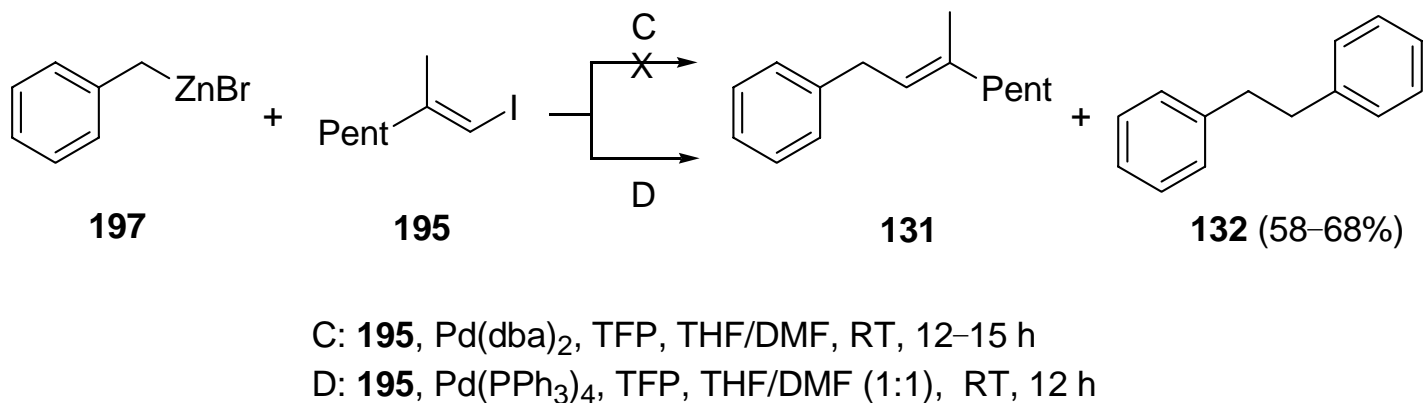

Der durchgeführte Versuch bei Raumtemperatur (vgl. Tabelle 37, Eintrag 3) ergab neben 10\% unumgesetzten Benzylbromid 130 ein Singulett bei 5.10 ppm, welches auf ein Produkt hinwies, das in großer Menge gebildet wurde, aber nicht identifiziert werden konnte. Der bei 60 ${ }^{\circ} \mathrm{C}$ durchgeführte Versuch (vgl. Tabelle 37, Eintrag 4) wies neben dem Bromid 130 (71\% Anteil) die Bildung von Toluol (29\% Bildungsteil bezogen auf Bromid) auf. Das Erscheinen des Bromids 130 und des Toluols wurde auf die unvollständige Umwandlung des Bromids 130 zu dem Zinkorganyl 197 sowie eine Hydrolyse des gebildeten Zinkorganyls 197 zurückgeführt, welches trotz unvollständiger Metallierung die Bildung der Organozinkspezies 197 verdeutlicht. Als Konsequenz wurde zur weiteren Überprüfung der Zinkorganyllösung eine nach der Variante A generierte Organozinklösung mit $\mathrm{D}_{2} \mathrm{O}$ hydrolysiert. ${ }^{[90]}$ Die Auswertung der Deuterolyse zeigte die Bildung geringer Mengen an Deuterotoluol (Multiplett) bei 2.35 ppm sowie des als Hauptprodukt isolierten Homokupplungsproduktes 132 bei 2.92 ppm an. Da jedoch bei den Versuchen 3 und 4 (vgl. Tabelle 37, Einträge 3 u. 4) sowie bei den Versuchen 1, 2 und 5 (vgl. Tabelle 37, Einträge 1, 2 u. 5) das gewünschte Kreuzkupplungsprodukt 130 nicht beobachtet werden konnte, wurde $\left[\mathrm{Pd}\left(\mathrm{PPh}_{3}\right)_{4}\right]$ anstatt $\left[\mathrm{Pd}(\mathrm{dba})_{2}\right]$ als Katalysator eingesetzt. Die hiernach erfolgten Umsetzungen (vgl. Tabelle 37, Einträge 6-8) ergaben Anteile des Kreuzkupplungsprodukts 131 von $58-68 \%$. Offensichtlich ist also $\left[\mathrm{Pd}\left(\mathrm{PPh}_{3}\right)_{4}\right]$ wesentlich besser für die Kreuzkupplung geeignet. Darauf aufbauend wurde die entsprechende Umsetzung mit dem aus Brommethylpyron 96 generierten Zinkorganyl 198 untersucht. Diese Verknüpfungsreaktion mit dem Iodid 195 in Anwesenheit von $5 \mathrm{~mol} \%$ des $\left[\mathrm{Pd}\left(\mathrm{PPh}_{3}\right)_{4}\right]$ ergab die Verbindung 89 als Produkt des Debromierungsprozesses mit einem Bildungsanteil von 66\% (vgl. Schema 69, Tabelle 37, Eintrag 9). Das Entstehen der Verbindung 89 zeigt einmal mehr, dass diese Reduktion am Pyronsystem sehr rasch erfolgen kann. 
Schema 69. Pd-katalysierte Kreuzkupplung des aus dem Brommethylpyron 96 generierten Zinkorganyls 198 mit 1-Heptenyliodid 195 zu dem Kreuzkupplungsprodukt 125.

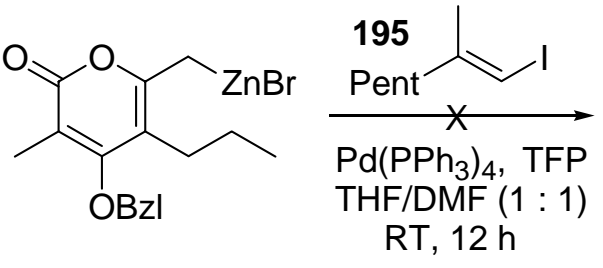

198<smiles>CCCc1c(C/C=C(\C)[Po])oc(=O)c(C)c1OCC</smiles>

125

Tabelle 37. Pd-katalysierte Kreuzkupplungsreaktionen der Zinkorganyle 197 bzw. 198 mit 1-Heptenyliodid 195 zu den Kreuzkupplungsprodukten 131 bzw. 125.

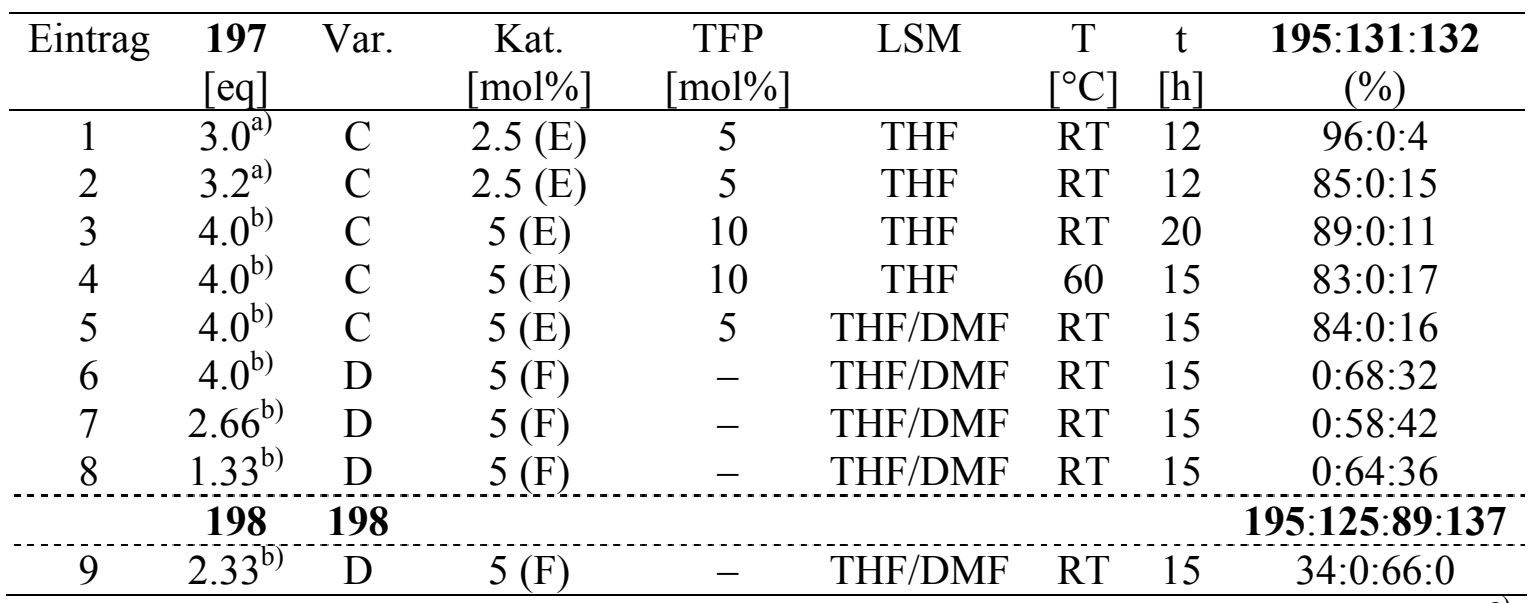

Bei allen Reaktionen wurde 1.0 eq Iodid 195 eingesetzt. E: [Pd(dba $\left.)_{2}\right] . F$ : $\left[\mathrm{Pd}\left(\mathrm{PPh}_{3}\right)_{4}\right]^{\text {a) }}$

Zinkorganyl nach Variante A hergestellt. ${ }^{\text {b) }}$ Zinkorganyl nach Variante B hergestellt.

\subsubsection{Versuche zu Pd-katalysierten Kreuzkupplungsreaktionen von $\boldsymbol{\gamma}$-Stannylorganyl- und $\gamma$-Zinkorganyl-maskierten Trienolaten mit Alkenyliodid}

Yamamoto et al. ${ }^{[93]}$ beschrieben die C-C-Bindungsverknüpfung von $\alpha$ - $\beta$-ungesättigten $\gamma$ stannyl Carbonsäureestern mit Acyl-, Aryl- und Vinylhalogeniden. So überführten sie einen $\alpha$ - $\beta$-ungesättigten Ester unter Deprotonierung an der $\gamma$-Position in das entsprechende LithiumDienolat. Die Li-Dienolate reagierten mit $\mathrm{Bu}_{3} \mathrm{SnCl} z u \alpha$ - $\beta$-ungesättigten $\gamma$-Stannylestern, sog. $\gamma$-Stannyl-maskierten Dienolaten. Die Pd-katalysierte Umsetzung der $\gamma$-StannylVerbindungen mit Acyl-, Aryl- bzw. Vinylhalogeniden führte unter C-C-Bindungsbildung hoch regioselektiv zu $\gamma$-substituierten $\alpha$ - $\beta$-ungesättigten Estern (Ausbeute 44-83\%). Im Rahmen der vorliegenden Arbeit wurde daher die Deprotonierung des Pyronbenzoats 89 unter 
Behandlung mit LDA zu dem Lithium-Organyl 199, die Generierung des $\gamma$-Stannylierten Pyrons 200 und die anschließende Pd-katalysierte Kreuzkupplung mit 1-Heptenyliodid 195 unter regioselektiver C-C-Bindungsverknüpfung zu dem Kreuzkupplungsprodukt 125 geplant (vgl. Schema 70).

Schema 70. Pd-katalysierte Kreuzkupplungsreaktionen des $\gamma$-Stannanorganyls 200 bzw. des

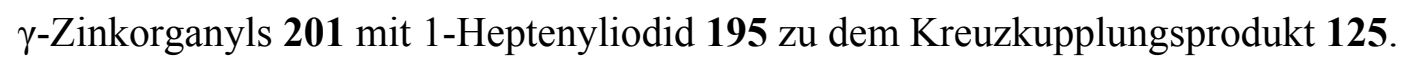

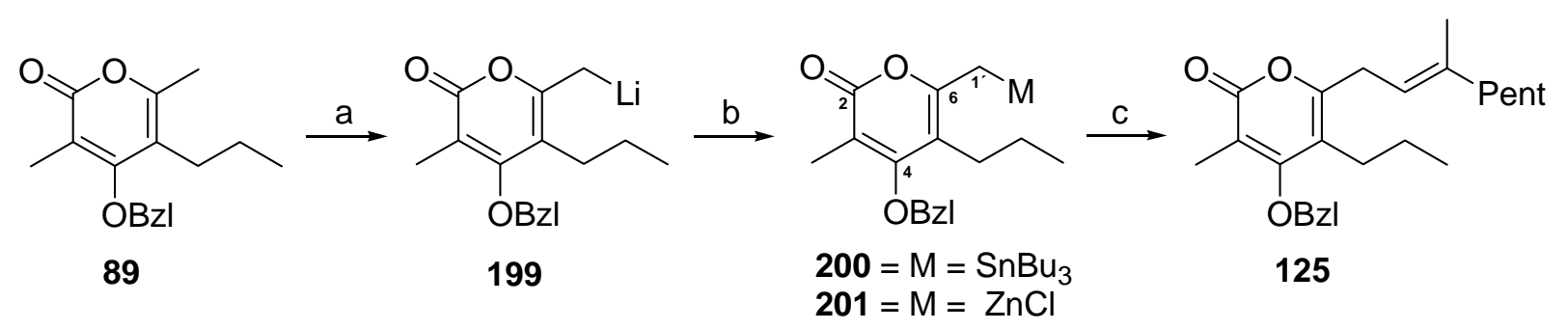

für 200:
a) LDA, HMPTA, THF, $-78^{\circ} \mathrm{C}, 1 \mathrm{~h}$
b) $\mathrm{Bu}_{3} \mathrm{SnCl}, \mathrm{THF},-78^{\circ} \mathrm{C}, 1 \mathrm{~h}$
a) LDA, THF, $-78^{\circ} \mathrm{C}, 1 \mathrm{~h}$
b) $\mathrm{ZnCl}_{2}, \mathrm{THF},-78^{\circ} \mathrm{C}, 30 \mathrm{~min}$
c) $195, \mathrm{Pd}(\mathrm{dba})_{2}, \mathrm{PPh}_{3}, \mathrm{DMF}, \mathrm{RT}, 15 \mathrm{~h}$
c) $195, \mathrm{Pd}\left(\mathrm{PPh}_{3}\right)_{4}, \mathrm{DMF}, 0{ }^{\circ} \mathrm{C}, 15 \mathrm{~h}$

für 201:

Die für eine Lithierung erforderliche Reaktivität des C-1'-Kohlenstoffzentrums des Pyrons ist schon aus dem Funktionalisierungsschritt mit $\mathrm{SeO}_{2}$ bekannt. ${ }^{[35][38][39]}$ Einer Deprotonierung am C-1'-Kohlenstoffzentrum des 6-Methylpyrons 89 wurde daher eine realistische Chance beigemessen. Zur Überprüfung wurde das 6-Methylpyron 89 nach der Deprotonierung durch LDA mit $\mathrm{D}_{2} \mathrm{O}$ deuteriert. Die Auswertung des unscharfen Spektrums ließ aufgrund vorhandener Verunreinigungen, von einem geringfügigen Debenzoylierungsanteil abgesehen, keine schlüssige Aussage über das Ausmaß der Deuterierungsreaktion zu, d.h., ob eine vollständige Deprotonierung erfolgt war oder nicht, blieb offen. Die im Verlauf der Reaktion erfolgte dünnschichtchromatographische Detektion deutete jedoch auf die Deprotonierung des C-1'Kohlenstoffs des Pyrons 89 hin.

Im Rahmen der Versuche zur Pd-katalysierten Kreuzkupplung des $\gamma$-Stannanorganyls 200 mit dem 1-Heptenyliodid 155 wurde zunächst die Generierung des $\gamma$-Stannanorganyls 200 aus dem Lithiumorganyl 199 untersucht, in dem das lithierte Pyron 199 bei $-78{ }^{\circ} \mathrm{C}$ mit Tributylzinnchlorid (1.0 eq) umgesetzt wurde (vgl. Schema 70). Im ${ }^{1} \mathrm{H}-\mathrm{NMR}$ des Rohproduktes wurde ein Signal bei 2.02 ppm den Protonen an der C-1'-Position im Zielprodukt 200 (Anteil 36\%) 
zugeordnet. Daneben wurden das debenzoylierte Edukt (Anteil 30\%) und das eingesetzte Edukt 89 (Anteil 34\%) beobachtet. Die chromatographische Reinigung des Produktes 200 führte zur vollständigen Zersetzung, die wahrscheinlich auf die Hydrolyse des Stannans während der Reinigung zurückzuführen ist. Als Konsequenz wurde der Pd-katalysierte Kreuzkupplungsversuch des Stannans 200 in situ (ohne Zwischenisolierung) in Anwesenheit von Hexamethylphosphorsäuretriamid (HMPTA) mit 1-Heptenyliodid 195 durchgeführt. Das Benzoat 89 wurde hiernach bei $-78{ }^{\circ} \mathrm{C}$ in Gegenwart von jeweils 1.15 eq LDA und HMPTA (zur Solvatisierung der Kationen) lithiiert und anschließend mit $\mathrm{Bu}_{3} \mathrm{SnCl}$ (1.0 eq) versetzt. Die Auswertung der Kreuzkupplungsreaktion in Gegenwart von 1.0 eq Iodid 195, 5 mol\% [Pd(dba) $\left.{ }_{2}\right]$ und $5 \mathrm{~mol} \% \mathrm{PPh}_{3}$ in DMF zeigte die vollständige Zersetzung des Pyronbenzoates 89 und die Rückgewinnung des Iodids 195 sowie des Tributylzinnhalogenids an. Die Gründe für das Scheitern wurden nicht näher untersucht.

Im Weiteren wurde untersucht, ob eine Kupplung anstatt über das Stannan 200 auch über ein in situ generiertes Zinkorganyl 201 erfolgen kann. Die Generierung des Lithiumorganyls 199 aus dem Benzoat 89 wurde hier ohne Einsatz von HMPTA mit LDA durchgeführt und anschließend mit wasserfreiem $\mathrm{ZnCl}_{2}$ (1.2 eq) umgesetzt. Die Kreuzkupplungsreaktion der generierten Organyllösung wurde dann mit 1.0 eq Iodid 195 und $5 \mathrm{~mol} \%\left[\mathrm{Pd}\left(\mathrm{PPh}_{3}\right)_{4}\right]$ in DMF untersucht. Es konnte auch hier keine Bildung des Kreuzkupplungsproduktes beobachtet werden. Die Auswertung der Reaktion zeigte den Erhalt des eingesetzen Iodids 195, des Benzoats 89 und dessen korrespondierenden debenzoylierten Produkts in Anteilen von 14:17:69 an. Die Bildung des entschützten Produktes kann eventuell auf die hohe Lewisacidität des $\mathrm{ZnCl}_{2} \mathrm{zu}$ rückgeführt werden, wodurch durch Komplexierung der Benzoatgruppe eine Entschützung erleichtern werden könnte. Hierfür spricht auch die Isolierung von Benzoesäure als Nebenprodukt. Der hohe Debenzoylierungsanteil sowie annähernd gleiche Anteile an unumgesetzten Iodid 195 (14\%) und Benzoat 89 (17\%) dürften die Annahme stützen, dass zwar eine Deprotonierung des Benzoats an der C-1'-Position und eine Bildung des Lithiumorganyls 199 erfolgt ist, aber keine Ummetallierung auf Zink stattgefunden hat. Das Zinksalz könnte so in starkem Maße an die beiden Carbonylgruppen des Benzoates 89 koordiniert worden sein, dass es auf diese Weise verbraucht wurde und nicht für eine Transmetallierung zur Verfügung stand. Daher wäre auch ein nucleophiler Angriff des Zinkorganyls 201 auf das 1Hepenyliodid 195 ausgeblieben, was die fehlende Bildung des Kreuzkupplungsproduktes 125 erklären würde. 


\subsection{Ni-katalysierte Kreuzkupplung mit Alanen}

Ni-katalysierte Kreuzkupplungsreaktionen verlaufen ähnlich dem Pd-katalysierten Katalysezyklus und sind ebenfalls in vier Teilschritte unterteilt. Der mechanistische Verlauf des Katalysezylus ist im Schema 71 am Beispiel einer Kumada-Kupplung dargestellt. Über den Nikatalysierten Reaktionsmechanismus können Alkenylbromide oder -iodide sowie Aryltriflate, -bromide oder -iodide mit Grignard-Verbindungen Substitutionsreaktionen eingehen. ${ }^{[94]}$ Die Kumada-Kupplung ist die Methode der Wahl, da sie die Durchführung preisgünstiger Kreuzkupplungsreaktionen bei sehr guten Umsätzen ermöglicht und deshalb z.B. auch bei der industriellen Darstellung von asymmetrischen Biarylen zur Anwendung kommt. Ni-Katalysatoren sind im Vergleich zu ihren analogen Pd-Katalysatoren sowohl reaktiver als auch stabiler gegen eine Oxidation durch Sauerstoffatome.

Schema 71. Teilschritte einer Ni-katalysierten Kreuzkupplung zwischen einem Arylhalogenid und einer Grignard-Verbindung (Kumada-Kupplung).

$$
\begin{aligned}
& \mathrm{R}^{\prime} \mathrm{X}+\mathrm{RMgX} \stackrel{\text { "Ni" }}{\longrightarrow} \mathrm{R}-\mathrm{R}^{\prime} \quad \mathrm{R}=\text { Aryl, Vinyl, Alkyl } \\
& \mathrm{R}^{\prime}=\text { Aryl, Vinyl } \\
& \mathrm{X}=\mathrm{Cl}>\mathrm{Br}>\mathrm{I}
\end{aligned}
$$

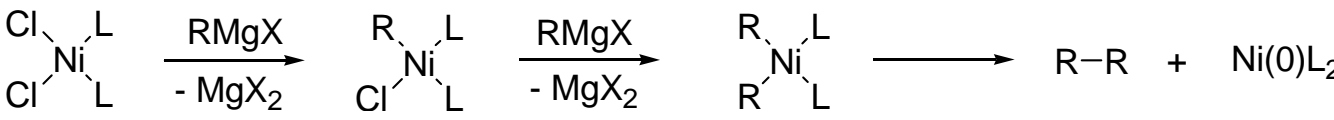

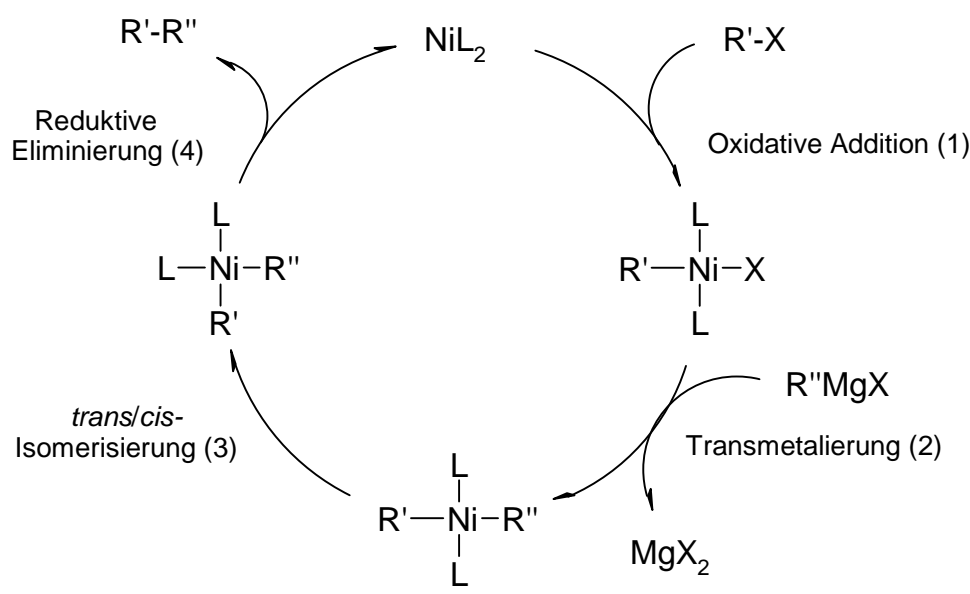


Die einzelnen Reaktionsschritte können in Komplexierung, oxidative Addition, Ummetallierung, reduktive Eliminierung und schließlich Dissoziation unterteilt werden. ${ }^{[94]}$ Die Reaktion wird durch Ni(0)-Komplexe katalysiert, welche auch in situ gebildet werden können. Der entscheidende katalytische Reaktionsschritt läuft über eine oxidative Addition, bei der sich ein Ni-(II)-Organyl bildet, welches in einer Transmetallierung zu einem Diorganyl umgewandelt wird. Der reduktive Eliminierungsschritt setzt das gekuppelte Produkt und den Katalysator in der Oxidationsstufe Null wieder frei.

\subsubsection{Ni-katalysierte Kreuzkupplung der Benzylhalogenide 130 und 222 sowie der Py- ronhalogenide 96 und 135 mit dem Alan 123}

Lipshutz et al. ${ }^{[19][57]}$ führten erfolgreich die Ni-katalysierte Kreuzkupplung von ortho-, metaund para-substituierten Benzylchloriden mit Ethenylalanen unter C-C-Bindungsbildung durch und berichteten von Ausbeuten in Höhe 77-93\%. Auch Negishi et al. ${ }^{[95]}$ berichteten über eine Ni-vermittelte C-C-Bindungsverknüpfung von Alkenylalanen mit Benzylhalogeniden in exzellenten Ausbeuten bis zu 99\%. Daher wurden zunächst nach Negishi unter identischen Bedingungen Vorversuche mit Benzylchlorid 222 und Benzylbromid 130 untersucht (vgl. Schema 72, Exp. Teil, Allg. Arbeitsvorschrift A28).

Schema 72. Ni-katalysierte Kupplung der Benzylhalogenide 130 bzw. 222 mit dem Alan 123.

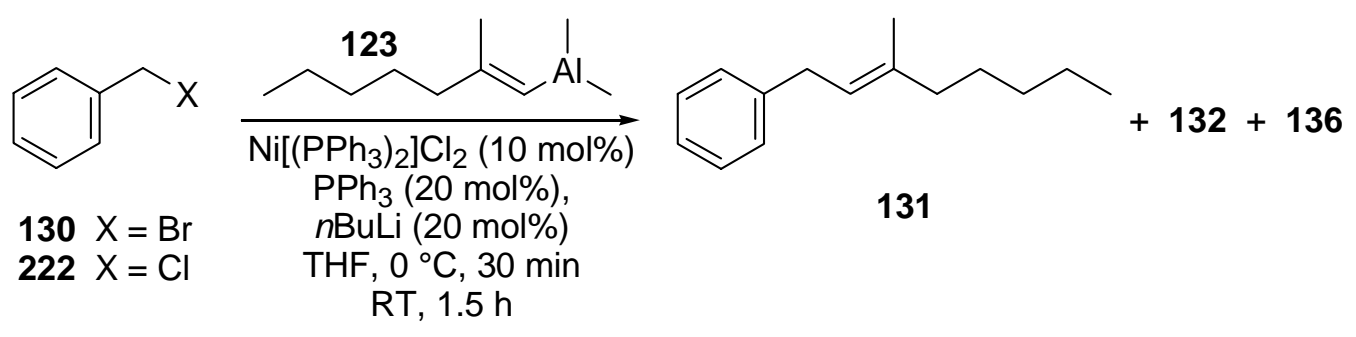

Die Umsetzungen ergaben das Kreuzkupplungsprodukt 131 in guten bis exzellenten Anteilen von 72\% (Benzylbromid 130) und 96\% (Benzylchlorid 222) als Hauptprodukt (vgl. Tabelle 38, Einträge 1 u. 2). Desweiteren wurde das Homokupplungsprodukt 132 bei dem ersten Versuch (Eintrag 1) in einem Anteil von 22\% und bei dem zweiten Versuch (Eintrag 2) in einem Anteil von 4\% als Nebenprodukt gebildet. Bei der ersten Umsetzung entstand als weiteres 
Nebenprodukt zudem das Dien 136 in geringer Menge von 6\%. Der hohe Anteil am Kreuzkupplungsprodukt 131 sowie der geringe Bildungsanteil an dem Homokupplungsprodukt 132 und das Ausbleiben des Diens 136 bei dem zweiten Versuch zeigte die höhere Chemoselektivität der Chloride bei den Ni-katalysierten Kreuzkupplungsreaktionen im Vergleich zu den entsprechenden Bromiden.

Tabelle 38. Ni-katalysierte Kreuzkupplung der Benzylhalogenide 130 bzw. 222 mit dem Alan 123 zu dem Kreuzkupplungsprodukt 131.

\begin{tabular}{|c|c|c|c|c|c|c|c|c|}
\hline Eintrag & $\mathbf{X}$ & $\begin{array}{l}\text { Kat } \\
{[\mathrm{eq}]}\end{array}$ & $\begin{array}{l}\mathrm{PPh}_{3} \\
\text { [eq] }\end{array}$ & $\begin{array}{c}n \mathrm{BuLi} \\
{[\mathrm{eq}]}\end{array}$ & Lsm. & $\begin{array}{c}\mathrm{T} \\
{\left[{ }^{\circ} \mathrm{C}\right]}\end{array}$ & $\begin{array}{c}\mathrm{t} \\
{[\mathrm{h}]}\end{array}$ & $\begin{array}{c}130: 131: 132: 136 \\
(\%)\end{array}$ \\
\hline 1 & $\mathrm{Br}$ & 0.1 & 0.2 & 0.2 & THF & $0 \rightarrow \mathrm{RT}$ & 1.5 & $0: 72: 22: 6$ \\
\hline & & & & & & & & $222: 131: 132: 136$ \\
\hline 2 & $\mathrm{Cl}$ & 0.1 & 0.2 & 0.2 & THF & $0 \rightarrow \mathrm{RT}$ & 1.5 & $0: 96: 4: 0$ \\
\hline
\end{tabular}

Die Reaktionen wurden in THF durchgeführt.

Daraufhin wurden die entsprechenden Pyronderivate, das Bromid 96 und das Chlorid 135, nach den in dem Schema 73 und der Tabelle 39 dargestellten Reaktionsbedingungen mit Ni${ }^{\mathrm{II}}\left[\left(\mathrm{PPh}_{3}\right)_{2}\right] \mathrm{Cl}_{2}$ und $\mathrm{Ni}^{0}\left[(\mathrm{COD})_{2}\right]$ untersucht. Bei dem Versuch 1 (vgl. Tabelle 39, Eintrag 1) wurde die Reduzierung des Ni(II)-Katalysators zum aktiven Nickel(0)-Katalysator unter Zugabe von $n \mathrm{BuLi}$ zu einer Suspension aus $\mathrm{Ni}^{\mathrm{II}}\left[\left(\mathrm{PPh}_{3}\right)_{2}\right] \mathrm{Cl}_{2}$ und $\mathrm{PPh}_{3}$ durchgeführt und anschließend wurde die Reaktionslösung mit Brommethylpyron 96 versetzt. Die Reaktionen wurden mit Ausnahme des Versuchs 3 (Eintrag 3, 3.5 eq des Alans 123) in Gegenwart von 1.5 eq des Alans 123 durchgeführt.

Schema 73. Ni-katalysierte Kreuzkupplung des Brommethylpyrons 96 bzw. Chlormethylpyrons 135 mit dem Alan 123 zu dem Kreuzkupplungsprodukt 125.

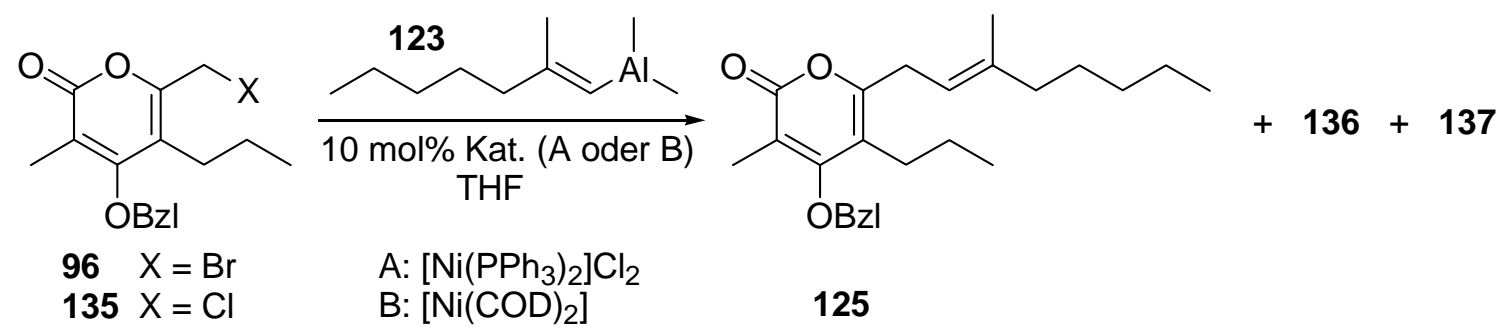


Tabelle 39. Ni-katalysierte Kreuzkupplung des Brommethylpyrons 96 bzw. Chlormethylpyrons 135 mit dem Alan 123 zu dem Kreuzkupplungsprodukt 125.

\begin{tabular}{|c|c|c|c|c|c|c|c|c|}
\hline Eintrag & $\mathbf{X}$ & Kat. & Additiv & [eq] & $n \mathrm{BuLi}[\mathrm{eq}]$ & $\mathrm{T}\left[{ }^{\circ} \mathrm{C}\right]$ & $\mathrm{t}[\mathrm{h}]$ & 96:125:136:137 \\
\hline 1 & $\mathrm{Br}$ & A & $\mathrm{PPh}_{3}$ & 0.2 & 0.2 & $0 \rightarrow \mathrm{RT}$ & 1.5 & $0: 0: 44: 56$ \\
\hline \multirow[t]{2}{*}{2} & $\mathrm{Br}$ & A & $\mathrm{LiCl}$ & 1.0 & 0.2 & $0(\mathrm{RT})$ & $1.5(3)$ & $0: 0: 85: 15$ \\
\hline & & & & & & & & $135: 125: 136: 137$ \\
\hline $3^{a)^{-}}$ & $\mathrm{Cl}$ & $\mathrm{A}$ & - & - & 0.2 & $0 \rightarrow \mathrm{RT}$ & 7 & $0: 20: 80: 0$ \\
\hline $4^{a)}$ & $\mathrm{Cl}$ & A & - & - & 0.2 & $0 \rightarrow \mathrm{RT}$ & 6 & $73: 15^{\mathrm{b})}: 12: 0$ \\
\hline 5 & $\mathrm{Cl}$ & B & $\mathrm{PPh}_{3}$ & 0.2 & - & RT & 4.5 & $0: 0: 88: 12$ \\
\hline
\end{tabular}

Das Spektrum des Rohproduktes des ersten Versuches (vgl. Tabelle 39, Eintrag 1) zeigte bei vollständigem Ausbleiben des gewünschten Kreuzkupplungsprodukts 125 ein Gemisch der Homokupplungsprodukte 136 (Anteil 44\%, vgl. Schema 34) und 137 (Anteil 56\%, vgl. Schema 34) sowie eine geringe Menge des debromierten Eduktes 89. Die vorgenannten, bereits aus anderen Versuchen bekannten Produkte wurden massenspektroskopisch nachgewiesen. Lipshutz et al. ${ }^{[19]}$ berichteten ebenfalls von der Bildung entsprechender Homokupplungsprodukte und beschrieben, dass ein höherer Gehalt an $\mathrm{PPh}_{3} \mathrm{zu}$ einem höheren Bildungsanteil der Homokupplungsprodukte führen würde (je geringer der Anteil an $\mathrm{PPh}_{3}$, umso höher der Bildungsanteil des Kreuzkupplungsprodukts und umso geringer der Bildungsanteil der Homokupplungsprodukte). Bei dem zweiten Versuch (vgl. Tabelle 39, Eintrag 2) wurde daher das $\mathrm{LiCl}$ als Additiv in stöchiometrischer Menge eingesetzt. Es wurde das Dien 136 als Hauptprodukt in einem Anteil von 85\% und das Dipyronethan 137 in einem Anteil von 15\% erhalten. Als weiteres Nebenprodukt wurde das debromierte Edukt 89 gebildet, dessen Anteil aufgrund von Verunreinigungen nicht angegeben werden konnte. Die in Gegenwart von $\left[\mathrm{Ni}\left(\mathrm{PPh}_{3}\right)_{2}\right] \mathrm{Cl}_{2}$ durchgeführten Versuche (vgl. Tabelle 39, Einträge 3 u. 4) wurden mit Chlormethylpyron 135 ohne Ligandenzusatz (in Abwesenheit von $\mathrm{PPh}_{3}$ und $\mathrm{LiCl}$ ) in THF und DMF durchgeführt (s. Exp. Teil, Allg. Arbeitsvorschrift A29). Während bei dem Versuch 3 das Dien 136 als Hauptprodukt (Anteil 80\%) und das gewünschte Kreuzkupplungsprodukt 125 als Nebenprodukt (Anteil 20\%) erhalten wurden, wurde bei der Umsetzung 4 ein Gemisch aus dem Edukt 135 (Anteil 73\%), dem Kreuzkupplungsprodukt 125 (Anteil 15\%) und dem Dien 136 (Anteil 12\%) erzielt. Die Bildung des Kreuzkupplungsprodukts 125 wurde massenspektroskopisch untersucht und nachgewiesen. 
Lipshutz et al. ${ }^{[19]}$ hatten in ihrer Veröffentlichung von der exzellenten Ausbeutesteigerung der Kreuzkupplungsbildung unter Verwendung von $\mathrm{Ni}(0)-\mathrm{Katalysatoren}$ wie $\left[\mathrm{Ni}^{0}(\mathrm{COD})_{2}\right]$ in Anwesenheit von 5-10 mol\% an $\mathrm{PPh}_{3}$ bei $50{ }^{\circ} \mathrm{C}$ berichtet, wodurch der Bildungsanteil der Homokupplungsprodukte bis auf 5-10\% herabgesetzt werden konnte. Der Versuch 5 (vgl. Tabelle 39, Eintrag 5) wurde daher unter Verwendung von $\left[\mathrm{Ni}^{0}(\mathrm{COD})_{2}\right]$ und $\mathrm{PPh}_{3}$ untersucht. Hierbei wurde das Dien 136 (vgl. Schema 34) in einem Anteil von 88\% als Hauptprodukt gebildet. Daneben ergab die Versuchsauswertung die geringfügige Bildung einer dechlorierten Verbindung.

\subsubsection{Ni-katalysierte Kreuzkupplung der Chlormethylpyridine 223 und 179 mit dem} Alan 123

Neben den Pyronen wurden auch Pyridine zur Ni-katalysierten Kreuzkupplung mit dem Alan 123 eingesetzt. Hierbei handelte es sich um die Chlormethylpyridine 223 (vgl. Schema 74) und 179 (vgl. Schema 75). Der Ni-katalysierte Kreuzkupplungsversuch mit Chlormethylpyridin 223 und dem Alan 123 wurde in vollständiger Analogie zu dem Versuch mit $\left[\mathrm{Ni}\left(\mathrm{PPh}_{3}\right)_{2}\right] \mathrm{Cl}_{2}, \mathrm{PPh}_{3}, n \mathrm{BuLi}$ und 1.5 eq Alan 123 nach Lipshutz ${ }^{[19]}$ durchgeführt (vgl. Tabelle 39, Eintrag 1). Hierbei wurden in einer sauberen Umsetzung das Kreuzkupplungsprodukt 131 in einem Anteil von 69\% und das Dien 136 in einem Anteil von 31\% als Produkte erhalten.

Schema 74. Ni-katalysierte Kreuzkupplung des Chlormethylpyridins 223 mit dem Alan 123 zu dem Kreuzkupplungsprodukt 223.<smiles>ClCc1ccccn1</smiles>

223

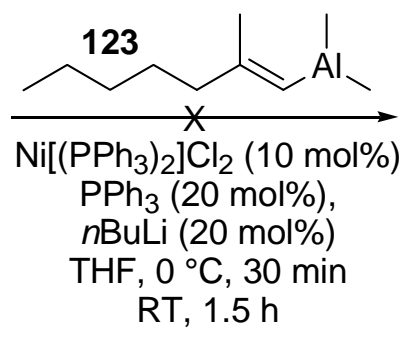

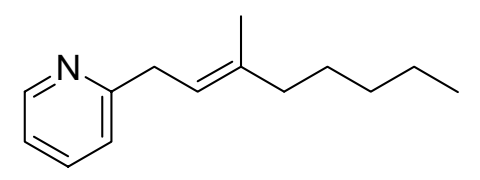

224

Die mit dem Chlormethylpyridin 179 durchgeführte Ni-katalysierte Umsetzung mit $\left[\mathrm{Ni}\left(\mathrm{PPh}_{3}\right)_{2}\right] \mathrm{Cl}_{2}, \mathrm{PPh}_{3}, n$ BuLi und dem Alan 123 (2.25 eq) blieb erfolglos (vgl. Schema 75). Es konnten keine Spuren des gewünschten Kreuzkupplungsproduktes 225 erhalten werden. Die 
Umsetzung lieferte lediglich ein Gemisch aus dem eingesetzten Edukt 179 in einem Anteil von 52\% und dem Homokupplungsprodukt 136 (vgl. Schema 34) als Dien in einem Anteil von $48 \%$.

Schema 75. Ni-katalysierte Kreuzkupplung des Chlormethylpyridins 179 mit dem Alan 123 zu dem Kreuzkupplungsprodukt 225.<smiles>CCCc1c(CCl)nc(OC(C)=O)c(C)c1OC(C)=O</smiles>

179

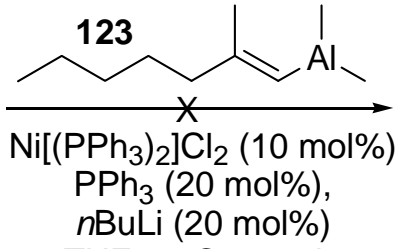

THF, $0^{\circ} \mathrm{C}, 30 \mathrm{~min}$ RT, 2 h<smiles>CCCCC/C(C)=C/Cc1nc(OC(C)=O)c(C)c(OC(C)=O)c1CCC</smiles>

225

\subsection{Cu-katalysierte Kreuzkupplungsreaktionen}

Organokupferreagentien werden im Allgemeinen als die nützlichsten Synthesereagentien unter den übergangsmetallorganischen Verbindungen angesehen. ${ }^{[96]}$ Insbesondere Alkylierungen und Allylierungen lassen sich häufig mit Organocupraten besser als mit anderen metallorganischen Reagentien realisieren. ${ }^{[96]}$ Daher wurde auch im Rahmen dieser Dissertation die Untersuchung Cu-katalysierter Kreuzkupplungsreaktionen geplant.

In der Literatur berichtete unter anderem Nakamura über $\mathrm{Cu}$-katalysierte $\mathrm{S}_{N} 2-$ bzw. $\mathrm{S}_{N} 2^{\prime}-$ Substitutionsreaktionen. Nach seiner Ansicht lässt sich die Substitutionsreaktion eines Alkylhalogenids mit einem harten Nucleophil wie einem Alkylanion am einfachsten mit Hilfe der Organokupferchemie realisieren. Für den Ablauf einer $\mathrm{S}_{N} 2$-Reaktion eines Alkylhalogenids (227 bzw. 230) mit einem Alkylanion sind zwei Mechanismen diskutiert worden (vgl. Schema 76) ${ }^{[96]}$ Der Erste verläuft über eine einfache $S_{N} 2$-Substitution durch die anionische Gruppe R 226. Der zweite Vorschlag nimmt eine geschwindigkeitsbestimmende Verdrängung der Abgangsgruppe, bei der Kupfer 238 eine negative Formalladung trägt, und die anschließende Bildung des Trialkylkupfer(III)-Intermediates $\mathbf{2 3 8}$ an, aus welchem sich durch reduktive Eliminierung das Kreuzkupplungsprodukt 241 bildet. 
Schema 76. Mögliche Mechanismen der Alkylierung durch Kupfer-Reagentien.

Mech. 1:
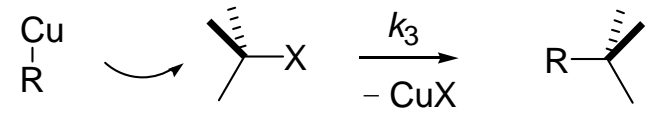

226

227

228

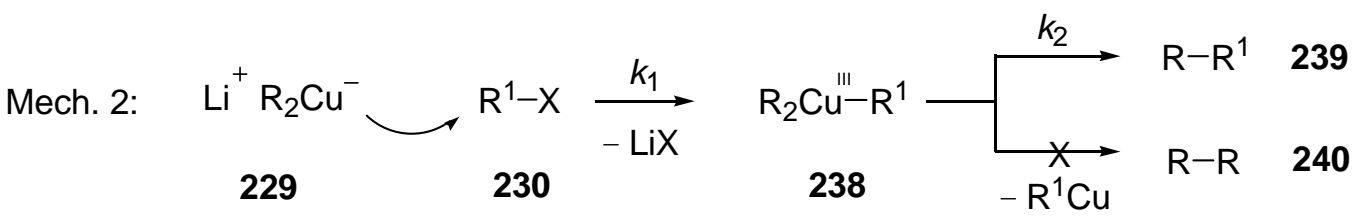

Für die Cu-katalysierte allylische Substitutionsreaktion wird ein Übergangszustand entsprechend dem im Schema 77 dargestellten Mechanismus vertreten. ${ }^{[96]}$ Hiernach führt der Angriff des anionischen Kupfer(I)-Intermediates 242 auf das Allylhalogenid 241 zur Bildung des $\pi$ Komplexes 245. Der $\mathrm{S}_{N} 2$ '-Angriff des Cuprates führt unter Umlagerung der Doppelbindung und Verdrängung der Abgangsgruppe zur Bildung der $\mathrm{Cu}(\mathrm{III})$-Spezies 246. Parallel führt der unter direkter Verdrängung der Abgangsgruppe verlaufende $\mathrm{S}_{N} 2$-Angriff zur Bildung der $\mathrm{Cu}(\mathrm{III})$-Spezies 247, die mit der Organokupfer-Verbindung 246 im dynamischen Gleichgewicht steht. Die abschließende reduktive Eliminierung führt schließlich zur Bildung eines Gemisches aus dem $\mathrm{S}_{N} 2$-Produkt 243 und dem $\mathrm{S}_{N} 2$-Produkt 244.

Schema 77. $\mathrm{S}_{N} 2$-Allylierung mit konkurrierendem $\mathrm{S}_{N} 2$-Reaktionsweg.

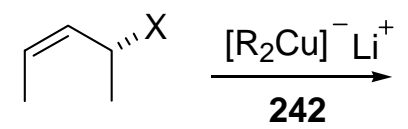

241<smiles>[V][Tl]</smiles><smiles>[R20][Cl+]/C(C)=C/[C@@H]([X])C</smiles>

245<smiles>[R]C(C)/C=C\C</smiles>

243

$\uparrow S_{N}{ }^{\prime}$<smiles>[R]C([R])[GeH2]C(C)/C=C\C</smiles>

246<smiles>[R]C(C)/C=C\C</smiles>

244

$\uparrow \mathrm{s}_{N} 2$

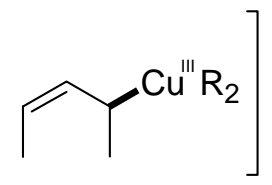

247

$$
\mathrm{X}=\mathrm{I}, \mathrm{Br}, \mathrm{Cl}, \mathrm{OAc}, \mathrm{OP}(\mathrm{O}) \mathrm{Y}_{2}
$$




\subsubsection{Cu-katalysierte Kreuzkupplung des Brommethylpyrons 96 mit Alanen}

Flemming et al. ${ }^{[97]}$ beschrieben die CuCN-katalysierte und hoch selektive $\mathrm{S}_{\mathrm{N}}{ }^{\prime}$-Reaktion von Allylphosphaten mit verschiedenen Alkylalanen unter C-C-Bindungsbildung. Die von ihnen durchgeführte Umsetzung des Geraniolphosphats mit 2.0 eq $\mathrm{AlMe}_{3}$ unter Verwendung von 10 mol\% des Katalysators $\mathrm{CuCN}$ in $\mathrm{THF}$ bei $-15^{\circ} \mathrm{C}$ lieferte die gewünschte $\mathrm{C}-\mathrm{C}$ Bindungsverknüpfung in 93\%iger Ausbeute, wobei ausschließlich das $\mathrm{S}_{\mathrm{N}}$-Produkt und nicht das $\mathrm{S}_{\mathrm{N}}$-Produkt erhalten wurde. Fleming et al. berichteten desweiteren von der leicht durchführbaren Synthese der Ethenylalane durch Hydro- bzw. Carboaluminierungsreaktionen der Alkine. Ihnen gelang die Cu-katalytische Übertragung der aus DIBAH generierten Ethenylalane auf das Geraniolphosphat in einer Ausbeute von $62 \%$.

Schema 78. Cu-katalysierte Kreuzkupplung des Brommethylpyrons 96 mit dem in situ generierten (E)-1-Heptenyldiisobutylalan 202 zu dem Kreuzkupplungsprodukt 203.

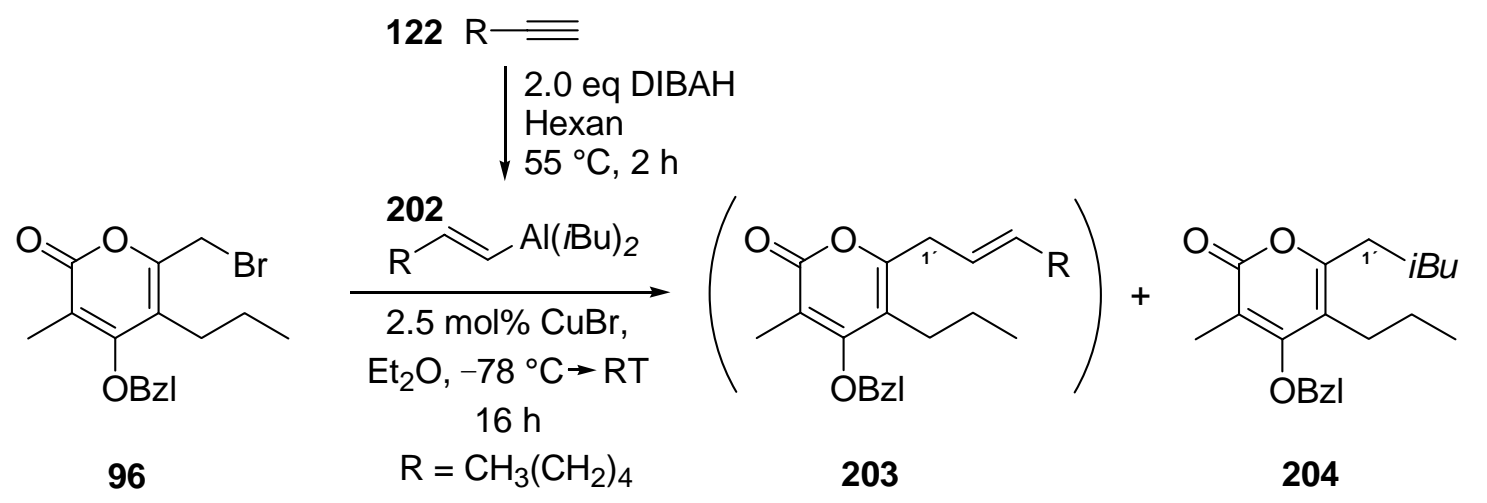

Tabelle 40. Cu-katalysierte Kreuzkupplung des Brommethylpyrons 96 mit den in situ generierten Alanen 202 bzw. 123 zu den Kreuzkupplungsprodukten 203 bzw. 125.

\begin{tabular}{|c|c|c|c|c|}
\hline Eintrag & $202[\mathrm{eq}]$ & $\mathrm{CuBr}[\mathrm{mol} \%]$ & $\mathrm{t}\left[{ }^{\circ} \mathrm{C}\right]$ & 96:203:136:137:204 (\%) \\
\hline 1 & 2.0 & 2.5 & $-78 \rightarrow \mathrm{RT}$ & $35: 0: 0: 2: 63$ \\
\hline & 123 [eq] & & & $96: 125: 136: 137: 138$ \\
\hline $2^{\mathrm{a})}$ & 2.0 & 2.5 & $-100 \rightarrow \mathrm{RT}$ & $0: 0: 11: 2: 87$ \\
\hline 3 & 2.0 & 2.5 & $-100 \rightarrow \mathrm{RT}$ & $8: 0: 0: 2: 80$ \\
\hline $4^{\text {b) }}$ & 2.0 & 1.25 & $-78 \rightarrow \mathrm{RT}$ & $90: 0: 0: 0: 10$ \\
\hline
\end{tabular}

a) Die Reaktion wurde in Anwesenheit von 5 mol\% $\mathrm{ZnCl}_{2}$ durchgeführt. b) Das Alan 123 wurde unter Verwendung von 1 eq $\mathrm{AlMe}_{3}$ generiert. 
Der Vorversuch zur Cu-katalytischen C-C-Bindungsbildung wurde mit Brommethylpyron 96 und (E)-1-Heptenyldiisobutylalan 202 nach Flemming durchgeführt (vgl. Schema 78, Tabelle 40, Eintrag 1). Die Umsetzung lieferte ein Gemisch aus dem eingesetzten Edukt 96 (Anteil 35\%), dem Dipyronethan 137 (Anteil 2\%) als Homokupplungsprodukt des Brommethylpyrons 96 und der an der C-1'-Position isobutylierten Verbindung 204 (Anteil 63\%) als unerwünschtes Hauptprodukt (vgl. Schema 78). Für das 1'-Protonensignal der Isobutylgruppe wurde eine Multiplett bei 2.38-2.51 ppm beobachtet. Es wurde keine weitere Reinigung und Charakterisierung der unerwünschten Verbindung 204 unternommen.

Die Cu-katalysierten Kreuzkupplungsreaktionen des Brommethylpyrons 96 mit dem Dimethylalan 123 wurden nach den in der Tabelle 40 (Einträge 2-5) angegebenen Reaktionsbedingungen durchgeführt (vgl. Schema 79). Die Generierung des eingesetzten Dimethylalans 123 wurde unter Zugabe von $\mathrm{AlMe}_{3}$ bei $0{ }^{\circ} \mathrm{C}$ mit 1-Heptin (122) und $\mathrm{Cp}_{2} \mathrm{ZrCl}_{2}$ durchgeführt. Die zitronengelbe Alanlösung wurde anschließend bei tiefer Temperatur (vgl. Tabelle 40) zu einer Suspension aus Brommethylpyron 96 und $\mathrm{CuBr}$ transferiert und bei Raumtemperatur über Nacht gerührt.

Schema 79. Cu-katalysierte Kreuzkupplung des Brommethylpyrons 96 mit dem in situ generierten Dimethylalan 123 zu dem Kreuzkupplungsprodukt 125.

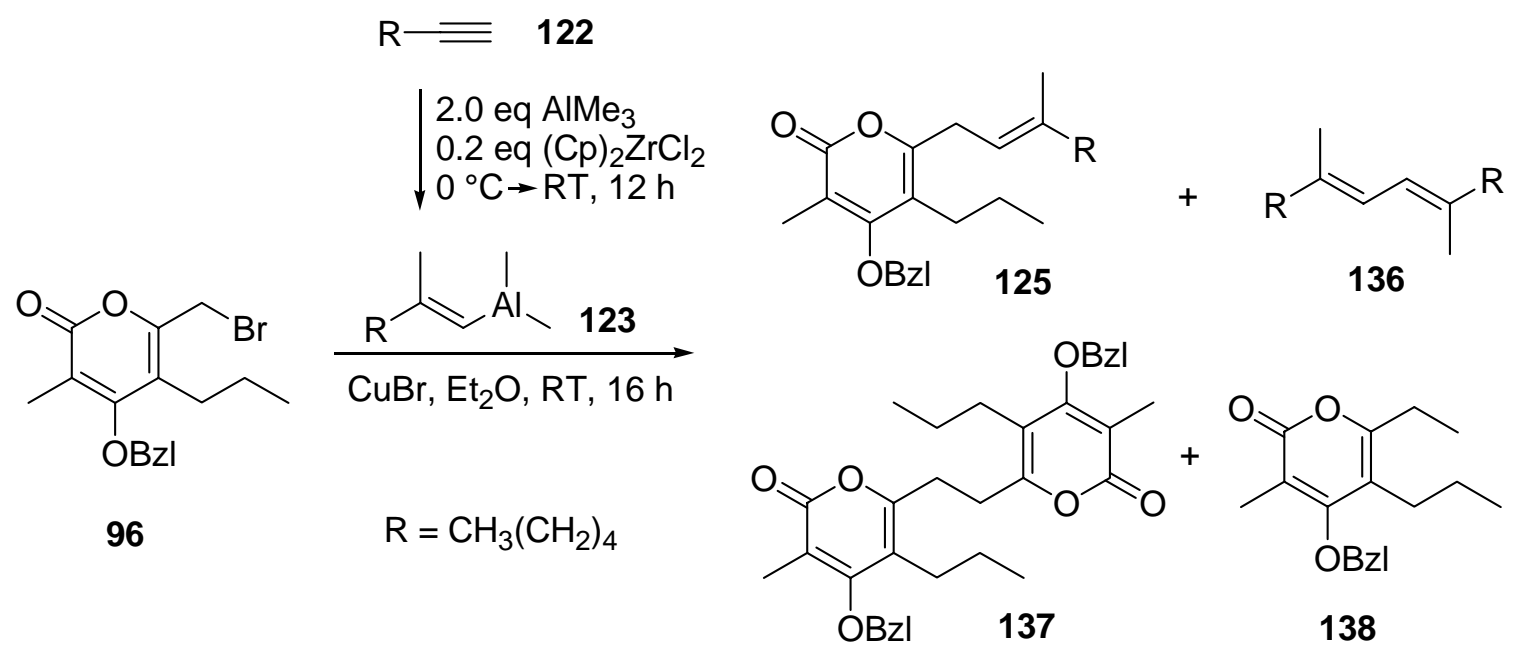

Es konnte bei keiner der Umsetzungen mit Dimethylalan 123 das gewünschte Kreuzkupplungsprodukt 125 beobachtet werden (vgl. Tabelle 40, Einträge 2-4). Während die Versuche 2 
und 3 (Einträge 2 u. 3) hauptsächlich das methylierte Produkt 138 (Quartett bei 2.58 ppm für das 1'-Protonensignal) in Anteilen von $87 \%$ und $80 \%$ ergaben, wurde bei dem Versuch 4 (Eintrag 4) neben der methylierten Verbindung 138 (Anteil 10\%) das unumgesetzte Brommethylpyron 96 (Anteil 90\%) zurückerhalten. Das Problem der unerwünschten Methylierung war auch bei den Kreuzkupplungsreaktionen des Brommethylpyrons 96 mit dem Alanat 124 aufgetreten (vgl. Abschnitt 2.2.2.2). Der geringe Anteil an dem Methylierungsprodukt 138 bei dem Versuch 4 wurde darauf zurückgeführt, dass die Carboaluminierung zu dem Alan 123 wohl nur teilweise erfolgte, so dass auch in diesem Versuch noch unverbrauchtes $\mathrm{AlMe}_{3}$ vorlag, worüber die Methylierung verlaufen sein könnte.

Zur Klärung des Einflusses des Cu-Katalysators auf die Bildung der unerwünschten Produkte wurde eine Cu-katalysatorfreie Umsetzung des Brommethylpyrons 96 mit dem Dimethylalan 123 in THF bei einer Temperatur von $0{ }^{\circ} \mathrm{C}$ durchgeführt. Die Reaktion führte weder zur Bildung des gewünschten Kreuzkupplungsproduktes noch zur Bildung des bei den vorherigen Versuchen erhaltenen unerwünschten Methylierungsprodukt 138. Stattdessen wurden das 1,3Dien 136 als Homokupplungsprodukt in einem Anteil von 83\% und das unumgesetzte Edukt 96 in einem Anteil von 17\% erhalten. Durch die unerwartete Bildung des Homokupplungsproduktes konnten weder der Einfluss des $\mathrm{Cu}$-Katalysators noch die Ursache des Methylierungseffektes geklärt werden.

Zur Überprüfung, ob auch in Abwesenheit von $\mathrm{AlMe}_{3}$ eine Alkylübertragung stattfindet, wurde zudem ein Cu-katalysierter Alkylübertragungsversuch mit Diethylzink $\left(\mathrm{ZnEt}_{2}\right)$ unter im übrigen identischen Reaktionsbedingungen durchgeführt (vgl. Schema 80). Das Ergebnis dieses Versuches, bei dem neben einer geringen Menge des Homokupplungsprodukts 137 (Anteil 4\%) hauptsächlich nur das eingesetzte Bromid 96 (Anteil 96\%) zurückerhalten wurde, entsprach dem Ergebnis des Versuchs 4 (vgl. Tabelle 40, Eintrag 4). Das Ausbleiben des alkylierten Produktes 205 wurde darauf zurückgeführt, dass wahrscheinlich keine Übertragung der Ethylgruppe auf das Kupfer (Ummetallierung) stattgefunden hatte. Dies könnte mit der im Vergleich zu Aluminium schwächeren Lewis-Acidität des Zinks erklärt werden. Zum Gelingen der Ummetallierungsreaktion wäre im Falle des Zinks vermutlich die Unterstützung einer Lewissäure nötig gewesen. 
Schema 80. Cu-katalysierter Alkylierungsversuch des Brommethylpyrons 96 mit Diethylzink zu dem Kreuzkupplungsprodukt 205.

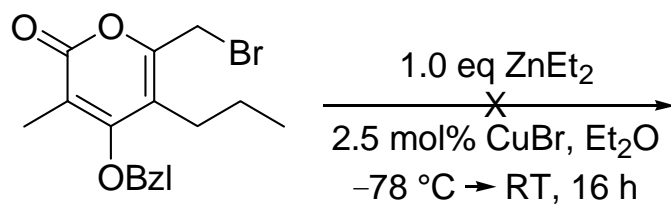

96<smiles>CCCOc1c(CCC)c(CCC)oc(=O)c1C</smiles>

205

Im Hinblick auf die zuvor dargestellten Ergebnisse der Versuche zu den Cu-katalysierten Kreuzkupplungsreaktionen des Brommethylpyrons 96 mit in situ generierten Alanen, bei denen anstatt der Bildung des Kreuzkupplungsproduktes fast ausschließlich die unerwünschte methylierte Verbindung 138 gebildet wurde, wurde zur Vermeidung des Alkylierungsprozesses der Einsatz $\mathrm{AlMe}_{3}$-freien Alans 123 in Betracht gezogen (vgl. Schema 81). Hierzu wurde das $\mathrm{AlMe}_{3}$-freie Dimethylalan 123 nach der allgemeinen Arbeitsvorschrift A5 (s. Exp. Teil, Variante 1 bzw 2) dargestellt. Die Kreuzkupplungsreaktionen wurden unter Einsatz der $\mathrm{Cu}-$ Katalysatoren $\mathrm{CuCl}, \mathrm{CuBr}, \mathrm{CuI}$ und $\mathrm{CuCN}$ untersucht. Eine Lösung von Dimethylalan 123 wurde unter den in der Tabelle 41 angegebenen Reaktionstemperaturen zu einer Lösung von Brommethylpyron 96 und dem Katalysator zugegeben und die Reaktion bei Raumtemperatur durchgeführt.

Schema 81. Cu-katalysierte Kreuzkupplung des Brommethylpyrons 96 mit dem $\mathrm{AlMe}_{3}-$ freiem Alan 123 zu dem Kreuzkupplungsprodukt 125.<smiles>CCCOc1c(CCC)c(CBr)oc(=O)c1C</smiles>

96

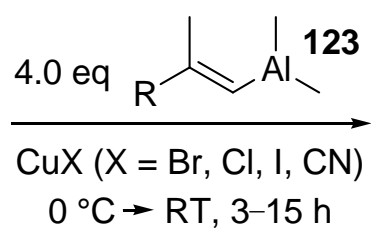

$0{ }^{\circ} \mathrm{C} \rightarrow \mathrm{RT}, 3-15 \mathrm{~h}$<smiles>CCCc1c(C/C=C(\C)[Pb])oc(=O)c(C)c1OCC</smiles>

125 
Tabelle 41. Cu-katalysierte Kupplung des Brommethylpyrons 96 mit dem $\mathrm{AlMe}_{3}$ freiem Alan 123 zu dem Kreuzkupplungsprodukt 125.

\begin{tabular}{ccccccc}
\hline Eintrag & $\begin{array}{c}\mathrm{CuX}- \\
\text { Katalysator }\end{array}$ & $\begin{array}{c}\mathrm{CuX} \\
{[\mathrm{mol} \%]}\end{array}$ & $\mathrm{LSM}$ & $\begin{array}{c}\mathrm{T} \\
{\left[{ }^{\circ} \mathrm{C}\right]}\end{array}$ & $\begin{array}{c}\mathrm{t} \\
{[\mathrm{h}]}\end{array}$ & $\begin{array}{c}\mathbf{9 6 : 1 2 5 : 1 3 6 : 1 3 7} \\
(\%)\end{array}$ \\
\hline 1 & $\mathrm{CuBr}$ & 10 & $\mathrm{Et}_{2} \mathrm{O}$ & $0 \rightarrow \mathrm{RT}$ & 3.0 & $0: 0: 100: 0$ \\
2 & $\mathrm{CuBr}$ & 10 & $\mathrm{THF}$ & $0 \rightarrow \mathrm{RT}$ & 4.5 & $0: 0: 89: 11$ \\
3 & $\mathrm{CuCN} \cdot 2 \mathrm{LiCl}$ & 10 & $\mathrm{THF}$ & $0 \rightarrow \mathrm{RT}$ & 15 & $0: 0: 71: 29$ \\
4 & $\mathrm{CuBr}$ & 2.5 & $\mathrm{Et}_{2} \mathrm{O}$ & $-78 \rightarrow \mathrm{RT}$ & 15 & $0: 0: 89: 11$ \\
5 & $\mathrm{CuCl}$ & 2.5 & $\mathrm{Toluol}$ & $-78 \rightarrow \mathrm{RT}$ & 15 & $\begin{array}{c}\text { Zersetzung } \\
6\end{array}$ \\
\hline
\end{tabular}

Bei keiner der durchgeführten Umsetzungen konnte das gewünschte Kreuzkupplungsprodukt 125 erhalten werden (vgl. Tabelle 41, Einträge 1-6). Während die Umsetzung in Anwesenheit von $\mathrm{CuCl}$ (vgl. Tabelle 41, Eintrag 5) zur vollständigen Zersetzung des Brommethylpyrons 96 führte, bildeten sich bei allen anderen Umsetzungen das Homokupplungsprodukt 137 (vgl. Schema 34) als Neben- sowie das Dien 136 (vgl. Schema 34) als Hauptprodukt. Das vollständige Ausbleiben des Kreuzkupplungsprodukts 125 bei allen in Tabelle 41 aufgeführten Umsetzungen dürfte auch hier damit zu begründen sein, dass Alkenylreste unter diesen Bedingungen im Gegensatz zu den Alkylresten nicht übertragen werden. Daher erfolgt bei Einsatz des $\mathrm{AlMe}_{3}$-freien Alans 123 die Homokupplung der Alkenylreste, was Kupfer-katalytisch unterstützt bzw. gesteuert worden sein könnte. Die Ergebnisse der Versuche unter Einsatz von $\mathrm{AlMe}_{3}$-freien Alanen, bei denen die Bildung des methylierten Produktes ausblieb, stützten zudem die zuvor aufgestellte Annahme, dass die Gründe für die Bildung der alkylierten Produkte 138 und 204 bei den Versuchen mit in situ generierten Alanen (vgl. Schema 78) auf die Anwesenheit von unverbrauchtem freiem $\mathrm{AlMe}_{3}$ bzw. DIBAH in dem Reaktionsmedium zurückzuführen ist.

Die Cu-katalysierte Synthese von 1,3-Dienen, die mit dem bei den Versuchen mit $\mathrm{AlMe}_{3}$ freien Alanen als Hauptprodukt erhaltenen Dien 136 vergleichbar sind, wurde auch in der Literatur beschrieben. Zweifel et al. ${ }^{[98]}$ berichteten von der Cu-katalysierten stereoselektiven Synthese von 1,3-Dienen aus Alkinen über eine Addition des Kupferchlorids (20 mol\%) an die Ethenylalane, bei der die trans,trans-1,3-Diene erfolgreich in Ausbeuten von 67-73\% synthetisiert wurden. Die Bildung der 1,3-Diene soll hiernach unter Ausscheidung von schwarzem Kupfer $\left(\mathrm{Cu}^{0}\right)$ erfolgt sein (vgl. Schema 82). 
Schema 82. Cu-katalysierte Bildung des Diens 136 aus dem Alan 123 nach Zweifel.

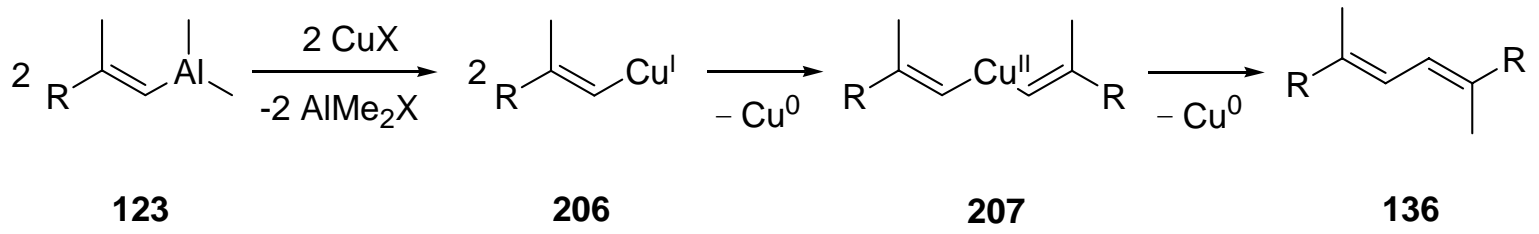

Unter der Annahme einer Bildung der 1,3-Diene unter Ausscheidung von schwarzem Kupfer $\left(\mathrm{Cu}^{0}\right)$ nach Zweifel müsste das Alkenylalan 123 im ersten Teilschritt eine Transmetallierung auf $\mathrm{CuX}$ eingehen, deren Ursache die starke Affinität des Aluminiums zu den Halogeniden sein sollte (vgl. Schema 82). Unter Ausscheidung von $\mathrm{AlMe}_{2} \mathrm{X}$ führt dies zur Bildung des $\mathrm{Cu}(\mathrm{I})$-Intermediates 206. Im nächsten Schritt der Katalyse würde das gebildete $\mathrm{Cu}(\mathrm{I})$ Intermediat 206 mit einem weiteren Cuprat(I)organyl 206 unter Ausscheidung des $\mathrm{Cu}^{0}$ zur Bildung des Cu(II) 207 führen. Die anschließende reduktive Eliminierung unter Ausscheidung von $\mathrm{Cu}(0)$ würde dann die Bildung des Diens 136 ergeben. Die von Zweifel angenommene Bildung des Diens unter Ausscheidung von $\mathrm{Cu}(0)$ lässt sich jedoch nicht mit den Ergebnissen der in der Tabelle 41 aufgeführten Versuche vereinbaren. Zum einen kann der von Zweifel behauptete Mechanismus nur unter stöchiometrischer Menge an Katalysator ablaufen. Die in der Tabelle 41 aufgeführten Versuche wurden jedoch alle in Gegenwart von 2.5-10 mol\% des $\mathrm{Cu}$-Katalysators durchgeführt. Desweiteren spricht gegen die Annahme von Zweifel, dass bei dem oben beschriebenen Versuch ohne Einsatz eines Cu-Katalysators das 1,3-Dien 136 in einem Anteil von 83\% gebildet wurde. Schließlich könnte der von Zweifel beschriebene Reaktionsweg nur dann erklärt werden, wenn das entstandene schwarze Kupfer durch in der Reaktionslösung vorhandene geeignete Oxidationsmittel in das $\mathrm{Cu}(\mathrm{I})$ regeneriert worden wäre. Nach den Reaktionsbedingungen der in der Tabelle 41 aufgeführten Versuche dürfte diese Voraussetzung aber mangels Vorhandenseins eines Oxidationsmittels nicht vorgelegen haben. Zur Ursache der Bildung des Diens 136 wurden allerdings keine weiteren Untersuchungen durchgeführt.

Als Zwischenergebnis der obigen Versuche konnte festgehalten werden, dass unter den bisherigen Versuchsbedingungen (insb. Einsatz von aus stöchiometrischen bzw. überschüssigen Mengen $\mathrm{AlMe}_{3}$ in situ generierten Alanen sowie von $\mathrm{AlMe}_{3}$-freien Alanen) nur eine Übertragung von Alkylresten nicht jedoch die für die Iromycinsynthese erforderliche Übertragung der langkettigen Alkenylreste erzielt werden konnte. Zur Klärung der Frage, ob eine generelle 
Möglichkeit der Übertragung von Alkenylresten auf das Brommethylpyron 96 unter CuKatalyse besteht, wurden weitere $\mathrm{Cu}$-katalysierte Versuche zur Übertragbarkeit diverser organischer Reste von aus Aluminiumchloriden hergestellten Alanen durchgeführt.

Zunächst wurden Cu-katalysierte Kreuzkupplungsreaktionen mit aus Grignard-Reagentien und Aluminiumchloriden generierten trisubstituierten Alanen untersucht. Die hierzu benötigten Dialkylaluminiummonochloride bzw. Alkylaluminiumdichloride wurden aus Synproportionierungsreaktionen der entsprechenden Aluminiumtrialkyle mit Trichloraluminium $\left(\mathrm{AlCl}_{3}\right)$ nach Arbeitsvorschriften von Pawlenko ${ }^{[99]}$ und Collette ${ }^{[100]}$ synthetisiert. Die Alane $\mathrm{Et}_{2} \mathrm{AlCl}$, $\mathrm{EtAlCl}_{2}$, (Isobutyl) ${ }_{2} \mathrm{AlCl}$, (Isobutyl) $)_{1.5} \mathrm{Al}(\mathrm{Cl})_{1.5}$ und $\mathrm{MeAlCl}_{2}$ wurden entsprechend dargestellt und nach Herstellung einer jeweils 1.0 M Lösung der jeweiligen Triorganylalane mit Brommethylpyron 96 und $\mathrm{Cu}$-Katalysator $(2.5 \mathrm{~mol} \% \mathrm{CuBr}$ ) umgesetzt (vgl. Schemata 83 und 84, Exp. Teil, Allg. Arbeitsvorschrift A26).

Zur Feststellung, ob eine Transmetallierung zwischen dem Aluminiumchlorid und den Grignard-Reagenzien stattfindet, wurde zunächst die im Schema 83 dargestellte Alkylierung des Brommethylpyrons 96 untersucht. Der durchgeführte Propylierungsversuch (vgl. Tabelle 42, Eintrag 1) ergab ein Gemisch aus dem Bromid 96 (Anteil 11\%), dem Homokupplungsprodukt 137 (Anteil 21\%) und dem propylierten Produkt 212 (Anteil 68\%), dessen Erhalt als Nachweis für die erfolgte Transmetallierung gewertet wurde. Für die Bildung des propylierten Produktes 212 sprach das erhaltene Multiplett im ${ }^{1} \mathrm{H}-\mathrm{NMR}-$ Spektrum bei 2.55 ppm, welches der C-1'-Position zugeordnet wurde und trotz einer geringen Chemoselektivität die C-CBindungsbildung bestätigte.

Schema 83. Cu-katalysierte Kupplung des Brommethylpyrons 96 mit dem aus der Ummetallierung von Grignard-Reagenzien und Monochlordiethylalan 231 generierten Propylalan 232.

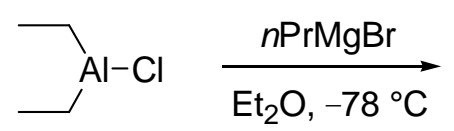

$1 \mathrm{~h}$

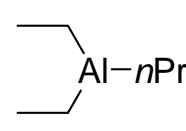

232
$\underset{\mathrm{Et}_{2} \mathrm{O},-78^{\circ} \mathrm{C} \rightarrow \mathrm{RT}}{\stackrel{96,2.5 \mathrm{~mol} \% \mathrm{CuBr}}{\longrightarrow}}$

$16 \mathrm{~h}$<smiles>CCCCc1oc(=O)c(C)c(OC(C)C)c1CCC</smiles>

212 
Tabelle 42. Cu-katalysierte Kreuzkupplung des Bromids 96 mit aus der Ummetallierung von Grignard-Reagenzien und Mono- bzw. Dichloraluminium generierten Alanen.

\begin{tabular}{|c|c|c|c|c|c|}
\hline Eintrag & $\begin{array}{l}\text { Aluminium- } \\
\text { chlorid }\end{array}$ & $\begin{array}{c}\text { Generiertes } \\
\text { Alan }\end{array}$ & [eq] & $\begin{array}{c}\text { Übertragene } \\
\text { Gruppen }\end{array}$ & $\begin{array}{c}\text { 96:205:212:137 } \\
(\%)\end{array}$ \\
\hline 1 & $\mathrm{Et}_{2} \mathrm{AlCl}$ & $\mathrm{Et}_{2} \mathrm{AlPr}$ & 2.0 & Propyl & $11: 0: 68: 21$ \\
\hline & & & & & $96: 205: 214: 137$ \\
\hline 2 & $\mathrm{Et}_{2} \mathrm{AlCl}$ & $\mathrm{Et}_{2} \mathrm{Al}-\mathrm{Vinyl}$ & 2.0 & Ethyl & $90: 10: 0: 0$ \\
\hline 3 & $\mathrm{Et}_{2} \mathrm{AlCl}$ & $\mathrm{Et}_{2} \mathrm{Al}$-Vinyl & 4.0 & Ethyl/Vinyl & $6: 41: 42: 11$ \\
\hline 4 & $\mathrm{EtAlCl}_{2}$ & EtAl-(Vinyl $)_{2}$ & 4.0 & Vinyl & $66: 0: 32: 2$ \\
\hline & & & & & $96: 204: 214: 137$ \\
\hline 5 & (Isobutyl) ${ }_{2} \mathrm{AlCl}$ & (Isobutyl) 2 Al-Vinyl & 4.0 & $i \mathrm{Bu} / \mathrm{Vinyl}$ & $47: 18: 30: 5$ \\
\hline 6 & (Isobutyl) $\mathrm{AlCl}_{2}$ & (Isobutyl)Al-(Vinyl) & 2.0 & $i \mathrm{Bu} / \mathrm{Vinyl}$ & $0: 48: 42: 10$ \\
\hline & & & & & $96: 138: 214: 137$ \\
\hline 7 & $\mathrm{MeAlCl}_{2}$ & MeAl-(Vinyl) $)_{2}$ & 2.0 & Vinyl & $0: 0: 91: 9$ \\
\hline
\end{tabular}

Der Vinylierungsversuch mit 2.0 eq Diethylvinylalan [(Et $\left.)_{2} \mathrm{Al}-\mathrm{Vinyl}\right]$ führte fast nur zur Reisolierung des eingesetzten Brommethylpyrons 96 (vgl. Tabelle 42, Eintrag 2). Die angestrebte Vinylierung des Bromids 96 unter Verwendung von 4.0 eq des Diethylvinylalans [(Et) ${ }_{2} \mathrm{Al}-$ Vinyl] (vgl. Tabelle 42, Eintrag 3) verlief ebenfalls unter geringer Selektivität. Es wurde bei unvollständiger Umsetzung neben dem Homokupplungsprodukt 137 (Anteil 11\%) ein Gemisch aus der ethylierten Verbindung 205 (Anteil 41\%, vgl. Schema 80) und dem vinylierten Produkt 214 (Anteil 42\%, vgl. Schema 84) erhalten.

Schema 84. Cu-katalysierte Kupplung des Brommethylpyrons 96 mit den aus der Ummetallierung von Grignard-Reagenzien und Mono- bzw. Dichloraluminium generierten Alanen 217 und 216.

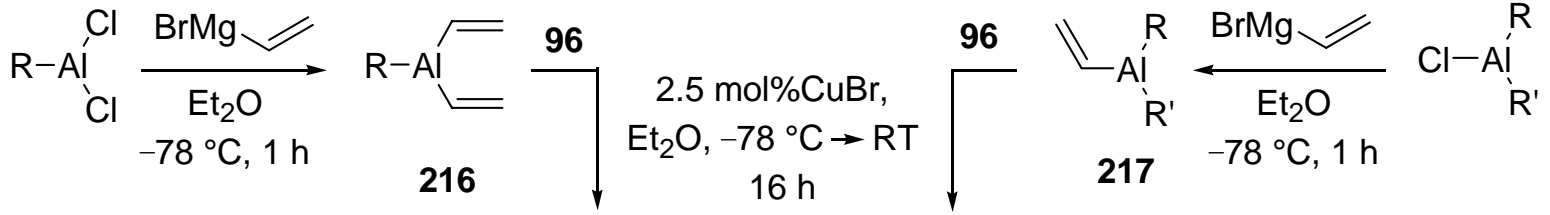

$$
\begin{aligned}
& 233 \mathrm{R}=\mathrm{Me} \\
& 234 \mathrm{R}=\mathrm{Et} \\
& 235 \mathrm{R}=i \mathrm{Bu}
\end{aligned}
$$


Der in dem Versuch 4 (vgl. Tabelle 42, Eintrag 4) erfolgte Einsatz von 4.0 eq Divinylethylalan EthylAl-(Vinyl) $)_{2}$ zur Erhöhung der Übertragungsrate der Vinylierungsgruppe führte zwar zu einer Selektivitätssteigerung, es konnte aber kein vollständiger Umsatz erreicht werden. Die Versuche 5 und 6 (vgl. Tabelle 42, Einträge 5 u. 6) unter Verwendung der mit sterisch anspruchsvolleren Alkylgruppen substituierten Mono- und Divinylalane (isoBu) ${ }_{2} \mathrm{Al}$-Vinyl bzw. (isoBu)Al-(Vinyl) ${ }_{2}$ verliefen ebenfalls unselektiv. Es wurde neben dem Vinylierungsprodukt 214 (Anteile 30\% und 42\%) auch die Verbindung 204 (vgl. Schema 78) in Anteilen von $18 \%$ und $48 \%$ gebildet. Unter Verwendung von 2.0 eq MeAl(Vinyl) $)_{2}$ bei der Reaktion 7 (vgl. Tabelle 42, Eintrag 7) konnte das Brommethylpyron 96 entgegen der Erfahrungen aus den Versuchen 5 und 6 - trotz geringem sterischen Anspruch wurde keine Methylübertragung beobachetet - hoch selektiv in die vinylierte Verbindung 214 (Anteil 91\%) überführt werden. Daneben wurde nur eine geringe Menge an Homokupplungsprodukt 137 (9\%) gebildet. Eine Bildung des Diens 136 (vgl. Schema 34) konnte nicht sicher bestätigt werden, wird jedoch aufgrund der Überlappung der olefinischen Signale der Vinylgruppe bei 5.9-6.02 ppm mit dem olefinischen Singulettsignal des Diens 136 bei 6.0 ppm auch nicht definitiv ausgeschlossen.

Als Gesamtergebnis der Versuche mit den aus Aluminiumchloriden dargestellten Alanen ließ sich feststellen, dass die der Alkenylgruppe vergleichbare olefinische Vinylgruppe gegenüber den Alkylgruppen im Gegensatz zu den Ergebnissen der Versuche aus den Tabellen 40 und 41 am Besten übertragen werden konnte (Rangfolge der Übertragbarkeit nach Tabelle 42: Vinyl $>$ Propyl $>$ Ethyl $>$ Isobutyl $>$ Methyl).

Aufgrund des gelungenen Cu-katalysierten Vinylierungsversuches des Brommethylpyrons 96 (vgl. Tabelle 42, Eintrag 7), der die grundsätzliche Übertragbarkeit von Alkenylgruppen aufzeigte, wurden die Untersuchungen zu den $\mathrm{Cu}$-katalysierten Kreuzkupplungsversuchen des Brommethylpyrons 96 fortgesetzt. Die Ummetallierung wurde allerdings aufgrund der höheren Reaktivität an Stelle von Grignard-Reagentien mit tert-BuLi durchgeführt. In die Cukatalysierten Kreuzkupplungsversuche wurden daher die aus $\mathrm{Et}_{2} \mathrm{AlCl}$ bzw $\mathrm{EtAlCl}_{2}$ und Heptenyllithium 209 generierten Alane 219 bzw. 220 eingesetzt (vgl. Schema 85). Das für die Darstellung des Heptenyllithiums 209 als Ausgangssubstanz benötigte Heptenylbromid 196 wurde nach der Allg. Arbeitsvorschrift A22 (s. Exp. Teil) dargestellt. 
Schema 85. Cu-katalysierte Kupplung des Bromids 96 mit den Alanen 219 bzw. 220.<smiles>[Z4]C(C)=CBr</smiles>

196

8.0 eq tBuLi, THF

$-78{ }^{\circ} \mathrm{C}, 2.5 \mathrm{~h}$
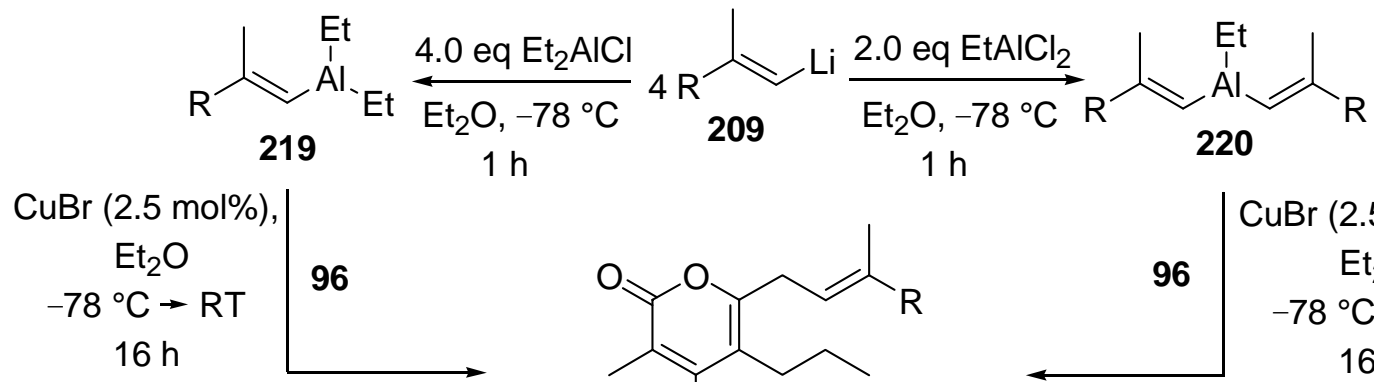<smiles>[R]C(C)=CCc1oc(=O)c(C)c(OCC)c1CCC</smiles>

$96 \mid \begin{gathered}\mathrm{CuBr}(2.5 \mathrm{~mol} \%), \\ \mathrm{Et}_{2} \mathrm{O} \\ -78{ }^{\circ} \mathrm{C} \rightarrow \mathrm{RT} \\ 16 \mathrm{~h}\end{gathered}$

125 mit $\mathrm{R}=\mathrm{CH}_{3}\left(\mathrm{CH}_{2}\right)_{4}$

Zur Generierung von 4.0 eq Heptenyllithium 209 wurden 4.0 eq Heptenylbromid 196 mit 8.0 eq $t \mathrm{BuLi}$ versetzt. Der Lithiumorganyllösung 209 wurde eine Lösung aus $\mathrm{Et}_{2} \mathrm{AlCl}$ (4.0 eq) bzw. EtAlCl 2 (2.0 eq) zugegeben. Anschließend wurden die generierten Alanlösungen 219 bzw. 220 jeweils zu einer auf $-78^{\circ} \mathrm{C}$ vorgekühlten Suspension von Brommethylpyron 96 und $\mathrm{CuBr}(2.5 \mathrm{~mol} \%)$ transferiert (vgl. Schema 85 u. Exp. Teil, Allg. Arbeitsvorschrift A27). Das gewünschte Kreuzkupplungsprodukt 125 blieb bei beiden durchgeführten Umsetzungen aus (vgl. Tabelle 43, Einträge 1 u. 2). Die Versuche ergaben jeweils ein Gemisch aus dem Homokupplungsprodukt 137 in Anteilen von 20\% bzw. 3\% und dem debromierten Produkt 89 in Anteilen von 80\% bzw. 42\%. Daneben wurde bei dem Versuch mit dem Alan 220 (Eintrag 2) das Bromid 96 teilweise unumgesetzt zurückerhalten (Anteil 55\%). Da das erwünschte Kreuzkupplungsprodukt bei diesen Versuchen noch nicht einmal in Spuren gebildet wurde, schien die Übertragung der für die Iromycinsynthese erforderlichen langkettigen Alkenylgruppen auf dem Weg über eine Cu-katalysierte Kreuzkupplung mit Alanen nicht realisierbar zu sein, so dass keine weiteren Versuche durchgeführt wurden.

Tabelle 43. Cu-katalysierte Kupplung des Bromids 96 mit den Alanen 219 bzw. 220.

\begin{tabular}{cccccc}
\hline Eintrag & $\begin{array}{c}\text { Aluminium- } \\
\text { chlorid }\end{array}$ & $\begin{array}{c}\text { Aluminium- } \\
\text { chlorid [eq] }\end{array}$ & $\begin{array}{c}\text { Generiertes } \\
\text { Alan }\end{array}$ & $\begin{array}{c}\text { Generiertes } \\
\text { Alan [eq] }\end{array}$ & $\begin{array}{c}\mathbf{9 6 : 1 2 5 : 1 3 7 : 8 9} \\
(\%)\end{array}$ \\
\hline 1 & $\mathrm{Et}_{2} \mathrm{AlCl}$ & 4.0 & $\mathbf{2 1 9}$ & 4.0 & $0: 0: 20: 80$ \\
2 & $\mathrm{EtAlCl}_{2}$ & 2.0 & $\mathbf{2 2 0}$ & 2.0 & $55: 0: 3: 42$ \\
\hline
\end{tabular}




\subsubsection{Cu-katalysierte Kreuzkupplung von Lithium-Alkenylen mit Benzylbromid bzw. Brommethylpyron}

Högeberg et al. ${ }^{[101]}$ berichteten von der erfolgreichen Synthese von 1,4-Dienen durch die Kreuzkupplung vinylischer Cuprate mit Allylhalogeniden unter Brom-Lithium-Austausch. Die Lithium-bis(organyl)-Cuprate generierten sie mit tert-Butylammonium-Kupferdibromid $\left[(\mathrm{TBA}) \mathrm{Cu}(\mathrm{I}) \mathrm{Br}_{2}\right]$ und entsprechenden Vinyl-Lithiumorganylen, die durch Behandlung von Vinylbromid mit Lithiumorganylen dargestellt wurden. Seebach et al. ${ }^{[102]}$ beschrieben ebenfalls die erfolgreiche Umwandlung von Vinylhalogeniden in Alkenyllithiumverbindungen durch Brom-Lithium-Austausch. Zudem berichteten Nicolaou et al. ${ }^{[103]}$ von einer katalysatorfreien C-C-Bindungsverknüpfung eines Lithiumorganyls bei der Synthese von Pheromonen mit Vinylbromiden (Ausbeute von 82\%), bei der das Lithiumorganyl unter Anwendung von $t$ BuLi aus Allylbromid generiert wurde.

Die Vorversuche zur geplanten C-C-Bindungsverknüpfung auf dem Weg zur Synthese des Iromycin-Vorläufers 125 wurden zunächst mit Benzylbromid 130 und Heptenyliodid 195 in Gegenwart von katalytischen sowie stöchiometrischen Mengen an Kupfer durchgeführt (vgl. Schema 86). Nach dem im Schema 86 dargestellten Reaktionsablauf wird durch den Einsatz von tert-Butyllithium vinylisch gebundenes Iod gegen Lithium ausgetauscht. Die Umsetzung des Heptenyllithiums 209 führt in Anwesenheit des Cu-Katalysators zur Bildung des Cuprats als Intermediat (in katalytisch bzw. stöchiometrisch gebildeter Menge), das als Nucleophil in einer $\mathrm{S}_{N}$-Reaktion das Bromid des Benzylbromids 130 als Abgangsgruppe verdrängt und das gewünschte Kreuzkupplungsprodukt 131 liefert. Seebach wies bei seinen Untersuchungen nach, dass zum vollständigen Austausch von 1.0 eq vinylisch gebundenem Brom bei $-78^{\circ} \mathrm{C}$ 2.0 eq des tert-Butyllithiums erforderlich sind. Als Grund hierfür wurden die schnelle Reaktion des intermediär entstehenden tert-Butylbromids mit tert-Butyllithium zu LiBr und die daraus resultierende Bildung von Isobuten und Isobutan genannt. Entsprechend der Vorgaben von Seebach wurde das Lithiumorganyl 209 ausgehend von 1.0 eq Heptenyliodid 195 und 2.0 eq tert-BuLi bei $-78{ }^{\circ} \mathrm{C}$ generiert. Anschließend wurde eine Lösung aus Benzylbromid 130 (0.85 eq) und dem Cu-Katalysator (entsprechend der in der Tabelle 44 angegebenen Menge) zur orangen Lithiumorganyllösung 209 gegeben. 
Schema 86. Cu-katalysierte Kreuzkupplung des Heptenyllithiums 209 mit dem Benzylbromid 130 zu dem Kreuzkupplungsprodukt 131.

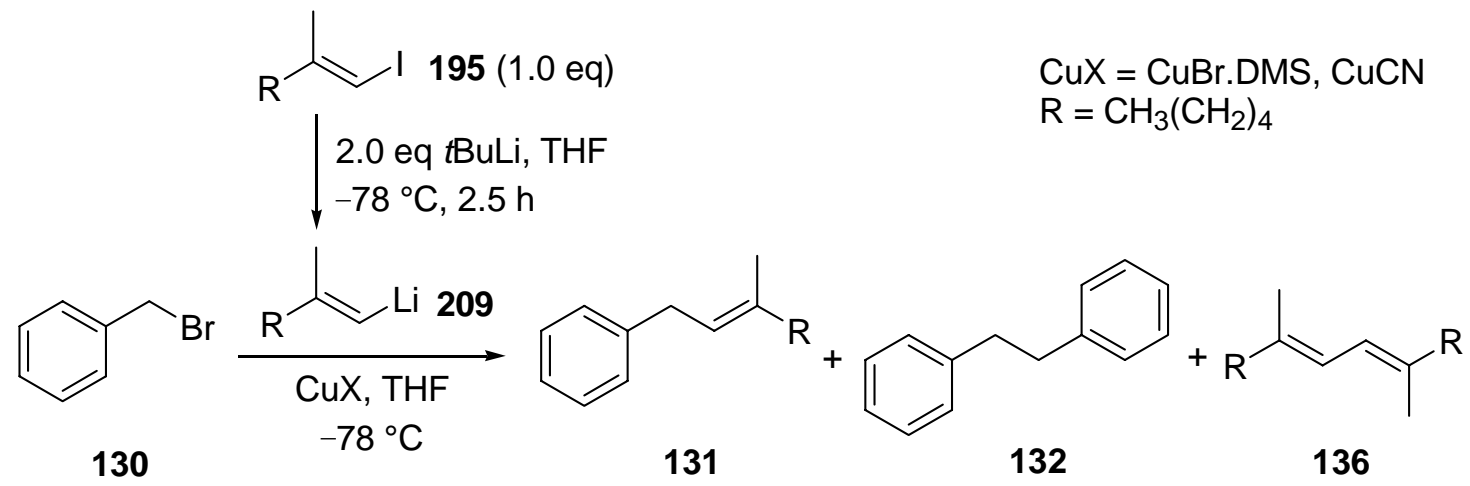

Tabelle 44. Cu-katalysierte Kreuzkupplung des Heptenyllithiums 209 mit dem Benzylbromid 130 zu dem Kreuzkupplungsprodukt 131.

\begin{tabular}{cccc}
\hline Eintrag & Kat. [eq] & $\mathrm{t}[\mathrm{h}]$ & $\mathbf{1 3 0 : 1 3 1 : 1 3 2 : 1 3 6 ( \% )}$ \\
\hline 1 & $0.1(\mathrm{~A})$ & 1.5 & $0: 0: 95: 5$ \\
2 & $1.0(\mathrm{~A})$ & 15 & $40: 0: 22: 38$ \\
3 & $0.1(\mathrm{~B})$ & 1.5 & $0: 0: 80: 20$ \\
\hline
\end{tabular}

A: CuBr·DMS. B: CuCN.

Bei keiner der Cu-vermittelten Umsetzungen (vgl. Tabelle 44, Einträge 1-3) konnte die Bildung des angestrebten Kreuzkupplungsprodukts 131 beobachtet werden. Während die mit 0.1 eq an $\mathrm{Cu}$-Katalysatoren (CuBr-DMS und $\mathrm{CuCN}$ ) durchgeführten Versuche (Einträge 1 u. 3) die Homokupplungsverbindung 132 als Hauptprodukt (Anteil 95\% bzw. 80\%) lieferten, ergab der in stöchiometrischer Menge an $\mathrm{CuBr}$ durchgeführte Versuch (Eintrag 2) nach $15 \mathrm{~h}$ ein Gemisch aus dem unumgesetzten Benzylbromid 130 (Anteil 40\%), dem Homokupplungsprodukt 132 (Anteil 22\%) und dem Dien 136 (Anteil 38\%). Desweiteren erhielt man bei allen Versuchen das Dien 136 als Homokupplungsprodukt der Seitenkette in Bildungsanteilen von 5\%, 38\% und 20\% (vgl. Tabelle 44). Die Bildung des Homokupplungsprodukts 132 in hohen Anteilen (Eintrag 1: 95\%, Eintrag 3: 80\%) wird auf den Einsatz einer katalytischen Menge an Kupfer (10 mol\%) zurückgeführt (vgl. Schema 87). Die fast ausschließliche Bildung des Homokupplungsprodukts 132 bei dem über den Einsatz von $\mathrm{CuX}(0.1 \mathrm{eq} \mathrm{CuBr} \cdot \mathrm{DMS}$ und $\mathrm{CuCN})$ verlaufenden Reaktionsmechanismus könnte gemäß dem Schema 87 mit der Entstehung des über den Weg 1 gebildeten Benzyllithiums 133 begründet werden. Die Bildung des Benzyllithiums 133 dürfte unter Bildung des Alkenylbromids 196 (Weg 1, Schema 87) erfolgt sein. 
Da Toluol und Ethen identische $p$ Ks-Werte aufweisen, sollte ein solcher Brom-LithiumAustausch möglich sein. Das in Anwesenheit des $\mathrm{Cu}$-Katalysators gebildete Kupferorganyl 211 kann dann in einer nucleophilen $\mathrm{S}_{N} 2$-Reaktion das Benzylbromid 130 als Elektrophil angreifen, wodurch bevorzugt das Homokupplungsprodukt 132 gebildet wird. Das über den Weg 2 gebildete Kupferalkenyl 210 kann dagegen nach den oben beschriebenen Ergebnissen von Zweifel zum Produkt 136 abreagieren.

Schema 87. Mechanismus der Cu-katalysierten Bildung der Homokupplungsprodukte 132 und 136.

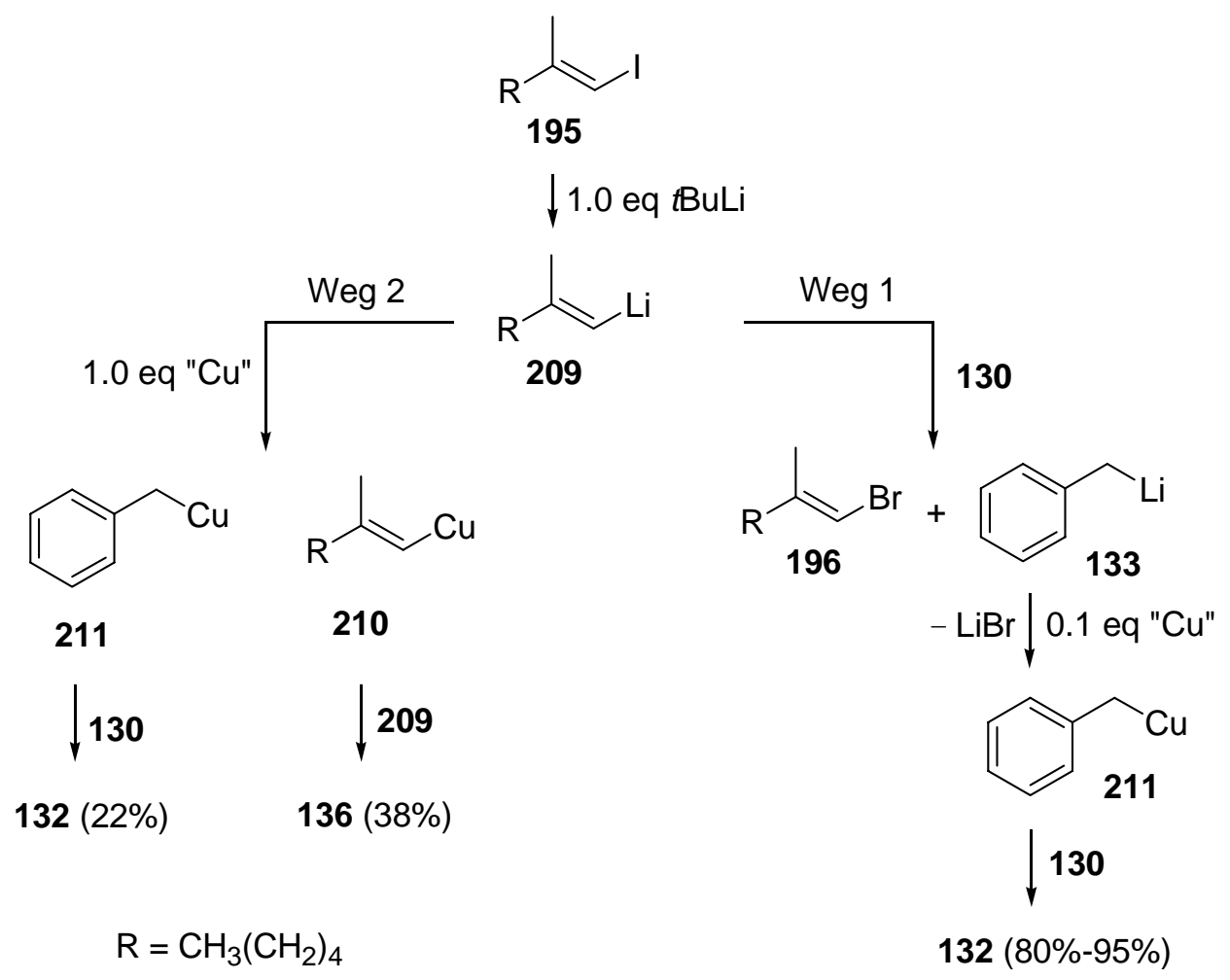

\subsubsection{Cu-katalysierte Kreuzkupplung von Grignard-Reagentien mit Bromiden}

Desweiteren wurde angedacht, die Verwendung von Alkenylmagnesiumbromiden als Grignard-Reagentien zur Übergangsmetall-katalysierten Kreuzkupplung mit Brommethylpyron $96 \mathrm{zu}$ untersuchen. Yamaoto et al. ${ }^{[104]}$ beschrieben die erfolgreiche $\mathrm{S}_{N} 2^{\prime}$-selektive Grignard-Kupplung (Übergangsmetall-katalysierte Substitutionsreaktion von Alkylhalogeniden mit Grignard-Reagenzien nach Kharasch) mit primären Allylphosphaten an der geringer 
substituierten $\gamma$-Position in Gegenwart katalytischer Mengen an $\mathrm{Cu}$-Katalysatoren $(\mathrm{CuCN} \cdot 2 \mathrm{LiCl})$. Yamaoto et al. berichteten zudem von der Ni- bzw. Cu-katalysierten Kreuzkupplung von Vinylgrignardorganylen mit Allylphosphaten, die das Produkt der $\mathrm{S}_{N} 2-$ bzw. $\mathrm{S}_{N} 2$ '-selektiven Reaktion in einem Verhältnis von 99:1 (Ni) bzw. 95:5 (Cu) ergab. Hierfür wurden 1.0 eq der Allylphosphate mit 2.0 eq Grignard-Reagenz in THF bei $-78^{\circ} \mathrm{C}$ in Anwesenheit von $5 \mathrm{~mol} \%$ an $\mathrm{CuCN} \cdot 2 \mathrm{LiCl}$ bzw. Ni(acac) $)_{2}$ umgesetzt.

Um einen geeigneten Katalysator für die geplante Kreuzkupplung zu finden, wurde zunächst das Brommethylpyron 96 in Gegenwart der Übergangsmetall-Katalysatoren $\mathrm{CuBr}, \mathrm{CuCN}$ und $\mathrm{Ni}(\mathrm{acac})_{2}$ untersucht (vgl. Schema 88). 1.5 eq Propylmagnesiumbromid wurde bei $-78^{\circ} \mathrm{C} \mathrm{zu}$ einer Lösung von Brommethylpyron 96 und dem Katalysator (10 mol\%) in THF gegeben und $1.5 \mathrm{~h}$ bei dieser Temperatur gerührt. Während die mit $\mathrm{CuBr}$ durchgeführten Alkylierungsreaktionen ein Gemisch aus dem debromierten Edukt 89 (Anteil 55\%) und dem propylierten Produkt 212 (Anteil 45\%) lieferten, führten die mit $\mathrm{CuCN}$ und $\mathrm{Ni}(\mathrm{acac})_{2}$ umgesetzten Versuche zur sauberen und vollständigen Debromierung zu dem Produkt 89. Aufgrund der erzielten Ergebnisse erwies sich der Übergangsmetallkatalysator $\mathrm{CuBr}$ im Vergleich zu $\mathrm{CuCN}$ und $\mathrm{Ni}(\mathrm{acac})_{2}$ als besser geeignet.

Schema 88. Übergangsmetall-katalysierte Kreuzkupplung von Alkylmagnesiumbromiden mit dem Brommethylpyron 96.

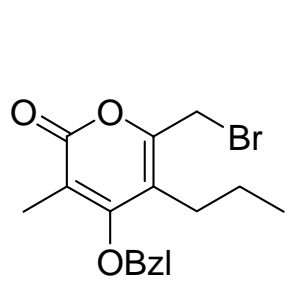

96

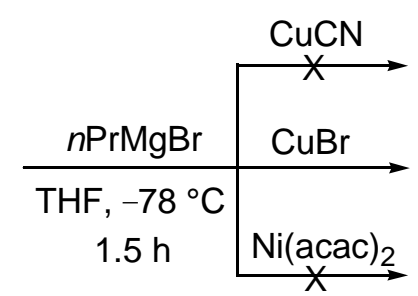

$1.5 \mathrm{~h}$

$212(45 \%)$<smiles>CCCCc1oc(=O)c(C)c(O)c1CCC</smiles>

Zunächst wurden Vorversuche zur Vinylierung des Benzylbromids 130 entsprechend der in der Tabelle 45 angegebenen Reaktionsbedingungen in Gegenwart der Cu-Katalysatoren $\mathrm{CuBr} \cdot \mathrm{DMS}$ und $\mathrm{CuCN}$ durchgeführt (vgl. Schema 89). Während bei den mit $\mathrm{CuCN}$ und $\mathrm{CuBr} \cdot \mathrm{DMS}$ durchgeführten katalytischen Versuchen (vgl. Tabelle 45, Einträge 1 u. 2) keine Umsetzung des Bromids 130 beobachtet wurde, konnte bei den Reaktionen 3, 4 u. 5 (vgl. Ta- 
belle 45, Einträge 3-5) ein Gemisch aus dem Kreuzkupplungsprodukt 213 und dem Homokupplungsprodukt 132 in Anteilen von 13:1, 21:1 und 2:1 erhalten werden. Das Ausbleiben der Umsetzung bei dem Versuch 1 (Eintrag 1) wurde auf die Verwendung von CuCN (vgl. Schema 88) zurückgeführt, bei dem Versuch 2 (Eintrag 2) wurde hingegen die kurze Reaktionszeit von $1.5 \mathrm{~h}$ hierfür verantwortlich gemacht. Letzteres wird durch den Vergleich mit der Reaktionszeit sowie der Reaktionstemperatur bei dem Versuch 3 (Eintrag 3) deutlich, der unter gleichen katalytischen Bedingungen von ebenfalls 10 mol\%, aber bei einer längeren Reaktionszeit von $15 \mathrm{~h}$ über Nacht bei Raumtemperatur durchgeführt wurde und der zumindest zu einer anteiligen Bildung des Kreuzkupplungsproduktes führte. Der Bildungsanteil des Kreuzkupplungsproduktes 213 konnte bei dem Versuch 4 (Eintrag 4) unter stöchiometrischem Einsatz von 1.0 eq des Cu-Katalysators auf einen fast quantitativen Anteil von 95\% erhöht werden. Die unter Verwendung von 1.0 eq des Katalysators bei $0{ }^{\circ} \mathrm{C}$ umgesetzte Reaktion (Eintrag 5) führte zu einem höheren Bildungsanteil an Homokupplungsprodukt 132, das scheinbar bei erhöhten Temperaturen begünstigt gebildet wird.

Schema 89. Cu-katalysierte Kreuzkupplungsversuche des Benzylbromids 130 mit Vinylmagnesiumbromid zu dem Allylbenzol 213.<smiles>BrCc1ccccc1</smiles>

130
1) 2.0 eq VinylMgBr, CuX, 2) 130 CuX = CuCN, CuBr.DMS 213:132 (95:5)\%<smiles>C=CCc1ccccc1</smiles>

132

Tabelle 45. Cu-katalysierte Kreuzkupplung des Benzylbromids 130 mit Vinylmagnesiumbromid zu dem Allylbenzol 213.

\begin{tabular}{cccccc}
\hline Eintrag & Kat. & {$[\mathrm{eq}]$} & $\mathrm{T}\left[{ }^{\circ} \mathrm{C}\right]$ & $\mathrm{t}[\mathrm{h}]$ & $\mathbf{1 3 0 : 2 1 3 : 1 3 2}$ \\
\hline 1 & $\mathrm{CuCN}$ & 0.1 & -78 & 1.5 & $1: 0: 0$ \\
2 & CuBr$\cdot \mathrm{DMS}$ & 0.1 & -78 & 1.5 & $36: 0: 1$ \\
3 & CuBr$\cdot \mathrm{DMS}$ & 0.1 & $-78 \rightarrow \mathrm{RT}$ & 15 & $0: 13: 1$ \\
4 & $\mathrm{CuBr} \cdot \mathrm{DMS}$ & 1.0 & $-78 \rightarrow \mathrm{RT}$ & 15 & $0: 21: 1$ \\
5 & $\mathrm{CuBr} \cdot \mathrm{DMS}$ & 1.0 & $0 \rightarrow \mathrm{RT}$ & 15 & $0: 2: 1$ \\
\hline
\end{tabular}


Da bei den Vorversuchen mit dem Benzylbromid 130 die Bildung des Kreuzkupplungsproduktes 213 in Anteilen von 95\% erzielt werden konnte, wurden analoge Vinylierungsexperimente mit Brommethylpyron 96 untersucht. Aufgrund der Ergebnisse der Tabelle 45 wurden die Versuche zur Vinylierung der C-1'-Position des Brommethylpyrons 96 ausschließlich mit Kupferbromiden $(\mathrm{CuBr}$-DMS und $\mathrm{CuBr})$ als Katalysator in Abwesenheit bzw. in Anwesenheit von $\mathrm{ZnCl}_{2}$ zur Erhöhung der Metallaktivitäten untersucht (vgl. Schema 90). Die Vinylierungsreaktionen mit Brommethylpyron 96 wurden entsprechend den in der Tabelle 46 angegebenen Reaktionsbedingungen durchgeführt (s. Exp. Teil, Allg. Arbeitsvorschrift A25).

Schema 90. Cu-katalysierte Kreuzkupplungsversuche des Brommethylpyrons 96 mit Vinylmagnesiumbromid zu dem 6-Allylpyron 214.<smiles>C=COC(=O)/C(C)=C(\OCCC)C(CCC)C(C)C=CC</smiles>

Tabelle 46. Versuche zur Cu-katalysierten Kreuzkupplung des Brommethylpyrons 96 mit Vinylmagnesiumbromid in Anwesenheit bzw. Abwesenheit von $\mathrm{ZnCl}_{2}$.

\begin{tabular}{|c|c|c|c|c|c|c|c|}
\hline Eintrag & $\begin{array}{l}\text { Vinylcuprat } \\
{[\mathrm{eq}], \mathrm{T}_{1}\left[{ }^{\circ} \mathrm{C}\right]}\end{array}$ & $\begin{array}{l}\text { Kat. } \\
\text { [eq] }\end{array}$ & $\begin{array}{c}\mathrm{ZnCl}_{2} \\
\text { [eq] }\end{array}$ & Lsm. & $\begin{array}{c}\mathrm{T}_{2} \\
{\left[{ }^{\circ} \mathrm{C}\right]}\end{array}$ & $\begin{array}{c}\mathrm{t} \\
{[\mathrm{h}]}\end{array}$ & $\begin{array}{c}\text { 96:214:137:89:208 } \\
(\%)\end{array}$ \\
\hline 1 & $2.0,-78$ & $1.0(\mathrm{~A})$ & - & THF & $-78 \rightarrow \mathrm{RT}$ & 15 & 0:0:0:100:0 \\
\hline 2 & $2.0,0$ & $1.0(\mathrm{~A})$ & - & THF & $0 \rightarrow \mathrm{RT}$ & 15 & Zersetzung \\
\hline 3 & $1.5,-78$ & 0.1 (B) & - & THF & -78 & 1 & 0:0:0:100:0 \\
\hline 4 & $1.5,-78$ & $0.025(\mathrm{~B})$ & - & $\mathrm{Et}_{2} \mathrm{O}$ & -78 & 1 & 1:0:1:98:0 \\
\hline 5 & $1.5,-78$ & $0.025(\mathrm{~B})$ & 1.0 & THF & -78 & 1 & 91:0:9:0:0 \\
\hline 6 & $1.5,-78$ & $0.025(\mathrm{~B})$ & 1.0 & $\mathrm{Et}_{2} \mathrm{O}$ & -78 & 1 & 75:0:25:0:0 \\
\hline \multirow[t]{2}{*}{7} & $2.0,-100$ & 0.025 (B) & 1.0 & Toluol & -100 & 3 & \\
\hline & & & & $/ \mathrm{Et}_{2} \mathrm{O}^{\mathrm{a})}$ & -78 & 0.5 & 0:0:0:15:85 \\
\hline \multirow[t]{2}{*}{8} & $2.0,-100$ & $0.025(\mathrm{~B})$ & 0.1 & $\mathrm{Et}_{2} \mathrm{O}$ & -100 & 3 & \\
\hline & & & & & -78 & 0.5 & $\left.0: 0: 0: 16: 84^{b}\right)$ \\
\hline \multirow[t]{2}{*}{9} & $2.0,-100$ & $0.025(\mathrm{~B})$ & 1.0 & $\mathrm{Et}_{2} \mathrm{O}$ & -100 & 3 & \\
\hline & & & & & -78 & 0.5 & $5: 0: 0: 45: 50$ \\
\hline
\end{tabular}

A: CuBr-DMS. B: $\mathrm{CuBr}^{\text {a) }}$ Toluol:Et ${ }_{2} \mathrm{O}-$ Verhältnis 4:1. ${ }^{\text {b) }}$ Die Masse des Trienesters 208 wurde massenspektroskopisch nachgewiesen. 
Die in Abwesenheit von $\mathrm{ZnCl}_{2}$ durchgeführten CuBr -DMS- und $\mathrm{CuBr}$-katalysierten Versuche (vgl. Tabelle 46, Einträge 1, 3 u. 4) bei $-78{ }^{\circ} \mathrm{C}$ führten zur vollständigen Debromierung des Eduktes 96 und Bildung der unerwünschten Verbindung 89 (vgl. Schema 19). Der Vinylierungsversuch bei $0{ }^{\circ} \mathrm{C}$ (vgl. Tabelle 46, Eintrag 2) führte zur vollständigen Zersetzung des Eduktes 96, sodaß eine Auswertung ausblieb. Die Versuche 3 und 4 (Einträge 3 u. 4) zeigten, dass der Debromierungsprozeß schon nach $1 \mathrm{~h}$ abgeschlossen war. Daraus ist eine Parallele zu den Debromierungseffekten bei den in Anwesenheit von Lithiumorganylen durchgeführten Umsetzungen mit Benzylbromid 130 und Brommethylpyron 96 zu ziehen. Die Magnesiumorganyle weisen ähnlich ihrer analogen Lithiumorganyle die Tendenz auf, als Nucleophile zu agieren. Für den Debromierungsprozess bei den Versuchen 3 und 4 könnte ein $\mathrm{Cu}$ katalysierter Prozeß über die Umwandlung des $\mathrm{Cu}(\mathrm{I}) \mathrm{Br}$ zu $\mathrm{Cu}(\mathrm{II}) \mathrm{Br}$ verantwortlich sein. Wahrscheinlicher ist jedoch, dass die Debromierung durch das Vinylmagnesiumbromid, das große Reaktivitätsähnlichkeiten zu den Lithiumorganylen aufweist, initialisiert wurde, wobei Vinylbromid gebildet werden würde. Hierfür spricht auch, dass das Brommethylpyron 96 im Vergleich zu dem stabileren Benzylbromid 130 durch seine doppelt vinyloge Eigenschaft an der C-1'-Position stärker reaktiv und somit stärker anfällig für die erfolgte Debromierung ist. Ein ähnlicher Debromierungsprozeß bei der Cu-katalysierten Vinylierung des Benzylbromids 130 mit Vinyl-Grignard hätte zur Bildung von Toluol führen müssen (vgl. Tabelle 45), was jedoch nicht beobachtet wurde.

Desweiteren wurde durch Versuche in Anwesenheit von $\mathrm{ZnCl}_{2}$ untersucht, ob eine Transmetallierungskaskade von Magnesium zunächst auf Zink und erst dann auf Kupfer möglich ist (vgl. Tabelle 46, Einträge 5-9). Mit Ausnahme des Versuchs 8 (Eintrag 8) wurden alle mit $\mathrm{ZnCl}_{2}$ durchgeführten Umsetzungen unter stöchiometrischem Einsatz des $\mathrm{Cu}$-Katalysators durchgeführt. Bei keinem der Versuche konnte die Bildung des gewünschten Vinylierungsprodukts 214 beobachtet werden. Aufgrund der Auswertung der ${ }^{1}$ H-NMR-Spektren wurde bei den Umsetzungen 7 und 8 (Einträge 7 u. 8) auf die Entstehung eines offenkettigen Esterderivates 208 geschlossen, das über eine Ringöffnung an der ehemaligen C-6-Position und eine Debromierung an dem ehemaligen C-1'-Kohlenstoffzentrum aus dem Brommethylpyron 96 gebildet wurde. Die Ringöffnung wurde durch den nucleophilen Angriff des Vinylgrignards an der C-6-Position des Brommethylpyrons 96 initialisiert. Die anschließende Protonierung nach der Hydrolyse führte zur Bildung einer vinylierten Triensäureesterverbindung 208. Die Auswertung der bei $-100{ }^{\circ} \mathrm{C}$ durchgeführten Umsetzungen (Einträge 7-9) ergab ein Gemisch aus dem Debromierungsprodukt 89 und einer Verbindung, die als oben be- 
reits beschriebener Trienester angenommen wurde. Die nach säulenchromatographischer Reinigung unternommene massenspektroskopische Untersuchung der bei dem Versuch 8 (Eintrag 8) isolierten unbekannten Verbindung wies die Masse des mit einer Vinylgruppe zweifach funktionalisierten Trienesters $\mathbf{2 0 8}$ nach.

Die Cu-katalysierten Kreuzkupplungsversuche mit Benzylbromid 130 bzw. Brommethylpyron 96 wurden schließlich auch in Gegenwart von 2.0 eq des Grignard-Reagenz 215 und stöchiometrischer Menge des $\mathrm{CuBr} \cdot \mathrm{SMe}_{2}$ als Katalysator durchgeführt (vgl. Schema 91). Der Katalysator wurde aufgrund der befürchteten Bildung des Homokupplungsproduktes (vgl. Abschnitt 5.3.2, Tabelle 44) in stöchiometrischer Menge eingesetzt. Das Grignard-Reagenz 215 wurde nach bekannten Literaturvorschriften ${ }^{[105]}$ dargestellt. Die hiermit durchgeführten Umsetzungen (vgl. Tabelle 47, Einträge 1 u. 2) verliefen sehr unsauber und schienen daher für die geplante Synthese nicht als geeignet (s. Exp. Teil, Allg. Arbeitsvorschrift A25).

Schema 91. Cu-katalysierte Kreuzkupplung des Heptenylmagnesiumiodids 215 mit dem Benzylbromid 130 bzw. Brommethylpyron 96 zu den Kreuzkupplungsprodukten 131 bzw. 125.

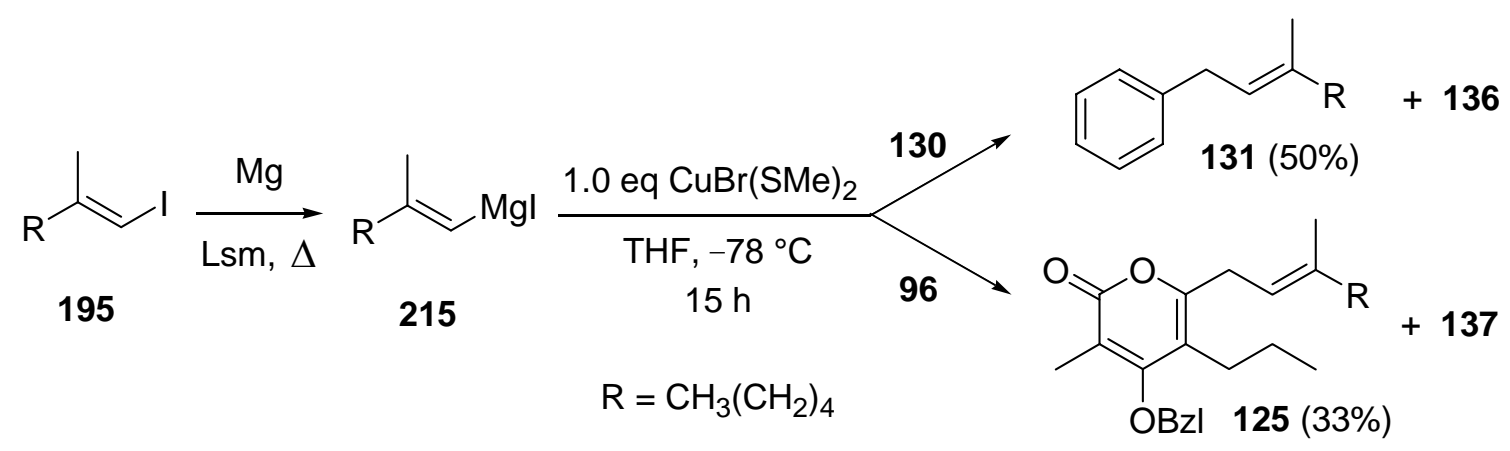

Tabelle 47. Cu-katalysierte Kreuzkupplung des Heptenylmagnesiumiodids 215 mit dem Benzylbromid 130 bzw. Brommethylpyron 96 zu den Produkten 131 bzw. 125.

\begin{tabular}{|c|c|c|c|c|c|c|}
\hline Eintrag & Bromid & [eq] & $\mathrm{CuBr} \cdot \mathrm{DMS}$ [eq] & $\mathrm{T}\left[{ }^{\circ} \mathrm{C}\right]$ & $\mathrm{t}[\mathrm{h}]$ & $130: 131: 136(\%)$ \\
\hline 1 & 130 & 1.0 & 1.0 & -78 & 15 & $40: 50: 10$ \\
\hline & & & & & & $96: 125: 137(\%)$ \\
\hline 2 & 96 & 1.0 & 1.0 & -78 & 15 & $\left.61: 33^{a}\right): 6$ \\
\hline
\end{tabular}

a) Die Bildung des Kreuzkupplungsprodukts 125 (33\%) wurde nicht durch Isolierung nachgewiesen. Der erhaltene Wert basiert nur auf ${ }^{1} \mathrm{H}-\mathrm{NMR}$-spektroskopischen Daten. 
Während die Auswertung des ${ }^{1}$ H-NMR-Spektrums des Rohproduktes bei dem ersten Versuch (vgl. Tabelle 47, Eintrag 1) ein Gemisch aus dem Edukt 130, dem Kreuzkupplungsprodukt 131 und dem Dien 136 im Verhältnis 40:50:10 ergab, konnte der zweite Versuch (vgl. Tabelle 47, Eintrag 2) nicht eindeutig ausgewertet werden. Die letztgenannte Reaktion ergab das Bromid 96 (Anteil 61\%), das Dien 136 (Anteil 6\%) sowie vermutlich das Kreuzkupplungsprodukt 125 (Anteil 33\%). Zusätzlich wurden weitere unbekannte Nebenprodukte beobachtet, die als wahrscheinliche Hydrolyseprodukte des Brommethylpyrons 96 gewertet wurden. Da die Umsetzungen insgesamt sehr unsauber verliefen und die Signale im aliphatischen Bereich eine unverhältnismäßig große Protonenintensität aufwiesen, wurde den theoretisch berechneten Bildungsanteilen der Kreuzkupplungsprodukte (vgl. Tabelle 47) keine Relevanz beigemessen und daher keine weiteren analytischen Untersuchungen mehr vorgenommen. Die unvollständige Umsetzung wurde hauptsächlich auf das eingesetzte Grignard-Reagenz zurückgeführt. Die Versuche zu dessen Darstellung führten stets zur Bildung eines weißen unlöslichen Niederschlags, der trotz großer Verdünnung mit polaren Lösungsmitteln $\left(\mathrm{Et}_{2} \mathrm{O}\right.$ oder THF) nicht aufgelöst werden konnte. Die Versuche zur Konzentrationsbestimmung der Grignard-Lösungen wiesen stets sehr große Abweichungen zu ihren korrespondierenden SollWerten auf.

\subsubsection{Versuche zur $\mathrm{Cu}$-katalysierten Alkinylierung des Brommethylpyrons 96}

Jeffery et al. ${ }^{[106]}$ berichteten von der $\mathrm{Cu}(\mathrm{I})$ - und Phasentransfer-katalysierten Allylsubstitution von substituierten und unsubstituierten Allylhalogeniden mit terminalen Alkinen. Jeffery et al. hoben die große Toleranz der Reaktionsbedingungen gegenüber vielen funktionellen Gruppen hervor, da die Alkinylierungsprozesse der Allylhalogenide im Allgemeinen sehr effizient bei sehr milden Reaktionsbedingungen unter fest-flüssigen Phasentransfer-katalysierten Konditionen abliefen. Die Alkinylierungsreaktion zur C-C-Bindungsbildung des Brommethylpyrons 96 wurde im Rahmen der vorliegenden Arbeit in Gegenwart von Alkin (1.1 eq), $\mathrm{K}_{2} \mathrm{CO}_{3}(1.5$ eq), $\mathrm{NEt}_{4} \mathrm{Cl}$ (0.1 eq) CuI (5 mol\%) durchgeführt (vgl. Schema 92, Tabelle 48). Während bei der bei Raumtemperatur durchgeführten Reaktion (vgl. Tabelle 48, Eintrag 1) neben geringfügigen Verunreinigungen das Bromid 96 unumgesetzt zurückgewonnen wurde, führte der bei erhöhter Temperatur untersuchte Versuch (vgl. Tabelle 48, Eintrag 2) zur Zersetzung und Bildung einer unbekannten Verbindung. 
Schema 92. $\mathrm{Cu}$ - und Phasentransfer-katalysierte Allylsubstitution bei terminalen Alkinen.

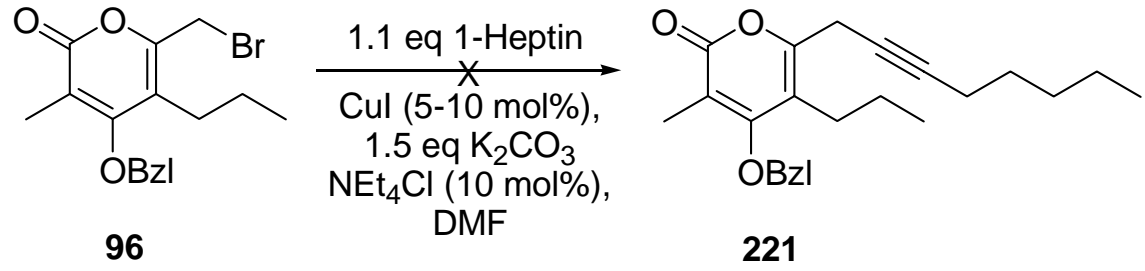

Tabelle 48. Cu- und Phasentransfer-katalysierte Allylsubstitution mit terminalen Alkinen.

\begin{tabular}{ccccc}
\hline Eintrag & $\mathrm{CuI}[\mathrm{mol} \%]$ & $\mathrm{T}\left[{ }^{\circ} \mathrm{C}\right]$ & $\mathrm{t}[\mathrm{h}]$ & $\mathbf{9 6 : 2 2 1}$ \\
\hline 1 & 5 & $\mathrm{RT}$ & 22 & $1: 0$ \\
2 & 10 & 55 & 54 & $0: 0$ \\
\hline
\end{tabular}

Sato et al. ${ }^{[50]}$ führten eine Cu-katalysierte Kupplung von Allylbromiden mit terminalen Alkinen unter Verwendung von Grignardlösungen als Base durch. Die Alkinylierung des Brommethylpyrons 96 wurde entsprechend den Vorgaben von Sato durchgeführt (vgl. Schema 93, Tabelle 49). Die bei $0{ }^{\circ} \mathrm{C}$ bzw. bei Raumtemperatur durchgeführten Cu-katalysierten Alkinylierungsversuche des Brommethylpyrons 96 (vgl. Tabelle 49, Einträge 1 u. 2) mißlangen ebenso wie die zuvor dargestellten Alkinylierungsversuche. Es konnte bei vollständiger Umsetzung des Eduktes jeweils nur ein Gemisch aus dem propylierten Hauptgrundgerüst 212 als Substitutionsprodukt (vgl. Schema 83) und der Verbindung 137 (vgl. Schema 34) als Homokupplungsprodukts des Brommethylpyrons 96 erhalten werden. Die über ${ }^{1} \mathrm{H}-\mathrm{NMR}$ charakterisierte Verbindung 212 wurde zusätzlich über massenspektrometrische Methoden nachgewiesen. Das ${ }^{1}$ H-NMR-Signal als Triplett bei 2.56 ppm wurde der C-1'-Allylposition zugeordnet.

Schema 93. Cu-katalysierte Alkinylierung des Brommethylpyrons 96 mit GrignardReagenzien.<smiles>CCCc1c(CBr)oc(=O)c(C)c1OC(=O)OCc1ccccc1</smiles>

96
1) 1.1 eq 1-Heptin, $1.2 \mathrm{eq} n \mathrm{PrMgBr}$ THF, $0{ }^{\circ} \mathrm{C}, 1 \mathrm{~h}$

2) $96, \mathrm{CuBr}(10 \mathrm{~mol} \%)$, $-10 \rightarrow 0{ }^{\circ} \mathrm{C}, 1 \mathrm{~h}$, $\rightarrow \mathrm{RT}, 1 \mathrm{~h}$<smiles>CCCCCC#CCc1oc(=O)c(C)c(O)c1CCC</smiles>

221 
Tabelle 49. Cu-katalysierte Alkinylierung des Brommethylpyrons 96.

\begin{tabular}{ccccc}
\hline Eintrag & $\mathrm{CuBr}[\mathrm{mol} \%]$ & $\mathrm{T}\left[{ }^{\circ} \mathrm{C}\right]$ & $\mathrm{t}[\mathrm{h}]$ & $\mathbf{9 6 : 2 2 1 : 2 1 2 : 1 3 7 ( \% )}$ \\
\hline 1 & 10 & $-10 \rightarrow 0$ & 1 & $0: 0: 85: 15$ \\
$2^{\text {a) }}$ & 10 & $-10 \rightarrow 0 \rightarrow \mathrm{RT}$ & $1(1)$ & $0: 0: 83: 17$ \\
\hline
\end{tabular}

a) Säulenchromatographische Reinigung.

Die Bildung des propylierten Benzoats 212 wird mit der Bildung des zweifach substituierten Gilman-Cuprats $\left[\mathrm{CH}_{3}\left(\mathrm{CH}_{2}\right)_{4} \mathrm{C} \equiv \mathrm{CCu}\left(\mathrm{CH}_{2}\right)_{2} \mathrm{CH}_{3}\right] \mathrm{MgBr}$ erklärt, bei dem die Alkylgruppe leichter als die Alkinylgruppe auf die Allylposition übertragen werden kann. Dies ist wahrscheinlich auf die Stabilisierung durch die Wechselwirkung des $d$-Orbitals des Kupfers mit dem $p$ Orbital der Dreifachbindung zurückzuführen, wodurch die Nucleophilie der Alkinylgruppe im Vergleich zu Alkylgruppe herabgesetzt wäre.

Nach dem Scheitern der Cu-katalysierten Alkinylierungsversuche wurden keine weiteren Untersuchungen zu Cu-katalysierten Kreuzkupplungen des Brommethylpyrons 96 mehr durchgeführt. Ein Vergleich der Ergebnisse aller Cu-katalysierten Reaktionen erbrachte eine Analogie zu den Pd- u. Ni-katalysierten Kreuzkupplungsversuchen, bei denen ebenfalls die aus Benzylderivaten angestrebten Kreuzkupplungsprodukte in mehr oder minder guten Anteilen erfolgreich synthetisiert werden konnten, während die aus Pyron-, Pyridon- sowie Pyridinhalogeniden ausgehenden gewünschten Kreuzkupplungsprodukte allenfalls nur in sehr geringen Mengen realisiert werden konnten. Damit erwiesen sich Übergangsmetall-katalysierte Kreuzkupplungen im Gegensatz zu den erfolgreichen Kreuzkupplungen mit Brommethylpyron 96 und Alanaten für die Iromycinsynthese insgesamt als ungeeignet. 


\section{Hauptteil 2: Stand der Arbeiten zur Synthese von Collinolacton}

\section{Retrosynthese und Reaktionsmechanismen der Collinolactonsynthese}

Wie schon am Beginn dieser Arbeit erwähnt, befasst sich der Hauptteil 2 mit der Synthese des Naturstoffs Collinolacton (24). Da die synthetische Darstellung des Moleküls 24 im zeitlichen Rahmen dieser Arbeit nicht abgeschlossen werden konnte, behandelt der zweite Teil die retrosynthetischen Überlegungen zur Erstellung eines allgemeingültigen Syntheseplans des Collinolactons (24) sowie den Stand der Synthesearbeiten.

Der retrosynthetische Plan des Collinolactons (24) wurde auf der Grundlage von im Rahmen anderer Arbeiten gewonnenen Informationen und Erkenntnissen über den biosynthetischen Aufbau des Naturstoffes erstellt, nach denen die Biosynthese mit einem aus sechs Acetat- und drei Propionateinheiten aufgebauten Makrolidring initialisiert wird. Nach der Biosynthesehypothese $^{[107]}$ führt die Umsetzung des Makrolidringes in einer [4+6]-Cycloaddition zur Bildung des tricyclischen Collinoketons, dessen Cyclohexanonring durch eine enzymatische Baeyer-Villiger-Oxidation in einen siebengliedrigen Ring überführt wird. Hieraus entsteht das 7-10-6-Ringgerüst des Collinolactons (24). ${ }^{[107]}$ Nach dem aufgestellten allgemeinen Syntheseplan für das Collinolacton (24) (vgl. Schema 94) soll der synthetische Aufbau des Moleküls 24 in vier Schlüsselschritten über eine enantioselektive Aldol-Reaktion, eine diastereoselektive Diels-Alder-Reaktion, eine Oxy-Cope-Umlagerung sowie eine Baeyer-Villiger-Oxidation verlaufen. Hierzu wurde als erster Schlüsselschritt der Synthese der Aufbau des Triens 254 über eine enantioselektive Aldolreaktion des Aldehyds 255 mit dem Hexenon 256 geplant, das im nächsten Schritt als Ausgangssubstanz für eine diasteroselektive Bildung des Bicyclo[4.4.0]decenons 253 über eine intramolekulare Diels-Alder-Reaktion dienen sollte. Nach anschließender Entschützung der funktionellen Gruppen sollten über eine Wittig-Reaktion bzw. eine Reaktion mit einer Alkenyllithium-Verbindung an den Positionen 9 und 10 der Verbindung 253 Alkenylsubstituenten eingeführt werden, welche zur Bildung des gewünschten Intermediats 252 führen. Im nächsten relevanten Schritt folgt dann eine sigmatrope Umlagerung, bei der das Dialkenylcyclohexenol 252 über eine sogenannte anionische Oxy-CopeUmlagerung in das zehngliedrige Cyclodecadienon 251 umgewandelt werden sollte. Anschließend sollte die Ketofunktion des Cyclodecadienons 251 in eine trisubstituierte Doppelbindung überführt werden. Ein Ringschluss zum sechsgliedrigen Lactonring ergäbe dann die Verbindung 250 als Collinolactonvorstufe. Die abschließende Baeyer-Villiger-Oxidation soll- 
te schließlich das sechsgliedrige Cyclohexanon 250 in die siebengliedrige Lactonverbindung überführen, woraus sich das gewünschte tricyclische Collinolacton (24) als Zielprodukt der Totalsynthese ergeben sollte.

Schema 94. Retrosynthetischer Aufbau des Collinolactons (24).

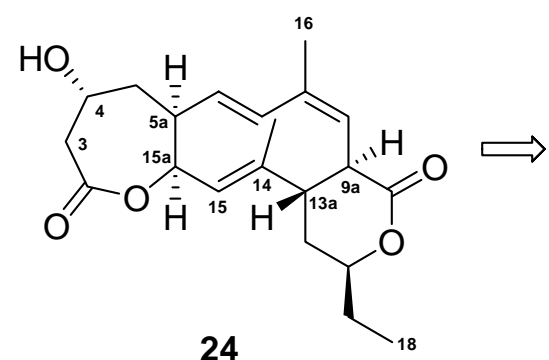

24<smiles>[R]O/C=C/C=C\CC(=O)C[C@@H](O)/C=C/C([R2])([R2])O[R]</smiles>

254<smiles>CC[C@H]1C[C@H]2C=C(C)C=C[C@H]2C(=O)O[C@H]1CC(=O)O</smiles>

250<smiles>[R]O[C@H]1C=C[C@H]2C[C@@H](O)CC(=O)[C@@]2(C)[C@H]1C([R2])(C)O[R]</smiles>

253

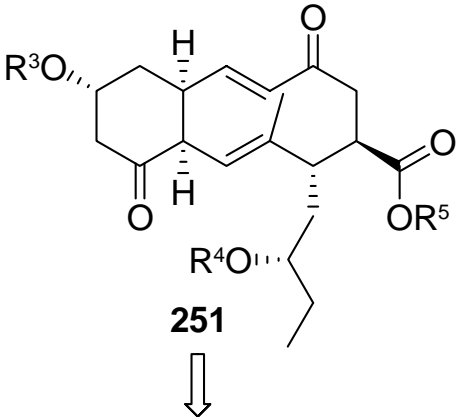<smiles>C=C</smiles>

\1<smiles>C1=CCCCC1</smiles><smiles>[134Sb]</smiles><smiles>[R]O[K]</smiles><smiles>CC=CC=CCC=O</smiles>

255

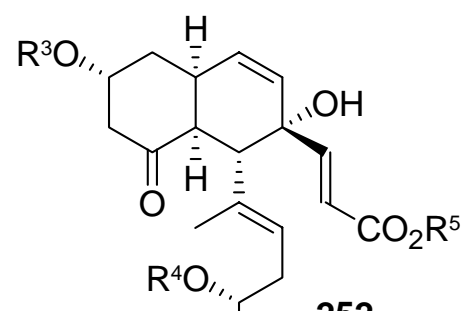

252

Die bereits erwähnten Schlüsselschritte bei der Synthese des Collinolactons sollen vorab aus mechanistischer Sicht näher dargestellt werden, um einen Einstieg in die zentralen Reaktionsschritte zu erleichtern. Den ersten Schritt zur geplanten Synthese des Collinolactons (24) stellt die enantioselektive Aldol-Reaktion dar, die im Abschnitt 3 ausführlich behandelt wird. Demgegenüber konnte die geplante Untersuchung der Diels-Alder-Reaktion, der Oxy-CopeUmlagerung (sigmatrope Umlagerung) und der Baeyer-Villiger-Oxidation im Rahmen dieser Arbeit aus zeitlichen Gründen nicht mehr durchgeführt werden, weshalb diese Syntheseschritte zur mechanistischen Verdeutlichung des geplanten Syntheseablaufs lediglich in einem kurzen Überblick vorgestellt werden. 
Als Aldol-Reaktionen bezeichnet man die Reaktionen von Carbonylverbindungen (Aldehyden oder Ketonen) untereinander, von denen die eine als Nucleophil (Methylenkomponente) und die andere als Elektrophil (Carbonylkomponente) in die Reaktion eintritt. ${ }^{[94]}$ Die Reaktionen können basen- oder säurekatalysiert verlaufen. Bei der Basenkatalyse läuft die Reaktion über das Enolat, das durch die Deprotonierung des $\alpha$-ständigen aciden Protons der Carbonylgruppe generiert wird. Dabei reagiert die elektronenreiche Doppelbindung des Enolats als Nucleophil mit dem elektrophilen Carbonyl-C-Atom einer weiteren Carbonylverbindung. Der beschriebene Reaktionstyp sollte nach den retrosynthetischen Überlegungen in dem ersten Schlüsselschritt der Synthese ausgehend von dem Aldehydderivat 255 und dem Hexenonderivat 256 (vgl. Schema 95) unter C-C-Verknüpfung zur enantioselektiven Bildung des Schlüsselintermediats der Diels-Alder-Vorstufe 254 führen.

Schema 95. Aldolreaktion des Ketoacetals 256 mit dem Hexadienal 255 zu dem Aldoladditionsprodukt 254 als Diels-Alder-Vorstufe.

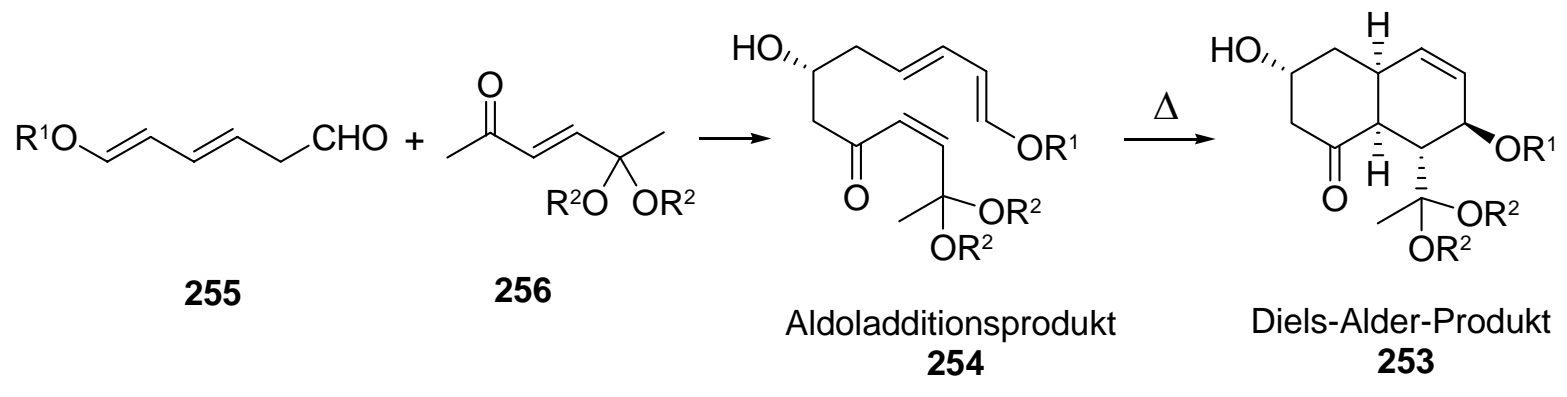

Über eine intramolekulare Diels-Alder-Reaktion (Otto Diels u. Kurt Alder, Nobelpreis 1950) sollte das durch die Aldol-Reaktion verknüpfte Trienderivat 254 in das Bicyclo[4.4.0]decenon 253 (vgl. Schema 95) überführt werden. Bei der Diels-Alder-Reaktion reagiert ein Dien als $4 \pi$-Elektronensystem mit einem Dienophil als $2 \pi$-Elektronensystem. Die Diels-AlderReaktion ist somit eine (4+2)-Cycloaddition, die unter supra-supra-facialer Annäherung von Dien und Dienophil stattfindet. Sie verläuft über einen hochgeordneten Übergangszustand und einen konzertierten Reaktionsmechanismus. Bei einer Diels-Alder-Reaktion können bis zu vier Stereozentren auf einmal aufgebaut werden. ${ }^{[94]}$ Die relative Konfiguration der Edukte bleibt während der Reaktion erhalten. Aus der (4+2)-Cycloaddition eines cis,trans-1,4disubstituierten 1,3-Butadiens 257 mit einem Dien 258 entsteht ein Cyclohexen 259 mit einer 
trans-Anordnung. Ein trans,trans-1,4-disubstituiertes Dien 260 liefert demgegenüber ein Cyclohexen 261, in dem die Substituenten cis-ständig zueinander stehen (vgl. Schema 96).

Schema 96. Schematische Darstellung der Diels-Alder-Reaktion von Dienen mit Dienophilen.

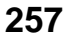

258

260

258

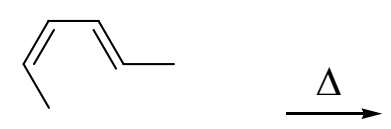

$\overline{\bar{z}}$

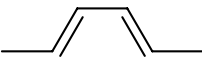

$\overline{=}$

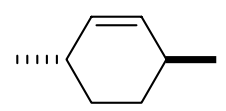

259
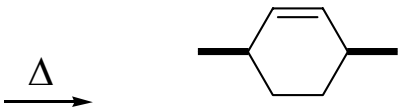

261

Der sigmatrope Umlagerungsschritt der Synthese initialisiert die Überführung des Dialkenylcyclohexenols 252 in das Cyclodecadienon 251 als zehngliedrige Ringverbindung (vgl. Schema 94). Die Oxy-Cope-Umlagerung zeigt eine Verwandtschaft zu der bekannten thermischen Cope-Umlagerung ${ }^{[108]}$, die zur Isomerisierung von 1,5-Dienen angewandt wird. Bei diesem Reaktionstyp handelt sich um eine konzertiert verlaufende [3.3]sigmatrope Umlagerung, bei der zunächst eine C-C-Bindung gelöst und anschließend wieder an einer anderen Stelle ein neue C-C-Bindung geknüpft wird. Aufgrund der Reversibilität dieser Gleichgewichtsreaktion wird hierbei stets das thermodynamisch stabilere Isomer gebildet. Die ausgehend von 3-Hydroxy-1,5-dienen 262 oder 1,2-Divinylcyclohexanolen 265 verlaufende Variante wird als Oxy-Cope-Umlagerung bezeichnet (vgl. Schema 97). ${ }^{[109]}$ Diese Reaktionen sind sowohl thermisch als auch photochemisch durchführbar. Analog der Cope-Umlagerung wird auch hier eine $\sigma$-Bindung gelöst und dafür eine neue $\sigma$-Bindung gebildet. Die Oxy-CopeUmlagerung wird meist zur Darstellung der zehngliedrigen Ringe 266 und 267 (vgl. Schema 97) angewandt. Allgemein kann so ausgehend von geeigneten kleineren Ringen eine Erweiterung um vier Ringglieder ermöglicht werden. Der Vorteil der Oxy-Cope-Umlagerung gegenüber der Cope-Umlagerung ist, dass die Reaktion nicht mehr reversibel verläuft. Erhitzt man beispielweise 3-Hydroxy-1,5-dien 262 (vgl. Schema 97), wird das resultierende Produkt 263 $\mathrm{zu}$ einem Keton tautomerisiert und hierdurch dem Gleichgewicht entzogen. Vorteilhaft ist desweiteren, dass sterische Information aus dem Edukt in das Produkt überführt wird (Chiralitätstransfer) und dass die Entstehung der Carbonylgruppe zudem eine vielfältige Folgechemie 
ermöglicht. Die Triebkraft der Reaktion ist die Bildung der $\mathrm{C}=\mathrm{O}$-Doppelbindung, welches aus energetischer Sicht gegenüber dem Verlust der $\mathrm{C}=\mathrm{C}$-Doppelbindung einen Gewinn darstellt.

Schema 97. Bildung eines zehngliedrigen Cyclodecenon-Ringes über eine Oxy-CopeUmlagerung.

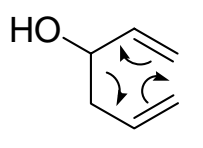

262

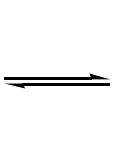<smiles>OC1=CCCC=C1</smiles>

263

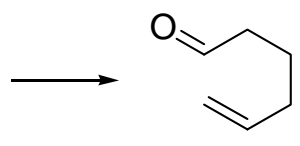

264

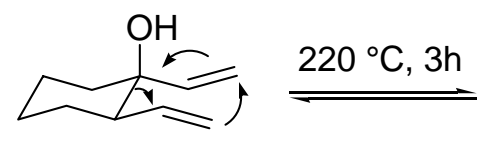

265

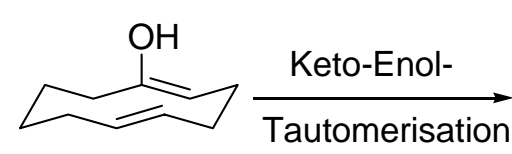

266<smiles>O=C1CCCCCCCCCCCC1</smiles>

267

Die Baeyer-Villiger-Oxidation, die allgemein zur oxidativen Ringerweiterung eines Cyclohexanons zu einem siebengliedrigen Lactonring angewandt wird, wurde als letzter Schlüsselschritt der Synthese zur Umfunktionalisierung der Ketonverbindung 250 zum gewünschten Naturstoff Collinolacton (24) vorgesehen (vgl. Schema 94). Diese Reaktion wandelt Ketone in Ester um (Baeyer, Nobelpreis 1905), was am Beispiel der Umwandlung von 3,3Dimethylbutan-2-on (268) zu Essigsäure-tert-butylester (270) im Schema 98 dargestellt ist. Aus cyclischen Ketonen entstehen unter Erweiterung des Rings die entsprechenden Lactone. Von den beiden Seitenketten des Ketons wandert bevorzugt der höher substituierte Rest zum Sauerstoff hin. Die hierfür angewandten Persäuren sind vor allem Peroxytrifluoressigsäure (TFPOA) und $m$-Chlorperbenzoesäure (MCPBA).

Schema 98. Mechanismus der Baeyer-Villiger-Oxidation.<smiles>CC(=O)C(C)(C)C</smiles>

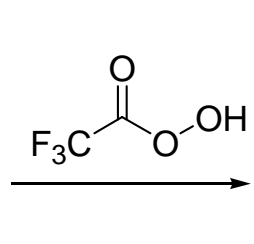<smiles>CC1(C)OOC1(C)OC(=O)C(F)(F)F</smiles><smiles>[C-]C([CH2+])([CH2-])O</smiles><smiles>CC(=O)OC(C)(C)C</smiles>

270 


\section{Darstellung der Ausgangssubstanzen}

Die für die geplante Aldol-Reaktion benötigten zwei Fragmente mussten zunächst als Ausgangssubstanzen synthetisiert werden. Bei allen Additionsreaktionen sollte jeweils Hexadienal 281 als Reaktant eingesetzt werden, welcher ausgehend von dem kommerziell erhältlichen (Z)-But-2-en-1,4-diol (276) dargestellt wurde.

Schema 99. Syntheseroute der Ausgangsverbindungen zur Synthese des Collinolactons (24).<smiles>Cc1ccc(C)o1</smiles>

271

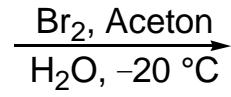

$5 \mathrm{~h}$ $62 \%$

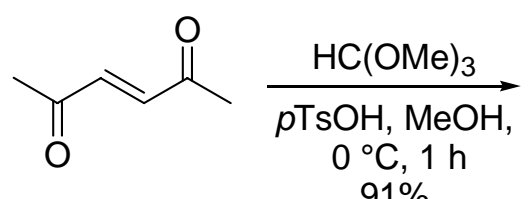
$91 \%$

272<smiles>COC(C)(/C=C/C(C)=O)OC</smiles>

273

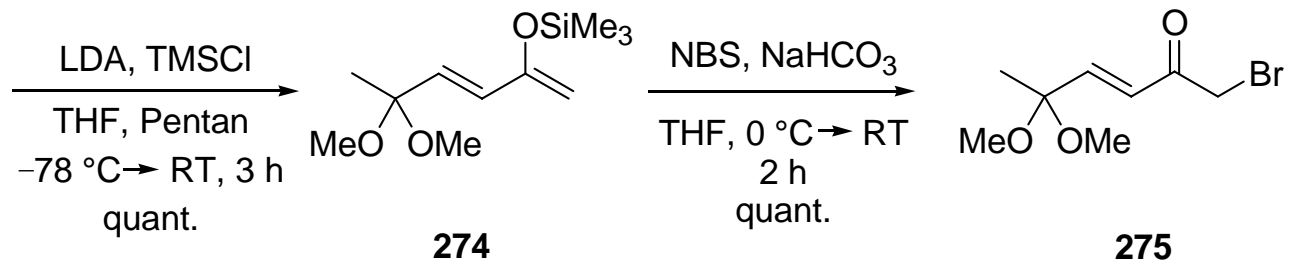

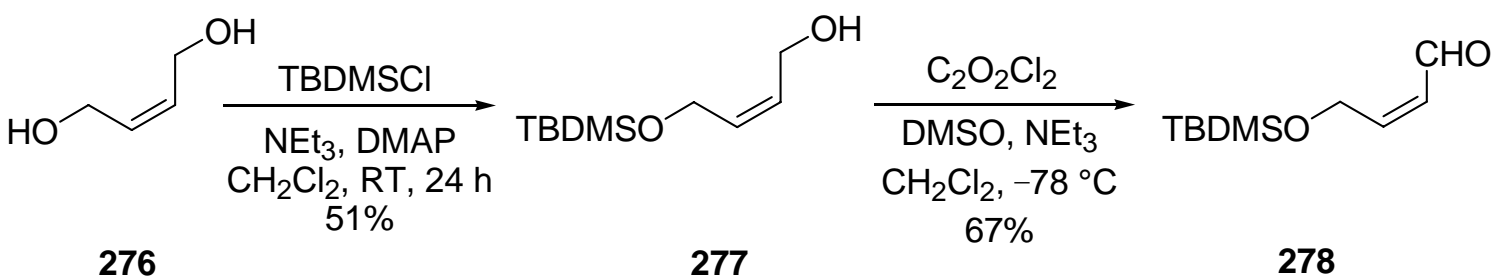

276 277<smiles>CCOC(=O)CP(=O)(OCC)OCC</smiles>

$50 \%$<smiles>CCOC(=O)OCC=CC=CCOS(=O)(=O)OCC</smiles>

279 TBDMSO<smiles>CC=CC=CCC(=O)OCC</smiles>

280

DIBAH, $\mathrm{CH}_{2} \mathrm{Cl}_{2}$ $-78^{\circ} \mathrm{C}, 1 \mathrm{~h}$ 94\%<smiles>O=CCC=CC=CO[AsH3+]</smiles>

281 
Da die Aldol-Reaktionen im Rahmen dieser Arbeit auf drei verschiedenen Wegen untersucht wurden, nämlich unter Verwendung von Basen, Lewissäuren sowie Metallorganylen (vgl. Abschnitt 3, Schema 115), wurden für die Umsetzung mit Hexadienal 281, abhängig von der jeweils angewandten Methode, $(E)-5,5$-Dimethoxyhex-3-en-2-on (273), $(E)$-5,5-Dimethoxy2-trimethylsilyloxyhexa-1,3-dien (274) und (E)-1-Brom-5,5-dimethoxyhex-3-en-2-on (275) als weitere Ausgangssubstanzen benötigt. Die eingesetzten Ausgangsverbindungen wurden gemäß dem im Schema 99 illustrierten Syntheseweg synthetisiert und dargestellt.

\subsection{Synthese der Methylenkomponenten 273, 274 und 275}

Für die Synthese der Verbindung (E)-5,5-Dimethoxy-hex-3-en-2-on (273) musste zunächst als Ausgangssubstanz die Verbindung (E)-Hex-3-en-2,5-dion (272) dargestellt werden (vgl. Schema 100). Das Endion 272 wurde ausgehend von 2,5-Dimethylfuran (271) nach einer Methode zur Furan-Spaltung nach Garcia et al. ${ }^{[110]}$ synthetisiert. Hierzu wurde 1.0 eq Brom mit einer Lösung von Dimethylfuran 271 in einem Aceton/Wasser-Gemisch umgesetzt (vgl. Tabelle 50, Einträge 1-4).

Schema 100. Synthese von E-Hex-3-en-2,5-dion (272) über Furanspaltung mit $\mathrm{Br}_{2}$.

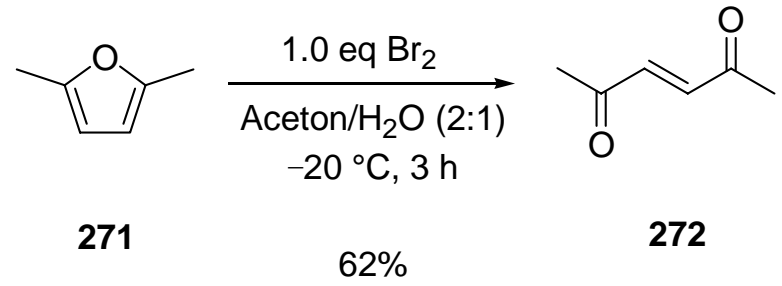

Tabelle 50. Synthese von E-Hex-3-en-2,5-dion (272) über Furanspaltung mit $\mathrm{Br}_{2}$.

\begin{tabular}{ccccc}
\hline Eintrag & $\mathrm{t}[\mathrm{h}]$ & Aufreinigung & Massenbilanz (\%) & Ausbeute (\%) \\
\hline 1 & 3 & $\mathrm{CC}^{\mathrm{a})}$ & 66 & 35 \\
2 & 5 & $\mathrm{UK}^{\mathrm{b})}$ & 72 & 33 \\
3 & 3 & $\mathrm{CF}^{\mathrm{c})}$ & 73 & 62 \\
4 & 6 & - & 78 & - \\
\hline${ }^{\text {a) }}$ Säulenchromatische Reinigung $(\mathrm{CC}) .{ }^{\text {b) }}$ & Umkristallisation (UK) aus $n$ Pentan und \\
EtOAc. ${ }^{c)}$ Reinigung über säulenchromatographische Filtration an $\mathrm{SiO}_{2}(\mathrm{CF})$.
\end{tabular}


Die Reaktionen verliefen in der Regel sehr sauber und wurden daher teilweise ohne weitere Aufreinigung im nächsten Syntheseschritt eingesetzt. Das Produkt fiel dabei häufig als rötlicher Feststoff aus. Dies wurde auf das Vorhandensein von Bromrückständen im Rohprodukt zurückgeführt. Die Behandlung des zunächst dunkelroten Feststoffes 272 mit $\mathrm{Na}_{2} \mathrm{~S}_{2} \mathrm{O}_{3}$ zur Reduktion des Broms zu Bromid erwies sich als effektive Vorreinigung. Die Versuche der säulenchromatographischen Reinigung und der Umkristallisation aus $n$ Pentan und EtOAc waren stets mit massiven Massenverlusten des gewünschten Produktes 272 verbunden (vgl. Tabelle 50). Dies wird aus dem Vergleich der erhaltenen Ausbeute ersichtlich. Am Effektivsten erwies sich die säulenchromatographische Filtration, bei der gelbe Kristalle isoliert werden konnten. Armstrong et al. ${ }^{[111]}$ sowie Conant und Lutz ${ }^{[12]}$ berichteten von dem Erhalt gelber $(E)$ - und weißer $(Z)$-Isomere der Endione als Feststoff. Die entsprechenden ${ }^{1} \mathrm{H}-\mathrm{NMR}$ Spektren zeigten bei 2.35 und 6.74 ppm jeweils ein Singulett für die methyl- und olefinischen Protonen (s. Exp. Teil, Allg. Arbeitsvorschrift B1). Daher konnte die Verbindung 272 offensichtlich isomerenrein gewonnen werden.

Zur Darstellung des (E)-5,5-Dimethoxyhex-3-en-2-ons (273) wurde Hex-3-en-2,5-dion (272) einer $p$-Toluolsulfonsäure-katalysierten Schützung mit Orthoameisensäureester nach Jurczak et al. ${ }^{[113]}$ unterzogen (vgl. Schema 101). Hierzu wurde eine Lösung des Endions 272 und Orthoameisensäureester mit $p$-Toluolsulfonsäure als Katalysator versetzt und entsprechend den in der Tabelle 51 angegebenen Reaktionsbedingungen umgesetzt. Nach Beendigung der Reaktion wurde die $p$-Toluolsulfonsäure durch Zugabe von $\mathrm{NEt}_{3}$ neutralisiert und anschließend aufgearbeitet (s. Exp. Teil, Allg. Arbeitsvorschrift B2).

Schema 101. $p$-Toluolsulfonsäure-katalysierte Synthese von (E)-5,5-Dimethoxyhex-3-en-2on (273) mit Orthoameisensäureester.

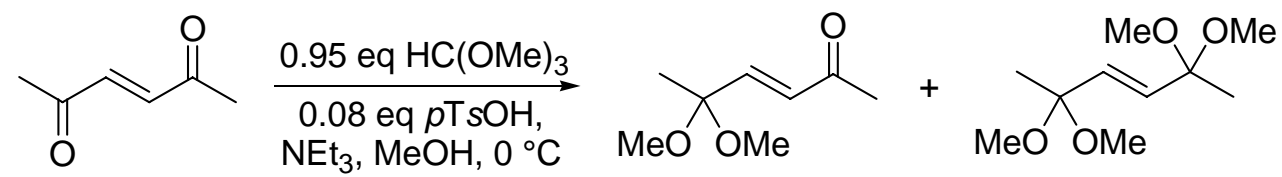


Tabelle 51. $p$-Toluolsulfonsäure-katalysierte Synthese von E-5,5-Dimethoxyhex-3-en-2-on (273) mit Orthoameisensäureester.

\begin{tabular}{ccccccc}
\hline Eintrag & $\begin{array}{c}\mathrm{HC}(\mathrm{OMe})_{3} \\
{[\mathrm{eq}]}\end{array}$ & $\begin{array}{c}p \text {-Toluol- } \\
\text { sulfonsäure[eq] }\end{array}$ & $\begin{array}{c}\mathrm{NEt}_{3} \\
{[\mathrm{eq}]}\end{array}$ & $\begin{array}{c}\mathrm{t} \\
{[\mathrm{h}]}\end{array}$ & $\begin{array}{c}\text { Massenbilanz } \\
\mathbf{2 7 3}(\%)^{\mathrm{c})}\end{array}$ & $\begin{array}{c}\text { Ausbeute } \\
\mathbf{2 7 3}(\%)^{\mathrm{d})}\end{array}$ \\
\hline $1^{\text {a) }}$ & 0.9 & 0.08 & 0.09 & 27 & -6 & - \\
$2^{\text {a) }}$ & 0.9 & 0.085 & 0.12 & 1 & - & - \\
$3^{\text {a) }}$ & 0.9 & 0.17 & 0.12 & 1 & - & - \\
4 & 0.95 & 0.08 & 0.08 & 1 & 100 & 91 \\
5 & 0.95 & 0.08 & 0.08 & 1 & 95 & 90 \\
6 & 1.0 & 0.08 & 0.08 & 1 & 79 & 63 \\
\hline
\end{tabular}

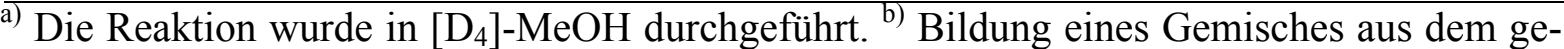
wünschten Produkt 273 und einer Diacetal-Verbindung. ${ }^{c}$ Verhältnis des erhaltenen Rohproduktes und des eingesetzten Eduktes. ${ }^{\text {d) }}$ Ausbeute nach destillativer Reinigung.

Zur Beobachtung des Reaktionsverlaufs wurden analog der oben beschriebenen Arbeitsvorschrift Schützungsversuche im Mikromaßstab im NMR-Röhrchen mit [ $\left.\mathrm{D}_{4}\right]-\mathrm{MeOH}$ als Lösungsmittel durchgeführt (vgl. Tabelle 51, Einträge 1-3). Die Reaktionen wurden zur Optimierung der Reaktionsbedingungen unter geringfügigem Unterschuß an Orthoameisensäureester (0.9 eq) und ansteigenden Mengen von 0.08, 0.085 und 0.17 eq an $p$-Toluolsulfonsäure als Katalysator untersucht. Alle o.g. Schützungsversuche führten zum gewünschten Produkt 273. Während die Umsetzungen 2 und 3 (Einträge 2 u. 3) bereits nach $1 \mathrm{~h}$ in qualitativ sehr sauber verlaufenden Reaktionen zu vollständigen Umsetzungen führten, zeigte die Auswertung des Versuchs 1 (Eintrag 1) nach $27 \mathrm{~h}$ einen vergleichweise hohen Anteil an Verunreinigung an. Die Auswertung des ${ }^{13} \mathrm{C}-\mathrm{NMR}$-Spektrums des letztgenannten Versuchs zeigte die Bildung eines Gemischs aus dem monogeschützten gewünschten Produkt 273 und einem an den beiden Oxo-Gruppen geschützten Molekül 283 an (vgl. Schema 101). Die ${ }^{13} \mathrm{C}-\mathrm{NMR}$ Signale (50.3 MHz, $\mathrm{CDCl}_{3}$ ) bei 23.50, 27.60, 49.07, 99.28, 131.36, 146.25 und 198.48 ppm wurden dem gewünschten Produkt 273 zugeordnet (s. Exp. Teil, Allg. Arbeitsvorschrift B2), die Signale bei 23.49, 48.75, 99.29 und 173.41 ppm wurden als Hinweis auf die Bildung der Diacetal-Verbindung 283 interpretiert. Die weiteren Schützungsversuche (vgl. Tabelle 51, Einträge 4-6) unter katalytischem Einsatz von 0.08 eq $p$-Toluolsulfonsäure und 0.95-1.0 eq Orthoameisensäureester verliefen qualitativ sehr zufriedenstellend und ergaben nach entsprechender destillativer Reinigung akzeptable bis sehr gute Ausbeuten von 63-91\%. Die Bildung der Diacetal-Verbindung $\mathbf{2 8 3}$ blieb bei den in kürzerer Reaktionszeit von $1 \mathrm{~h}$ durchgeführten Versuchen (Einträge 2-6) vollständig aus und wurde daher auf die verlängerte Reaktionszeit der Umsetzung 1 von 27 h (Eintrag 1) zurückgeführt. Die säulenchromatographische Reinigung einer Probe des Rohproduktes 273 (160 mg) führte zur Bildung unbekannter Nebenpro- 
dukte und einem starken Massenverlust des Rohproduktes von bis zu 75\%. Die erhaltenen Rohprodukte wurden daher bevorzugt einer destillativen Reinigung unterzogen. Für die in kleinerem Maßstab zu reinigenden Rohprodukte erwies sich eine Kugelrohrdestillation, für die größeren Ansätze eine Normaldestillation als vorteilhaft.

Die Synthese des Silylenolethers 274 durch Silylierung des Ketoacetals 273 wurde zunächst ausgehend von einer Vorschrift nach Demuth und Fuchs et al. ${ }^{[14]}$ untersucht. Diese berichteten von der O-Silylierung von 4-Oxopent-2-ensäuremethylester mit Chlortrimethylsilan unter Verwendung von $\mathrm{NEt}_{3}$ als Base. Die entsprechend durchgeführte Umsetzung (vgl. Schema 102, Tabelle 52, Eintrag 1) ergab ein Gemisch aus dem eingesetzten Edukt 273 und dem gewünschten Silylenolether 274 im Verhältnis 60:40, so dass nur eine isolierte Ausbeute von $33 \%$ erhalten wurde (s. Exp. Teil, Allg. Arbeitsvorschrift B3, Variante 2).

Schema 102. Synthese des Silylenolethers 274 mit Chlortrimethylsilan und $\mathrm{NEt}_{3}$ als Base.

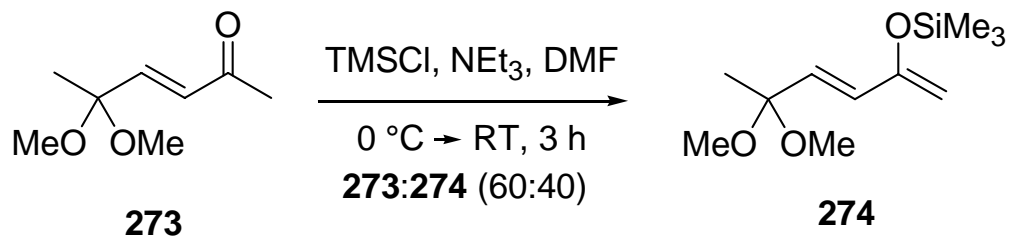

273

1) $\mathrm{TMSCl}_{\mathrm{NEt}}$, $\mathrm{Nal}, \mathrm{MeCN}, \mathrm{O}^{\circ} \mathrm{C}$

2) $\Delta, 20 \mathrm{~h}$

274

273:274 (78:22)

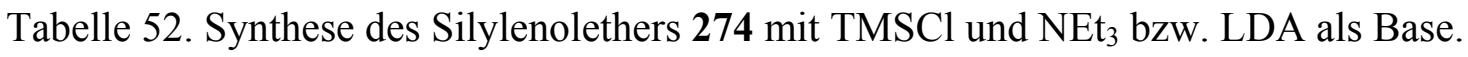

\begin{tabular}{cccccccc}
\hline Eintrag & $\begin{array}{c}\text { TMSCl } \\
{[\mathrm{eq}]}\end{array}$ & $\begin{array}{c}\text { Base } \\
{[\mathrm{eq}]}\end{array}$ & Lsm. & $\begin{array}{c}\mathrm{T} \\
{\left[{ }^{\circ} \mathrm{C}\right]}\end{array}$ & $\begin{array}{c}\mathrm{t} \\
{[\mathrm{h}]}\end{array}$ & $\begin{array}{c}\mathbf{2 7 3 : 2 7 4} \\
(\%)\end{array}$ & $\begin{array}{c}\text { Ausbeute } \\
(\%)\end{array}$ \\
\hline 1 & 1.25 & $\mathrm{NEt}_{3}(1.4)$ & $\mathrm{DMF}$ & $0 \rightarrow \mathrm{RT}$ & 12 & $60: 40$ & 33 \\
2 & 1.6 & $\mathrm{NEt}_{3}(1.65)$ & MeCN & Rückfluß & 20 & $78: 22$ & 42 \\
3 & 1.4 & LDA (1.2) & THF & $-78 \rightarrow \mathrm{RT}$ & 3 & $0: 100$ & quant. \\
4 & 1.4 & LDA (1.2) & THF & $-78 \rightarrow \mathrm{RT}$ & 3 & $0: 100$ & 98 \\
\hline
\end{tabular}


Aufgrund der geringen Ausbeute des Versuchs nach Demuth und Fuchs (vgl. Tabelle 52, Eintrag 1) wurde die Silylierung der Verbindung 273 alternativ nach einer Methode von Reissig et al. ${ }^{[115]}$ untersucht. Diese berichteten, dass das gewünschte Silylierungsprodukt des analogen Benzyloxypenten-2-ons mit $\mathrm{TMSCl}, \mathrm{NEt}_{3}$ und $\mathrm{NaI}$ in Acetonitril unter Rückflußbedingungen in einer Ausbeute von 72\% isoliert werden konnte (vgl. Schema 102). Die zur Ausbeutesteigerung angewandte Methode nach Reissig ergab ein Gemisch aus dem Edukt 273 und dem Silylenolether 274 im Verhältnis von 78:22 (vgl. Tabelle 52, Eintrag 2). Die Umsetzung verlief analog dem Versuch nach Demuth und Fuchs (vgl. Tabelle 52, Eintrag 1) ebenfalls unsauber und unter Bildung unbekannter Nebenprodukte (s. Exp. Teil, Allg. Arbeitsvorschrift B3, Variante 3).

Die Synthese des gewünschten Silylenolethers 274 gelang schließlich unter Verwendung von 1.2 eq LDA als Base in THF (vgl. Schema 103, Tabelle 52, Einträge 3 u. 4). Zur Darstellung der Silylverbindung 274 wurde die Ketoacetal-Verbindung 273 bei $-78{ }^{\circ} \mathrm{C}$ durch Deprotonierung nach Langer ${ }^{[116]}$ in das entsprechende Enolat überführt und anschließend mit TMSCl (1.4 eq) versetzt (s. Exp. Teil, Allg. Arbeitsvorschrift B3, Variante 1). Die auf diese Weise durchgeführten Silylierungsversuche (vgl. Tabelle 52, Einträge 3 u. 4) verliefen qualitativ sehr sauber. Das gewünschte silylierte Produkt 274 wurde nach 3 h bei Raumtemperatur bei der Umsetzung 4 (Eintrag 4) in einer exzellenten Ausbeute von 98\% und bei der Umsetzung 3 (Eintrag 3) sogar in quantitativer Ausbeute erhalten.

Schema 103. Synthese des Silylenolethers 274 mit Chlortrimethylsilan und LDA als Base.

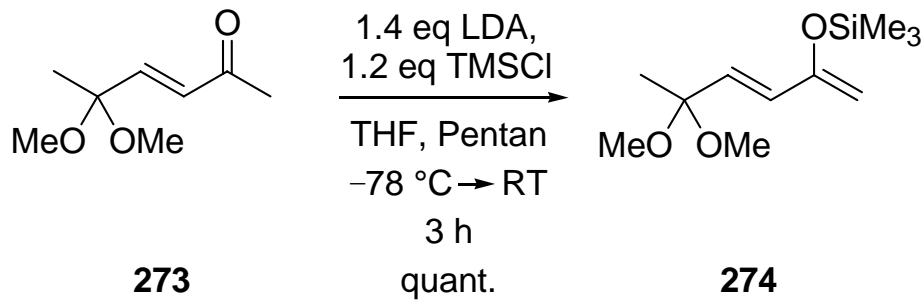

Die Synthese von 1-Brom-5,5-dimethoxyhex-3-en-2-on (275) gelang schließlich sehr erfolgreich über eine Bromierung mit NBS nach Webber et al. ${ }^{[17]}$, die Silylisoindoldione in Gegenwart von NBS und $\mathrm{NaHCO}_{3}$ an der C-1-Position bromierten (Ausbeute 81\%). Die Bromierung im Rahmen der Synthese des $\alpha$-Bromketons 275 wurde demnach mit einer Suspension 
des Silylenolethers 274 und $\mathrm{NaHCO}_{3}$ unter Zugabe von 1.0 eq $N$-Bromsuccinimid (NBS) durchgeführt (vgl. Schema 104). Der Einsatz des $\mathrm{NaHCO}_{3}$ erfolgte zum Abfangen der gebildeten Säurespuren während des Reaktionsablaufs, um eine Abspaltung der Schutzgruppen zu verhindern. Die Bromierungsversuche verliefen komplikationslos und sehr sauber (s. Exp. Teil, Allg. Arbeitsvorschrift B4). Bei den durchgeführten Umsetzungen konnte das gewünschte Bromierungsprodukt 275 stets in quantitativen Ausbeuten erhalten werden

Schema 104. Synthese des $\alpha$-Bromketons 275 über eine Bromierung mit NBS.

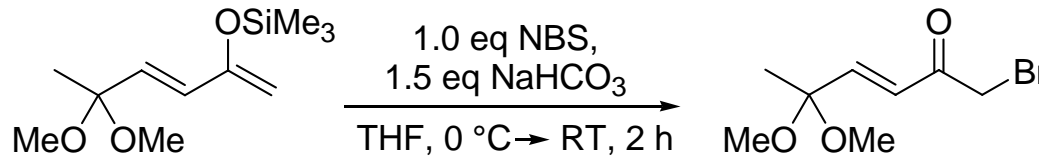

274

quant.

275

\subsection{Synthese des (E,E)-6-(tert-Butyldimethylsilanoxy)hexa-3,5-dienals (281)}

Zur Synthese des Hexadienals $\mathbf{2 8 1}$ als Carbonylkomponente für die geplante AldoladditionsReaktion (vgl. Schema 99) wurde zunächst das kommerziell erhältliche (Z)-But-2-en-1,4-diol (276) zur Synthese der bekannten Verbindung But-2-enol 277 eingesetzt (vgl. Schema 105). Salomon et al. $^{[118]}$ schützten das Endiol 276 (2.0 eq) unter Verwendung von 1.0 eq TBDMSCl, 1.2 eq $\mathrm{NEt}_{3}$ sowie 5 mol\% DMAP und erhielten das monosilylierte Produkt 277 in einer Ausbeute von 70\%. Entsprechend der von Salomon et al. angegebenen Arbeitsvorschrift wurde die Schützung des (Z)-But-2-en-1,4-diols (276) unter Verwendung von TBDMSCl in Gegenwart von DMAP und $\mathrm{NEt}_{3}$ durchgeführt. Die Reaktion verlief sehr sauber und komplikationslos. Das erhaltene Rohprodukt wurde destillativ gereinigt. Der Silylierungsversuch ergab im Gegensatz zu den Literaturangaben nur eine Ausbeute von 51\% (s. Exp. Teil, Allg. Arbeitsvorschrift B5). Da die Reaktionsbedingungen den Vorgaben von Salomon et al. entsprachen, wurde die geringe Ausbeute an erhaltenem Silan mit der im Gegensatz zu ihnen (säulenchromatographische Reinigung) durchgeführten fraktionierenden Destillation erklärt. 
Schema 105. Synthese von (Z)-4-(tert-Butyldimethylsilanyloxy)but-2-en-1-ol (277).<smiles>OC/C=C\CO</smiles>

276

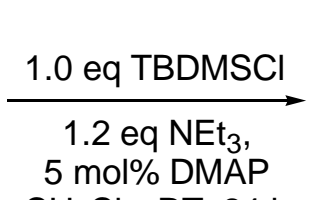

$\mathrm{CH}_{2} \mathrm{Cl}_{2}, \mathrm{RT}, 24 \mathrm{~h}$

$51 \%$<smiles>CCCCOC/C=C\CO</smiles>

277

Als zweiter Schritt zur Synthese des Hexadienals 281 musste das But-2-enol 277 in das (Z)-4(tert-Butyldimethylsilanyloxy)but-2-enal (278) überführt werden (vgl. Schema 99). Der erste Oxidationsversuch des (Z)-4-(tert-Butyldimethylsilanyloxy)but-2-en-1-ols (277) wurde mit Pyridiniumchlorochromat (PCC) nach Ipaktschi et al. ${ }^{[119]}$ durchgeführt (vgl. Schema 106), die diese Oxidation des Alkohols 277 zum entsprechenden Aldehyd bereits beschrieben hatten. Das für die Synthese verwendete Oxidationsmittel PCC wurde auf bekannte Weise ${ }^{[105 a]}$ dargestellt. Zur Synthese des Aldehyds 278 wurde eine Suspension von PCC und Celite mit einer Lösung des Alkohols 277 zur Reaktion gebracht. Der gewünschte Aldehyd 278 konnte in einer sehr sauberen Reaktion in einer Ausbeute von 50\% erhalten werden (s. Exp. Teil, Allg. Arbeitsvorschrift B6, Variante 2).

Schema 106. Synthese des But-2-enals 278 mit PCC.

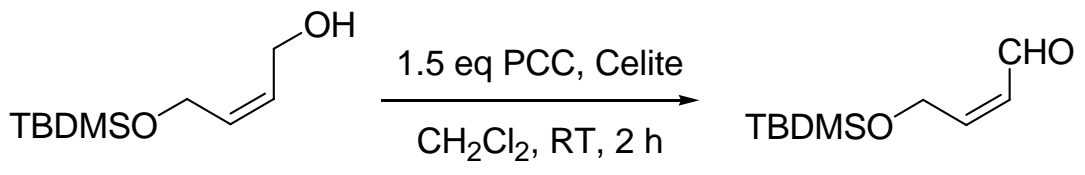

Trotz der sehr sauberen Umsetzung der Oxidationsreaktion mit PCC wurden zur Ausbeutesteigerung des Aldehyds 278 und wegen der hochgiftigen Eigenschaft des Oxidationsmittels weitere Versuche mittels einer Swern-Oxidation nach Uenishi et al. ${ }^{[120]}$ und Suh et al. ${ }^{[121]}$ untersucht. Erstere erzielten eine Ausbeute von 93\% für den Aldehyd 278. Die entsprechenden Swern-Oxidationsreaktionen des Alkohols 277 wurden mit Oxalylchlorid, Dimethylsulfoxid und $\mathrm{NEt}_{3}$ in $\mathrm{CH}_{2} \mathrm{Cl}_{2}$ bei $-78{ }^{\circ} \mathrm{C}$ durchgeführt (vgl. Schema 107), wobei nach entsprechender 
säulenchromatographischer Reinigung eine Ausbeute von 67\% erzielt werden konnte (s. Exp. Teil, Allg. Arbeitsvorschrift B6, Variante 1). Im Vergleich zu den durchgeführten Oxidationsreaktionen mit PCC verliefen die Umsetzungen über die Swern-Oxidation unsauber und ergaben Anteile der Nebenprodukte 284, 285 und 286 (vgl. Schema 107), deren Bildung auf einen Angriff des aktivierten DMSO auf die olefinische Doppelbindung zurückgeführt wurde. Dies erklärt auch die erhaltenen reduzierten Ausbeuten an Aldehyd 278. Dennoch erschien die Darstellung des Aldehyds 278 über eine Swern-Oxidation sowohl wegen der erzielten Ausbeutesteigerung von 50\% auf $67 \%$ als auch wegen der Vermeidung des hochgiftigen PCC als Oxidationsmittel gegenüber den mit PCC durchgeführten Oxidationsreaktionen als vorteilhaft.

Schema 107. Synthese des But-2-enals 278 über eine Swern-Oxidation.
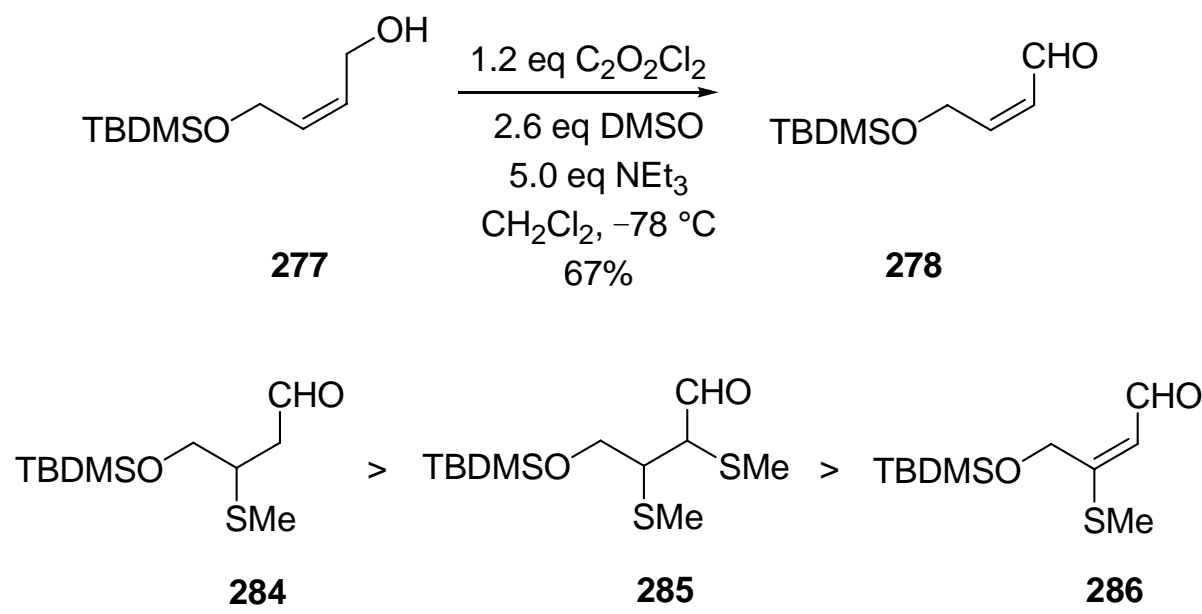

Die Umsetzung des Butenals 278 zu dem 2,4-Hexadiensäureethylester 279 auf dem Weg zur Synthese des Hexadienals 281 (vgl. Schema 99) wurde mit Phosphonessigsäuretriethylester und $\mathrm{NaOH}$ als Base nach Stambouli et al. ${ }^{[122]}$ in einer Horner-Wadsworth-Emmons-Reaktion durchgeführt (vgl. Schema 108). Während in der Literatur $\mathrm{H}_{2} \mathrm{O}$ und $\mathrm{CH}_{2} \mathrm{Cl}_{2}$ als Lösungsmittel verwendet wurden, wurde die Reaktion der Verbindung 278 zur Vermeidung eines wässrigen Mediums in modifizierter Form unter Einsatz von THF ohne Zusatz von $\mathrm{H}_{2} \mathrm{O}$ geplant. Hierzu wurde eine Lösung von Phosphonessigsäuretriethylester (1.0 eq) und dem Aldehyd 278 (1.0 eq) in THF mit einer Suspension von 2.0 eq NaOH in THF umgesetzt (s. Exp. Teil, Allg. Arbeitsvorschrift B7, Variante 2). Die in Gegenwart von $\mathrm{NaOH}$ als Base durchgeführten Umsetzungen (vgl. Tabelle 53, Einträge 1 u. 2) verliefen sehr sauber, ergaben jedoch nach entspre- 
chenden Reinigungsversuchen lediglich mäßige Ausbeuten von 35\% und 39\% (Lit. $^{[122]}$ für die Umsetzung in wässriger Phase: 54\%).

Schema 108. Umsetzung des Aldehyds 278 zu dem Ester 279 in einer Horner-WadsworthEmmons-Reaktion mit Phosphonessigsäuretriethylester und $\mathrm{NaOH}$ als Base.<smiles>CCCCOS(=O)OC/C=C\C=O</smiles>

278

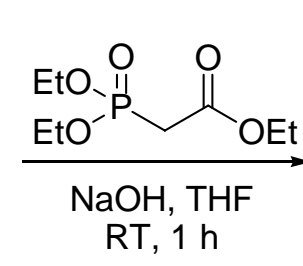

$39 \%$<smiles>CCOC(=O)/C=C/C=C\COS(C)(C)CC</smiles>

279

Tabelle 53. Umsetzung des Aldehyds 278 zu dem Ester 279 in einer Horner-WadsworthEmmons-Reaktion mit Phosphonessigsäuretriethylester.

\begin{tabular}{|c|c|c|c|c|c|c|}
\hline Eintrag & Base & $\mathrm{T}\left[{ }^{\circ} \mathrm{C}\right]$ & $\mathrm{t}[\mathrm{min}]$ & Massenbilanz (\%) & Isolierung & Ausbeute $(\%)$ \\
\hline 1 & $\mathrm{NaOH}$ & RT & 60 & 95 & $\mathrm{FD}^{\mathrm{b})}$ & 35 \\
\hline 2 & $\mathrm{NaOH}$ & RT & 60 & quant. & $\mathrm{CC}^{\mathrm{c})}$ & 39 \\
\hline 3 & $\mathrm{NaH}$ & $-20 \rightarrow \mathrm{RT}$ & 60 & quant. & $\mathrm{CC}^{\mathrm{c})}$ & 50 \\
\hline 4 & $\mathrm{NaH}$ & $-20 \rightarrow \mathrm{RT}$ & 60 & quant. & $\mathrm{CC}^{\mathrm{c})}$ & 45 \\
\hline
\end{tabular}

Zur Optimierung und Ausbeutesteigerung wurden weitere Versuche zur Olefinierung des Aldehyds 278 nach Marquet et al. ${ }^{[123]}$ durchgeführt. Marquet et al. berichteten von der Durchführung der Horner-Wadsworth-Emmons-Reaktion unter Verwendung von Phosphonessigsäuretriethylester und $\mathrm{NaH}$ als Base. Für die entsprechend durchgeführten Umsetzungen (vgl. Schema 109, Tabelle 53, Einträge 3, 4) wurden 1.5 eq Phosphonessigsäuretriethylester und 1.5 eq NaH als Base eingesetzt (s. Exp. Teil, Allg. Arbeitsvorschrift B7, Variante 1). Diese Umsetzungen lieferten nach Aufreinigung Ausbeuten von 50\% und 45\% (vgl. Tabelle 53, Einträge 3 u. 4). Die Reaktion 3 (Eintrag 3) lieferte zudem neben dem gewünschten Silyldienester 279 (Anteil 96\%) das im Rahmen des nächsten Syntheseschrittes angestrebte Isomerisierungsprodukt 280 (vgl. Schema 110) in einem Anteil von 6\%. 
Schema 109. Umsetzung des Aldehyds 278 zu dem Ester 279 in einer Horner-WadsworthEmmons-Reaktion mit Phosphonessigsäuretriethylester und $\mathrm{NaH}$ als Base.

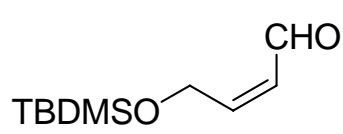

278

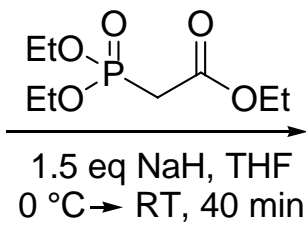

$50 \%$<smiles>CCOC(=O)/C=C/C=C\COS(C)(=O)=O</smiles>

279

Die Bildung des Isomerisierungsproduktes 280 wurde auf die Deprotonierung des allylischen Protons der gebildeten Esterverbindung 279 während des Reaktionsverlaufs durch die Base $\mathrm{NaH}$ und die anschließende Reprotonierung der Verbindung zurückgeführt, bei der das $(E, E)-$ Isomer 280 gebildet werden konnte. Die Isomerisierung könnte auf dem Überschuß von 0.5 eq an $\mathrm{NaH}$ oder auf dem geringeren Gehalt an Phosphonessigsäuretriethylester beruhen. Auch der geringere Gehalt an Phosphonessigsäuretriethylester in dem Reaktionsmedium würde das Vorliegen unverbrauchten $\mathrm{NaH}$ bedingen, welches die Isomerisierung des Diens 279 initialisiert haben könnte, denn bei einem ausreichenden Gehalt an Phosphonessigsäuretriethylester würde die Deprotonierung an dessen methylenaktivem Proton durch $\mathrm{NaH}$ aufgrund der starken Acidität der methylenaktiven Position leichter stattfinden als der Angriff des $\mathrm{NaH}$ auf die allylischen Protonen des Diens 279.

Als nächster Schritt der Synthese des Hexadienals 281 wurde die geplante intramolekulare Doppelbindungsisomerisierung des konjugierten Esters 279 zum entsprechenden Hexadiensäureester 280 durchgeführt. Shea et al. ${ }^{[124]}$ führten die Dekonjugierung verwandter Hexadiencarbonsäuren unter Verwendung von LDA durch. Zur geplanten Isomerisierung wurde eine Lösung des Dienesters 279 in THF nach Shea et al. bei $-78{ }^{\circ} \mathrm{C}$ mit einer Lösung von 1.2 eq LDA umgesetzt (vgl. Schema 110; Tabelle 54, Eintrag 1). Die Reaktion wurde bei $-78{ }^{\circ} \mathrm{C}$ mit einer 10\%-igen HOAc-Lösung hydrolysiert und nach Erwärmung auf Raumtemperatur mit ges. $\mathrm{NH}_{4} \mathrm{Cl}$-Lösung und anschließend mit ges. NaCl-Lösung aufgearbeitet. Die Auswertung der ${ }^{1} \mathrm{H}$-NMR- und ${ }^{13} \mathrm{C}$-NMR-Spektren des Versuchs belegte eine vollständige Rückgewinnung des Eduktes 279. 
Schema 110. Basische Isomerisierung des Hexadiensäureesters 279 zu dem Hexadiensäureester $\mathbf{2 8 0}$ mit LDA.<smiles>CCOC(=O)/C=C/C=C\COS(C)(C)C</smiles>

279

1) 1.2 eq LDA, THF,

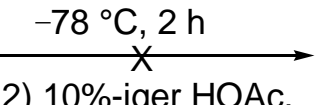
$-78{ }^{\circ} \mathrm{C}$<smiles>CCOC(=O)CC=CC=COS(=O)(=O)[18O]CC</smiles>

280

Tabelle 54. Isomerisierung des Hexadiensäureesters 279 zum Hexadiensäureester 280.

\begin{tabular}{ccccc}
\hline Eintrag & DMPU $[\mathrm{eq}]$ & $\mathrm{t}[\mathrm{h}]$ & $\mathbf{2 7 9 : 2 8 0}(\%)$ & Ausbeute an $\mathbf{2 8 0}(\%)^{\mathrm{a})}$ \\
\hline 1 & - & 2 & $100: 0$ & 0 \\
2 & 2.5 & 3 & $40: 60^{\mathrm{b})}$ & 53 \\
3 & 5.0 & 3 & $41: 59^{\mathrm{b})}$ & 50 \\
4 & 2.0 & 3 & $0: 100$ & 93 \\
\hline
\end{tabular}

a) Ausbeute nach säulenchromatographischer Reinigung. ${ }^{\text {b) }}$ Es wurde zusätzlich eine geringe Menge einer unbekannten Verunreinigung erhalten.

Shea et al. ${ }^{[125]}$ berichteten desweiteren von der erfolgreichen Isomerisierung der analogen Hexadiensäuren unter Verwendung von LDA in Gegenwart von DMPU. Die Doppelbindungsumlagerung des Hexadiensäureesters $279 \mathrm{zu}$ dem gewünschten Isomerisierungsprodukt 280 gelang unter Reproduzierung und Optimierung der Reaktionsbedingungen nach Shea et al. mit DMPU sehr erfolgreich (vgl. Schema 111).

Schema 111. Basische Isomerisierung des Diensäureesters $279 \mathrm{zu}$ dem Hexadiensäureester 280 mit LDA und DMPU.<smiles>CCOC(=O)/C=C\C=C/COS(C)(=O)=O</smiles>

279

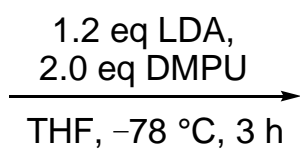

$93 \%$<smiles>[3H][SbH]([3H])([3H])OC=CC=CCC(=O)OCC</smiles>

280 
Hierzu wurde LDA in Gegenwart von DMPU in den in der Tabelle 54 angegebenen Mengen generiert und bei $-78{ }^{\circ} \mathrm{C}$ mit einer Lösung des Esters 279 in THF versetzt (vgl. Tabelle 54, Einträge 2-4). Die Reaktionen wurden mit 10\%-iger HCl-Lösung beendet (s. Exp. Teil, Allg. Arbeitsvorschrift B8). Der Isomerisierungsversuch unter Verwendung von 2.5 eq DMPU (Eintrag 2) lieferte ein Gemisch aus dem gewünschten Produkt 280 (Anteil 60\%), dem unumgesetzten Edukt 279 (Anteil 40\%) und einer geringen Menge einer unbekannten Verunreinigung. Der als Referenzreaktion in Gegenwart von 5.0 eq an DMPU durchgeführte Doppelbindungsumlagerungsversuch (Eintrag 3) führte zur Reproduktion der bei Versuch 2 (Eintrag 2) erzielten Ergebnisse. Die annähernd gleichen Ergebnisse dieser beiden Umsetzungen sind auf die Tatsache zurückzuführen, dass scheinbar der erhöhte Überschuß an DMPU (Erhöhung von 2.5 auf 5.0 eq) keinen merklichen Beitrag zur vollständigen Umsetzung der Isomerisierung erbringen konnte. Die geringere Ausbeute der Doppelbindungsumlagerungsversuche $2 \mathrm{u}$. 3 (Einträge 2 u. 3) dürfte neben der unvollständigen Umsetzung auf die abgewandelte Aufarbeitung der Reaktionslösung mit einem Gemisch aus Hexan $/ \mathrm{H}_{2} \mathrm{O}$ zurückzuführen sein. Zur Entfernung der Base (DMPU ist stark wasserlöslich) aus dem Reaktionsmedium wurden die erhaltenen Rohprodukte zusätzlich mit $\mathrm{H}_{2} \mathrm{O}$ (2 Mal) aufgearbeitet. Zur Ausbeutesteigerung wurde ein Isomerisierungsversuch unter Einsatz von 2.0 eq DMPU durchgeführt (Eintrag 4). Zudem wurde im Gegensatz zu den Versuchen 2 und 3 keine zusätzliche Aufarbeitung mit $\mathrm{H}_{2} \mathrm{O}$ zur Entfernung des DMPU durchgeführt. Bei diesem Versuch konnte das gewünschte Isomerisierungsprodukt $\mathbf{2 8 0}$ mit einer exzellenten Ausbeute von 93\% erfolgreich isoliert werden.

Das als Ausgangssubstanz der geplanten Aldolreaktion erforderliche Hexadienal 281 sollte schließlich aus dem Hexadiensäureester 280 dargestellt werden (vgl. Schema 99). Baker et al. ${ }^{[126]}$ berichteten über die Reduktion des (2S)-2-[(tert-Butyldimethylsilanyl)oxy]propionsäuremethylesters zu dem entsprechenden Aldehyd unter Verwendung von DIBAH. Die Reduktion des Hexadiensäureesters 280 nach Baker zum gewünschten Dienal 281 wurde entsprechend der Angaben des Schemas 112 und den in der Tabelle 55 angegebenen Reaktionsbedingungen untersucht. Das gewünschte Produkt 281 blieb bei dem durchgeführten Reduktionsversuch in Hexan vollständig aus (vgl. Schema 112, Tabelle 55, Eintrag 1). Ebenfalls scheiterte die Reduktion des Esters 280 bei dem nach Otera et al. ${ }^{[127]}$ unter Verwendung von Toluol als Lösungsmittel durchgeführten Versuch (vgl. Schema 112; Tabelle 55, Eintrag 2). Bei den vorgenannten Versuchen wurde der eingesetzte Ester $\mathbf{2 8 0}$ jeweils vollständig zurückerhalten. 
Schema 112. Reduktion des Hexadiensäureesters 280 zu dem Hexadienal 281 mit DIBAH.

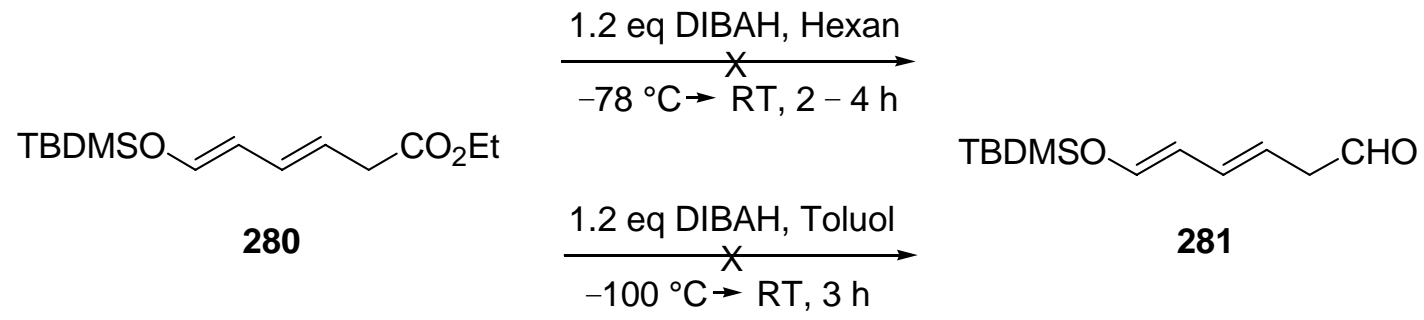

Tabelle 55. Reduktion des Hexadiensäureesters 280 zu dem Hexadienal 281 mit DIBAH.

\begin{tabular}{|c|c|c|c|c|c|c|c|c|}
\hline Eintrag & $\begin{array}{c}\text { DIBAH } \\
\text { [eq] }\end{array}$ & Hydrolyse & [eq] & Lsm. & $\begin{array}{c}\mathrm{T} \\
{\left[{ }^{\circ} \mathrm{C}\right]}\end{array}$ & $\begin{array}{c}\mathrm{t} \\
{[\mathrm{min}]}\end{array}$ & $\begin{array}{c}280: 281 \\
(\%)\end{array}$ & $\begin{array}{c}\text { Ausbeute } \\
(\%)\end{array}$ \\
\hline \multirow[t]{2}{*}{1} & 1.2 & NaCl-Lsg. & - & Hexan & -78 & 120 & & \\
\hline & & & & & RT & 240 & 100:0 & - \\
\hline 2 & 1.0 & NaCl-Lsg. & - & Toluol & -100 & 180 & 100:0 & - \\
\hline 3 & 1.0 & $\mathrm{CH}_{3} \mathrm{COOH}$ & 1.0 & $\mathrm{CH}_{2} \mathrm{Cl}_{2}$ & -78 & 60 & $40: 60$ & - \\
\hline 4 & 1.05 & $\mathrm{CH}_{3} \mathrm{COOH}$ & 1.05 & $\mathrm{CH}_{2} \mathrm{Cl}_{2}$ & -78 & 60 & $0: 100$ & 70 \\
\hline 5 & 1.2 & $\mathrm{CH}_{3} \mathrm{COOH}$ & 1.2 & $\mathrm{CH}_{2} \mathrm{Cl}_{2}$ & -78 & 60 & $0: 100$ & 66 \\
\hline 6 & 1.6 & $\mathrm{CH}_{3} \mathrm{COOH}$ & 1.6 & $\mathrm{CH}_{2} \mathrm{Cl}_{2}$ & -78 & 60 & $0: 100$ & 61 \\
\hline \multirow[t]{3}{*}{7} & 1.0 & Weinsäure & 2.0 & $\mathrm{CH}_{2} \mathrm{Cl}_{2}$ & $-78 \rightarrow$ & 60 & & \\
\hline & & & & $/ \mathrm{EtOH}$ & $-30 \rightarrow$ & 120 & & \\
\hline & & & & & RT & 30 & $28: 72$ & - \\
\hline \multirow[t]{3}{*}{8} & 1.05 & Weinsäure & 2.1 & $\mathrm{CH}_{2} \mathrm{Cl}_{2}$ & $-78 \rightarrow$ & 60 & & \\
\hline & & & & $/ \mathrm{EtOH}$ & -30 & 10 & & \\
\hline & & & & & $\mathrm{RT}$ & 5 & $0: 100$ & 94 \\
\hline
\end{tabular}

Shing et al. ${ }^{[128]}$ berichteten von der Überführung eines Hexadiensäureesters in das entsprechende Hexadienal über die Reduktion der Verbindung mit DIBAH und die anschließende Hydrolyse der Reaktionslösung mit konz. Essigsäure. Im Rahmen von Referenz- und Optimierungsversuchen wurde die Reduktion des Esters $\mathbf{2 8 0}$ mit 1.0, 1.05, 1.2 und 1.6 eq an DIBAH durchgeführt (vgl. Schema 113; Tabelle 55, Einträge 3-6). Analog der eingesetzten DIBAH-Mengen wurden die Reaktionslösungen bei $-78{ }^{\circ} \mathrm{C}$ mit jeweils $1.0,1.05,1.2$ bzw. 1.6 eq an konz. $\mathrm{CH}_{3} \mathrm{COOH}$ hydrolysiert und beendet (s. Exp. Teil, Allg. Arbeitsvorschrift B9, Variante 2). Während die Umsetzung unter Verwendung von 1.0 eq DIBAH (Eintrag 3) ein Gemisch aus dem eingesetzten Edukt 280 und dem Aldehyd 281 im Verhältnis 40:60 lieferte, führten die Reduktionsversuche unter dem Einsatz von 1.05, 1.2 bzw. 1.6 eq des Reduktionsmittels (Einträge 4-6) bei vollständiger Umsetzung (100\%) zur Bildung des gewünschten Dienals 281 in isolierten Ausbeuten von 61\%, 66\% und 70\%. 
Schema 113. Reduktion des Hexadiensäureesters 280 zu dem Hexadienal 281 mit DIBAH.

1) 1.05 eq DIBAH,

TBDMSO $\mathrm{CO}_{2} \mathrm{Et} \underset{\text { 2) } 1.05 \mathrm{eq} \mathrm{CH}_{3} \mathrm{COOH}}{\stackrel{\mathrm{CH}_{2} \mathrm{Cl}_{2},-78^{\circ} \mathrm{C}, 1 \mathrm{~h}}{\longrightarrow}}$

280
$70 \%$
TBDMSO手CHO

281

Zum Zwecke der Ausbeutesteigerung wurde die Reduktion des Esters 280 unter Verwendung von Weinsäure zur Hydrolyse nach Viala et al. ${ }^{[129]}$ untersucht. Viala et al. berichteten von der Reduktion des (Z)-6,6-Dimethoxy-3-hexensäuremethylesters zu dem entsprechenden 3Hexenal unter Verwendung von DIBAH und der Hydrolyse mit Weinsäure in einer Ausbeute von 95\%. Die Vorgaben von Viala wurden bei den Reduktionsversuchen unter dem Einsatz von Weinsäure (vgl. Tabelle 55, Einträge 7 u. 8) analog angewandt. Hierzu wurde zunächst analog der im Schema 114 dargestellten Variante eine Lösung des Hexadiensäureesters 280 in $\mathrm{CH}_{2} \mathrm{Cl}_{2}$ bei $-78{ }^{\circ} \mathrm{C}$ mit DIBAH versetzt. Die Reaktion wurde mit einer ges. WeinsäureLösung bei $-78{ }^{\circ} \mathrm{C}$ beendet. Zur Reaktionsmischung wurde nach deren Erwärmung auf -30 ${ }^{\circ} \mathrm{C}$ eine Gemisch aus $\mathrm{Na}_{2} \mathrm{SO}_{4}$ und Celite (Verhältnis 1:1) gegeben, die Lösung auf Raumtemperatur erwärmt und aufgearbeitet (s. Exp. Teil, Allg. Arbeitsvorschrift B9, Variante 1). Der Versuch unter Verwendung von 1.0 eq DIBAH und 2.0 eq Weinsäure lieferte ein Gemisch aus dem Edukt 280 und dem Produkt 281 im Verhältnis 28:72 (vgl. Tabelle 55, Eintrag 7).

Schema 114. Reduktion des Hexadiensäureesters 280 zu dem Hexadienal 281 mit DIBAH.

1) $280,1.05$ eq DIBAH

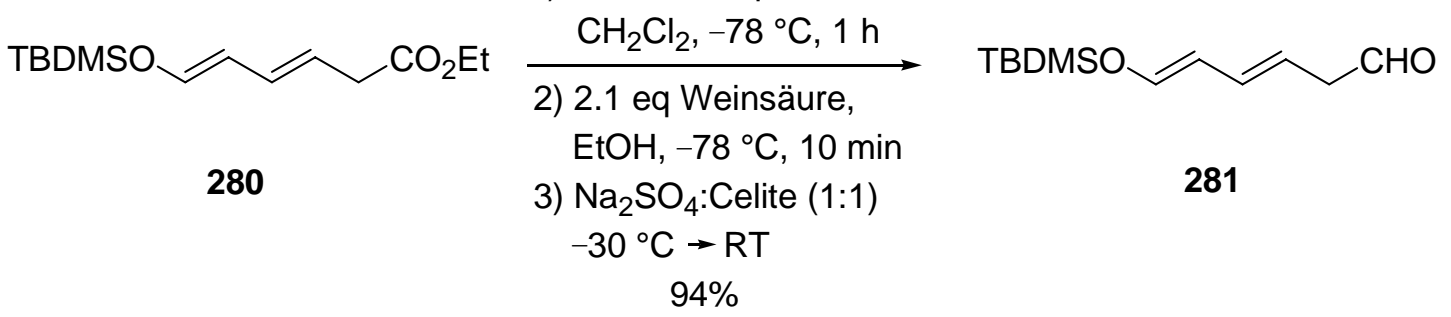

Zur Optimierung der Reaktionsbedingungen und Erzielung besserer Umsätze wurde ein weiterer Versuch (Tabelle 55, Eintrag 8) unter geringfügigem Überschuß an DIBAH (1.05 eq) und Weinsäure (2.1 eq) durchgeführt (vgl. Schema 114, Exp. Teil, Allg. Arbeitsvorschrift B9, Variante 1). Hierbei konnte der Silylester 280 unter vollständiger Umsetzung in sehr exzellen- 
ter Ausbeute von 94\% in das gewünschte Dienal 281 überführt werden. Obwohl der Silylester 280 auch unter Protolyten-Beigabe von $\mathrm{CH}_{3} \mathrm{COOH}$ unter vollständiger Umsetzung zum Dienaldehyd 281 umfunktionalisiert werden konnte, ist der Durchführung der Reaktion mit Weinsäure aufgrund der erzielten deutlich höheren Ausbeuten der Vorzug einzuräumen.

\section{C-C-Kupplungsversuche zur Darstellung des Aldoladditionsproduktes}

Die geplante C-C-Verknüpfung der Fragmente zum Aldoladditionsprodukt 282 als Edukt der intramolekularen Diels-Alder-Reaktion zur Synthese des Collinolactons wurde unter Verwendung von Basen und Lewissäuren sowie als Additionsreaktion eines Metallorganyls an die Carbonylgruppe des Aldehyds untersucht. Hierbei fanden einerseits das Hexadienal 281 sowie andererseits in Abhängigkeit von den jeweiligen Reaktionsbedingungen das Hexenon 273, das $\alpha$-Bromketon 275 sowie der Trimethylsilylenolether 274 Verwendung (vgl. Schema 115). Im Rahmen von Optimierungs- bzw. Vorversuchen wurden anstelle des Hexadienals 281 auch diverse kommerziell erhältliche Aldehyde als Modellsubstrate getestet.

Schema 115. C-C-Kupplung der Kettenfragmente zu dem Aldoladditionsprodukt 282.
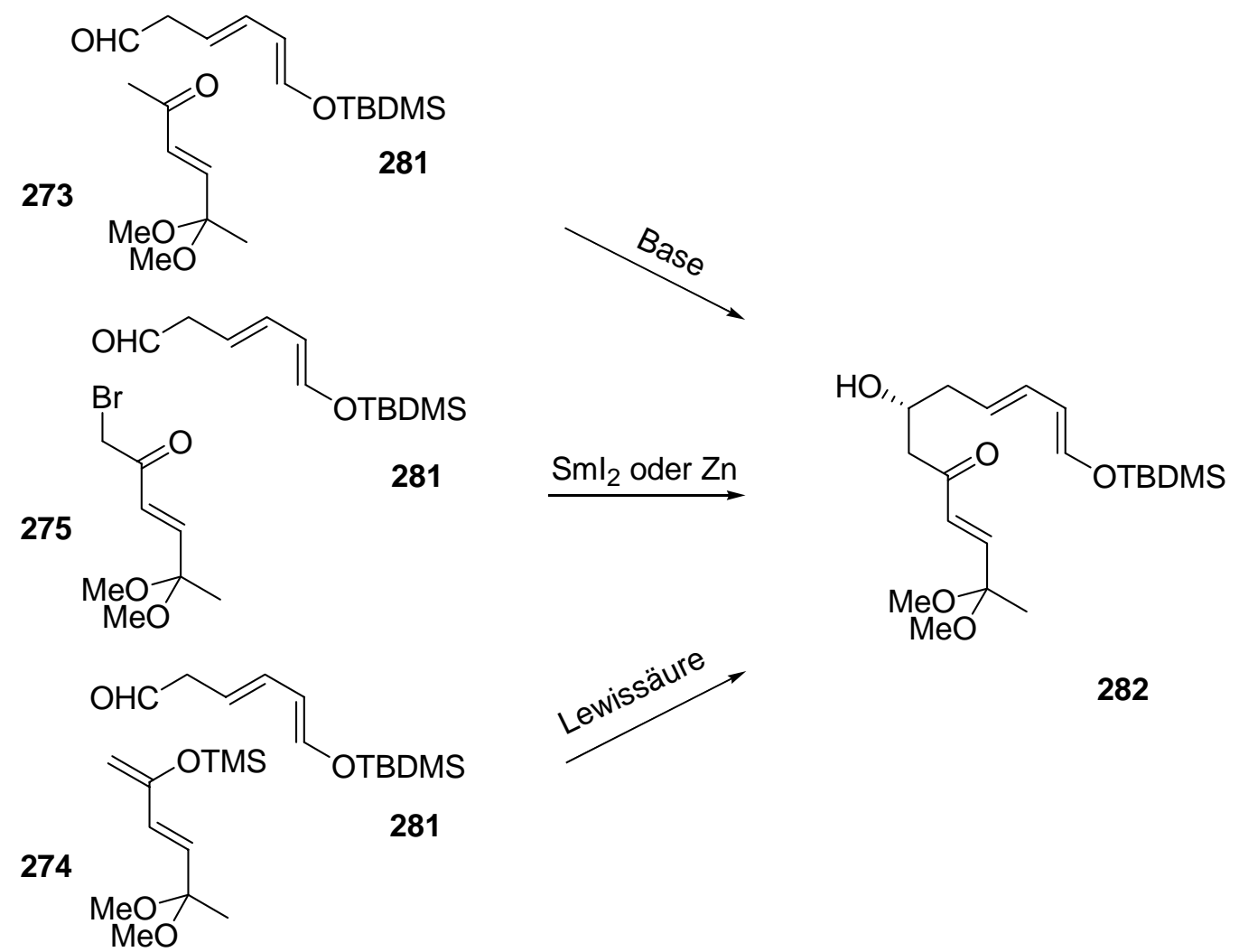

282 


\subsection{Versuche zur Aldoladdition unter basischen Bedingungen}

Shing et al. ${ }^{[128]}$ beschrieben die Synthese von Tricyclen über eine stereoselektive AldolReaktion und eine anschließende intramolekulare Diels-Alder-Reaktion (IMDA). Die Aldolreaktion wurde mit einem $\beta, \gamma$-ungesättigten Aldehyd und einem aus Methylcarvon unter kinetischer Kontrolle generierten $(E)$-Enolat durchgeführt. Shing et al. gelang die Darstellung des Aldolprodukts durch Deprotonierung des Methylcarvons unter Verwendung von 1.6 eq LDA in THF bei $-78{ }^{\circ} \mathrm{C}$ in Gegenwart von DMPU (1.6 eq bezogen auf das Keton). Die Zugabe des Dienaldehyds bei $-100{ }^{\circ} \mathrm{C}$ und die anschließende Aufarbeitung lieferte das anti-Aldolprodukt (Ausbeute 60\%). Shing et al. berichteten, dass das anti-Aldolprodukt durch einen Angriff des Aldehyds auf die sterisch geringer gehinderter Seite des $(E)$-Enolats entsteht. Diese stereochemische Induktion begründeten sie damit, dass die $(E)$-Enolate generell mit achiralen Aldehyden zu anti-Aldolprodukten reagieren. ${ }^{[128]}$ Als Nebenprodukt erhielten Shing et al. zudem eine geringe Menge des Diels-Alder-Produkts. Die anschließende IMDA-Reaktion führten sie unter Erwärmung durch und erhielten das tricyclische Produkt in einer Ausbeute von 80\%.

Schema 116. Aldolreaktion des Ketals 273 mit dem Dienaldehyd 281 zu dem Produkt 282.

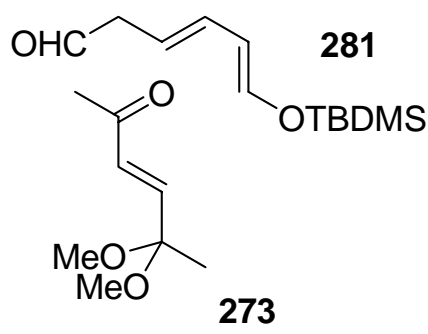

1) $\mathrm{HN}(\text { isoPr })_{2}, n \mathrm{BuLi}$

$\frac{-78^{\circ} \mathrm{C}, \mathrm{THF}, 10 \mathrm{~min}}{\text { 2) (DMPU), 273, THF, } 1 \mathrm{~h}}$

3) $281,-78^{\circ} \mathrm{C}, 5 \mathrm{~min}$

282:273 (1.7:1)

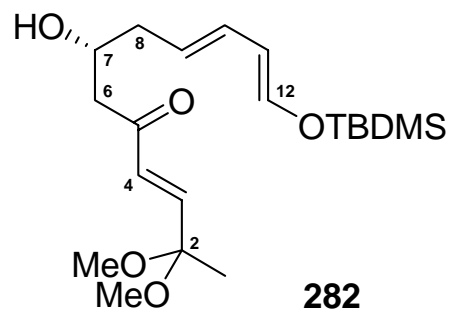

Aufgrund der von Shing et al. berichteten positiven Ergebnisse wurde die geplante Aldoladditionsreaktion unter den in der Tabelle 56 angegebenen Bedingungen zunächst bei $-100{ }^{\circ} \mathrm{C}$ untersucht. Zur Generierung des hierzu benötigten $(E)$-Enolats wurde das Ketal 273 mit 1.2 eq LDA in Gegenwart von DMPU bei $-100{ }^{\circ} \mathrm{C}$ deprotoniert. Dann wurde der Aldehyd zugegeben und nach 5 min mit ges. $\mathrm{NH}_{4}$ Cl-Lösung hydrolysiert. Die Auswertung des komplexen Rohprodukts dieses Versuchs (vgl. Tabelle 56, Eintrag 1) ließ keine sichere Bestätigung der Bildung des Aldolproduktes 282 zu (vgl. Schema 116). Durch das Fehlen des Aldehydsignals bei 9.66 ppm wurde eine vollständige Umsetzung des Aldehyds 281 festgestellt. Die Protonensignale eines AB-Systems bei 2.17 und 2.38 ppm wurden der Position 8 und diejenigen 
bei 2.63-2.80 ppm der neugebildeten Position 6 des Aldolprodukts 282 zugeordnet. Hiernach ließ sich der Erhalt eines Gemisches aus dem Edukt 273 und dem Aldolprodukt 282 in einem Verhältnis von 3.5:1 ermitteln. Bei dem Versuch zur säulenchromatographischen Reinigung trat jedoch Zersetzung ein.

Tabelle 56. C-C-Kupplung der Kettenfragmente Ketoacetal 273 und Dienaldehyd 281 zu dem Aldoladditionsprodukt 282 unter Verwendung von Basen.

\begin{tabular}{ccccccccc}
\hline Eintrag & $\begin{array}{c}\mathbf{2 8 1} \\
{[\mathrm{eq}]}\end{array}$ & $\begin{array}{c}\mathbf{2 7 3} \\
{[\mathrm{eq}]}\end{array}$ & $\begin{array}{c}\text { DMPU } \\
{[\mathrm{eq}]}\end{array}$ & $\begin{array}{c}\text { LDA } \\
{[\mathrm{eq}]}\end{array}$ & Lsm & $\begin{array}{c}\mathrm{T} \\
{\left[{ }^{\circ} \mathrm{C}\right]}\end{array}$ & $\begin{array}{c}\mathrm{t} \\
{[\mathrm{min}]}\end{array}$ & $\mathbf{2 8 2 : 2 7 3}$ \\
\hline 1 & 1.0 & 1.0 & 1.6 & 1.2 & THF & -100 & 5 & $1: 3.5$ \\
2 & 1.0 & 1.0 & 1.6 & 1.2 & THF & -78 & 5 & $1: 2.3$ \\
3 & 1.0 & 1.5 & 2.5 & 1.65 & THF & -78 & 5 & $1.7: 1$ \\
4 & 1.0 & 1.2 & - & 1.3 & Hexan & -78 & 60 & $1.5: 1$ \\
\hline
\end{tabular}

Ein zweiter Versuch wurde bei $-78^{\circ} \mathrm{C}$ unter im übrigen gleichen stöchiometrischen Einsätzen wie bei Versuch 1 durchgeführt (vgl. Tabelle 56, Eintrag 2). Bei dem dritten Versuch wurden hingegen 1.0 eq Aldehyd 281, 1.5 eq Ketal 273, 2.5 eq DMPU und 1.65 eq LDA bei $-78^{\circ} \mathrm{C}$ eingesetzt (vgl. Tabelle 56, Eintrag 3). Während die Umsetzung 2 das Aldolprodukt 282 in einem Anteil von 30\% ergab (Eintrag 2), konnte der Anteil des gewünschten Aldolprodukts 282 bei dem Versuch mit einem Überschuss an Enolat des Ketons 273 auf 63\% gesteigert werden (Eintrag 3). Signale im ${ }^{13}$ C-NMR-Spektrum des Rohproduktes bei 48.57 und 67.48 ppm wurden den C-8- und C-7-Atomen des Aldolproduktes 282 zugeordnet.

Ito et al. ${ }^{[130]}$ berichteten von der Durchführung einer Aldol-Additionsreaktion von Aceton mit einem Aldehyd entsprechend der in der Tabelle 56 angegebenen Bedingungen, aber unter Verzicht auf die Beigabe von DMPU als Lithium-Chelator. Im Rahmen dieser Arbeit wurde nach Ito et al. LDA in Abwesenheit von DMPU bei $-78^{\circ} \mathrm{C}$ mit dem Acetal 273 und anschließend mit dem Dienal 281 umgesetzt. Dieser in Hexan durchgeführte Versuch (vgl. Tabelle 56, Eintrag 4) führte zur Bildung eines Gemisches aus dem eingesetzten Edukt 273 und dem Aldolprodukt 282 als Hauptprodukt (Anteil 60\%) im Verhältnis von 1.5:1. Dieses Ergebnis blieb nur geringfügig unter dem erzielten Resultat des Versuchs 3 (Eintrag 3), bei dem das Aldolprodukt 282 in einem Anteil von 63\% gebildet wurde. Auch bei dem Versuch 4 führte die entsprechende Aufreinigung des Rohproduktes zur Rückgewinnung des Eduktes 273. Insgesamt erwies sich die Aufreinigung neben der unvollständigen Umsetzung als eines der Haupt- 
probleme bei den basenvermittelten Aldol-Additionsreaktionen. Obwohl die SpektrenAuswertung der Rohprodukte Hinweise auf die Bildung des gewünschten Aldolproduktes lieferte, scheiterten alle durchgeführten Reinigungsversuche in Form von säulenchromatographischer und destillativer Reinigung sowie Kugelrohr-Destillation, da sich das erhaltene Rohprodukt stets größtenteils oder sogar vollständig zersetzte. Das Scheitern der Isolierung des gewünschten Aldoladditionsproduktes 282 erschwerte den Nachweis für dessen Bildung. Der Grund für die unvollständige Bildung des Aldoladditionsproduktes 282 könnte in der geringen Stabilität des Hexadienals 281 liegen, dessen hohe Reaktivität mit den beiden C-CDoppelbindungen des Aldehyds im Zusammenhang steht.

Zur Klärung der Frage, ob der geringe Bildungsanteil des Aldolproduktes in der Instabilität des Hexadienals 281 begründet lag, wurden basenvermittelte Aldolreaktionen als Testversuche mit dem Ketoacetal 273 und den kommerziell erhältlichen Aldehyden Butyraldehyd 290, Hydrozimtaldehyd 291, Pivaldehyd 292, Benzaldehyd 293 und Phenylacetaldehyd 294 durchgeführt (vgl. Schema 117). Die Reaktionen wurden entsprechend den in der Tabelle 57 angegebenen Reaktionsbedingungen sowohl in Anwesenheit als auch in Abwesenheit von DMPU untersucht (Einträge 1-10). Bei allen diesen Testreaktionen konnte keine vollständige Umsetzung der Aldehyde 290-294 zu den jeweiligen Aldoladditionsprodukten 295-299 erzielt werden. Während die mit Butyraldehyd 290 und Benzaldehyd 293 untersuchten Reaktionen (vgl. Tabelle 57, Einträge 1, 2 u. 9) zufriedenstellende Produktanteile von 2.4:1, 3.9:1 und 3.8:1 zugunsten der Aldolprodukte 295 und 298 ergaben, konnten die aus Hydrozimtaldehyd 291, Pivaldehyd 292 und Phenylacetaldehyd 294 stammenden Aldoladditionsprodukte 296, 297, 299 nur in recht mäßigen Anteilen erhalten werden (vgl. Tabelle 57, Einträge 3-7, 8 u. 10). Die in Gegenwart von DMPU durchgeführten Versuche zeigten (vgl. Tabelle 57, Einträge $1 \mathrm{u}$. 7) entgegen der Erwartung nachteilige Auswirkungen des Komplexbildners auf die Anteile der Aldoladditionsprodukte (vgl. Tabelle 56). Aufgrund der Ergebnisse der Testreaktionen konnte die eingangs aufgeworfene Frage, ob die unzureichenden Bildungsanteile des gewünschten Aldolproduktes auf der Instabilität des Hexadienals 281 beruhten, nicht geklärt werden. Da sämtliche als Testreagenzien eingesetzten Aldehyde wesentlich stabiler sind als die Verbindung 281, aber nur im Falle des Einsatzes von Butyraldehyd 290 und Benzaldehyd 293 bessere Anteile an Aldolprodukten erzielt werden konnten, während bei den übrigen Aldehyden im Vergleich zu der basenvermittelten Umsetzung des Hexadienals 281 sogar schlechtere Bildungsanteile erhalten wurden, ließen die Ergebnisse der Testreaktionen keine eindeutigen Schlussfolgerungen zu. 
Schema 117. Basenvermittelte Aldol-Reaktion des Ketoacetals 273 mit Butyraldehyd 290, Hydrozimtaldehyd 291, Pivaldehyd 292, Benzaldehyd 293 und Phenylacetaldehyd 294.<smiles>COC(C)(/C=C/C(C)=O)OC</smiles>

273
1) $\mathrm{HN}(\text { isoPr })_{2}, n \mathrm{BuLi}$ $-78^{\circ} \mathrm{C}$, THF, $10 \mathrm{~min}$ 2) (DMPU), 273, THF, $1 \mathrm{~h}$

3) Aldehyd (290-294) $-78{ }^{\circ} \mathrm{C}, 5 \mathrm{~min}$<smiles>[R]C(O)CC(=O)/C=C/C(C)(OC)OC</smiles>

295-299

\begin{tabular}{l|c|cl}
\multicolumn{1}{c|}{ Aldehyd } & Eintrag & \multicolumn{2}{|c}{ Aldolprodukt } \\
\hline Butyraldehyd & 290 & 295 & $\mathrm{R}=\mathrm{CH}_{3} \mathrm{CH}_{2} \mathrm{CH}_{2}$ \\
Hydrozimtaldehyd & 291 & 296 & $\mathrm{R}=\mathrm{C}_{6} \mathrm{H}_{5} \mathrm{CH}_{2} \mathrm{CH}_{2}$ \\
Pivaldehyd & 292 & 297 & $\mathrm{R}=\mathrm{C}\left(\mathrm{CH}_{3}\right)_{3}$ \\
Benzaldehyd & 293 & 298 & $\mathrm{R}=\mathrm{C}_{6} \mathrm{H}_{5}$ \\
Phenylacetaldehyd & 294 & 299 & $\mathrm{R}=\mathrm{C}_{6} \mathrm{H}_{5} \mathrm{CH}_{2}$
\end{tabular}

Tabelle 57. Basenvermittelte Aldol-Reaktion des Ketoacetals 273 mit Butyraldehyd 290, Hydrozimtaldehyd 291, Pivaldehyd 292, Benzaldehyd 293 und Phenylacetaldehyd 294 zu den Aldoladditionsprodukten 295-299.

\begin{tabular}{|c|c|c|c|c|c|}
\hline Eintrag & Aldehyd [eq] & 273 [eq] & LDA [eq] & DMPU [eq] & Auswertung \\
\hline & 290 & & & & 295:273 \\
\hline 1 & 1.0 & 1.0 & 1.2 & 1.6 & $2.4: 1$ \\
\hline \multirow[t]{2}{*}{2} & 1.0 & 1.0 & 1.2 & - & $3.9: 1$ \\
\hline & 291 & & & & $296: 273$ \\
\hline 3 & 1.0 & 1.0 & 1.2 & - & $1: 1$ \\
\hline 4 & 1.0 & 1.0 & 1.8 & - & $1: 2.5$ \\
\hline 5 & 1.0 & 1.0 & 2.4 & - & $1: 3.3$ \\
\hline 6 & 1.0 & 2.0 & 2.2 & - & $1: 1.7$ \\
\hline \multirow[t]{2}{*}{7} & 1.0 & 1.5 & 1.65 & 2.5 & $1: 1$ \\
\hline & 292 & & & & $297: 273$ \\
\hline \multirow[t]{2}{*}{8} & 1.0 & 1.2 & 1.25 & - & $1: 2$ \\
\hline & 293 & & & & $298: 273$ \\
\hline \multirow[t]{2}{*}{9} & 1.0 & 1.2 & 1.25 & - & $3.8: 1$ \\
\hline & 294 & & & & 299:273 \\
\hline 10 & 1.0 & 1.2 & 1.25 & - & $1.37: 1$ \\
\hline
\end{tabular}

Zusammenfassend erwiesen sich die basenvermittelten Aldoladditionsreaktionen zur Darstellung des ersten Schlüsselintermediates der Synthese des Collinolactons (24) nicht als geeignet, da keine vollständigen Umsetzungen und nur unbefriedigende Bildungsanteile erzielt 
werden konnten. Darüber hinaus konnte das gewünschte Aldolprodukt nicht isoliert werden, da sämtliche Reinigungsversuche zu Zersetzungen führten.

\subsection{Versuche zur C-C-Kupplung über das $\alpha$-Bromketon 275}

Ohta et al. ${ }^{[131]}$ untersuchten $\mathrm{SmI}_{2}$-vermittelte, intermolekulare Aldoladditionsreaktionen von Phenylacylbromiden mit Carbonylverbindungen, bei denen die gewünschten $\beta$ Hydroxyketone in moderaten bis sehr guten Ausbeuten synthetisiert werden konnten. Die von Ohta et al. beschriebene, über eine radikalische Zwischenstufe verlaufende Reaktion des Aldol-Typs wurde im Rahmen dieser Arbeit zunächst mit dem $\alpha$-Bromketon 275 (vgl. Abschnitt 2.1, Exp. Teil, Allg. Arbeitsvorschrift B4) und dem kommerziell erhältlichen Hydrozimtaldehyd 291 im Wege einer Testreaktion untersucht (vgl. Schema 118). Hierzu wurde zu einem Lösungsgemisch aus $\alpha$-Bromketon 275, Hydrozimtaldehyd 291 und dem Additiv $\mathrm{Et}_{2} \mathrm{AlCl}$ eine Lösung von $\mathrm{SmI}_{2}$ gegeben und nach $1 \mathrm{~h}$ bei $-78^{\circ} \mathrm{C}$ mit $10 \%$-iger HCl-Lösung hydrolysiert und aufgearbeitet.

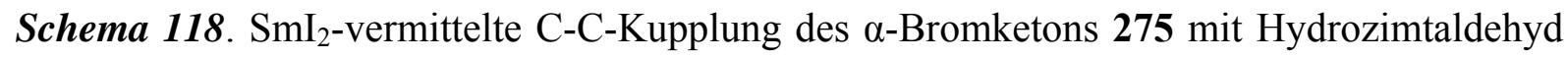
291 zu dem Aldolprodukt 296 über eine radikalische Reaktion des Aldol-Typs.<smiles>COC(C)(/C=C/C(=O)CBr)OC</smiles>

275
1) 1.0 eq 275, 1.0 eq 291

$\frac{2.1 \text { eq } \mathrm{Et}_{2} \mathrm{AlCl}, \mathrm{THF},}{\text { 2) } 2.1 \mathrm{eq} \mathrm{Sml}_{2},-78^{\circ} \mathrm{C}, 1 \mathrm{~h}}$

3) $\mathrm{HCl}, \mathrm{Na}_{2} \mathrm{~S}_{2} \mathrm{O}_{3}$

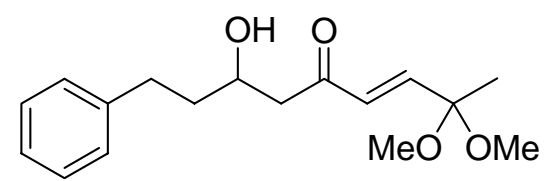

$296(11 \%)$

Die Auswertung des Rohproduktes der radikalischen Umsetzung zeigte ein Gemisch aus dem Aldehyd 291 (Anteil 63\%), einer p-disubstituierten aromatischen Verbindung (Anteil 25\%), dem Aldolprodukt 296 (Anteil 11\%) und dem unter Verlust der Brom- und Acetalgruppe in geringer Menge gebildeten Diketon 272 (vgl. Schema 100), dessen Anteil aufgrund starker Verunreinigung nicht exakt berechnet werden konnte. Die Bildung des aus dem Hydrozimtaldehyd 291 stammenden, $p$-disubstituierten Derivates wurde aus der Aufspaltung des Multiplett-Signals des aromatischen Rings von Hydrozimtaldehyd 291 bei 7.25 auf zwei Multiplett-Signale bei 7.51 ppm und 7.72 ppm hergeleitet. Die Entschützung der Acetalgruppe zur 
Ketogruppe dürfte auf die Gegenwart der oxophilen Lewissäure $\mathrm{Et}_{2} \mathrm{AlCl}$ zurückzuführen sein. Da der radikalische Vorversuch nur zu einem geringfügigen Bildungsanteil des Aldolproduktes führte, wurde die Additionsreaktion unter Verwendung von $\mathrm{SmI}_{2}$ als nicht erfolgversprechend eingestuft und deshalb nicht weiter untersucht.

Nazaki et al. ${ }^{[132]}$ gelang in Analogie zur Reformatsky-Reaktion die regioselektive Bildung von $\beta$-Hydroxyketonen in exzellenten Ausbeuten von 92-100\%. Die unter Einsatz von metallischem Zink- und $\mathrm{Cu}$-Salzen durchgeführte Reaktion verläuft über die Lewissäure-Eigenschaft des $\mathrm{Et}_{2} \mathrm{AlCl}$ als Additiv, die zur Bildung entsprechender Aluminium-Enolate führt. Unter Anwendung des Verfahrens nach Nazaki et al. ${ }^{[132 a]}$ wurde zunächst als Vorversuch die Kupplung des $\alpha$-Bromketons 275 mit Hydrozimtaldehyd 291 zu dem Aldolprodukt 296 (vgl. Schema 119) untersucht. Hierzu wurde eine Suspension von Zink und einer katalytischen Menge $\mathrm{CuBr}_{2}$ in THF mit einer Lösung von $\mathrm{Et}_{2} \mathrm{AlCl}$ versetzt. Der hieraus resultierenden, auf $-20{ }^{\circ} \mathrm{C}$ heruntergekühlten Reaktionsmischung wurde eine Lösung aus dem $\alpha$-Bromketon 275 und dem Hydrozimtaldehyd 291 in THF zugegeben. Die Reaktion wurde durch Zugabe von 1.1 eq Pyridin und anschließender Zugabe von $1 \mathrm{M}$ HCl-Lösung beendet und aufgearbeitet. Hierbei wurde bei Ausbleiben des gewünschten Aldoladditionsprodukts 296 hauptsächlich der eingesetzte Hydrozimtaldehyd 291 als Edukt zurückgewonnen. Die Auswertung der Umsetzung ergab zusätzlich die Bildung von Nebenprodukten, die nicht identifiziert werden konnten. Da der mit Zink und $\mathrm{CuBr}_{2}$ durchgeführte Additionsversuch aufgrund des Ausbleibens des gewünschten Aldolproduktes noch schlechter verlief als derjenige unter Einsatz von $\mathrm{SmI}_{2}$, wurde das reduktive Additionsverfahren insgesamt als ungeeignet befunden und nicht weiter verfolgt.

Schema 119. C-C-Kupplung des $\alpha$-Bromketons 275 mit Hydrozimtaldehyd 291 zu dem Aldolprodukt 296 unter Verwendung von $\mathrm{Zn}$ und $\mathrm{CuBr}_{2}$ als Reduktionsmittel.<smiles>COC(C)(/C=C/C(=O)CBr)OC</smiles>

275
1) 1.1 eq $\mathrm{Et}_{2} \mathrm{AlCl}, 1.5 \mathrm{eq} \mathrm{Zn}$

$\mathrm{CuBr}_{2}$ (5 mol\%), THF

$20^{\circ} \mathrm{C}, 1 \mathrm{~h}$

2) $1.0 \mathrm{eq} 275,1.1 \mathrm{eq} 291$ $\mathrm{THF},-20^{\circ} \mathrm{C}, 15 \mathrm{~min}$

3) 1.1 eq Pyridin, RT<smiles>COC(C)(/C=C/C(=O)CC(O)CCc1ccccc1)OC</smiles>

296 


\subsection{Versuche zur Mukaiyama-Aldoladdition unter Verwendung von Lewissäuren}

Anders als bei den im Abschnitt 3.1. behandelten Aldoladditionsreaktionen, die über LiEnolate verliefen, werden bei den unter der Bezeichnung "Mukaiyama-Reaktion" bekannten Aldolreaktionen anstelle von Enolaten Silylenolether als Nucleophile verwendet, die auch als "maskierte Anionen" bezeichnet werden. Die nach Mukaiyama benannte Reaktion verläuft unter Katalyse von Lewissäuren. Setzt man entsprechend chirale Lewissäuren ein, kann die Stereochemie der Aldolreaktion effektiv gesteuert werden. ${ }^{[133]}$ Im Rahmen der Darstellung des Aldolproduktes 282 als erstes Schlüsselintermediat zur Synthese des Collinolactons (24) wurden Versuche zur Aldoladdition des Silylenolethers 274 mit dem Hexadienal 281 nach Mukaiyama unter Verwendung von Lewissäuren durchgeführt. Das Mukaiyama-Verfahren wurde dabei unter Verwendung von $\mathrm{TiCl}_{4}, \mathrm{ZnCl}_{2}, \mathrm{Yb}(\mathrm{OTf})_{3}$, TBAF und ( \pm )-BINOL/Ti(O$i \operatorname{Pr})_{4}$ als Lewissäuren untersucht (vgl. Tabelle 58).

Tabelle 58. Versuche zur Mukaiyama-Aldoladdition des Silylenolethers 274 mit dem Hexadienal 281 zu dem Aldolprodukt 282 unter Verwendung von Lewissäuren.

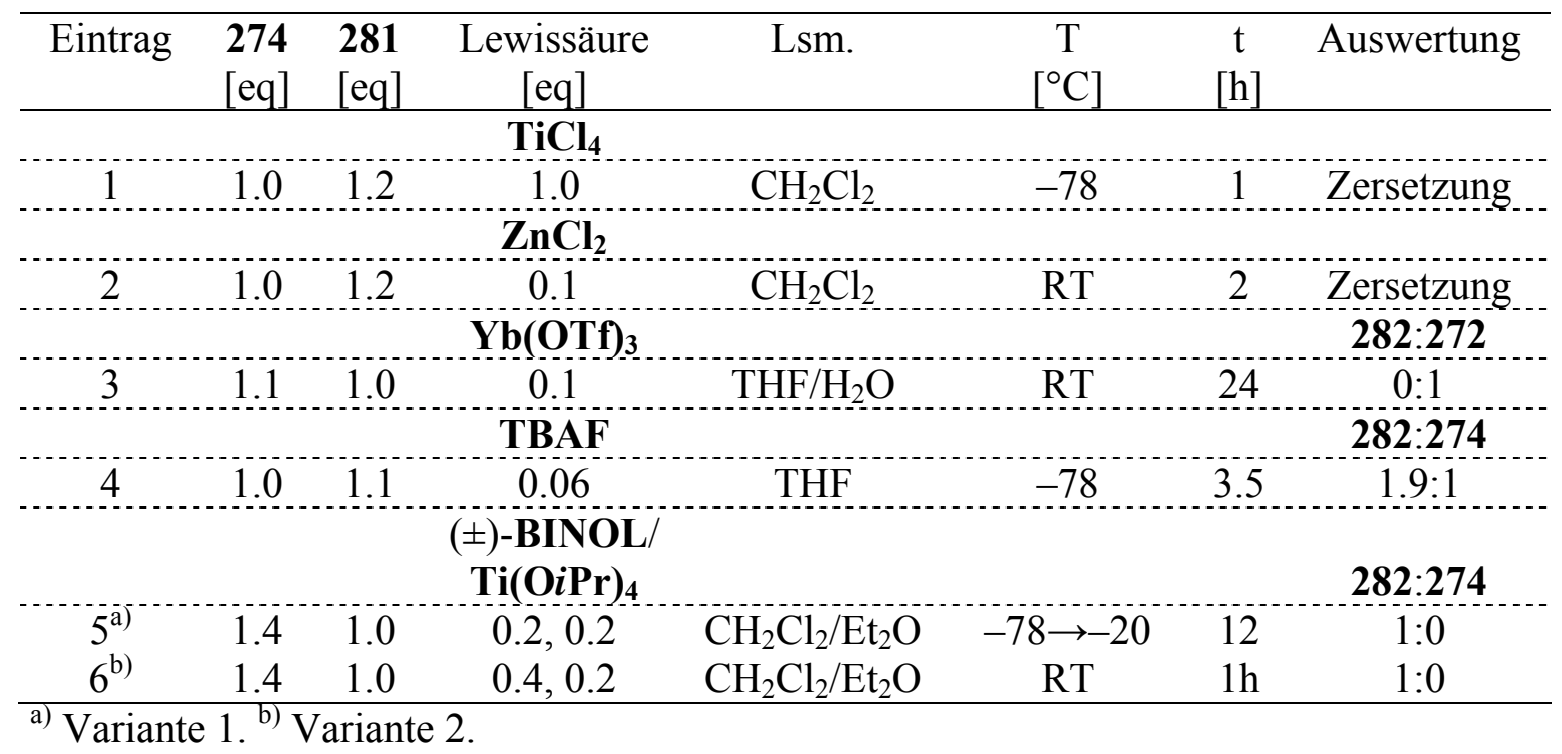

\subsubsection{Versuch zur Mukaiyama-Aldoladdition mit $\mathbf{T i C l}_{4}$ als Lewissäure}

Mukaiyama et al. ${ }^{[133][134]}$ berichteten über Aldolreaktionen von Trimethylsilylenolethern als Ketonderivate mit Aldehyden unter Einsatz von $\mathrm{TiCl}_{4}$ als Lewissäure, bei der ähnliche $\beta$ Hydroxyketone in sehr guten Ausbeuten dargestellt wurden. Zur Synthese des Aldoladditi- 
onsprodukts 282 wurden der Dienaldehyd 281 und der dargestellte Silylenolether 274 (vgl. Abschnitt 2.1, Exp. Teil, Allg. Arbeitsvorschrift B3) als Nucleophile eingesetzt (vgl. Schema 120). Entsprechend der Vorgaben von Mukaiyama et al. ${ }^{[133]}$ wurde die Aldol-Reaktion des Silylenolethers 274 (1.0 eq) mit einer Mischung aus dem Hexadienal 281 (1.2 eq) und $\mathrm{TiCl}_{4}$ als Lewissäure untersucht (vgl. Tabelle 58, Eintrag 1). Der Versuch führte zur vollständigen Zersetzung der Edukte 274 und 281, so daß eine Auswertung gänzlich ausblieb.

Schema 120. Versuch zur Mukaiyama-Aldoladdition des Hexadienals 281 mit dem Silylenolether 274 zu dem Aldolprodukt 282 mit $\mathrm{TiCl}_{4}$ als Lewissäure.
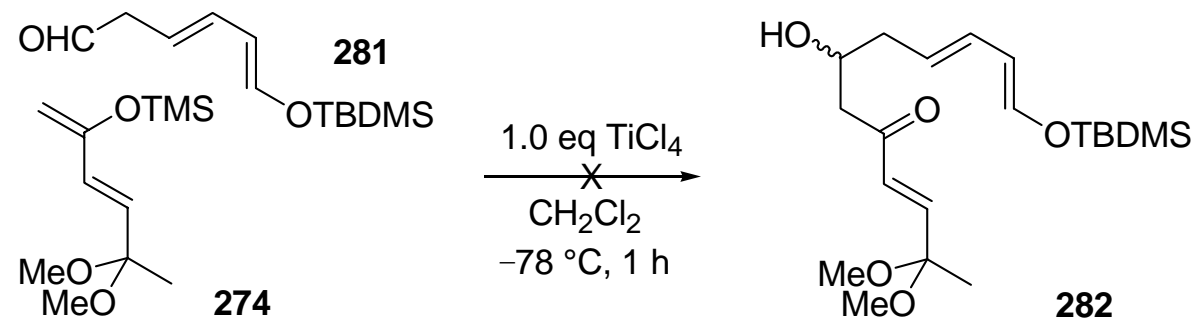

\subsubsection{Versuch zur Mukaiyama-Aldoladdition mit $\mathrm{ZnCl}_{2}$ als Lewissäure}

Hagiwara et al. ${ }^{[135]}$ berichteten über Lewissäure-vermittelte Aldol-Kondensationsreaktionen von Bistrimethylsilylenolethern mit Acetessigsäuremethylestern und 2-Benzyloxyhexanal. Bei der unter Chelat-Kontrolle verlaufenden Aldolreaktion setzten Hagiwara et al. unter anderem Zinkchlorid $\left(\mathrm{ZnCl}_{2}\right)$ als Lewissäure in stöchiometrischer Menge ein, welches zur Bildung eines Gemisches aus syn- und anti-Aldolprodukt in einer Ausbeute von 37\% führte. Trotz der geringen von Hagiwara et al. erzielten Ausbeute erschien die Untersuchung der Aldolreaktion unter Einsatz von $\mathrm{ZnCl}_{2}$ wegen dessen gegenüber $\mathrm{TiCl}_{4}$ schwächeren Reaktivität sinnvoll. Eine Lösung des Dienaldehyds 281 (1.2 eq) in $\mathrm{CH}_{2} \mathrm{Cl}_{2}$ wurde bei Raumtemperatur zu einer Suspension von $\mathrm{ZnCl}_{2}$ (1.0 eq) gegeben (vgl. Schema 121; Tabelle 58, Eintrag 2). Anschliessend wurde die Lösung des Silylenolethers 274 (1.0 eq) tropfenweise zugegeben und $2 \mathrm{~h}$ gerührt. Die klargelbe Reaktionslösung verfärbte sich nach $45 \mathrm{~min}$ zu einer dunkelbraunen Lösung, die nach $2 \mathrm{~h}$ mit $\mathrm{H}_{2} \mathrm{O}$ hydrolysiert und mit $\mathrm{Et}_{2} \mathrm{O}$ aufgearbeitet wurde. Die Auswertung des Versuchs ergab ebenfalls eine vollständige Zersetzung der eingesetzten Edukte 274 und 281 (vgl. Tabelle 58, Eintrag 2), wobei die Zersetzungsprodukte denen des Versuchs 1 ähnelten. 
Schema 121. Versuch zur Mukaiyama-Aldoladdition des Hexadienals 281 mit dem Silylenolether 274 zu dem Aldolprodukt 282 mit $\mathrm{ZnCl}_{2}$ als Lewissäure.

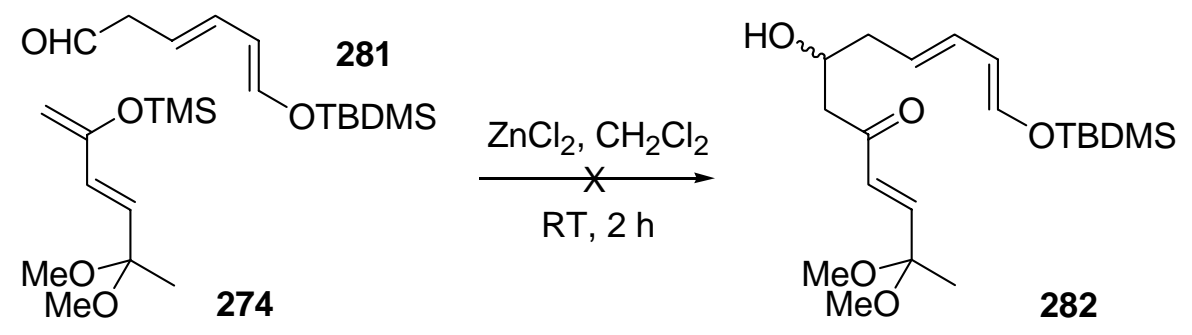

\subsubsection{Versuch zur Mukaiyama-Aldoladdition mit $\mathrm{Yb}(\mathrm{OTf})_{3}$ als Lewissäure}

Kobayashi et al. ${ }^{[136]}$ berichteten über die Hydroxymethylierungsreaktionen von Silylenolether mit wässrigen Formalaldehydlösungen. Sie verwendeten hierzu unter anderem Ytterbium(III)triflat $\left(\mathrm{Yb}(\mathrm{OTf})_{3}\right)$, das sich im Gegensatz zu den meisten Lewissäuren, die sofort mit Wasser reagieren und als entsprechende Hydrate keine Reaktivität mehr besitzen, in wässriger Lösung als sehr stabil erwies. Die über eine Aldolreaktion angestrebten Hydroxymethylierungen führten Kobayashi et al. erfolgreich mit Silylenolether und Formaldehyd in Gegenwart von katalytischen Mengen an $\mathrm{Yb}(\mathrm{OTf})_{3}(10 \mathrm{~mol} \%)$ in wässrigem Medium durch. Die $\mathrm{Yb}(\mathrm{OTf})_{3^{-}}$ katalysierte Aldolreaktion nach Kobayashi et al. wurde auch bei der C-C-Bindungsbildung des Hexadienals 281 mit dem Silylenolether 274 untersucht (vgl. Schema 122; Tabelle 58, Eintrag 3). Hierzu wurde zu einer Lösung von $\mathrm{Yb}(\mathrm{OTf})_{3}(10 \mathrm{~mol} \%)$ in einem $\mathrm{THF} / \mathrm{H}_{2} \mathrm{O}-$ Gemisch ein Gemisch aus dem Hexadienal 281 (1.0 eq) und dem Silylenolether 274 (1.1 eq) in THF (genaue Volumenabmessung, so daß sich für die Gesamtreaktionslösung ein Verhältnis von $\mathrm{THF} / \mathrm{H}_{2} \mathrm{O}$ von 4:1 ergab) zugetropft und 24 h gerührt. Die Auswertung des Rohproduktes zeigte bei Ausbleiben des Aldolprodukts 282 die vollständige Entschützung des Silylenolethers 274 zu (E)-Hex-3-en-2,5-dion (272) (vgl. Schema 100). Dies wurde auf die Chelatisierung der Acetalgruppen der Silylether-Verbindung 274 durch Yt(OTf) 3 zurückgeführt, die den Angriff des Lösungsmittels $\mathrm{H}_{2} \mathrm{O}$ erleichterte und so zur vollständigen Hydrolyse des Acetals führte. 
Schema 122. Versuch zur Mukaiyama-Aldoladdition des Hexadienals 281 mit dem Silylenolether 274 zu dem Aldolprodukt 282 mit $\mathrm{Yb}(\mathrm{OTf})_{3}$ als Lewissäure.

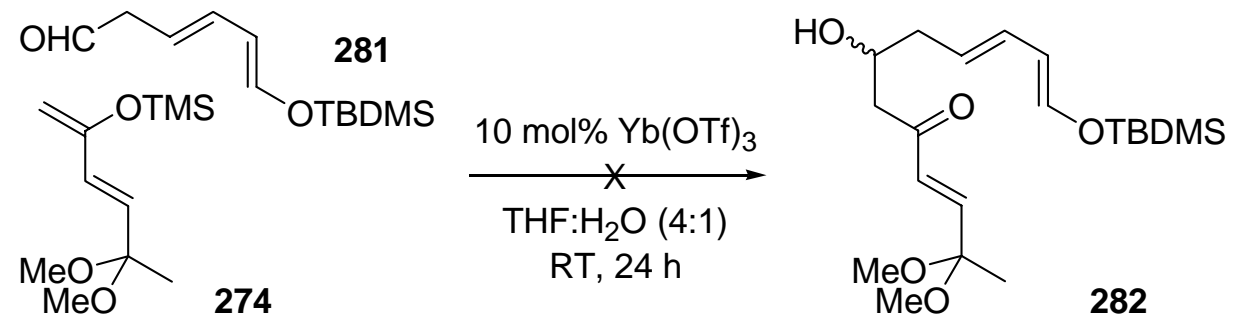

\subsubsection{Versuch zur Mukaiyama-Aldoladdition mit TBAF als Lewissäure}

Noyori et al. ${ }^{[137]}$ untersuchten Fluorid-katalysierte Aldol-Reaktionen von Trimethylsilylenolethern mit Benzaldehyden. Die Untersuchungen zeigten, dass die gewünschten $\beta$ Hydroxyketone am Effektivsten unter Verwendung von Tetrabutylammoniumfluorid (TBAF) in THF verliefen (Ausbeuten 64-84\%). Zur experimentellen Durchführung der AldolReaktionen gaben Noyori et al. eine Lösung von Benzaldehyd (1.1 eq) und Silylether (1.0 eq) bei $-78^{\circ} \mathrm{C}$ tropfenweise zu einer Lösung von TBAF (6 mol\%) und rührten die Reaktion bei dieser Temperatur 3.5 h. Anschließend hydrolysierten sie die Reaktionslösung bei $-78{ }^{\circ} \mathrm{C}$ mit $\mathrm{H}_{2} \mathrm{O}$, erwärmten auf Raumtemperatur und extrahierten mit $\mathrm{Et}_{2} \mathrm{O}$. Die geplante Aldoladdition des Hexadienals 281 (1.1 eq) mit dem Silylenolether 274 (1.0 eq) wurde nach Noyori et al. unter identischen Reaktionsbedingungen bei $-78^{\circ} \mathrm{C}$ unter dem Einsatz von TBAF (6 mol\%) durchgeführt (vgl. Schema 123).

Schema 123. Versuch zur Mukaiyama-Aldoladdition des Hexadienals 281 mit dem Silylenolether 274 zu dem Aldolprodukt 282 mit TBAF als Lewissäure.

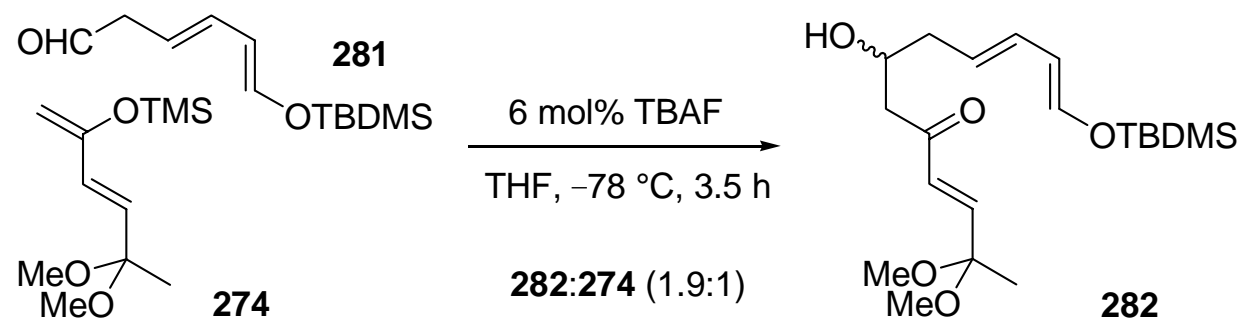


Auch bei dem Versuch nach Noyori et al. konnte keine vollständige Umsetzung zum Aldolprodukt erreicht werden. Die unter katalytischem Einsatz von TBAF durchgeführte Aldolreaktion (vgl. Tabelle 58, Eintrag 4) führte zu einem Gemisch aus dem Aldoladditionsprodukt 282 (Anteil 66\%) als Hauptprodukt und dem Silylenolether 274 als Edukt im Verhältnis 1.9:1. Trotz der unvollständigen Umsetzung stellte dieses Ergebnis eine geringfügige Verbesserung des Produktanteils im Vergleich zu den Basen-initialisierten Aldol-Reaktionen dar (vgl. Abschnitt 3.1, Tabelle 56, Einträge 3 u. 4), bei denen das Aldolprodukt 282 in Anteilen von 63\% und $60 \%$ gebildet wurde.

\subsubsection{Versuche zur Mukaiyama-Aldoladdition mit BINOL/Ti(O-iPr) $)_{4}$ als Lewissäure- Katalysator}

Keck et al. ${ }^{[138]}$ untersuchten katalytische, enantioselektive Mukaiyama-Aldol-Kondensationsreaktionen von Thioester-substituierten Silylethern und Aldehyden, die in sehr hohen Ausbeuten sowie mit exzellenter Enantioselektivität zur Bildung von $\beta$-Hydroxythioestern führten. Zur geplanten Mukaiyama-Aldoladdition von Hexadienal 281 und Silylenolether 274 zu dem Aldolprodukt 282 wurden die Versuche 5 und 6 (vgl. Tabelle 58, Einträge 5 u. 6) entsprechend der angegebenen Arbeitsvorschriften nach Keck et al. mit $( \pm)-\mathrm{BINOL} / \mathrm{Ti}(\mathrm{O} i \mathrm{Pr})_{4}$ als Lewissäure-Katalysator durchgeführt (vgl. Schema 124, s. Exp. Teil, Allg. Arbeitsvorschrift B10, Varianten 1 und 2). Als Hauptziel dieser Reaktionen wurde das Erreichen einer vollständigen Umsetzung angestrebt, weshalb zunächst racemisches BINOL eingesetzt wurde.

Schema 124. Mukaiyama-Aldoladdition des Hexadienals 281 mit dem Silylenolether $274 \mathrm{zu}$ dem Produkt 282 mit BINOL und Ti $(\mathrm{O} i \mathrm{Pr})_{4}$.
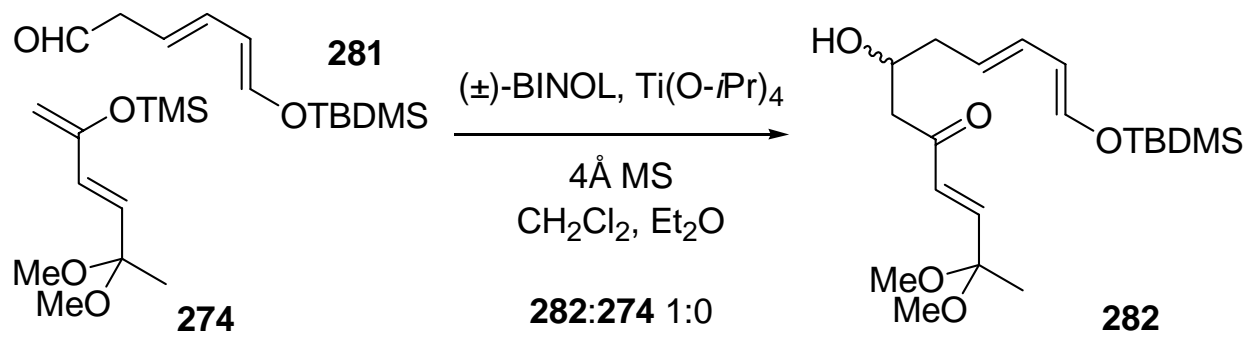
Die Mukaiyama-Aldoladditions-Reaktionen der nach Variante 1 bei $-78{ }^{\circ} \mathrm{C}$ und nach Variante 2 bei Raumtemperatur durchgeführten Versuche führten unter quantitativer Umsetzung zur Bildung des erwünschten Aldolproduktes 282 (vgl. Tabelle 58, Einträge 5 u. 6).

Das erfolgreich als racemisches Gemisch synthetisierte Aldoladditionsprodukt 282 eröffnet die Chance für die selektive Synthese des gewünschten $(R)$-Enantiomers, das als Vorstufe für die Diels-Alder-Cyclisierung notwendig ist. Die diastereoselektive Diels-Alder-Reaktion führt zur Cyclisierung und damit zur Bildung des Bicyclo[4.4.0]decenons 253 (vgl. Schema 94), so daß aus einem Molekül mit einem stereogenen Zentrum eine Verbindung mit vier Stereozentren entsteht. Daher ist die Darstellung des $(R)$-konfigurierten Aldolprodukts 282 für den erfolgreichen stereoselektiven Aufbau des Bicyclodecenons 253 die nächste Herausforderung zur Synthese des Collinolactons, die unter Einsatz von enantiomerenreinem BINOL unter Stereokontrolle zu bewerkstelligen sein dürfte, aber im Rahmen dieser Arbeit aus zeitlichen Gründen nicht mehr fortgeführt werden konnte. Dies muß noch in weiteren Arbeiten untersucht und realisiert werden. 


\section{Experimenteller Teil}

\section{Angewandte Methoden}

\section{Spektroskopische Methoden}

${ }^{1}$ H-NMR-Spektroskopie: Bruker AM 250 (250 MHz), Varian Unity-200 (200 MHz), Varian Mercury 200 (200 MHz), Varian Unity 300 (300 MHz), Bruker AMX 300 (300 MHz), Varian Inova 500 (500 MHz); Referenzen: $\delta=0$ für Tetramethylsilan, 7.16 für [D $\mathrm{D}_{5}$ Benzol, $7.26 \mathrm{ppm}$ für Chloroform- $\mathrm{d}_{1}$. Charakterisierung der Signalaufspaltung: $\mathrm{s}=$ Singulett, $\mathrm{br}=$ breites Singulett, $\mathrm{d}=$ Dublett, $\mathrm{t}=$ Triplett, $\mathrm{q}=$ Quartett, quint $=$ Quintett, $\mathrm{m}=$ Multiplett, $\mathrm{m}_{\mathrm{c}}=$ zentriertes Multiplett, $\mathrm{dd}=$ doppeltes Dublett, $\mathrm{dd}=$ Dublett eines Dubletts, $\mathrm{ddd}=$ doppeltes Dublett eines Dubletts, $\mathrm{dt}=$ doppeltes Triplett. $\mathrm{AB}=$ Signalaufspaltung entspricht einem AB-Spektrum. Die Spektren wurden nach erster Ordnung ausgewertet. Die entsprechenden Kupplungskonstanten sind als Betrag angegeben. Bei nicht eindeutiger Zuordnung werden jeweils alle möglichen Wasserstoffatome angegeben.

${ }^{13}$ C-NMR-Spektroskopie: Bruker AM 250 (62.9 MHz), Varian Unity 200 (50.3 MHz), Varian Mercury 200 (50.3 MHz), Varian Unity 300 (75.5 MHz), Bruker AMX 300 (75.5 MHz), Varian Inova 500 (125.7 MHz); Referenzen: $\delta=77.0$ für Deuterochloroform- $\mathrm{d}_{1}$, 128.0 für $\left[\mathrm{D}_{5}\right]$ Benzol. Die Multiplizität der Signale wurde durch die APT-Aufnahmetechnik (APT = attached proton test) oder die DEPT-Aufnahmetechnik $(\mathrm{DEPT}=$ distortionless enhancement by polarisation transfer) bestimmt und wie folgt angegeben: APT: $+=$ positives APT-Signal [primäre $\left(\mathrm{CH}_{3}\right)$ oder tertiäre $(\mathrm{CH})$ ], - = negatives APT-Signal [sekundäre $\left(\mathrm{CH}_{2}\right)$ oder quartäre C-Atome $\left(\mathrm{C}_{\text {quart. }}\right)$ ]; DEPT: $+=$ positives DEPT-Signal [primäre $\left(\mathrm{CH}_{3}\right)$ oder tertiäre $(\mathrm{CH})$ ], - = negatives DEPT-Signal [sekundäre $\left(\mathrm{CH}_{2}\right)$ oder quartäre C-Atome $\left(\mathrm{C}_{\text {quart. }}\right)$, DEPTSignalintensität Null]. Abkürzungen für Zuordnungen der Signale: C-Ar $=$ Aromatisch. Vertauschbare Zuordnungen werden durch "*" gekennzeichnet. Bei nicht eindeutiger Zuordnung werden jeweils alle möglichen Kohlenstoffatome angegeben.

Massenspektrometrie: Finnigan MAT 95 [Elektronenstoßionisation EI, 70 eV, Direkte Chemische Ionisation DCI, $200 \mathrm{eV}$ und HRMS (High Resolution MS)], Finnigan LC-Q (Electrospray-Ionisation ESI).

Infrarot-Spektroskopie: FTIR-Spektrometer: Bruker IFS, Bruker Vector 22.

\section{Chromatographische Methoden}

Säulenchromatographie (SC): Chromatographische Trennungen erfolgten an Merck Kieselgel 60 (0.063-0.200 mm, 230-400 mesh) als Normalchromatographie bzw. an Macherey- 
Nagel Kieselgel 60 (0.040-0.063 mm, 200-400 mesh) als Flash-Chromatographie nach Still et al. $^{[139]}$ oder an Aluminiumoxid (ICN Alumina - Super I). Alle Laufmittel wurden vor Verwendung destilliert.

Dünnschichtchromatographie (DC): Merck DC-Fertigfolien Kieselgel 60 Sil G/UV 254 auf Aluminiumfolien oder Fertigfolien Polyfram Aluminiumoxid N/UV 254 . Detektion unter Normallicht und UV-Licht bei $254 \mathrm{~nm}$, Entwicklung mit Molybdatophosphat ${ }^{[140]}$ (5 proz. Phosphormolybdänsäure in EtOH) als Tauchreagenz.

\section{Elementaranalysen}

Die Elementaranalysen wurden im Mikroanalytischen Laboratorium des Instituts für Organische Chemie der Universität Göttingen ausgeführt. Geräte: Leco CHN 2000, Heraeus Verbrennungsapparatur Mikro U/D.

\section{Chemikalien und Arbeitstechniken}

Die verwendeten Chemikalien Weinrebamid ${ }^{[26]}, N$-Acetylimidazol ${ }^{[29]}$ u. akt. $\mathrm{SeO}_{2}{ }^{[40]}$ wurden nach bekannten Arbeitsvorschriften hergestellt. Wasserfreie Lösungsmittel wurden nach üblichen Laboratoriumsmethoden ${ }^{[141]}$ gewonnen und unter Stickstoff destilliert (Trocknung: THF, Diethylether, Dioxan, Benzol, DMF, DME, Toluol und Pentan mit Natrium/Benzophenon, HMPA, NMP, $\mathrm{HNEt}_{2}, \mathrm{NEt}_{3}$, Pyridin, DMF, DMSO, $\mathrm{CCl}_{4}$ und $\mathrm{CH}_{2} \mathrm{Cl}_{2}$ mit $\mathrm{CaH}_{2}$, 1,2Dichlorethan, Aceton und Acetonitril mit $\mathrm{P}_{4} \mathrm{O}_{10}$, EtOH und Hexan mit Natrium, $\mathrm{MeOH}$ über $\mathrm{Mg}$ ). Alle Reaktionen wurden, soweit nicht anders erwähnt, unter Sauerstoff- und Feuchtigkeitsausschluss und unter inerter Atmosphäre (Argon oder Stickstoff) durchgeführt. Wenn nicht anders angegeben, sind Lösungen von $\mathrm{NH} 4 \mathrm{Cl}, \mathrm{NaCl}, \mathrm{Na}_{2} \mathrm{SO}_{4}, \mathrm{NaHCO}_{3}, \mathrm{Na}_{2} \mathrm{CO}_{3}$, $\mathrm{KHSO}_{4}$ und Zitronensäure gesättigte wässrige Lösungen. Nicht gesondert aufgeführte Chemikalien sind kommerziell erhältlich und wurden ohne weitere Reinigung eingesetzt. 


\section{Darstellung der Verbindungen (Hauptteil 1)}

\section{Synthese der Seitenkette}

\section{Allgemeine Arbeitsvorschrift A1 \\ 4-Methyl-1-penten-3-ol (99A)}

$\mathrm{Zu}$ einer Lösung von Ethenylmagnesiumbromid $(260 \mathrm{~mL}, 0.218 \mathrm{~mol}, 0.840 \mathrm{M}$ in
$\mathrm{mL}, 15 \mathrm{~g}, 0.21 \mathrm{~mol})$ in THF $(20 \mathrm{~mL})$ innerhalb von $15 \mathrm{~min}$. zugegeben und bei Raumtemperatur 30 min. gerührt. Zu der Reaktionslösung wurde ges. $\mathrm{NH}_{4} \mathrm{Cl}$-Lösung gegeben (schäumt stark) und mit Diethylether extrahiert. Die organische Phase wurde getrennt, die wässrige Phase mit Diethylether $(2 \times 50 \mathrm{~mL})$ reextrahiert. Die vereinigte organische Phase wurde über $\mathrm{MgSO}_{4}$ getrocknet. Das Lösungsmittel wurde erst am Rotationsverdampfer ( $\mathrm{P}=$ 200 mbar) eingeengt und das verbliebene THF über eine Destillationsbrücke $(10 \mathrm{~cm})$ entfernt. Das ölige Rohprodukt wurde fraktionierend destilliert. Man erhielt 20.800 g (quant.) (Lit. ${ }^{[45]}$ $73 \%$ ) des gewünschten Allylalkohols 99A, Sdp.100mbar $63-65{ }^{\circ} \mathrm{C}$ (Lit. ${ }^{[4]}$ Sdp.760mmHg $122-$ 123), als farblose Flüssigkeit. - IR (Film): $v=3601 \mathrm{~cm}^{-1}, 3414(\mathrm{OH}), 3053,2962,2875$, 2360, 2336, $1645(\mathrm{C}=\mathrm{CH}), 1560,1470,1265,1019,993,921,740 .-{ }^{1} \mathrm{H}-\mathrm{NMR}(250 \mathrm{MHz}$, $\left.\mathrm{CDCl}_{3}\right): \delta=0.87\left(\mathrm{~d}, J=6.7 \mathrm{~Hz}, 3 \mathrm{H}, 1^{\prime}-\mathrm{H}\right), 0.90(\mathrm{~d}, J=6.7 \mathrm{~Hz}, 3 \mathrm{H}, 5-\mathrm{H}), 1.70\left(\mathrm{~m}_{\mathrm{c}}, 1 \mathrm{H}, 4-\right.$ H), $1.87\left(\mathrm{~s}_{\mathrm{br}}, 1 \mathrm{H}, \mathrm{OH}\right), 3.84(\mathrm{t}, 7.1 \mathrm{~Hz}, 1 \mathrm{H}, 3-\mathrm{H}), 5.06-5.27$ [m, $\left.2 \mathrm{H}, 1(\mathrm{cis})(\operatorname{trans})-\mathrm{H}\right], 5.85$ (m, $1 \mathrm{H}, 2-\mathrm{H}) .-{ }^{13} \mathrm{C}-\mathrm{NMR}\left(62.9 \mathrm{MHz}, \mathrm{CDCl}_{3}\right.$, zusätzl. DEPT): $\delta=17.83$ (-, C*-1'), 17.95 (-, C*-5), 33.38 (-, C-4), 78.05 (-, C-3), 115.34 (+, C-1), 139.35 (-, C-2). - MS (EI, 70 eV), m/z (\%): 127 (2), 101 (4), 100 (20), 98 (3), 86 (<1), 85 (2), 72 (1), 71 (4), 59 (1), 58 (18), 57 (100), $55(2), 45(<1), 43(16), 41(10)$.

\section{4,4-Dimethylpent-1-en-3-ol (99R)}

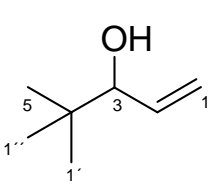

Die Reaktion wurde gemäß der allgemeinen Arbeitsvorschrift A1 mit Pivalinaldehyd (19.0 mL, $15.0 \mathrm{~g}, 174 \mathrm{mmol})$ und Ethenmagnesiumbromid (550 mL, $183 \mathrm{mmol}, 0.33 \mathrm{M}$ in THF) durchgeführt. Das ölige Rohprodukt wurde fraktionierend destilliert. Man erhielt $12.97 \mathrm{~g}$, (65\%) des Allylalkohols 99R, Sdp.100mbar $67-68{ }^{\circ} \mathrm{C}$ (Lit. ${ }^{[47]} \mathrm{Sdp} .700 \mathrm{mmHg} 128-129{ }^{\circ} \mathrm{C}$ ) als farblose Flüssigkeit. $-{ }^{1} \mathrm{H}-\mathrm{NMR}^{[142]}\left(250 \mathrm{MHz}, \mathrm{CDCl}_{3}\right): \delta$ $=0.91\left[\mathrm{~s}, 9 \mathrm{H}, 5\left(1^{\prime}\right)\left(1^{\prime \prime}\right)-\mathrm{H}\right], 1.59(\mathrm{~s}, 1 \mathrm{H}, \mathrm{OH}), 3.80(\mathrm{~d}, J=6.51 \mathrm{~Hz}, 1 \mathrm{H}, 3-\mathrm{H}), 5.15-5.28(\mathrm{~m}$, $2 \mathrm{H}, 1-\mathrm{H})$, 5.84-6.00 (m, $1 \mathrm{H}, 2-\mathrm{H}) .-{ }^{13} \mathrm{C}-\mathrm{NMR}\left(62.9 \mathrm{MHz}, \mathrm{CDCl}_{3}\right.$, zusätzl. DEPT): $\delta=$ 
$25.56\left[-\right.$, C-5(1')(1")], $34.60\left(\mathrm{C}_{\text {quart. }}-4\right), 81.12$ (-, C-3), 116.29 (+, C-1), 138.03 (-, C-2). - MS (EI, $70 \mathrm{eV}), m / z$ (\%): 242 (10), 158 (5), 149 (3), 132 (62) [M + $\left.\mathrm{NH}_{4}\right]^{+}, 131$ (39) [M + $\left.\mathrm{NH}_{3}\right]$, $114(100)\left[\mathrm{M}-\mathrm{H}_{2} \mathrm{O}+\mathrm{NH}_{4}\right]^{+}$.

\section{Allgemeine Arbeitsvorschrift A2}

(2E)-1-Brom-4-methylpent-2-en (100A) und 3-Brom-4-methylpent-1-en (101A)<smiles>C=CC(Br)C(C)C</smiles>

Eine Lösung von $\mathrm{PBr}_{3}(9.71 \mathrm{~mL}, 27.9 \mathrm{~g}, 103 \mathrm{mmol})$ in trockenem Hexan $(60 \mathrm{~mL})$ wurde unter Stickstoffatmosphäre mit 4

Tropfen wasserfreiem Pyridin versetzt und auf $-30{ }^{\circ} \mathrm{C}$ abgekühlt. Eine Lösung von 4-Methyl-1-penten-3-ol (99A) (20.7 g, 207 mmol) und wasserfreiem Pyridin $(6.69 \mathrm{~mL}, 6.54 \mathrm{~g}, 82.7 \mathrm{mmol})$ in trockenem Hexan $(40 \mathrm{~mL})$ wurde innerhalb von 40 min zugetropft, das Kältebad entfernt und bei Raumtemperatur 1 h gerührt. Die Reaktionslösung wurde auf eisgekühlte ges. $\mathrm{NaHCO}_{3}$-Lösung gegeben und mit Pentan extrahiert. Die wässrige Phase $(\mathrm{pH}=8)$ wurde mit Diethylether $(2 \times 50 \mathrm{~mL})$ reextrahiert. Die vereinigte organische Phase wurde über $\mathrm{MgSO}_{4}$ getrocknet. Das Lösungsmittel wurde am Rotationsverdampfer ( $\mathrm{P}=150$ mbar) entfernt. Das ölige Rohprodukt wurde fraktionierend destilliert. Man erhielt $10.71 \mathrm{~g}(32 \%)$ eines Gemisches aus $\mathrm{S}_{N^{\prime}}$ - und $\mathrm{S}_{N^{-}}$-Produkt (3.7:1) ${ }^{[48][49]}$ der gewünschten Bromide 100A und 101A als farblose Flüssigkeit, Sdp. ${ }_{100 \mathrm{mbar}} 70-75{ }^{\circ} \mathrm{C}$ (Lit. ${ }^{[46]} \mathrm{Sdp} \cdot 20 \mathrm{mmHg} 58$ $\left.{ }^{\circ} \mathrm{C}\right) .-$ IR (Film): $v=3053 \mathrm{~cm}^{-1}, 2966,1560,1421,1206,970,896,706 .-{ }^{1} \mathrm{H}-\mathrm{NMR}$ (250 $\left.\mathrm{MHz}, \mathrm{CDCl}_{3}\right): \delta=1.0\left(\mathrm{~d}, J=6.8 \mathrm{~Hz}, 6 \mathrm{H}, 5\left(1^{\prime}\right)-\mathrm{H}\right), 2.35\left(\mathrm{~m}_{\mathrm{c}}, 1 \mathrm{H}, 4-\mathrm{H}\right), 3.96(\mathrm{~d}, J=6.5 \mathrm{~Hz}, 2$ $\mathrm{H}, 1-\mathrm{H}), 5.58-5.84$ [m, $2 \mathrm{H}, 3(2)-\mathrm{H}] .-{ }^{13} \mathrm{C}-\mathrm{NMR}\left(62.9 \mathrm{MHz}, \mathrm{CDCl}_{3}\right.$, zusätzl. DEPT): $\delta=$ $21.87\left[-, C^{*}-5\left(1^{\prime}\right)\right], 30.61(-, C-4), 33.74(+, C-1), 123.47$ (-, C-2), $143.16(-$, C-3). - MS (EI, $70 \mathrm{eV}), m / z(\%): 165$ (3), 164 (40), $162(46), 149$ (11), $136(6), 120(<1), 108(<1), 91(<1)$, 84 (4), 83 (100), 71 (1), 69 (2), 67 (13), 65 (2), 55 (64), 53 (3), $51(<1), 43$ (11), 41 (35).

\section{(2E)-1-Brom-4,4-dimethylpent-2-en (100R) und 3-Brom-4,4-dimethylpent-1-en (101R)}

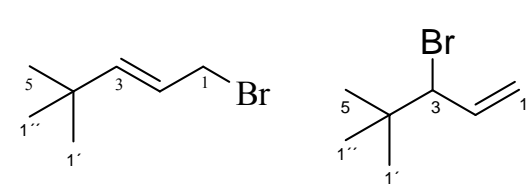

Die Reaktion wurde gemäß der allgemeinen Arbeitsvorschrift A2 mit 4.4-Dimethylpent-1-en-3-ol (99R) (12.5 g, 110 mmol), Phosphortribromid (5.2 mL, 15 g, $55 \mathrm{mmol}$ ) und Pyridin (3.5 mL, 3.5 g, $44 \mathrm{mmol}$ ) durchgeführt. Das Rohprodukt wurde einer fraktionierenden Destillation unterzogen. Man erhielt $10.7 \mathrm{~g}$ (55\%) eines Gemisches ${ }^{[143]}$ aus $\mathrm{S}_{N}$-Produkt (Hauptprodukt) und $\mathrm{S}_{N}$-Produkt (Spuren) der gewünschten Bromide 100R und 101R als farb- 
lose Flüssigkeit, Sdp.35mbar $58-59{ }^{\circ} \mathrm{C}$ (Lit. ${ }^{[47][143]}$ Sdp.40Torr $75-77{ }^{\circ} \mathrm{C}$ ). Das erhaltene Bromidgemisch 100R/101R wurde ohne weitere Trennung der Isomere umgesetzt. $-{ }^{1} \mathrm{H}-\mathrm{NMR}^{[46][47]}$ $\left(250 \mathrm{MHz}, \mathrm{CDCl}_{3}\right): \delta=1.02$ [s, $\left.9 \mathrm{H}, 5\left(1^{\prime}\right)\left(1^{\prime \prime}\right)-\mathrm{H}\right], 3.96(\mathrm{~d}, J=7.5 \mathrm{~Hz}, 2 \mathrm{H}, 1-\mathrm{H}), 5.50-5.65$ (m, $1 \mathrm{H}, 2-\mathrm{H}), 5.98$ (d, $J=15.5 \mathrm{~Hz}, 1 \mathrm{H}, 3-\mathrm{H}) .-{ }^{13} \mathrm{C}-\mathrm{NMR}\left(62.9 \mathrm{MHz}, \mathrm{CDCl}_{3}\right.$, zusätzl. DEPT): $\delta=29.15$ [-, C-5(1')(1")], $33.02\left(\mathrm{C}_{\text {quart. }}-4\right), 34.16$ (-, C-1), 121.37 (-, C-2), 147.02 (-, C-3).

\section{Allgemeine Arbeitsvorschrift A3}

(2E)-1-Trimethylsilyl-6-methylhept-4-en-1-in (102A)

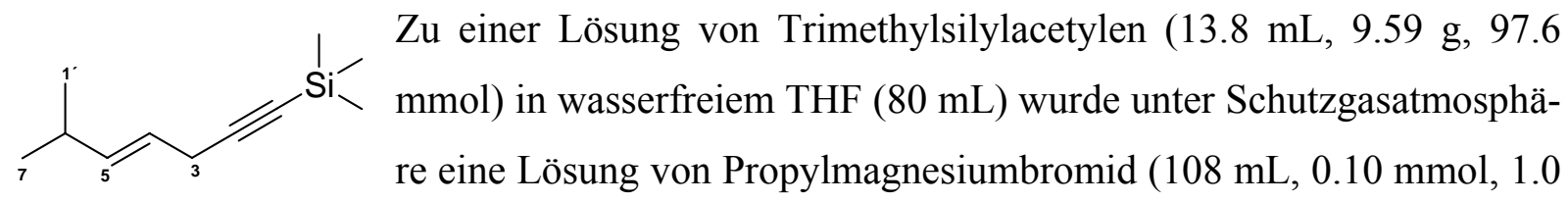
$\mathrm{M}$ in $\mathrm{Et}_{2} \mathrm{O}$ ) bei $0{ }^{\circ} \mathrm{C}$ tropfenweise zugegeben, das Eisbad entfernt und bei Raumtemperatur $2 \mathrm{~h}$ gerührt. Die generierte Trimethylsilylethinylmagnesiumbromid-Lösung wurde bei $-10{ }^{\circ} \mathrm{C}$ über einer Transferkanüle zu der Suspension eines Gemisches von (2E)-1-Brom-4methylpent-2-en (100A) und 3-Brom-4-methylpent-1-en (101A) (10.60 g, 3.7:1-Verhältnis, $65.01 \mathrm{mmol})$ sowie $\mathrm{CuCN}(0.29 \mathrm{~g}, 3.3 \mathrm{mmol})$ in wasserfreiem THF (100 mL) gegeben, das Kältebad entfernt und $2.5 \mathrm{~h}$ bei Raumtemperatur gerührt. Die Reaktionslösung wurde mit ges. $\mathrm{NH}_{4} \mathrm{Cl}$-Lösung versetzt und mit $\mathrm{Et}_{2} \mathrm{O}$ extrahiert. Die wässrige Phase $(\mathrm{pH}=7)$ wurde mit $\mathrm{Et}_{2} \mathrm{O}$ $(2 \times 50 \mathrm{~mL})$ reextrahiert. Die vereinigte organische Phase wurde über $\mathrm{MgSO}_{4}$ getrocknet. Das Lösungsmittel wurde am Rotationsverdampfer $(\mathrm{P}=10 \mathrm{mbar})$ entfernt. Das erhaltene Rohprodukt wurde über einer Kieselgelfiltration $\left(150 \mathrm{~g} \mathrm{SiO}_{2}\right.$, Pentan, Phosphormolybdänsäure in EtOH als Tauchreagenz) gereinigt. Man erhielt $11.72 \mathrm{~g}(95 \%)$ des gewünschten Silans 102A $\left(R_{f}=0.46\right)$ als farblose Flüssigkeit. - IR (film): $v=3033 \mathrm{~cm}^{-1}, 2960(\mathrm{C}-\mathrm{H}), 2871,2177$ $(\mathrm{C} \equiv \mathrm{C}), 1669$ (C=C), 1467, 1420, 1321, 1250, 1102, 1050, 1008, 969, 910, 845, 760, 736, 698, 672, 643. $-{ }^{1} \mathrm{H}-\mathrm{NMR}\left(250 \mathrm{MHz}, \mathrm{CDCl}_{3}\right): \delta=0.18$ [s, $\left.9 \mathrm{H}, \mathrm{Si}\left(\mathrm{CH}_{3}\right)_{3}\right], 0.98[\mathrm{~d}, J=6.6 \mathrm{~Hz}, 6 \mathrm{H}$, 7(1')-H], 2.28 (m $\left.\mathrm{m}_{\mathrm{c}}, 1 \mathrm{H}, 6-\mathrm{H}\right), 2.94(\mathrm{~d}, J=5.5 \mathrm{~Hz}, 2 \mathrm{H}, 3-\mathrm{H}), 5.31$ (dt, $J=15.5, J=5.5 \mathrm{~Hz}, 1$ H, 4-H), 5.65 (ddt, $J=15.5, J=5.5 \mathrm{~Hz}, J=1.5 \mathrm{~Hz}, 1 \mathrm{H}, 5-\mathrm{H}) .-{ }^{13} \mathrm{C}-\mathrm{NMR}(62.9 \mathrm{MHz}$, $\mathrm{CDCl}_{3}$, zusätzl. DEPT): $\delta=0.05\left[+, \mathrm{Si}\left(\mathrm{CH}_{3}\right)_{3}\right], 22.26\left[+, \mathrm{C}-7\left(1^{\prime}\right)\right], 22.97(-, \mathrm{C}-3), 30.69(+, \mathrm{C}-$ 6), 85.95 ( $\left.\mathrm{C}_{\text {quart }}, \mathrm{C}-1\right), 104.72$ ( $\left.\mathrm{C}_{\text {quart }}, \mathrm{C}-2\right), 120.61$ (+, C-4), $139.24(+, \mathrm{C}-5) .-\mathrm{MS}$ (EI, 70 $\mathrm{eV}), m / z(\%): 180(8)\left[\mathrm{M}^{+}\right], 165$ (100) $\left[\mathrm{M}^{+}-\mathrm{CH}_{3}\right], 147$ (3), $135(9)\left[\mathrm{M}^{+}-3 \mathrm{CH}_{3}\right], 123$ (25), 109 (17), 106 (30), 97 (10), 83 (14), 73 (100), 69 (20), 59 (32). 


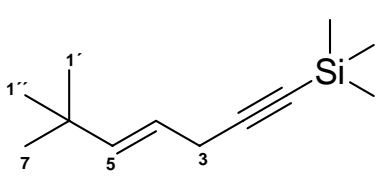

Die Reaktion wurde gemäß der allgemeinen Arbeitsvorschrift A3 mit Trimethylsilylacetylen $(12.6 \mathrm{~mL}, 8.74 \mathrm{~g}, 88.9 \mathrm{mmol})$, Ethylmagnesiumbromid (123 mL, $88.9 \mathrm{mmol}, 0.66 \mathrm{M}$ in THF), (2E)-1-Brom-4,4dimethylpent-2-en (100R) und 3-Brom-4,4-dimethylpent-1-en (101R) (10.5 g, $59.3 \mathrm{mmol})$ sowie $\mathrm{CuCN}$ (0.270 g, $2.96 \mathrm{mmol})$ durchgeführt und die Reaktionslösung $2.5 \mathrm{~h}$ bei Raumtemperatur gerührt. Die Reaktionslösung wurde mit ges. $\mathrm{NH}_{4} \mathrm{Cl}$-Lösung versetzt und mit $\mathrm{Et}_{2} \mathrm{O}$ ex-trahiert. Die wässrige Phase wurde mit $\mathrm{Et}_{2} \mathrm{O}(2 \times 50 \mathrm{~mL})$ reextrahiert. Die vereinigte organische Phase wurde über $\mathrm{Na}_{2} \mathrm{SO}_{4}$ getrocknet. Das Lösungsmittel wurde am Rotationsverdampfer ( $\mathrm{P}=100$ mbar) entfernt. Das erhaltene Rohprodukt wurde einer fraktionierenden Destillation unterzogen. Man erhielt 6.08 g (53\%) des Eninsilans 102R $\left(R_{f}=0.38\right.$, Pentan), Sdp. 15 mbar $60-70{ }^{\circ} \mathrm{C}$, als farblose Flüssigkeit. - IR (Film): $v=2960 \mathrm{~cm}^{-1}(\mathrm{CH}), 2177(\mathrm{C} \equiv \mathrm{C})$, 1464, 1363, 1250, 971, 842, 760. - ${ }^{1} \mathrm{H}-\mathrm{NMR}\left(250 \mathrm{MHz}, \mathrm{CDCl}_{3}\right): \delta=0.19$ [s, $\left.9 \mathrm{H}, \mathrm{Si}\left(\mathrm{CH}_{3}\right)_{3}\right]$, 1.00 [s, 9 H, 7(1")(1')-H], 2.95 (d, J=6.6 Hz, 2 H, 3-H), 5.24-5.36 (m, 1 H, 4-H), 5.65 (d, $J=$ $15.5 \mathrm{~Hz}, 1 \mathrm{H}, 5-\mathrm{H}) .-{ }^{13} \mathrm{C}-\mathrm{NMR}\left(62.9 \mathrm{MHz}, \mathrm{CDCl}_{3}\right.$, zusätzl. DEPT): $\delta=0.07\left[-, \mathrm{Si}\left(\mathrm{CH}_{3}\right)_{3}\right]$, $23.02\left(+\right.$, C-3), $29.50\left[-\right.$, C-7(1")(1')], 32.79 (C-6), $85.99\left(\mathrm{C}_{\text {quart }}-1\right), 104.85\left(\mathrm{C}_{\text {quart }}-2\right), 118.44$ (-, C-4), 143.09 (-, C-5). - MS (EI, $70 \mathrm{eV}), m / z(\%): 194$ (7) $\left[\mathrm{M}^{+}\right], 181$ (2), 180 (11), 179 (70) $\left[\mathrm{M}^{+}-\mathrm{CH}_{3}\right], 163$ (>1), 151 (1), 149 (2), 138 (4), 135 (2), 121 (4), 120 (11), 109 (3), 97 (6), 84 (2), 83 (58), 73 (100), 70 (14), 59 (14), 57 (12), 41 (7).

\section{1-Trimethylsilyl-(4E)-5-phenylpent-4-en-1-in (115)}

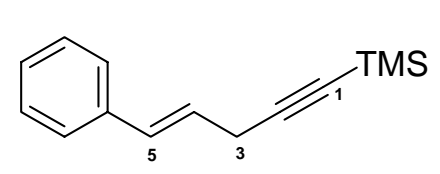

Die Reaktion wurde gemäß der allgemeinen Arbeitsvorschrift A3 mit Trimethylsilylacetylen $(6.45 \mathrm{~mL}, 4.485 \mathrm{~g}, 45.7 \mathrm{mmol})$, Ethylmagnesiumbromid (53.0 mL, $45.7 \mathrm{mmol}, 0.86 \mathrm{M}$ in THF), Cinnamylbromid 114 (4.50 mL, $6.0 \mathrm{~g}, 30.4 \mathrm{mmol})$ und $\mathrm{CuCN}$ (0.136 g, $1.52 \mathrm{mmol})$ durchgeführt und die Reaktionslösung 2.5 h bei Raumtemperatur gerührt. Die Reaktionslösung wurde mit ges. $\mathrm{NH}_{4} \mathrm{Cl}$-Lösung versetzt und mit $\mathrm{Et}_{2} \mathrm{O}$ extrahiert. Die wässrige Phase wurde mit $\mathrm{Et}_{2} \mathrm{O}(2 \mathrm{x}$ $50 \mathrm{~mL}$ ) reextrahiert. Die vereinigte organische Phase wurde über $\mathrm{Na}_{2} \mathrm{SO}_{4}$ getrocknet. Das Lösungsmittel wurde am Rotationsverdampfer $(\mathrm{P}=30 \mathrm{mbar})$ entfernt. Man erhielt $6.52 \mathrm{~g}$ (quant.) des Eninsilans $115\left(R_{f}=0.41\right.$, Pentan) als rotbraune Flüssigkeit. - ${ }^{1} \mathrm{H}-\mathrm{NMR}(250$ $\left.\mathrm{MHz}, \mathrm{CDCl}_{3}\right): \delta=0.21\left[\mathrm{~s}, 9 \mathrm{H}, \mathrm{Si}\left(\mathrm{CH}_{3}\right)_{3}\right], 3.21(\mathrm{~d}, J=6.4 \mathrm{~Hz}, 2 \mathrm{H}, 3-\mathrm{H}), 6.11-6.22(\mathrm{~m}, 1 \mathrm{H}$, 4-H), 6.65 (d, $J=16.1 \mathrm{~Hz}, 1 \mathrm{H}, 5-\mathrm{H}), 7.18-7.39$ (m, $5 \mathrm{H}$, Arom.-H). 


\section{Allgemeine Arbeitsvorschrift A4 (Variante 1) \\ (2E)-6-Methylhept-4-en-1-in (103A)}

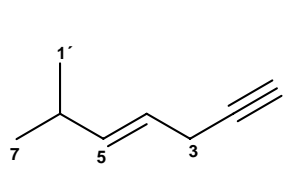

$\mathrm{Zu}$ einer Lösung von (2E)-1-Trimethylsilyl-6-methylhept-4-en-1-in (102A) (10.80 g, $59.88 \mathrm{mmol})$ in $\mathrm{MeOH}(200 \mathrm{~mL})$ wurde bei Raumtemperatur $\mathrm{NaOH}\left(96 \mathrm{~mL}, 96 \mathrm{mmol}, 1.0 \mathrm{M}\right.$ in $\mathrm{H}_{2} \mathrm{O}$ ) zugegeben und $3.5 \mathrm{~h}$ gerührt. Die Reaktionslösung wurde mit ges. $\mathrm{NH}_{4} \mathrm{Cl}$-Lösung versetzt und mit Pentan extrahiert. Die wässrige Phase $(\mathrm{pH}=6)$ wurde mit Pentan $(3 \times 50 \mathrm{~mL})$ reextrahiert. Die vereinigte organische Phase wurde über $\mathrm{MgSO}_{4}$ getrocknet. Das Lösungsmittel wurde destillativ über eine Vigreuxkolonne (20 cm, P = 200 mbar) entfernt. Das Rohprodukt wurde fraktioniert destilliert. Man erhielt $6.43 \mathrm{~g}(99 \%)$ des Enins $\mathbf{1 0 3 A}$ als farblose Flüssigkeit, Sdp.200mbar $65-70{ }^{\circ} \mathrm{C}$ (Lit. ${ }^{[52]}$ Sdp.11Torr $\left.72-73{ }^{\circ} \mathrm{C}\right) .-$ IR (film): $v=3309 \mathrm{~cm}^{-1}(\mathrm{C} \equiv \mathrm{CH}), 2961,2872,2252(\mathrm{C} \equiv \mathrm{C}), 1653$ $(\mathrm{C}=\mathrm{C}), 1559,1466,1419,1384,1254,1056,972,909,847,735,668,651 .-{ }^{1} \mathrm{H}$ NMR $(250$ $\left.\mathrm{MHz}_{\mathrm{CDCl}}\right): \delta=0.99$ [d, $\left.J=6.6 \mathrm{~Hz}, 6 \mathrm{H}, 7\left(1^{\prime}\right)-\mathrm{H}\right], 2.05\left(\mathrm{~m}_{\mathrm{c}}, 1 \mathrm{H}, 1-\mathrm{H}\right), 2.30\left(\mathrm{~m}_{\mathrm{c}}, 1 \mathrm{H}, 6-\mathrm{H}\right)$, $2.88\left(\mathrm{~m}_{\mathrm{c}}, 2 \mathrm{H}, 3-\mathrm{H}\right), 5.31$ (dt, $\left.J=15.5, J=5.5 \mathrm{~Hz}, 1 \mathrm{H}, 4-\mathrm{H}\right), 5.65$ (ddt, $J=15.5, J=5.5 \mathrm{~Hz}, J$ $=1.5 \mathrm{~Hz}, 1 \mathrm{H}, 5-\mathrm{H}) .-{ }^{13} \mathrm{C}-\mathrm{NMR}\left(62.9 \mathrm{MHz}, \mathrm{CDCl}_{3}\right.$, zusätzl. DEPT): $\delta=21.48$ (-, C-3), $22.21\left[+, \mathrm{C}-7\left(1^{\prime}\right)\right], 30.71(+, \mathrm{C}-6), 69.69(+, \mathrm{C}-1), 82.05\left(\mathrm{C}_{\text {quart }}, \mathrm{C}-2\right), 120.37(+, \mathrm{C}-4), 139.40$ $(+, \mathrm{C}-5)$.

\section{Allgemeine Arbeitsvorschrift A4 (Variante 1)}

\section{(2E)-6,6-Dimethylhept-4-en-1-in (103R) (Variante 1)}

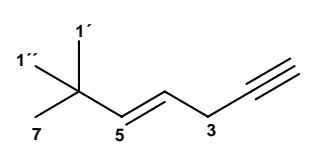

Die Reaktion wurde gemäß der allgemeinen Arbeitsvorschrift A4 mit (2E)1-Trimethylsilyl-6,6-dimethylhept-4-en-1-in $\mathbf{1 0 2 R}$ (6.08 g, $31.1 \mathrm{mmol})$ und $\mathrm{NaOH}\left(50 \mathrm{~mL}, 50 \mathrm{mmol}, 1.0 \mathrm{M}\right.$ in $\left.\mathrm{H}_{2} \mathrm{O}\right)$ durchgeführt und 4 h gerührt.

Die Reaktionslösung wurde mit ges. $\mathrm{NH}_{4} \mathrm{Cl}$-Lösung versetzt und mit Pentan extrahiert. Die wässrige Phase $(\mathrm{pH}=6)$ wurde mit Pentan $(3 \times 50 \mathrm{~mL})$ reextrahiert. Die vereinigte organische Phase wurde über $\mathrm{MgSO}_{4}$ getrocknet und das Lösungsmittel destillativ über eine lange Vigreuxkolonne $(20 \mathrm{~cm}, \mathrm{P}=300 \mathrm{mbar}$ ) entfernt. Das Rohprodukt wurde über fraktionierende Destillation gereinigt. Man erhielt 2.24 g (59\%) des Enins 103R als farblose Flüssigkeit, Sdp. 65 mbar $50-55^{\circ} \mathrm{C} .-{ }^{1} \mathrm{H}-\mathrm{NMR}\left(250 \mathrm{MHz}, \mathrm{CDCl}_{3}\right): \delta=1.01$ [s, $\left.9 \mathrm{H}, 7\left(1^{\prime \prime}\right)\left(1^{\prime}\right)-\mathrm{H}\right], 2.08\left(\mathrm{~m}_{\mathrm{c}}, 1\right.$ H, 1-H), $2.95\left(\mathrm{~m}_{\mathrm{c}}, 2 \mathrm{H}, 3-\mathrm{H}\right)$, 5.25-5.38 (m, $\left.1 \mathrm{H}, 4-\mathrm{H}\right), 5.68$ (m, $\left.1 \mathrm{H}, 5-\mathrm{H}\right) .-{ }^{13} \mathrm{C}-\mathrm{NMR}$ (62.9 $\mathrm{MHz}, \mathrm{CDCl}_{3}$, zusätzl. DEPT): $\delta=21.61(+, \mathrm{C}-3), 29.47$ [-, C-7(1")(1')], $32.83\left(\mathrm{C}_{\text {quart }}-6\right)$, 69.71 (C-1), 82.33 (C-2), 118.18 (-, C-4), 143.33 (-, C-5). 


\section{Allgemeine Arbeitsvorschrift A4 (Variante 1)}

\section{(4E)-5-Phenylpent-4-en-1-in 116}

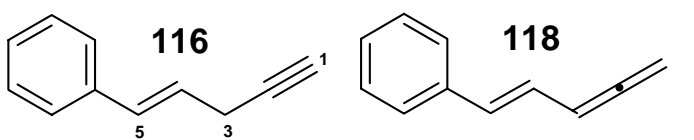

Die Reaktion wurde gemäß der allgemeinen Arbeitsvorschrift A4 (Variante 1) mit 1-Trimethylsilyl(4E)-5-phenylpent-4-en-1-in 115 (10.0 g, $47.0 \mathrm{mmol})$ und NaOH (75 mL, $75 \mathrm{mmol}, 1.0 \mathrm{M}$ in $\left.\mathrm{H}_{2} \mathrm{O}\right)$ in $\mathrm{MeOH}(200 \mathrm{~mL})$ durchgeführt und aufgearbeitet. Das Ende der Reaktion wurde über eine DC-Detektion festgestellt. Die Trimethylsilylverbindungen waren nach Oxidation mit Molybdänphosphorsäure schwach sichtbar, während die flüchtigen Enine nicht detektiert wurden. Nach Entfernung des Lösungsmittels bei 400 mbar wurde der Rückstand einer fraktionierenden Destillation ( $\mathrm{P}=200$ mbar) unterzogen, welches zur Niederschlagbildung führte. Der Umkondensationsversuch (bei $10 \mathrm{mbar}, 80^{\circ} \mathrm{C}$ ) blieb erfolglos. Die analytische Auswertung zeigte ein Gemisch des gewünschten Enins 116 (Ausbeute 39\%) und des unerwünschten trans-Isomeren (2E)-Penta-1,3,4-trienylbenzols 118 (Ausbeute 61\%) im Verhältnis 1:1.6 Durch Charakterisierung einer Testprobe nach säulenchromatographischer Reinigung konnte die Bildung des Allens bestätigt werden. ${ }^{[52]}$

\section{Allgemeine Arbeitsvorschrift A4 (Variante 2)}

\section{(4E)-5-Phenylpent-4-en-1-in 116}

$\mathrm{Zu}$ einer Lösung aus 1-Trimethylsilyl-(4E)-5-phenylpent-4-en-1-in 115 (52.1 mg, 0.244 mmol) in THF ( $5 \mathrm{~mL})$ wurde unter Argonatmosphäre bei $0{ }^{\circ} \mathrm{C}$ langsam $\mathrm{Bu}_{4} \mathrm{NF}(288 \mu \mathrm{L}, 0.488$ mmol, $1 \mathrm{M}$ in THF) zugetropft, $15 \mathrm{~min}$ bei $0{ }^{\circ} \mathrm{C}$ und $2 \mathrm{~h}$ bei Raumtemperatur gerührt. Zur Aufarbeitung wurde die Mischung mit gesättigter NaCl-Lösung versetzt. Die wässrige Phase wurde mit Diethylether reextrahiert. Die vereinigte organische Phase wurde über $\mathrm{MgSO}_{4}$ getrocknet, das Lösungsmittel im Vakuum entfert. Man erhielt ein braunes Öl, das ein Gemisch aus dem gewünschten Enin 116 (16\%) und unerwünschten trans-Isomeren (2E)-Penta-1,3,4trienylbenzol $118(61 \%)$ enthielt.

\section{Allgemeine Arbeitsvorschrift A4 (Variante 3)}

\section{(4E)-5-Phenylpent-4-en-1-in 116}

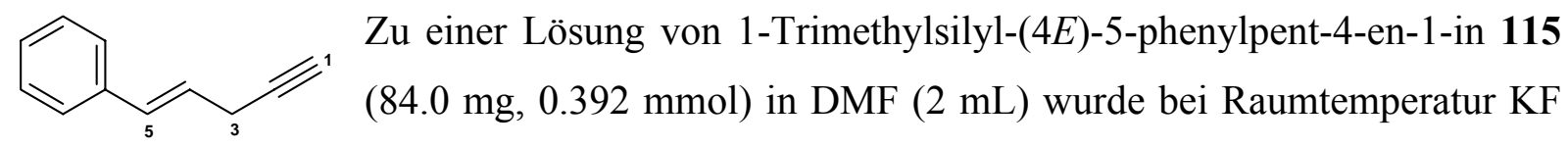


(45.6 mL, $0.784 \mathrm{mmol}$ ) zugegeben und $5 \mathrm{~h}$ gerührt. Zur Aufarbeitung wurde die Mischung mit Pentan verdünnt und mit gesättigter NaCl-Lösung gewaschen. Die vereinigte organische Phase wurde über $\mathrm{MgSO}_{4}$ getrocknet und das Lösungsmittel im Vakuum entfernt. Man erhielt $42.9 \mathrm{mg}(77 \%)$ des Enins 116 als farblose Flüssigkeit. $-{ }^{1} \mathrm{H}-\mathrm{NMR}\left(250 \mathrm{MHz}, \mathrm{CDCl}_{3}\right): \delta=$ 2.10 (t, $1 \mathrm{H}, 1-\mathrm{H}), 3.15$ (dd, 2 H, 3-H), 6.20 (dt, 1 H, 4-H), 6.65 (d, 1 H, 5-H), 7.20-7.40 (m, 5 H, Arom.-H).

\section{Allgemeine Arbeitsvorschrift A5 (Variante 1)}

\section{(E)-2-Methylhepta-1-enyldimethylalan (123)}

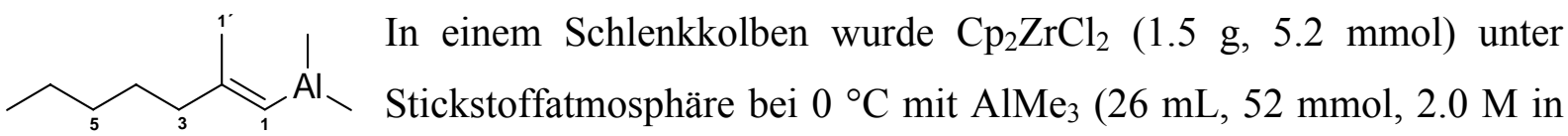
Hexan) versetzt. Das Hexan wurde bei $0{ }^{\circ} \mathrm{C}$ unter vermindertem Druck $(\mathrm{P}=0.002 \mathrm{mbar})$ entfernt. Anschließend wurde wasserfreies 1,2-Dichlorethan $(200 \mathrm{~mL})$ zugegeben und die zitronengelbe Reaktionslösung $30 \mathrm{~min}$ bei $0{ }^{\circ} \mathrm{C}$ gerührt. Eine Lösung von 1-Heptin (122) $(3.4 \mathrm{~mL}$, $2.5 \mathrm{~g}, 26 \mathrm{mmol})$ in 1,2-Dichlorethan $(10 \mathrm{~mL})$ wurde bei $0{ }^{\circ} \mathrm{C}$ zugetropft, die Reaktionslösung auf Raumtemperatur erwärmt und 15 h gerührt. Nach Beendigung der Reaktion (GCanalytische Untersuchung, 70-150 ${ }^{\circ} \mathrm{C}$, Steigerungsrate: 10\%, 5-8-5 min) wurde 1,2Dichlorethan und überschüssiges $\mathrm{AlMe}_{3}$ i.V. $\left(\mathrm{P}=0.002 \mathrm{mbar}, 0^{\circ} \mathrm{C}, 1.5 \mathrm{~h}\right)$ entfernt. Wasserfreies Hexan $(5 \mathrm{~mL})$ wurde bei $0{ }^{\circ} \mathrm{C}$ zugegeben, um das Zirconiumsalz auszufällen. Die gelbe Suspension wurde über eine Umkehrfritte unter Stickstoffatmosphäre abfiltriert und mit wasserfreiem Hexan $(10 \mathrm{~mL})$ nachgewaschen. Das Hexan wurde i.V. entfernt. Man erhielt $4.68 \mathrm{~g}$ (quant.) des Alans 123 als gelbes Öl. $-{ }^{1} \mathrm{H}-\mathrm{NMR}\left(250 \mathrm{MHz}, \mathrm{CDCl}_{3}\right): \delta=-0.78[\mathrm{~s}, 6 \mathrm{H}$, $\left.\mathrm{Al}\left(\mathrm{CH}_{3}\right)_{2}\right], 0.90(\mathrm{t}, J=6.5 \mathrm{~Hz}, 3 \mathrm{H}, 7-\mathrm{H}), 1.28\left[\mathrm{~m}_{\mathrm{c}}, 4 \mathrm{H}, 5(6)-\mathrm{H}\right], 1.50\left(\mathrm{~m}_{\mathrm{c}}, 2 \mathrm{H}, 4-\mathrm{H}\right) 2.02(\mathrm{~s}$, $\left.3 \mathrm{H}, 1^{\prime}-\mathrm{H}\right), 2.30$ (t, J=7.2 Hz, $\left.2 \mathrm{H}, 3-\mathrm{H}\right), 5.34$ (s, $\left.1 \mathrm{H}, 1-\mathrm{H}\right) .-{ }^{13} \mathrm{C}-\mathrm{NMR}\left(75.5 \mathrm{MHz}, \mathrm{CDCl}_{3}\right.$, zusätzl. DEPT): $\delta=0.20$ [+, $\left.\mathrm{Al}\left(\mathrm{CH}_{3}\right)_{2}\right], 13.92(+, \mathrm{C}-7), 22.40$ (-, C-6), $27.46(-, \mathrm{C}-* 4), 27,84$ (+, C-1'), 31.27 (-, C-*5), 44.96 (-, C-3), 119.90 (+, C-1), 156.50 (+, C quart, C-2).

\section{Allgemeine Arbeitsvorschrift A5 (Variante 2)}

\section{(E,E)-2,6-Dimethylhepta-1,4-dienyldimethylalan (123A)}

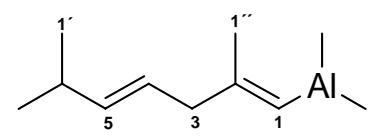

In einem Schlenkkolben wurde $\mathrm{Cp}_{2} \mathrm{ZrCl}_{2}(0.400 \mathrm{~g}, 1.37 \mathrm{mmol})$ unter

Stickstoffatmosphäre bei $0{ }^{\circ} \mathrm{C}$ mit $\mathrm{AlMe}_{3}(6.92 \mathrm{~mL}, 14 \mathrm{mmol}, 2.0 \mathrm{M}$ in Hexan) versetzt. Das Hexan wurde bei $0{ }^{\circ} \mathrm{C}$ unter vermindertem Druck $(\mathrm{P}=0.002$ mbar) ent- 
fernt. Anschließend wurde wasserfreies 1,2-Dichlorethan (5 mL) zugegeben und die zitronengelbe Reaktionslösung 30 min bei $0{ }^{\circ} \mathrm{C}$ gerührt. Eine Lösung von (2E)-6-Methylhept-4-en-1in 103A (0.75 g, $6.9 \mathrm{mmol})$ in 1,2-Dichlorethan $(10 \mathrm{~mL})$ wurde bei $0{ }^{\circ} \mathrm{C}$ zugetropft, das Kältebad entfernt und $2 \mathrm{~h}$ bei Raumtemperatur gerührt. Nach Beendigung der Reaktion (GCanalytische Untersuchung, 70-150 ${ }^{\circ} \mathrm{C}$, Steigerungsrate: $10 \%, 5-8-5 \mathrm{~min}$ ) wurden 1,2Dichlorethan und überschüssiges $\mathrm{AlMe}_{3}$ i.V. $\left(\mathrm{P}=0.002 \mathrm{mbar}, 0{ }^{\circ} \mathrm{C}, 1.5 \mathrm{~h}\right)$ entfernt. Wasserfreies Hexan $(1 \mathrm{~mL})$ wurde bei $0{ }^{\circ} \mathrm{C}$ zugegeben, um das Zirconiumsalz auszufällen. Die gelbe Suspension wurde über eine Umkehrfritte unter Stickstoffatmosphäre abfiltriert und mit wasserfreiem Hexan $(1 \mathrm{~mL})$ nachgewaschen. Das Hexan wurde i.V. entfernt. Man erhielt $0.85 \mathrm{~g}$ $(68 \%)$ des Alans 123A als gelbes Öl. $-{ }^{1} \mathrm{H}-\mathrm{NMR}\left(250 \mathrm{MHz}, \mathrm{CDCl}_{3}\right): \delta=-0.78[\mathrm{~s}, 6 \mathrm{H}$, $\left.\mathrm{Al}\left(\mathrm{CH}_{3}\right)_{2}\right], 0.98$ [d, J = 6.6 Hz, $\left.6 \mathrm{H}, 7\left(1^{\prime}\right)-\mathrm{H}\right], 2.01$ (s, $\left.3 \mathrm{H}, 1 "-\mathrm{H}\right), 2.30\left(\mathrm{~m}_{\mathrm{c}}, 1 \mathrm{H}, 6-\mathrm{H}\right), 2.92$ (d, $J=5.0 \mathrm{~Hz}, 2 \mathrm{H}, 3-\mathrm{H}), 5.26-5.54$ [m, $3 \mathrm{H}, 1(4,5)-\mathrm{H}]$.

\section{(E,E)-2,6,6-Trimethylhepta-1,4-dienyldimethylalan (123R)}

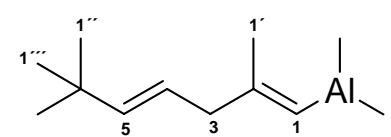

Die Reaktion wurde gemäß der allgemeinen Arbeitsvorschrift A5

(Variante 2) mit (2E)-6,6-Dimethylhept-4-en-1-in 103R (0.572 g, $4.68 \mathrm{mmol}), \mathrm{Cp}_{2} \mathrm{ZrCl}_{2}(0.274 \mathrm{~g}, 0.936 \mathrm{mmol})$ und $\mathrm{AlMe}_{3}(4.68 \mathrm{~mL}, 9.36 \mathrm{mmol}, 2.0 \mathrm{M}$ in $\mathrm{He}-$ xan) in 1,2-Dichlorethan (5 mL) durchgeführt. Man erhielt $0.813 \mathrm{~g}(89 \%)$ des Alans 123R als gelbes Öl. $-{ }^{1} \mathrm{H}$ NMR $\left(250 \mathrm{MHz}, \mathrm{CDCl}_{3}\right): \delta=0.08$ [s, $\left.6 \mathrm{H}, \mathrm{Al}\left(\mathrm{CH}_{3}\right)_{2}\right], 1.0$ [s, $9 \mathrm{H}, 7\left(1^{\prime \prime}\right)\left(1^{\prime \prime \prime}\right)-$ H], 2.01 (s, 3 H, 1'-H), 2.95 (d, $J=5.0 \mathrm{~Hz}, 2$ H, 3-H), 5.24-5.60 [m, 3 H, 1(4)(5)-H].

\section{Optimierung der $\mathrm{AlMe}_{3}$-freien Alan-Herstellung}

Die Darstellung der $\mathrm{AlMe}_{3}$-freien Alane wurde wie folgt durchgeführt und optimiert: In einem trockenen Schlenkkolben wurde unter Schutzgas 0.25 eq $\mathrm{Cp}_{2} \mathrm{ZrCl}_{2}$ in wasserfreiem Dichlorethan bei $0{ }^{\circ} \mathrm{C}$ mit 2.0 eq AlMe - Lösung in Hexan versetzt und 30 min bei $0{ }^{\circ} \mathrm{C}$ gerührt. Dichlorethan wurde gerade soviel zugegeben, bis eine homogene Lösung vorlag. Die eingesetzten $\mathrm{AlMe}_{3}$-Lösungen wurden bevorzugt auf 2.0 M Hexanlösung eingestellt, um größere Verdünnungen zu vermeiden. Die Entfernung des im Reaktionskolben befindlichen Hexans unter vermindertem Druck, wie von Lipshutz et al. ${ }^{[57]}$ angegeben, erwies sich experimentell als nicht vorteilhaft, da ein nicht unerheblicher Anteil an $\mathrm{AlMe}_{3}$ frühzeitig aus der Reaktionslösung entfernt wurde und sich dadurch im Schlenkhahn niederschlug. Der Einsatz von 1.52.0 eq $\mathrm{AlMe}_{3}$ erschien aufgrund der durchgeführten Untersuchungen notwendig, da die entsprechenden in situ durchgeführten Umsetzungen mit 1.25 eq $\mathrm{AlMe}_{3}$ nicht die erhofften Re- 
sultate ergaben und mit auffällig geringen Ausbeuten an Kreuzkupplungsprodukten verbunden waren. Eine Lösung entsprechender Alkine wurde bei $0{ }^{\circ} \mathrm{C}$ zugegeben und gerührt. Eine vollständige Umsetzung wurde erst nach einer Reaktionszeit von 2 h erreicht. Die Qualität der Alane sowie das Ende der Umsetzung wurden stets GC-analytisch sowie spektroskopisch $\left({ }^{1} \mathrm{H}\right.$ sowie ${ }^{13} \mathrm{C}$-NMR) überprüft. Hierzu wurden $50 \mu \mathrm{L}$ der Reaktionslösung durch Zugabe eines Gemisches aus 1.0 M HCl und Pentan hydrolysiert. Die Pentanschicht wurde gegen die entsprechenden Alkine als Referenz analysiert. Für die Alkine 103A, 103R und 122 bzw. ihre Hydrolyseprodukte 126, 126A und 126R (siehe unten) ergaben sich folgende Werte: für 1Heptin (122) $1.488 \mathrm{~min}$, für das 1-Alken $1262.148 \mathrm{~min}$ (bei $50-120{ }^{\circ} \mathrm{C}$, Steigerungsrate 10 ${ }^{\circ} \mathrm{C} / \mathrm{min}$, 5-10-5 min), für (2E)-6-Methylhept-4-en-1-in (103A) $1.4 \mathrm{~min}$, für das Dien 126A 1.7 $\min$ (bei $50-150{ }^{\circ} \mathrm{C}$, Steigerungsrate $10{ }^{\circ} \mathrm{C} / \mathrm{min}, 5-8-5 \mathrm{~min}$ ), für (2E)-6,6-Dimethylhept-4-en1-in (103R) $1.8 \mathrm{~min}$ und für das Dien 126R $2.2 \mathrm{~min}$ (bei 70-150 ${ }^{\circ} \mathrm{C}$, Steigerungsrate 10 $\left.{ }^{\circ} \mathrm{C} / \mathrm{min}, 5-8-5 \mathrm{~min}\right)$.

Zur spektroskopischen Untersuchung der Alane während des Reaktionsverlaufs wurden 100 $\mu \mathrm{L}$ der Reaktionslösung unter Argonatmosphäre in einem trockenen $10 \mathrm{~mL}$ Schlenkkolben aufgenommen und das Lösungsmittel entfernt. Das Rohprodukt wurde mit $\mathrm{CDCl}_{3}$ versetzt und anschließend in einem NMRRöhrchen mit Schutzgasvorrichtung der Messung überführt. Durch dieses Analytikverfahren ließen sich wertvolle Informationen über Zustand und Qualität der besonders luft- und hydrolyseempfindlichen Alane vor und nach der Isolierung als Referenz gewinnen. Nach Reaktionsende wurden das Lösungsmittel Dichlorethan und das überschüssige $\mathrm{AlMe}_{3}$ bei $0{ }^{\circ} \mathrm{C}$ unter vermindertem Druck (0.002 mbar, $1.5 \mathrm{~h}$ ) und ständigem Rühren entfernt. Wasserfreies Hexan wurde bei $0{ }^{\circ} \mathrm{C}$ zugegeben, um das Zirconiumsalz auszufällen. Die gelbe Suspension wurde über einer Umkehrfritte unter Stickstoffatmosphäre abfiltriert und mit wenig wasserfreiem Hexan nachgewaschen. Das Hexan wurde unter vermindertem Druck entfernt. Die isolierten gelb- bis gelborange-farbenen, öligen Alane wurden als molare Lösungen in trockenem Hexan eingeeicht und eingesetzt. Die isolierten Alane waren 4-6 Wochen haltbar. 


\section{Synthese der Pyronderivate}

\section{Allgemeine Arbeitsvorschrift A6}

\section{2-Methyl-3-oxoheptansäureethylester (84)}

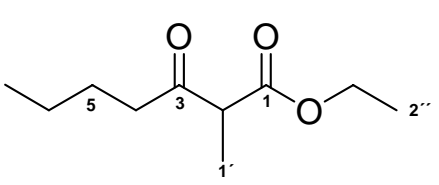

$\mathrm{Zu}$ einer Lösung von Diisopropylamin $(30.50 \mathrm{~mL}, 22.02 \mathrm{~g}, 0.2176$ mol) in wasserfreiem THF $(380 \mathrm{~mL})$ wurde bei $0{ }^{\circ} \mathrm{C}$ unter Stickstoffatmosphäre $n \mathrm{BuLi}(95 \mathrm{~mL}, 0.22 \mathrm{~mol}, 2.27 \mathrm{M}$ in Hexan) langsam zugegeben und $30 \mathrm{~min}$. gerührt. Eine Lösung von 2-Methyl-3-oxo-butansäureethylester (14) (14.12 g, $97.94 \mathrm{mmol})$ in wasserfreiem THF (5 mL) wurde tropfenweise zugegeben und $30 \mathrm{~min}$ gerührt. Eine Lösung von Propyliodid $(10.5 \mathrm{~mL}, 18.3 \mathrm{~g}, 0.108 \mathrm{~mol})$ in THF $(5 \mathrm{~mL})$ wurde bei $0{ }^{\circ} \mathrm{C}$ zugetropft und $2.5 \mathrm{~h}$ gerührt. Die Reaktionslösung wurde auf Raumtemperatur erwärmt und langsam mit konz. $\mathrm{HCl}(12 \mathrm{~mL})$ versetzt. Die organische Phase wurde abge-

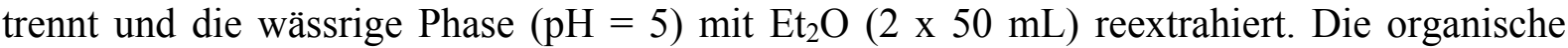
Phase wurde mit $\mathrm{H}_{2} \mathrm{O}(2 \times 20 \mathrm{~mL})$ gewaschen $(\mathrm{pH}=7)$ und extrahiert. Die vereinigte organische Phase wurde über $\mathrm{MgSO}_{4}$ getrocknet und das Lösungsmittel i. V. entfernt. Das Rohprodukt wurde fraktioniert destilliert. Man erhielt 10.23 g (56\%) des Ketoesters 84, Sdp.8mbar 90 ${ }^{\circ} \mathrm{C}$ (Lit. ${ }^{[144]} \mathrm{Sdp} .10 \mathrm{mmHg} 112-124{ }^{\circ} \mathrm{C}$ ), als farblose Flüssigkeit. - IR (film): v $=2984 \mathrm{~cm}^{-1}, 2961$, 2875, $1749(\mathrm{C}=\mathrm{O}), 1716(\mathrm{C}=\mathrm{O}), 1653,1457,1377,1327,1270,1239,1198,1115,1069$, 1027, 860, 739, 704. - ${ }^{1} \mathrm{H}-\mathrm{NMR}\left(250 \mathrm{MHz}, \mathrm{CDCl}_{3}\right): \delta=0.91(\mathrm{t}, J=6.4 \mathrm{~Hz}, 3 \mathrm{H}, 7-\mathrm{H}), 1.26$ (t, $J=6.8 \mathrm{~Hz}, 3 \mathrm{H}, 2 "-\mathrm{H}), 1.29$ (d, $\left.J=6.8 \mathrm{~Hz}, 3 \mathrm{H}, 1^{\prime}-\mathrm{H}\right), 1.31\left(\mathrm{~m}_{\mathrm{c}}, 2 \mathrm{H}, 6-\mathrm{H}\right), 1.54\left(\mathrm{~m}_{\mathrm{c}}, 2 \mathrm{H}\right.$, 5-H), $2.54\left(\mathrm{~m}_{\mathrm{c}}, 2 \mathrm{H}, 4-\mathrm{H}\right), 3.50$ (q, J=7.4 Hz, $\left.1 \mathrm{H}, 2-\mathrm{H}\right), 4.18$ (q, J= $\left.7.4 \mathrm{~Hz}, 2 \mathrm{H}, 1 "-\mathrm{H}\right)$. ${ }^{13} \mathrm{C}-\mathrm{NMR}\left(62.9 \mathrm{MHz}, \mathrm{CDCl}_{3}\right.$, zusätzl. DEPT): $\delta=12.53(+, \mathrm{C}-1$ '), $13.61(+, \mathrm{C}-7), 13.86(+$, C-2"), 21.97 (-, C-6), 25.40 (-, C-5), 40.88 (-, C-4), 52.58 (+, C-2), 61.02 (-, C-1"), 170.40 $\left(\mathrm{C}_{\text {quart }}, \mathrm{C}-1\right), 205.73\left(\mathrm{C}_{\text {quart }}, \mathrm{C}-3\right) .-\mathrm{MS}(\mathrm{EI}, 70 \mathrm{eV}), \mathrm{m} / z(\%): 186(10)\left[\mathrm{M}^{+}\right], 157$ (3) $\left[\mathrm{M}^{+}-\right.$ $\left.\mathrm{CH}_{2} \mathrm{CH}_{3}\right], 141(8)\left[\mathrm{M}^{+}-\mathrm{OCH}_{2} \mathrm{CH}_{3}\right], 129$ (6), 102 (28), 85 (100) $\left[\mathrm{C}_{5} \mathrm{H}_{9} \mathrm{O}^{+}\right], 74$ (6), 57 (23). $\mathrm{C}_{10} \mathrm{H}_{18} \mathrm{O}_{3}$ (186.3): ber. C 64.49, H 9.74; gef. C 64.28, H 9.49.

\section{Allgemeine Arbeitsvorschrift A7}

\section{4-Acetyl-2-methyl-3-oxoheptansäureethylester (64)}

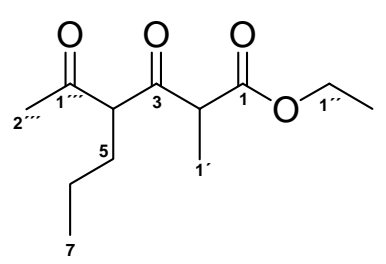

$\mathrm{Zu}$ einer Lösung von Diisopropylamin (30.8 mL, $22.2 \mathrm{~g}, 0.220 \mathrm{~mol})$ in wasserfreiem THF $(500 \mathrm{~mL})$ wurde unter Stickstoffatmosphäre bei $0{ }^{\circ} \mathrm{C} n \mathrm{BuLi}(87 \mathrm{~mL}, 0.22 \mathrm{mmol}, 2.5 \mathrm{M}$ in Cyclohexan) zugegeben 
und 30 min gerührt. Eine Lösung von 2-Methyl-3-oxoheptansäureethylester (84) (15.60 g, $83.76 \mathrm{mmol})$ in wasserfreiem THF $(10 \mathrm{~mL})$ wurde bei $0{ }^{\circ} \mathrm{C}$ tropfenweise zugegeben und $1 \mathrm{~h}$ gerührt. Die Reaktionslösung wurde auf $-78{ }^{\circ} \mathrm{C}$ abgekühlt und eine Lösung von $N$-Acetylimidazol $^{[29]}(13.84$ g, $0.1257 \mathrm{~mol})$ in trockenem THF $(150 \mathrm{~mL})$ zugegeben. Die Reaktionslösung wurde $2 \mathrm{~h}$ bei $-78{ }^{\circ} \mathrm{C}$ gerührt. Die Reaktion wurde nach Hydrolyse mit ges. $\mathrm{NH}_{4} \mathrm{Cl}-$ Lösung auf Raumtemperatur erwärmt. Die wässrige Phase $(\mathrm{pH}<7)$ wurde abgetrennt und mit EtOAc $(2 \times 50 \mathrm{~mL})$ extrahiert. Die vereinigte organische Phase wurde mit $\mathrm{H}_{2} \mathrm{O}(100 \mathrm{~mL})$ sowie ges. NaCl-Lösung $(100 \mathrm{~mL})$ gewaschen. Die wässrige Phase wurde mit EtOAc $(2 \times 50$ $\mathrm{mL})$ reextrahiert. Die vereinigte organische Phase wurde über $\mathrm{MgSO}_{4}$ getrocknet und i.V. konzentriert. Man erhielt 20.84 g des $\beta, \delta$-Ketoesters 64 als viskoses braunes Öl. Das Rohprodukt wurde aufgrund starker Zersetzung bei Säulenchromatographie ohne weitere Reinigung umgesetzt. - IR (film): $v=3057 \mathrm{~cm}^{-1}, 2987,2963,2937,2875,1720(\mathrm{C}=\mathrm{O}), 1700(\mathrm{C}=\mathrm{O})$, 1653, 1457, 1379, 1266, 737, 704. - ${ }^{1} \mathrm{H}-\mathrm{NMR}\left(250 \mathrm{MHz}, \mathrm{CDCl}_{3}\right): \delta=0.93\left(\mathrm{~m}_{\mathrm{c}}, 3 \mathrm{H}, 7-\mathrm{H}\right)$, 1.10-1.60 [m, 8 H, 1'(2")(6)-H], 1.68-2.01 (m, 2 H, 5-H), 2.15 (m, 3 H, 2"'-H), $3.65\left(\mathrm{~m}_{\mathrm{c}}, 1 \mathrm{H}\right.$, 2-H), 3.80-3.96 (m, $1 \mathrm{H}, 4-\mathrm{H}), 4.15\left(\mathrm{~m}_{\mathrm{c}}, 2 \mathrm{H}, 1 "-\mathrm{H}\right) .-\mathrm{MS}$ (ESI), m/z (\%): 479 (46) $[2 \mathrm{M}+\mathrm{Na}]^{+}, 292(100), 251(18)[\mathrm{M}+\mathrm{Na}]^{+}$.

\section{Allgemeine Arbeitsvorschrift A8}

\section{3,6-Dimethyl-4-hydroxy-5-propylpyran-2-on (63)}<smiles>CCCc1c(I)oc(=O)c(I)c1O</smiles>

$\mathrm{Zu}$ einer Lösung von 4-Acetyl-2-methyl-3-oxo-heptansäureethylester (64) (20.73 g, $90.79 \mathrm{mmol})$ in wasserfreiem Benzol $(300 \mathrm{~mL})$ wurde DBU (16.42 mL, $16.59 \mathrm{~g}, 108.9 \mathrm{mmol})$ gegeben und unter Rückfluß $\left(95^{\circ} \mathrm{C}\right) 6 \mathrm{~h}$ gerührt. Die Reaktionslösung wurde mit ges. $\mathrm{NaHCO}_{3}$-Lösung versetzt und mit $\mathrm{Et}_{2} \mathrm{O}$ extrahiert. Die wässrige Phase $(\mathrm{pH}=9)$ wurde $\mathrm{mit}^{\mathrm{Et}} \mathrm{Et}_{2} \mathrm{O}(2 \times 100 \mathrm{~mL})$ reextrahiert. Die vereinigte organische Phase wurde über $\mathrm{Na}_{2} \mathrm{SO}_{4}$ getrocknet und i. V. konzentriert. Man erhielt $7.552 \mathrm{~g}$ des Zersetzungsproduktes 2-Methyl-3-oxoheptansäureethylester (84) als Nebenprodukt. Die erhaltene wässrige Phase wurde erst mit $\mathrm{Et}_{2} \mathrm{O}(300 \mathrm{~mL})$ und anschließend mit konz. $\mathrm{HCl}(38$ \%ige Lsg) versetzt und der pH-Wert der wässrigen Phase auf 2 eingestellt. Die organische Phase wurde getrennt und die wässrige Phase mit $\mathrm{Et}_{2} \mathrm{O}(3 \times 50 \mathrm{~mL})$ reextrahiert. Die vereinigte organische Phase wurde über $\mathrm{Na}_{2} \mathrm{SO}_{4}$ getrocknet und i. V. konzentriert. Das Rohprodukt wurde durch Säulenchromatographie an Kieselgel gereinigt (800 g, Hex/EE 2:1 + 5\% Me$\mathrm{OH})$. Man erhielt $9.71 \mathrm{~g}\left(64 \%\right.$ über 2 Stufen) des Pyrons $63\left(R_{f}=0.35\right)$ als weißen Feststoff (Smp. $\left.81{ }^{\circ} \mathrm{C}\right) .-$ IR (film): $v=3205 \mathrm{~cm}^{-1}(\mathrm{OH}), 2962,2873,1669(\mathrm{C}=\mathrm{O}), 1569,1456,1223$, 
1174, 1131, 1056, 945, 761. - ${ }^{1} \mathrm{H}-\mathrm{NMR}\left(250 \mathrm{MHz}, \mathrm{CDCl}_{3}\right): \delta=0.92(\mathrm{t}, J=7.4 \mathrm{~Hz}, 3 "-\mathrm{H})$, 1.49 (m $\left.\mathrm{m}_{\mathrm{c}}, 2 \mathrm{H}, 2^{\prime \prime}-\mathrm{H}\right), 1.99$ (s, 3 H, 1"'-H), 2.21 (s, 3 H, 1'-H), 2.36 (t, J= 8.0 Hz, 2 H, 1"-H). ${ }^{13} \mathrm{C}-\mathrm{NMR}\left(62.9 \mathrm{MHz}, \mathrm{CDCl}_{3}\right.$, zusätzl. DEPT): $\delta=8.65\left(+, \mathrm{C}-1{ }^{\prime \prime \prime}\right), 13.70(+, \mathrm{C}-3 "), 16.89(+$,

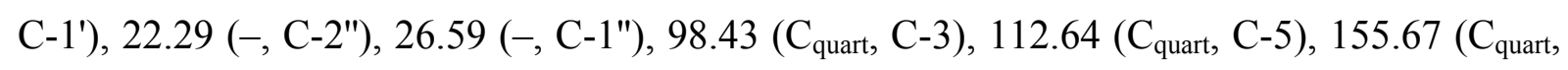
C-6), 166.37 ( $\left.\mathrm{C}_{\text {quart }}, \mathrm{C}-4\right), 167.18\left(\mathrm{C}_{\text {quart }}, \mathrm{C}-2\right) .-\mathrm{MS}(\mathrm{EI}, 70 \mathrm{eV}), m / z(\%): 182(16)\left[\mathrm{M}^{+}\right], 154$ (16) $\left[\mathrm{M}^{+}-\mathrm{C}_{2} \mathrm{H}_{4}\right], 127$ (9) $\left[\mathrm{M}^{+}-\mathrm{C}_{4} \mathrm{H}_{7}\right], 125$ (18) $\left[\mathrm{M}^{+}-\mathrm{C}_{4} \mathrm{H}_{9}\right], 97$ (20), 86 (20), 84 (35), 83 (12), $71(30), 69(18), 57(53)\left[\mathrm{C}_{3} \mathrm{H}_{5} \mathrm{O}^{+}\right], 55(26), 47(10), 43(100)\left[\mathrm{C}_{3} \mathrm{H}_{7}^{+}\right], 41$ (37).

\section{Allgemeine Arbeitsvorschrift A9 (Variante 1)}

\section{Benzoesäure-3,6-dimethyl-2-oxo-5-propyl-2H-pyran-4-ylester (89)}

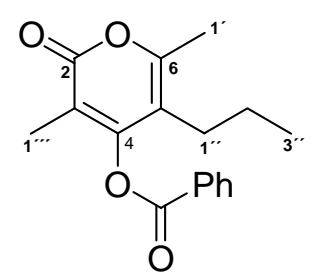

Eine Mischung aus dem Pyron 63 (5.17 g, $28.4 \mathrm{mmol})$ und Benzoylchlorid (3.63 mL, $4.40 \mathrm{~g}, 31.3 \mathrm{mmol})$ in Pyridin $(50 \mathrm{~mL})$ wurde unter Stickstoffatmosphäre bei Raumtemperatur $44 \mathrm{~h}$ gerührt. Es bildete sich sofort ein weißer Niederschlag, der auf die Bildung der $N$-Acetylpyridiniumchlorid-

Zwischenstufe hindeutete. Die rote Reaktionslösung wurde mit ges. $\mathrm{NaHCO}_{3}$-Lösung (50 $\mathrm{mL}$ ) versetzt und mit EtOAc extrahiert. Die organische Phase wurde getrennt, die wässrige Phase $(\mathrm{pH}=9)$ mit EtOAc $(2 \times 100 \mathrm{~mL})$ reextrahiert. Die vereinigte organische Phase wurde über $\mathrm{Na}_{2} \mathrm{SO}_{4}$ getrocknet und i. V. konzentriert. Das Rohprodukt wurde säulenchromatographisch an Kieselgel gereinigt (550 g, Hex/EE 8:1 + 5\% MeOH). Man erhielt 7.21 g (89\%) des Benzoats $89\left(R_{f}=0.30\right)$ als farbloses Öl. - IR (film): $v=2965 \mathrm{~cm}^{-1}, 1743(\mathrm{C}=\mathrm{O})$, $1707(\mathrm{C}=\mathrm{O}), 1577,1387,1256,1178,1114,1066,908,733 .-{ }^{1} \mathrm{H}-\mathrm{NMR}\left(250 \mathrm{MHz}, \mathrm{CDCl}_{3}\right): \delta$ $=0.87(\mathrm{t}, J=7.4 \mathrm{~Hz}, 3 \mathrm{H}, 3 "-\mathrm{H}), 1.47\left(\mathrm{~m}_{\mathrm{c}}, 2 \mathrm{H}, 2^{\prime \prime}-\mathrm{H}\right), 1.92$ (s, $\left.3 \mathrm{H}, 1 " '-\mathrm{H}\right), 2.22$ (t, $J=7.4 \mathrm{~Hz}$, $2 \mathrm{H}, 1 "-\mathrm{H}), 2.28$ (s, $\left.3 \mathrm{H}, 1^{\prime}-\mathrm{H}\right), 7.54\left(\mathrm{~m}_{\mathrm{c}}, 2 \mathrm{H}, \mathrm{Ar}-\mathrm{H}\right), 7.70\left(\mathrm{~m}_{\mathrm{c}}, 1 \mathrm{H}, \mathrm{Ar}-\mathrm{H}\right), 8.15\left(\mathrm{~m}_{\mathrm{c}}, 2 \mathrm{H}, \mathrm{Ar}-\right.$ H). $-{ }^{13} \mathrm{C}-\mathrm{NMR}\left(62.9 \mathrm{MHz}, \mathrm{CDCl}_{3}\right.$, zusätzl. DEPT): $\delta=10.34$ (+, C-1"'), 13.71 (+, C-3"), 17.21 (+, C-1'), 22.69 (-, C-2"), 27.43 (-, C-1"), 112.77 (C $\left.\mathrm{C}_{\text {quart }}, \mathrm{C}-3\right), 113.36$ (C quart $\left._{\text {, }} \mathrm{C}-5\right)$, $127.70\left(\mathrm{C}_{\text {quart }}, \mathrm{C}-\mathrm{Ar}\right), 128.91(+, \mathrm{C}-\mathrm{Ar}), 130.23(+, \mathrm{C}-\mathrm{Ar}), 134.42(+, \mathrm{C}-\mathrm{Ar}), 156.32\left(\mathrm{C}_{\text {quart }}, \mathrm{C}-\right.$ 6), $159.46\left(\mathrm{C}_{\text {quart }}, \mathrm{C}-4\right), 162.57 \quad\left[\mathrm{C}_{\text {quart }}, \mathrm{O}(\mathrm{CO}) \mathrm{Ph}\right], 164.50\left(\mathrm{C}_{\text {quart }}, \mathrm{C}-2\right) .-\mathrm{MS}$ (ESI, $\left.\mathrm{MeOH} / \mathrm{NH}_{4} \mathrm{OAc}\right): 573(46)[2 \mathrm{M}+\mathrm{H}]^{+}, 555(77)[2 \mathrm{M}-\mathrm{OH}]^{+}, 433(23), 287(100)[\mathrm{M}+\mathrm{H}]^{+}$. $-\mathrm{C}_{17} \mathrm{H}_{18} \mathrm{O}_{4}$ (286.3): ber. C 71.31, H 6.34; gef. C 71.12, H 6.02. 


\section{Allgemeine Arbeitsvorschrift A9 (Variante 2)}

\section{Essigsäure-3,6-dimethyl-2-oxo-5-propyl-2H-pyran-4-ylester (88)}

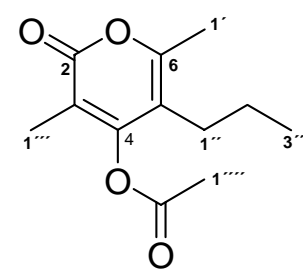

$\mathrm{Zu}$ einer Lösung von Acetylchlorid (1.84 mL, $2.03 \mathrm{~g}, 25.9 \mathrm{mmol})$ in Dioxan $(140 \mathrm{~mL})$ wurde Pyridin $(2.09 \mathrm{~mL}, 2.05 \mathrm{~g}, 25.9 \mathrm{mmol})$ bei $0{ }^{\circ} \mathrm{C}$ zugegeben und 10 min gerührt. Nach Zugabe einer Lösung des Pyrons 63 (4.7068 g, $25.862 \mathrm{mmol})$ in Dioxan $(20 \mathrm{~mL})$ bei $0{ }^{\circ} \mathrm{C}$ wurde das Kältebad entfernt und $20 \mathrm{~h}$ bei Raumtemperatur gerührt. Die Reaktionsmischung wurde über eine Celite/Aktivkohle-Schicht durch eine Umkehrfritte filtriert und mit EtOH (20 mL) und $\mathrm{Et}_{2} \mathrm{O}(100$ mL) nachgewaschen. Das Filtrat wurde i.V. konzentriert. Das Rohprodukt wurde säulenchromatographisch an Kieselgel gereinigt (800 g, Hex/EE 2:1). Man erhielt 3.996 g (69\%) des Acetatesters $88\left(R_{f}=0.55\right)$ als farbloses Öl. - IR (film): $v=2964 \mathrm{~cm}^{-1}, 2932,1772(\mathrm{C}=\mathrm{O})$, $1713(\mathrm{C}=\mathrm{O}), 1580,1366,1201,1180,1112,1062,908,732 .-{ }^{1} \mathrm{H}-\mathrm{NMR}\left(250 \mathrm{MHz}, \mathrm{CDCl}_{3}\right): \delta$ $=0.90(\mathrm{t}, J=7.1 \mathrm{~Hz}, 3 \mathrm{H}, 3 "-\mathrm{H}), 1.42\left(\mathrm{~m}_{\mathrm{c}}, 2 \mathrm{H}, 2^{\prime \prime}-\mathrm{H}\right), 1.83$ (s, $\left.3 \mathrm{H}, 1 " '-\mathrm{H}\right), 2.14$ (t, $J=7.8 \mathrm{~Hz}$, 2 H, 1"-H), 2.20 (s, 3 H, 1'"'-H), 2.30 (s, 3 H, 1'-H). - ${ }^{13}$ C-NMR (50.3 MHz, CDCl 3 , zusätzl. APT): $\delta=10.26$ (+, C-1"'), 13.85 (+, C-3"), 17.19 (+, C-1'), 20.39 (+, C-1'"'), 22.55 (-, C-2"), 27.48 (-, C-1"), 112.55 (-, C quart, $\left.C^{*}-3\right), 113.09$ (-, C $\left.\mathrm{Cuart}_{\text {qu }} \mathrm{C}^{*}-5\right), 156.30$ (-, $\left.\mathrm{C}_{\text {quart }}, \mathrm{C}-6\right)$, 159.10 (-, $\left.\mathrm{C}_{\text {quart }}, \mathrm{C}-4\right), 164.46\left[-, \mathrm{C}_{\text {quart }}, \mathrm{O}\left(\mathrm{C}^{*} \mathrm{O}\right) \mathrm{CH}_{3}\right], 166.69\left(\mathrm{C}_{\text {quart }}, \mathrm{C}^{*}-2\right) .-\mathrm{MS}(\mathrm{EI}$, $70 \mathrm{eV}), m / z(\%): 252(<1), 225(4)[\mathrm{M}+\mathrm{H}]^{+}, 224(26)[\mathrm{M}]^{+}, 206(<1), 183(8), 182(100), 167$ (3), 155 (6), 154 (81), 140 (<1), 126 (8), 125 (30), 111 (4), 97 (21), 83 (9), 67 (2), 55 (9), 43 (92), 41 (5). $-\mathrm{C}_{12} \mathrm{H}_{16} \mathrm{O}_{4}$ (224.26): ber. C 64.27, H 7.19, gef. C 64.39, H 7.24.

\section{Essigsäure-6-brommethyl-3-methyl-2-oxo-5-propyl-2H-pyran-4-ylester (183)}

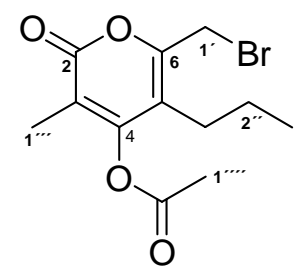

Die Reaktion wurde gemäß der allgemeinen Arbeitsvorschrift A9 (Variante 2) mit 6-Brommethyl-4-hydroxy-3-methyl-2-oxo-5-propyl-2H-pyran (97) (145 mg, $0.556 \mathrm{mmol})$, Acetylchlorid $(39.5 \mu \mathrm{L}, 43.6 \mathrm{mg}, 0.556 \mathrm{mmol})$ und Pyridin $(44.9 \mu \mathrm{L}, 43.9 \mathrm{mg}, 0.556 \mathrm{mmol})$ in Dioxan $(3 \mathrm{~mL})$ durchgeführt und 38 h gerührt. Das Rohprodukt wurde säulenchromatographisch an akt. ALOX gereinigt (10 g, Hex/EE 7:1). Man erhielt $8.37 \mathrm{mg}(50 \%)$ des Bromesters $183\left(R_{f}=0.33\right)$ als weißen Feststoff. $-{ }^{1} \mathrm{H}-\mathrm{NMR}\left(250 \mathrm{MHz}, \mathrm{CDCl}_{3}\right): \delta=1.05$ (t, $\left.J=7.2 \mathrm{~Hz}, 3 \mathrm{H}, 3 "-\mathrm{H}\right), 1.60\left(\mathrm{~m}_{\mathrm{c}}, 2 \mathrm{H}\right.$, 2"-H), 1.95 (s, 3 H, 1'"-H), 2.38-2.48 [m, 5 H, 1"(1"')-H], 4.60 (s, 2 H, 1'-H). 


\section{Allgemeine Arbeitsvorschrift A10}

\section{Benzoesäure-6-formyl-3-methyl-2-oxo-5-propyl-2H-pyran-4-ylester (94)}<smiles>CCCc1c(C=O)oc(=O)c(I)c1OC(=O)c1ccccc1</smiles>

In einem Bombenrohr wurde $\mathrm{zu}$ einer Lösung von Benzoesäure-3,6dimethyl-2-oxo-5-propyl-2H-pyran-4-ylester 89 (9.76 g, $34.1 \mathrm{mmol})$ in wasserfreiem Dioxan $(200 \mathrm{~mL})$ akt. Selendioxid ${ }^{[77]}(20.0 \mathrm{~g}, 0.180 \mathrm{~mol})$ in 3 Portionen je 2.0 eq gegeben und bei $130{ }^{\circ} \mathrm{C} 18 \mathrm{~h}$ gerührt. Die Reaktionsmischung wurde auf Raumtemperatur abgekühlt, über eine $\mathrm{Na}_{2} \mathrm{SO}_{4} /$ CeliteSchicht abfiltriert und mit $\mathrm{Et}_{2} \mathrm{O}(200 \mathrm{~mL})$ nachgewaschen. Das Filtrat wurde unter vermindertem Druck konzentriert. Man erhielt 10.16 g (99\%) des Aldehyds 94 (Hex/EE 4:1, $R_{f}=0.32$ ) als weißen Feststoff $\left(\mathrm{Smp} .=97{ }^{\circ} \mathrm{C}\right)$ mit einer Reinheit von $>95 \% .-$ IR $(\mathrm{KBr}): v=3055 \mathrm{~cm}^{-1}$, 2969, $1748(\mathrm{C}=\mathrm{O}), 1728(\mathrm{C}=\mathrm{O}), 1748(\mathrm{C}=\mathrm{O}), 1636,1266,1242,1193,1177,1045,739,705$. $-{ }^{1} \mathrm{H}-\mathrm{NMR}\left(300 \mathrm{MHz}, \mathrm{CDCl}_{3}\right.$ ): $\delta=0.89$ (t, $J=7.4 \mathrm{~Hz}, 3 \mathrm{H}, 3$ "-H), 1.54 (sext, $J=7.4 \mathrm{~Hz}, 2 \mathrm{H}$, 2"-H), 2.01 (s, 3 H, 1"'-H), 2.68 (bs, 2 H, 1"-H), 7.55 (t, J = 7.8 Hz, 2 H, Ar-H), 7.70 (m, 1 H, Ar-H), 8.15 (m $\left.\mathrm{m}_{\mathrm{c}}, 2 \mathrm{H}, \mathrm{Ar}-\mathrm{H}\right), 9.78$ (1 H, 1'-H). $-{ }^{13} \mathrm{C}-\mathrm{NMR}$ (75.5 MHz, $\mathrm{CDCl}_{3}$, zusätzl. APT): $\delta=11.52$ (+, C-1"'), 13.74 (+, C-3"), 23.27 (-, C-2"), 25,15 (-, C-1"), 123.35 (C quart, C-3), 124.73 ( $\left.\mathrm{C}_{\text {quart }}, \mathrm{C}-5\right), 127.05$ (C $\left.\mathrm{C}_{\text {quart }}, \mathrm{C}-\mathrm{Ar}\right), 129.07$ (+, C-Ar), 130.35 (+, C-Ar), 134.85 (+, C$\operatorname{Ar}$ ), 147.19 ( $\left.\mathrm{C}_{\text {quart }}, \mathrm{C}-6\right), 157.53$ ( $\left.\mathrm{C}_{\text {quart }}, \mathrm{C}-4\right), 161.42$ (C $\left.\mathrm{C}_{\text {quart }}, \mathrm{C}-2\right), 162.37$ [C $\mathrm{C}_{\text {quart }}, \mathrm{O}(\mathrm{CO}) \mathrm{Ph}$ ], 183.43 (+, C-1'). - MS (EI, $70 \mathrm{eV}), m / z(\%): 300(6)\left[\mathrm{M}^{+}\right], 105$ (100), 77 (16). $-\mathrm{C}_{17} \mathrm{H}_{16} \mathrm{O}_{5}$ (300.3): ber. C 67.99, H 5.37; gef. C 67.70, H 5.24.

Essigsäure-6-formyl-3-methyl-2-oxo-5-propyl-2H-pyran-4-ylester (92) und Essigsäure-6hydroxymethyl-3-methyl-2-oxo-5-propyl-2H-pyran-4-ylester (93)
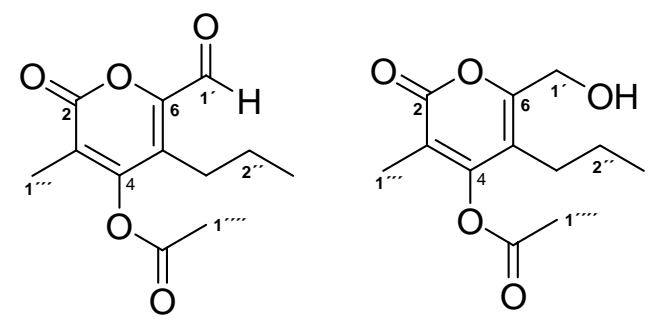

Die Reaktion wurde gemäß der allgemeinen Arbeitsvorschrift A10 mit Essigsäure-3,6-dimethyl-2-oxo-5propyl-2H-pyran-4-ylester (88) $(2.151 \mathrm{~g}, 9.689 \mathrm{mmol})$ und akt. Selendioxid (3.196 g, $28.77 \mathrm{~mol}$ ) in wasserfreiem Dioxan $(30 \mathrm{~mL})$ durchgeführt und $27 \mathrm{~h}$ gerührt.

Man erhielt 2.7 g (quant.) eines Gemisches aus dem Aldehyd 92 und dem Alkohol 93 in einem Verhältnis von 2.6:1 als weißen Feststoff. Das Rohprodukt wurde ohne weitere Reinigung umgesetzt. - ${ }^{1} \mathrm{H}-\mathrm{NMR}\left(250 \mathrm{MHz}, \mathrm{CDCl}_{3}\right): \delta=0.95\left(\mathrm{~m}_{\mathrm{c}}, 3 \mathrm{H}, 3 "-\mathrm{H}\right), 1.52\left(\mathrm{~m}_{\mathrm{c}}, 2 \mathrm{H}, 2^{2}-\right.$ H), 2.01 (s, 3 H, 1'"-H), 2.41 (s, 3 H, 1'"'-H), 2.67 (t, J = 7.8 Hz, 2 H, 1"-H), 4.46 (s, 2 H, 1'H), 9.98 (s, $1 \mathrm{H}, 1$ '-H). 


\section{Allgemeine Arbeitsvorschrift A11}

\section{Benzoesäure-6-hydroxymethyl-3-methyl-2-oxo-5-propyl-2H-pyran-4-ylester (95)}<smiles>CCCc1c(CO)oc(=O)c(I)c1OC(=O)c1ccccc1</smiles>

$\mathrm{Zu}$ einer Lösung von Benzoesäure-6-formyl-3-methyl-2-oxo-5-propyl-2Hpyran-4-ylester 94 (0.5622 g, $1.872 \mathrm{mmol})$ in abs. EtOH (15 mL) wurde bei $0{ }^{\circ} \mathrm{C}$ und unter Schutzgasatmosphäre $\mathrm{NaBH}_{4}(85 \mathrm{mg}, 2.2 \mathrm{mmol})$ gegeben, das Kältebad entfernt und $4 \mathrm{~h}$ bei Raumtemperatur gerührt. Bei der Zugabe von $\mathrm{NaBH}_{4}$ trat eine Rotfärbung auf, die auf das Einsetzen des Reduktionsbeginns hinwies. Nach vollständigem Reduktionsprozeß trat eine teil- bzw. vollständige Entfärbung der Reaktionslösung in Erscheinung. Die Reaktionsmischung wurde mit ges. $\mathrm{NH}_{4} \mathrm{Cl}$-Lösung $(10 \mathrm{~mL})$ beendet, unter vermindertem Druck eingeengt und mit EtOAc extrahiert. Die wässrige Phase $(\mathrm{pH}=5.6)$ wurde mit EtOAc $(5 \times 25 \mathrm{~mL})$ reextrahiert. Die vereinigte organische Phase wurde über $\mathrm{Na}_{2} \mathrm{SO}_{4}$ getrocknet, das Lösungsmittel i.V. entfernt. Man erhielt $0.513 \mathrm{~g}(91 \%)$ des Alkohols 95 als weißen Feststoff $\left(\mathrm{Smp} .=110^{\circ} \mathrm{C}\right) .-\mathrm{IR}(\mathrm{KBr}): v=3423 \mathrm{~cm}^{-1}(\mathrm{OH}), 2962,2930$, 2874, $1746(\mathrm{C}=\mathrm{O}), 1717(\mathrm{C}=\mathrm{O}), 1582,1243,1176,1112,1046,1022,706 .-{ }^{1} \mathrm{H}-\mathrm{NMR}$ (250 $\mathrm{MHz}, \mathrm{CDCl}_{3}$ ): $\delta=0.89$ (t, $J=7.4,3$ H, 3"-H), 1.50 (m $\mathrm{c}_{\mathrm{c}}, 2 \mathrm{H}, 2$ "-H), 1.97 (s, $\left.3 \mathrm{H}, 1 " '-\mathrm{H}\right), 2.30$ $\left(\mathrm{m}_{\mathrm{c}}, 2 \mathrm{H}, 1 "-\mathrm{H}\right), 4.52$ (s, $\left.2 \mathrm{H}, 1^{\prime}-\mathrm{H}\right), 7.55$ (t, $\left.J=7.1 \mathrm{~Hz}, 2 \mathrm{H}, \mathrm{Ar}-\mathrm{H}\right), 7.70$ (t, J= 7.1 Hz, $1 \mathrm{H}$, Ar-H), 8.15 (d, $J=7.1 \mathrm{~Hz}, 2 \mathrm{H}, \mathrm{Ar}-\mathrm{H}) .-{ }^{13} \mathrm{C}-\mathrm{NMR}\left(75.5 \mathrm{MHz}, \mathrm{CDCl}_{3}\right.$, zusätzl. APT): $\delta=$ 10.67 (+, C-1"'), 13.72 (+, C-3"), 23.43 (-, C-2"), 26.86 (-, C-1"), 58.96 (-, C-1'), 114.33 (C $\left.\mathrm{C}_{\text {quart }}, \mathrm{C}-3\right), 115.92$ ( $\left.\mathrm{C}_{\text {quart }}, \mathrm{C}-5\right), 127.57$ ( $\left.\mathrm{C}_{\text {quart }}, \mathrm{C}-\mathrm{Ar}\right), 128.99$ (+, C-Ar), $130.32(+, \mathrm{C}-\mathrm{Ar})$, 134.57 (+, C-Ar), 155.76 ( $\left.\mathrm{C}_{\text {quart }}, \mathrm{C}-6\right), 158.98$ (C $\left.\mathrm{C}_{\text {quart }}, \mathrm{C}-4\right), 162.49$ ( $\left.\mathrm{C}_{\text {quart }}, \mathrm{C}-2\right), 163.83$ [C $\mathrm{C}_{\text {quart }}$, $\mathrm{O}(\mathrm{CO}) \mathrm{Ph}] .-\mathrm{MS}(\mathrm{EI}, 70 \mathrm{eV}), \mathrm{m} / z$ (\%): 302 (4) $\left[\mathrm{M}^{+}\right], 105$ (100), 77 (16), 69 (3), 43 (3). $\mathrm{C}_{17} \mathrm{H}_{18} \mathrm{O}_{5}$ (302.3): ber. C 67.54, H 6.00; gef. C 67.61, H 6.04.

\section{4-Hydroxy-6-hydroxymethyl-3-methyl-2-oxo-5-propyl-2H-pyran (91)}<smiles>CCCc1c(CO)oc(=O)c(I)c1O</smiles>

Die Reaktion wurde gemäß der allgemeinen Arbeitsvorschrift A11 mit einem Gemisch (2.6:1) aus Essigsäure-6-formyl-3-methyl-2-oxo-5-propyl2H-pyran-4-ylester (92) und Essigsäure-6-hydroxymethyl-3-methyl-2-oxo5-propyl-2H-pyran-4-ylester (93) $(2.80 \mathrm{~g}, 11.8 \mathrm{mmol})$ sowie mit $\mathrm{NaBH}_{4}(0.534 \mathrm{~g}, 14.1 \mathrm{mmol})$ in abs. EtOH $(80 \mathrm{~mL})$ bei $0{ }^{\circ} \mathrm{C}$ durchgeführt und $2.5 \mathrm{~h}$ bei Raumtemperatur gerührt. Das Rohprodukt wurde aus heißem EtOAc umkristallisiert. Man erhielt $2.33 \mathrm{~g}$ (100\%) des entschützten Diols 91 als weißen Feststoff. $-{ }^{1} \mathrm{H}-\mathrm{NMR}\left(250 \mathrm{MHz}, \mathrm{CDCl}_{3}\right): \delta=1.0(\mathrm{t}, J=7.2 \mathrm{~Hz}, 3 \mathrm{H}$, 
3"-H), 1.57 (m, 2 H, 2"-H), 1.98 (s, 3 H, 1"'-H), 2.52 (t, J= 7.8 Hz, 2 H, 1"-H), 4.43 (s, 2 H, $\left.1^{\prime}-\mathrm{H}\right)$.

\section{Allgemeine Arbeitsvorschrift A12 (Variante 1)}

\section{Benzoesäure-6-brommethyl-3-methyl-2-oxo-5-propyl-2H-pyran-4-ylester (96)}

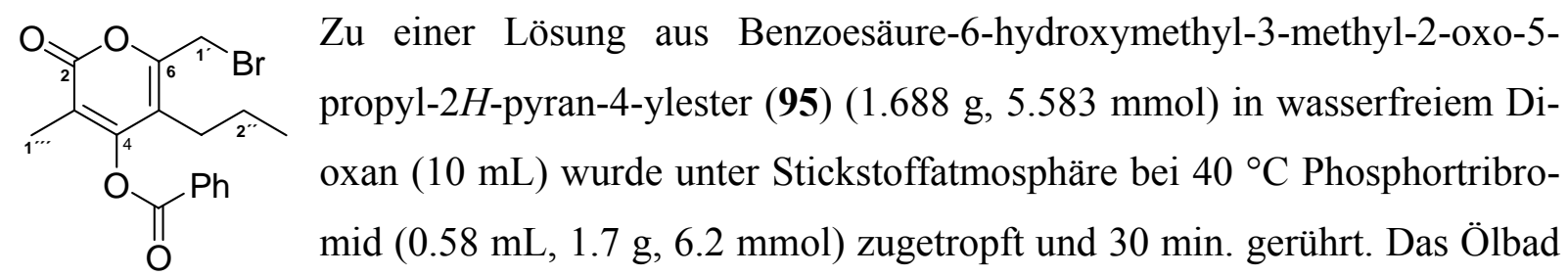
wurde entfernt und die Reaktionslösung $15 \mathrm{~h}$ bei Raumtemperatur gerührt. Zur trüben Reaktionsmischung wurde ges. $\mathrm{NaHCO}_{3}$-Lösung $(20 \mathrm{~mL})$ gegeben und mit EtOAc extrahiert. Die wässrige Phase $(\mathrm{pH}=8)$ wurde mit EtOAc $(2 \times 20 \mathrm{~mL})$ reextrahiert. Die vereinigte organische Phase wurde über $\mathrm{MgSO}_{4}$ getrocknet. Das Rohprodukt wurde säulenchromatographisch an Kieselgel gereinigt (250 g, Hex/EE 5:1). Man erhielt $2.04 \mathrm{~g}$ (quant.) des gewünschten Bromids $96\left(R_{f}=0.30\right)$ als weißen Feststoff (Smp. $\left.84^{\circ} \mathrm{C}\right)$. - IR $(\mathrm{KBr}): v=3055 \mathrm{~cm}^{-1}, 2964$, 2874, $1747(\mathrm{C}=\mathrm{O}), 1718(\mathrm{C}=\mathrm{O}), 1576,1265,1178,1066,1046,739,705,658 .-{ }^{1} \mathrm{H}-\mathrm{NMR}$ $\left(250 \mathrm{MHz}, \mathrm{CDCl}_{3}\right): \delta=0.92$ (t, J=7.4 Hz, $3 \mathrm{H}, 3$ "-H), 1.60 (m $\left.\mathrm{m}_{\mathrm{c}}, 2 \mathrm{H}, 2^{\prime \prime}-\mathrm{H}\right), 1.97$ (s, $3 \mathrm{H}, 1$ "'H), 2.30 (t, J= 7.4 Hz, 2 H, 1"-H), 4.30 (s, 2 H, 1'-H), 7.54 (t, J=7.1 Hz, 2 H, Ar-H), 7.70 (t, $J=7.1 \mathrm{~Hz}, 1 \mathrm{H}, \mathrm{Ar}-\mathrm{H}), 8.15(\mathrm{~d}, J=7.1 \mathrm{~Hz}, 2 \mathrm{H}, \mathrm{Ar}-\mathrm{H}) .-{ }^{13} \mathrm{C}-\mathrm{NMR}\left(75.5 \mathrm{MHz}, \mathrm{CDCl}_{3}\right.$, zusätzl. APT): $\delta=10.87$ (+, C-1"'), 13.96 (+, C-3"), 22.62 (-, C-1'), 24.58 (-, C-2"), 27.59 (-, C1"), 115.89 ( $\left.\mathrm{C}_{\text {quart }}, \mathrm{C}-3\right), 117.25$ (C $\left.\mathrm{C}_{\text {quart }}, \mathrm{C}-5\right), 127.44$ (C $\left.\mathrm{C}_{\text {quart }}, \mathrm{C}-\mathrm{Ar}\right), 129.02$ (+, C-Ar), 130.31 (+, C-Ar), 134.64 (+, C-Ar), 152.60 ( $\left.\mathrm{C}_{\text {quart }}, \mathrm{C}-6\right), 158.46\left(\mathrm{C}_{\text {quart }}, \mathrm{C}-4\right), 162.40\left(\mathrm{C}_{\text {quart }}, \mathrm{C}-2\right)$, $163.11\left[\mathrm{C}_{\text {quart }}, \mathrm{O}(\mathrm{CO}) \mathrm{Ph}\right] .-\mathrm{MS}\left(\mathrm{DCI}, \mathrm{NH}_{3}\right), m / z(\%): 748$ (38) $\left[2 \mathrm{M}+\mathrm{NH}_{4}\right]^{+}, 670(20), 401$ (4) $\left[\mathrm{M}+\mathrm{NH}_{3}+\mathrm{NH}_{4}\right]^{+}, 382(100)\left[\mathrm{M}+\mathrm{NH}_{4}\right]^{+}, 365(7)[\mathrm{M}+\mathrm{H}]^{+}, 304(70) .-\mathrm{C}_{17} \mathrm{H}_{17} \mathrm{BrO}_{4}$ (365.2): ber. C 55.91, H 4.69; gef. C 56.21, H 4.40.

\section{6-Brommethyl-4-hydroxy-3-methyl-2-oxo-5-propyl-2H-pyran (97)}<smiles>CCCc1c(CBr)oc(=O)c(I)c1O</smiles>

Die Reaktion wurde gemäß der allgemeinen Arbeitsvorschrift A12 (Variante 1) mit 4-Hydroxy-6-hydroxymethyl-3-methyl-2-oxo-5-propyl-2H-pyran

(91) (0.9351 g, $4.717 \mathrm{mmol})$ und $\operatorname{PBr}_{3}(0.488 \mathrm{~mL}, 1.41 \mathrm{~g}, 5.19 \mathrm{mmol})$ in Dioxan $(5 \mathrm{~mL})$ durchgeführt und $18 \mathrm{~h}$ gerührt. Man erhielt $0.6275 \mathrm{~g}(50 \%)$ des gewünschten Bromalkohols $97\left(R_{f}=0.30\right)$ als weißen Feststoff. Das Rohprodukt wurde ohne weitere Rei- 
nigung umgesetzt. - ${ }^{1} \mathrm{H}-\mathrm{NMR}\left(250 \mathrm{MHz}, \mathrm{CDCl}_{3}\right): \delta=1.05(\mathrm{t}, J=7.2 \mathrm{~Hz}, 3 \mathrm{H}, 3$ "-H), 1.64 (me, 2 H, 2"-H), 2.0 (s, 3 H, 1"'-H), 2.51 (t, J= 7.8 Hz, 2 H, 1"-H), 4.45 (s, 2 H, 1'-H).

\section{Allgemeine Arbeitsvorschrift A12 (Variante 3)}

\section{Benzoesäure-6-chlormethyl-3-methyl-2-oxo-5-propyl-2H-pyran-4-ylester (135)}<smiles>CCCc1c(CCl)oc(=O)c(I)c1OC(=O)c1ccccc1</smiles>

$\mathrm{Zu}$ wasserfreiem $\mathrm{NEt}_{4} \mathrm{Cl}(0.91 \mathrm{~g}, 5.5 \mathrm{mmol})$ wurde bei $-30{ }^{\circ} \mathrm{C}$ eine Lösung von Benzoesäure-6-brommethyl-3-methyl-2-oxo-5-propyl-2H-pyran4-ylester (96) (200 mg, $0.548 \mathrm{mmol}$ ) in trockenem $\mathrm{CH}_{2} \mathrm{CH}_{2}(5 \mathrm{~mL})$ zugegeben und gerührt. Die Reaktionslösung wurde innerhalb von $2 \mathrm{~h}$ auf Raumtemperatur erwärmt, mit ges. NaCl-Lösung $(5 \mathrm{~mL})$ versetzt und mit $\mathrm{Et}_{2} \mathrm{O}(2 \times 15 \mathrm{~mL})$ extrahiert. Die vereinigte organische Phase wurde über $\mathrm{MgSO}_{4}$ getrocknet. Das Lösungsmittel wurde i.V. entfernt. Man erhielt 183 mg (quant.) des Chlormethylpyrons 135 als weißen Feststoff. Das Rohprodukt wurde ohne weitere Reinigung umgesetzt. - ${ }^{1} \mathrm{H}-\mathrm{NMR}(250 \mathrm{MHz}$, $\left.\mathrm{CDCl}_{3}\right): \delta=0.91(\mathrm{t}, J=7.2 \mathrm{~Hz}, 3 \mathrm{H}, 3 "-\mathrm{H}), 1.56\left(\mathrm{~m}_{\mathrm{c}}, 2 \mathrm{H}, 2 "-\mathrm{H}\right), 1.98$ (s, $3 \mathrm{H}, 1$ "'-H), 2.30 (t, $\left.J=7.6 \mathrm{~Hz}, 2 \mathrm{H}, 1^{\prime \prime}-\mathrm{H}\right), 4.0$ (s, $\left.2 \mathrm{H}, 1^{\prime}-\mathrm{H}\right), 7.59$ (t, $\left.J=7.1 \mathrm{~Hz}, 2 \mathrm{H}, \mathrm{Ar}-\mathrm{H}\right), 7.72$ (t, $J=7.1 \mathrm{~Hz}, 1$ $\mathrm{H}, \mathrm{Ar}-\mathrm{H}), 8.17(\mathrm{~d}, J=7.1 \mathrm{~Hz}, 2 \mathrm{H}, \mathrm{Ar}-\mathrm{H}) .-{ }^{13} \mathrm{C}-\mathrm{NMR}\left(75.5 \mathrm{MHz}, \mathrm{CDCl}_{3}\right): \delta=10.82(+, \mathrm{C}-$ 1"'), 13.88 (+, C-3"), 23.02 (-, C-2"), 27.40 (-, C-1"), 38.91 (-, C-1'), 116.13 (C quart, C-3), 117.31 ( $\left.\mathrm{C}_{\text {quart }}, \mathrm{C}-5\right), 127.37$ (C $\left.\mathrm{C}_{\text {quart }}, \mathrm{C}-\mathrm{Ar}\right), 129.00$ (+, C-Ar), 130.29 (+, C-Ar), $134.64(+, \mathrm{C}-$ $\mathrm{Ar}), 152.24$ ( $\left.\mathrm{C}_{\text {quart }}, \mathrm{C}-6\right), 158.37$ ( $\left.\mathrm{C}_{\text {quart }}, \mathrm{C}-4\right), 162.40$ ( $\left.\mathrm{C}_{\text {quart }}, \mathrm{C}-2\right), 163.13$ [ $\mathrm{C}_{\text {quart }}, \mathrm{O}(\mathrm{CO}) \mathrm{Ph}$ ]. MS (EI, $70 \mathrm{eV}), m / z(\%): 323(<1), 320(4)\left[\mathrm{M}^{+}\right], 285(<1), 265(<1), 235(<1), 216(<1), 187$ $(<1), 153(<1), 123(<1), 105$ (100), 83 (2), 77 (21), 55 (1), 51 (4), 41 (2).

\section{Allgemeine Arbeitsvorschrift A13}

Benzoesäure-6-[(E)-3'-methylocta-2'-enyl]-3-methyl-2-oxo-5-propyl-2H-pyran-4-ylester (125)

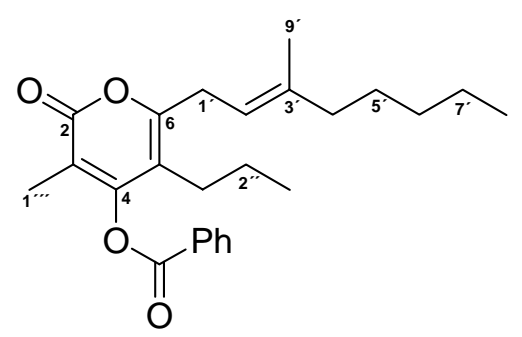

$\mathrm{Zu}$ einer Lösung von (E)-2-Methylhepta-1-enyldimethylalan (123) (0.82 mL, $0.82 \mathrm{mmol}, 1.0 \mathrm{M}$ in Hexan) in THF (5.0 mL) wurde unter Stickstoffatmosphäre bei $0{ }^{\circ} \mathrm{C} n \mathrm{BuLi}(0.31 \mathrm{~mL}$, $0.82 \mathrm{mmol}, 2.7 \mathrm{M}$ in Hexan) zugegeben und $30 \mathrm{~min}$ gerührt.

Eine Lösung des Bromids 96 (150 mg, $0.411 \mathrm{mmol})$ in THF $(2.0 \mathrm{~mL})$ wurde bei $0{ }^{\circ} \mathrm{C}$ der vorgenerierten, zitronengelben Alanatlösung langsam zugetropft und 2.5 h gerührt. Die Reaktionslösung wurde mit ges. $\mathrm{NH}_{4} \mathrm{Cl}$-Lösung $(5 \mathrm{~mL})$ versetzt und 
mit EtOAc $(15 \mathrm{~mL})$ extrahiert. Die organische Phase wurde getrennt und die wässrige Phase mit EtOAc $(2 \times 15 \mathrm{~mL})$ reextrahiert. Die vereinigte organische Phase wurde über $\mathrm{MgSO}_{4}$ getrocknet und das Lösungsmittel i.V. entfernt. Das erhaltene Rohprodukt wurde säulenchromatographisch an Kieselgel gereinigt $(10 \mathrm{~g}, \mathrm{Hex} / \mathrm{EE} 6: 1 \rightarrow 3: 1 \rightarrow 1: 1)$. Man erhielt $135 \mathrm{mg}$ $(83 \%)$ des Dienesters $125\left(R_{f}=0.38\right)$ als farbloses Öl. Zusätzlich wurden $26.4 \mathrm{mg}(11 \%)$ des Ethandipyronylbenzoats $137\left(R_{f}=0.11\right)$ als weißen Feststoff isoliert. - IR (Film): $v=3054$ $\mathrm{cm}^{-1}$, 2932, $1785(\mathrm{C}=\mathrm{O}), 1717(\mathrm{C}=\mathrm{O}), 1653,1576,1265,1218,1177,1107,1078,1023,737$. $-{ }^{1} \mathrm{H}-\mathrm{NMR}\left(250 \mathrm{MHz}, \mathrm{CDCl}_{3}\right): \delta=0.88\left[\mathrm{~m}_{\mathrm{c}}, 6 \mathrm{H}, 3^{\prime \prime}\left(8^{\prime}\right)-\mathrm{H}\right], 1.13-1.53\left[\mathrm{~m}, 8 \mathrm{H}, 2^{\prime \prime}\left(5^{\prime}\right)\left(6^{\prime}\right)\left(7^{\prime}\right)-\right.$ H], 1.68 (s, 3 H, 9'-H), 1.90 (s, 3 H, 1"'-H), 1.98 (t, J=7.0 Hz, 2 H, 4'-H), 2.2 (m, 2 H, 1"-H), $3.25\left(\mathrm{~d}, J=7.0 \mathrm{~Hz}, 2 \mathrm{H}, 1^{\prime}-\mathrm{H}\right), 5.21$ (t, $\left.J=7.0 \mathrm{~Hz}, 1 \mathrm{H}, 2^{\prime}-\mathrm{H}\right), 7.54$ (t, $\left.J=7.1 \mathrm{~Hz}, 2 \mathrm{H}, \mathrm{Ar}-\mathrm{H}\right)$, 7.68 (t, $J=7.1 \mathrm{~Hz}, 1 \mathrm{H}, \mathrm{Ar}-\mathrm{H}), 8.16(\mathrm{~d}, J=7.1 \mathrm{~Hz}, 2 \mathrm{H}, \mathrm{Ar}-\mathrm{H}) .-{ }^{13} \mathrm{C}-\mathrm{NMR}(75.5 \mathrm{MHz}$, $\mathrm{CDCl}_{3}$, zusätzl. APT): $\delta=10.44$ (+, C-1"'), 13.92 (+, C*-3"), 14.03 (+, C*-8'), 16.32(+, C-9'), $22.50\left(-, C^{*}-1^{\prime}\right), 23.20\left(-, C^{*}-2^{\prime \prime}\right), 27.35\left(-, C_{-} * 7^{\prime}\right), 27.37\left(-, C^{*}-6^{\prime}\right), 30.19\left(+, C^{*}-5^{\prime}\right), 31.47$ (-, C-1"), 39.52 (-, C*-4'), 112.44 (-, C quart, $\left.C^{*}-5\right), 113.50$ (-, C quart, $\left.C^{*}-3\right), 117.42$ (+, C-2'), 127.81(-, C quart, C-Ar), 128.92 (+, C-Ar), 130.25 (+, C-Ar), 134.39 (+, C-Ar), 138.84 (-, $\left.\mathrm{C}_{\text {quart }}, \mathrm{C}-3^{\prime}\right), 158.86$ (-, $\left.\mathrm{C}_{\text {quart }}, \mathrm{C}^{*}-6\right), 159.49$ (-, $\left.\mathrm{C}_{\text {quart }}, \mathrm{C}^{*}-4\right), 162.55$ (-, $\left.\mathrm{C}_{\text {quart }}, \mathrm{C}^{*}-2\right), 164.57$ [-, $\left.\mathrm{C}_{\text {quart }}, \mathrm{O}(\mathrm{CO}) \mathrm{Ph}\right] .-\mathrm{MS}(\mathrm{EI}), m / z(\%): 398(2)[\mathrm{M}+2 \mathrm{H}]^{+}, 396(6)\left[\mathrm{M}^{+}\right], 342(<1), 307$ $(<1), 291(<1), 260(<1), 223(<1), 191(<1), 163(<1), 149$ (2), 129 (4), 105 (100), 83 (3), 77 (11), 57 (8).

\section{Benzoesäure-6-(2'-benzoesäure-3-methyl-2-oxo-5-propyl-2H-pyran-4-ylesterethylyl)-3- methyl-2-oxo-5-propyl-2H-pyran-4-ylester (137)}

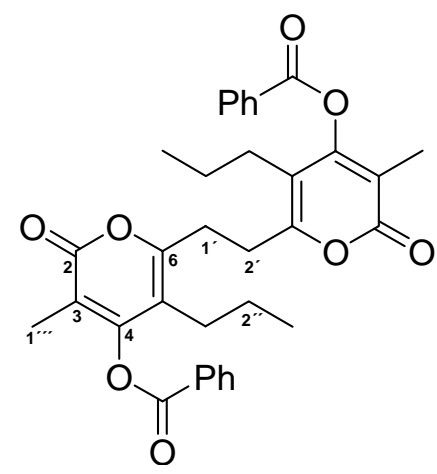

Die Verbindung wurde bei der Kreuzkupplungsreaktion der Alanate mit dem Brommethylpyron 96 erhalten. $-{ }^{1} \mathrm{H}-\mathrm{NMR}(250 \mathrm{MHz}$, $\left.\mathrm{CDCl}_{3}\right): \delta=0.86(\mathrm{t}, J=7.8 \mathrm{~Hz}, 6 \mathrm{H}, 2 \times 3 "-\mathrm{H}), 1.45\left(\mathrm{~m}_{\mathrm{c}}, 4 \mathrm{H}, 2 \mathrm{x}\right.$ 2"-H), 1.94 (s, 6 H, 2 x 1"'-H), 2.23 (t, J= 7.8 Hz, 4 H, 2 x 1"-H), $2.98\left[\mathrm{~s}, 4 \mathrm{H}, 1^{\prime}\left(2^{\prime}\right)-\mathrm{H}\right], 7.58(\mathrm{t}, J=7.1 \mathrm{~Hz}, 4 \mathrm{H}, 2 \mathrm{x} \mathrm{Ar-H}), 7.72(\mathrm{t}, J$ $=7.1 \mathrm{~Hz}, 2 \mathrm{H}, 2 \times \mathrm{Ar}-\mathrm{H}), 8.16(\mathrm{~d}, J=7.1 \mathrm{~Hz}, 4 \mathrm{H}, 2 \times \mathrm{Ar}-\mathrm{H}) .-$ ${ }^{13} \mathrm{C}-\mathrm{NMR}\left(75.5 \mathrm{MHz}, \mathrm{CDCl}_{3}\right.$, zusätzl. DEPT, APT): $\delta=10.55(+$, 2 x C-1"'), 13.73 (+, 2 x C-3"), 23.23 (-, 2 x C*-1'), 28.22 (-, 2 x C*-2"), 29.09 (-, 2 x C-1"), 113.92 (-, $\mathrm{C}_{\text {quart }}, 2$ x C-3), 114.65 (-, $\mathrm{C}_{\text {quart }}, 2$ x C-5), 127.61 (-, $\mathrm{C}_{\text {quart }}, 2$ x C-Ar), 128.93 (+, 2 x C-Ar), 130.27 (+, 2 x C-Ar), 134.48 (+, 2 x C-Ar), 156.53 (-, C quart, 2 x C-4), 159.14 (-, $\mathrm{C}_{\text {quart }}, 2$ x C-6), 162.43 (-, $\mathrm{C}_{\text {quart }}, 2$ x C-2), 164.07 [-, $\mathrm{C}_{\text {quart }}, 2$ x O(CO)Ph]. - MS (DCI, $\left.\mathrm{NH}_{3}\right)$, 
$m / z(\%): 872(<1), 681(<1), 604(<1), 587(33)\left[\mathrm{M}+\mathrm{NH}_{3}\right], 588(100)\left[\mathrm{M}+\mathrm{NH}_{4}\right]^{+}, 571(18)$ $[\mathrm{M}+\mathrm{H}]^{+}, 458(>1), 389(>1), 345(>1), 304$ (6), 244 (2), 195 (6), 139 (14).

\section{6,9-Dimethyltetradeca-6,8-dien (136)}

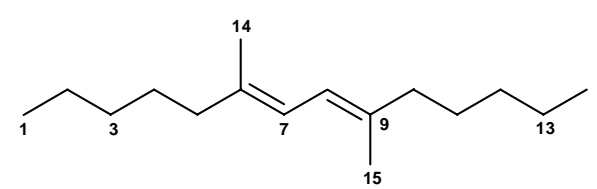

Die Verbindung wurde bei der Kreuzkupplungsreaktion der Alanate mit dem Brommethylpyron 96 erhalten. $-{ }^{1} \mathrm{H}$ NMR $\left(250 \mathrm{MHz}, \mathrm{CDCl}_{3}\right): \delta=0.92[\mathrm{t}, J=6.5 \mathrm{~Hz}, 6 \mathrm{H}$, 1(14)-H], 1.18-1.36 [m, 8 H, 2(3)(12)(13)-H], 1.42 [m $\left.\mathrm{m}_{\mathrm{c}}, 4 \mathrm{H}, 4(11)-\mathrm{H}\right], 1.71$ [s, $6 \mathrm{H}, 14(15)-$ H], 2.04 [t, $J=7.0 \mathrm{~Hz}, 4 \mathrm{H}, 5(10)-\mathrm{H}], 6.0$ [s, $2 \mathrm{H}, 7(8)-\mathrm{H}]$. - MS (DCI, $\left.\mathrm{NH}_{3}\right), m / z(\%): 222$ (12) $\left[\mathrm{M}^{+}\right], 179(<1), 165(8), 151$ (3), 137 (>1), 123 (4), 109 (20), 95 (94), 91 (13), 81 (38), 69 (20), 67 (26), 57 (26), 55 (67), 41 (100).

\section{Benzoesäure-6-[(E,E)-3',7'-dimethylocta-2',5'-dienyl]-3-methyl-2-oxo-5-propyl-2H- pyran-4-ylester (125A)}

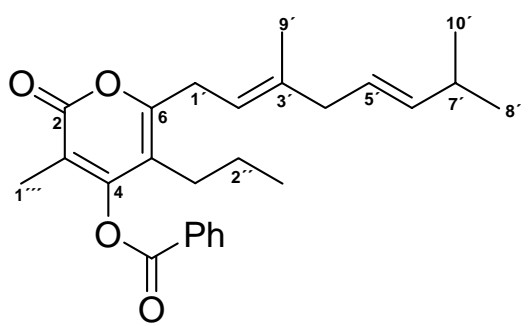

Die Reaktion wurde gemäß der allgemeinen Arbeitsvorschrift A13 mit dem Bromid 96 (150 mg, $0.411 \mathrm{mmol})$ in THF $(2.0 \mathrm{~mL}), \quad(E, E)-2,6$-Dimethylhepta-1,4-dienyldimethylalan (123A) (1.23 mL, $1.23 \mathrm{mmol}, 1.0 \mathrm{M}$ in Hexan) in THF $(1.0 \mathrm{~mL})$ und $n \mathrm{BuLi}(0.39 \mathrm{~mL}, 0.83 \mathrm{mmol}, 2.13 \mathrm{M}$

in Hexan) durchgeführt. Die Reaktionslösung wurde $2 \mathrm{~h}$ bei $0{ }^{\circ} \mathrm{C}$ gerührt. Nach anschließender Aufarbeitung wurde das erhaltene Rohprodukt säulenchromatographisch an Kieselgel gereinigt (10 g, Hex/EE 6:1). Man erhielt $0.16 \mathrm{~g}(95 \%)$ des Dienesters 125A $\left(R_{f}=0.45\right)$ als farbloses Öl. - IR (film): $v=2963 \mathrm{~cm}^{-1}, 1747(\mathrm{C}=\mathrm{O}), 1714(\mathrm{C}=\mathrm{O}), 1576,1457,1374,1243$, 1108, 1063, 910, 733, 706. - ${ }^{1} \mathrm{H}-\mathrm{NMR}\left(250 \mathrm{MHz}, \mathrm{CDCl}_{3}\right): \delta=0.85$ (t, $\left.J=7.4 \mathrm{~Hz}, 3 \mathrm{H}, 3 "-\mathrm{H}\right)$, 0.95 [d, $\left.J=7.1 \mathrm{~Hz}, 6 \mathrm{H}, 8^{\prime}\left(10^{\prime}\right)-\mathrm{H}\right], 1.46$ (m $\mathrm{c}, 2$ H, 2"-H), 1.66 (s, 3 H, 9'-H), 1.93 (s, 3 H, 1"'H), 2.23 [m $\left.\mathrm{c}, 3 \mathrm{H}, 7^{\prime}\left(1^{\prime \prime}\right)-\mathrm{H}\right], 2.65$ (d, $\left.J=5.3 \mathrm{~Hz}, 2 \mathrm{H}, 4^{\prime}-\mathrm{H}\right), 3.27$ (d, J=7.0 Hz, $\left.2 \mathrm{H}, 1^{\prime}-\mathrm{H}\right)$, 5.20-5.50 [m, 3 H, 2'(5')(6')-H], $7.56(\mathrm{t}, J=7.1 \mathrm{~Hz}, 2 \mathrm{H}, \mathrm{Ar}-\mathrm{H}), 7.70$ (t, $J=7.1 \mathrm{~Hz}, 1 \mathrm{H}, \mathrm{Ar}-$ $\mathrm{H}), 8.15$ (d, $J=7.1 \mathrm{~Hz}, 2 \mathrm{H}, \mathrm{Ar}-\mathrm{H}) .-{ }^{13} \mathrm{C}-\mathrm{NMR}\left(75.5 \mathrm{MHz}, \mathrm{CDCl}_{3}\right.$, zusätzl. APT): $\delta=10.46$ (+, C-1"'), 13.94 (+, C-3"), 16.33 (+, C-9'), 22.55 [+, C-8'(10')], 23.21 (-, C-2"), 27.38 (-, C1"), 30.30 (-, C-1'), 30.96 (+, C-7'), 42.71 (-, C-4'), 112.56 ( $\left.\mathrm{C}_{\text {quart }}, \mathrm{C}-3\right), 113.58$ (C $\left.\mathrm{C}_{\text {quart }}, \mathrm{C}-5\right)$, $118.10\left(+, \mathrm{C}^{\prime} 2^{\prime}\right), 124.20$ (+, C-5'), 127.81 (C $\left.\mathrm{C}_{\text {quart }}, \mathrm{C}-\mathrm{Ar}\right), 128.92$ (+, C-Ar), 130.26 (+, C-Ar), 134.40 (+, C-Ar), 137.90 (C $\left.\mathrm{C}_{\text {quart }}, \mathrm{C}-3^{\prime}\right), 139.77$ (+, C-6'), $158.68\left(\mathrm{C}_{\text {quart }}, \mathrm{C}-6\right), 159.49$ (C quart, $\mathrm{C}-$ 
4), $162.56\left(\mathrm{C}_{\text {quart }}, \mathrm{C}-2\right), 164.56$ [C $\left.\mathrm{C}_{\text {quart }}, \mathrm{O}(\mathrm{CO}) \mathrm{Ph}\right] .-\mathrm{MS}$ (ESI), $\mathrm{m} / \mathrm{z}(\%): 839$ (100) $[2 \mathrm{M}+\mathrm{Na}]^{+}, 431(21)\left[\mathrm{M}+\mathrm{Na}^{+}\right], 409(1)\left[\mathrm{M}+\mathrm{H}^{+}\right]$.

\section{Benzoesäure-6-[(E,E)-3',7',7'-trimethylocta-2',5'-dienyl]-3-methyl-2-oxo-5-propyl-2H- pyran-4-ylester (125R)}

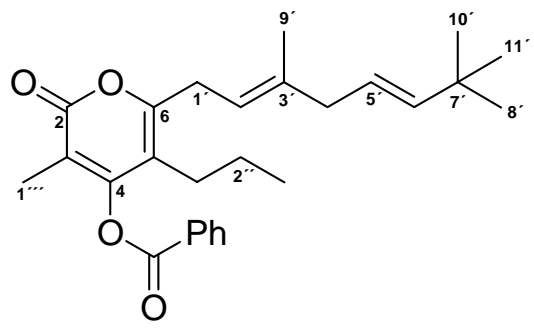

Die Reaktion wurde gemäß der allgemeinen Arbeitsvorschrift A13 mit dem Bromid 96 (150 mg, $0.411 \mathrm{mmol}$ ), (E,E)-2,6,6-Trimethylhepta-1,4-dienyldimethylalan (123R) (2.46 mL, 1.23 mmol, 0.5 M in Hexan) und $n \operatorname{BuLi}(0.35 \mathrm{~mL}$, $0.82 \mathrm{mmol}, 2.3 \mathrm{M}$ in Hexan) durchgeführt. Das erhaltene Rohprodukt wurde säulenchromatographisch an Kieselgel gereinigt (10 g, Hex/EE 6:1). Man erhielt $61.7 \mathrm{mg}(45 \%)$ des Dienesters 125R $\left(R_{f}=0.45\right)$ als farbloses Öl. Es wurden $30.6 \mathrm{mg}$ (26\%) des Bromids zurückerhalten. - IR (film): $v=2959 \mathrm{~cm}^{-1}, 1747(\mathrm{C}=\mathrm{O}), 1718(\mathrm{C}=\mathrm{O})$, 1577, 1452, 1363, 1243, 1175, 1107, 1063, 1022, 973, 706. - ${ }^{1} \mathrm{H}-\mathrm{NMR}\left(250 \mathrm{MHz}, \mathrm{CDCl}_{3}\right): \delta$ $=0.87\left(\mathrm{t}, J=7.4 \mathrm{~Hz}, 3 \mathrm{H}, 3^{\prime \prime}-\mathrm{H}\right), 1.0$ [s, 9 H, 8'(10') (11')-H], 1.47 (m, 2 H, 2"-H), 1.68 (s, 3 H, 9'-H), 1.95 (s, 3 H, 1'"-H), 2.23 [m, 2 H, 1"-H], 2.67 (d, J=5.3 Hz, 2 H, 4'-H), 3.30 (d, J= $\left.7.0 \mathrm{~Hz}, 2 \mathrm{H}, 1^{\prime}-\mathrm{H}\right), 5.20-5.55$ [m, 3 H, 2'(5')(6')-H], 7.57 (t, J=7.1 Hz, $\left.2 \mathrm{H}, \mathrm{Ar}-\mathrm{H}\right), 7.70$ (t, $J=$ $7.1 \mathrm{~Hz}, 1 \mathrm{H}, \mathrm{Ar}-\mathrm{H}), 8.16$ (d, J=7.1 Hz, $2 \mathrm{H}, \mathrm{Ar}-\mathrm{H}) .-{ }^{13} \mathrm{C}-\mathrm{NMR}\left(75.5 \mathrm{MHz}, \mathrm{CD}_{3} \mathrm{OD}\right.$, zusätzl. APT): $\delta=10.52$ (+, C-1'"), 14.31 (+, C*-3"), 16.54 (+, C*-9'), 24.24 (-, C-1'), 28.26 (-, C-2"),

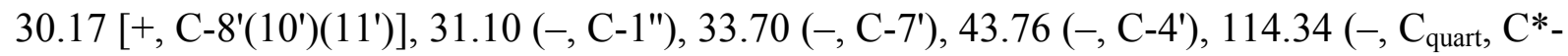
5), 114.43 (-, $\left.\mathrm{C}_{\text {quart }}, \mathrm{C}^{*}-3\right), 119.36\left(+, \mathrm{C}-2^{\prime}\right), 123.31$ (+, C-5'), 128.97 (-, C quart, $\left.\mathrm{C}-\mathrm{Ar}\right), 130.25$ (+, C-Ar), 131.28 (+, C-Ar), 135.83 (+, C-Ar), 139.46 (-, C quart $_{1}$ C-3'), 144.70 (+, C-6'), 160.35 (-, $\left.\mathrm{C}_{\text {quart }}, \mathrm{C}^{*}-6\right), 161.48$ (-, $\left.\mathrm{C}_{\text {quart }}, \mathrm{C}^{*}-4\right), 163.81$ (-, $\left.\mathrm{C}_{\text {quart }}, \mathrm{C}^{*}-2\right), 166.25$ [-, $\mathrm{C}_{\text {quart }}$, $\left.\mathrm{O}\left(\mathrm{C}^{*} \mathrm{O}\right) \mathrm{Ph}\right] .-\mathrm{MS}(\mathrm{ESI}), m / z(\%): 422(7)\left[\mathrm{M}^{+}\right], 105$ (100), 77 (21), 41 (7).

\section{Allgemeine Arbeitsvorschrift A14 (Variante 1)}

\section{4-Hydroxy-3-methyl-6-(3'-methylocta-2'-enyl)-5-propyl-1H-pyridin-2-on (23)}

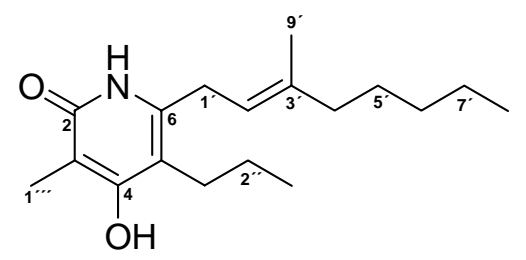

In einem Autoklave wurde der Dienester 125 (12.2 mg, 0.031 mmol) in liq. $\mathrm{NH}_{3}$ (Überschuß) $48 \mathrm{~h}$ bei $70{ }^{\circ} \mathrm{C}$ und einem Druck von 30 bar erhitzt und gerührt. Hierzu wurde der Autoklave erst auf $-78{ }^{\circ} \mathrm{C}$ vorgekühlt und über eine $\mathrm{KOH}$ Trockenvorrichtung $1 \mathrm{~h}$ gasförmiges $\mathrm{NH}_{3}$ eingeleitet und einkondensiert. Nach Beendigung 
der $\mathrm{NH}_{3}$-Zufuhr wurde die Reaktionsapparatur auf $70{ }^{\circ} \mathrm{C}$ erhitzt. Nach Ende der Umsetzung wurde das Reaktionsgefäß auf Raumtemperatur abgekühlt und vorsichtig entgast. $\mathrm{Et}_{2} \mathrm{O}$ (20 $\mathrm{mL}$ ) und 1.0 $\mathrm{M} \mathrm{KHSO}_{4}$-Lösung $(3 \mathrm{~mL})$ wurden zugegeben und extrahiert. Die organische

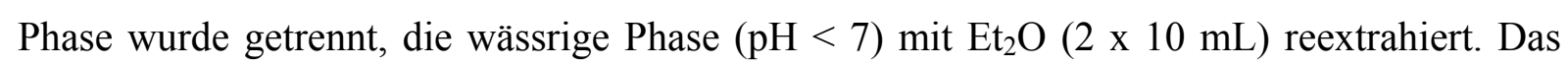
Rohprodukt wurde säulenchromatographisch an Kieselgel gereinigt (8 g, Hex/EE 2:1 + 1\% HOAc). Man erhielt $5.2 \mathrm{mg}(58 \%)$ des Iromycin-Analogons $23\left(R_{f}=0.40\right)$ als weißen Feststoff. $-{ }^{1} \mathrm{H}-\mathrm{NMR}\left(250 \mathrm{MHz}, \mathrm{CD}_{3} \mathrm{OD}\right): \delta=0.88$ (t, $\left.J=6.9 \mathrm{~Hz}, 3 \mathrm{H}, 8^{\prime}-\mathrm{H}\right), 0.95$ (t, $J=7.0 \mathrm{~Hz}$, 3 H, 3"-H), 1.20-1.37 [m, 4 H, 6'(7')-H], 1.41 (m, 2 H, 5'-H), 1.49 [m $\mathrm{m}_{\mathrm{c}}, 2$ H, 2"-H), 1.72 (s, 3 H, 9'-H), 1.98 (s, 3 H, 1'"-H), 2.02 (t, $J=7.0$ Hz, 2 H, 4'-H), 2.41 (m, 2 H, 1"-H), 3.30 (d, $J=$ $\left.7.0 \mathrm{~Hz}, 2 \mathrm{H}, 1^{\prime}-\mathrm{H}\right), 5.15$ (t, $\left.J=7.0 \mathrm{~Hz}, 1 \mathrm{H}, 2^{\prime}-\mathrm{H}\right) .-{ }^{13} \mathrm{C}-\mathrm{NMR}\left(75.5 \mathrm{MHz}, \mathrm{CD}_{3} \mathrm{OD}\right): \delta=8.70$ (C-1"'), 14.38 (C*-3"), 14.47 (C*-8'), 16.37(C-9'), 23.57 (C*-1'), 24.09 (C*-2"), 27.90 (C-*7'), $28.55\left(\mathrm{C}^{*}-6^{\prime}\right), 30.29\left(\mathrm{C}^{*}-5^{\prime}\right), 32.59\left(\mathrm{C}-1^{\prime \prime}\right), 40.57\left(\mathrm{C}^{*}-4^{\prime}\right), 105.95\left(\mathrm{C}_{\text {quart }}, \mathrm{C}^{*}-5\right), 113.58\left(\mathrm{C}_{\text {quart }}\right.$, $\left.\mathrm{C}^{*-3}\right), 120.46$ (C-2'), $139.83\left(\mathrm{C}_{\text {quart }}, \mathrm{C}^{\prime} 3^{\prime}\right), 143.08$ ( $\left.\mathrm{C}_{\text {quart }}, \mathrm{C}^{*}-6\right), 165.94\left(\mathrm{C}_{\text {quart }}, \mathrm{C}^{*}-4\right), 166.01$ $\left(-, \mathrm{C}_{\text {quart }}, \mathrm{C}^{*}-2\right)$. - MS (EI), $m / z(\%): 1300$ (39), 1189 (23) $[4 \mathrm{M}+\mathrm{Na}+\mathrm{H}]^{+}, 1188$ (35) [4M + $\mathrm{Na}]^{+}, 1187(47)[4 \mathrm{M}+\mathrm{Na}-\mathrm{H}]^{+}, 1010(31), 942(12), 896(25)[3 \mathrm{M}+\mathrm{Na}-\mathrm{H}]^{+}, 895$ (39) [3M $+\mathrm{Na}-2 \mathrm{H}]^{+}, 606(37)[2 \mathrm{M}+\mathrm{Na}+\mathrm{H}]^{+}, 605(100)[2 \mathrm{M}+\mathrm{Na}]^{+}, 583(15)[2 \mathrm{M}+\mathrm{H}]^{+}, 292$ (9) $[\mathrm{M}+\mathrm{H}]^{+}, 238(5)$.

\section{Allgemeine Arbeitsvorschrift A14 (Variante 1)}

\section{6-(3',7'-Dimethylocta-2',5'-dienyl)-4-hydroxy-3-methyl-5-propyl-1H-pyridin-2-on (23A)}

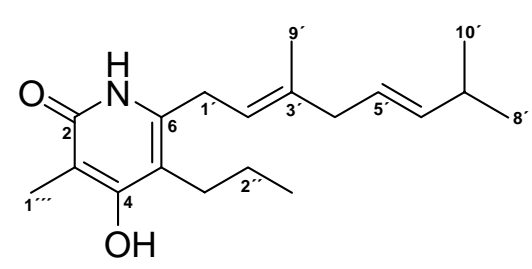

Die Reaktion wurde gemäß der allgemeinen Arbeitsvorschrift A14 (Variante 1) mit dem Dienester 125A (60.0 mg, 0.147 mmol) in liq. $\mathrm{NH}_{3}$ (Überschuß) durchgeführt. Das Rohprodukt wurde säulenchromatographisch an Kieselgel gereinigt

(8 g, Hex/EE 2:1 + 1\% HOAc). Man erhielt $28.8 \mathrm{mg}$ (65\%) des Zielproduktes Iromycin A (23A) $\left(R_{f}=0.38\right)$ als weißen Feststoff. $-{ }^{1} \mathrm{H}-\mathrm{NMR}\left(250 \mathrm{MHz}, \mathrm{CD}_{3} \mathrm{OD}\right): \delta=1.0[\mathrm{~m}, 9 \mathrm{H}$, 3"(8')(10')-H], 1.53 (m, 2 H, 2"-H), 1.75 (s, 3 H, 9'-H), 2.02 (s, 3 H, 1"'-H), 2.28 (m, 1 H, 7'H), 2.45 (t, $\left.J=8.0 \mathrm{~Hz}, 2 \mathrm{H}, 1^{\prime \prime}-\mathrm{H}\right), 2.72$ (d, $\left.J=6.5 \mathrm{~Hz}, 2 \mathrm{H}, 4^{\prime}-\mathrm{H}\right), 3.33$ (d, J=7.5 Hz, $2 \mathrm{H}, 1^{\prime}-$ H), $5.21\left(\mathrm{t}, J=7.0 \mathrm{~Hz}, 1 \mathrm{H}, 2^{\prime}-\mathrm{H}\right), 5.30-5.55\left[\mathrm{~m}, 2 \mathrm{H}, 5^{\prime}\left(6^{\prime}\right)-\mathrm{H}\right] .-{ }^{13} \mathrm{C}-\mathrm{NMR}(75.5 \mathrm{MHz}$, $\mathrm{CD}_{3} \mathrm{OD}$, zusätzl. APT): $\delta=8.72\left(+, \mathrm{C}-1^{\prime \prime \prime}\right), 14.54$ (+, C-3"), 16.47 (+, C-9'), 23.03 [+, C8'(10')], $24.10\left(-, C^{\prime}-2^{\prime}\right), 27.97\left(-, C^{\prime} 1^{\prime}\right), 30.41$ (-, C-1"), 32.33 (+, C-7'), 43.67 (-, C-4'), $105.96\left(-, \mathrm{C}_{\text {quart }}, \mathrm{C}^{*}-3\right), 113.81\left(-, \mathrm{C}_{\text {quart }}, \mathrm{C}^{*}-5\right), 121.02\left(+, \mathrm{C}-2^{\prime}\right), 125.70$ (+, C-5'), 138.79 (-, 
$\left.\mathrm{C}_{\text {quart }}, \mathrm{C}^{-3} 3^{\prime}\right), 140.83$ (+, C-6'), 142.93 (-, $\left.\mathrm{C}_{\text {quart }}, \mathrm{C}-6\right), 165.93$ (-, $\left.\mathrm{C}_{\text {quart }}, \mathrm{C}-4\right), 166.34$ (-, $\mathrm{C}_{\text {quart }}$, C-2).

\section{Allgemeine Arbeitsvorschrift A14 (Variante 1)}

6-(3',7',7'-Trimethylocta-2',5'-dienyl)-4-hydroxy-3-methyl-5-propyl-1H-pyridin-2-on (23R)

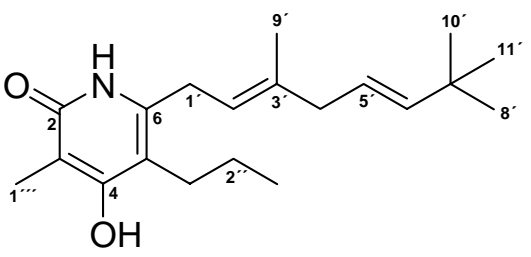

Die Reaktion wurde gemäß der allgemeinen Arbeitsvorschrift A14 (Variante 1) mit dem Dienester 125R (61.7 mg, $0.146 \mathrm{mmol}$ ) durchgeführt. Das Rohprodukt wurde säulenchromatographisch an Kieselgel gereinigt (8 g, Hex/EE 2:1+ $1 \%$ HOAc). Man erhielt $46.3 \mathrm{mg}(63 \%)$ des Iromycins R (23R) $\left(R_{f}=0.33\right)$ als weißen Feststoff. - IR (KBr): $v=2958 \mathrm{~cm}^{-1}, 1627(\mathrm{C}=\mathrm{O}), 1456,1362,1218,1163,1113,1029,972$. ${ }^{1} \mathrm{H}-\mathrm{NMR}\left(250 \mathrm{MHz}, \mathrm{CD}_{3} \mathrm{OD}\right): \delta=0.95$ (t, $\left.J=7.0 \mathrm{~Hz}, 3 \mathrm{H}, 3^{\prime \prime}-\mathrm{H}\right), 099$ [s, $9 \mathrm{H}, 8^{\prime}\left(10^{\prime}\right)\left(11^{\prime}\right)-$ H], 1.47 (m, 2 H, 2"-H), 1.70 (s, 3 H, 9'-H), 1.98 (s, 3 H, 1"'-H), 2.40 (t, J=8.0 Hz, 2 H, 1"H), $2.65\left(\mathrm{~d}, J=5.5 \mathrm{~Hz}, 2 \mathrm{H}, 4^{\prime}-\mathrm{H}\right), 3.28$ (d, $\left.J=7.5 \mathrm{~Hz}, 2 \mathrm{H}, 1^{\prime}-\mathrm{H}\right), 5.15$ (t, $J=7.0 \mathrm{~Hz}, 1 \mathrm{H}, 2^{\prime}-$ H), 5.21-5.35 (m, $1 \mathrm{H}, 5$ '-H), $5.50\left(\mathrm{~d}, J=15.5 \mathrm{~Hz}, 1 \mathrm{H}, 6{ }^{\prime}-\mathrm{H}\right] .-{ }^{13} \mathrm{C}-\mathrm{NMR}(75.5 \mathrm{MHz}$, $\mathrm{CD}_{3} \mathrm{OD}$, zusätzl. APT): $\delta=8.73$ (+, C-1"'), 14.59 (+, C*-3"), 16.47 (+, C*-9'), 24.09 (-, C-2"), 27.97 (-, C-1'), 30.14 [+, C-8'(10')(11')], 30.44 (-, C-1"), 33.69 (-, C-7'), 43.79 (-, C-4'), $105.96\left(-, \mathrm{C}_{\text {quart }}, \mathrm{C}^{*}-3\right), 113.65$ (-, $\left.\mathrm{C}_{\text {quart }}, \mathrm{C}^{*}-5\right), 121.05\left(+, \mathrm{C}-2^{\prime}\right), 123.36\left(+, \mathrm{C}-5^{\prime}\right), 138.73$ (-, C $_{\text {quart, }}$ C-3'), 143.00 (-, C quart, C-6), 144.69 (+, C-6'), 165.96 (-, C quart, C*-4), 165.99 (-, C quart, $\mathrm{C}^{*}$-2). - MS (ESI, MeOH/Na): 975 (8) $[3 \mathrm{M}+\mathrm{Na}]^{+}, 974$ (32) $[3 \mathrm{M}+\mathrm{Na}-1 \mathrm{H}]^{+}, 973$ (42) $[3 \mathrm{M}$ $+\mathrm{Na}-2 \mathrm{H}]^{+}, 659(8)[2 \mathrm{M}+\mathrm{Na}+2 \mathrm{H}]^{+}, 658(34)[2 \mathrm{M}+\mathrm{Na}+1 \mathrm{H}]^{+}, 657(100)[2 \mathrm{M}+\mathrm{Na}]^{+}$, $340(10)[\mathrm{M}+\mathrm{Na}]^{+}$.

\section{Allgemeine Arbeitsvorschrift A14 (Variante 2)}

\section{4-Hydroxy-3-methyl-6-(3'-methylocta-2'-enyl)-5-propylpyran-2-on (150)}

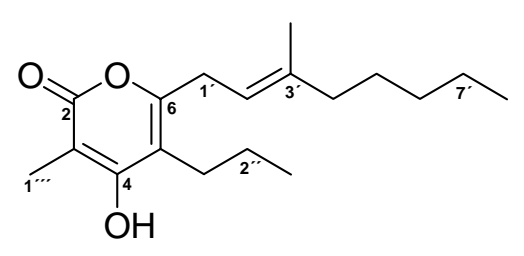

Zur Durchführung des O-N-Austausches wurde das Kreuzkupplungsprodukt 125 mit einer übersättigten methanolischen $\mathrm{NH}_{3}$-Lösung im Überschuß versetzt und gerührt. Zur Herstellung der übersättigten methanolischen $\mathrm{NH}_{3}$-Lösung $(200 \mathrm{~mL})$ wurde ein trockener $500 \mathrm{~mL}$ Schlenkkolben jeweils für 100 und $200 \mathrm{~mL}$ Lösung markiert. Nach Befüllung des Reaktionskolbens mit $100 \mathrm{~mL}$ wasserfreiem $\mathrm{MeOH}\left(\mathrm{Smp} .-98^{\circ} \mathrm{C}\right.$ ) wurde 
Ammoniak über eine $\mathrm{KOH}$-Trockenvorrichtung bei $-30{ }^{\circ} \mathrm{C}$ in das Reaktionsgefäß einkondensiert. Der Lösungskolben wurde an einen Blasenzähler angeschlossen und über Nacht auf Raumtemperatur erwärmt. Die O-N-Austauschreaktionen wurden in einem Schlenkkolben, einem Bombenrohr sowie einem Autoklave durchgeführt. Aufgrund der zweifachen Umfunktionalisierung (Debenzoylierung und Umwandlung zu cyclischem Amid) und des hierdurch bedingten Polaritätszuwachses im Vergleich zu dem eingesetzten Edukt kann die Bildung des Iromycin-Analogons effektiv über Dünnschichtchromatographie detektiert werden. Die Reaktionslösungen wurden nach Beendigung der Reaktion zunächst bis zu 1/4 des ursprünglichen Volumens einkonzentriert und mit ges. $\mathrm{NH}_{4} \mathrm{Cl}$-Lösung und EtOAC extrahiert. Eine Referenzauftragung (DC) zeigte die Bildung des Benzamids (Hex/EtOAc, 2:1 + 1\% HOAc, $R_{f}=0.22$ ) an, die die erwartete Entschützung im basischen Medium belegte. Die in Gegenwart von ges. methanol. $\mathrm{NH}_{3}$ im Überschuß und 1.0 eq des Benzoates 125 durchgeführten Umsetzungen in einem Autoklave (RT, 18 h), einem Schlenkkolben (RT, 3 d) sowie einem Bombenrohr (60 ${ }^{\circ} \mathrm{C}, 72 \mathrm{~h}, 80^{\circ} \mathrm{C}, 3 \mathrm{~d}$ ) führten lediglich zur vollständigen Entschützung des Benzoates 125 und zur Bildung des Entschützungsproduktes 150 (Hex/EtOAC 2:1 + 1\% HOAc, $R_{f}=0.45$ ). Es konnte keine Bildung des Iromycin-Analogons 23 festgestellt werden.

\section{Allgemeine Arbeitsvorschrift A14 (Variante 3)}

\section{4-Hydroxy-3-methyl-6-(3'-methylocta-2'-enyl)-5-propylpyran-2-on (150)}

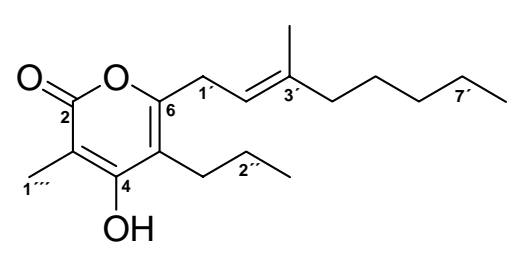

Zur einer Lösung des Benzoates 125 (10.000 mg, 0.025) in abs. MeOH $(1 \mathrm{~mL})$ wurde bei Raumtemperatur eine Lösung von $\mathrm{NH}_{4} \mathrm{OAc}(19.5 \mathrm{mg}, 0.25 \mathrm{mmol})$ in abs. $\mathrm{MeOH}(1 \mathrm{~mL})$ zugegeben und $4 \mathrm{~d}$ gerührt. Die Reaktion wurde mit $\mathrm{H}_{2} \mathrm{O}$ versetzt und mit EtOAc $(2 \times 10 \mathrm{~mL})$ extrahiert. Die vereinigte organische Phase wurde über $\mathrm{Na}_{2} \mathrm{SO}_{4}$ getrocknet und das Lösungsmittel i.V. entfernt. Man erhielt $3.3 \mathrm{mg}$ (45\%) des Entschützungsproduktes 150 (Hex/EtOAC 2:1 + 1\% HOAc, $\left.R_{f}=0.45\right)$. Es konnte keine Bildung des Iromycin-Analogons $\mathbf{2 3}$ festgestellt werden. 


\section{Synthese der Pyridonderivate}

\section{Allgemeine Arbeitsvorschrift A15 (Variante 1) \\ 4-Hydroxy-3,6-dimethyl-5-propyl-1H-Pyridin-2-on (62)}<smiles>CCCc1c(I)[nH]c(=O)c(I)c1O</smiles>

Eine Mischung des Pyrons 63 (2.63 g, 14.4 mmol) und konz. $\mathrm{NH}_{4} \mathrm{OH}$ (48 $\mathrm{mL}, 0.361 \mathrm{~mol}, 28-30 \mathrm{Gew} \%)$ in Dioxan $(35 \mathrm{~mL}, 0.361 \mathrm{~mol})$ wurde in einem Bombenrohr auf $120{ }^{\circ} \mathrm{C}$ erhitzt und $2 \mathrm{~h}$ gerührt. Eine dünnschichtchromatographische Detektion des Pyridons 62 war aufgrund seiner schwach UV-löschenden Eigenschaft sowie der Acidität der Hydroxyfunktion, die in der basischen Reaktionslösung als Salz vorlag, nur schwach erfassbar und konnte durch Ansäuern (pH-Wert 6-7) und Einsatz eines Anfärbemittels (Molybdänphosphorsäure-Lösung) leichter durchgeführt werden. Die Reaktionslösungen wurden im Allgemeinen aufgrund der stark polaren Eigenschaft des Pyridons $62\left(\mathrm{Hex} /\right.$ EtOAc $\left.2: 1+10 \% \mathrm{MeOH}, R_{f}=0.37\right)$ und seiner hohen Löslichkeit in alkoholischen Lösungen sowie in Wasser bevorzugt stark eingeengt und anschließend mit 1.0 $\mathrm{M} \mathrm{KHSO}_{4}$-Lösung und EtOAc aufgearbeitet. So wurde die Reaktionsmischung zunächst auf Raumtemperatur abgekühlt und das Lösungsmittel i.V. entfernt. $1 \mathrm{M}$ $\mathrm{KHSO}_{4}$-Lösung $(20 \mathrm{~mL})$ wurde zugegeben und mit EtOAc $(3 \times 100 \mathrm{~mL})$ extrahiert. Die vereinigte organische Phase wurde über $\mathrm{Na}_{2} \mathrm{SO}_{4}$ getrocknet. Das Rohprodukt wurde aus Aceton umkristallisiert. Man erhielt $1.952 \mathrm{~g}(75 \%)$ des Pyridons $62\left(R_{f}=0.37\right.$, Hex/EtOAc $2: 1+10 \%$ $\mathrm{MeOH}$ ) als weißen Feststoff (Smp. $237^{\circ} \mathrm{C}$ ). Die angestrebten Umkristallisationen waren mit hohen Verlusten an Ausbeute verbunden, weshalb in der Regel die Produkte bei sauberen Umsetzungen direkt ohne weitere Reinigungsversuche in den nächsten Schritt eingesetzt wurden. - IR (film): $v=1646 \mathrm{~cm}^{-1}(\mathrm{C}=\mathrm{O}), 1602(\mathrm{NH}), 1559,1463,1392,1374,1282,1032,896$, 850, 761, 646, 609. - ${ }^{1} \mathrm{H}-\mathrm{NMR}\left(250 \mathrm{MHz}, \mathrm{CD}_{3} \mathrm{OD}\right): \delta=0.98$ (t, $\left.J=7.2 \mathrm{~Hz}, 3 "-\mathrm{H}\right), 1.52\left(\mathrm{~m}_{\mathrm{c}}\right.$, 2 H, 2"-H), 2.0 (s, 3 H, 1"'-H), 2.29 (s, 3 H, 1'-H), 2.50 (t, J=8.0 Hz, 2 H, 1"-H). - ${ }^{13} \mathrm{C}-\mathrm{NMR}$ (62.9 MHz, $\mathrm{CD}_{3} \mathrm{OD}$, zusätzl. DEPT): $\delta=9.02$ (+, C-1"'), 14.51 (+, C-3"), $16.53\left(+, \mathrm{C}-1^{\prime}\right)$, 23.99 (-, C-2"), 28.14 (-, C-1"), 105.97 (C quart, $\mathrm{C}-3), 113.86$ (C quart $_{\text {, }}$ C-5), 140.56 (C quart $_{\text {, }}$ C-6), $166.08\left(\mathrm{C}_{\text {quart }}, \mathrm{C}-4\right), 166.19$ (C $\left.\mathrm{C}_{\text {quart }}, \mathrm{C}-2\right) .-\mathrm{MS}(\mathrm{EI}, 70 \mathrm{eV}), \mathrm{m} / z(\%): 182(3)[\mathrm{M}+\mathrm{H}]^{+}, 181$ (35) $\left[\mathrm{M}^{+}\right], 168(<1), 166(2), 154(<1), 152(100)\left[\mathrm{M}^{+}-\mathrm{C}_{2} \mathrm{H}_{5}\right], 139(<1), 125(1)\left[\mathrm{M}^{+}-\mathrm{C}_{4} \mathrm{H}_{8}\right]$, $124(10)\left[\mathrm{M}^{+}-\mathrm{C}_{4} \mathrm{H}_{9}\right], 110(<1), 97$ (3), 96 (28), 94 (2), 83 (7), 69 (4), 57 (2) $\left[\mathrm{C}_{3} \mathrm{H}_{5} \mathrm{O}^{+}\right], 55$ (11), 43 (3) $\left[\mathrm{C}_{3} \mathrm{H}_{7}^{+}\right], 42$ (12). $-\mathrm{C}_{10} \mathrm{H}_{15} \mathrm{NO}_{2}$ (181.23): ber. C 66.27, H 8.34, N 7.73; gef. C 65.98, H 8.32, N 7.65. 


\section{Allgemeine Arbeitsvorschrift A15 (Variante 2) \\ 4-Hydroxy-3,6-dimethyl-5-propyl-1H-Pyridin-2-on (62)}

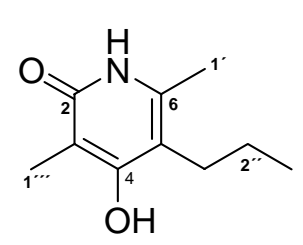

In einem NMR-Röhrchen wurden $N$-Aminopyridon 162 (4.00 mg, 0.02 mmol) und $\mathrm{NaNO}_{2}(2.81 \mathrm{mg}, 0.04 \mathrm{mmol})$ vorgelegt und mit einem Rührschwein versehen. Der Mischung wurde bei Raumtemperatur $d^{4}$-HOAc langsam zugegeben und gerührt. Die homogene, farblose Reaktionslösung begann sich unter Blasenbildung zu verfärben (Bildung von Salpetersäure). Die gelbe Reaktionslösung wurde bis zum Ende der Gasentwicklung gerührt (5 min). Die anschließende spektroskopische Untersuchung wies das Pyridon $62\left(R_{f}=0.37\right.$, Hex/EtOAc 2:1 $\left.+10 \% \mathrm{MeOH}\right)$ als weißen Feststoff (Smp. $237{ }^{\circ} \mathrm{C}$ ) nach. - IR (film): $v=2963 \mathrm{~cm}^{-1}, 2361,2339,1714$ $(\mathrm{C}=\mathrm{O}), 1558(\mathrm{NH}), 1409,1299,1170,1045,909,831,734 .-{ }^{1} \mathrm{H}-\mathrm{NMR}\left(250 \mathrm{MHz}, \mathrm{CDCl}_{3}\right): \delta$ $=0.91\left(\mathrm{t}, J=7.2 \mathrm{~Hz}, 3^{\prime \prime}-\mathrm{H}\right), 1.47$ (m, 2 H, 2"-H), 1.98 (s, 3 H, 1"'-H), 2.21 (s, 3 H, 1'-H), 2.39 (t, $J=8.0 \mathrm{~Hz}, 2 \mathrm{H}, 1$ "-H). - MS (EI, $70 \mathrm{eV}), m / z(\%): 629$ (58), 385 (22) [2M + Na] $]^{+}, 346$ (72), 235 (100), $182(26)[\mathrm{M}+\mathrm{H}]^{+}$. Zur Optimierung der Darstellung des Pyridons 62 wurde eine Lösung des $N$-Aminopyridons $162(40 \mathrm{mg})$ in Essigsäure $(1 \mathrm{~mL})$ gelöst und gerührt. Eine homogene Lösung von $\mathrm{NaNO}_{2}$ in $\mathrm{H}_{2} \mathrm{O}(1 \mathrm{~mL})$ wurde bei Raumtemperatur langsam zugetropft und $3 \mathrm{~h}$ gerührt. Die Bildung von salpetriger Säure $\left(\mathrm{HNO}_{2}\right)$ wurde durch eine gelbe Verfärbung der zunächst farblosen Reaktionslösung und durch eine Gasentwicklung (Beginn des Reduktionsprozesses) angezeigt. Nach $3 \mathrm{~h}$ wurde der Reaktionslösung ein Gemisch aus eisgekühlter ges. NaCl-Lösung und $\mathrm{Et}_{2} \mathrm{O}$ zugegeben und extrahiert. Die Umsetzungen führten zur vollständigen Zersetzung. In einem weiteren Optimierungsversuch wurde zur Verfolgung des Reaktionsgeschehens die Umsetzung in einem NMR-Röhrchen durchgeführt. Hierzu wurden $N$-Aminopyridon 162 (4.0 mg, $0.02 \mathrm{mmol})$ und $\mathrm{NaNO}_{2}(2.81 \mathrm{mg}, 0.04 \mathrm{mmol})$ vorgelegt und mit einem Rührschwein versehen. Die Reaktionsmischung wurde bei Raumtemperatur tropfenweise mit $\mathrm{d}^{4}$-HOAc $(0.42 \mathrm{~mL})$ unter Einsetzen einer Blasenbildung versetzt, bis zur Beendigung der Gasentwicklung (5-6 min) gerührt und anschließend analytisch untersucht. Die analytischen Daten ( ${ }^{1} \mathrm{H}-\mathrm{NMR}$, IR- und Massenspektroskopie) entsprachen den aus der Darstellung des Pyrons 63 mit Ammoniak gewonnenen analytischen Werten des Pyridons 62 (Allgemeine Arbeitsvorschrift A15, Variante 1). 


\section{Allgemeine Arbeitsvorschrift A15 (Variante 3)}

\section{4-Hydroxy-3,6-dimethyl-5-propyl-1H-Pyridin-2-on (62)}

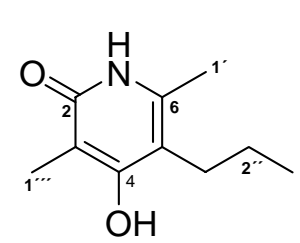

Zur Darstellung des Pyridons 62 wurde eine Suspension des $\mathrm{N}$ Benzylpyridons 163 (0.112 g, $0.412 \mathrm{mmol})$ und eines PearlmanKatalysators [20 mg, $1.43 \mathrm{mmol}, \mathrm{Pd}(\mathrm{OH})_{2} /$ Aktivkohle, 20\%] in EtOH (10 $\mathrm{mL}$ ) bei $100{ }^{\circ} \mathrm{C}$ und einem Druck von $3.515 \mathrm{~kg} / \mathrm{cm}^{2}$ an der Parr-Apparatur hydriert und $44 \mathrm{~h}$ gerührt. Die Reaktionslösung wurde über Celite abfiltriert und mit EtOH (20 mL) nachgewaschen. Die erhaltene ethanolische Lösung wurde unter vermindertem Druck konzentriert. Man erhielt 74 mg (quant.) des Pyridons 62 als Rohprodukt. - IR (film): $v$ $=2934 \mathrm{~cm}^{-1}, 1601(\mathrm{NH}), 1435,1391,1342,1282,1232,1117,1057,895,849,759,646,608$. $-{ }^{1} \mathrm{H}-\mathrm{NMR}$ (250 MHz, DMSO): $\delta=0.89$ (t, $\left.J=7.2 \mathrm{~Hz}, 3 "-\mathrm{H}\right), 1.39\left(\mathrm{~m}_{\mathrm{c}}, 2 \mathrm{H}, 2 "-\mathrm{H}\right), 1.81$ (s, 3 H, 1"'-H), 2.08 (s, 3 H, 1'-H), 2.29 (t, $J=8.0$ Hz, 2 H, 1"-H). - MS (EI, 70 eV), m/z (\%): 213 $(<1), 181(42)\left[\mathrm{M}^{+}\right], 152(100)\left[\mathrm{M}^{+}-\mathrm{C}_{2} \mathrm{H}_{5}\right], 124(5)\left[\mathrm{M}^{+}-\mathrm{C}_{4} \mathrm{H}_{9}\right], 96$ (21), 69 (5), 42 (5). Der verwendete Pearlman-Katalysator $\left[\mathrm{Pd}(\mathrm{OH})_{2}\right]$ wurde nach Pearlman ${ }^{[145]}$ hergestellt. Hierzu wurde eine Mischung von $\mathrm{PdCl}_{2}(2.0 \mathrm{~g}, 11 \mathrm{mmol})$ und Aktivkohle $(4.8 \mathrm{~g}, 0.4 \mathrm{~mol})$ in dem. $\mathrm{H}_{2} \mathrm{O}(40 \mathrm{~mL})$ auf $80{ }^{\circ} \mathrm{C}$ erhitzt und gerührt. Eine Lösung von $\mathrm{LiOH}^{\cdot} \mathrm{H}_{2} \mathrm{O}(1.0 \mathrm{~g}, 17 \mathrm{mmol})$ in dem. $\mathrm{H}_{2} \mathrm{O}(10 \mathrm{~mL})$ wurde in einer Portion zugegeben und über Nacht bei Raumtemperatur gerührt. Die Reaktionslösung wurde abfiltriert und der Rückstand mit $0.5 \mathrm{~V} / \mathrm{V} \%$ an wässriger Essigsäure (2.5 mL 100\%-ige HOAc in $500 \mathrm{~mL} \mathrm{dem.} \mathrm{H}_{2} \mathrm{O}$ ) gewaschen. Das erhaltene $\mathrm{Pd}(\mathrm{OH})_{2} /$ Aktivkohle-Gemisch (quant.) wurde am Rotorvapor bei $60{ }^{\circ} \mathrm{C}$ getrocknet.

\section{1-Amin-3,6-dimethyl-4-hydroxy-2-oxo-5-propyl-1H-pyridin (162)}

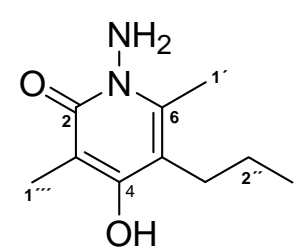

Die Reaktion wurde gemäß der allgemeinen Arbeitsvorschrift A15 (Variante 1) mit dem Pyron 63 (0.15 g, $0.82 \mathrm{mmol})$ und Hydrazin (1.5 mL) in Dioxan $(1.5 \mathrm{~mL})$ bei $60{ }^{\circ} \mathrm{C}$ durchgeführt und $5 \mathrm{~h}$ gerührt. Der Reaktionslösung wurde zunächst unter vermindertem Druck vollständig einkonzentriert und $1 \mathrm{M} \mathrm{KHSO}_{4}$-Lösung $(1.5 \mathrm{~mL})$ zugegeben und mit EtOAc $(3 \times 10 \mathrm{~mL})$ extrahiert. Die vereinigte organische Phase wurde über $\mathrm{MgSO}_{4}$ getrocknet. Man erhielt $0.16 \mathrm{~g}(99 \%) \mathrm{des} \mathrm{N}$ Aminopyridons $162\left(R_{f}=0.2\right.$, EtOAc) als weißen Feststoff. $-{ }^{1} \mathrm{H}-\mathrm{NMR}\left(250 \mathrm{MHz}, \mathrm{CD}_{3} \mathrm{OD}\right)$ : $\delta=0.95$ (t, $J=7.2 \mathrm{~Hz}, 3 "-\mathrm{H}), 1.45$ (m, 2 H, 2"-H), 2.03 (s, 3 H, 1"'-H), 2.41 (s, 3 H, 1'-H), $2.47(\mathrm{t}, J=8.0 \mathrm{~Hz}, 2 \mathrm{H}, 1 "-\mathrm{H}) .-{ }^{13} \mathrm{C}-\mathrm{NMR}\left(62.9 \mathrm{MHz}, \mathrm{CD}_{3} \mathrm{OD}\right.$, zusätzl. APT): $\delta=9.64(+$, C-1"'), 14.15 (+, C-3"), 15.12 (+, C-1'), 24.01 (-, C-2"), 28.54 (-, C-1"), 104.81 (C quart, C-3), 
$113.08\left(\mathrm{C}_{\text {quart }}, \mathrm{C}-5\right), 142.30\left(\mathrm{C}_{\text {quart }}, \mathrm{C}-6\right), 162.42\left(\mathrm{C}_{\text {quart }}, \mathrm{C}-4\right), 162.76\left(\mathrm{C}_{\text {quart }}, \mathrm{C}-2\right) .-\mathrm{MS}$ (hochauflösende MS, $\mathrm{MeOH} / \mathrm{H}_{2} \mathrm{O}$ ) m/z (\%): 197 (34) $[\mathrm{M}+\mathrm{H}]^{+}, 454$ (39), 456 (17).

\section{Essigsäure-3,6-dimethyl-2-oxo-5-propyl-1,2-dihydropyridin-4-ylester (156)}

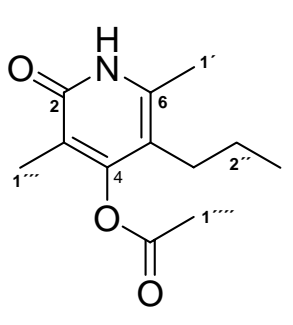

Die Reaktion wurde gemäß der allgemeinen Arbeitsvorschrift A9 (Variante

2) mit 4-Hydroxy-3,6-dimethyl-5-propyl-1H-Pyridin-2-on (62) (500 mg, $2.76 \mathrm{mmol}$ ), Acetylchlorid (196 $\mu \mathrm{L}, 0.217 \mathrm{~g}, 2.76 \mathrm{mmol}$ ) und Pyridin $(0.223 \mu \mathrm{L}, 0.218 \mathrm{~g}, 2.76 \mathrm{mmol})$ in Dioxan $(15 \mathrm{~mL})$ bei $0{ }^{\circ} \mathrm{C}$ durchgeführt und $63 \mathrm{~h}$ bei Raumtemperatur gerührt. Zur Feststellung des Reaktionsendes wurde stets eine Probe der Suspensionslösung sowohl dünnschichtchromatographisch $\left(\mathrm{SiO}_{2}\right)$ als auch spektroskopisch untersucht. Das Rohprodukt wurde säulenchromatographisch an Kieselgel gereinigt (70 g, Hex/EE 2:1 + 10\% MeOH). Man erhielt 0.424 g (69\%) des Pyridonesters $156\left(R_{f}=0.33\right)$ als weißen Feststoff (Smp. $\left.205{ }^{\circ} \mathrm{C}\right) .-\mathrm{IR}(\mathrm{KBr}): v=2986 \mathrm{~cm}^{-1}$, 2410, $1767(\mathrm{C}=\mathrm{O}), 1650(\mathrm{C}=\mathrm{O}), 1623(\mathrm{NH}), 1576,1473,1265,1195,1112,1060,909,739$, 736. $-{ }^{1} \mathrm{H}-\mathrm{NMR}\left(250 \mathrm{MHz}, \mathrm{CDCl}_{3}\right): \delta=0.92$ (t, $\left.J=7.1 \mathrm{~Hz}, 3 \mathrm{H}, 3 "-\mathrm{H}\right), 1.47\left(\mathrm{~m}_{\mathrm{c}}, 2 \mathrm{H}, 2 "-\mathrm{H}\right)$, 1.93 (s, 3 H, 1"'-H), 2.25 (t, $J=7.8$ Hz, 2 H, 1"-H), 2.35 [s, 5 H, 1'"'(1')-H]. - ${ }^{13} \mathrm{C}-\mathrm{NMR}(50.3$ MHz, $\mathrm{CD}_{3} \mathrm{OD}$, zusätzl. APT): $\delta=9.89$ (+, C-1"'), 14.20 (+, C-3"), 16.31 (+, C-1'), 20.32 (+, C-1"''), 23.90 (-, C-2"), 28.60 (-, C-1"), 115.18 (-, C quart, C*-3), 117.82 (-, C quart, $\left.C^{*}-5\right)$, 141.46 (-, $\left.\mathrm{C}_{\text {quart }}, \mathrm{C}-6\right), 159.84$ (-, $\left.\mathrm{C}_{\text {quart }}, \mathrm{C}-4\right), 165.72$ [-, $\left.\mathrm{C}_{\text {quart }}, \mathrm{O}(\mathrm{CO}) \mathrm{CH}_{3}\right], 169.01$ (-, $\mathrm{C}_{\text {quart }}$, C-2). - MS (EI, $70 \mathrm{eV}), m / z(\%): 224$ (3) [M + H] $]^{+}, 223$ (19) [M] $]^{+}, 209(<1), 194(<1), 182$ (3), $181(42)\left[\mathrm{M}-\mathrm{C}_{3} \mathrm{H}_{5}\right]^{+},, 166$ (3), 153 (10), 152 (100), 139 (<1), 124 (1), $108(<1), 96(9)$, 82 (7), 68 (1), 55 (4), 43 (12), 41 (2). $-\mathrm{C}_{12} \mathrm{H}_{17} \mathrm{NO}_{3}$ (223.27): ber. C 64.56, H 7.67, N 6.27; gef. C 64.72, H 7.63, N 6.36.

\section{Essigsäure-6-formyl-3-methyl-2-oxo-5-propyl-1,2-dihydropyridin-4-ylester (157)}<smiles>CCCc1c(C=O)[nH]c(=O)c(I)c1OC(=O)F</smiles>

Die Reaktion wurde entsprechend der allgemeinen Arbeitsvorschrift A10 mit Essigsäure-3,6-dimethyl-2-oxo-5-propyl-1,2-dihydropyridin-4-ylester (156) $(1.19 \mathrm{~g}, 5.33 \mathrm{mmol})$ und akt. Selendioxid (5.91 g, $53.3 \mathrm{mmol})$ in wasserfreiem Dioxan $(50 \mathrm{~mL})$ durchgeführt und $10 \mathrm{~h}$ gerührt. Es wurde $1.04 \mathrm{~g}$ des Aldehyds 157 (82\%) als sauberes Rohprodukt erhalten. Die Umkristallisation aus EtOAc lieferte $0.443 \mathrm{~g}$ (35\%) des Aldehyds $157\left(R_{f}=0.33\right.$, Hex/EE 2:1 $+10 \% \mathrm{MeOH})$ als weißen Feststoff $\left(\mathrm{Smp} .168^{\circ} \mathrm{C}\right) .-\mathrm{IR}(\mathrm{KBr}): v=2961 \mathrm{~cm}^{-1}, 1768(\mathrm{C}=\mathrm{O})$, 
$1728(\mathrm{C}=\mathrm{O}), 1692(\mathrm{C}=\mathrm{O}), 1649,1612(\mathrm{NH}), 1472,1330,1238,1221,1194,1150,1045,924$, 772, 743. - ${ }^{1} \mathrm{H}-\mathrm{NMR}\left(250 \mathrm{MHz}, \mathrm{CDCl}_{3}\right): \delta=0.97$ (t, $\left.J=7.2 \mathrm{~Hz}, 3 \mathrm{H}, 3 "-\mathrm{H}\right), 1.59\left(\mathrm{~m}_{\mathrm{c}}, 2 \mathrm{H}\right.$, 2"-H), 2.02 (s, 3 H, 1'"-H), 2.40 (s, 3 H, 1'"'-H), 2.62 (t, J= 7.8 Hz, 2 H, 1"-H), 9.94 (s, 1 H, 1'-H). $-{ }^{13} \mathrm{C}-\mathrm{NMR}\left(62.9 \mathrm{MHz}, \mathrm{CDCl}_{3}\right.$, zusätzl. DEPT): $\delta=11.13$ (+, C-1"'), 13.73 (+, C-3"), 20.34 (+, C-1"''), 24.70 (-, C-2"), 25.16 (-, C-1"), 124.67 (C quart, $\mathrm{C}-3), 130.40$ (C $\left.\mathrm{C}_{\text {quart }}, \mathrm{C}-5\right)$, $132.63\left(\mathrm{C}_{\text {quart }}, \mathrm{C}-6\right), 155.73\left(\mathrm{C}_{\text {quart }}, \mathrm{C}-4\right), 161.44\left[\mathrm{C}_{\text {quart }}, \mathrm{O}(\mathrm{CO}) \mathrm{CH}_{3}\right], 167.05\left(\mathrm{C}_{\text {quart }}, \mathrm{C}-2\right)$,

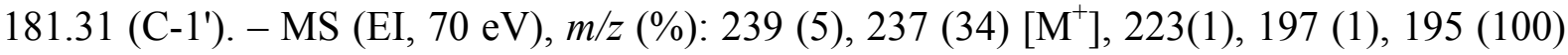
$\left[\mathrm{M}-\mathrm{C}_{3} \mathrm{H}_{6}\right]^{+}, 193(<1), 181$ (2), 180 (10), 167 (6), 166 (54), $162(<1), 152$ (8), 139 (29), 138 (4), 110 (6), 94 (1), 83 (14), 43 (36). $-\mathrm{C}_{12} \mathrm{H}_{15} \mathrm{NO}_{4}$ (237.26): ber. C 60.75, H 6.37, N 5.90; gef. C 60.57, H 6.29, N 5.77.

\section{Essigsäure-6-hydroxymethyl-3-methyl-2-oxo-5-propyl-1,2-dihydropyridin-4-ylester} (158)

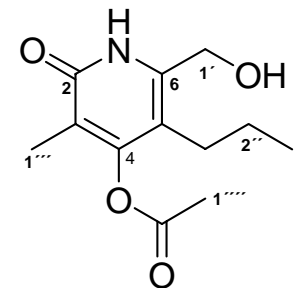

Die Reaktion wurde entsprechend der allgemeinen Arbeitsvorschrift A11 mit Essigsäure-6-formyl-3-methyl-2-oxo-5-propyl-1,2-dihydropyridin-4ylester (157) $(0.6615 \mathrm{~g}, 2.788 \mathrm{mmol})$ und $\mathrm{NaBH}_{4}(0.127 \mathrm{~g}, 3.35 \mathrm{mmol})$ in abs. EtOH $(18 \mathrm{~mL})$ bei $0{ }^{\circ} \mathrm{C}$ durchgeführt und $2.5 \mathrm{~h}$ bei Raumtemperatur gerührt. Die Reaktionslösung wurde mit $1.0 \mathrm{M} \mathrm{HCl}$ vorsichtig hydrolysiert bis ein $\mathrm{pH}-$ Wert von 6 erreicht wurde. Das Rohprodukt wurde aus heißem EtOAc umkristallisiert. Man erhielt $0.58 \mathrm{~g}(87 \%)$ des Alkohols $158\left(R_{f}=0.43\right.$, Hex/EE $\left.2: 1+10 \% \mathrm{MeOH}\right)$ als weißen Feststoff. ${ }^{1} \mathrm{H}-\mathrm{NMR}\left(250 \mathrm{MHz}, \mathrm{CDCl}_{3}\right): \delta=0.92(\mathrm{t}, J=7.2 \mathrm{~Hz}, 3 \mathrm{H}, 3 "-\mathrm{H}), 1.41\left(\mathrm{~m}_{\mathrm{c}}, 2 \mathrm{H}, 2 "-\mathrm{H}\right), 1.91$ (s, 3 H, 1"'-H), 2.22 (t, J= 7.8 Hz, 2 H, 1"-H), 2.31 (s, 3 H, 1'"'-H), 4.65 (s, 2 H, 1'-H).

\section{Allgemeine Arbeitsvorschrift A12 (Variante 1)}

Essigsäure-6-brommethyl-3-methyl-2-oxo-5-propyl-1,2-dihydropyridin-4-ylester (159)<smiles>CCCc1c(CBr)[nH]c(=O)c(I)c1OC(=O)F</smiles>

Die Reaktion wurde gemäß der allgemeinen Arbeitsvorschrift A12 (Variante 1) mit Essigsäure-6-hydroxymethyl-3-methyl-2-oxo-5-propyl-1,2dihydropyridin-4-ylester (158) (17 mg, 0.07 mmol) und $\mathrm{PBr}_{3}(7.35 \mu \mathrm{L}, 21.2$ g, $0.078 \mathrm{mmol})$ in $\mathrm{Et}_{2} \mathrm{O}(2 \mathrm{~mL})$ durchgeführt und $20 \mathrm{~h}$ gerührt. Man erhielt $21.5 \mathrm{mg}$ (quant.) des Brommethylpyridons 159 (Hex/EtOAc 6:1, $R_{f}=0.50$ ) als weißen Feststoff (Smp. $\left.190{ }^{\circ} \mathrm{C}\right) .-{ }^{1} \mathrm{H}-\mathrm{NMR}\left(250 \mathrm{MHz}, \mathrm{CDCl}_{3}\right): \delta=0.96(\mathrm{t}, J=7.2 \mathrm{~Hz}, 3 \mathrm{H}, 3$ "-H), 1.52 $\left(\mathrm{m}_{\mathrm{c}}, 2 \mathrm{H}, 2^{\prime \prime}-\mathrm{H}\right), 1.97$ (s, 3 H, 1"'-H), 2.30-2.40 [m, 5 H, 1"'"(1")-H], 4.40 (s, 2 H, 1'-H). - ${ }^{13} \mathrm{C}-$ 
NMR (62.9 MHz, $\mathrm{CDCl}_{3}$, zusätzl. DEPT): $\delta=10.14$ (+, C-1"'), 14.22 (+, C-3"), 20.43 (+, C1'"'), 22.97 (-, C-2"), 24.86 (-, C-1"), 27.76 (-, C-1'), 115.67 (C quart, $\mathrm{C}-3), 137.86$ (C $\left.\mathrm{C}_{\text {quart }}, \mathrm{C}-5\right)$, $157.38\left(2 \times \mathrm{C}_{\text {quart }}, \mathrm{C}^{*}-6, \mathrm{C}^{*}-4\right), 167.14$ [2 x C quart, $\left.\mathrm{O}\left(\mathrm{C}^{*} \mathrm{O}\right) \mathrm{CH}_{3}, \mathrm{C}^{*}-2\right] .-\mathrm{MS}\left(\mathrm{DCI}, \mathrm{NH}_{3}\right), m / z$ (\%): $447.5(1)\left[2 \mathrm{M}+\mathrm{NH}_{4}\right]^{+}, 398(<1), 320(1)\left[\mathrm{M}+\mathrm{NH}_{4}\right]^{+}, 302(4)\left[\mathrm{M}^{+}\right], 242$ (3), $241(20)$, $224(100), 181(<1), 152(1), 134(<1), 106(<1)$.

\section{Allgemeine Arbeitsvorschrift A12 (Variante 2)}

Essigsäure-6-brommethyl-3-methyl-2-oxo-5-propyl-1,2-dihydropyridin-4-ylester (159)<smiles>CCCc1c(CBr)[nH]c(=O)c(I)c1OC(=O)F</smiles>

Zur Durchführung der radikalischen Bromierung wurde eine Mischung aus dem Pyridonester 156, Bromsuccinimid (NBS) und Benzoylperoxid (DBPO) als Radikalstarter in $\mathrm{CCl}_{4}$ gelöst und unter Rückfluß bei $100{ }^{\circ} \mathrm{C}$ gerührt. (s. Theoretischer Teil, Tabelle 25). Die Suspensionslösung wurde zusätzlich mit Lichtbestrahlung $(1000 \mathrm{~W})$ aktiviert. Die Reaktionsmischung veränderte zeitweilig das Farbspektrum (nacheinander folgender Farbumschlag von farblos zu gelb, dann zu rot und anschließend wieder zu gelb). Das Ende der Reaktion wurde durch das Aufschwimmen des Succinimids auf das $\mathrm{CCl}_{4}$ als Lösungsmittel angezeigt. Da das NBS vor der Übertragung der Bromidgruppe eine höhere Dichte als $\mathrm{CCl}_{4}$ besitzt, schwamm das Bromierungsmittel während der Umsetzung als Suspension innerhalb der Reaktionslösung. Als Folge des ProtonBromid-Austausches besitzt das korrespondierende Succinimid eine geringere Dichte als das Lösungsmittel und schwimmt folglich als weißer Feststoff oberhalb der Reaktionslösung. Die Reaktionsmischung wurde nach Beendigung durch eine Celite-Aktivkohle-Schicht abfiltriert und mit $\mathrm{CCl}_{4}$ nachgewaschen. Das Lösungsmittel wurde i.V. entfernt. Es wurde ein Gemisch aus dem gewünschten Brommethylpyridon 159 als Nebenprodukt (9-25\%) und einer unerwünschten mehrfach bromierten Verbindung ${ }^{[39]}$ (91-75\%) als Hauptprodukt erhalten.

\section{Allgemeine Arbeitsvorschrift A16}

\section{Essigsäure-6-brommethyl-3-methyl-2-oxo-5-propyl-1-trimethylsilanyl-1,2-dihydropyri-}

\section{din-4-ylester(169)}

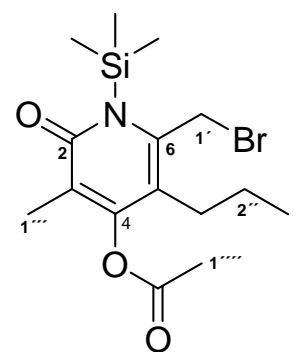

Eine Lösung von Chlortrimethylsilan $(100 \mu \mathrm{l}, 0.049 \mathrm{mmol}, 0.5 \mathrm{M}$ in $\mathrm{CDCl}_{3}$ ) wurde bei Raumtemperatur zu einer Lösung des Brommethylpyridons 159 (10 $\mathrm{mg}, 0.03 \mathrm{mmol})$ und $\mathrm{NEt}_{3}(13.8 \mu \mathrm{l}, 0.01 \mathrm{~g}, 0.10 \mathrm{mmol})$ in Benzol (2 mL) gegeben, die Reaktionslösung auf $40{ }^{\circ} \mathrm{C}$ erwärmt und $2 \mathrm{~h}$ gerührt. Nach Beendigung der Umsetzung wurde das Reaktionsgemisch 
i.V. einkonzentriert. Der Rückstand wurde in Benzol aufgenommen, über einen Spritzenfilter extrahiert und mit Chloroform $(0.5 \mathrm{~mL})$ nachgewaschen. Die erhaltene organische Phase wurde i.V. konzentriert. Man erhielt $12.3 \mathrm{mg}$ (quant.) der Silylverbindung 169 als weißen Feststoff. $-{ }^{1} \mathrm{H}-\mathrm{NMR}\left(250 \mathrm{MHz}, \mathrm{CDCl}_{3}\right): \delta=0.41\left[\mathrm{~s}, 9 \mathrm{H}, \mathrm{Si}\left(\mathrm{CH}_{3}\right)_{3}\right], 0.81\left(\mathrm{t}, J=7.2 \mathrm{~Hz}, 3 \mathrm{H}, 3^{\prime \prime}-\right.$ H), 1.45 (m, 2 H, 2"-H), 1.70 (s, 3 H, 1"'-H), 2.0 (s, 3 H, 1"'"-H), 2.33 (t, 2 H, 1"-H), 4.15 (s, 2 H, 1'-H). $-{ }^{13} \mathrm{C}-\mathrm{NMR}\left(62.9 \mathrm{MHz}, \mathrm{CDCl}_{3}\right.$, zusätzl. APT): $\delta=1.91\left[+, \mathrm{Si}\left(\mathrm{CH}_{3}\right)_{3}\right], 10.17(+, \mathrm{C}-$ 1"'), 14.23 (+, C-3"), 20.40 (+, C-1"''), 23.00 (-, C-2"), 24.88 (-, C-1"), 27.79 (-, C-1'), 115.56 (-, $\left.\mathrm{C}_{\text {quart }}, \mathrm{C}-3\right), 137.72$ (-, $\left.\mathrm{C}_{\text {quart }}, \mathrm{C}-5\right), 157.35$ (-, 2 x $\left.\mathrm{C}_{\text {quart }}, \mathrm{C}^{*}-6, \mathrm{C}^{*}-4\right), 164.90$ [-, $\mathrm{C}_{\text {quart }}$, $\left.\mathrm{O}\left(\mathrm{C}^{*} \mathrm{O}\right) \mathrm{CH}_{3}\right], 167.13\left(-, \mathrm{C}_{\text {quart }}, \mathrm{C}^{*}-2\right)$.

\section{Allgemeine Arbeitsvorschrift A17}

Essigsäure-3-methyl-6-(3'-methylocta-2'-enyl)-2-oxo-5-propyl-1,2-dihydropyridin-4ylester (165)

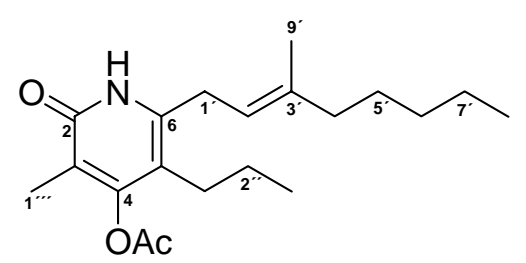

Die Vorversuche zu den Deprotonierungs- bzw. Protonierungsschritten wurden mit $\mathrm{AlMe}_{3}$-Lösung und ges. $\mathrm{NH}_{4} \mathrm{Cl}$ Lösung untersucht. Hierzu wurde ein NMR-Röhrchen mit einer Verschlußschraube als Reaktionsmedium verwendet und $\mathrm{AlMe}_{3}(5 \mu \mathrm{L}, 0.01 \mathrm{mmol}, 2.0 \mathrm{M}$ in Hexan) durch die Verschlußschraube über eine lange Kanüle bei $0{ }^{\circ} \mathrm{C}$ zu einer Lösung des Brommethylpyridons 159 (3.1 mg, $\left.0.01 \mathrm{mmol}\right)$ in THF (0.5 $\mathrm{mL}$ ) gegeben und gerührt. Die Blasenbildung zeigte die Gasentwicklung (Freisetzung von Methangas) und somit die Deprotonierung des Stickstoffprotons an. Zur Hydrolyse der Reaktionslösung wurden nach 0.5 h bzw. 1 h zwei Proben der Reaktionslösung mit ges. $\mathrm{NH}_{4} \mathrm{Cl}-$ Lösung versetzt und dünnschichtchromatographisch $\left(\mathrm{SiO}_{2}-\mathrm{Karten}\right)$ untersucht. Hierbei konnte keine Zersetzung des Bromids 159 festgestellt werden. Analoge Untersuchungsverfahren wurde mit 1.0 eq Brommethylpyridon 159 und 1.1 eq $\mathrm{Zn}(\mathrm{Et})_{2}$ (1.0 M in Hexan) in THF bei 0 ${ }^{\circ} \mathrm{C}$ durchgeführt und $7 \mathrm{~h}$ bei Raumtemperatur gerührt. Die Reaktionsproben wurden ebenfalls mit ges. $\mathrm{NH}_{4} \mathrm{Cl}$-Lösung aufgearbeitet und untersucht (DC-Kontrolle), wobei keine Zersetzung beobachtet wurde.

Die geplanten Kreuzkupplungsreaktionen unter Einsatz von $\mathrm{AlMe}_{3}, \mathrm{Al}(\mathrm{iBu})_{3}$ bzw. $\mathrm{Zn}(\mathrm{Et})_{2}$ wurden wie folgt durchgeführt: Für die Generierung der Reaktionslösung 1 wurde zu einer Lösung von 1.0 eq Brommethylpyridon 159 bei $0{ }^{\circ} \mathrm{C}$ eine Metallorganyl-Lösung [ $\mathrm{AlMe}_{3}$, $\mathrm{Al}(\mathrm{iBu})_{3}$ oder $\left.\mathrm{Zn}(\mathrm{Et})_{2}\right]$ entsprechend den in der Tabelle 27 angegebenen Äquivalenten an Base zugegeben und 30 min gerührt. Für die Reaktionslösung 2 wurde zur Generierung des Ala- 
nats $124 n \mathrm{BuLi}$ bei $0{ }^{\circ} \mathrm{C}$ zu einer Lösung des Alans 123 in THF gegeben und 30 min gerührt. Anschließend wurde die Reaktionslösung 1 gemäß der in der Tabelle 27 angegebenen Reaktionsbedingungen zu der Reaktionslösung 2 gegeben und gerührt. Die Reaktionslösungen wurden nach Beendigung der Versuche mit ges. $\mathrm{NH}_{4} \mathrm{Cl}$-Lösung hydrolysiert und mit EtOAc extrahiert. Die vereinigte organische Phase wurde über $\mathrm{MgSO}_{4}$ getrocknet und anschließend i.V. konzentriert. Im Rahmen dieser Untersuchung konnte das gewünschte Kreuzkupplungsprodukt 165 in einem Anteil von 23\% erhalten werden.

Die durchgeführten Kreuzkupplungsversuche unter Einsatz von $n \mathrm{BuLi}$ und $\mathrm{NaH}$ wurden unter den in der Tabelle 27 angegebenen Reaktionsbedingungen wie folgt durchgeführt: Für die Generierung der Reaktionslösung 1A wurde zu einer Lösung von 1.0 eq Brommethylpyridon 159 in THF 1.0 eq $n \mathrm{BuLi}$ bei $0^{\circ} \mathrm{C}$ zugegeben und $30 \mathrm{~min}$ gerührt. Für die Reaktionslösung 1B wurde zu einer Suspension von NaH ( $>99 \%$-ig, fettfrei) und 1.0 eq 18-Krone-6 in THF bei $-30{ }^{\circ} \mathrm{C}$ eine Lösung des Bromids 159 in THF zugegeben und $1 \mathrm{~h}$ gerührt. Das Alanat 124 (Reaktionslösung 2) wurde entsprechend der oben bei den lewissauren Metallorganylen angeführten Arbeitsvorschrift generiert und bei $-30{ }^{\circ} \mathrm{C} z \mathrm{zu}$ den Reaktionslösungen 1A bzw. 1B transferiert. Die Reaktionslösungen wurden nach Reaktionsabbruch ebenfalls mit ges. $\mathrm{NH}_{4} \mathrm{Cl}-$ Lösung und mit EtOAc aufgearbeitet. Im Rahmen dieser Untersuchung konnte das gewünschte Kreuzkupplungsprodukt 165 in einem Anteil von 20\% sowie das unerwünschte Debromierungsprodukt 156 (Anteil 74\%) erhalten werden.

\section{Allgemeine Arbeitsvorschrift A18}

\section{Essigsäure-6-brommethyl-1,3-dimethyl-2-oxo-5-propyl-1,2-dihydropyridin-4-ylester}

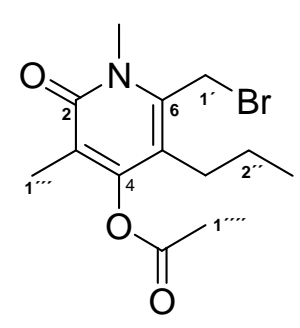

Zu einer Suspension des Brommethylpyridons 159 (50 mg, $0.17 \mathrm{mmol})$ und Kieselgel $(10 \mathrm{mg})$ in THF $(4 \mathrm{~mL})$ wurde bei $0{ }^{\circ} \mathrm{C}$ Diazomethan $(8.3 \mathrm{~mL}$, $3.3 \mathrm{mmol}, 0.4 \mathrm{M}$ in $\mathrm{Et}_{2} \mathrm{O}$ ) innerhalb von $2 \mathrm{~min}$ zugetropft und $20 \mathrm{~h}$ gerührt. Die Reaktionslösung wurde abfiltriert, das erhaltene Filtrat unter vermindertem Druck konzentriert. Man erhielt $52 \mathrm{mg}$ (quant.) eines Gemisches aus dem $N$-Methyl- 174 und dem O-Methyl-Isomer 177 im Verhältnis von 53:47 zugunsten des $N$-Isomers. Die Isomere wurden über säulenchromatographische Reinigung [SiO2, Hex/EtOAc 2:1 $+10 \% \mathrm{MeOH}, R_{f}(\mathrm{OMe})=0.94$ und $\left.\left.R_{f}(\mathrm{NMe})=0.47\right)\right]$ getrennt. Man erhielt $14 \mathrm{mg}(26 \%)$ der $N$-methylierten Verbindung 174 als weißen Feststoff. - ${ }^{1} \mathrm{H}-\mathrm{NMR}(250 \mathrm{MHz}$, $\left.\mathrm{CDCl}_{3}\right): \delta=0.98$ (t, J=7.2 Hz, 3 H, 3"-H), 1.54 (m, 2 H, 2"-H), 1.96 (s, 3 H, 1"'-H), 2.30 (m, 
3 H, 1"'-H), 2.45 (t, $J=8.0$ Hz, 2 H, 1"-H), 3.67 (s, 3 H, NCH $), 4.41$ (s, 2 H, 1'-H). - MS (EI, $70 \mathrm{eV}), m / z(\%): 317$ (16) [M + H], 273 (3), 244 (8), 236 (24), 194 (100), 178 (4), 166 (29), 150 (2), $136(2), 106(1), 87$ (1), 56 (5), 43 (21).

\section{Synthese der Pyridinderivate}

\section{Allgemeine Arbeitsvorschrift A19}

\section{Essigsäure-2-acetat-3,6-dimethyl-5-propylpyridin-4-ylester (178)}

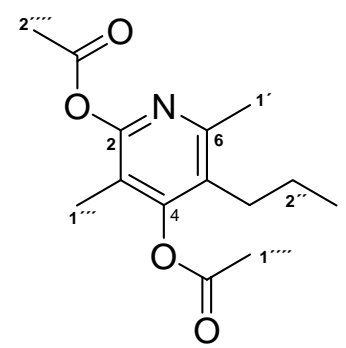

Eine Lösung des Pyridons 62 (51.5 mg, $0.285 \mathrm{mmol})$ in Essigsäureanhydrid $(3.00 \mathrm{~mL}, 3.22 \mathrm{~g}, 31.3 \mathrm{mmol})$ wurde $2 \mathrm{~h}$ auf $140{ }^{\circ} \mathrm{C}$ erhitzt und gerührt. Die Reaktionslösung wurde auf Raumtemperatur abgekühlt und mit ges. $\mathrm{NaHCO}_{3}$-Lösung $(5 \mathrm{~mL})$ und EtOAc $(15 \mathrm{~mL})$ versetzt. Die organische Phase wurde getrennt und die wässrige Phase mit EtOAc ( $2 \mathrm{x}$

$15 \mathrm{~mL}$ ) reextrahiert. Die vereinigte organische Phase wurde über $\mathrm{NaSO}_{4}$ getrocknet und das Lösungsmittel i.V. entfernt. Das Rohprodukt wurde säulenchromatographisch an Kieselgel gereinigt (10 g, Hex/EE 3:1 + 10\% MeOH). Man erhielt 0.0401 g (53\%) des Pyridondiacetats $178\left(R_{f}=0.42\right)$ als weißen Feststoff. $-{ }^{1} \mathrm{H}-\mathrm{NMR}\left(250 \mathrm{MHz}, \mathrm{CDCl}_{3}\right): \delta=0.98(\mathrm{t}, J=7.1 \mathrm{~Hz}, 3$ H, 3"-H), 1.48 (m c $_{\text {c }} 2$ H, 2"-H), 1.93 (s, 3 H, 1"'-H), 2.33 (s, 3 H, 2"'-H*), 2.36 (s, 3 H, 1'"'$\left.\mathrm{H}^{*}\right), 2.38-2.51$ [m, $\left.2 \mathrm{H}, 1^{\prime \prime}\left(1^{\prime}\right)-\mathrm{H}\right] .-{ }^{13} \mathrm{C}-\mathrm{NMR}\left(50.3 \mathrm{MHz}, \mathrm{C}_{6} \mathrm{D}_{6}\right.$, zusätzl. APT): $\delta=10.04(+$, C-1"'), 14.35 (+, C-3"), 19.68 (+, C*-2'"'), 20.26 (+, C*-1"'"), 21.73 (+, C-1'), 22.38 (-, C-2"), 29.05 (-, C-1"), 116.24 (-, C quart, $\left.C^{*}-3\right), 127.34$ (-, $\left.\mathrm{C}_{\text {quart }}, \mathrm{C}^{*}-5\right), 154.80$ (-, $\left.\mathrm{C}_{\text {quart }}, \mathrm{C}-6\right)$, 155.48 (-, $\left.\mathrm{C}_{\text {quart }}, \mathrm{C}-4\right), 157.33\left(-, \mathrm{C}_{\text {quart }}, \mathrm{C}-2\right), 167.06\left[-, \mathrm{C}_{\text {quart }}, \mathrm{O}\left(\mathrm{C}^{*} \mathrm{O}\right) \mathrm{CH}_{3}\right], 168.26\left[-, \mathrm{C}_{\text {quart }}\right.$, $\left.\mathrm{O}\left(\mathrm{C}^{*} \mathrm{O}\right) \mathrm{CH}_{3}\right]$.

\section{Essigsäure-2-acetoxy-6-formyl-3-methyl-5-propylpyridin-4-ylester (180)}<smiles>[3H]C(=O)Oc1c(F)c(OC(C)=O)nc(C=O)c1CCC</smiles>

Versuch 1: Die Reaktion wurde gemäß der allgemeinen Arbeitsvorschrift A10 mit Essigsäure-2-acetat-3,6-dimethyl-5-propylpyridin-4-ylester (178) $(17.9 \mathrm{mg}, 0.068 \mathrm{mmol})$ und akt. Selendioxid $(22.5 \mathrm{mg}, 0.203$ mmol) in wasserfreiem Dioxan $(5 \mathrm{~mL})$ bei einer Temperatur von $110{ }^{\circ} \mathrm{C}$ durchgeführt und $18.5 \mathrm{~h}$ gerührt. Es wurde ein Gemisch aus dem eingesetzten Diacetat 178 und dem gewünschten Pyridincarbaldehyd 180 im Verhältnis von 1.3:1 erhalten. 
Versuch 2: Die Reaktion wurde gemäß der allgemeinen Arbeitsvorschrift A10 mit Essigsäure2-acetat-3,6-dimethyl-5-propylpyridin-4-ylester (178) (18.8 mg, $0.071 \mathrm{mmol})$ und akt. Selendioxid (23.6 mg, $0.213 \mathrm{mmol})$ in wasserfreiem Dioxan $(5 \mathrm{~mL})$ bei einer Temperatur von 170 ${ }^{\circ} \mathrm{C}$ durchgeführt und $1.5 \mathrm{~h}$ gerührt. Die Reaktion führte zwar zur vollständigen Funktionalisierung der Seitenkette zum Aldehyd, es fand jedoch eine Abspaltung beider Acetatgruppen zum 4-Hydroxypyridonaldehyd statt.

\section{Allgemeine Arbeitsvorschrift A20}

\section{Essigsäure-2-acetoxy-6-chlormethyl-3-methyl-5-propylpyridin-4-ylester (179)}

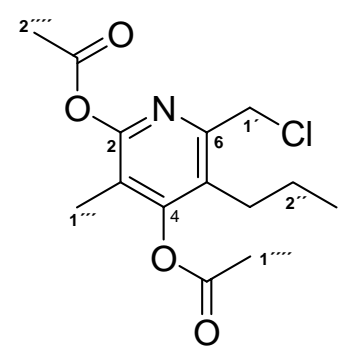

Für die Darstellung von 2-(Chlormethyl)pyridin 179 wurde eine Mischung von Diacetat 178 (80 mg, $0.30 \mathrm{mmol})$ und Benzamid $(1.1 \mathrm{mg}$, $9.0 \mu \mathrm{mol})$ in $\mathrm{CHCl}_{3}(4 \mathrm{~mL})$ gelöst und auf Rückfluß erhitzt. Innerhalb von $4 \mathrm{~h}$ wurde dann Trichlorisocyansäure (TCC) (39 mg, $0.17 \mathrm{mmol}$ ) portionsweise $(4 \mathrm{x})$ zugegeben und weiter unter Rückfluß gerührt. Die Reaktionslösung wurde nach $2 \mathrm{~h}$ auf Raumtemperatur abgekühlt und mit $\mathrm{CH}_{2} \mathrm{Cl}_{2}(20 \mathrm{~mL})$ verdünnt. Die Reaktionsmischung wurde über Celite abfiltriert und mit $\mathrm{CH}_{2} \mathrm{Cl}_{2}(40 \mathrm{~mL})$ gewaschen. Das Filtrat wurde mit ges. NaCl-Lösung $(10 \mathrm{~mL})$ versetzt und mit $\mathrm{CH}_{2} \mathrm{Cl}_{2}(2 \times 30$ $\mathrm{mL}$ ) extrahiert. Die vereinigte organische Phase wurde über $\mathrm{MgSO}_{4}$ getrocknet. Die durchgeführte säulenchromatographische Reinigung (Hex/EtOAC 4:1) ergab ein Gemisch aus $8.7 \mathrm{mg}$ (9\%) Dichlordiacetat $\left(R_{f}=0.62\right), 19 \mathrm{mg}(21 \%)$ Monochlordiacetat $179\left(R_{f}=0.44\right)$ und 29.5 mg (34\%) Dichlormonoacetat $\left(R_{f}=0.35\right)$. Das ${ }^{1} \mathrm{H}-\mathrm{NMR}-$ Spektrum des Monochlordiacetats 179 wurde wie folgt ausgewertet. - ${ }^{1} \mathrm{H}-\mathrm{NMR}\left(250 \mathrm{MHz}, \mathrm{CDCl}_{3}\right): \delta=0.99$ (t, $J=7.2 \mathrm{~Hz}, 3 \mathrm{H}$, 3"-H), 1.58 (m, 2 H, 2"-H), 1.96 (s, 3 H, 1"'-H), 2.33 (s, 3 H, 2"''-H*), 2.36 (s, 3 H, 1"''-H*), $2.54\left(\mathrm{~m}_{\mathrm{c}}, 2 \mathrm{H}, 1\right.$ "-H), 4.61 (s, 2 H, 1'-H).

\section{Essigsäure-2-acetoxy-6-brommethyl-3-methyl-5-propylpyridin-4-ylester (181)}

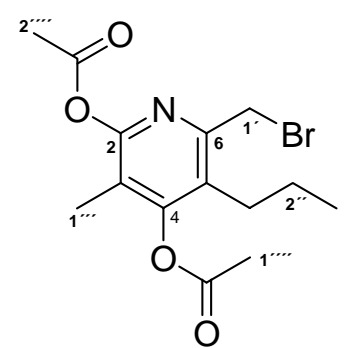

Die radikalische Bromierungsreaktion wurde gemäß der allgemeinen Arbeitsvorschrift A12 (Variante 2) mit Diacetoxypyridin 178 (40 mg, $0.15 \mathrm{mmol}$ ), NBS (26.4 mg, $0.148 \mathrm{mmol})$ und DBPO (5.5 mg, 0.02 mmol) in $\mathrm{CCl}_{4}$ bei $100{ }^{\circ} \mathrm{C}$ durchgeführt und $1 \mathrm{~h}$ gerührt. Die Reaktionslösung wurde nach Beendiung lediglich abfiltriert und unter verminder-

tem Druck konzentriert. Das erhaltene Rohprodukt wurde säulenchromatographisch an Kie- 
selgel (Hex:EE 3:1 + 5\% MeOH) gereinigt. Man erhielt $8.9 \mathrm{mg}(22 \%)$ des Bromids $181\left(R_{f}=\right.$ 0 36) als gelben Feststoff. Das ${ }^{1} \mathrm{H}-\mathrm{NMR}-$ Spektrum des Bromids 181 wurde wie folgt ausgewertet. - ${ }^{1} \mathrm{H}-\mathrm{NMR}\left(250 \mathrm{MHz}, \mathrm{CDCl}_{3}\right): \delta=0.94\left(\mathrm{~m}_{\mathrm{c}}, 3 \mathrm{H}, 3 "-\mathrm{H}\right), 1.55\left(\mathrm{~m}_{\mathrm{c}}, 2 \mathrm{H}, 2\right.$ "-H), 1.97 (s, 3 H, 1"'-H), 2.23-2.44 (m, 8 H, 1"(1"'")*(2"'')*-H), 4.42 (s, 2 H, 1'-H).

\section{Allgemeine Arbeitsvorschrift A21}

\section{1-Iod-2-methylhept-1-en 195}

$\mathrm{Zu}$ einer Suspension von $\mathrm{Cp}_{2} \mathrm{ZrCl}_{2}(0.30 \mathrm{~g}, 1.04 \mathrm{mmol})$ in wasserfreiem
1,2-Dichlorethan $(40 \mathrm{~mL})$ wurde 1-Heptin (122) $(0.68 \mathrm{~mL}, 0.50 \mathrm{~g}, 5.20$ mmol) zugegeben. $\mathrm{AlMe}_{3}\left(5.2 \mathrm{~mL}, 10.4 \mathrm{mmol}, 2.0 \mathrm{M}\right.$ in Hexan) wurde bei $0{ }^{\circ} \mathrm{C}$ zugegeben und $15 \mathrm{~h}$ gerührt. Die Reaktionslösung wurde mit einer Lösung von $\mathrm{I}_{2}$ (1.58 g, $6.24 \mathrm{mmol}$ ) in THF ( $8 \mathrm{~mL}$ ) versetzt und bis zur Entfärbung (von rot auf gelb) gerührt. Ein Gemisch aus $\mathrm{H}_{2} \mathrm{O} / \mathrm{Et}_{2} \mathrm{O}(50 \mathrm{~mL})$ wurde zugegeben und extrahiert. Die organische Phase wurde mit ges. $\mathrm{Na}_{2} \mathrm{~S}_{2} \mathrm{O}_{3}$-Lösung $(2 \times 20 \mathrm{~mL})$ gewaschen und die vereinigte organische Phase über $\mathrm{MgSO}_{4}$ getrocknet. Das erhaltene ölige Rohprodukt wurde über eine Kugelrohrdestillation gereinigt (0.01 mbar, $\left.55{ }^{\circ} \mathrm{C}\right)$. Man erhielt $0.694 \mathrm{~g}(56 \%)$ des Iodids 195 als farbloses Öl. $-{ }^{1} \mathrm{H}-\mathrm{NMR}$ $\left(250 \mathrm{MHz}, \mathrm{CDCl}_{3}\right): \delta=0.90(\mathrm{t}, J=6.5 \mathrm{~Hz}, 3 \mathrm{H}, 7-\mathrm{H}), 1.15-1.50$ [m, $\left.6 \mathrm{H}, 4(5)(6)-\mathrm{H}\right], 1.84$ (s, $\left.3 \mathrm{H}, 1^{\prime}-\mathrm{H}\right), 2.20\left(\mathrm{~m}_{\mathrm{c}}, 2 \mathrm{H}, 3-\mathrm{H}\right), 5.85$ (s, $\left.1 \mathrm{H}, 1-\mathrm{H}\right)$.

\section{Allgemeine Arbeitsvorschrift A22}

\section{1-Brom-2-methylhept-1-en 196}

$\underbrace{\mathrm{Zu} \text { einer Suspension von } \mathrm{Cp}_{2} \mathrm{ZrCl}_{2}(1.5 \mathrm{~g}, 5.2 \mathrm{mmol}) \text { in wasserfreiem }}_{1}$ mmol) zugegeben. $\mathrm{AlMe}_{3}\left(18.5 \mathrm{~mL}, 52.0 \mathrm{mmol}, 2.0 \mathrm{M}\right.$ in Hexan) wurde bei $0{ }^{\circ} \mathrm{C}$ zugegeben und $18 \mathrm{~h}$ gerührt. Die Reaktionslösung wurde auf $-30{ }^{\circ} \mathrm{C}$ abgekühlt, mit einer Lösung von $\mathrm{Br}_{2}$ ( $2.0 \mathrm{~mL}, 6.2 \mathrm{~g}, 39.0 \mathrm{mmol})$ in $\mathrm{CH}_{2} \mathrm{Cl}_{2}(10 \mathrm{~mL})$ versetzt und $3 \mathrm{~h}$ gerührt. Die Reaktionslösung wurde auf $0{ }^{\circ} \mathrm{C}$ (maximal $5{ }^{\circ} \mathrm{C}$ ) erwärmt, mit ges. $\mathrm{K}_{2} \mathrm{CO}_{3}$-Lösung $(5 \mathrm{~mL})$ versetzt und 5 min gerührt. Ein Gemisch aus $\mathrm{MgSO}_{4}$ und $\mathrm{Na}_{2} \mathrm{SO}_{4}(1: 1,24 \mathrm{~g})$ wurde zugegeben und 0.5 h gerührt. Die Reaktionslösung wurde über einer Fritte abfiltriert und mit $\mathrm{CH}_{2} \mathrm{Cl}_{2}(50 \mathrm{~mL})$ nachgewaschen. Die organische Phase wurde i.V. konzentriert. Das erhaltene ölige Rohprodukt wurde über eine Kugelrohrdestillation gereinigt (0.05 mbar, $\left.100{ }^{\circ} \mathrm{C}\right)$. Man erhielt $2.99 \mathrm{~g}(60 \%)$ des Bromids 196 als farbloses Öl. - ${ }^{1} \mathrm{H}-\mathrm{NMR}\left(250 \mathrm{MHz}, \mathrm{CDCl}_{3}\right): \delta=0.91(\mathrm{t}, J=6.5 \mathrm{~Hz}, 3 \mathrm{H}, 7-$ 
H), 1.20-1.44 [m, 4 H, 5(6)-H], 1.45-1.62 (m, 2 H, 4-H), 1.97 (s, 3 H, 1'-H), 1-98-2.10(m H, 3-H), 5.91 (s, $1 \mathrm{H}, 1-\mathrm{H})$.

\section{5. Übergangsmetall-katalysierte Reaktionen}

\section{Allgemeine Arbeitsvorschrift A23}

\section{(3,7-Dimethylocta-2,5-dienyl)benzol (131A) und (3-Methyloct-2-enyl)benzol (131)}

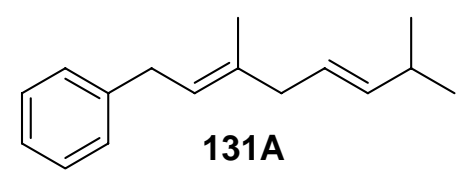

Die zur Darstellung der Verbindungen 131 bzw. 131A benötigten

Ethenylalane wurden aus den entsprechenden Verbindungen 1Heptin (122) und (2E)-6-Methylhept-4-en-1-in 103A in situ dar-<smiles>CCCCC/C(C)=C/Cc1ccccc1</smilesgestellt. Zur Carboaluminierung der Alkine 122 und 103A wurde bei $0{ }^{\circ} \mathrm{C}$ zu einer Lösung des Zirconocendichlorids (0.2 eq) in 1,2-Dichlorethan (DCE) 1.0 eq des Alkins (122 bzw. 103A) zugegeben und gerührt. Trimethylaluminium $\left(\mathrm{AlMe}_{3}\right)\left(2.0 \mathrm{eq}, 2.0 \mathrm{M}\right.$ in Toluol oder Hexan) wurde bei $0{ }^{\circ} \mathrm{C}$ zugegeben und $12 \mathrm{~h}$ bei Raumtemperatur gerührt. Die zitronengelbe Alan-Lösung (123 bzw. 123A) wurde dann bei Raumtemperatur mittels einer Transferkanüle zu einer Supension aus 0.05 eq $\left[\mathrm{Pd}\left(\mathrm{PPh}_{3}\right)_{4}\right]$ und Benzylbromid $\mathbf{1 3 0}$ in THF (bei einem Gesamtlösungmittelverhältnis THF:DCE von 50:15) überführt und gerührt. Nach Beendigung der Umsetzung (dünnschichtchromatographische Kontrolle) wurde die Reaktionslösung mit $3 \mathrm{~N} \mathrm{HCl}$ versetzt und mit Pentan, $\mathrm{H}_{2} \mathrm{O}, \mathrm{NaHCO}_{3}$ und $\mathrm{H}_{2} \mathrm{O}$ aufgearbeitet. Die vereinigte organische Phase wurde über $\mathrm{MgSO}_{4}$ getrocknet und i.V. konzentriert. Es wurden für die angestrebten Kreuzkupplungsprodukte 131A bzw. 131 Bildungsanteile von 55-88\% bzw. 79-97\% erhalten.

\section{Allgemeine Arbeitsvorschrift A24}

(3-Methyloct-2-enyl)benzol (131)

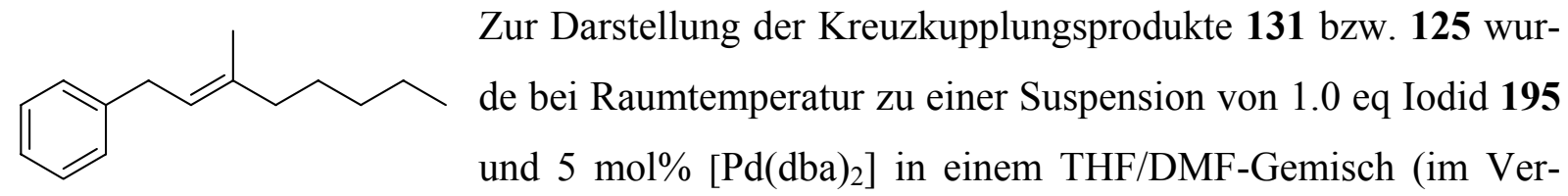

hältnis 1:1) eine Lösung der Zinkorganyle 197 (1.33-4.0 eq, $0.8 \mathrm{M}$ in THF) bzw. 198 (2.33 eq, $0.8 \mathrm{M}$ in THF) (zur Generierung der Zinkorganyle siehe unten) zugegeben und $15 \mathrm{~h}$ gerührt (vgl. Tabelle 37). Nach Abbruch der Umsetzungen wurden die Reaktionslösungen mit ges. $\mathrm{NH}_{4} \mathrm{Cl}$-Lösung, Pentan und anschließend mit ges. NaCl-Lösung aufgearbeitet. Die vereinigte 
organische Phase wurde über $\mathrm{MgSO}_{4}$ getrocknet. Das Lösungsmittel wurde i.V. entfernt. Die Umsetzungen ergaben die Bildung des Kreuzkupplungsprodukts 131 in Anteilen von 58-68\%. Als Nebenprodukt wurde das Homokupplungsprodukt 132 in Anteilen von 32-42\% erhalten. Die analog durchgeführten Pd-katalysierten Kreuzkupplungsreaktionen des aus dem Brommethylpyron 96 generierten Zinkorganyls 198 mit dem Iodid 195 zur Bildung des Kreuzkupplungsprodukts 125 blieben dagegen erfolglos.

\section{Generierung der Zinkorganyle 197 und 198}<smiles>CCCc1oc(=O)c(C)c(OCCBr)c1CCBr</smiles><smiles>Br[Mg]Cc1ccccc1</smiles>

Darstellung der Zinkorganyllösung 197 nach Variante 1: Zur Generierung der Zinkorganyllösung 197 nach der Variante 1 wurden in einem getrockneten Dreihalskolben mit Thermometer, Rührschwein und Rückflußkühler unter Argonatmosphäre 3.0 eq des Zn (Alderich-mesh 325) mit einer Lösung von Dibromethan (0.25 eq) in THF (genaue Volumenangabe) versetzt. Die Suspension wurde mit einem Fön 30 Sekunden lang auf Rückfluß erhitzt und anschließend auf Raumtemperatur abgekühlt (Vorgang wurde 3 Mal wiederholt). Anschließend wurde Chlortrimethylsilan (0.3 eq) zur Aktivierung und zum Anätzen der ZnÖberfläche zugegeben und 5 min gerührt. Die Reaktionsmischung wurde mit einem Eisbad auf $0{ }^{\circ} \mathrm{C}$ abgekühlt. Anschließend wurde 1.0 eq des Benzylbromids 130 in THF (genaue Volumenangabe) über eine Syring-Pumpe (1 Tropfen/5 sec) langsam zugegeben und nach Beendigung der Lösungszugabe weitere $30 \mathrm{~min}$ bei Raumtemperatur gerührt (nach Knochel $0.8 \mathrm{M}$ Zinkbromid-Lösung in $\mathrm{THF}^{[90]}$ ).

Darstellung der Zinkorganyllösungen 197 bzw. 198 nach Variante 2: Zur Generierung der Zinkorganyllösungen 197 bzw. 198 nach der Variante 2 wurde zu einer Mischung von 1.5 eq Zn (Alderich-mesh 325) und 1.0 eq der entsprechenden Bromidlösung (Benzylbromid 130 bzw. Brommethylpyron 96) in THF (genaue Volumenangabe) $I_{2}(0.15$ eq) zugegeben und gerührt. Die Reaktionsmischung wurde $45 \mathrm{~min}$ im Ultraschallbad aktiviert. Die vollständige Umsetzung des Bromids ist durch dünnschichtchromatographische Detektion überprüfbar (die Abwesenheit des Bromids zeigt vollständige Umsetzung zu den Zinkorganylen an). 


\section{Allgemeine Arbeitsvorschrift A25}

Allylbenzol (213)

1

Zur Generierung des Vinyl-Cuprats wurde bei $-78^{\circ} \mathrm{C}$ zu einer Suspension von $\mathrm{CuBr} \cdot \mathrm{SMe}_{2}(41 \mathrm{mg}, 0.20 \mathrm{mmol})$ in THF (1 mL) VinylmagnesiumbromidLösung (6.03 mL, 4.0 mmol, 0.665 M in THF) zugegeben und 30 min gerührt. Nach der gewünschten Ummetallierung wurde bei $-78^{\circ} \mathrm{C}$ eine Lösung des Benzylbromids $130(238 \mu \mathrm{L}$, $343 \mathrm{mg}, 2.00 \mathrm{mmol})$ in THF (1 mL) zugegeben und 1.5 gerührt. Die Reaktionslösung wurde bei $-78{ }^{\circ} \mathrm{C}$ mit $\mathrm{H}_{2} \mathrm{O}$ hydrolysiert, anschließend auf Raumtemperatur erwärmt und mit Pentan extrahiert (3 x $10 \mathrm{~mL})$. Die vereinigte organische Phase wurde nach Behandlung mit ges. $\mathrm{NaCl}$-Lösung und Extraktion über $\mathrm{MgSO}_{4}$ getrocknet und konzentriert. Man erhielt $237 \mathrm{mg}$ (quant.) eines Gemisches aus dem Allylbenzol 213 (95\%) und dem Homokupplungsprodukt $132(5 \%)$ als gelbes Öl. $-{ }^{1} \mathrm{H}-\mathrm{NMR}\left(250 \mathrm{MHz}, \mathrm{CDCl}_{3}\right): \delta=3.42\left(\mathrm{~m}_{\mathrm{c}}, 2 \mathrm{H}, 3-\mathrm{H}\right), 5.10\left(\mathrm{~m}_{\mathrm{c}}, 2\right.$ $\mathrm{H}, 1-\mathrm{H}), 6.00\left(\mathrm{~m}_{\mathrm{c}}, 1 \mathrm{H}, 2-\mathrm{H}\right)$, 7.11-7.43 (m, $5 \mathrm{H}$, Arom.-H).

\section{(3-Methyloct-2-enyl)benzol (131)}

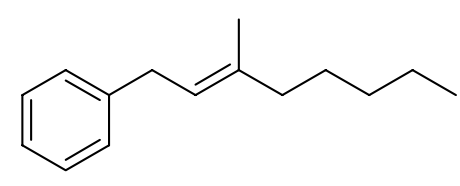

Die Kreuzkupplungsreaktion zur Darstellung der Verbindung 131 wurde gemäß der allgemeinen Arbeitsvorschrift A25 mit Benzylbromid 130 (35 $\mu \mathrm{L}, 50 \mathrm{mg}, 0.29 \mathrm{mmol}), \mathrm{CuBr} \cdot \mathrm{SMe}_{2}(60 \mathrm{mg}$, $0.29 \mathrm{mmol})$ und 1-Magnesiumiodid-2-methylhept-1-en (215) $(2.78 \mathrm{~mL}, 0.585 \mathrm{mmol}, 0.21 \mathrm{M}$ in $\left.\mathrm{Et}_{2} \mathrm{O} / \mathrm{THF}\right)$ in THF $(5 \mathrm{~mL})$ durchgeführt. Man erhielt ein Gemisch aus dem eingesetzten Edukt 130 (40\%), dem gewünschten Kreuzkupplungsprodukt 131 (50\%) und dem Homokupplungsprodukt 136 (10\%).

Benzoesäure-6-[(E)-3'-methylocta-2'-enyl]-3-methyl-2-oxo-5-propyl-2H-pyran-4-ylester (125)

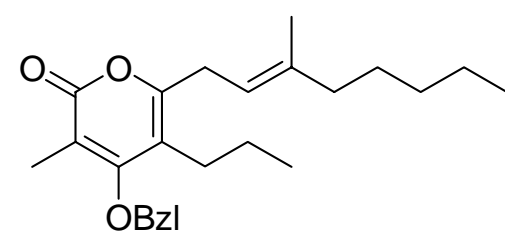

Die Kreuzkupplungsreaktion zur Darstellung der Verbindung 125 wurde gemäß der allgemeinen Arbeitsvorschrift A25 mit Brommethylpyron 96 (50 mg, $0.14 \mathrm{mmol}), \mathrm{CuBr} \cdot \mathrm{SMe}_{2}(28$ $\mathrm{mg}, 0.14 \mathrm{mmol}$ ) und 1-Magnesiumiodid-2-methylhept-1-en 
(215) (1.3 mL, $0.27 \mathrm{mmol}, 0.21 \mathrm{M}$ in $\left.\mathrm{Et}_{2} \mathrm{O} / \mathrm{THF}\right)$ in THF (5 mL) durchgeführt. Man erhielt ein Gemisch aus dem eingesetzten Edukt 96 (61\%), dem Kreuzkupplungsprodukt 125 (33\%) und dem Homokupplungsprodukt 137 (6\%).

\section{Allgemeine Arbeitsvorschrift A26}

\section{4-Benzoesäure-6-allyl-3-methyl-2-oxo-5-propyl-2H-pyran-4-ylester (214)}

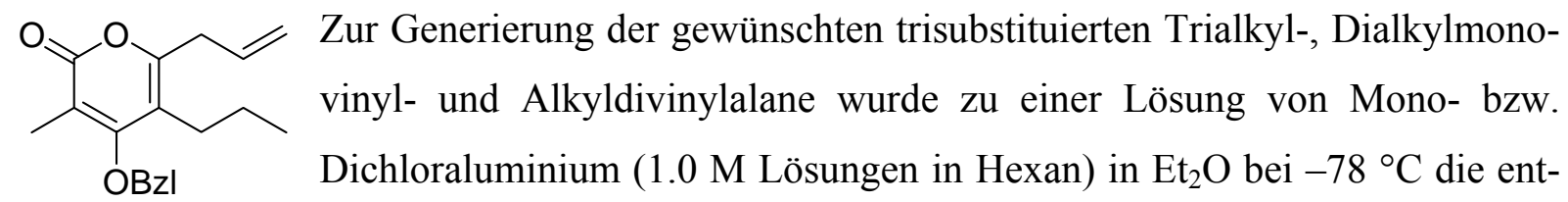
sprechende Alkyl- bzw. Vinylgrignard-Lösung (1.0 M Lösungen in THF oder Hexan) zugegeben und $1 \mathrm{~h}$ bei $-78{ }^{\circ} \mathrm{C}$ gerührt. Die Monochloride wurden stets mit $1.0 \mathrm{eq}$, die Dichloride mit 2.0 eq der Grignardlösung umgesetzt. Nach dem abschließenden Transmetallierungsprozeß (nach $1 \mathrm{~h}$ wurde weißer Niederschlag beobachtet, der die Bildung von $\mathrm{MgBrCl}$ und somit eine ablaufende Transmetallierung anzeigte) wurde die generierte Alanlösung bei $-78{ }^{\circ} \mathrm{C} \mathrm{zu}$ einer Suspension aus dem Brommethylpyron 96 (1.0 eq) und dem Cu-Katalysator (2.5 mol\% $\mathrm{CuBr}$ ) in $\mathrm{Et}_{2} \mathrm{O}$ transferiert und gerührt. Bei den durchgeführten Kreuzkupplungsversuchen wurde ein Gemisch aus dem gewünschten Kreuzkupplungsprodukt 214 (91\%) und dem Homokupplungsprodukt 137 (9\%) erhalten.

\section{Allgemeine Arbeitsvorschrift A27}

\section{Benzoesäure-6-[(E)-3'-methylocta-2'-enyl]-3-methyl-2-oxo-5-propyl-2H-pyran-4-ylester}

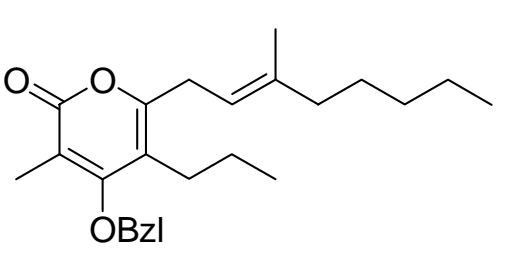

Zur Generierung von 4.0 eq 1-Lithium-2-methylhept-1-en

(209) wurde zu einer Lösung von 1-Brom-2-methylhept-1-en (196) (4.0 eq) in THF bei $-78^{\circ} \mathrm{C} t \operatorname{BuLi}(8.0$ eq, $1.54 \mathrm{M}$ in Pentan) gegeben und $2.5 \mathrm{~h}$ gerührt. Zur Lithiumorganyllösung 209 wurde bei $-78{ }^{\circ} \mathrm{C}$ eine Lösung aus $\mathrm{Et}_{2} \mathrm{AlCl}$ (4.0 eq, $1.0 \mathrm{M}$ in Hexan) bzw. $\mathrm{EtAlCl}_{2}$ (2.0 eq, $1.0 \mathrm{M}$ in Hexan) in $\mathrm{Et}_{2} \mathrm{O}$ gegeben und $1 \mathrm{~h}$ gerührt. Anschließend wurden die generierten Alanlösungen 219 bzw. 220 jeweils zu einer auf $-78{ }^{\circ} \mathrm{C}$ vorgekühlten Suspension von Brommethylpyron 96 und $\mathrm{CuBr}(2.5 \mathrm{~mol} \%)$ transferiert und über Nacht auf Raumtemperatur erwärmt und gerührt. Die Reaktionslösungen wurden mit ges. $\mathrm{NH}_{4} \mathrm{Cl}$-Lösung bzw. 1.0 M HClLösung auf $5<\mathrm{pH}>6$ eingestellt und mit EtOAc extrahiert. Das gewünschte Kreuzkupp- 
lungsprodukt 125 blieb bei den durchgeführten Versuchen aus. Als Hauptprodukte wurde ein Gemisch aus dem Homokupplungsprodukt 137 (3 bzw. 20\%) und dem debromierten Produkt 89 (80 bzw. 42\%) erhalten.

\section{Allgemeine Arbeitsvorschrift A28}

(3-Methyloct-2-enyl)benzol (131)

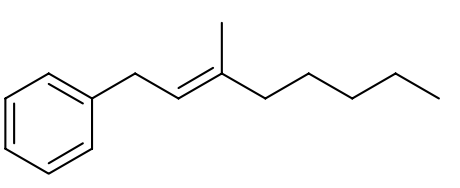

Zur Generierung des aktiven Ni-(0)-Katalysators wurde $n \mathrm{BuLi}$ (0.2 eq, 2.6 $\mathrm{M}$ in Cyclohexan) bei $0{ }^{\circ} \mathrm{C} \mathrm{zu}$ einer Suspension aus Dichlorbis(triphenylphosphin)nickel $\left[\mathrm{Ni}\left(\mathrm{PPh}_{3}\right)_{2} \mathrm{Cl}_{2}\right] \quad\left(\begin{array}{ll}10 & \mathrm{~mol} \%)\end{array}\right.$ und $\mathrm{PPh}_{3}(0.2$ eq) in THF zugegeben, das Kältebad entfernt und die Reaktionslösung bei Raumtemperatur 10 min gerührt. Die Reaktionslösungen wurden mit je 1.0 eq Benzylbromid 130 bzw. -chlorid 222 versetzt und für weitere 10 min gerührt. Anschließend wurde die Reaktionslösung bei $0^{\circ} \mathrm{C}$ zu einer Lösung des Alans 123 (1.5 eq, 1.0 M in Hexan) in THF zugegeben, 30 min bei $0{ }^{\circ} \mathrm{C}$ und anschließend $1.5 \mathrm{~h}$ bei Raumtemperatur gerührt. Die bräunlich gelben Reaktionslösungen wurden mit $1.0 \mathrm{M} \mathrm{HCl}$ und EtOAc aufgearbeitet. Die vereinigte organische Phase wurde über $\mathrm{MgSO}_{4}$ getrocknet und unter vermindertem Druck konzentriert. Während die Reaktion mit Benzylchlorid 222 zur Bildung eines Gemisches aus dem Kreuzkupplungsprodukt 131 (96\%) und dem Homokupplungsprodukt 132 (4\%) führte, ergab die Umsetzung mit Benzylbromid 130 ein Gemisch aus dem Kreuzkupplungsprodukt 131 (72\%) und den Homokupplungsprodukten 132 (22\%) und 136 (6\%).

\section{Allgemeine Arbeitsvorschrift A29}

Benzoesäure-6-[(E)-3'-methylocta-2'-enyl]-3-methyl-2-oxo-5-propyl-2H-pyran-4-ylester (125)

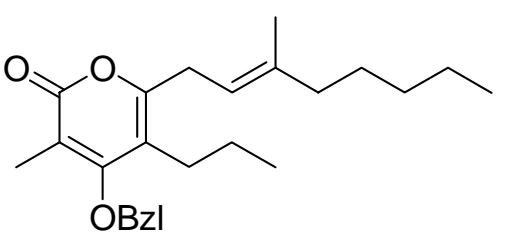

Zur Reduzierung des Ni(II)-Katalysators zum aktiven Ni(0)Katalysator wurde $n \mathrm{BuLi}(8.0 \mu \mathrm{L}, 0.02 \mathrm{mmol}, 2.6 \mathrm{M}$ in Cyclohexan) bei Raumtemperatur zu einer Suspension von NiII $\left[\left(\mathrm{PPh}_{3}\right)_{2}\right] \mathrm{Cl}_{2}(6.5 \mathrm{mg}, 10 \mu \mathrm{mol})$ in THF $(1 \mathrm{~mL})$ gegeben und

10 min gerührt. Der weinroten Reaktionslösung wurde eine Suspension von Chlormethylpyron 135 (32 mg, $0.1 \mathrm{mmol})$ in THF $(0.5 \mathrm{~mL})$ gegeben und diese für $10 \mathrm{~min}$ gerührt, wobei sich die Lösung grünlichgelb verfärbte. Die grünlichgelbe Ni(0)-Organyllösung wurde anschließend zu einer auf $0^{\circ} \mathrm{C}$ vorgekühlten Lösung von $\mathrm{AlMe}_{3}$-freiem Alan $\mathbf{1 2 3}(150 \mu \mathrm{L}, 0.150$ 
mmol, 1.0 M in Hexan) gegeben und 30 min gerührt (gelbe Reaktionslösung). Die Reaktionslösung wurde auf Raumtemperatur erwärmt und 7 gerührt. Nach Feststellung der vollständigen Umsetzung durch Detektion wurde die Reaktionslösung mit 1.0 M HCl und EtOAc $(2 \mathrm{x}$ $20 \mathrm{~mL}$ ) aufgearbeitet. Die vereinigte organische Phase wurde über $\mathrm{MgSO}_{4}$ getrocknet und unter vermindertem Druck konzentriert. Die Auswertung des ${ }^{1} \mathrm{H}-\mathrm{NMR}-\mathrm{Spektrums}$ des erhaltenen Rohproduktes zeigte ein Gemisch aus dem gewünschten Kreuzkupplungsprodukt 125 (20\%) und dem Homokupplungsprodukt 136 (80\%). Das Kreuzkupplungsprodukt 136 wurde massenspektroskopisch nachgewiesen. - MS (EI), m/z (\%): 396 (2) $\left[\mathrm{M}^{+}\right], 286$ (16), 221 (2), 181 (5), 115 (1), 109 (2), 105 (100), 77 (15), 71 (11), 43 (5).

\section{Darstellung der Ausgangssubstanzen zur Collinolactonteilsynthese (Hauptteil 2)}

\section{Allgemeine Arbeitsvorschrift B1}

\section{(E)-Hex-3-en-2,5-dion (272)}

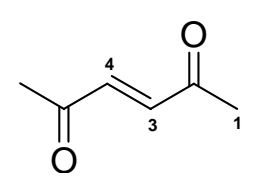

$\mathrm{Zu}$ einer Lösung von 2,5-Dimethylfuran (271) (15.5 g, $0.172 \mathrm{~mol}$ ) in einem Aceton/Wasser-Gemisch im Verhältnis 2:1 (140 mL:70 mL) wurde bei $-20{ }^{\circ} \mathrm{C}$ und unter starkem Rühren $\mathrm{Br}_{2}(8.81 \mathrm{~mL}, 27.48 \mathrm{~g}, 0.172 \mathrm{~mol})$ tropfenweise zugegeben. Die Reaktionslösung wurde nach anschließender Entfernung des Kältebads binnen $5 \mathrm{~h}$ auf Raumtemperatur erwärmt und gerührt, wobei sich eine Farbänderung von farblos nach bräunlich vollzog. Die Reaktionslösungen wurden dünnschichtchromatographisch detektiert ( $n$ Pentan:EtOAc 4:1, $R_{f}=0.33$ ), wodurch das Ende der Umsetzung aufgrund des stark UV-löschenden Charakters des Endions 272 leicht sichergestellt werden konnte. Die Reaktionslösung wurde mit einem Gemisch aus $\mathrm{Et}_{2} \mathrm{O}$ und ges. $\mathrm{NaHCO}_{3}$-Lösung $(2$ x je $150 \mathrm{~mL}$ ) versetzt $(\mathrm{pH}=9)$ und extrahiert. Die vereinigte organische Phase wurde mit ges. NaClLösung (150 mL) gewaschen und mit $\mathrm{Et}_{2} \mathrm{O}(2 \times 100 \mathrm{~mL})$ extrahiert. Die vereinigte organische Phase wurde über $\mathrm{Na}_{2} \mathrm{SO}_{4}$ getrocknet und i.V. konzentriert. Nach einer säulenchromatographischen Filtration an Kieselgel (120 g, $n$ Pentan:EtOAc 4:1) erhielt man $11.9 \mathrm{~g}(62 \%)$ der Diketon-Verbindung $272\left(R_{f}=0.33\right)$ als gelben Feststoff ${ }^{[111]}$. - ${ }^{1}$ H-NMR $(250 \mathrm{MHz}$, $\left.\mathrm{CDCl}_{3}\right): \delta=2.35[\mathrm{~s}, 6 \mathrm{H}, 1(6)-\mathrm{H}], 6.74[\mathrm{~s}, 2 \mathrm{H}, 3(4)-\mathrm{H}]$. 


\section{Allgemeine Arbeitsvorschrift B2}

(E)-5,5-Dimethoxyhex-3-en-2-on (273)

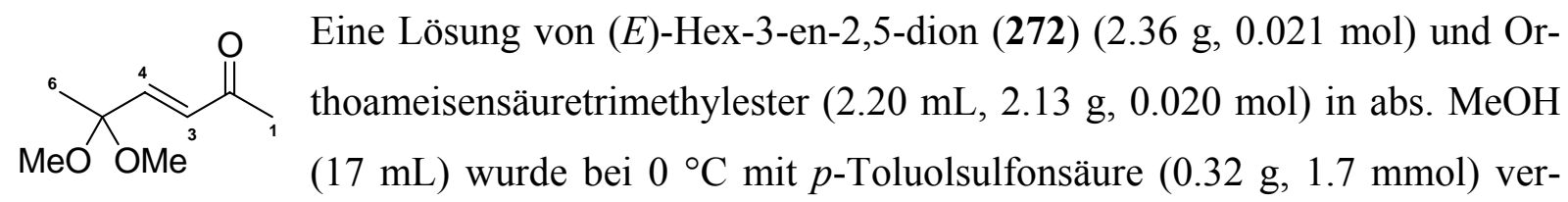
setzt und $1 \mathrm{~h}$ gerührt. Die braune Lösung wurde anschließend mit $\mathrm{NEt}_{3}(0.23 \mathrm{~mL}, 0.17 \mathrm{~g}, 1.7$ mmol) versetzt und für weitere 10 min gerührt. Die Reaktionslösung wurde mit $\mathrm{Et}_{2} \mathrm{O}$ und ges. $\mathrm{NaCl}$-Lösung extrahiert $\left(2 \times 150 \mathrm{~mL}\right.$ ). Die vereinigte organische Phase wurde mit $\mathrm{NaSO}_{4}$ getrocknet und i.V. konzentriert. Die braune Flüssigkeit wurde einer fraktionierenden Destillation unterzogen. Man erhielt 3.758 g (91\%) der Verbindung 272 als farblose Flüssigkeit (Sdp. 60-65 $\left.{ }^{\circ} \mathrm{C}, 0.001 \mathrm{mbar}\right) .-{ }^{1} \mathrm{H}-\mathrm{NMR}\left(250 \mathrm{MHz}, \mathrm{CDCl}_{3}\right): \delta=1.41$ (s, $\left.3 \mathrm{H}, 6-\mathrm{H}\right), 2.24$ (s, 3 H, 1-H), 3.18 (s, $6 \mathrm{H}, 2$ x OCH 3$), 6.48$ [dd, $J=15.8 \mathrm{~Hz}, 2 \mathrm{H}, 3(4)-\mathrm{H}] .-{ }^{13} \mathrm{C}-\mathrm{NMR}(50.3 \mathrm{MHz}$, $\left.\mathrm{CDCl}_{3}\right): \delta=23.50$ (C-6), $27.60(\mathrm{C}-1), 49.07$ (2 x $\left.\mathrm{OCH}_{3}\right), 99.28$ (C-5), $131.36(\mathrm{C}-3), 146.25$ (C-4), 198.48 (C-2).

\section{Allgemeine Arbeitsvorschrift B3 (Variante 1)}

(E)-5,5-Dimethoxy-2-trimethylsilyloxyhexa-1,3-dien (274)

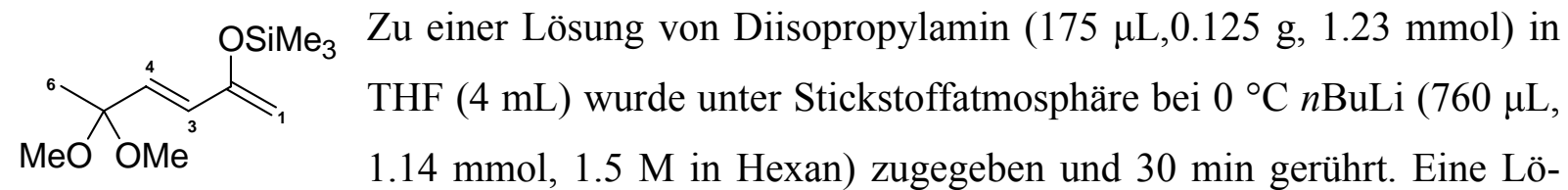
sung von (E)-5,5-Dimethoxyhex-3-en-2-on (273) (0.15 g, $0.95 \mathrm{mmol})$ in THF (1 mL) wurde bei $-78{ }^{\circ} \mathrm{C}$ zugetropft und für weitere 30 min gerührt. Dann wurde eine Lösung von Chlortrimethylsilan $(0.17 \mathrm{~mL}, 0.14 \mathrm{~g}, 1.3 \mathrm{mmol})$ in THF $(1 \mathrm{~mL})$ zugetropft und die Reaktionslösung innerhalb von $3 \mathrm{~h}$ auf Raumtemperatur erwärmt. Anschließend wurde das Lösungsmittel entfernt, das Rohprodukt abfiltriert, der Feststoff mit $n$ Pentan $(3 \times 50 \mathrm{~mL})$ gewaschen und das Lösungsmittel i.V. entfernt. Man erhielt $218 \mathrm{mg}$ (quant.) des Silylenolethers 274 als gelbes Ö1. - ${ }^{1} \mathrm{H}-\mathrm{NMR}\left(250 \mathrm{MHz}, \mathrm{CDCl}_{3}\right): \delta=0.20\left[\mathrm{~s}, 9 \mathrm{H}, \mathrm{Si}\left(\mathrm{CH}_{3}\right)_{3}\right], 1.37$ (s, $\left.3 \mathrm{H}, 6-\mathrm{H}\right), 3.16$ (s, 6 $\left.\mathrm{H}, 2 \times \mathrm{OCH}_{3}\right), 4.38$ (d, $\left.J_{2}=2 \mathrm{~Hz}, 2 \mathrm{H}, 1-\mathrm{H}\right), 5.89$ (d, $\left.J=15.8 \mathrm{~Hz}, 1 \mathrm{H}, 4-\mathrm{H}\right), 6.23$ (d, $J=15.8$ $\mathrm{Hz}, 1 \mathrm{H}, 3-\mathrm{H})$. 


\section{Allgemeine Arbeitsvorschrift B3 (Variante 2) \\ (E)-5,5-Dimethoxy-2-trimethylsilyloxyhexa-1,3-dien (274)}

Unter Schutzgasatmosphäre wurde eine Lösung von Chlortrimethylsilan $(0.40 \mathrm{~mL}, 0.34 \mathrm{~g}, 3.2$ $\mathrm{mmol})$ in DMF $(1 \mathrm{~mL})$ tropfenweise bei $0{ }^{\circ} \mathrm{C}$ zu einer Lösung des Ketoacetals 273 (400 mg, $2.53 \mathrm{mmol})$ und $\mathrm{NEt}_{3}(0.49 \mathrm{~mL}, 0.36 \mathrm{~g}, 3.5 \mathrm{mmol})$ in DMF $(5 \mathrm{~mL})$ gegeben und nach Entfernung des Kältebads $12 \mathrm{~h}$ bei Raumtemperatur gerührt. Nach Zugabe von ges. $\mathrm{NaHCO}_{3}$ Lösung (5 mL) wurde die Reaktionslösung mit $n$ Pentan $(3 \times 40 \mathrm{~mL})$ extrahiert. Die vereinigte organische Phase wurde über $\mathrm{Na}_{2} \mathrm{SO}_{4}$ getrocknet und unter vermindertem Druck konzentriert. Die Umsetzung ergab ein Gemisch aus dem eingesetzten Edukt 273 und dem gewünschten Silylenolether 274 im Verhältnis 60:40, welches einer Ausbeute von lediglich 33\% für das angestrebte Produkt 274 entsprach.

\section{Allgemeine Arbeitsvorschrift B3 (Variante 3) \\ (E)-5,5-Dimethoxy-2-trimethylsilyloxyhexa-1,3-dien (274)}

Bei $0{ }^{\circ} \mathrm{C}$ wurde zu einer Lösung von Ketoacetal 273 (0.15 g, 0.95 mmol) und $\mathrm{NEt}_{3}(220 \mu \mathrm{L}$, $158 \mathrm{mg}, 1.56 \mathrm{mmol})$ in Acetonitril (4 mL) eine Lösung von Chlortrimethylsilan (193 $\mu \mathrm{L}, 170$ $\mathrm{mg}, 1.52 \mathrm{mmol})$ in $\mathrm{MeCN}(2 \mathrm{~mL})$ innerhalb von $10 \mathrm{~min}$ tropfenweise zugegeben und $10 \mathrm{~min}$ gerührt (vgl. Tabelle 52). Anschließend wurde eine Lösung von NaI (180 mg, $1.19 \mathrm{mmol})$ in MeCN (7 mL) tropfenweise bei $0{ }^{\circ} \mathrm{C}$ zugefügt und das Kältebad entfernt. Die Reaktionslösung wurde 20 h unter Rückfluß gerührt. Nach Abkühlen auf Raumtemperatur wurde die Reaktionslösung auf Eiswasser $(10 \mathrm{~mL})$ gegeben und mit $n$ Pentan $(3 \times 30 \mathrm{~mL})$ extrahiert. Nach Auswaschen der vereinigten organischen Phase mit $\mathrm{H}_{2} \mathrm{O}(2 \times 20 \mathrm{~mL})$ wurde die organische Schicht über $\mathrm{Na}_{2} \mathrm{SO}_{4}$ getrocknet. Die Auswertung des Rohproduktes (93 mg, Ausbeute 42\%) des Versuchs ergab ein Gemisch aus dem Edukt 273 und dem Silylenolether 274 im Verhältnis von $78: 22$.

\section{Allgemeine Arbeitsvorschrift B4}

\section{(E)-1-Brom-5,5-dimethoxyhex-3-en-2-on (275)}

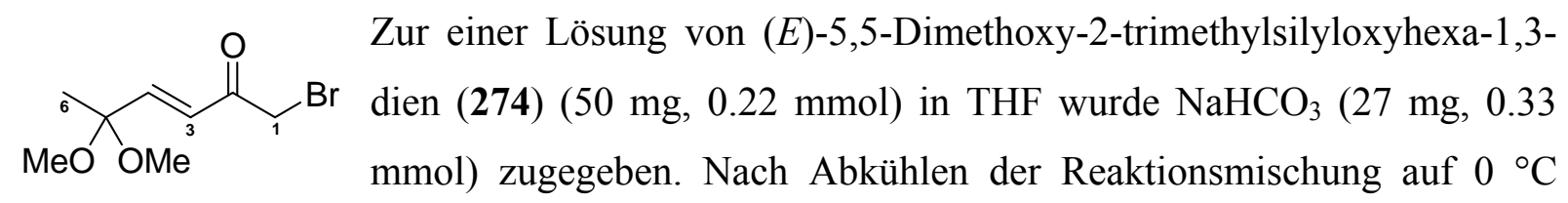


wurde $\mathrm{N}$-Bromsuccinimid (NBS) $(39 \mathrm{mg}, 0.22 \mathrm{mmol}$ ) zugegeben. Anschließend wurde die Reaktionsmischung innerhalb von $2 \mathrm{~h}$ auf Raumtemperatur erwärmt und gerührt. Das Ende der Reaktion wurde dünnschichtchromatographisch auf ALOX detektiert (Hex:EtOAc 8:1, $R_{f}$ $=0.33)$. Anschließend wurde die Reaktionsmischung mit ges. $\mathrm{NaHCO}_{3}$-Lösung und $\mathrm{Et}_{2} \mathrm{O}(3 \mathrm{x}$ $50 \mathrm{~mL})$ extrahiert. Die vereinigte organische Phase wurde mit $\mathrm{H}_{2} \mathrm{O}(10 \mathrm{~mL})$ gewaschen, über $\mathrm{Na}_{2} \mathrm{SO}_{4}$ getrocknet und das Lösungsmittel i.V. entfernt. Man erhielt $52 \mathrm{mg}$ (quant.) des Bromids 275 als farbloses Öl. - ${ }^{1} \mathrm{H}-\mathrm{NMR}\left(250 \mathrm{MHz}, \mathrm{CDCl}_{3}\right): \delta=1.40(\mathrm{~s}, 3 \mathrm{H}, 6-\mathrm{H}), 3.19(\mathrm{~s}, 6 \mathrm{H}$, $\left.2 \times \mathrm{OCH}_{3}\right), 4.01(\mathrm{~s}, 2 \mathrm{H}, 1-\mathrm{H}), 6.60(\mathrm{~d}, J=16 \mathrm{~Hz}, 1 \mathrm{H}, 3-\mathrm{H}), 6.73(\mathrm{~d}, J=16 \mathrm{~Hz}, 1 \mathrm{H}, 4-\mathrm{H})$.

\section{Allgemeine Arbeitsvorschrift B5}

\section{(Z)-4-(tert-Butyldimethylsilanyloxy)but-2-en-1-ol (277)}

TBDMSO ${ }_{4} \mathrm{Zu}$ einer Lösung von $(Z)$-But-2-en-1,4-diol 276 (35 g, $\left.0.40 \mathrm{~mol}\right)$, Di-
methylaminopyridon (DMAP) $(1.27 \mathrm{~g}, 9.95 \mathrm{mmol})$ und $\mathrm{NEt}_{3}(33.3 \mathrm{~mL}$,
$24.2 \mathrm{~g}, 239 \mathrm{mmol})$ in $\mathrm{CH}_{2} \mathrm{Cl}_{2}(650 \mathrm{~mL})$ wurde bei Raumtemperatur eine Lösung von tert-Butylchlordimethylsilan (40 mL, 0.20 mol, 50\%-ige Lösung in Hexan) über einer Syringpumpe innerhalb von $4 \mathrm{~h}$ zugetropft und $24 \mathrm{~h}$ gerührt. Die Reaktionslösung wurde mit $\mathrm{H}_{2} \mathrm{O}$, ges. $\mathrm{NH}_{4} \mathrm{Cl}$-Lösung und ges. NaCl-Lösung (je $200 \mathrm{~mL}$ ) gewaschen und mit $\mathrm{Et}_{2} \mathrm{O}(3 \times 250 \mathrm{~mL})$ extrahiert. Die vereinigte organische Phase wurde über $\mathrm{Na}_{2} \mathrm{SO}_{4}$ getrocknet und i.V. konzentriert. Das ölige Rohprodukt wurde einer fraktionierenden Destillation unterzogen. Man erhielt 20.6 g (51\%) des Silylethers 277 als farblose Flüssigkeit (Sdp. 85-86 ${ }^{\circ} \mathrm{C}$, 0.01 mbar). Die analytischen Daten entsprachen der Literatur. ${ }^{[118]}$

\section{Allgemeine Arbeitsvorschrift B6 (Variante 1)}

(Z)-4-(tert-Butyldimethylsilanyloxy)but-2-enal (278)

CHO In einem Schlenkkolben wurde unter Stickstoffatmosphäre eine Lösung
von Oxalylchlorid $(4.41 \mathrm{~mL}, 6.53 \mathrm{~g}, 51.4 \mathrm{mmol})$ in wasserfreiem $\mathrm{CH}_{2} \mathrm{Cl}_{2}(65 \mathrm{~mL})$ vorgelegt und auf $-78{ }^{\circ} \mathrm{C}$ heruntergekühlt. Eine Lösung von Dimethylsulfoxid $(7.9 \mathrm{~mL}, 8.7 \mathrm{~g}, 0.11 \mathrm{~mol})$ in wasserfreiem $\mathrm{CH}_{2} \mathrm{Cl}_{2}(15 \mathrm{~mL})$ gab man tropfenweise zu (Gasentwicklung) und rührte die Reaktionslösung $30 \mathrm{~min}$ bei $-78^{\circ} \mathrm{C}$. Eine Lösung des Alkohols 277 (8.69 g, $42.8 \mathrm{mmol})$ in $\mathrm{CH}_{2} \mathrm{Cl}_{2}(35 \mathrm{~mL})$ wurde bei $-78^{\circ} \mathrm{C}$ tropfenweise zugegeben und für weitere $30 \mathrm{~min}$ bei dieser Temperatur gerührt. Nach Zugabe von $\mathrm{NEt}_{3}(30 \mathrm{~mL}, 22 \mathrm{~g}, 0.21$ mol) entfernte man das Kältebad und ließ die Reaktionslösung auf Raumtemperatur erwär- 
men. Die Reaktionslösung wurde tropfenweise mit $\mathrm{H}_{2} \mathrm{O}(150 \mathrm{~mL})$ versetzt und $10 \mathrm{~min}$ gerührt. Nach Trennung der Phasen wurde die organische Phase mit $\mathrm{CH}_{2} \mathrm{Cl}_{2}$ (2 x $\left.250 \mathrm{~mL}\right)$ extrahiert. Die vereinigte organische Phase wurde über $\mathrm{Na}_{2} \mathrm{SO}_{4}$ getrocknet. Das Lösungsmittel wurde unter vermindertem Druck konzentriert. Das Rohprodukt wurde an Kieselgel (200 g, Petrolether/ $\left.\mathrm{Et}_{2} \mathrm{O} 40: 1 \rightarrow 20: 1 \rightarrow 10: 1\right)$ gereinigt. Man erhielt $5.71 \mathrm{~g}(67 \%)$ des Aldehyds 278 $\left(R_{f}=0.58\right)$ als gelbes Ö1. Die analytischen Daten entsprachen der Literatur. ${ }^{[120]}$

\section{Allgemeine Arbeitsvorschrift B6 (Variante 2)}

\section{(Z)-4-(tert-Butyldimethylsilanyloxy)but-2-enal (278)}

Zur Synthese des Aldehyds 278 wurde eine Suspension von PPC (8.21 g, $38.1 \mathrm{mmol})$ und Celite $(40 \mathrm{~g})$ in $\mathrm{CH}_{2} \mathrm{Cl}_{2}(150 \mathrm{~mL})$ bei Raumtemperatur tropfenweise mit einer Lösung des Silylethers 277 (5.15 g, $25.4 \mathrm{mmol})$ in $\mathrm{CH}_{2} \mathrm{Cl}_{2}(20 \mathrm{~mL})$ versetzt und $2 \mathrm{~h}$ gerührt. Das Ende der Reaktion wurde dünnschichtchromatographisch verfolgt (Permanganat-Lösung als Anfärbereagenz). Nach der Filtration der Reaktionslösung über einer Umkehrfritte wurde der Rückstand mit $\mathrm{CH}_{2} \mathrm{Cl}_{2}(200 \mathrm{~mL})$ nachgewaschen. Das erhaltene Rohprodukt wurde nach Entfernung der Lösungsmittel unter vermindertem Druck an Kieselgel $\left(R_{f}=0.38\right.$, Ether als Laufmittel) gereinigt. Man erhielt $2.56 \mathrm{~g}$ (50\%) des Aldehyds 278 als Feststoff (für die analytischen Daten vgl. Angaben bei Variante 1).

\section{Allgemeine Arbeitsvorschrift B7 (Variante 1)}

\section{(E,Z)-6-(tert-Butyldimethylsilanyloxy)hexa-2,4-dienessigsäureethylester (279)}

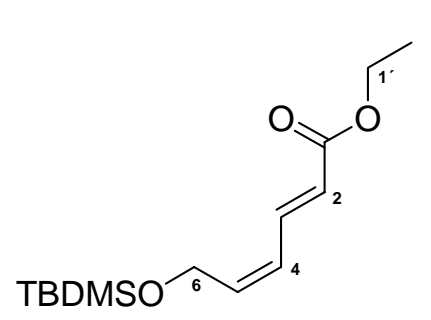

Eine Lösung von Phosphonessigsäuretriethylester (9.6 g, 43 mmol) in wasserfreiem THF $(10 \mathrm{~mL})$ wurde tropfenweise unter starkem Rühren bei $-20{ }^{\circ} \mathrm{C} \mathrm{zu}$ einer Dispersion von $\mathrm{NaH}$ (1.72 g, $43 \mathrm{mmol}$, 60\%-ige Suspension in Paraffinöl) gegeben und 30 min gerührt. Anschließend wurde bei $-20{ }^{\circ} \mathrm{C}$ eine Lösung des Aldehyds 278 (5.71 g, $29 \mathrm{mmol})$ in THF (10 mL) zugegeben und $20 \mathrm{~min}$ gerührt. Die Reaktionsmischung wurde nach Erwärmung auf Raumtemperatur für weitere 40 min gerührt und mit ges. $\mathrm{NH}_{4} \mathrm{Cl}$ Lösung und $\mathrm{Et}_{2} \mathrm{O}(2 \times 150 \mathrm{~mL})$ extrahiert. Die vereinigte organische Phase wurde zunächst mit ges. $\mathrm{Na}_{2} \mathrm{CO}_{3}$-Lösung $(3 \times 80 \mathrm{~mL})$ und anschließend mit ges. NaCl-Lösung $(3 \times 80 \mathrm{~mL})$ extrahiert. Die vereinigte organische Phase wurde über $\mathrm{Na}_{2} \mathrm{SO}_{4}$ getrocknet und unter vermindertem Druck konzentriert. Das Rohprodukt wurde säulenchromatographisch an Kieselgel $(600 \mathrm{~g}$, Petrolether:EtOAc 50:1 $\rightarrow 30: 1)$ gereinigt. Man erhielt $3.87 \mathrm{~g}(50 \%)$ der Ester- 
Verbindung $279\left(R_{f}=0.75\right)$ als farbloses Öl. $-{ }^{1} \mathrm{H}-\mathrm{NMR}\left(300 \mathrm{MHz}, \mathrm{CDCl}_{3}\right): \delta=0.05[\mathrm{~s}, 6 \mathrm{H}$, $\left.\mathrm{Si}\left(\mathrm{CH}_{3}\right)_{2} \mathrm{C}\left(\mathrm{CH}_{3}\right)_{3}\right], 0.89$ [s, $\left.9 \mathrm{H}, \mathrm{Si}\left(\mathrm{CH}_{3}\right)_{2} \mathrm{C}\left(\mathrm{CH}_{3}\right)_{3}\right], 1.26$ (t, $\left.J=7.1 \mathrm{~Hz}, 3 \mathrm{H}, 2^{\prime}-\mathrm{H}\right), 4.17$ (q, $J=$ $\left.7.1 \mathrm{~Hz}, 2 \mathrm{H}, 1^{\prime}-\mathrm{H}\right), 4.27$ (m $\left.\mathrm{c}, 2 \mathrm{H}, 6-\mathrm{H}\right), 5.83$ (d, J=15.3 Hz, $\left.1 \mathrm{H}, 2-\mathrm{H}\right), 6.14$ (dt, J = 15.1, 4.5 $\mathrm{Hz}, 1 \mathrm{H}, 5-\mathrm{H}), 6.37\left(\mathrm{~m}_{\mathrm{c}}, 1 \mathrm{H}, 4-\mathrm{H}\right), 7.25\left(\mathrm{~m}_{\mathrm{c}}, 1 \mathrm{H}, 3-\mathrm{H}\right) .-{ }^{13} \mathrm{C}-\mathrm{NMR}\left(75.5 \mathrm{MHz}, \mathrm{CDCl}_{3}\right.$, zu-

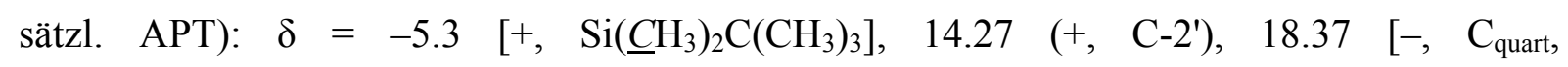
$\left.\mathrm{Si}\left(\mathrm{CH}_{3}\right)_{2} \underline{C}\left(\mathrm{CH}_{3}\right)_{3}\right], 25.86\left[+, \mathrm{Si}\left(\mathrm{CH}_{3}\right)_{2} \mathrm{C}\left(\underline{\mathrm{C}} \mathrm{H}_{3}\right)_{3}\right], 60.22\left(-, \mathrm{C}-1^{\prime}\right), 62.88$ (-, C-6), 120.75 (+, C2), 126.77 (+, C-4), $141.76(+, \mathrm{C}-5), 143.97$ (+, C-3), 167.09 (-, $\left.\mathrm{C}_{\text {quart }}, \mathrm{C}-1\right)$.

\section{Allgemeine Arbeitsvorschrift B7 (Variante 2)}

\section{(E,Z)-6-(tert-Butyldimethylsilanyloxy)hexa-2,4-dienessigsäureethylester (279)}

Zur Darstellung des Esters 279 wurde bei Raumtemperatur eine Lösung von Phosphonessigsäuretriethylester (2.18 g, $9.70 \mathrm{mmol})$ und dem Aldehyd 278 (1.94 g, $9.70 \mathrm{mmol})$ in THF (10 $\mathrm{mL})$ zu einer Suspension von $\mathrm{NaOH}(1.09 \mathrm{~g}, 19.4 \mathrm{mmol})$ in THF $(20 \mathrm{~mL})$ gegeben und $1 \mathrm{~h}$ gerührt. Die Reaktion wurde nach Beendigung der Umsetzung lediglich abfiltriert. Nach Entfernung des Lösungsmittels unter vermindertem Druck wurde das Rohprodukt säulenchromatographisch an Kieselgel (200 g, Petrolether:EtOAc 50:1 $\rightarrow 30: 1)$ gereinigt. Man erhielt $1.03 \mathrm{~g}$ (39\%) der Esterverbindung $279\left(R_{f}=0.75\right)$ als farbloses Öl (für die analytischen Daten vgl. Angaben bei Variante 1).

\section{Allgemeine Arbeitsvorschrift $\mathrm{B8}$}

\section{(E,E)-6-(tert-Butyldimethylsilanyloxy)hexa-3,5-dienessigsäureethylester (280)}

TBDMSO $\underbrace{}_{6} \begin{aligned} & \mathrm{Zu} \text { einer Lösung von Diisopropylamin }(2.45 \mathrm{~mL}, 1.75 \mathrm{~g} \text {, } \\ & 14.4 \mathrm{mmol}) \text { in wasserfreiem THF }(52 \mathrm{~mL}) \text { und DMPU }\end{aligned}$ (3.50 mL, $3.70 \mathrm{~g}, 28.8 \mathrm{mmol})$ wurde unter Schutzgasatmosphäre bei $-78^{\circ} \mathrm{C} n \mathrm{BuLi}(11.4 \mathrm{~mL}$, $17.3 \mathrm{mmol}, 1.52 \mathrm{M}$ in Hexan) zugegeben und $40 \mathrm{~min}$ gerührt. Eine Lösung des Dienesters 279 (3.89 g, $14.4 \mathrm{mmol})$ in THF (25 mL) wurde tropfenweise $(5 \mathrm{~min})$ bei $-78^{\circ} \mathrm{C}$ zugegeben und 3 h gerührt. Die Reaktionslösung wurde bei $-78{ }^{\circ} \mathrm{C}$ mit 10\%-iger HCl-Lösung $(20 \mathrm{~mL}, \mathrm{pH}<7)$ hydrolysiert und 5 min gerührt. Nach Zugabe von $\mathrm{Et}_{2} \mathrm{O}(2 \times 150 \mathrm{~mL})$ wurden die Phasen getrennt. Die vereinigte organische Phase wurde mit ges. $\mathrm{NH}_{4} \mathrm{Cl}$-Lösung $(1 \mathrm{x} 80 \mathrm{~mL})$ und anschließend mit ges. NaCl-Lösung $(1$ x $80 \mathrm{~mL})$ gewaschen. Die organische Phase wurde über $\mathrm{MgSO}_{4}$ getrocknet und i.V. konzentriert. Das Rohprodukt wurde säulenchromatographisch an Kieselgel (400 g, Petrolether:EtOAc 80:1) gereinigt. Man erhielt 3.63 g (93\%) des Isomerisie- 
rungsprodukts $280\left(R_{f}=0.63\right)$ als oranges Öl. $-{ }^{1} \mathrm{H}-\mathrm{NMR}\left(250 \mathrm{MHz}, \mathrm{CDCl}_{3}\right): \delta=0.11[\mathrm{~s}, 6 \mathrm{H}$, $\left.\mathrm{Si}\left(\mathrm{CH}_{3}\right)_{2} \mathrm{C}\left(\mathrm{CH}_{3}\right)_{3}\right], 0.90$ [s, $\left.9 \mathrm{H}, \mathrm{Si}\left(\mathrm{CH}_{3}\right)_{2} \mathrm{C}\left(\mathrm{CH}_{3}\right)_{3}\right], 1.23$ (t, $\left.J=7.1 \mathrm{~Hz}, 3 \mathrm{H}, 2^{\prime}-\mathrm{H}\right), 3.09$ (d, $J=$ $7.3 \mathrm{~Hz}, 2 \mathrm{H}, 2-\mathrm{H}), 4.15$ (q, J=7.1 Hz, $2 \mathrm{H}, 1 '-\mathrm{H}), 5.18\left(\mathrm{~m}_{\mathrm{c}}, 1 \mathrm{H}, 3-\mathrm{H}\right), 5.62\left(\mathrm{~m}_{\mathrm{c}}, 1 \mathrm{H}, 4-\mathrm{H}\right)$, $6.17(\mathrm{~d}, J=15.1,1 \mathrm{H}, 6-\mathrm{H}), 6.52\left(\mathrm{~m}_{\mathrm{c}}, 1 \mathrm{H}, 5-\mathrm{H}\right)$.

\section{Allgemeine Arbeitsvorschrift B9 (Variante 1)}

\section{(E,E)-6-(tert-Butyldimethylsilanyloxy)hexa-3,5-dienal (281)}

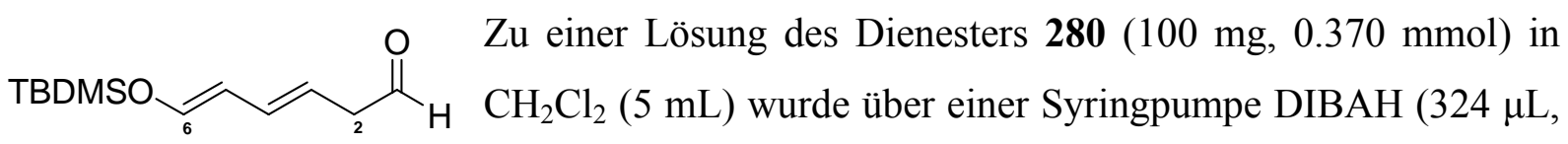
$0.389 \mathrm{mmol}, 1.2 \mathrm{M}$ in Toluol) tropfenweise bei $-78^{\circ} \mathrm{C}$ innerhalb von $15 \mathrm{~min}$ zugegeben und $1 \mathrm{~h}$ gerührt. Eine homogene Lösung von Weinsäure $(117 \mathrm{mg}, 0.778 \mathrm{mmol})$ in abs. EtOH (2 $\mathrm{mL}$ ) wurde bei $-78^{\circ} \mathrm{C}$ zugegeben und 10 min gerührt. Nach Erwärmung der Reaktionslösung auf $-30{ }^{\circ} \mathrm{C}$ wurde ein Gemisch aus $\mathrm{Na}_{2} \mathrm{SO}_{4} \cdot 10 \mathrm{H}_{2} \mathrm{O}$ :Celite $(1: 1$, je $0.5 \mathrm{~g}, 1.0 \mathrm{~g})$ zugegeben und 5 min gerührt. Die Reaktionslösung wurde auf Raumtemperatur erwärmt, über einer Umkehrfritte (Celiteschicht) abfiltriert und mit $n$ Pentan nachgewaschen $(180 \mathrm{~mL})$. Die organische Phase wurde nochmals über $\mathrm{Na}_{2} \mathrm{SO}_{4}$ getrocknet und i.V. konzentriert. Man erhielt $79.1 \mathrm{mg}$ (94\%) des Dienals 281 als gelbes Öl. $-{ }^{1} \mathrm{H}-\mathrm{NMR}\left(250 \mathrm{MHz}, \mathrm{CDCl}_{3}\right): \delta=0.12[\mathrm{~s}, 6 \mathrm{H}$, $\left.\mathrm{Si}\left(\mathrm{CH}_{3}\right)_{2} \mathrm{C}\left(\mathrm{CH}_{3}\right)_{3}\right], 0.90$ [s, $\left.9 \mathrm{H}, \mathrm{Si}\left(\mathrm{CH}_{3}\right)_{2} \mathrm{C}\left(\mathrm{CH}_{3}\right)_{3}\right], 3.01$ (d, $\left.J=4.8,2.2 \mathrm{~Hz}, 2 \mathrm{H}, 2-\mathrm{H}\right), 5.16$ $\left(\mathrm{m}_{\mathrm{c}}, 1 \mathrm{H}, 3-\mathrm{H}\right), 5.52\left(\mathrm{~m}_{\mathrm{c}}, 1 \mathrm{H}, 4-\mathrm{H}\right), 6.18(\mathrm{~d}, J=15.1,1 \mathrm{H}, 6-\mathrm{H}), 6.54\left(\mathrm{~m}_{\mathrm{c}}, 1 \mathrm{H}, 5-\mathrm{H}\right), 9.66(\mathrm{~s}$, $1 \mathrm{H}, \mathrm{CHO}) .-{ }^{13} \mathrm{C}-\mathrm{NMR}\left(50.3 \mathrm{MHz}, \mathrm{CDCl}_{3}\right): \delta=0.93\left[\mathrm{Si}\left(\mathrm{CH}_{3}\right)_{2} \mathrm{C}\left(\mathrm{CH}_{3}\right)_{3}\right], 18.20\left[\mathrm{C}_{\text {quart }}\right.$, $\left.\mathrm{Si}\left(\mathrm{CH}_{3}\right)_{2} \mathrm{C}\left(\mathrm{CH}_{3}\right)_{3}\right], 25.50$ [ $\left.\mathrm{Si}\left(\mathrm{CH}_{3}\right)_{2} \mathrm{C}\left(\mathrm{CH}_{3}\right)_{3}\right], 47.55$ (C-2), $109.61(\mathrm{C}-5), 117.92(\mathrm{C}-3), 128.33$ (C-4), 140.34 (C-6), 201.01 (C-1).

\section{Allgemeine Arbeitsvorschrift B9 (Variante 2)}

\section{(E,E)-6-(tert-Butyldimethylsilanyloxy)hexa-3,5-dienal (281)}

$\mathrm{Zu}$ einer Lösung des Dienesters 280 (100 mg, $0.370 \mathrm{mmol})$ in $\mathrm{CH}_{2} \mathrm{Cl}_{2}$ (5 mL) wurde über einer Syringpumpe DIBAH $\left(460 \mu \mathrm{L}, 0.389 \mathrm{mmol}, 1.52 \mathrm{M}\right.$ in Toluol) tropfenweise bei $-78{ }^{\circ} \mathrm{C}$ innerhalb von $30 \mathrm{~min}$ zugegeben und $1 \mathrm{~h}$ gerührt. Konz. Essigsäure (23.3 mg, $0.389 \mathrm{mmol})$ wurde bei $-78{ }^{\circ} \mathrm{C}$ zugegeben und 5 min gerührt. Die Reaktionslösung wurde auf Raumtemperatur erwärmt, mit ges. NaCl-Lösung versetzt und mit $n$ Pentan $(3 \times 50 \mathrm{~mL})$ extrahiert. Die vereinigte organische Phase wurde über $\mathrm{Na}_{2} \mathrm{SO}_{4}$ getrocknet und i.V. konzentriert. Man erhielt 54.6 mg (70\%) des Dienals 281 als gelbes Öl (für analytische Daten vgl. Variante 1). 


\section{Allgemeine Arbeitsvorschrift B10 (Variante 1)}

\section{2-(tert-Butyldimethylsilanyloxy)-7-hydroxy-2,2-dimethoxydodeca-3,9,11-trien-5-on}

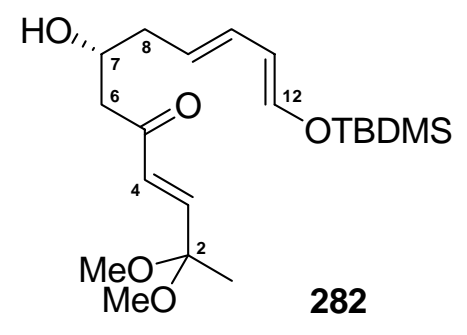

Für die Variante 1 wurde eine Mischung von ( \pm )-BINOL (0.2 eq), $\mathrm{Ti}(\mathrm{O}-\mathrm{i} \mathrm{Pr})_{4}\left(0.2 \mathrm{eq}, 1.0 \mathrm{M}\right.$ in $\left.\mathrm{CH}_{2} \mathrm{Cl}_{2}\right)$ und Molsieb $4 \AA$ (100 mg, ofengetrocknet) in $\mathrm{Et}_{2} \mathrm{O} 1 \mathrm{~h}$ unter Rückfluß gerührt. Nach Abkühlung der Reaktionslösung auf Raumtemperatur wurde das Hexadienals 281 (1.0 eq) zugegeben und 5 min gerührt. Die Reaktionslösung wurde auf $-78^{\circ} \mathrm{C}$ abgekühlt, mit dem Silylenolether 274 (1.4 eq) versetzt und 5 min gerührt. Anschließend wurde die Reaktionslösung in einem Gefrierschrank $12 \mathrm{~h}$ bei $-20{ }^{\circ} \mathrm{C}$ gelagert (ohne Rühren). Die Reaktionslösung wurde mit einer Puffer-Lösung ( $\mathrm{pH}=7$ ) hydrolysiert und 15 min gerührt. Die resultierende Reaktionsmischung wurde über einer Celiteschicht abfiltriert und mit $\mathrm{Et}_{2} \mathrm{O}$ extrahiert. Nach Entfernung der Lösungsmittel unter vermindertem Druck wurde der Rückstand mit 10\%-igem HCl-MeOH-Lösungsgemisch behandelt und die daraus resultierende Lösung unter vermindertem Druck konzentriert. Das Rohprodukt wurde abschließend mit $\mathrm{Et}_{2} \mathrm{O}$ extrahiert und das Lösungsmittel i.V. entfernt.

\section{Allgemeine Arbeitsvorschrift B10 (Variante 2)}

12-(tert-Butyldimethylsilanyloxy)-7-hydroxy-2,2-dimethoxydodeca-3,9,11-trien-5-on (282)

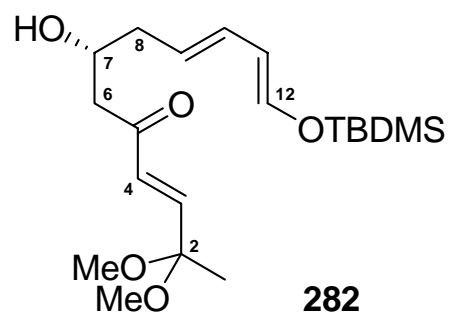

Für die Variante 2 wurde eine Mischung von ( \pm )-BINOL (0.4 eq) und $\mathrm{Ti}(\mathrm{O}-i \mathrm{Pr})_{4}\left(0.2 \mathrm{eq}, 1.0 \mathrm{M}\right.$ in $\left.\mathrm{CH}_{2} \mathrm{Cl}_{2}\right)$ in $\mathrm{Et}_{2} \mathrm{O} 1 \mathrm{~h}$ bei Raumtemperatur gerührt. Die rotbraune Reaktionslösung wurde mit dem Hexadienal 281 (1.0 eq) versetzt und anschließend auf $0{ }^{\circ} \mathrm{C}$ heruntergekühlt. Der Silylenolether 274 (1.4 eq) wurde bei $0{ }^{\circ} \mathrm{C}$ zugegeben und 10 min gerührt. Die Reaktionslösung wurde mit einer Puffer-Lösung (pHWert 7) hydrolysiert und entsprechend der schon im Rahmen der Variante 1 (Allgemeine Arbeitsvorschrift B10) dargestellten Methode aufgearbeitet. 


\section{Zusammenfassung}

Hauptziel dieser Promotionsarbeit war zunächst, die Möglichkeiten zur Totalsynthese des Naturstoffes Iromycin A (23A) und seines Analogons Iromycin R (23R) zu untersuchen. Zudem sollte als Modellsubstrat das Iromycin-Analogon 23 synthetisiert werden. Die Syntheseplanung wurde auf der Grundlage eines im Rahmen des retrosynthetischen Konzeptes angenommenen Bindungsbruches an der C-1'- und C-2'-Position der Seitenkette angelegt. Daraus folgend bot der strukturelle Aufbau des Iromycins das $\alpha$-Pyron, das $\alpha$-Pyridon sowie das $\alpha$ Pyridin als potentielle Ringsysteme und Grundbausteine für die Synthese an. Als Seitenkettenfragmente wurden die Alane 123, 123A und 123R bzw. die entsprechende Alanate 124, 124A und 124R eingesetzt.

Die Synthese des Iromycins A und seiner Analoga gelang hocheffizient in einer 13-stufigen Synthese auf dem Weg über die Kreuzkupplung des Brommethylpyrons 96 mit den Alanaten 124, 124A bzw. 124R. Die Alanate wurden aus $\mathrm{AlMe}_{3}$-freien Alanen in Gegenwart von $n$ Buli generiert. Die eingesetzten Alane 123, 123A und 123R wurden über eine $\mathrm{Cp}_{2} \mathrm{ZrCl}_{2-}$ katalysierte Carboaluminierungsreaktion mit $\mathrm{AlMe}_{3}$ aus 1-Heptin (122) bzw. den hierfür synthetisierten Alkinen 103A und 103R dargestellt. Das als eines der potentiellen Ringsysteme bei der Iromycinsynthese eingesetzte Brommethylpyron 96 konnte erfolgreich über 7 Stufen dargestellt werden. Der Butansäureethylester 83 wurde mit Propyliodid in den Heptansäureethylester 84 (Ausbeute 56\%) alkyliert und anschließend mit $N$-Acetylimidazol acyliert. Der erhaltene $\beta, \delta$-Diketoester 64 wurde in einer Cyclisierungsreaktion mit DBU in das entsprechende Pyron 63 überführt, welches dann mit Benzoylchlorid umgesetzt wurde. Der hieraus resultierende Benzoesäureester 89 (Ausbeute 89\%) konnte anschließend mit Selendioxid in das entsprechende Aldehyd 94 (Ausbeute 45\%) umfunktionalisiert werden. Der aus dem Formylester 94 mit $\mathrm{NaBH}_{4}$ hergestellte Alkohol 95 (Ausbeute 91\%) konnte abschließend mit $\mathrm{PBr}_{3}$ in das Brommethylpyron 96 in einer exzellenten Ausbeute von 93\% überführt werden.

Die über eine $\mathrm{S}_{\mathrm{N}} 2$-Reaktion verlaufenden Kreuzkupplungsreaktionen des Brommethylpyrons 96 mit den o.g. Alanaten konnten erfolgreich durchgeführt werden und ergaben die gewünschten Iromycinvorläufer 125, 125A und 125R. Der Iromycin A-Vorläufer 125A sowie dessen Analogon 125 konnten nach Optimierung der Reaktionsbedingungen in erfreulichen bis exzellenten Ausbeuten von 95\% bzw. 83\% synthetisiert werden, der Iromycin R-Vorläufer 125R in einer Ausbeute von 45\%. Durch Optimierungsversuche konnten als ideale Reakti- 
onsbedingungen der Einsatz von 3.0 eq Alanat in THF bei einer Temperatur von $0{ }^{\circ} \mathrm{C}$ und einer Reaktionszeit von $2 \mathrm{~h}$ festgestellt werden. Desweiteren zeigte sich im Rahmen der Untersuchungen, dass der Einsatz von $\mathrm{AlMe}_{3}$-freien Alanen eine unerlässliche Voraussetzung zur Erzielung einer vollständigen Umsetzung zu den gewünschten Iromycin-Vorläufern und zur Vermeidung der Bildung unerwünschter Nebenprodukte darstellt. Die Überführung der Kreuzkupplungsprodukte 125A, 125R und 125 in die gewünschte Iromycine A (23A) und R (23R) sowie das Iromycin-Analogon 23 konnte durch den O-N-Austausch der Kreuzkupplungsprodukte in Gegenwart von $\mathrm{NH}_{3}$ erfolgreich durchgeführt werden. Die Transformation mit flüssigem $\mathrm{NH}_{3}$ gelang bei einer Temperatur von $70{ }^{\circ} \mathrm{C}$ und einem Druck von 30 mbar. Die gewünschte Iromycine A (23A) und R (23R) sowie das Analogon 23 konnten in Ausbeuten von $64 \%$, 63\% sowie 58\% synthetisiert werden. Als entscheidende Reaktionsbedingung für die Effizienz des O-N-Austausches erwies sich der Einsatz von flüssigem $\mathrm{NH}_{3}$, da im $\mathrm{Ge}-$ gensatz zu untersuchten O-N-Austausch-Versuchen in Gegenwart von wässrigen bzw. methanolischen $\mathrm{NH}_{3}$-Lösungen hierdurch stets eine vollständige Umsetzung erzielt werden konnte. Der Vorteil der angewandten Synthesemethode besteht vor allem darin, dass die Seitenkette durch die Carboaluminierung eines Alkins in die funktionalisierte Form umgewandelt und anschließend an das Hauptgrundgerüst gekuppelt wird. Hierdurch konnte ein Synthesekonzept entwickelt werden, durch das sich die Kupplung der Seitenkette an die für eine nucleophile Substitution besonders geeignete Substanzklasse der allylischen Pyronhalogenide effektiv durchführen lässt. Durch die Carboaluminierung der leicht darzustellenden Alkine wird ein effektiver Zugang zu den Iromycinderivaten eröffnet, was besonders deshalb interessant ist, da die biologische Aktivität der Iromycine über die Seitenkettenenden bestimmt wird.

Als weiterer möglicher Weg zur Iromycin-Synthese wurde die C-C-Verknüpfung des Brommethylpyridons 159 als potentielles Ringsystem mit dem Alanat 124 untersucht. Zur Synthese des Brommethylpyridons 159 wurde das aus Pyron mit $\mathrm{NH}_{3}$ dargestellte Pyridon in Analogie zur Synthese des Brommethylpyrons zunächst mit AcCl in den Ester 156 (69\%), dann in den Aldehyd 157 und in den Alkohol 158 (95\%) umgewandelt und abschließend in das Brommethylpyridon (quant.) überführt. Die Kreuzkupplungsversuche mit Brommethylpyridon 159 wurden analog der Verfahrensweise bei der Kreuzkupplung mit Brommethylpyron 96 untersucht, führten jedoch stets zur Zersetzung des Eduktes 159. Alternativ wurde daher versucht, das Stickstoffproton des Brommethylpyridons 159 mit den Metallorganylen $\mathrm{AlMe}_{3}, \mathrm{Al}(\mathrm{iBu})_{3}$, $\mathrm{Zn}(\mathrm{Et})_{2}, n \mathrm{BuLi}$ und $\mathrm{NaH}$ in situ zu deprotonieren und anschließend mit dem Alanat $\mathbf{1 2 4} \mathrm{zu}$ dem Iromycinvorläufer umzusetzen. Auf diese Weise sollte das Stickstoffproton des Bromids 
159 als möglicher Hinderungsgrund der Kreuzkupplung ausgeschaltet werden. Die Umsetzung der aus der Deprotonierung des Brommethylpyridons 159 mit Metallorganylen resultierenden Lösungen mit Alanaten erwies sich jedoch nicht als geeignete Methode. Lediglich im Fall der aus $\mathrm{AlMe}_{3}$ generierten N-Metallorganyllösungen konnte überhaupt die Bildung eines geringen Anteils des gewünschten Kreuzkupplungsproduktes 165 (23\%) beobachtet werden. Auch eine untersuchte Kreuzkupplung über eine vorherige N-Schützung des Brommethylpyridons 159 stellte sich als nicht geeignete Synthesemethode dar. Bereits die Substitution des Stickstoffprotons erwies sich als schwierig und konnte nur im Fall des Einsatzes von TMSCl erfolgreich unter Bildung des N-geschützten Brommethylpyridons 169 in quantitativer Ausbeute durchgeführt werden. Die anschließende Kreuzkupplungsreaktion des N-geschützten Brommethylpyridons 169 mit dem Alanat 124 führte dann aber zur sofortigen Abspaltung der TMS-Schutzgruppe und damit zum Ausbleiben des Kreuzkupplungsproduktes.

Die entsprechend der Vorgehensweise bei der Kreuzkupplung mit Brommethylpyron 96 untersuchte Kreuzkupplung der Pyridine als weiteres potentielles Ringsystem, ausgehend von dem Brommethylpyridin 181, mit dem Alanat 124 erbrachten ebenfalls nicht die erhofften Resultate, da lediglich Spuren des gewünschten Kreuzkupplungsprodukts 182 beobachtet werden konnten.

Alternativ wurden schließlich Übergangsmetall-katalysierte Kreuzkupplungsversuche mit Pyron-, Pyridon- und Pyridinhalogeniden einerseits und den Alanen 123 bzw. 123A sowie Alkenylhalogeniden andererseits untersucht. Die Alkenylalane 123 bzw. 123A wurden in einer $\mathrm{Cp}_{2} \mathrm{ZrCl}_{2}$-vermittelten Reaktion mit $\mathrm{AlMe}_{3}$ aus den entsprechenden Alkinen 1-Heptin (122) bzw. 103A in situ generiert und mit Bromiden umgesetzt. Die Pd-katalysierten Kreuzkupplungsreaktionen wurden mit Brommethylpyridon 159 bzw. Brommethylpyron 183 im Sinne einer Kreuzkupplung und einer Stille-Kupplung sowie unter Einsatz des Zinkorganyls 198, des $\gamma$-Stannanorganyls 200 und des $\gamma$-Zinkorganyls 201 untersucht, blieben aber in allen Varianten ohne Erfolg. Bei allen angewandten Synthesemethoden trat eine Bildung der gewünschten Iromycinvorläufer als Kreuzkupplungsprodukte entweder überhaupt nicht oder nur in Spuren auf. Vielmehr wurde im Allgemeinen eine überwiegende bis vollständige Debromierung der als Edukte eingesetzten Pyron- und Pyridonhalogenide beobachtet. Die Versuche zur Cu-katalysierten Kreuzkupplung des Brommethylpyrons 96 wurden mit aus dem Heptin 122 generiertem Heptenyl-MgBr 215, mit in situ generiertem Diisobutyolalan 202 bzw. Dimethylalan 123 sowie unter Einsatz von $\mathrm{AlMe}_{3}$-freiem Alan 123 durchgeführt, ergaben aber 
lediglich im Fall der Kupplung mit dem Heptenyl-MgBr 215 einen geringen Bildungsanteil des Kreuzkupplungsproduktes von 33\%. Im Allgemeinen konnte bei den Cu-katalysierten Reaktionen überwiegend eine Übertragung der Alkylgruppen auf das Edukt Brommethylpyron 96 beobachtet werden. Die Ni-katalysierten Kreuzkupplungen des Brommethylpyrons 96 bzw. Chlormethylpyrons 135 mit dem Alan 123 erwiesen sich ebenfalls als nicht geeignete Syntheserouten zur Iromycindarstellung, da das gewünschte Kreuzkupplungsprodukt 125 nur in sehr geringen Bildungsanteilen von 15-20\% erhalten werden konnte. Es konnte stattdessen vor allem die Bildung der Homokupplungsprodukte 136 (12-85\% Anteil) bzw. 137 (15-56\% Anteil) beobachtet werden. Das überwiegende Ausbleiben der Kreuzkupplung bei den Übergangsmetall-katalysierten Versuchen wurde auf die Molekularstruktur der eingesetzten Edukte zurückgeführt, da mit Benzylbromid $\mathbf{1 3 0}$ analog durchgeführte Vorversuche vorwiegend erfolgreich verliefen.

Die Totalsynthese des Iromycins A und seiner Analoga konnte damit in 13 Stufen über eine nucleophile Substitutionsreaktion des Brommethylpyrons 96 mit den Alanaten 124, 124A und 124R erfolgreich abgeschlossen werden. Hierdurch wurde desweiteren die Überführung der Pyronanaloga in die Pyridon- sowie Pyridin-Analoga ermöglicht. Die Effizienz der aufgezeigten Methode ergab sich zum einen aus der Kürze der Syntheseroute. Zum anderen konnte festgestellt werden, dass sich die Methode hervorragend für eine C-C-Bindungsbildung der Pyrone eignet, bei denen die allylische Position aufgrund der doppelt-vinylogen Eigenschaft des Moleküls eine nucleophile Substitution ermöglicht. Hierdurch erwies sich die vorgeschlagene Synthesemethode insbesondere gegenüber Übergangsmetall-katalysierten Syntheseverfahren als überlegen.

Die Synthesearbeiten zur Darstellung des Collinolactons (24) konnten aus zeitlichen Gründen lediglich begonnen und nur die Durchführung der Aldolreaktion als erster Schlüsselschritt zur Synthese des Collinolactons (24) untersucht werden. In einer Teilsynthese wurden die hierzu benötigten Ausgangssubstanzen, und zwar für das erste Kettenfragment das Hexadienal 281 und für das zweite Kettenfragment das Acetal 273, der Silylenolether 274 sowie das Bromid 275 dargestellt. Die Additionsreaktionen wurden unter Verwendung von Basen und Lewissäuren sowie unter Einsatz von Zink und $\mathrm{SmI}_{2}$ untersucht. Um die Realisierbarkeit der Aldoladditionsreaktion zu überprüfen, wurden die Untersuchungen zunächst auf die racemische Darstellung des Aldolproduktes beschränkt. 
Die basenvermittelten Aldolreaktionen der Kettenfragmente Ketoacetal 273 und Hexadienaldehyd 281 unter Einsatz von LDA führten stets zur Bildung eines Gemisches aus dem gewünschten Aldoladditionsprodukt 282 und dem eingesetzten Ketoacetal 273, wobei der Bildungsanteil der Verbindung 282 in Anwesenheit von DMPU 63\% und in Abwesenheit von DMPU 60\% betrug. Die Ergebnisse der Versuche waren nicht zufriedenstellend, da keine vollständigen Umsetzungen erreicht werden konnten und sich die Rohprodukte als sehr unsauber erwiesen. Eine Reiniung der Rohprodukte war nicht möglich, da hierbei eine sofortige Zersetzung auftrat. Die unvollständige Bildung des Aldoladditionsproduktes 282 wurde auf die geringe Stabilität des Hexadienals 281 zurückgeführt.

Die Versuche zur Durchführung einer Additionsreaktion unter Verwendung von Zink bzw. $\mathrm{SmI}_{2}$ waren nicht erfolgreich. Im Rahmen von Vorversuchen konnte die Bildung des gewünschten Produktes 296 lediglich bei der Umsetzung des $\alpha$-Bromketons 275 mit Hydrozimtaldehyd 291 unter Verwendung von $\mathrm{SmI}_{2}$ in einem sehr geringen Anteil von 11\% beobachtet werden.

Als eine weitere Methode zur Darstellung des Aldolproduktes 282 wurden MukaiyamaAldoladditionen des Silylenolethers 274 und des Hexadienals 281 unter Einsatz von Lewissäuren untersucht. Die Kupplungsversuche unter Verwendung $\mathrm{TiCl}_{4}$ sowie $\mathrm{ZnCl}_{2}$ führten zur vollständigen Zersetzung der Edukte, der in der Gegenwart von Yb(OTf) 3 durchgeführte Versuch gelang ebenfalls nicht, da eine Rückbildung des Endions 272 eintrat. Hingegen konnte bei der Aldolreaktion unter Verwendung von TBAF ein Gemisch aus dem gewünschten Aldolprodukt 282 (Anteil 66\%) und dem Silylenolether 274 erreicht werden. Am Erfolgreichsten erwies sich die Mukaiyama-Aldoladdition unter Verwendung von $( \pm)$-BINOL und Ti(O$i \operatorname{Pr})_{4}$ als chirales Lewissäure-Katalysator-Gemisch, die zu einer vollständigen Umsetzung zu dem gewünschten Aldolprodukt $\mathbf{2 8 2}$ in einem racemischen Gemisch führte.

Das über eine Mukaiyama-Aldoladdition unter Einsatz von $( \pm)$-BINOL und Ti(O-iPr $)_{4}$ erfolgreich synthetisierte Aldoladditionsprodukt 282 als racemisches Gemisch eröffnet die Möglichkeit, in weiterführenden Arbeiten das als Intermediat zur Vorstufe der Diels-AlderCyclisierung notwendige Aldoladditionsprodukt 282 in der gewünschten $R$-Konfiguration darzustellen. Die durchgeführten Untersuchungen zur Teilsynthese des Aldoladditionsproduktes 282 liefern damit einen wertvollen Beitrag zur Ermöglichung einer späteren Totalsynthese des Collinolactons (24). 
<smiles>[R18]c1c(CC=C(C)CC=CC(C)C)[nH]c(=O)c(C)c1CC=C(C)CCCCC</smiles><smiles>[R10]CCc1c(C/C=C(\C)C/C=C/C(C)(C)C)[nH]c(=O)c(C)c1O</smiles><smiles>CCCC(C(C)=O)C(=O)C(C)C(=O)OCC</smiles>

64<smiles>[R20]C(C)C(=O)OCC</smiles>

83<smiles>CCCCC(=O)C(C)C(=O)OCC</smiles>

84<smiles>CCCc1c(C)oc(=O)c(C)c1OC(C)=O</smiles>

88<smiles>CCCOc1c(CCC)c(C)oc(=O)c1C</smiles>

89

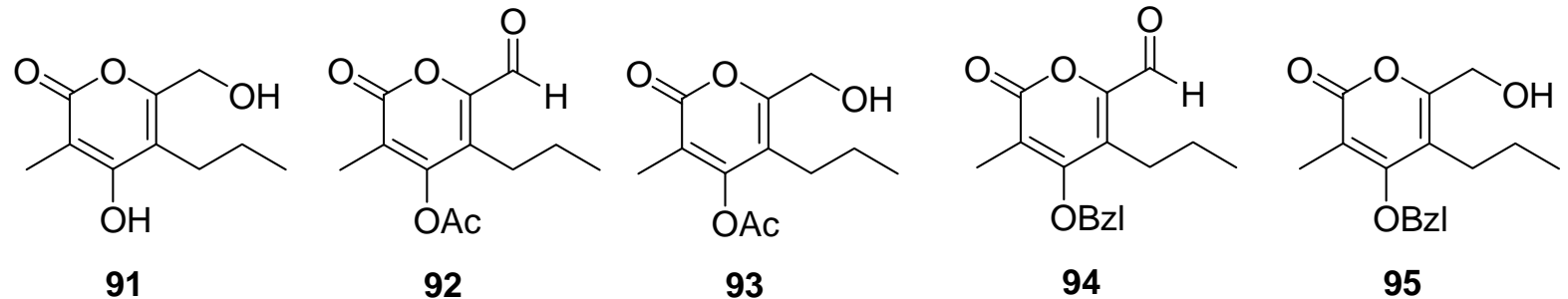<smiles>CCCOc1c(CCC)c(CBr)oc(=O)c1C</smiles>

96<smiles>CCCc1c(CBr)oc(=O)c(C)c1O</smiles>

97<smiles>C=CC(O)C(C)C</smiles>

$99 A$<smiles>C=CC(O)C(C)(C)C</smiles>

99R<smiles>CC(C)/C=C/CBr</smiles>

$100 \mathrm{~A}$<smiles>[R20][R10]#CC/C=C/C(C)C</smiles><smiles>C#CC/C=C/C(C)C</smiles>

103A<smiles>C#CC/C=C/C(C)(C)C</smiles>
103R<smiles>CC#CS(C)(C)(=O)C#CC/C=C/c1ccccc1</smiles>

115<smiles>C#CC/C=C/c1ccccc1</smiles>

116<smiles>[R10]C/C=C(/C)C/C=C(\C)C(C)(C)C</smiles> 
124, 131, 136, $\mathrm{R}=\mathrm{CH}_{3}\left(\mathrm{CH}_{2}\right)_{4}$

124A, 131A, 136A, $\mathrm{R}=\left(\mathrm{CH}_{3}\right)_{2} \mathrm{CHCH}=\mathrm{CHCH}_{2}$

124R, 131R, 136R, $\mathrm{R}=\left(\mathrm{CH}_{3}\right)_{3} \mathrm{CCH}=\mathrm{CHCH}_{2}$

$[{ }_{\mathrm{R}} \overbrace{\mathrm{Al}}{ }_{\mathrm{Bu}}]_{\mathrm{Li}}^{\ominus}$

124, 124A, 124R<smiles>CCCCCC/C(C)=C/Cc1oc(=O)c(C)c(OCCC)c1CCC</smiles>

125<smiles>CCCOc1c(CCC)c(C/C=C(\C)C/C=C/C(C)C)oc(=O)c1C</smiles>

125A<smiles>CCCOc1c(CCC)c(C/C=C(\C)C/C=C/C(C)(C)C)oc(=O)c1C</smiles>

125R

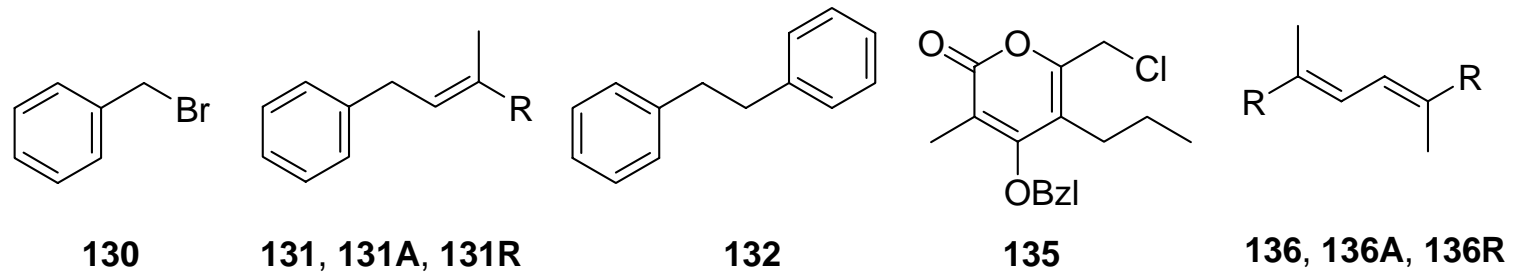<smiles>CCCOc1c(CCC)c(CCc2oc(=O)c(C)c(OCC)c2CCC)oc(=O)c1C</smiles><smiles></smiles>

138<smiles>CCCc1c(C/C=C(\C)[PH2]Cc2oc(=O)c(C)c(O)c2C)oc(=O)c(C)c1OC(C)=O</smiles>

150

156<smiles>CCCc1c(CO)[nH]c(=O)c(C=O)c1CCC</smiles><smiles>CCCc1c(C)c(O)c(C)c(=O)n1N</smiles>

162<smiles>CCCCC/C(C)=C/Cc1[nH]c(=O)c(C)c(OC(C)=O)c1CCC</smiles>

165<smiles>CCCc1[nH]c(=O)c(C)c(OC(C)=O)c1CCc1[nH]c(=O)c(C)c(OC(C)=O)c1CCC</smiles>

167<smiles>CCCc1c(CBr)c([Si](C)(C)C)[n+]([O-])c(C)c1OC(C)=O</smiles><smiles>CCCc1c(OC(C)=O)c(C)c(=O)n(C)c1CBr</smiles><smiles>CCCc1c(CBr)nc(OC)c(C)c1OC(C)=O</smiles><smiles>CCCc1c(C)nc(OC(C)=O)c(C)c1OC(C)=O</smiles><smiles>CCCc1c(CCl)nc(OC(C)=O)c(C)c1OC(C)=O</smiles>

169

174

177

178

179 
<smiles>CCCc1c(C=O)nc(OC(C)=O)c(C)c1OC(C)=O</smiles>

180<smiles>CCCc1c(CBr)nc(OC(C)=O)c(C)c1OC(C)=O</smiles>

181<smiles>CCCCCC/C(C)=C/Cc1nc(OC(C)=O)c(C)c(OC(C)=O)c1CCC</smiles>

182<smiles>CCCc1c(CBr)oc(=O)c(C)c1OC(C)=O</smiles>

183<smiles>CC([18OH])=CI</smiles>

195<smiles>CC(C=[Po])=CBr</smiles>

196<smiles>Br[Ga]Cc1ccccc1</smiles>

197<smiles>CCCOc1c(CCC)c(C[18Br])oc(=O)c1C</smiles>

198<smiles>CCCCCc1oc(=O)c(C)c(OCCC)c1CCC</smiles>

200<smiles>CCCOc1c(CCC)c(CC(Cl)Cl)oc(=O)c1C</smiles>

201

202<smiles>C=CCc1ccccc1</smiles>

213<smiles>C=CCc1oc(=O)c(C)c(OCC)c1CCC</smiles>

214<smiles>CC=C(C)C=[Mg]</smiles>

215<smiles>COC(C)(/C=C/C(C)=O)OC</smiles>

272

273<smiles>C=C(/C=C/C(C)(OC)OC)OC(C)(C)/C=C/C(=O)CBr</smiles><smiles>CCCCCS(C)(C)OC/C=C\CCOCCCC</smiles>

274 275 277 278

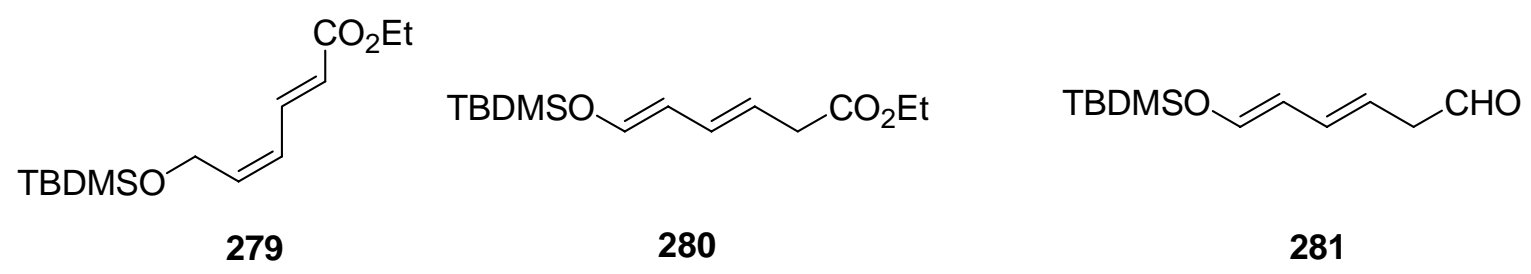

$\mathrm{HO}$,<smiles>CO[Sb]OC=CC=CCC(C)(C)OC</smiles><smiles>O=CCCc1ccccc1</smiles>

291<smiles>COC(C)(C)/C=C/C(=O)CC(O)CCc1ccccc1</smiles> 


\section{E. Abkürzungen}

A

$\begin{array}{ll}\mathrm{AcCl} & \text { Acetylchlorid } \\ \mathrm{AlMe}_{3} & \text { Trimethylalan } \\ \mathbf{B} & \end{array}$

$( \pm)$-BINOL $\quad \beta$-Binaphthol

$\mathrm{Boc}_{2} \mathrm{O} \quad$ Pyrokohlensäuredi-tbutylestercarbonat

$\mathrm{BrCH}_{2} \mathrm{CH}_{2} \mathrm{Br}$ Bibromethan

$\mathrm{Bu}_{4} \mathrm{NCl} \quad$ Tetrabutylammoniumchlorid

Bzl-NH $\mathrm{NH}_{2} \quad$ Benzylamin

C

$\mathrm{CH}_{2} \mathrm{~N}_{2} \quad$ Diazomethan

$\mathrm{ClCH}_{2} \mathrm{CH}_{2} \mathrm{Cl}$ Dichlorethan

$\mathrm{CpZrCl}_{2} \quad$ Zirconocendichlorid

D

DBPO Dibenzoylperoxid

DBU 1,8-Diazabicycl[5.4.0]ononan

DCC $\quad N$ - $N$-Dicyclohexylcarbdiimid

DCE 1,2-Dichlorethan

DIBAH DiIsobutylaluminiumhydrid

DMF Dimethylformamid

DMAP Dimethylaminopyridin

DME Dimethoxyethan

DMPU 1,3-Dimethyltetrahydro-2-(1H)-pyrimidinon

[D]-DMSO $d$-Dimethylsulfoxid

DMSO Dimethylsulfoxid

E

$\operatorname{Et}(i \mathrm{Pr})_{2} \quad$ Diiosopropylethylamin

$\mathrm{Et}_{2} \mathrm{O} \quad$ Diethylether

$\mathrm{H}$

HMDS Hexamethyldisilazid

HMPA Hexamethylphosphorsäuretriamid

L

LDA Lithiumdiisopropylamin

M

MCPBA m-Chlorperoxybenzoesäure

MeI Methyliodid

$p$ - $\mathrm{MeOBnNH}_{2} \quad p$-Methoxybenzylamin

$\mathrm{MOMCl} \quad$ Chlormethylmethylether

$\mathbf{N}$

NBS $\quad N$-Bromsuccinimid

$\mathrm{NEt}_{4} \mathrm{Cl} \quad$ Tetraethylammoniumchlorid

$\mathrm{NH}_{2} \mathrm{NH}_{2} \quad$ Hydrazin

$\mathrm{Ni}\left[(\mathrm{COD})_{2}\right] \quad$ Bis-(1,5-cyclooctadien)nickel

$\mathrm{Ni}\left[\left(\mathrm{PPh}_{3}\right)_{2}\right] \mathrm{Cl}_{2} \quad$ Dichlor-bis-(triphenylphosphin)nickel

$\mathbf{P}$

PCC Pyridiniumchlorochromat

$\left[\mathrm{Pd}(\mathrm{dba})_{2}\right] \quad$ Bis-(dibenzylidenaceton)palladium

$\left[\mathrm{Pd}\left(\mathrm{PPh}_{3}\right)_{4}\right] \quad$ Tetrakis(triphenylphosphin)palladium

$\mathrm{PPh}_{3} \quad$ Triphenylphosphin 
$\mathbf{S}$

$\mathrm{SnCl}_{4} \quad$ Zinntetrachlorid

T

TBAF

TBDMSCl

TCC

TFAA

TFP

TFPOA

THF

$\mathrm{Ti}(\mathrm{O}-i \mathrm{Pr})_{4}$

TMSCl

TMSI

TMSOTf

Y

$\mathrm{Yb}(\mathrm{OTf})_{3}$

Tetrahydrofuran
Tetrabutylammoniumfluorid

$t$ Butyldimethylsilylchlorid

Trichlor-s-triazin-2,4,6(1H, 3H, 5H)trion (Trichlorisocyansäure)

Trifluoressigsäureanhydrid

Tris-(2-furyl)phosphin

Trifluorperoxyessigsäure

Tetra-isopropoxytitanium (IV)

Trimethylsilylchlorid

Trimethylsilyliodid

Trimethylsilyltrifluormethansulfonat (Trimethylsilyltriflat)

Ytterbium(III)triflat 


\section{F. Literaturverzeichnis}

[1] D. Guo. Y. F. Su, J.G. Dai, H.Z. Guo, in Abstract Book, International Symposium on the Chemistry of Natural Products (23rd IUPAC), Florenz, Italien, 2002, 102.

[2] P. Nuhn, Naturstoffchemie, 3. Auflage, Hirzel, Stuttgart 1997.

[3] A. Zeeck, G. Bach, H. Terlau, Das medizinische Potential von Naturstoffen, Internist 2001, 42, 1422 - 1427.

[4] D. J. Newman, G. M. Cragg, K. M. Snader, J. Nat. Prod. 2003, 66, 1022 - 1037.

[5] F. v. Nussbaum, M. Brandis, B. Hinzen, S. Weigand, D. Häbich, Antibakterielle Natur stoffe in der medizinischen Chemie - Exodus oder Renaissance?, Angewandte Chemie, 2006, 118, 5194, 5254.

[6] G. T. Prance, Ethnobotany and the Search for new Drugs, Ciba Foundation Symposium 185, Wiley, Chichester, 1994, 4 - 24.

[7] K. Peter, C. Vollhardt, Organische Chemie, 1. Auflage, Basel 1988.

[8] K. C. Nicolaou, E. J. Sorensen, Classics in Total Synthesis, 1. Auflage, Weinheim 1996.

[9] J. C. Sheehan, K. R. Henery-Logan, J. Am. Chem. Soc. 1957, 79, 1262.

[10] O. Wagner, Dissertation, Universität Göttingen 2000.

[11] Japan Pat. 1998. 98237 044, CA, 129, 244203s.

${ }^{[12]}$ a) H. J. Schiewe, Dissertation Göttingen, 1997; b) H. B. Bode, B. Bethe, R. Höfs, A. Zeeck, Chem. Bio. Chem. 2002, 3, 619-627.

[13] R. Kind Dissertation Göttingen, 1991; H. J. Schiewe, Dissertation Göttingen, 1997.

[14] L. Hoffmann, Dissertation Göttingen, 2006.

[15] H. J. Schiewe, Dissertation Göttingen, 1997.

[16] W. Oppolzer, R. Moretti, G. Bernardinelli, Tetrahedron Letters 1986, 27, 39, 4713-4716.

[17] a) K. A. Keaton, A. J. Philips, J. Am. Chem. Soc. 2006, 128, 408 - 409; - b) F. P. Schmidtchen, H. Rapoport, J. Am. Chem. Soc. 1977, 99, 21, 7014-7019; - c) M. J. Schermann, D. L. Boger, J. Am. Chem. Soc. 2005, 127, 15704 - 15705.

[18] M. J. Schermann, D. L. Boger, J. Am. Chem. Soc. 2005, 127, 15704 - 15705.

[19] B. H. Lipshutz, G. Bülow, R. F. Lowe, K. L. Stevens; J. Am. Chem. Soc. 1996, 118, 5512 - 5513; -b) B. H. Lipshutz, G. Bülow, R. F. Lowe, K. L. Stevens; Tetrahedron 1996, 52, 21, $7265-7276$.

[20] a) H. Hagiwara, K. Kobayashi, S. Miya, T. Hoshi, T. Suzuki, M. Ando, T. Okamoto, M. Kobayashi, I. Yamamoto, S. Ohtsubo, M. Kato, H. Uda, J. Org. Chem. 2002, 67, 17, 5969 - 5976; -b) C. O. Kappe, T. Kappe, Monatsheft für Chemie; 1989, 120, 1095 - 
1100; -c) G. Köster, R. W. Hoffmann, Liebigs Ann. Chem., 1987, 987 - 990; - d) W. Op polzer, R. Moretti, G. Bernardinelli, Tetrahedron Letters 1986, 27, 39, 4713 - 4716; -e) A. A. Birkbeck, D. Euders; Tetrahedron Letters 1998, 39, 7823 - 7826; -f) Y. Ishibashi, S. Ohba, S. Nishiyama, S. Yamamura; Tetrahedron Letters 1996, 37, 17, 2997 - 3000; g) T. Kanazawa, Y. Ohkawa, T. Kuda, Y. Minobe, T. Tani, M. Nishizawa, Chem Pharm. Bull. 1997, 45, 6, 1046 - 1051;-h) R. W. Spencer, L. J. Copp, J. R. Pfister, J. Med. Chem. 1985, 28, $1828-1832$.

[21] T. Eicher, S. Hauptmann, Chemie der Heterocyclen, Georg Thieme Verlag, Stuttgart, New York 1994.

[22] T. L. Gilchrist, Heterocyclenchemie, VCH Weinheim, 1995.

[23] S. N. Huckin, L. Weiler, J. Am. Chem. Soc. 1974, 96, 4, 1082-1087.

[24] M. Wohlberg, A. Ji, W. Hummel, M. Müller, Synthesis 2001, 6, 937 - 942.

[25] T. A. Oster, T. M. Harris, Tetrahedron Letters 1983, 24, 18, 1851 - 1854.

[26] S. Nahm, S. M. Weinreb, Tetrahedron Letters 1981, 22, 39, 3815 - 3818.

[27] T. Hanamoto, T. Hiyama, Tetrahedron Letters 1988, 29, 49, 6467 - 6470.

[28] H. Hagiwara, K. Kobayashi, S. Miya, T. Hoshi, T. Suzuki, M. Ando, T. Okamoto, M. Kobayashi, I. Yamamoto, S. Ohtsubo, M. Kato, H. Uda, J. Org. Chem. 2002, 67, 17, $5969-5976$.

[29] a) M. W. Plath, H. D. Scharf; G. Raabe, C. Krüger, Synthesis 1990, 10, 951 - 955; -b) H. A. Staab, Angew. Chem., 1962, 74, 12, $407-423$.

[30] a) G. Köster, R. W. Hoffmann, Liebigs Ann. Chem. 1987, 987 - 990; -b) A. A. Birkbeck, D. Euders, Tetrahedron Letters 1998, 39, 7823 - 7826; -c) W. Oppolzer, R. Moretti, G. Bernardinelli, Tetrahedron Letters 1986, 27, 39, 4713 - 4716.

[31] F. A. Carey, R. J. Sundberg, Organische Chemie, VCH, Weinheim 1995.

[32] A. A.Birkbeck, D. Euders, Tetrahedron Letters 1998, 39, 7823 - 7826.

[33] W. Oppolzer, R. Moretti, G. Bernardinelli, Tetrahedron Letters 1986, 27, 39, 4713 4716.

[34] Y. Ishibashi, S. Ohba, S. Nishiyama, S. Yamamura; Tetrahedron Letters 1996, 37, 17, $2997-3000$.

[35] G. Köster, R. W. Hoffmann; Liebigs Ann. Chem. 1987, 987 - 990.

[36] T. Kappe, B. Schnell, J. Heterocyxclic Chem. 1996, 33, 663 - 670.

[37] C. O. Kappe, T. Kappe, Monatsheft für Chemie 1989, 120, 1095 - 1100.

[38] a) E. Suzuki, R. Hamajima, S. Inoue, Synthesis 1974, 192 - 194; -b) E. Suzuki, B. Katsuragawa, S. Inoue, J. Chem. Research 1982, 224 - 225. 
[39] R. Bacardit, M. Moreno-Maňas, R. Pleixats, J. Heterocyclic Chem. 1982, 19, 157 - 160.

[40] Organikum, 20 Aufl., Johann Ambrosius Barth Verlag, Heidelberg, Leipzig 1996.

[41] J. G. Bruno, M. N. Chang, Y. M. Choi-Sledeski, D. M. Green, D. G. McGarry, J. R. Regan, F. A. Volz; J. Org. Chem. 1997, 62, 15, 5174 - 5190.

[42] A. K. Bhattacharya, F. Stolz, Can. J. Chem. 2002, 80, 8, 973 - 982.

[43] N. Furukawa, H. Kishimoto, S. Ogawa, T. Kawai, H. Fujihara, S. Oae, Tetrahedron Letters 1981, 22, 44, 4409 - 4412.

[44] A. Amrollah-Madjdabadi, T. N. Pham, E. C. Ashby, Synthesis 1989, 8, 614-616.

[45] H. Kaga, K. Goto, T. Takahashi, M. Hino, T. Tokuhashi, K. Orito; Tetrahedron, 1996, $52,25,8451-8470$.

[46] A. J. Bloodworth, R. J. Curtis, M. D. Spencer, N. A. Tallant; Tetrahedron 1993, 49 13, $2729-2750$.

[47] R. W. Hoffmann, H. Brinkmann, G. Frenking; Chem. Ber. 1990, 123, 12, 2387 - 2394.

[48] D. Renneberg, H. Pfander, C. J. Leumann, J. Org. Chem. 2000, 65, 9069 - 9079.

[49] a) D. Seebach, L. Widler, Helv Chim Acta 1982, 65, 7, 1972 - 1981; -b) H. Kaga, K. Goto, M. Takahashi, M. Hino, T. Tokuhashi, K. Orito, Tetrahedron 1996, 52, 8451; -c) M. M. Bouis, Ann. Chim. 1928, 10, 9, 402.

[50] Y. Kawanaka, N. One, Y. Yoshida, S. Okamoto, F. Sato; J. Chem. Soc., Perkins Trans 1 1996, $715-718$.

[51] A. Commercon, J. Normant, J. Villieras, J. Organomet. Chem. 1975, 93, 415 - 421.

[52] a) J. Grimaldi, M. Bertrand, Bull. Soc. Chim. Fr. 1971, 947 - 957; -b) J. Grimaldi, M. Bertrand, Bull. Soc. Chim. Fr. 1971, 12, 4316 - 4320.

[53] D. Bergeron, B. Caron, P. Brassard, J. Org. Chem. 1993, 58, 509 - 511.

[54] D. Chemin, S. Gueugnot, G. Linstrumelle, Tetrahedron 1992, 48, 4369 - 4378.

[55] D. Enders, G. Geibel, S. Osborne, Chem. Eur. J. 2000, 6, 8, 1302 - 1309.

[56] E. Negishi, H. Matsushita, N. Okukado, Tetrahedron Letters. 1981, 22, 29, 2715 - 2718.

[57] B. H. Lipshutz, G. Bulow, R. F. Lowe, K. L. Stevens, J. Am. Chem. Soc. 1996, 118, $5512-5513$.

[58] a) K. Mori, M. Sakakibara, K. Okada, Tetrahedron 1984, 40, 10, 1767 - 1781; -b) K. Mori, T. Takeuchi; Liebigs Ann. Chem. 1988, 815 - 818.

[59] S. Baba, D. E. Horn, E. Negishi, Tetrahedron Letters 1976, 23, 1927 - 1930.

[60] K. Uchida, K. Utimoto, H. Nozaki, J. Org. Chem. 1976, 41, 12, 2215 - 2217.

[61] T. Inghardt, T. Frejd, Synthesis 1990, $285-291$.

[62] K. Mori, T. Takeuchi; Liebigs Ann. Chem. 1988, 815 - 816. 
[63] M. Cervera, M. Moreno-Maňas, R. Pleixats, Tetrahedron, 1990, 46, 23, 7885 - 7892.

[64] Zhen Li, Diplomarbeit, 2004, Universität Göttingen.

[65] S. Castillo, H. Ouadahi, V. Hérault, Bulletin de la Societe de France, 1982, 7 - 8, 257 261.

[66] K. D. Deodhar, M. B. Kekare, S. R. Pednekar; Synthesis 1985, 328 - 331.

[67] Chun-shan Wang, J. Heterocycl. Chem., 1970, 7, 389 - 392.

[68] W. C. Groutas, M. A. Stanga, M. J. Brubaker, T. L. Huang, Min K. Moi, R. T. Carroll, J. Med. Chem., 1985, 28, 8, 1106 - 1109.

[69] F. G. Baddar, F. H. Al-Hajjar, N. R. El-Rayyes, J. Heterocyclic Chem. 1976, 13, 195 203.

[70] a) I. El-Sayed El-Kholy, M. M. Mishrikey, S. L. Abdoul-Ela, J. Heterocyclic Chem., 1982, 19, 1329 - 1334; b) I. El-Sayed El-Kholy, M. M. Mishrikey, H. M. Fuid-Alla, J. Heterocyclic Chem. 1975, 12, 129 - 133.

[71] A. K. Kiang, S. F. Tan, W. S. Wong, J. Chem. Soc., 1971, $2721-2726$.

[72] R. H. Wiley, P. Beasley, L. H. Knabeschuh, J. Amer. Chem. Soc. 1954, 76, 625 - 627.

[73] T. Kappe, S. Ajili, W. Stadbauer, J. Heterocycl. Chem. 1988, 25, 463 - 468.

[74] T. Kurihara, H. Tanno, S. Takemura, S. Harusawa, R. Yoneda, J. Hetercycl. Chem., 1993, 30, 3, $643-652$.

[75] K. H. Chung, K. Y. Cho, Y. Asami, N. Takahashi, S. Yoshida, Heterocycles, 1991, 32, 1, $99-105$.

[76] K. Veres, J. Jonas, A. Horeni, Collect Czech Chem. Commun. 1959, 24, 3471 - 3474.

[77] Organikum, 20 Aufl., Johann Ambrosius Barth Verlag, Heidelberg, Leipzig, 1996.

[78] M. Oba, T. Terauchi, Y. Owari, Y. Imai, I. Motoyama, K. Nishiyama, J. Chem. Soc., Perkins Trans 1 1998, 7, 1275 - 1281.

[79] D. H. Hua, S. W. Miao, S. N. Bharathi; J. Org. Chem. 1990, 55, 11, $3682-3684$.

[80] D. J. Hart, C. S. Lee, J. Am. Chem. Soc. 1986, 108, 6054- 6056.

[81] P. J. Reider, E. J. Grabowski, Tetrahedron Letters 1982, 23, 22, 2293 - 2296.

[82] M. Mori, Y. Uozumi, M. Kimura, Y. Ban, Tetrahedron 1986, 42, 14; 3793 - 3806.

[83] A. Nadin, J. M. Sánches López, A. P. Owens, D. M. Howells, A. C. Talbot, T. Harrison, J. Org. Chem. 2003, 68, 7, $2844-2852$.

[84] A. B. Tomchin, S. P. Fradkina, I. M. Krylova, Z. A. Khromenkova, J. Org. Chem. USSR 1986, 22, 11, $2163-2172$.

[85] J. Buck, J. P. Madeley, G. Pattenden, J. Chem. Soc, Perkins Trans 1 1992, 67 - 73.

[86] V. M. Mukkala, J. J. Kankare, Helv. Chim. Acta. 1992, 75, 5, 1578 - 1591. 
[87] G. E. Jeromin, W. Orth, B. Rapp, W. Weiß; Chem. Bericht 1987, 120, 649 - 651.

[88] E. Negishi, N. Okukado, A. O. King, D. E. Van Horn, B. I. Spiegel, J. Am. Chem. Soc. 1978, 100, 7, $2254-2256$.

[89] E. P. v. Zezschwitz, Dissertation Göttingen, 1999.

[90] L. Berillon, R. Wagner, P. Knochel; J. Org. Chem. 1998, 63, 9117 - 9121.

[91] C. Santelli-Rouvier, C. Coin, L. Toupet, M. Santelli, J. of Organometallic Chemistry 1995, 495, $91-96$.

[92] E. Negishi, D. E. van Horn, T. Yoshida, J. Am. Chem. Soc. 1985, 107, 6639 - 6647.

[93] Y. Yamamoto, S. Hatsuya, J. Yamada; J. Org. Chem. 1990, 55, 3118 - 3128.

[94] R. Brückner, Reaktionsmechanismen, Spektrum Akademische Verlag, Heidelberg, 1996.

${ }^{[95]}$ E. Negishi, T. Takahashi, S. Baba, D. E. Van Horn, N. Okukado, J. Am. Chem. Soc. 1987, $109,2393-2401$.

[96] E. Nakamura, S. Mori, Angew. Chem. 2000, 112, $3902-3924$.

[97] S. Flemming, J. Kabbara, K. Nickisch, J. Westermann, J. Mohr, Synlett 1995, 183 - 185.

[98] G. Zweifel, R. L. Miller, J. Am. Chem. Soc. 1970, 92, 6678 - 6679.

[99] S. Pawlenko, Chem. Bericht 1969, 102, 1937 - 1943.

[100] J. W. Collette, J. Org. Chem. 1963, 28, 2489 - 2490.

${ }^{[101]}$ P. Baecström, F. Björkling, H. E. Högeberg, T. Norin, Acta Chemica Scandinavica 1984, $38,779-782$.

${ }^{[102]}$ D. Seebach, H. Neumann, Chem. Ber. 1974, 107, 847 - 853.

${ }^{[103]}$ B. E. Marron, K. C. Nicolaou, Synthesis 1987, 7, 537 - 539.

[104] A. Yanagisawa, N. Nomura, H. Yamaoto, Tetrahedron, 1994, 50, 20, 6017 - 6028.

[105] a) L. F. Tietze, T. Eicher, 2 Aufl., Reaktionen und Synthesen, Georg Thieme Verlag, Stuttgart, New York, 1991; b) Organikum, 20 Aufl., Johann Ambrosius Barth Verlag, Heidelberg, Leipzig, 1996.

[106] a) T. Jeffery, Tetrahedron Letters, 1989, 30, 17, 2225 - 2228; -b) T. Jeffery, S.

Gueugnot, G. Linstrumelle, Tetrahedron Letters, 1992, 33, 39, 5757 - 5760.

[107] a) H. J. Schiewe, Dissertation Göttingen, 1997; -b) W. Eisenreich, B. Menhard, M. S.

Lee, M. H. Zenk, A. Bacher, J. Am. Chem. Soc. 1998, 120, 9694 - 9695.

${ }^{[108]}$ A. C. Cope, E. M. Hardy, J. Am. Chem. Soc. 1940, 62, $441-444$.

${ }^{[109]}$ L. A. Paquette, Angew. Chem. 1990, 102, $642-660$.

${ }^{[110]}$ J. Bach, R. Berenguer, J. Garcia, M. López, J. Manzanal, J. Vilarrasa, Tetrahedron, 1998, 54, $14947-14962$. 
${ }^{[111]}$ K. F. Armstrong, R. Robinson, J. Chem. Soc. 1934, 1650 - 1652.

${ }^{[112]}$ Conant, Lutz, J. Am. Chem. Soc. 1923, 56, 1303.

[113] a) S. Pikul, J. Raczko, K. Ankner, J. Jurczak, J. Am. Chem. Soc. 1987, 109, 13, 3981 3987; -b) J. Jurczak, S. Pikul, K. Ankner, Tetrahedron Letters 1986, 27, 15, 1711 -1714.

[114] J. Oren, M. Demuth, B. Fuchs, Synthesis 1987, 9, 850 - 853.

[115] J. Schnaubelt, B. Frey, H. U. Reissig, Helv. Chim. Acta. 1999, 82, 666 - 676.

${ }^{[116]}$ Peter Langer, Universitäts Göttingen, 2000.

${ }^{[117]}$ T. L. Littel, S. E. Webber, J. Org. Chem. 1994, 59, 24, 7299 - 7305.

${ }^{[118]}$ M. Sun, Y. Deng, E. Batyreva, W. Sha, R. G. Salomon, J. Org. Chem. 2002, 67, 11, $3575-3584$.

[119] J. Ipaktschi, A. Heydari, H. O. Kalinowski, Chem. Ber. 1994, 127, 905 - 909.

${ }^{[120]}$ J. Uenishi, M. Motoyama, Y. Kimura, O. Yonemitsu, Heterocycles 1998, 47, 439 - 452.

${ }^{[121]}$ Y.G. Suh, K. H. Min, Y. S. Lee, S. Y. Seo, S. H. Kim, H. J. Park, Tetrahedron Letters 2002, 43, $3825-3828$.

${ }^{[122]}$ A. Stambouli, F. Chastrette, R. Amouroux, M. Chastrette, Tetrahedron Letters 1986, $27,35,4149-4152$.

${ }^{[123]}$ S. Mann, S. Carillon, O. Breyne, A. Marquet, Chem. Eur. J. 2002, 8, 2, 439 - 450.

${ }^{[124]}$ A. J. Gutierrez, K. J. Shea, J. J. Svoboda, J. Org. Chem. 1989, 54, 4335 - 4344.

${ }^{[125]}$ S. M. Sparks, K. J. Shea, Org. Lett. 2001, 3, 15, 2265 - 2267.

${ }^{[126]}$ S. K. Massad, L. D. Hawkins, D. C. Baker, J. Org. Chem. 1983, 48, 5180 - 5182.

${ }^{[127]}$ T. Sato, H. Okazaki, J. Otera, H. Nozaki, J. Am. Chem. Soc. 1988, 110, 15, 5209 - 5211.

${ }^{[128]}$ T. K. M. Shing, Ying Tang, Tetrahedron 1990, 46, 6, 2187 - 2194.

${ }^{[129]}$ J. Sandri, J. Viala, Synthesis 1995, 3, $271-275$.

${ }^{[130]}$ Y. Yamano, C. Tode, M. Ito, J. Chem. Soc. Perkins Trans 1, 1998, 16, 2569 - 2582.

${ }^{[131]}$ Y. Aoyagi, M. Yoshimura, M. Tsuda, T. Tsuchibuchi, S. Kawamata, H. Tateno, K. Asa no, H. Nakamura, M. Obokata, A. Ohta, Y. Kodama, J. Chem. Soc. Perkin Trans. 1, 1995, 6, $689-692$.

${ }^{[132]}$ a) K. Maruoka, S. Hashimoto, Y. Kitagawa, H. Yamamoto, H. Nazaki, Bull. Chem. Soc. $J P n .1980,53,3301$ - 3307; -b) K. Maruoka, S. Hashimoto, Y. Kitagawa, H. Yamamo to, H. Nazaki, J. Am. Chem. Soc. 1977, 99, $7705-7707$.

${ }^{[133]}$ T. Mukaiyama, K. Banno, K. Narasaka, J. Am. Chem. Soc. 1974, 96, 24, 7503 - 7509.

[134] T. Mukaiyama, K. Narasaka, K. Banno, Chem. Letters 1973, 1011 - 1014.

${ }^{[135]}$ H. Hagiwara, K. Kimura, H. Uda, J. Chem. Soc. Perkins Trans. 1 1992, 6, 693 - 700. 
[136] a) S. Kobayashi, I. Hachiya, J. Org. Chem. 1994, 59, 3590 - 3596; -b) S. Kobayashi, Chem. Letters 1991, 2087 - 2090.

${ }^{[137]}$ E. Nakamura, M. Shimizu, I. Kuwajima, J. Sakata, K. Yokoyama, R. Noyori, J. Org. Chem. 1983, 48, $932-945$.

${ }^{[138]}$ a) G. E. Keck, X. Y. Li, D. Krishnamurthy, J. Org. Chem. 1995, 60, 5998 - 5999; -b)

G. E. Keck, D. Krishnamurthy, J. Am. Chem. Soc. 1995, 117, 2363 - 2364.

[139] W. C. Still, M. Kahn, A. Mitra, J. Org. Chem. 1978, 43, 2923 - 2925.

${ }^{[140]}$ E. Merck, Anfärbereagenzien für Dünnschicht- und Papier-Chromatographie, E. Merck, Darmstadt, 1984.

${ }^{[141]}$ a) D. D. Perrin, W. L. F. Armarego, Purification of Laboratory Chemicals, Pergamon Press, New York, 1987; b) Organikum, 20 Aufl., Johann Ambrosius Barth Verlag, Heidelberg, Leipzig, 1996; c) L. F. Tietze, T. Eicher, Reaktionen und Synthesen, 2. Aufl., Georg Thieme verlag, Stuttgart, New York, 1991.

${ }^{[142]}$ C. Georgoulis, W. Smadja, G. Ville, Bull. Soc. Chim. Fr. 1984, 2, 7 - 8, $269-270$.

${ }^{[143]}$ de la Mare, E. D. Hughes, P. C. Merriman, L. Pichat, C. A. Vernon, J. Chem. Soc., 1958, 2563.

${ }^{[144]}$ R. Anderson, R. L. Edwards, A. J. S. Whalley; J. Chem. Soc. Perkin Trans 1; 1982, 215 -222 . 


\section{G. Spektrenanhang}

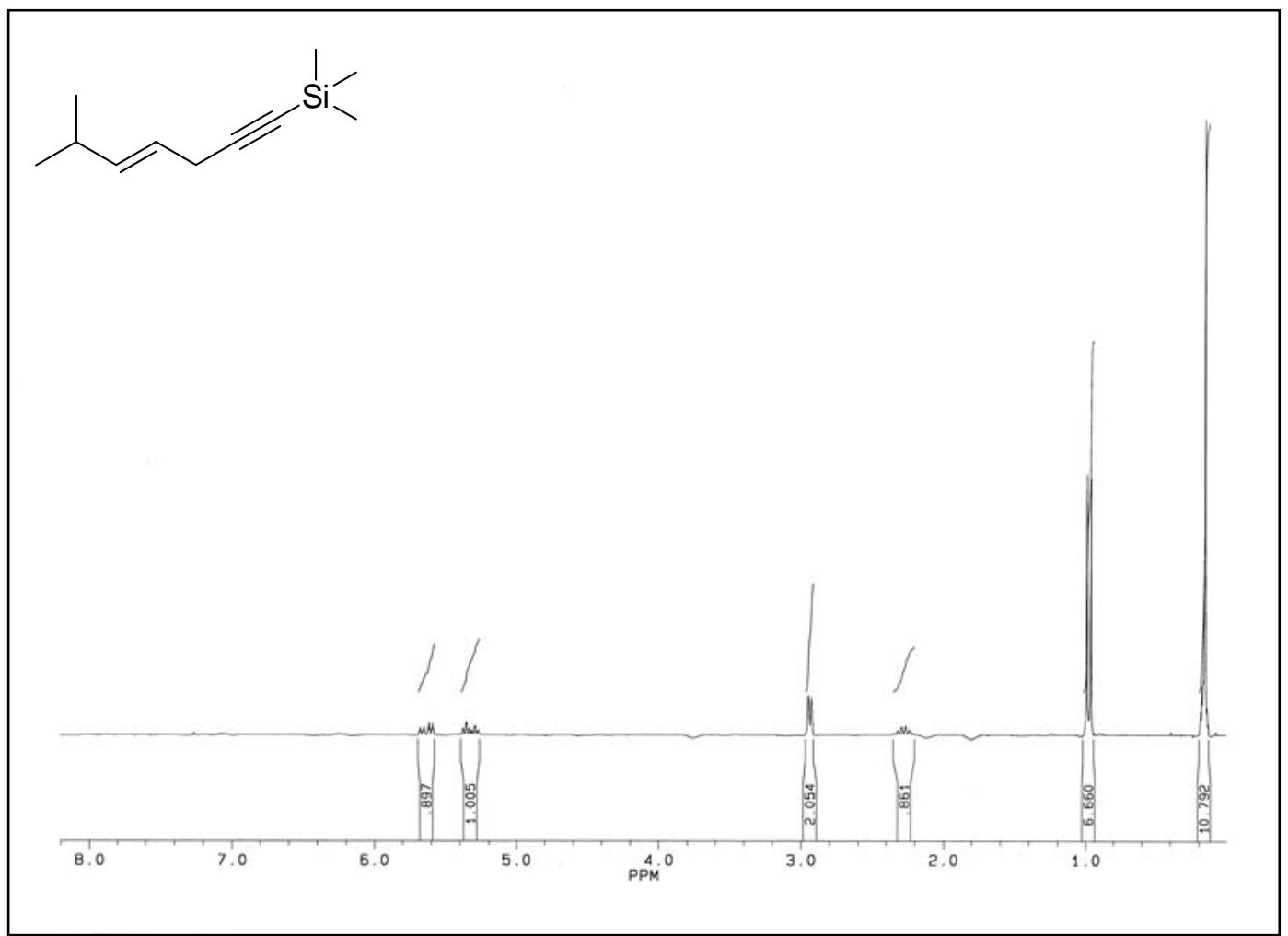

(2E)-1-Trimethylsilyl-6-methylhept-4-en-1-in (102A)

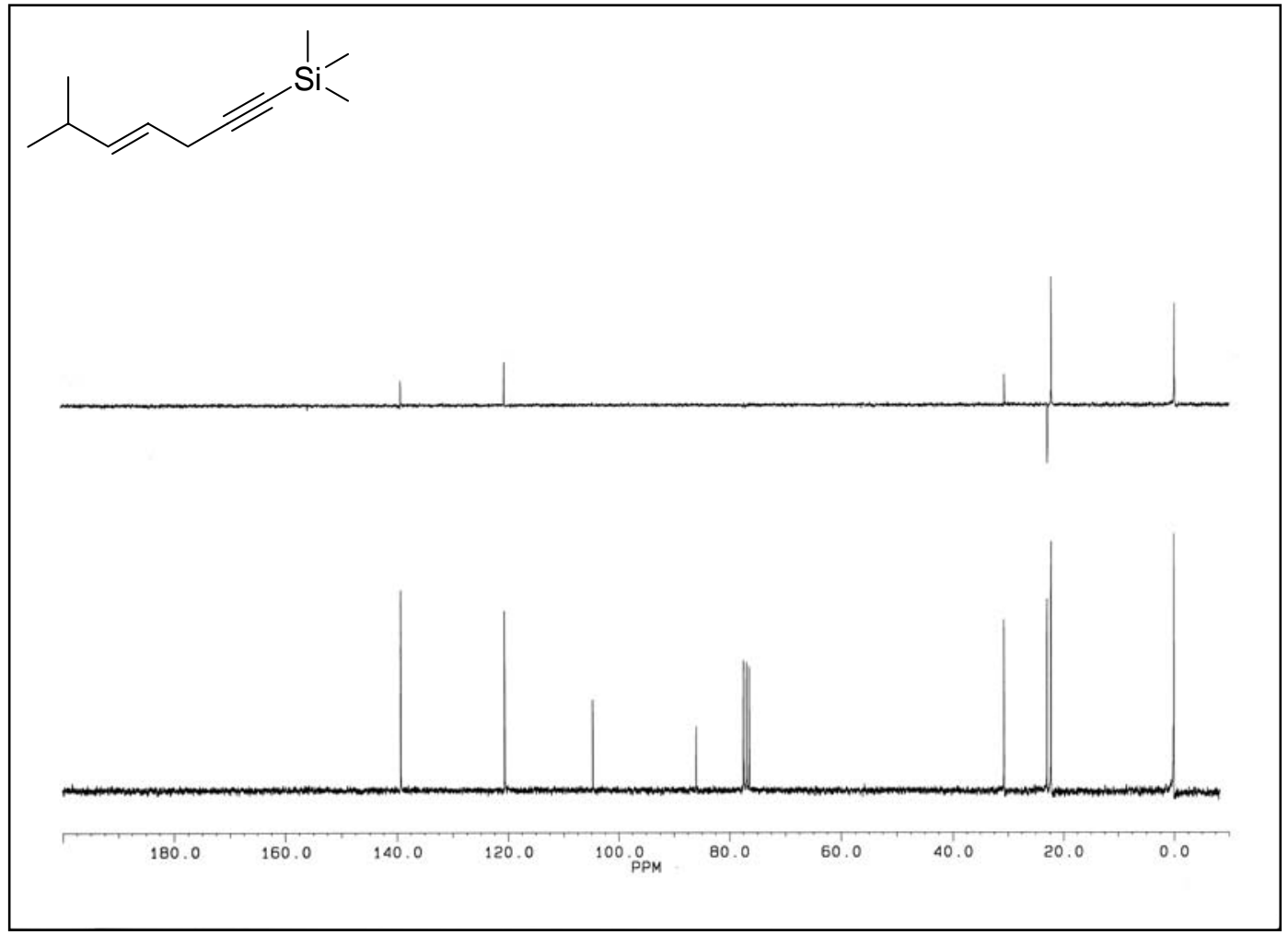

(2E)-1-Trimethylsilyl-6-methylhept-4-en-1-in (102A) 


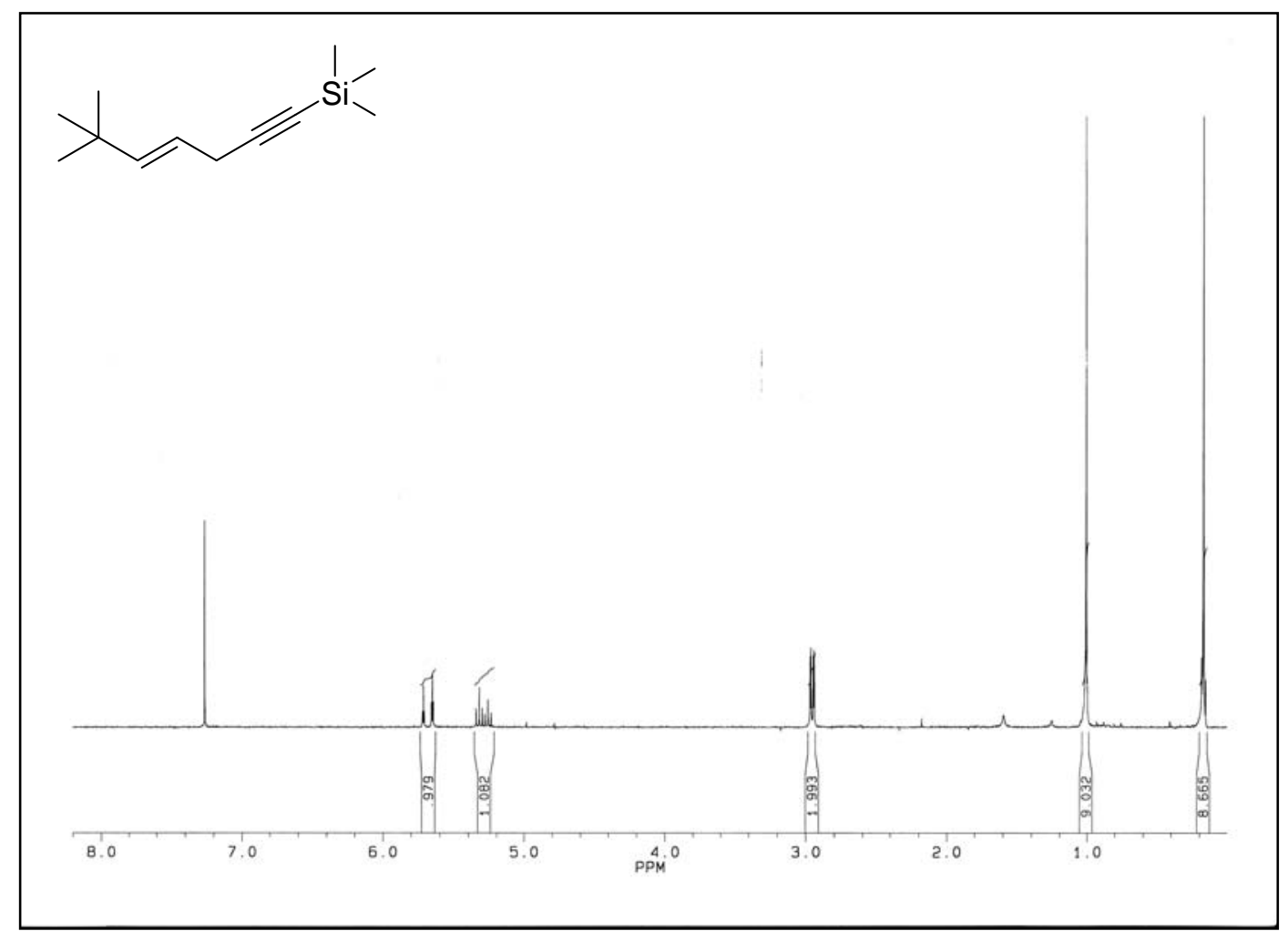

(2E)-1-Trimethylsilyl-6,6-Dimethylhept-4-en-1-in (102R)

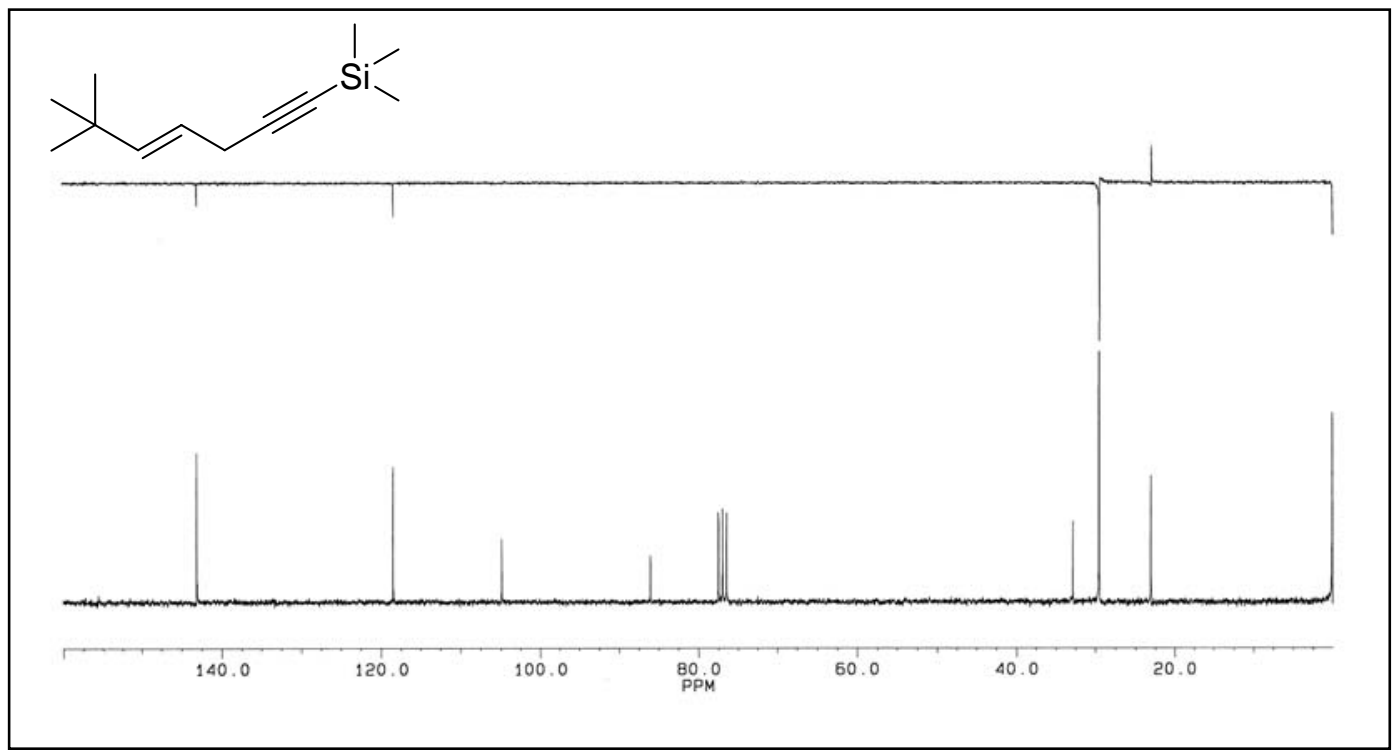

(2E)-1-Trimethylsilyl-6,6-Dimethylhept-4-en-1-in (102R) 


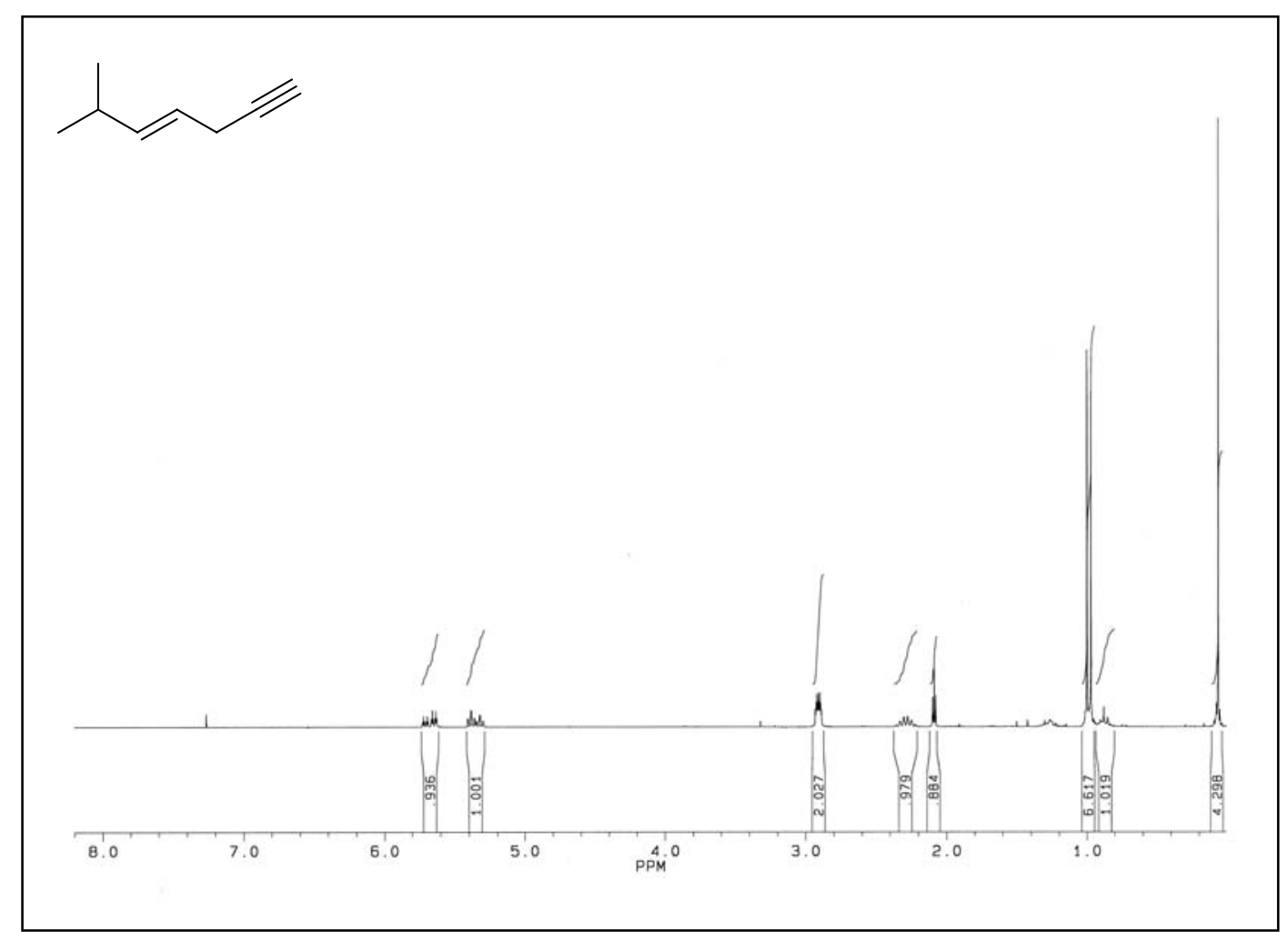

(2E)-6-Methylhept-4-en-1-in (103A)

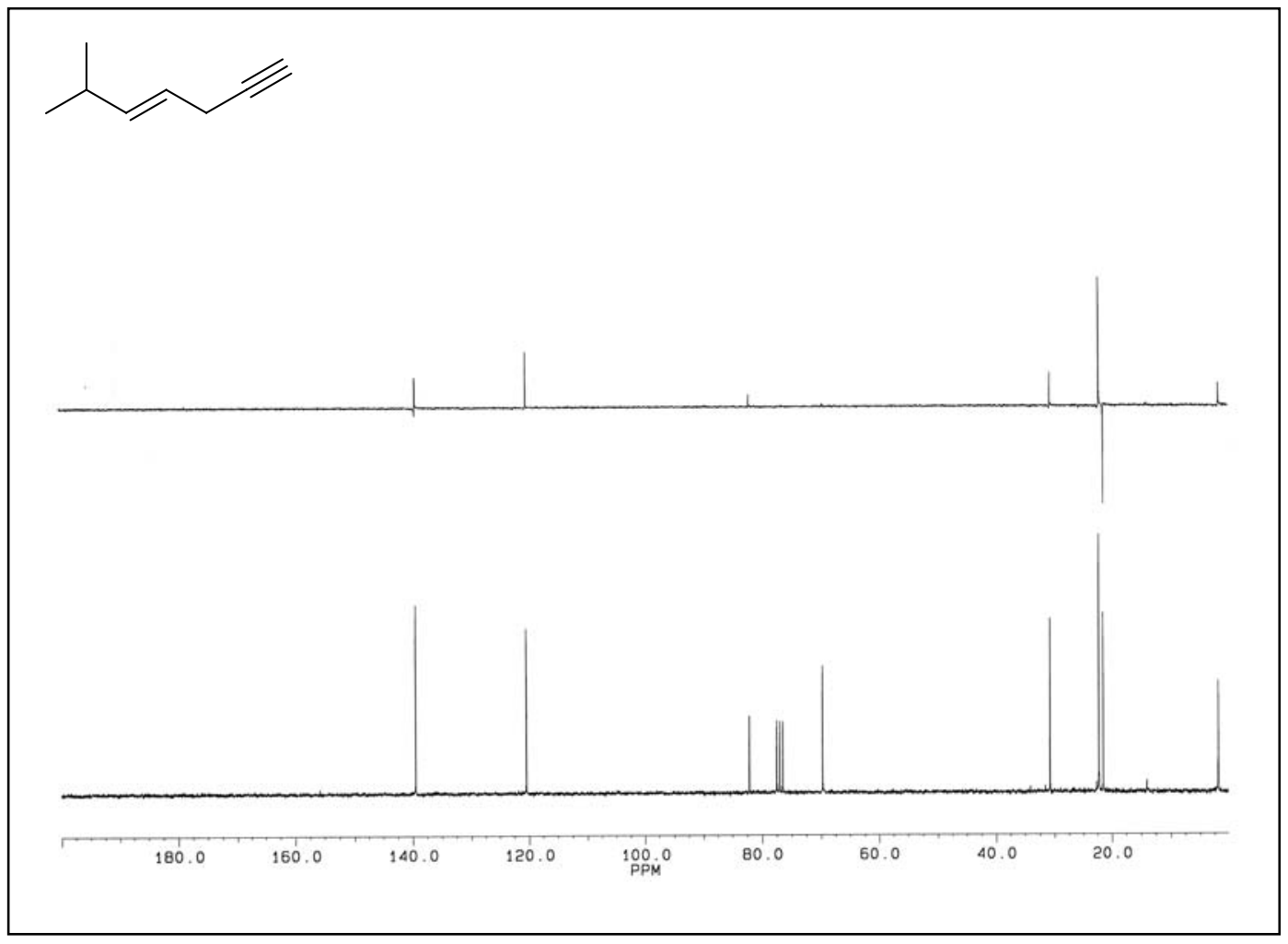

(2E)-6-Methylhept-4-en-1-in (103A) 


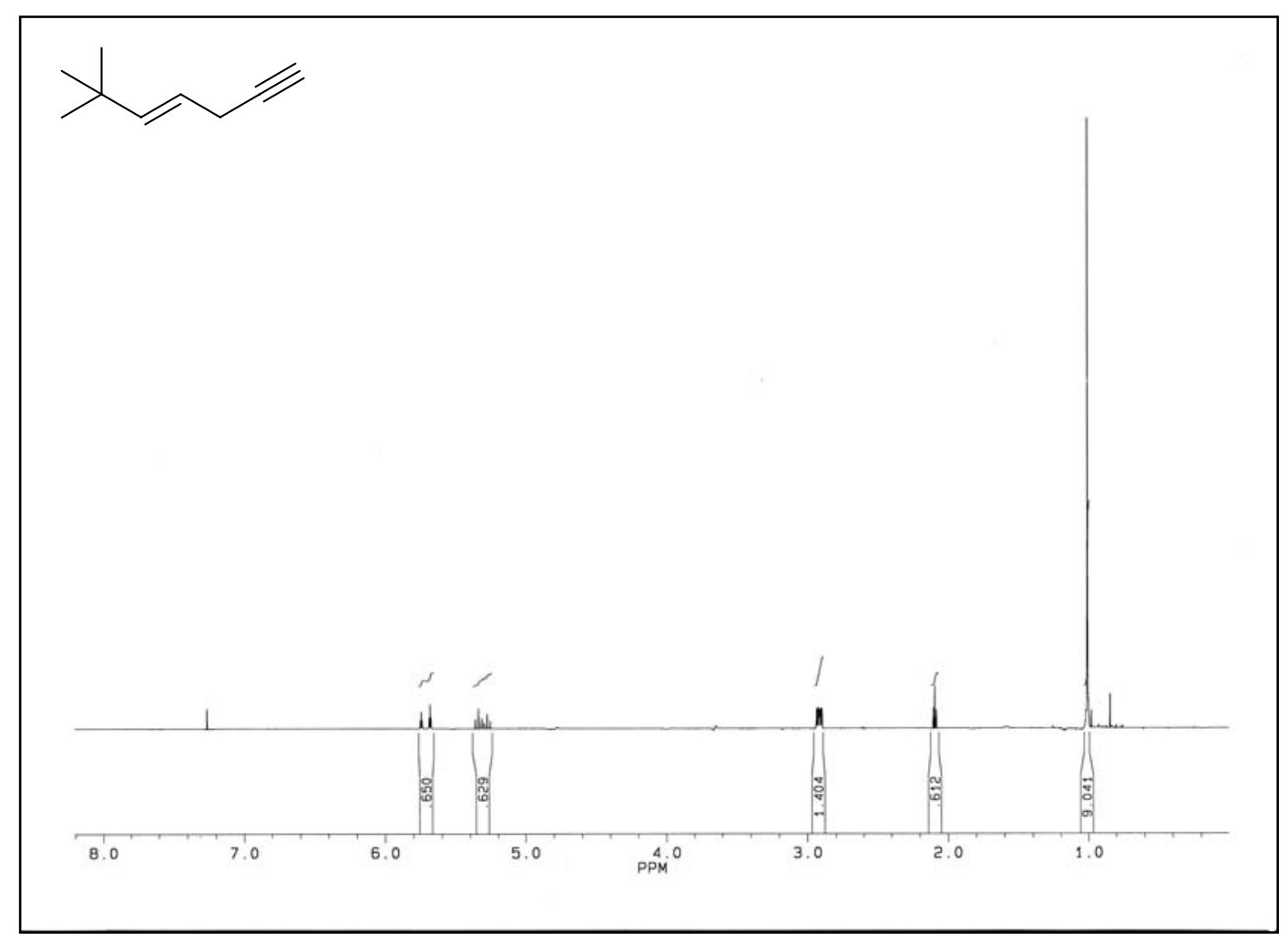

(2E)-6,6-Dimethylhept-4-en-1-in (103R)

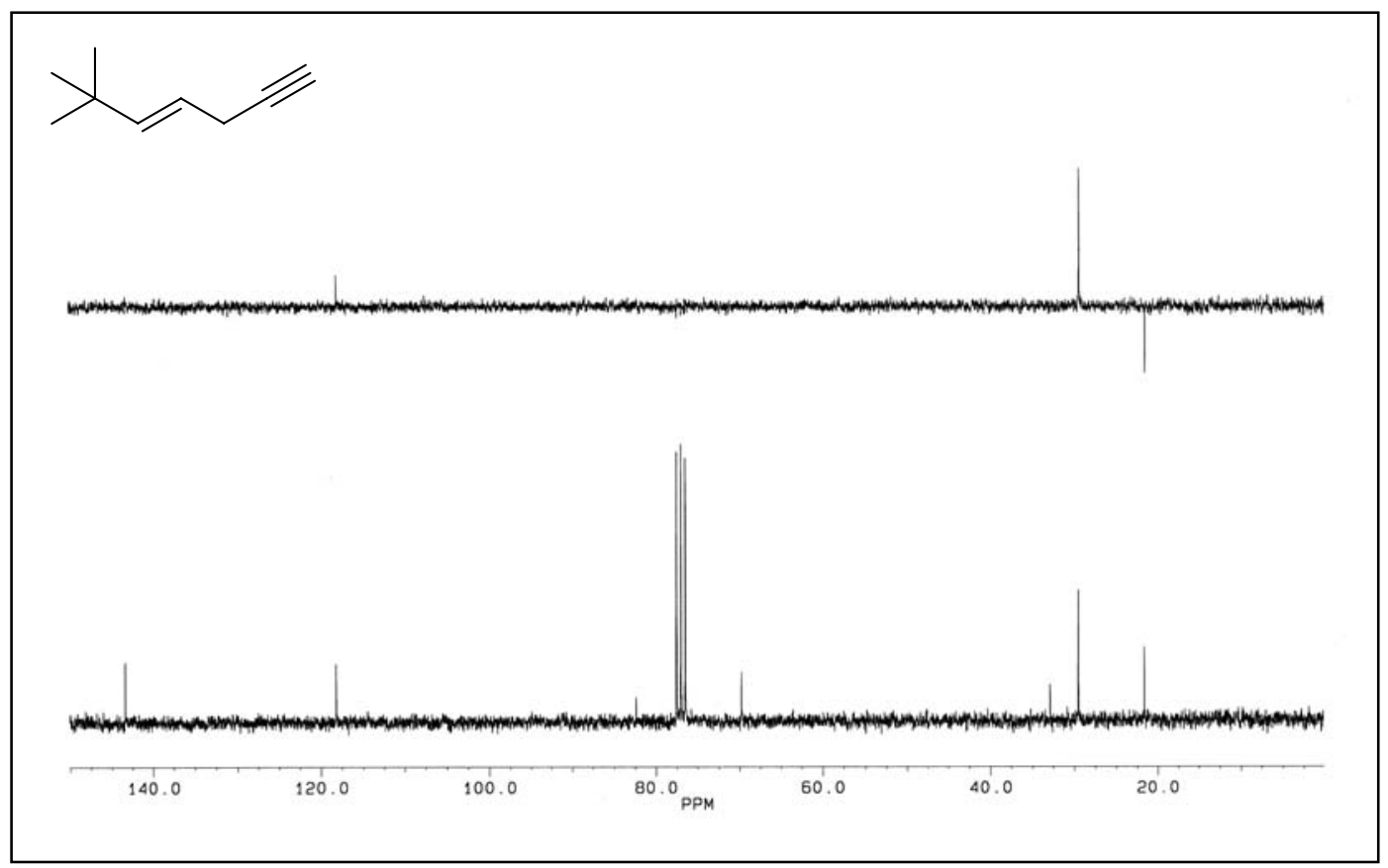

(2E)-6,6-Dimethylhept-4-en-1-in (103R) 


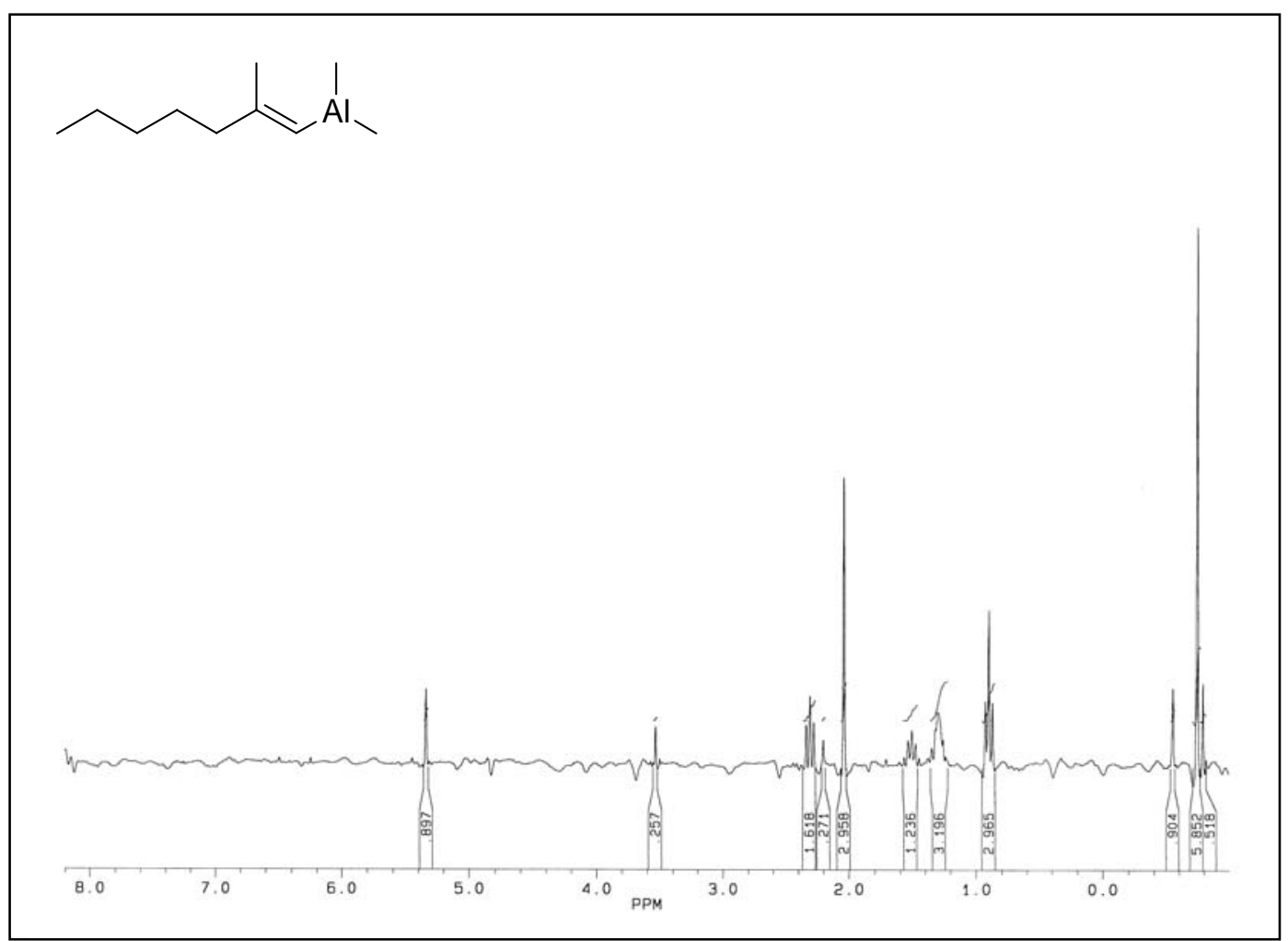

(E)-2-Methylhepta-1-enyldimethylalan (123)

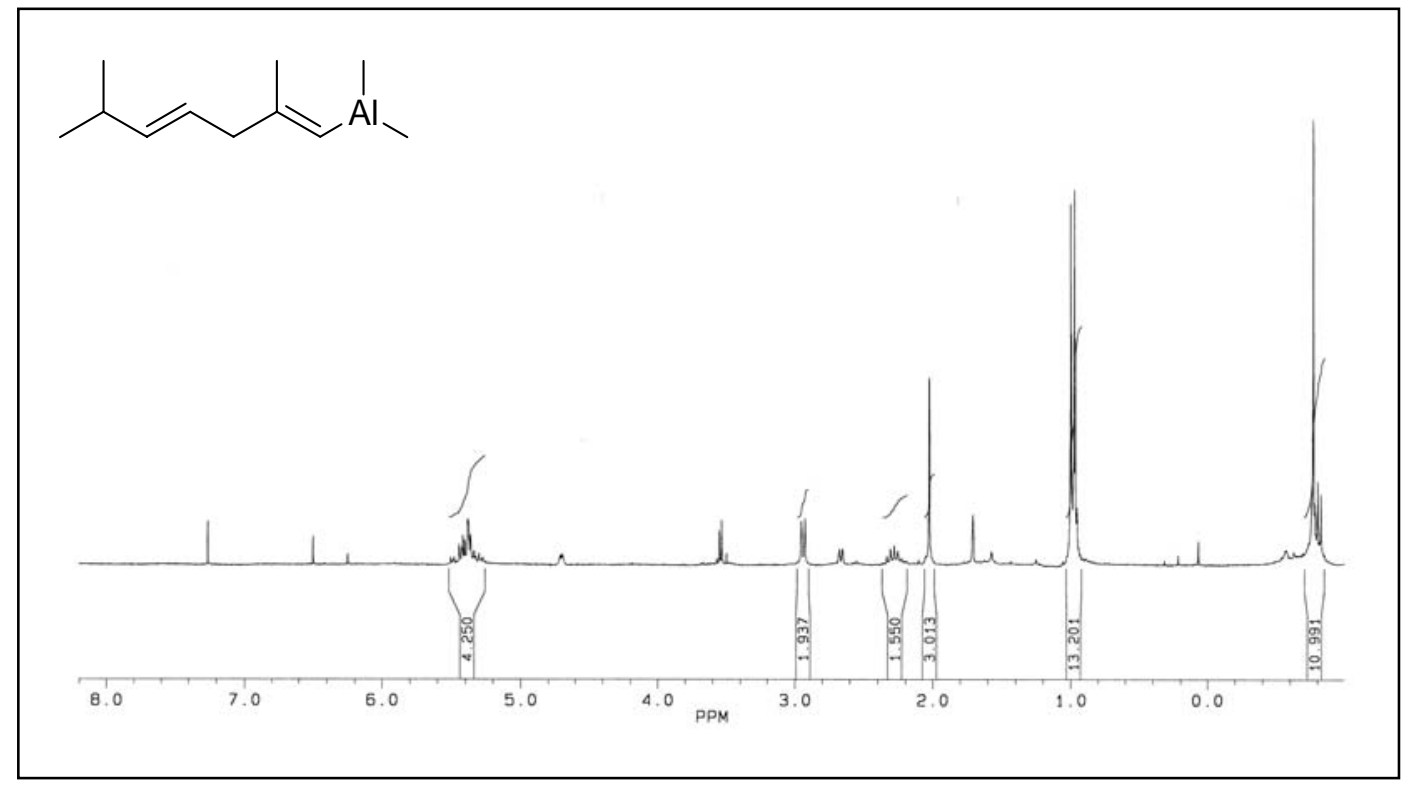

(E,E)-2,6-Dimethylhepta-1,4-dienyldimethylalan (123A) 


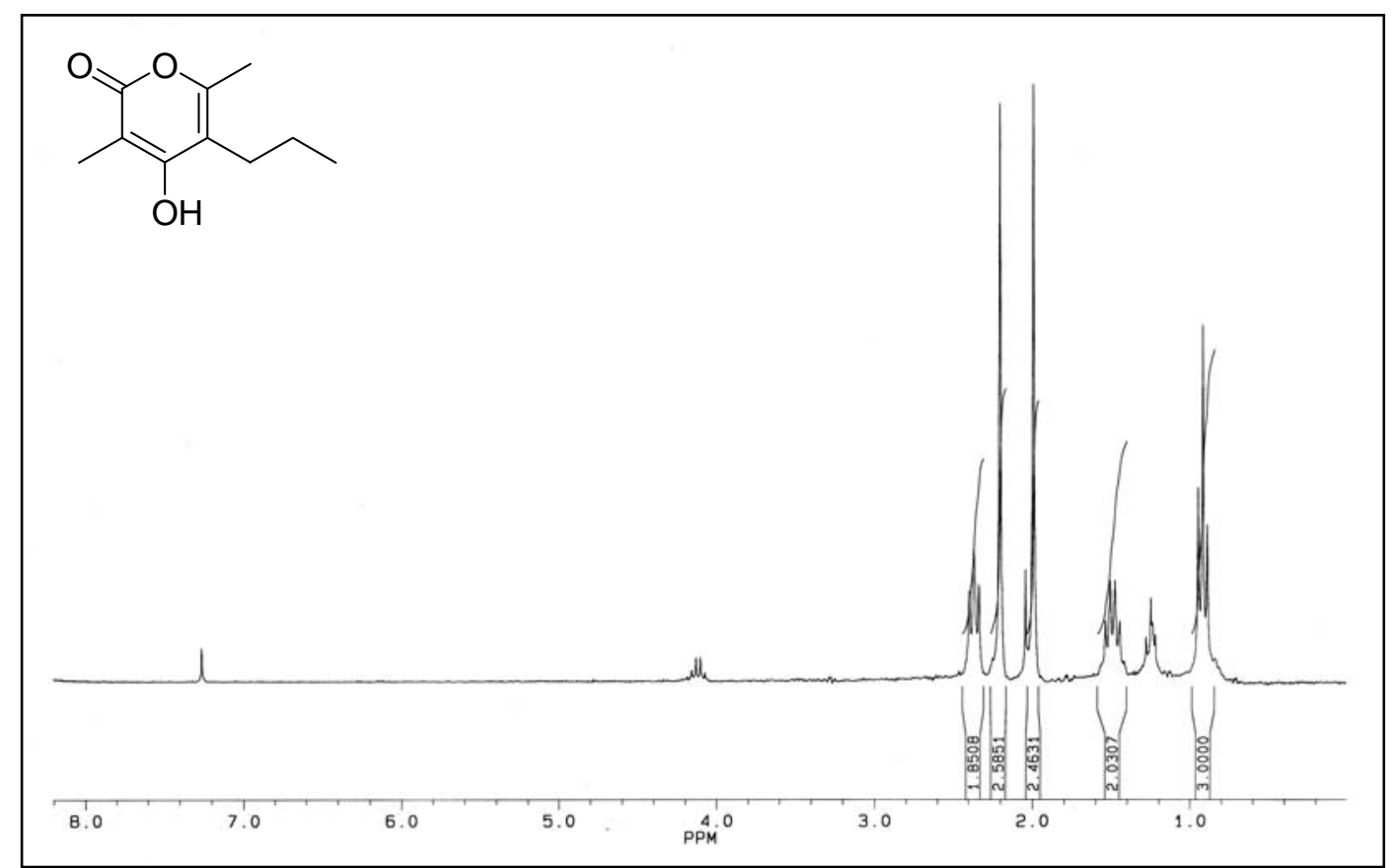

3,6-Dimethyl-4-hydroxy-5-propylpyran-2-on (63)

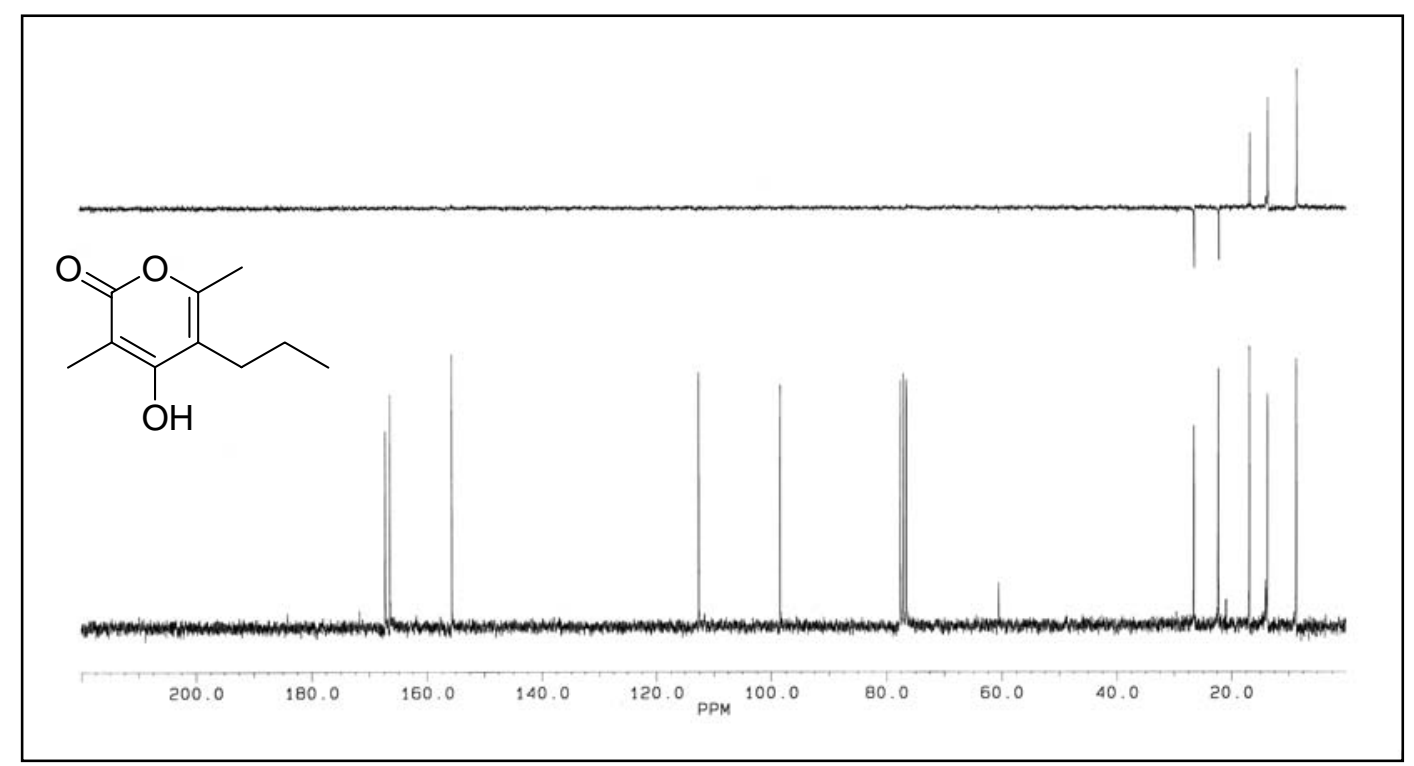

3,6-Dimethyl-4-hydroxy-5-propylpyran-2-on (63) 


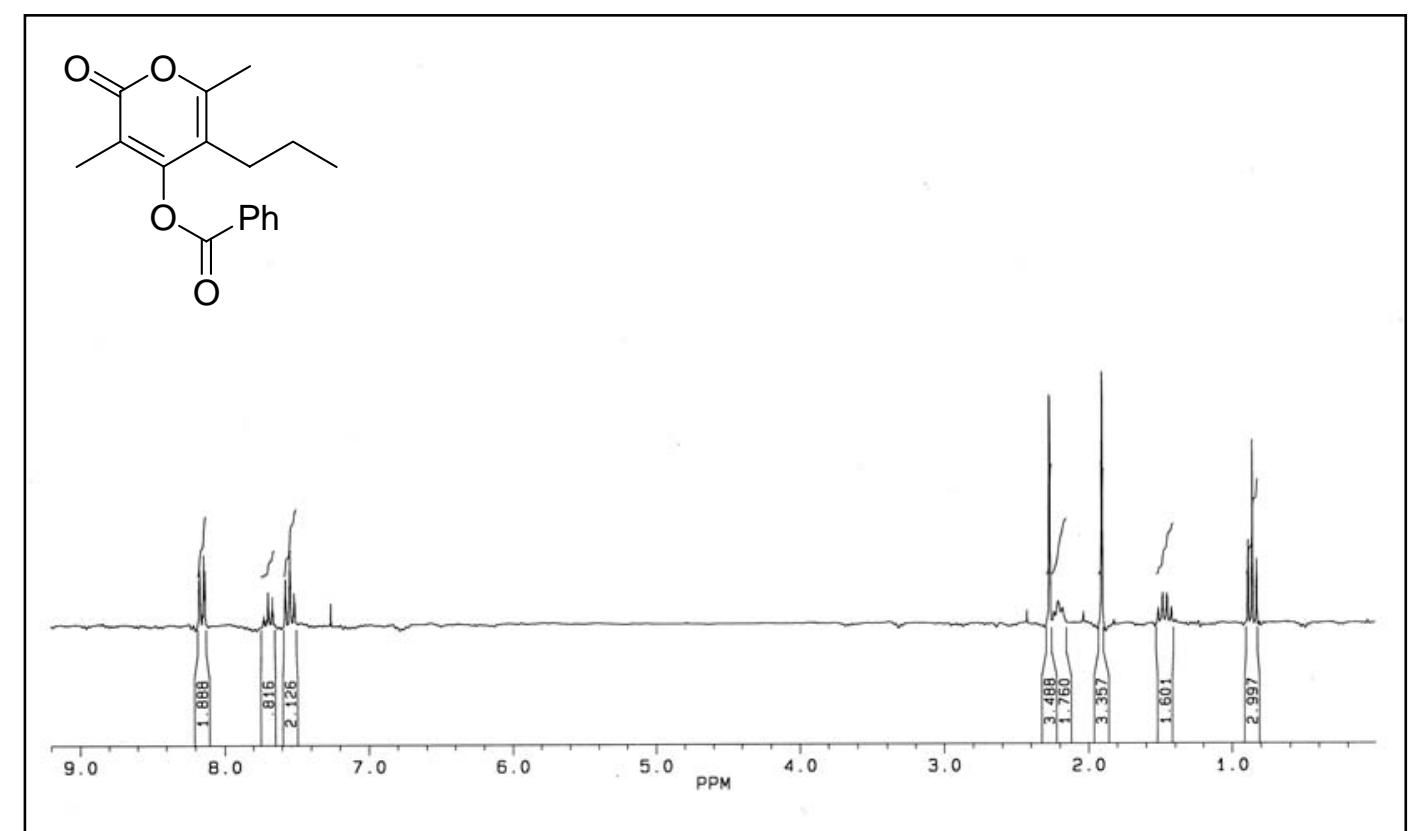

Benzoesäure-3,6-dimethyl-2-oxo-5-propyl-2H-pyran-4-ylester (89)

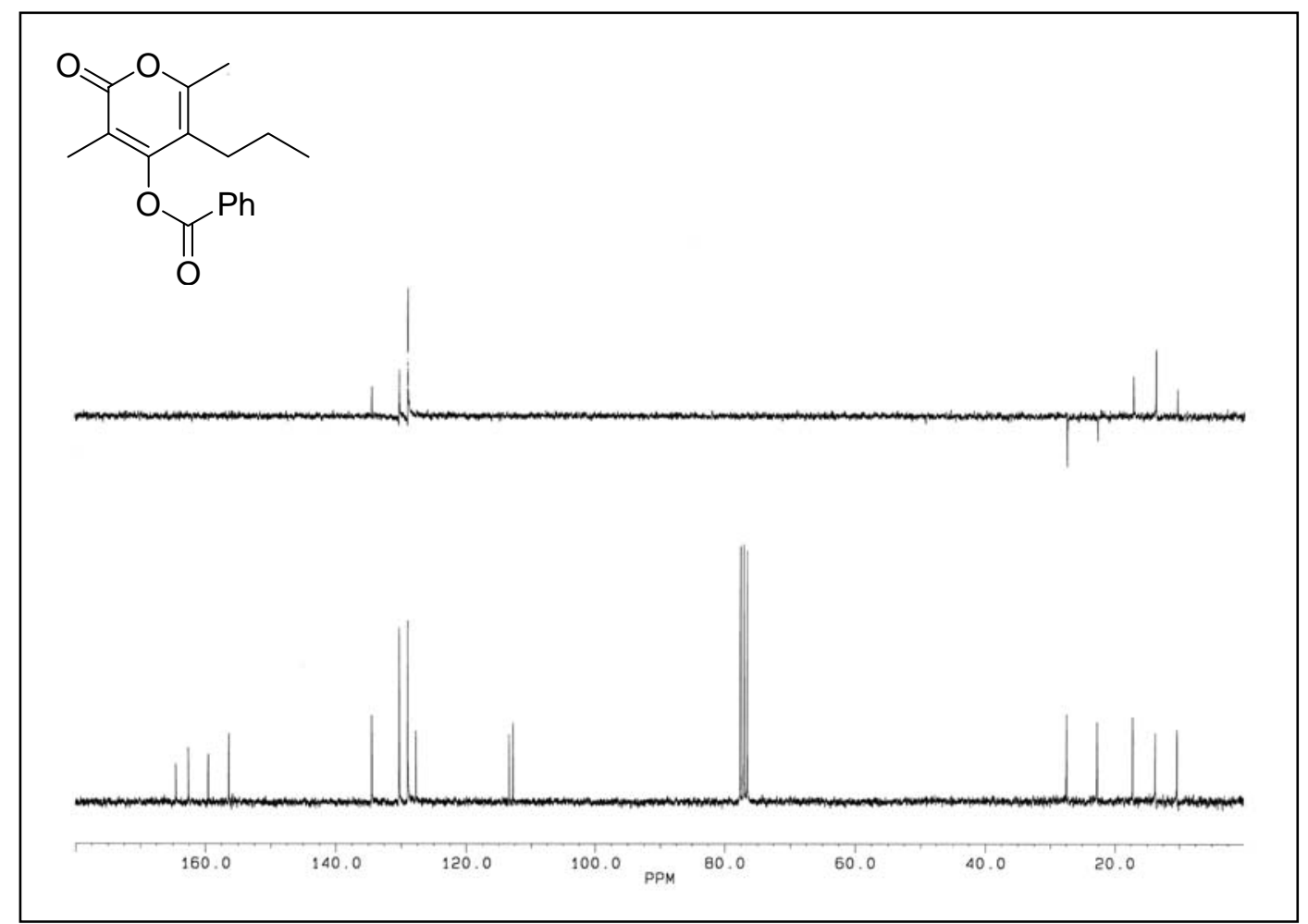

Benzoesäure-3,6-dimethyl-2-oxo-5-propyl-2H-pyran-4-ylester (89) 


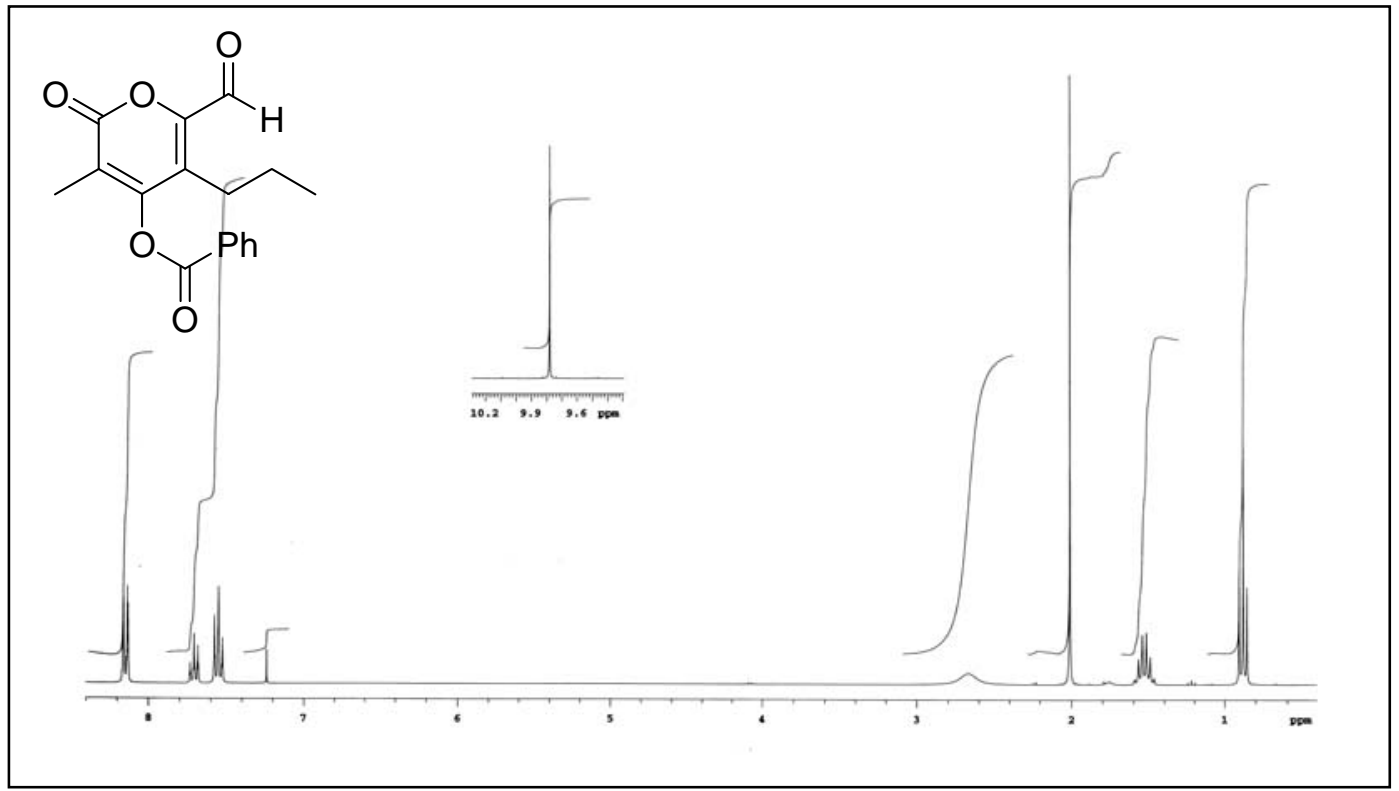

Benzoesäure-6-formyl-3-methyl-2-oxo-5-propyl-2H-pyran-4-ylester (94)

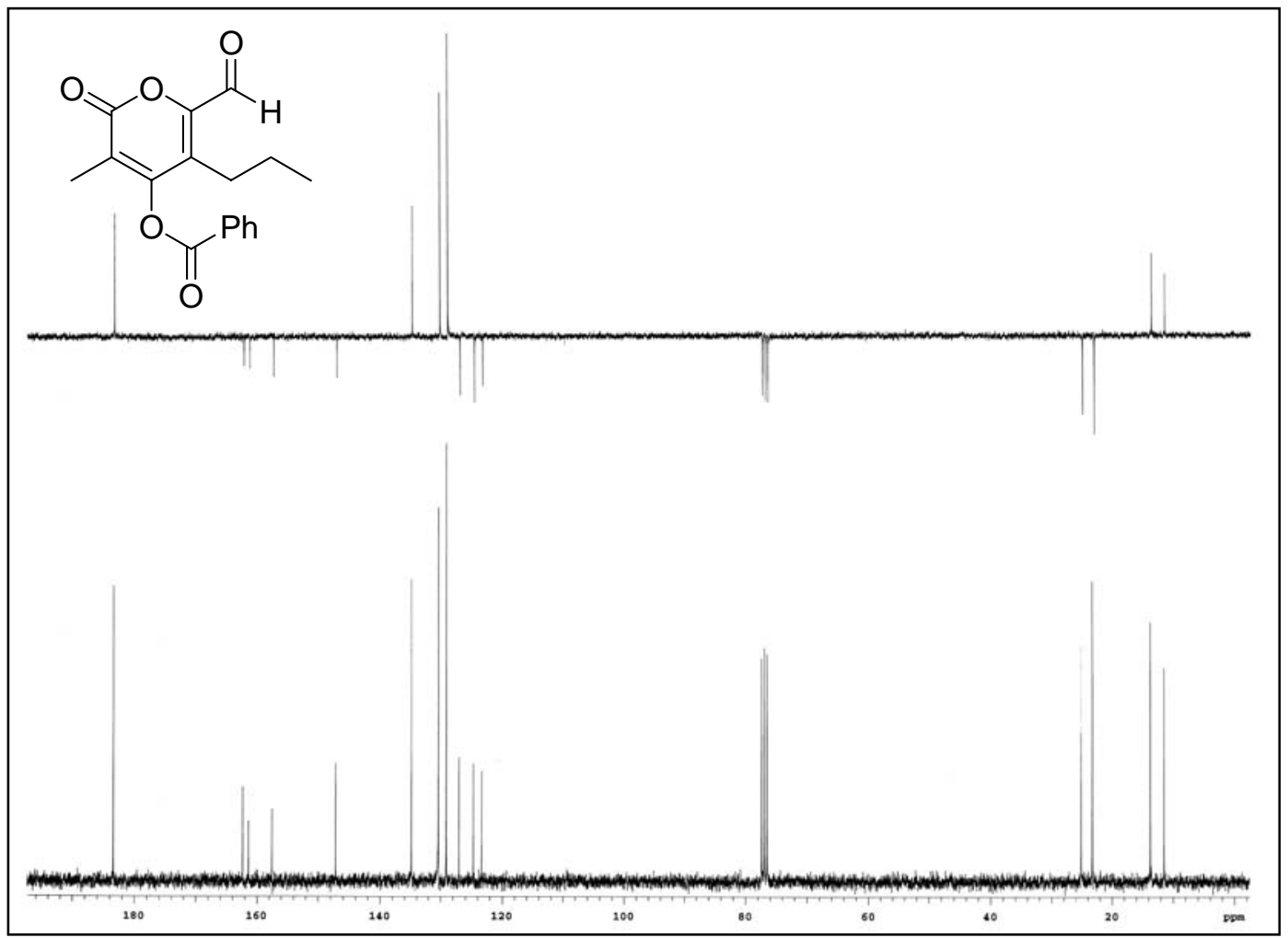

Benzoesäure-6-formyl-3-methyl-2-oxo-5-propyl-2H-pyran-4-ylester (94) 


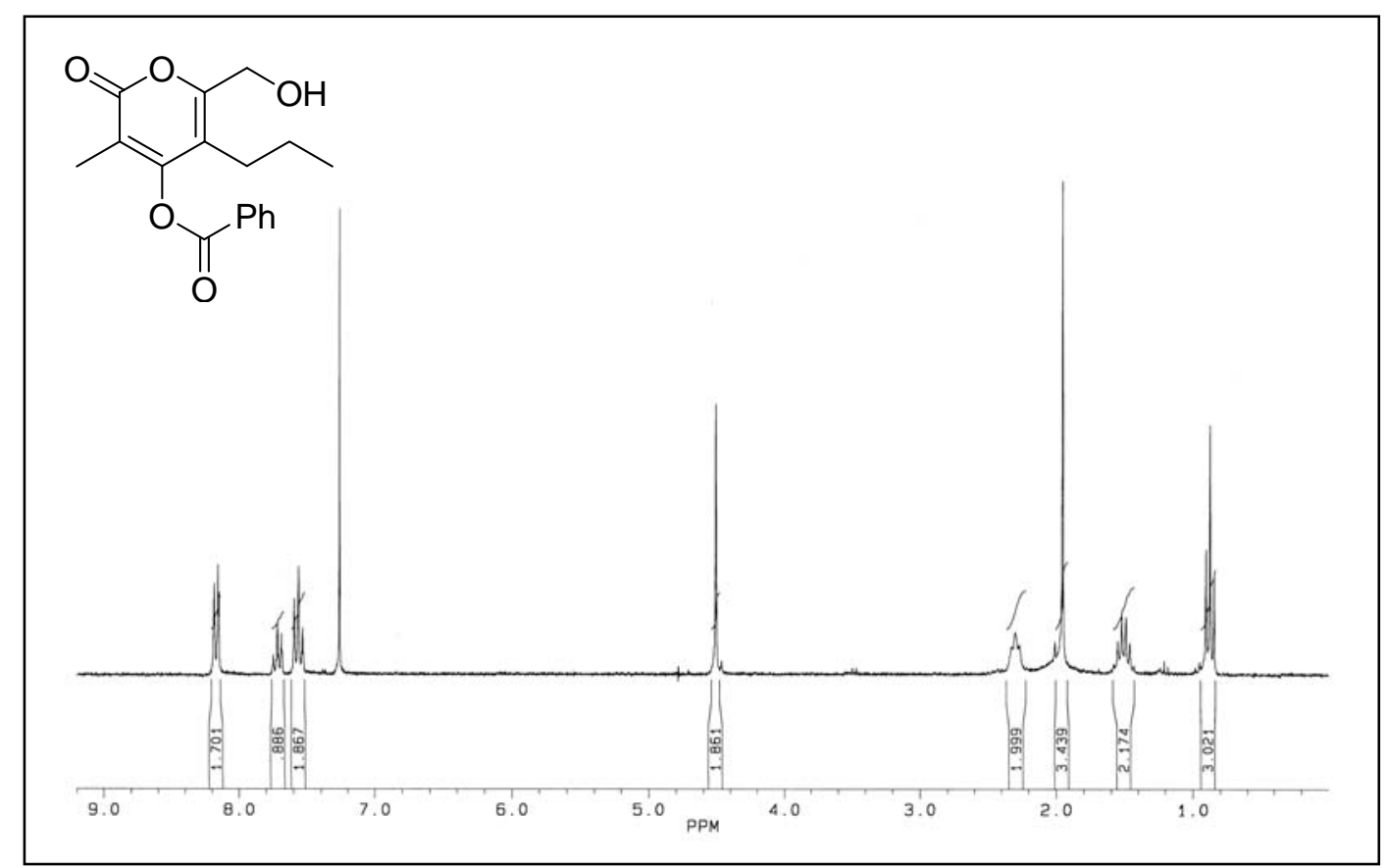

Benzoesäure-6-hydroxymethyl-3-methyl-2-oxo-5-propyl-2H-pyran-4-ylester (95)

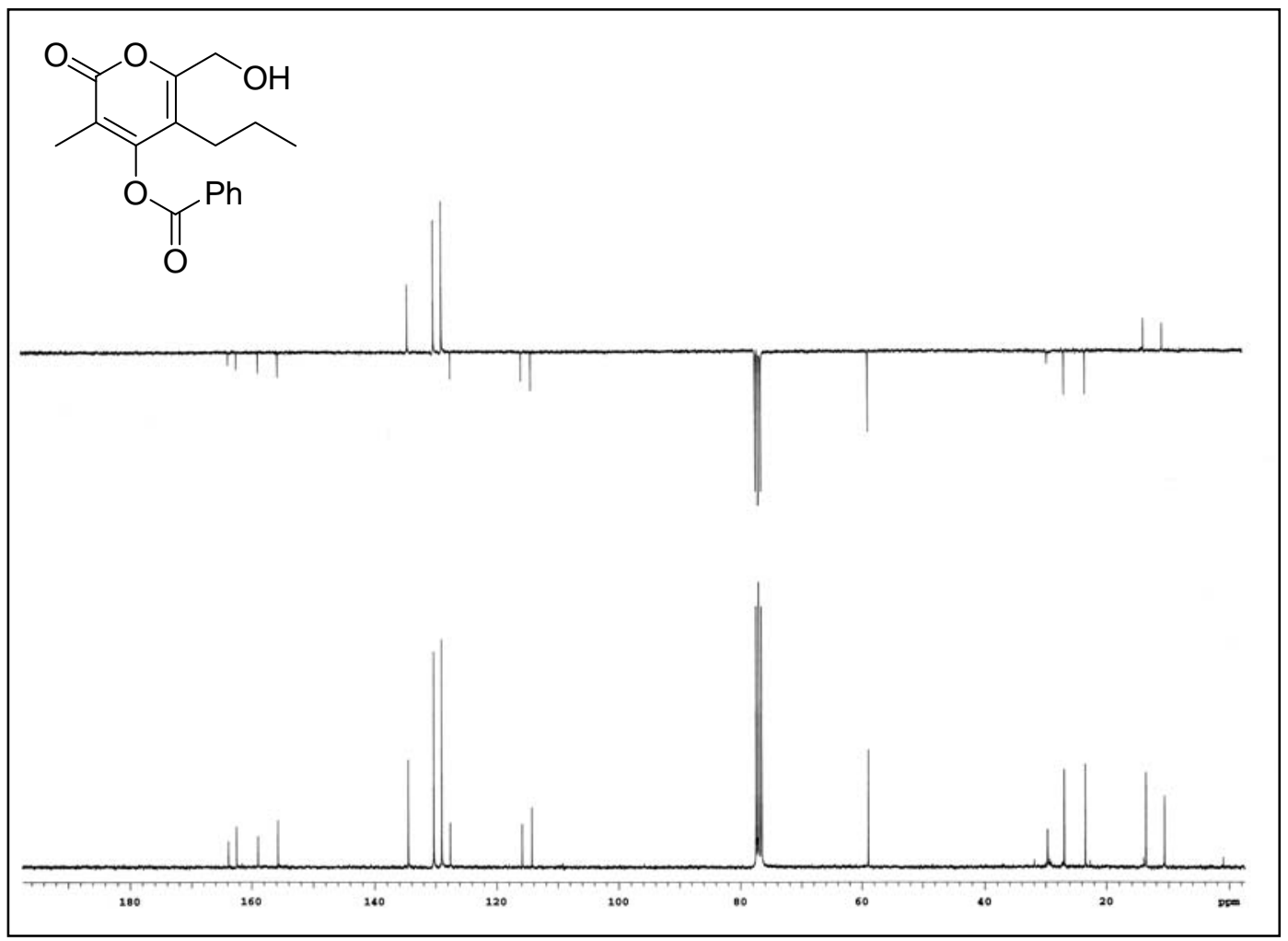

Benzoesäure-6-hydroxymethyl-3-methyl-2-oxo-5-propyl-2H-pyran-4-ylester (95) 


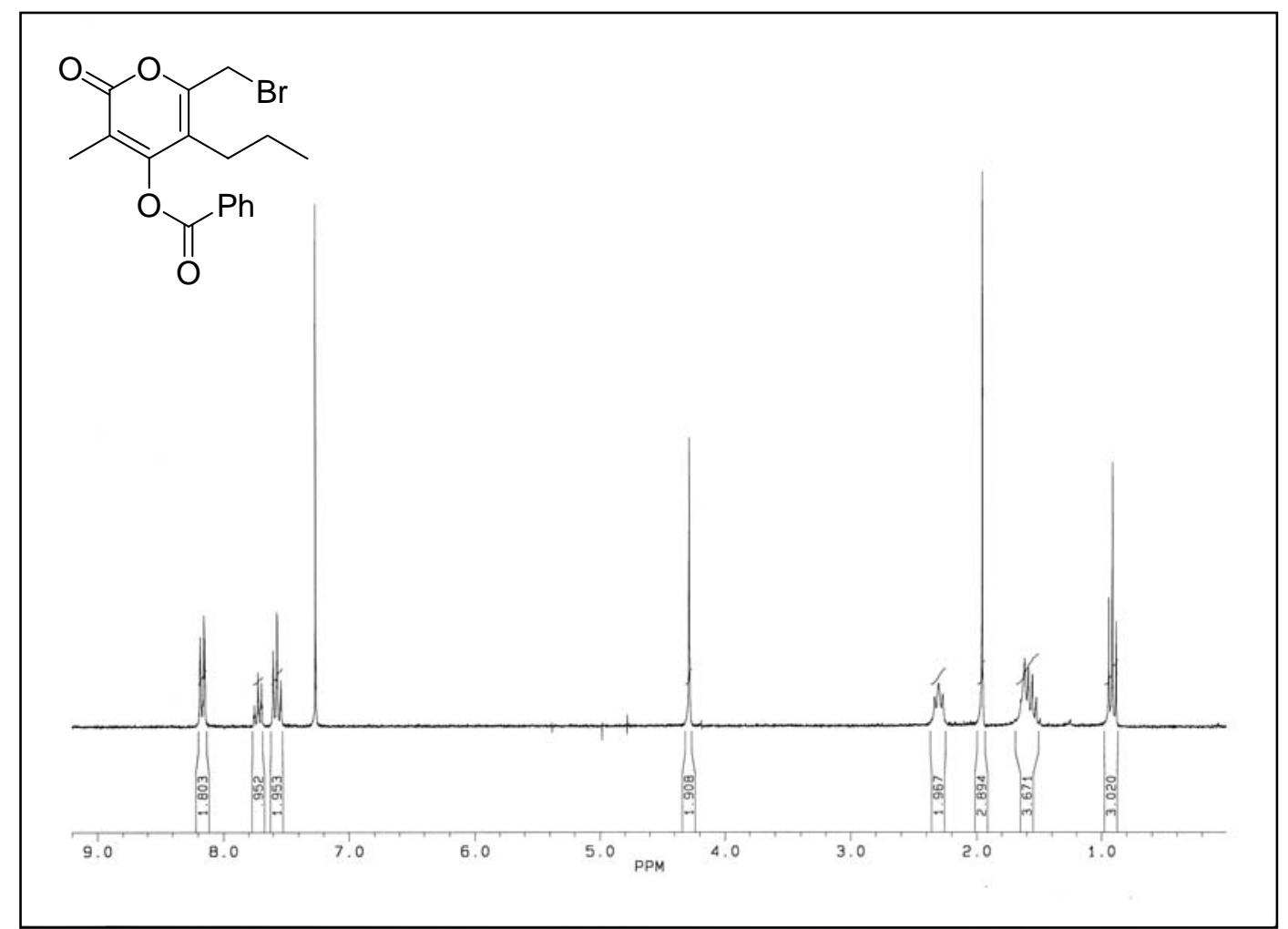

Benzoesäure-6-brommethyl-3-methyl-2-oxo-5-propyl-2H-pyran-4-ylester (96)

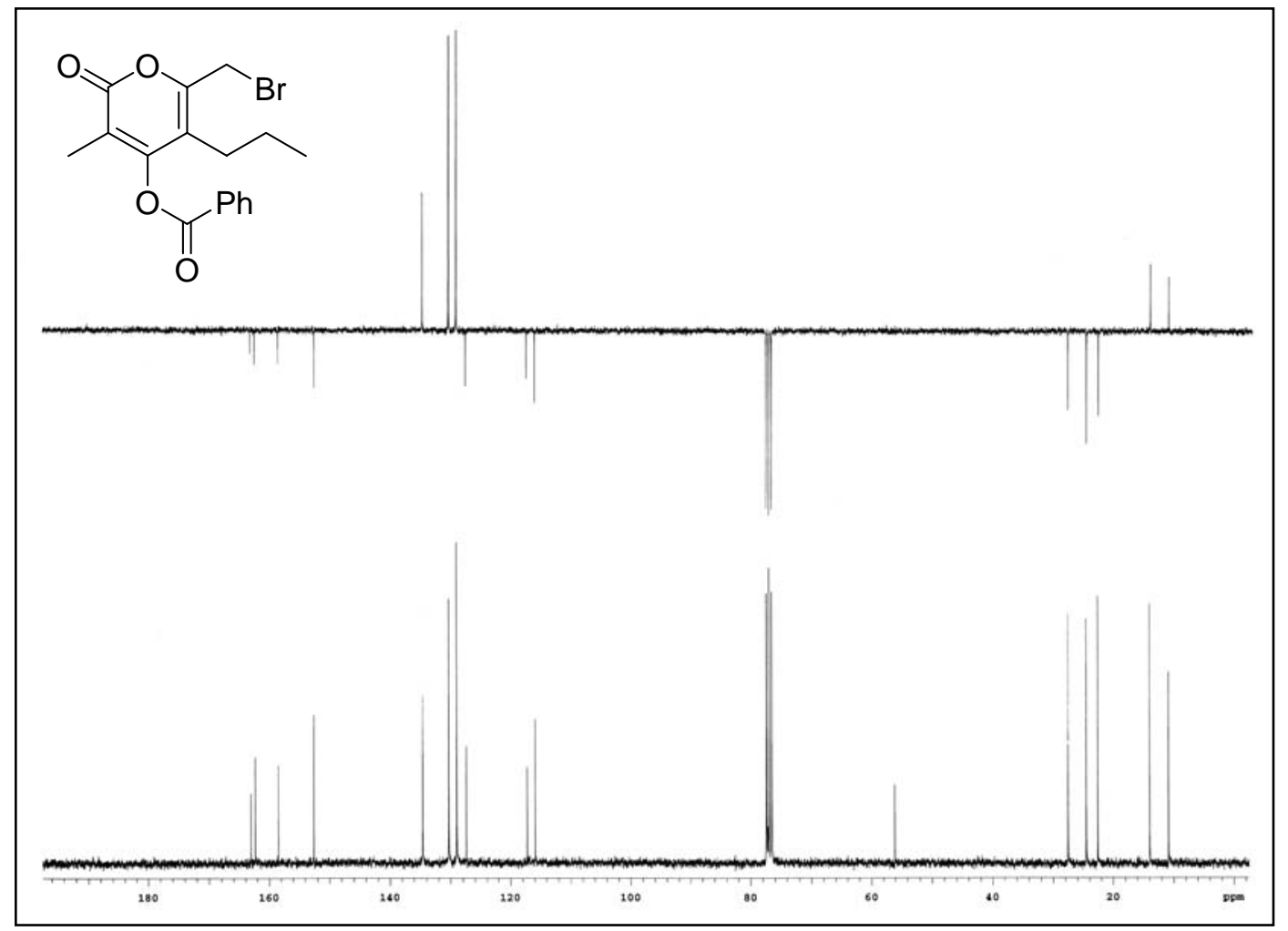

Benzoesäure-6-brommethyl-3-methyl-2-oxo-5-propyl-2H-pyran-4-ylester (96) 


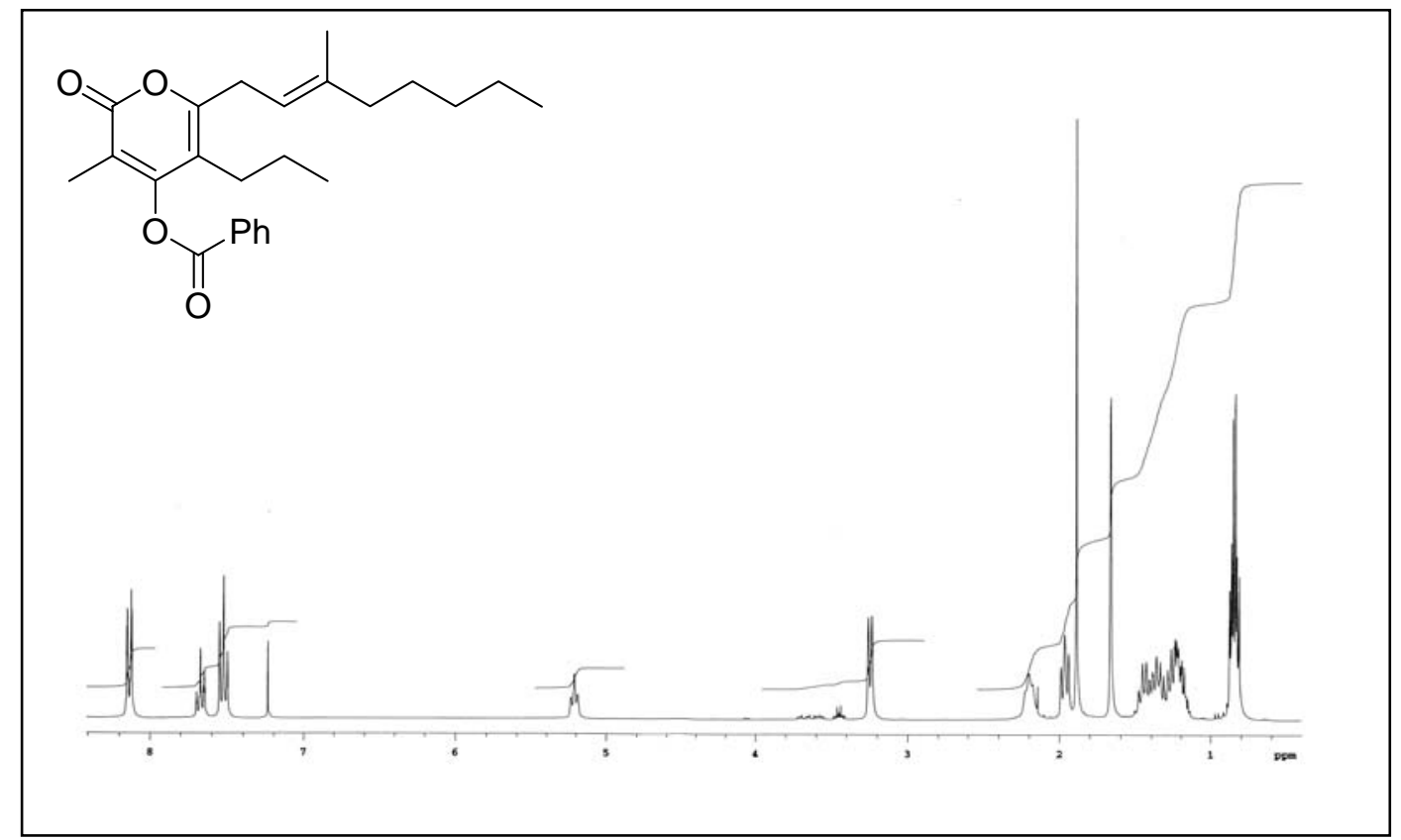

Benzoesäure-6-[(E)-3'-methylocta-2'-enyl]-3-methyl-2-oxo-5-propyl-2H-pyran-4ylester (125)

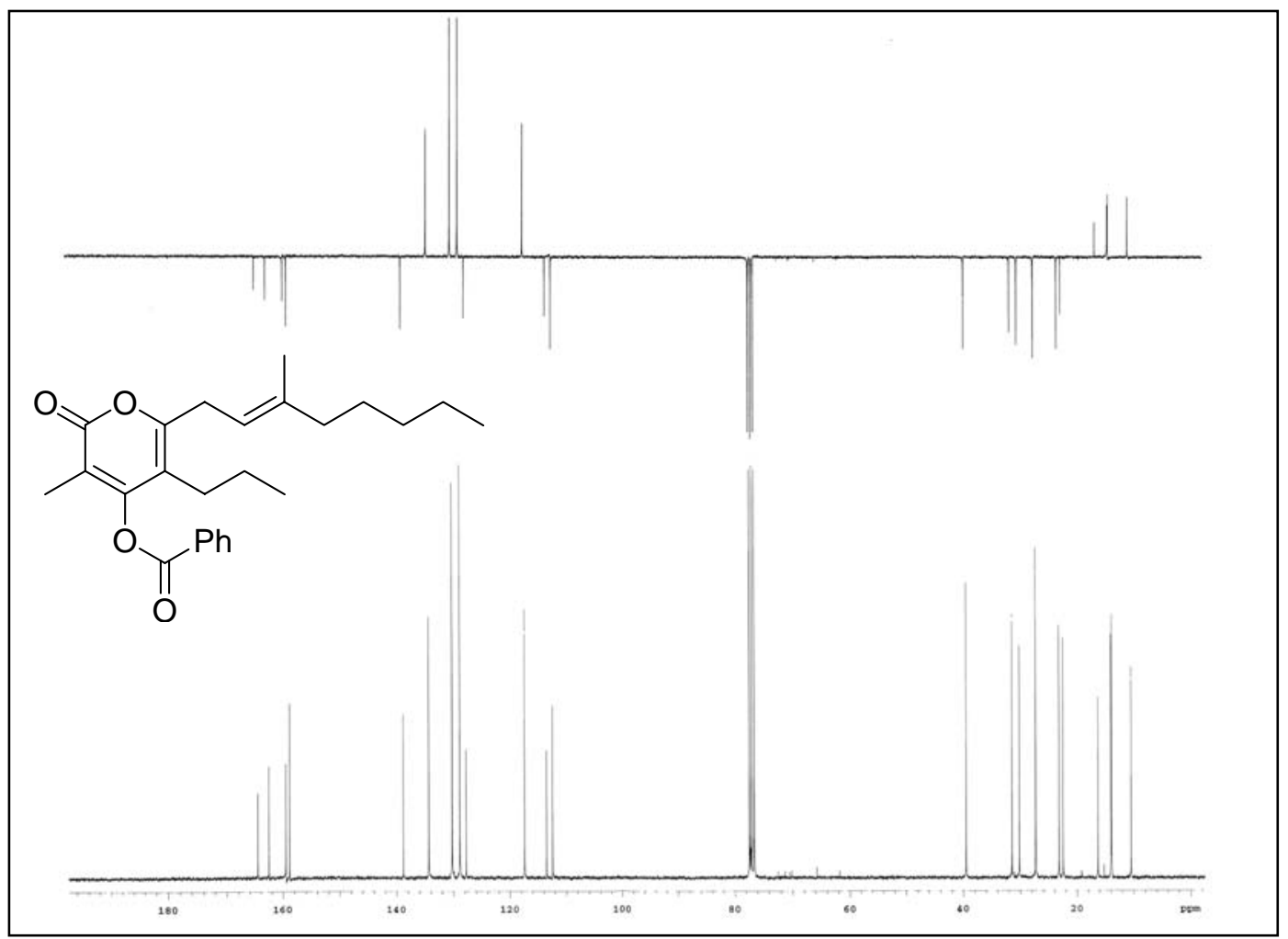

Benzoesäure-6-[(E)-3'-methylocta-2'-enyl]-3-methyl-2-oxo-5-propyl-2H-pyran-4ylester (125) 


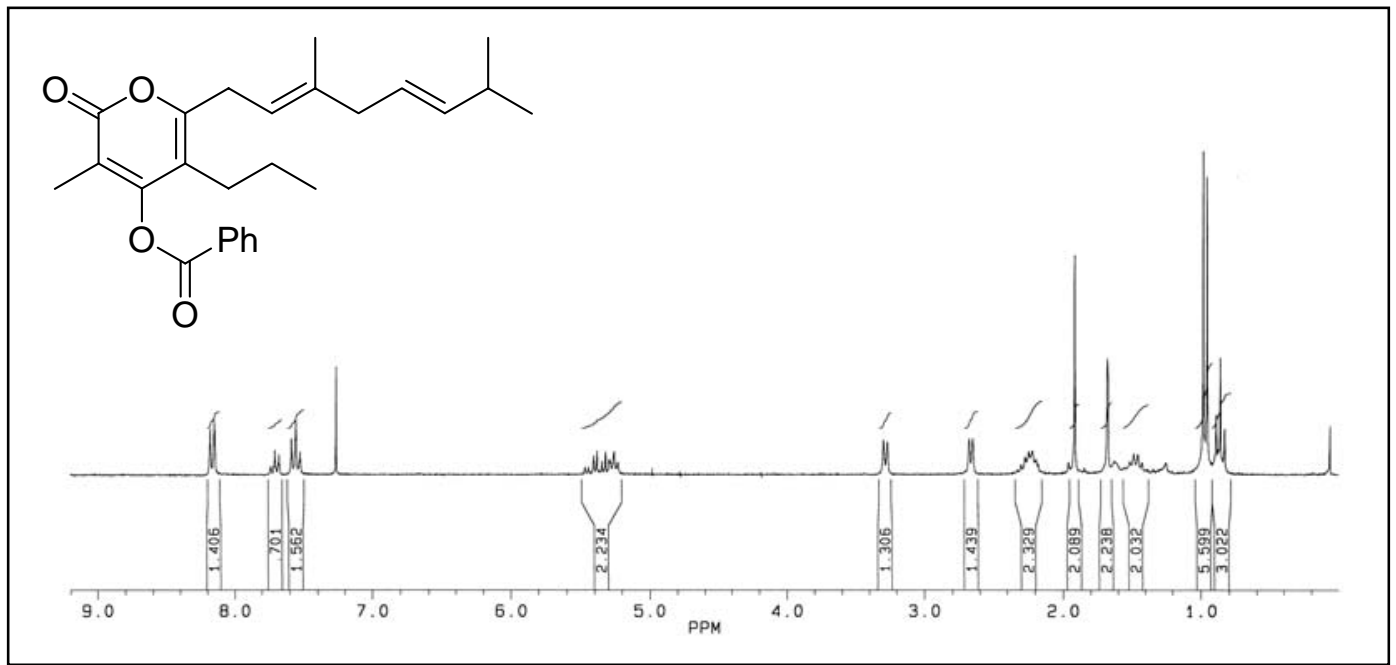

Benzoesäure-6-[(E,E)-3',7'-dimethylocta-2',5'-dienyl]-3-methyl-2-oxo-5-propyl2H-pyran-4-ylester (125A)

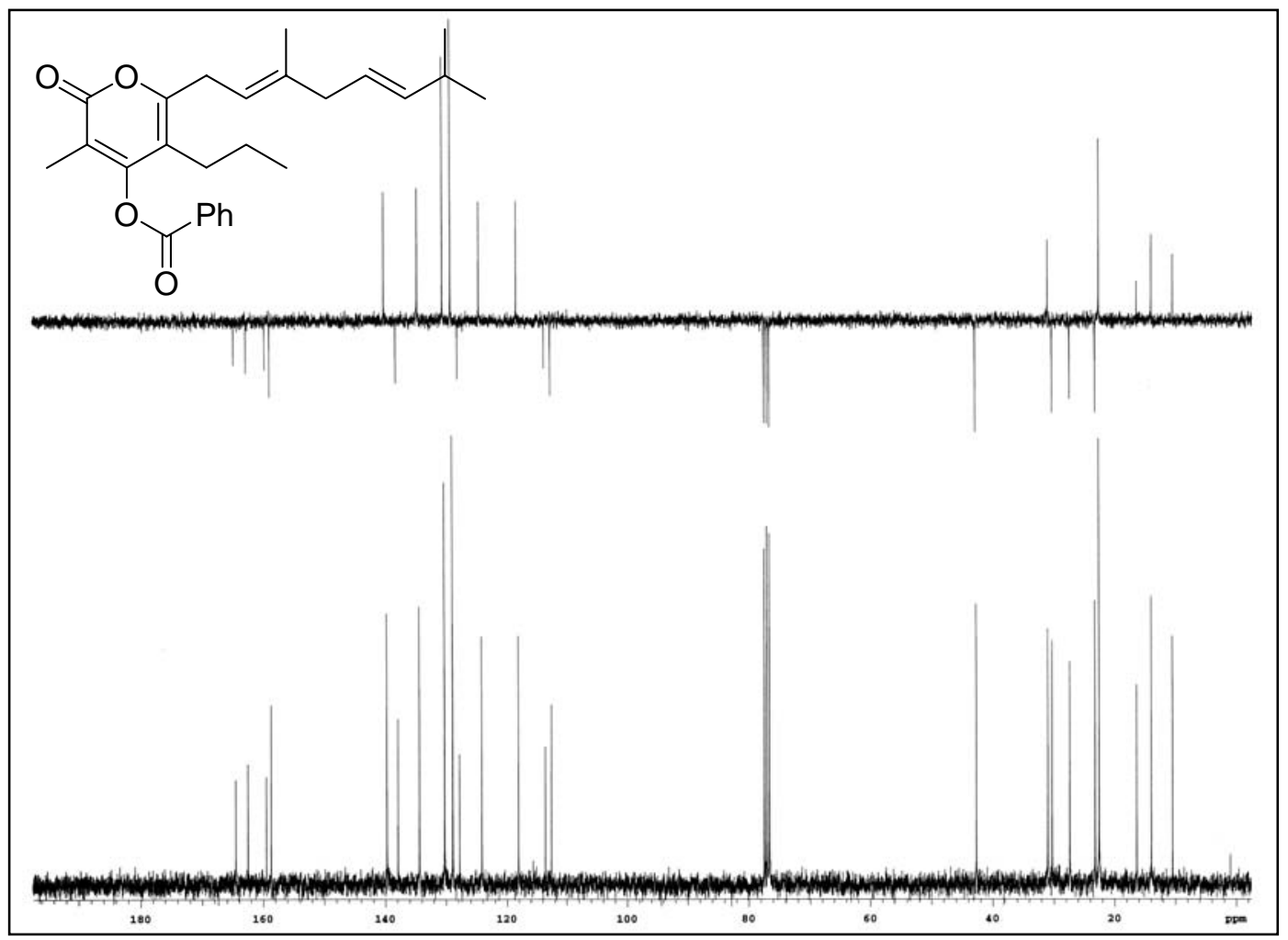

Benzoesäure-6-[(E,E)-3',7'-dimethylocta-2',5'-dienyl]-3-methyl-2-oxo-5-propyl2H-pyran-4-ylester (125A) 


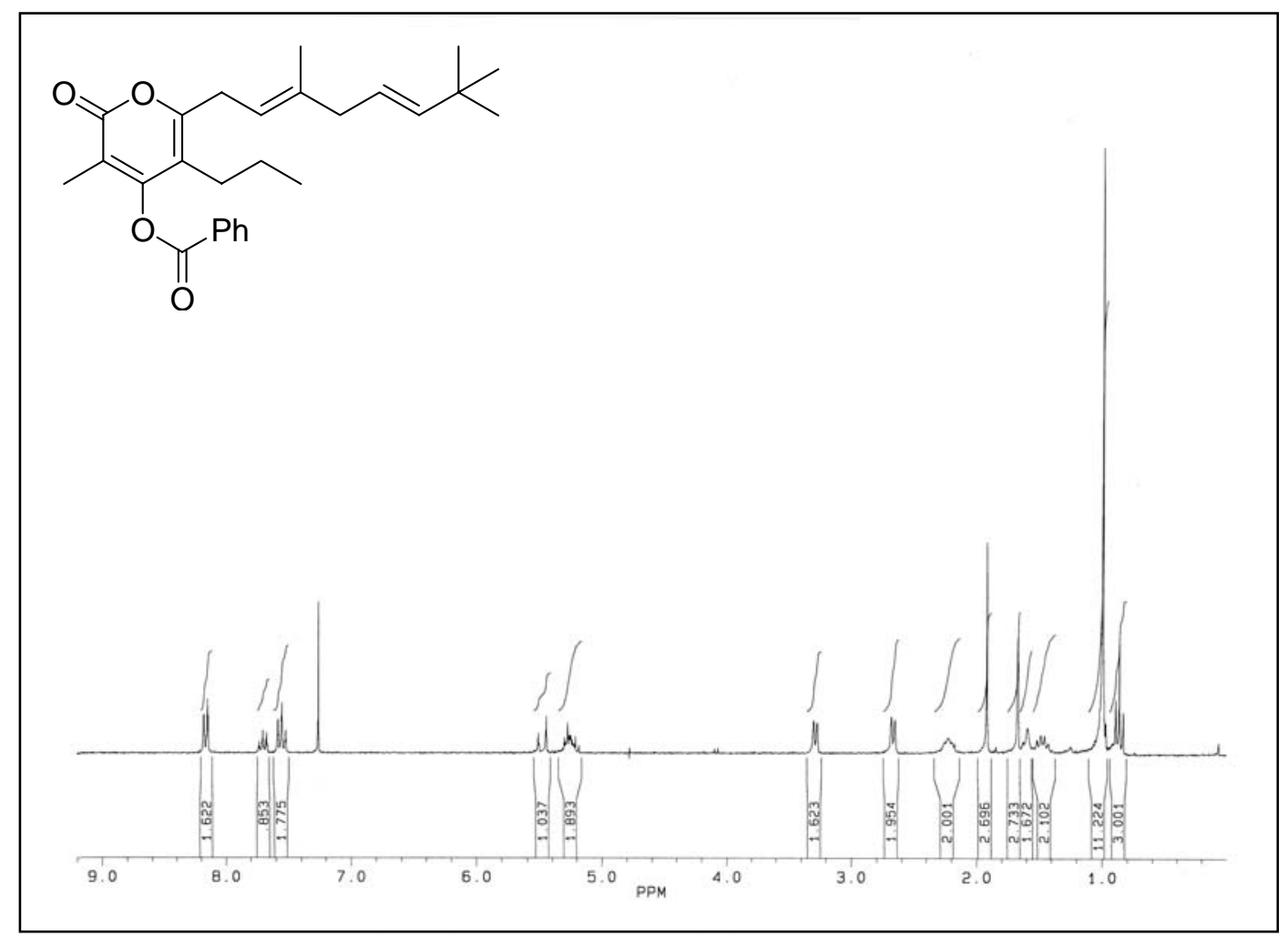

Benzoesäure-6-[(E,E)-3',7',7'-trimethylocta-2',5'-dienyl]-3-methyl-2-oxo-5-propyl2H-pyran-4-ylester (125R)

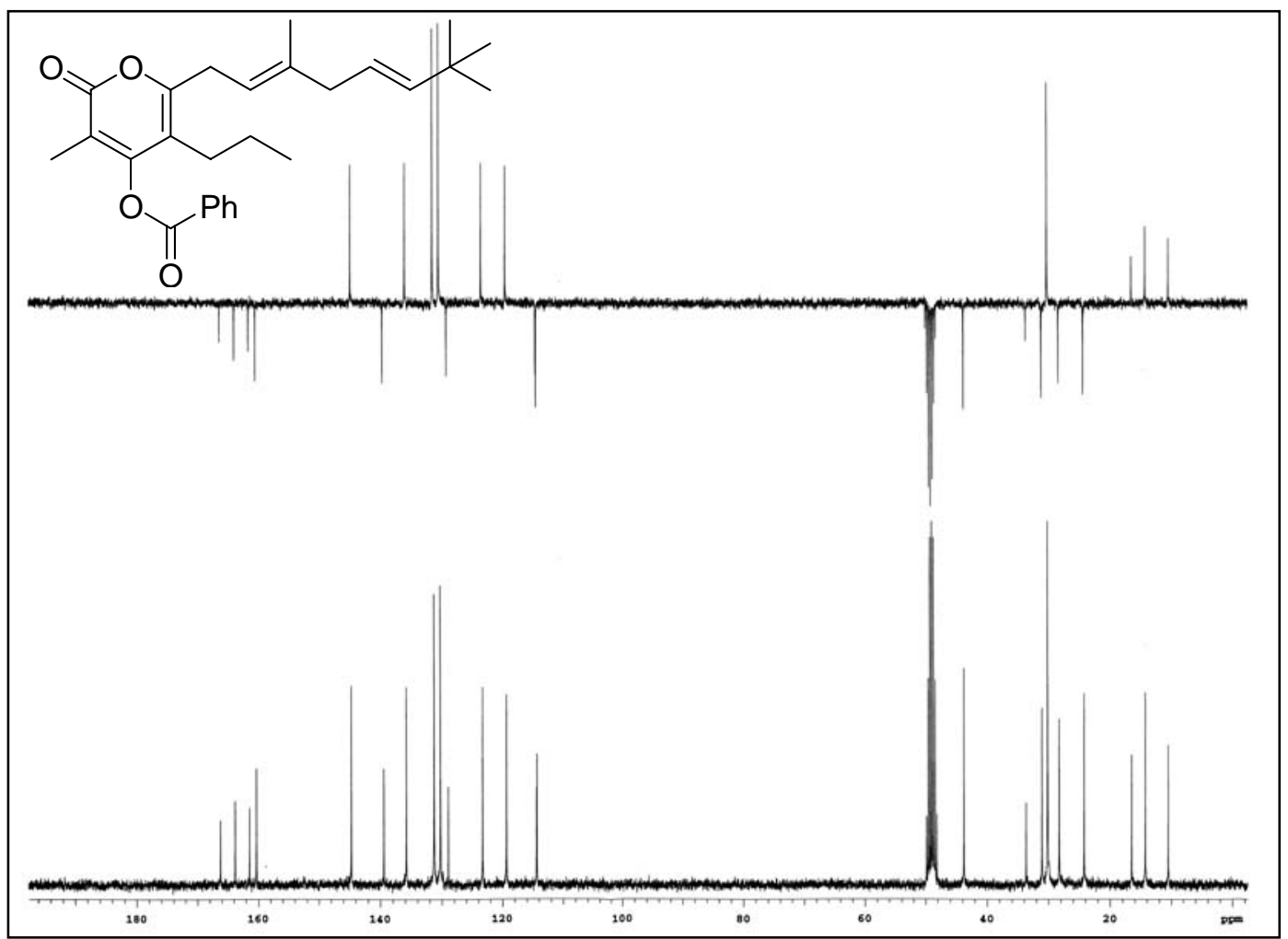

Benzoesäure-6-[(E,E)-3',7',7'-trimethylocta-2',5'-dienyl]-3-methyl-2-oxo-5-propyl2H-pyran-4-ylester (125R) 


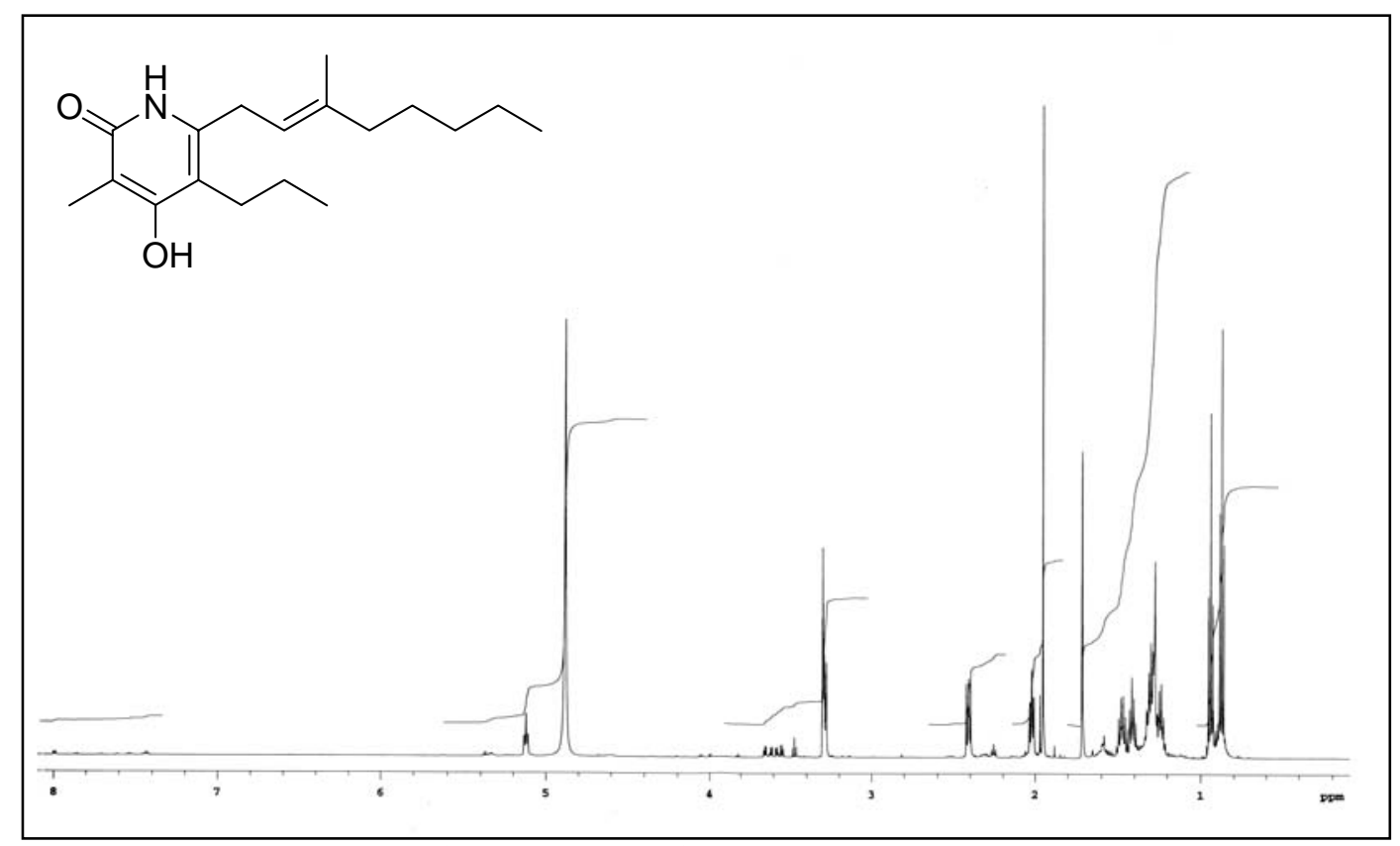

4-Hydroxy-3-methyl-6-(3'-methylocta-2'-enyl)-5-propyl-1H-pyridin-2-on (23)

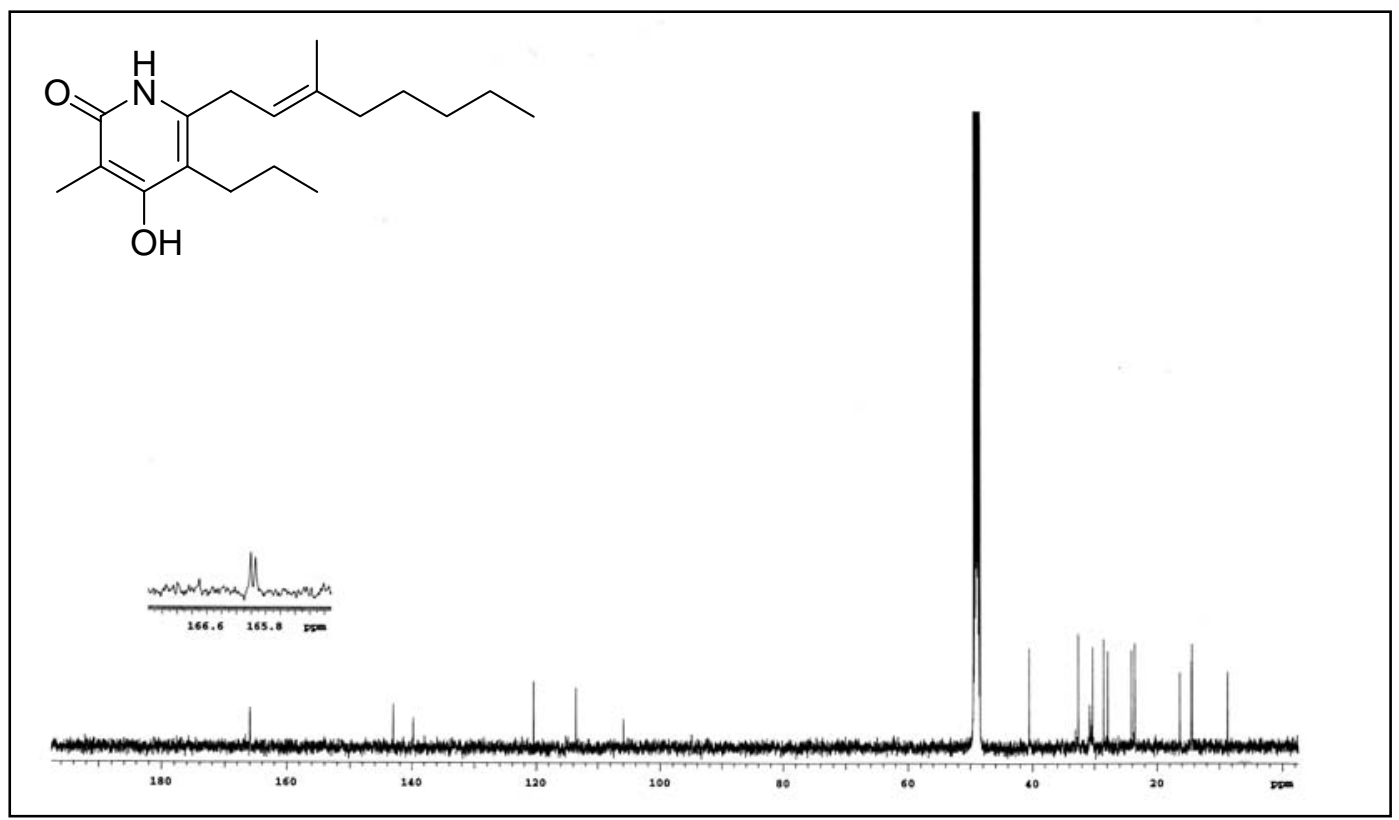

4-Hydroxy-3-methyl-6-(3'-methylocta-2'-enyl)-5-propyl-1H-pyridin-2-on (23) 


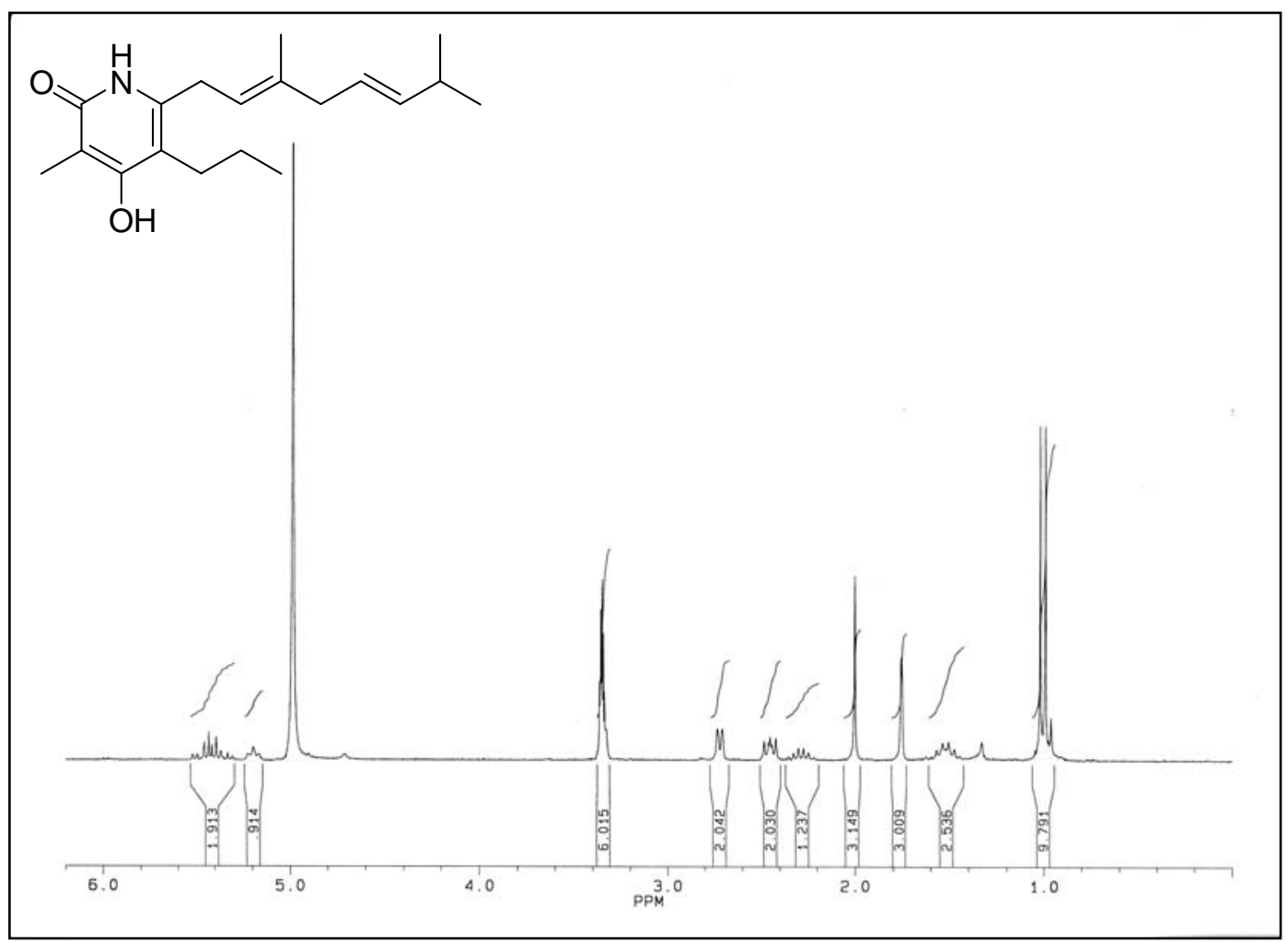

6-(3',7'-Dimethylocta-2',5'-dienyl)-4-hydroxy-3-methyl-5-propyl-1H-pyridin-2-on (23A)

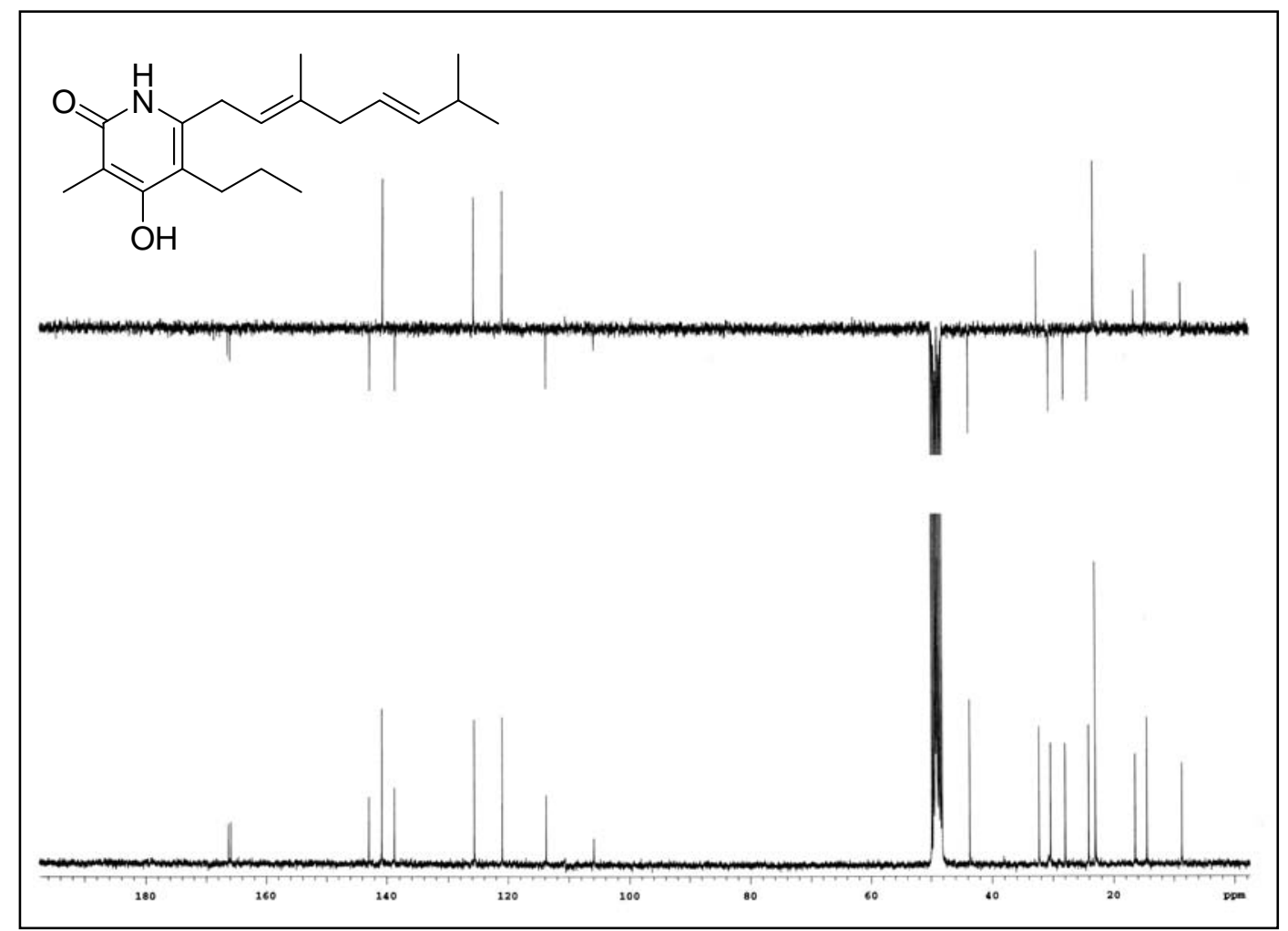

6-(3',7'-Dimethylocta-2',5'-dienyl)-4-hydroxy-3-methyl-5-propyl-1H-pyridin-2-on (23A) 


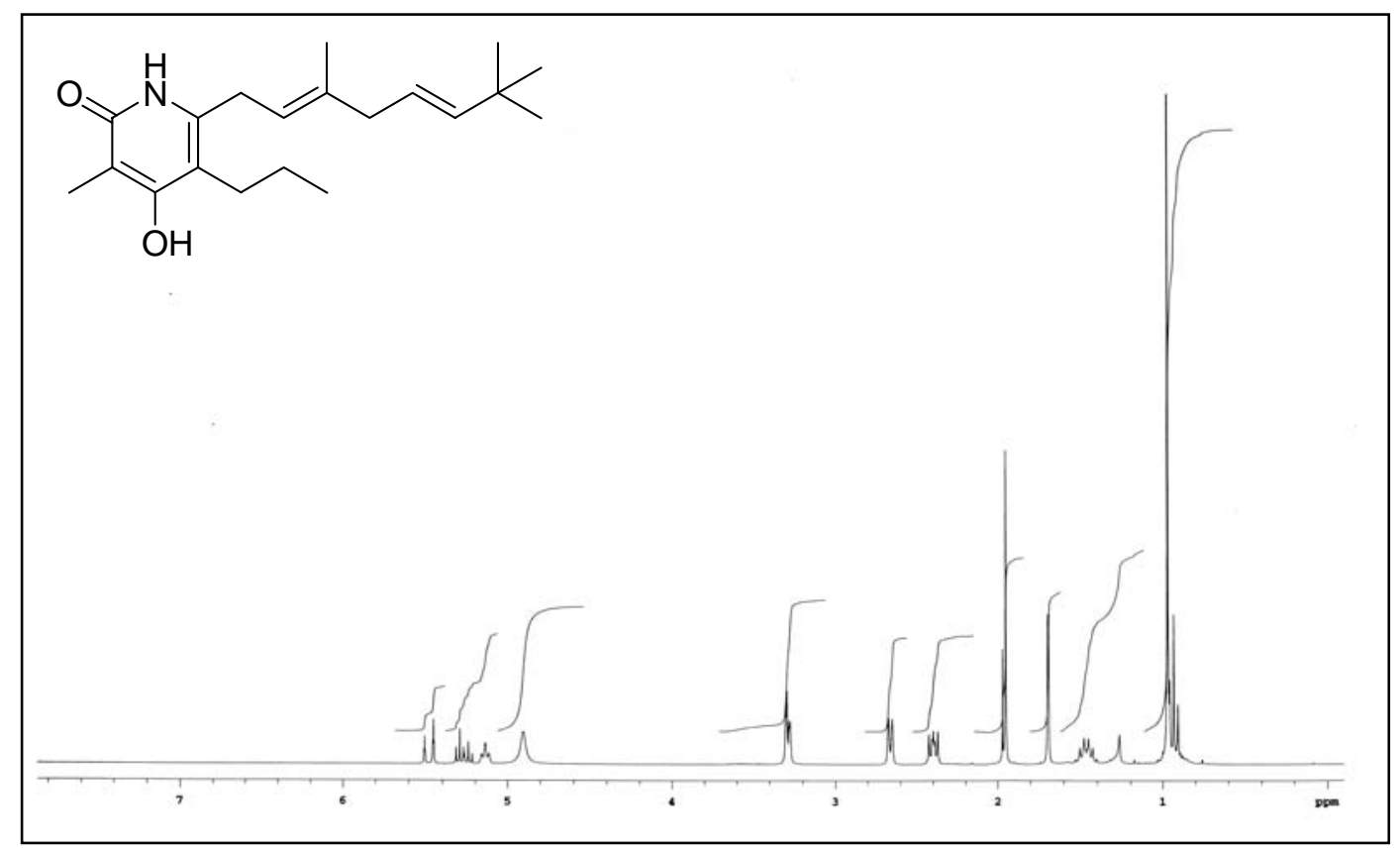

6-(3',7',7'-Trimethylocta-2',5'-dienyl)-4-hydroxy-3-methyl-5-propyl-1H-pyridin-2on $(23 R)$

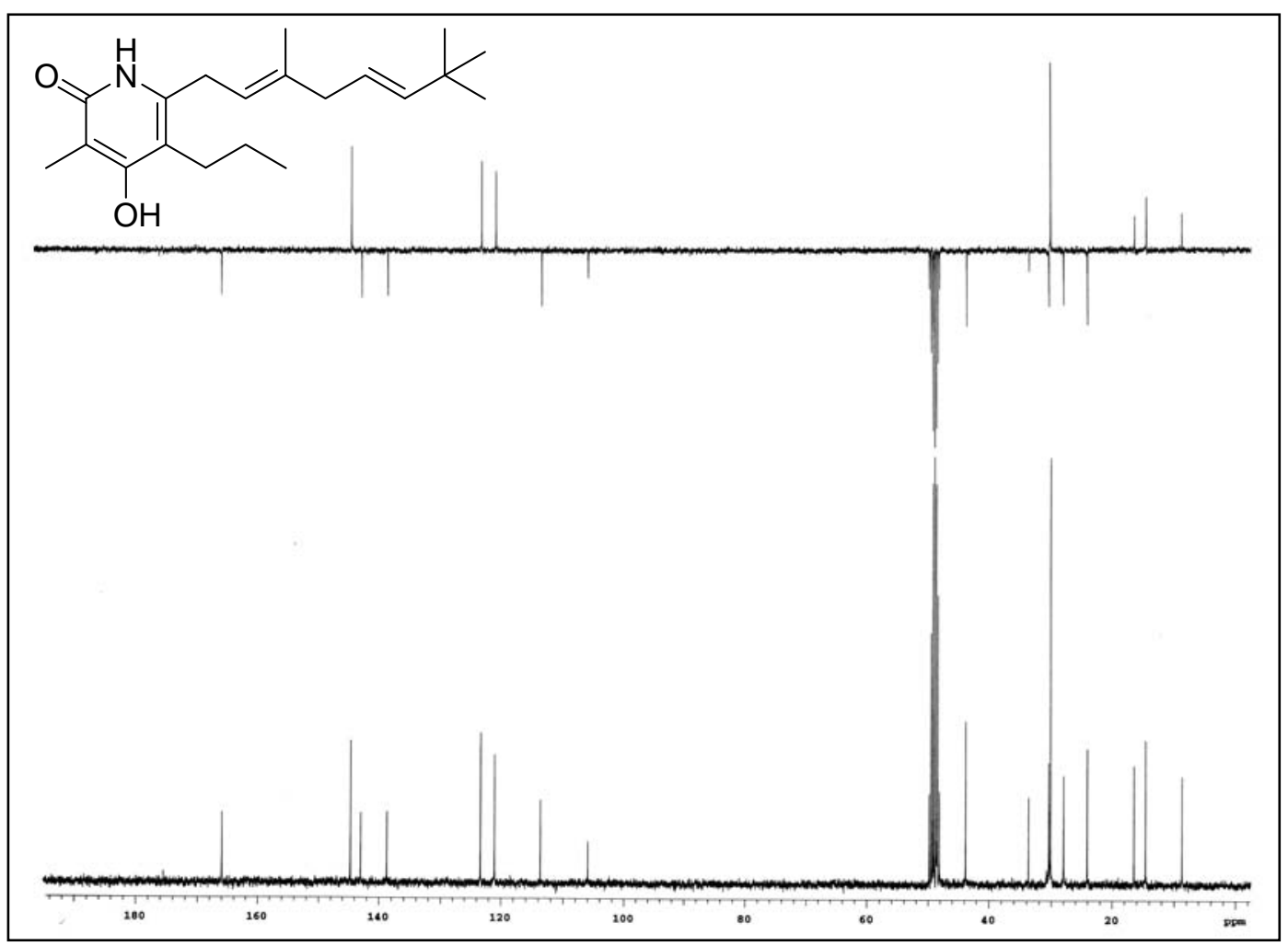

6-(3',7',7'-Trimethylocta-2',5'-dienyl)-4-hydroxy-3-methyl-5-propyl-1H-pyridin-2on $(23 R)$ 


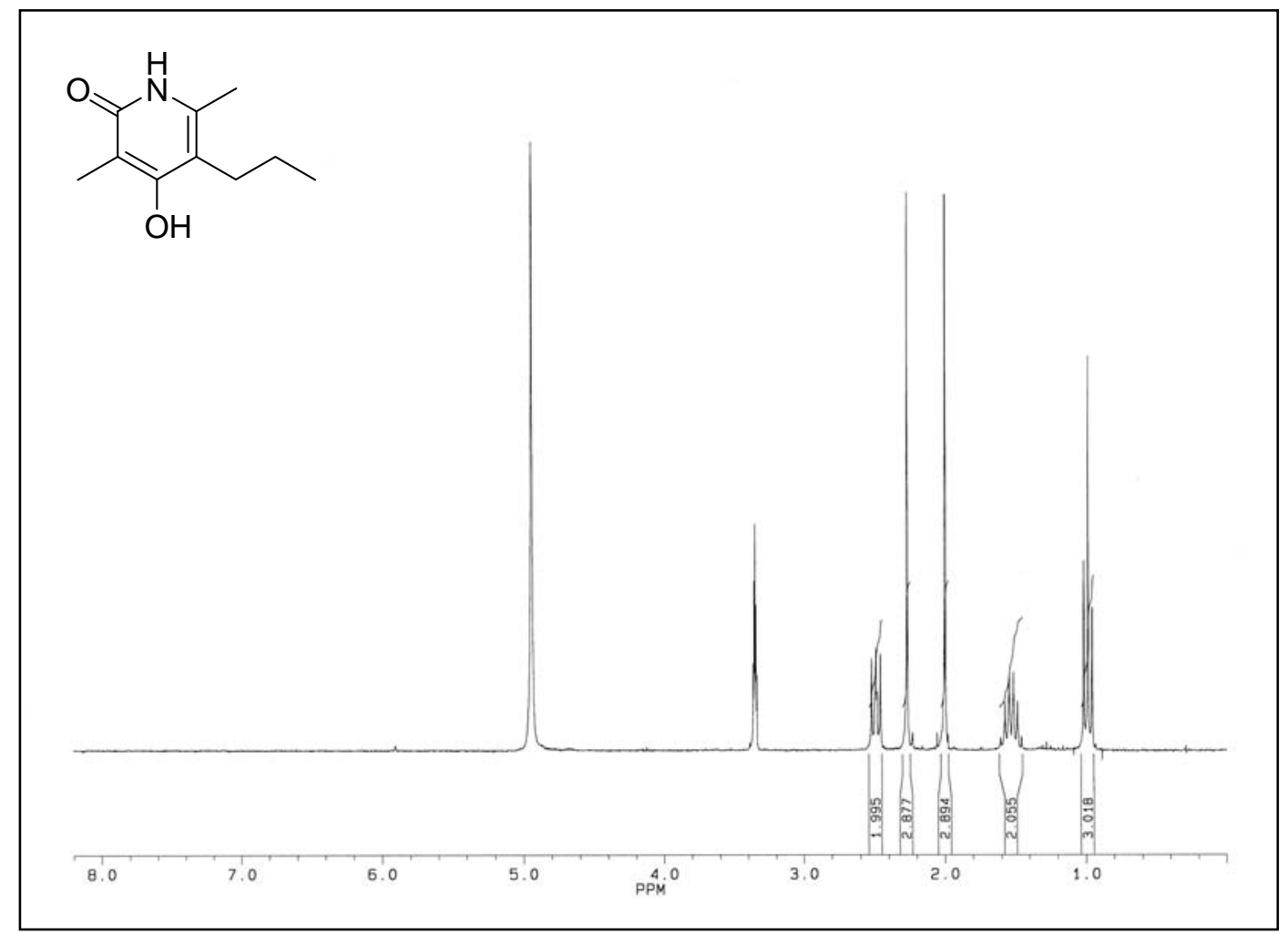

4-Hydroxy-3,6-dimethyl-5-propyl-1H-Pyridin-2-on (62)

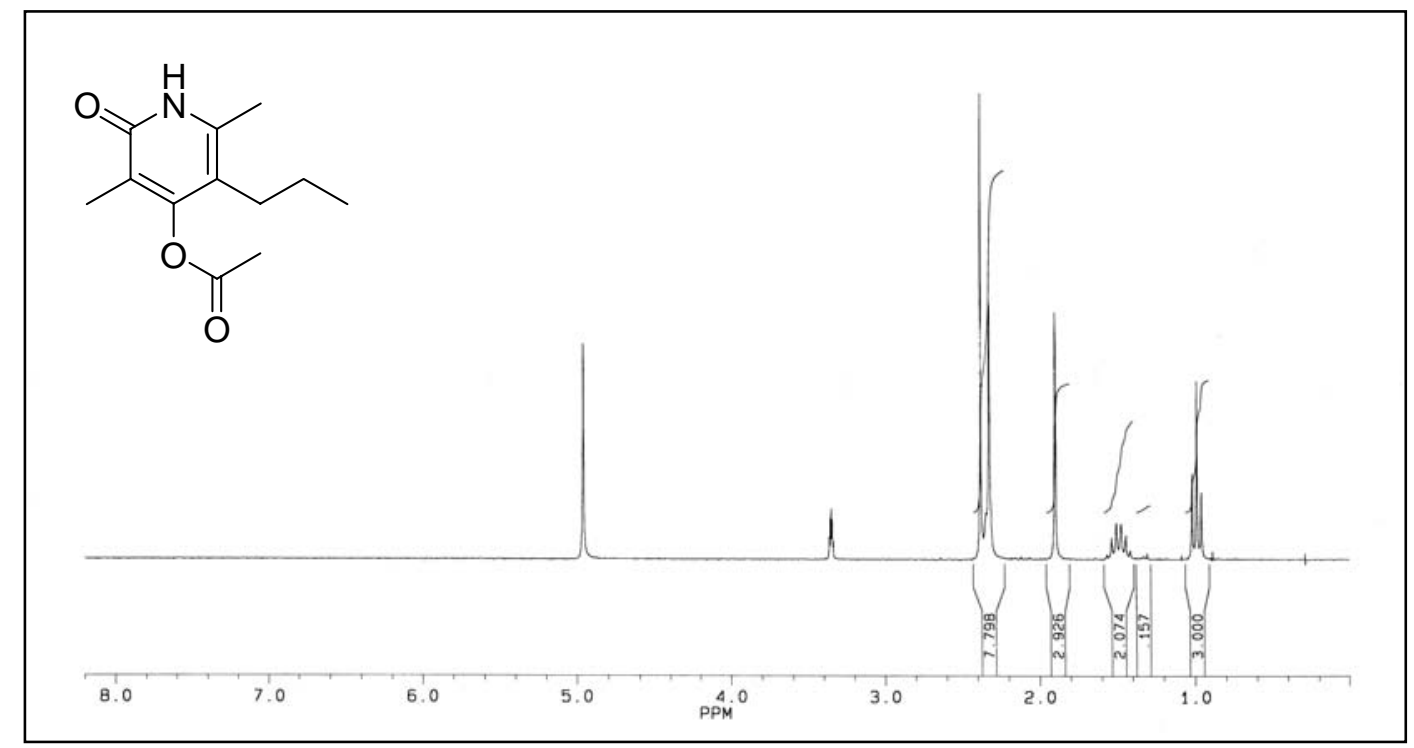

Essigsäure-3,6-dimethyl-2-oxo-5-propyl-1,2-dihydropyridin-4-ylester (156) 


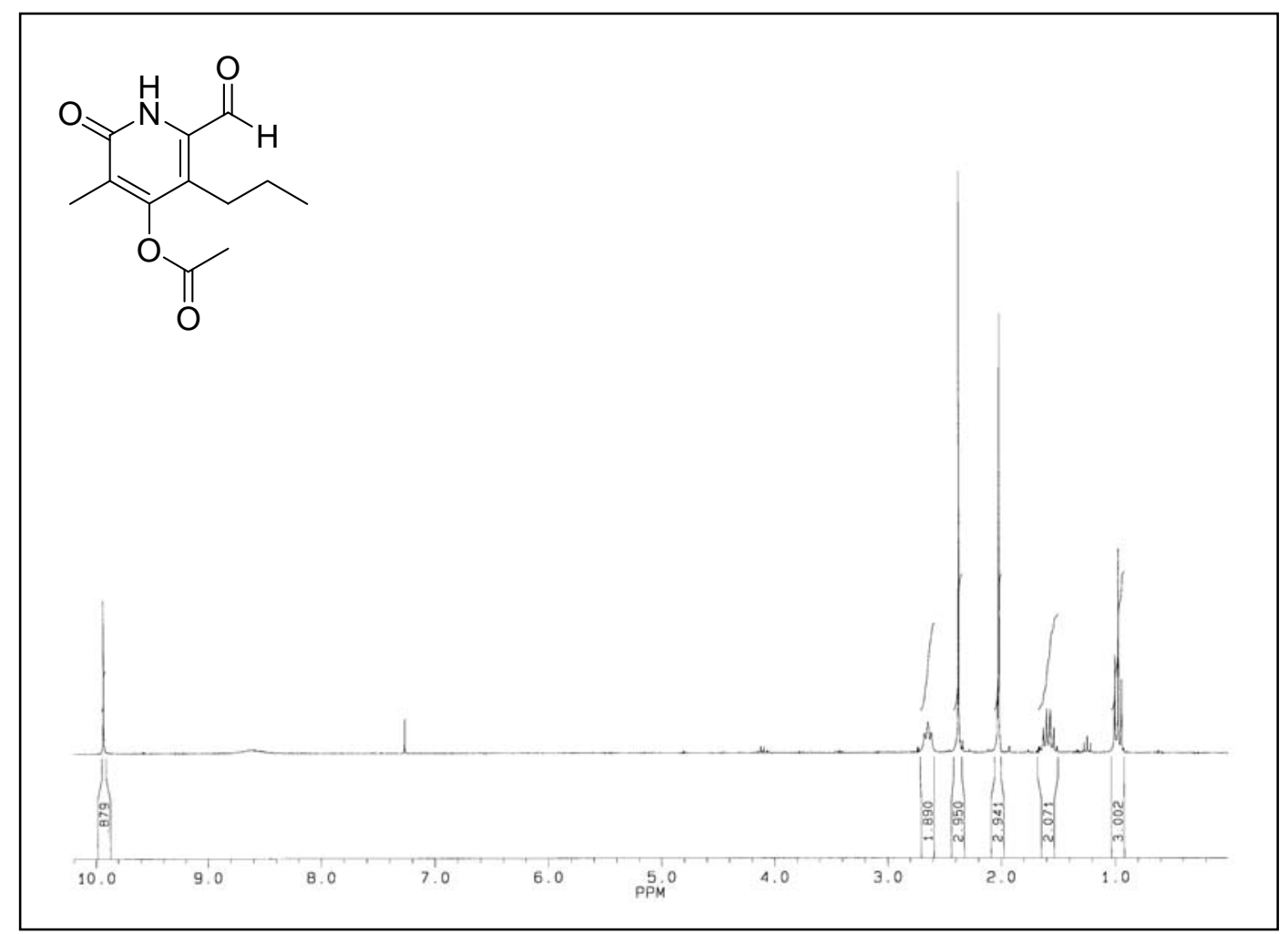

Essigsäure-6-formyl-3-methyl-2-oxo-5-propyl-1,2-dihydropyridin-4-ylester (157)

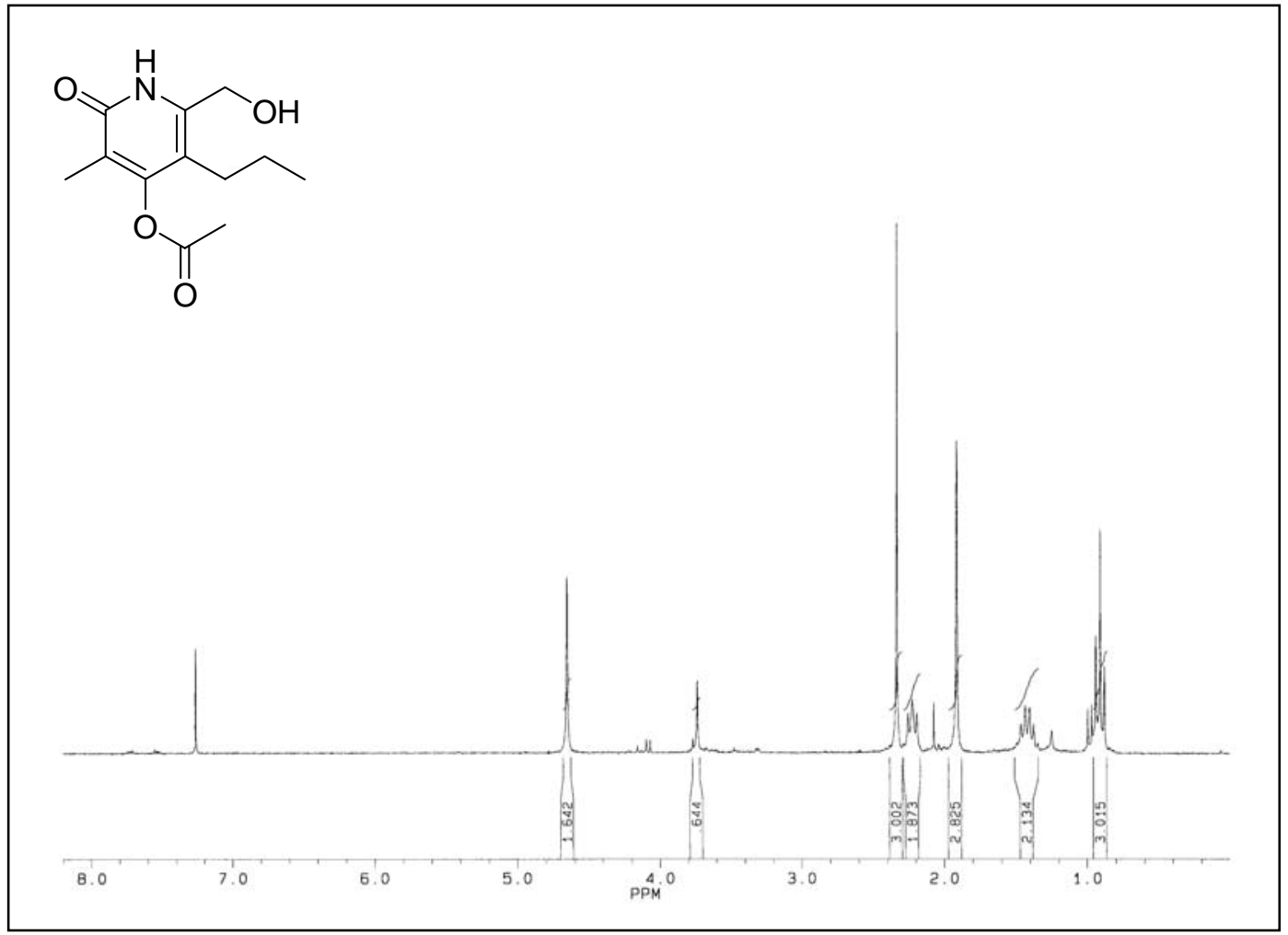

Essigsäure-6-hydroxymethyl-3-methyl-2-oxo-5-propyl-1,2-dihydropyridin-4ylester (158) 


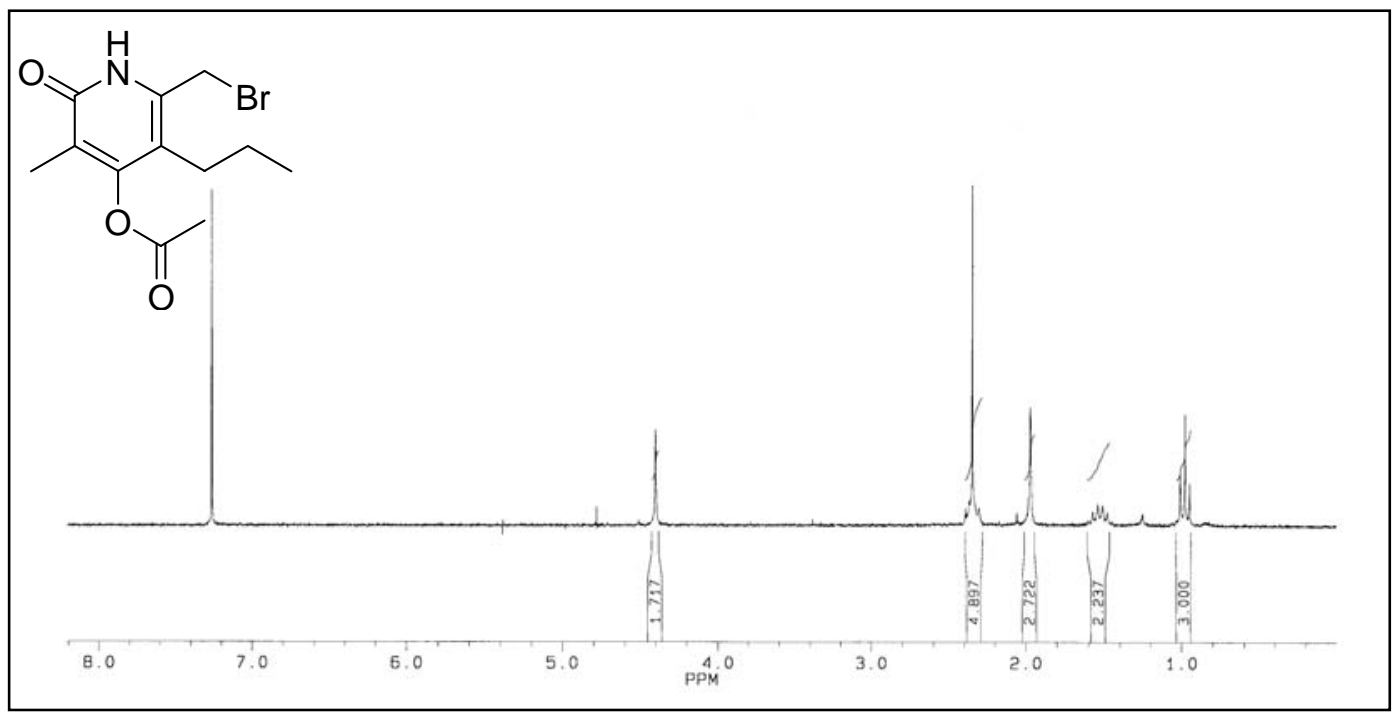

Essigsäure-6-brommethyl-3-methyl-2-oxo-5-propyl-1,2-dihydropyridin-4-ylester (159)

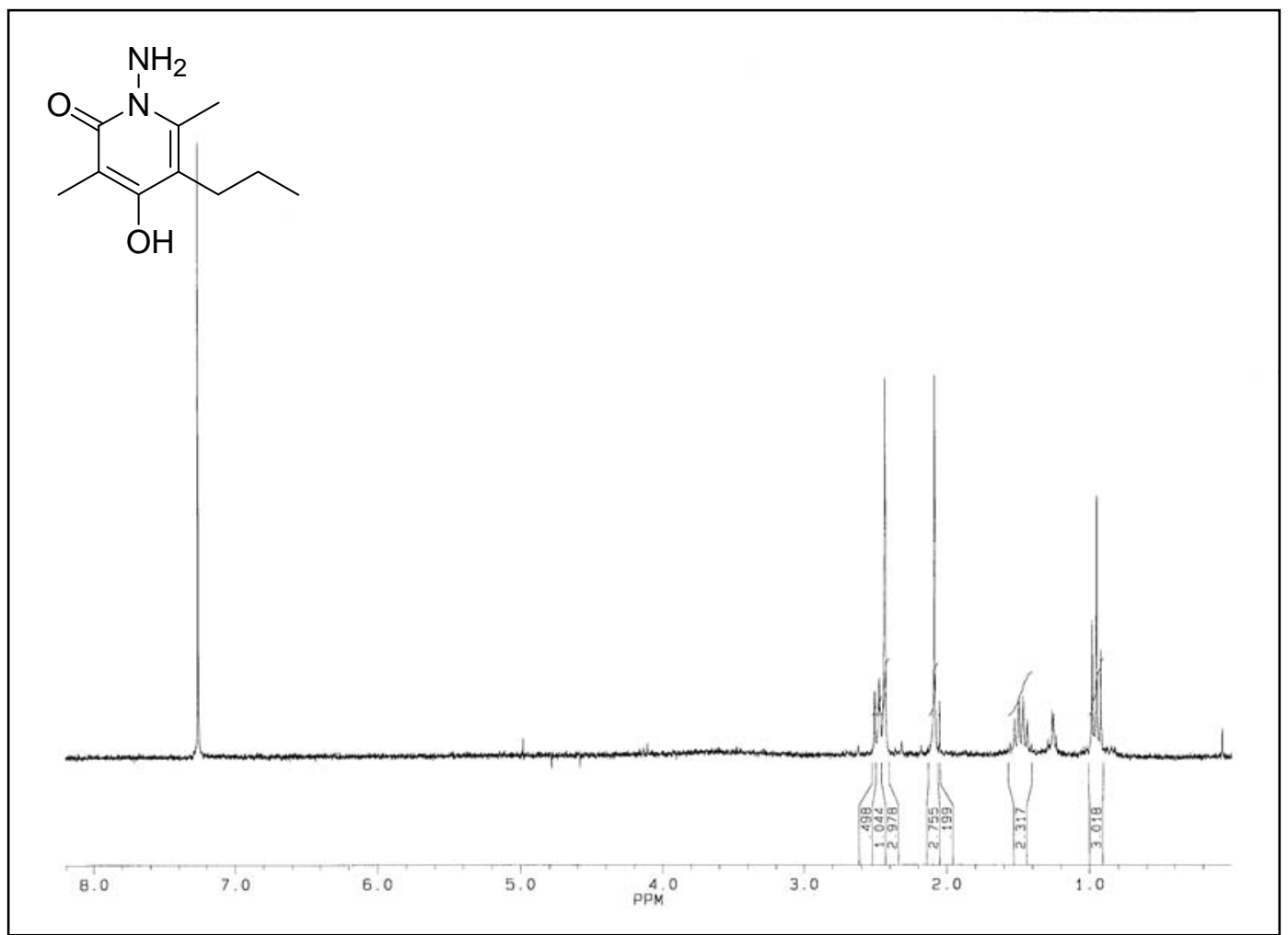

1-Amin-3,6-dimethyl-4-hydroxy-2-oxo-5-propyl-1H-pyridin (162) 


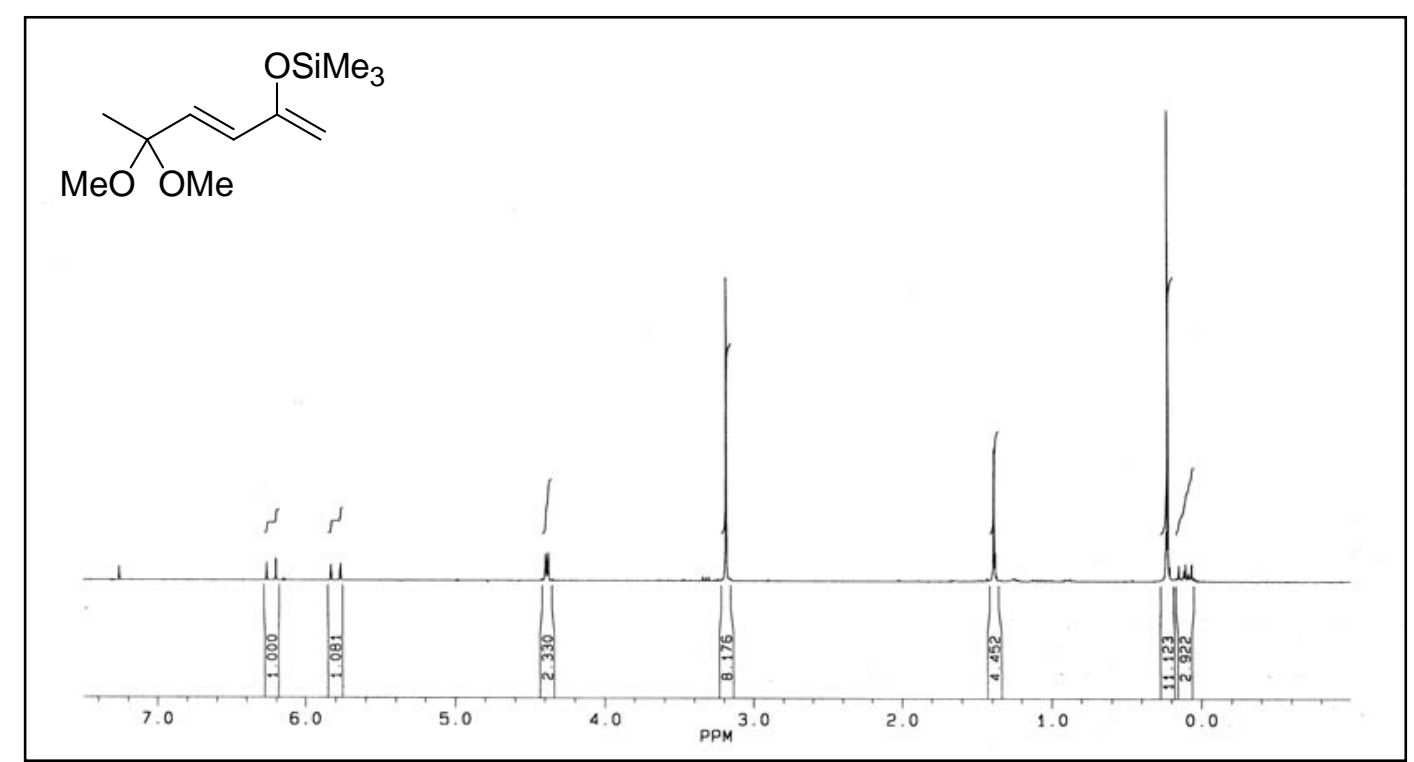

(E)-5,5-Dimethoxy-2-trimethylsilyloxyhexa-1,3-dien (274)

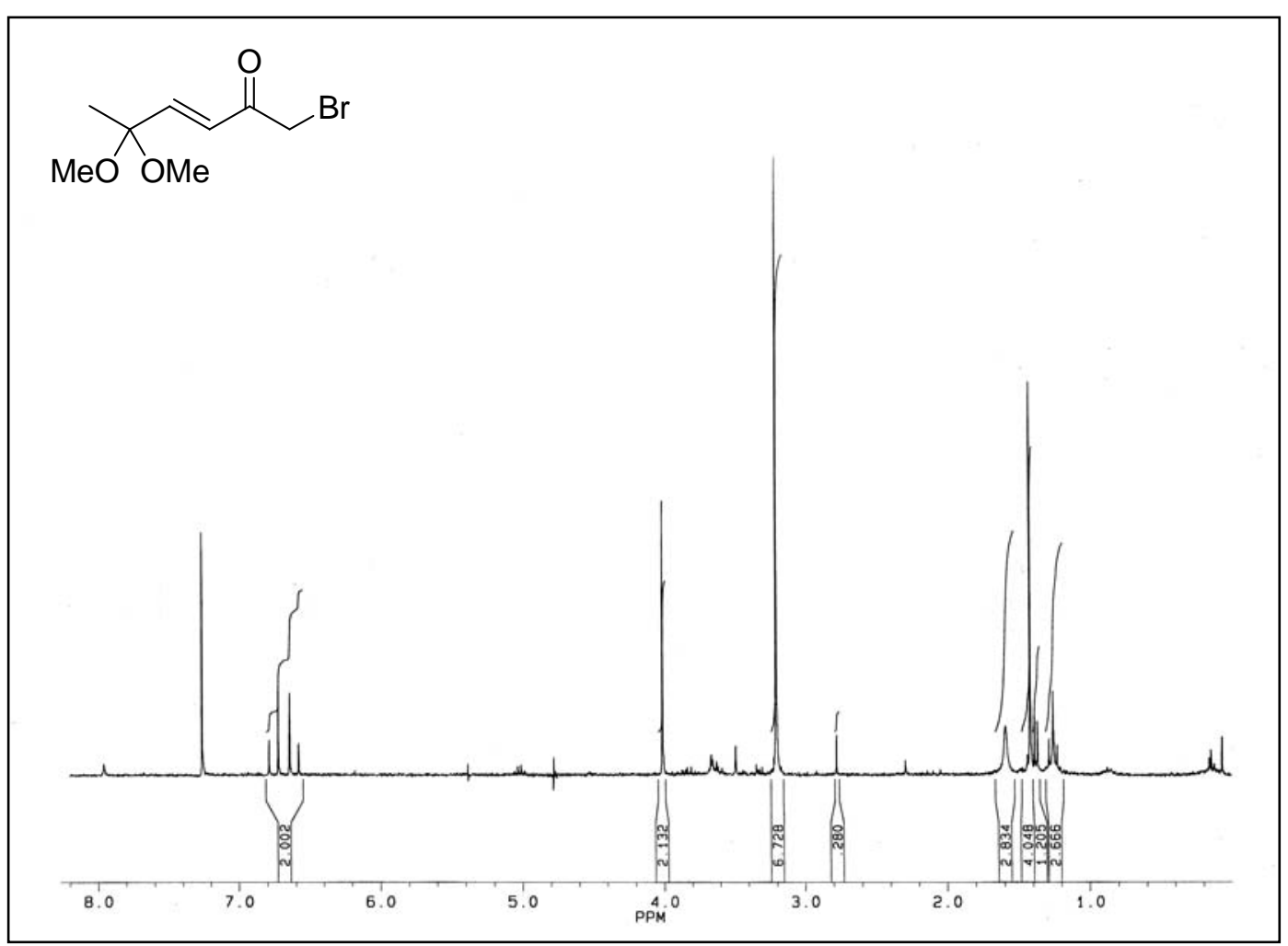

(E)-1-Brom-5,5-dimethoxyhex-3-en-2-on (275) 


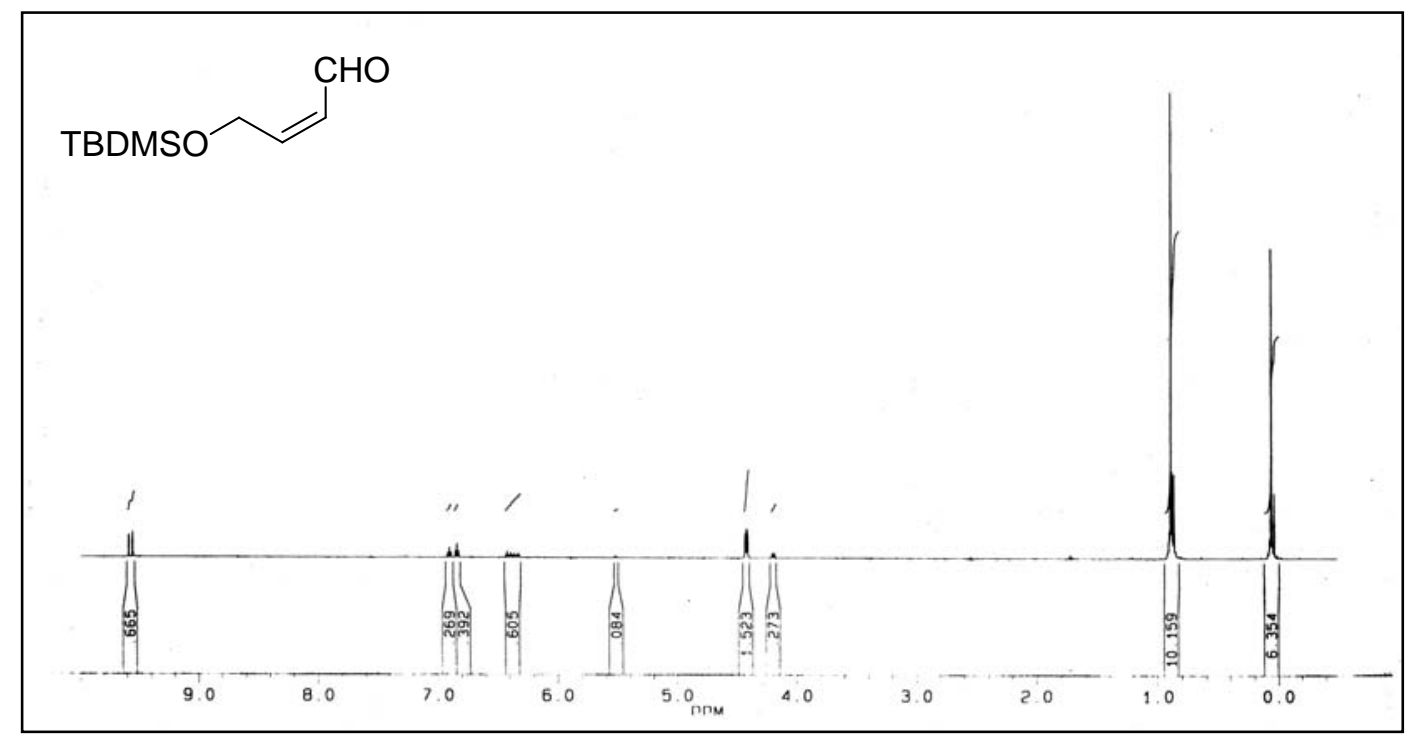

(Z)-4-(tert-Butyldimethylsilanyloxy)but-2-enal (278)

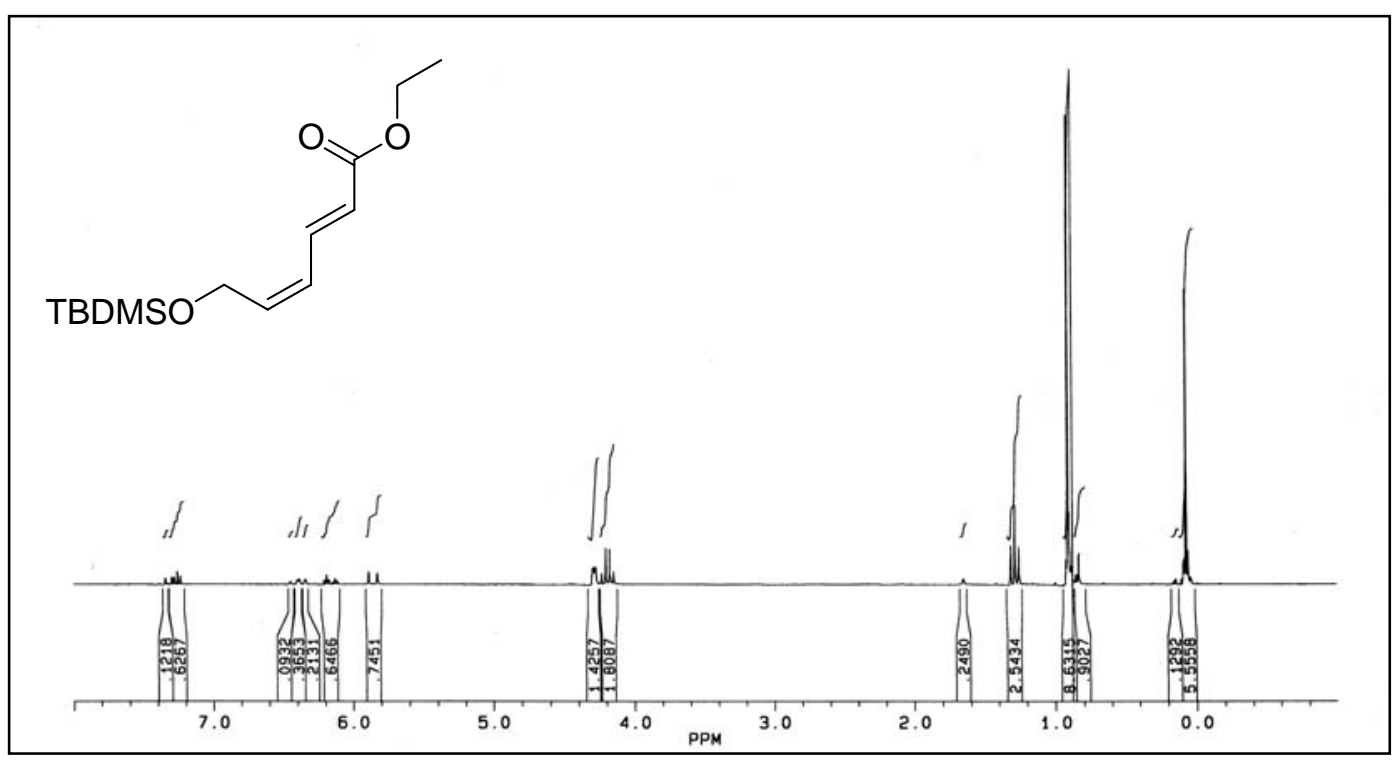

(E,Z)-6-(tert-Butyldimethylsilanyloxy)hexa-2,4-dienessigsäureehylester (279) 


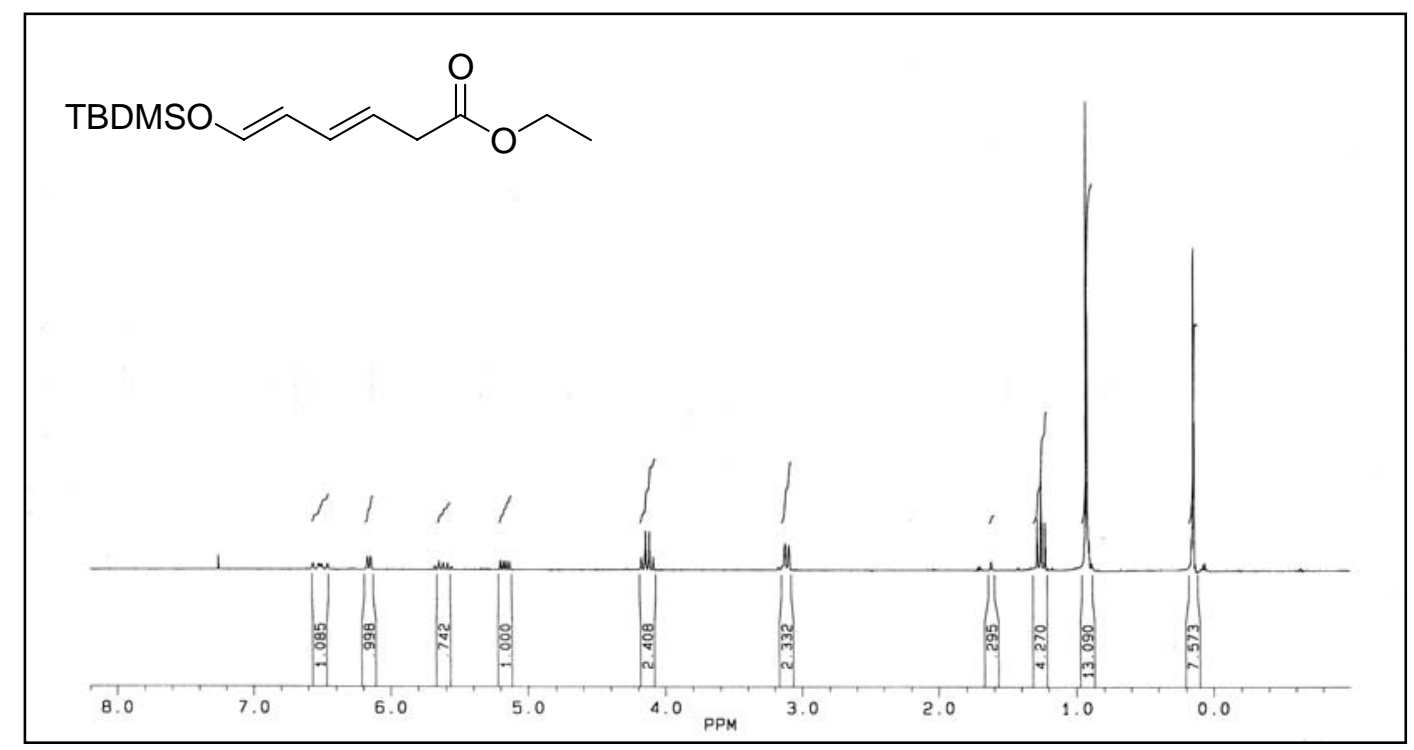

(E,E)-6-(tert-Butyldimethylsilanyloxy)hexa-3,5-dienessigsäureethylester (280)

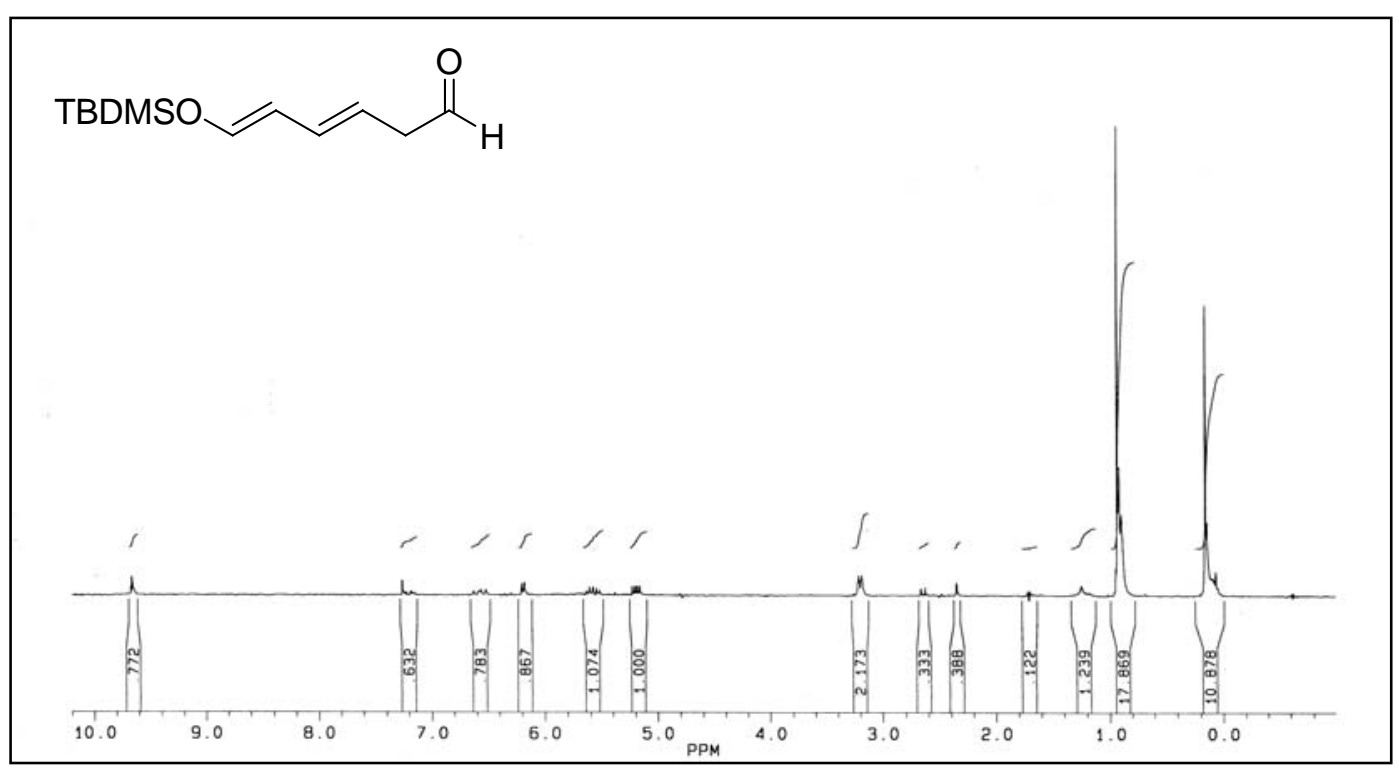

(E,E)-6-(tert-Butyldimethylsilanyloxy)hexa-3,5-dienal (281) 


\section{Publikationen und Präsentationen}

Artikel:

1) Domino-Staudinger-Aza-Wittig-1,5-Phosphonium-Rearrangement-FragmentationReactions of 1-Azido-2-hydroxy-4,6-dioxohexanes.

P. Langer*, I. Freifeld, H. Shojaei, Chem. Commun. 2003, 3044 - 3045.

2) Synthesis of 2-Alkylidenepyrrolidines and Pyrroles by Condensation of 1,3Dicarbonyl Dianions with $\alpha$-Azidoketones and subsequent Intramolecular StaudingerAza-Wittig-Reaction.

I. Freifeld, H. Shojaei, P. Langer*, J. Org. Chem. 2006, 71, 4965 - 4968.

3) Domino Staudinger / Semi-Aza-Wittig / Fragmentation Reactions of $\gamma$-Azido- $\beta$ hydroxyketones.

I. Freifeld, H. Shojaei, R. Dede, P. Langer*, J. Org. Chem. 2006, 71, 6165 - 6170.

4) Iromycins: A New Family of Pyridone Metabolites from Streptomyces sp. II. Convergent Total Synthesis.

H. Shojaei, Z. Li-Bohmer, P. von Zezschwitz*, J. Org. Chem. 2007, 72, 5091 - 5097.

5) In Veröffentlichung: Iromycins from Streptomyces sp. and Synthesis: New Inhibitors of the Mitochondrial Electron Transport Chain.

F. Surup, H. Shojaei, P. von Zezschwitz*, B. Kunze*, S. Grond*.

Poster:

1) Iromycin and Spirodionic Acid - Total Synthesis and Biosynthesis of two Novel Natural Products from Streptomyces sp.

H. Shojaei, J. Siewert, F. Surup, A. Textor, S. Grond*, P. von Zezschwitz*.

$1^{\text {st }}$ German-French Congress in Organic and Biomolecular Chemistry, Goslar, 2005.

2) Collinolactone - Chemistry and Biology of a 7-10-6-Tricyclic Metabolite from Streptomyces sp. 
L. Hoffmann, H. Shojaei, S. Grond* and P. von Zezschwitz*.

$1^{\text {st }}$ German-French Congress in Organic and Biomolecular Chemistry, Goslar, 2005. 


\section{Danksagung}

Zunächst möchte ich mich bei Herrn Prof. Dr. A. de Meijere für die Aufnahme in seinen Arbeitskreis und die damit verbundene Möglichkeit, alle technischen Einrichtungen seiner Abteilung nutzen zu dürfen, bedanken.

Weiterhin möchte ich insbesondere Herrn Dr. von Zezschwitz für die Betreuung meiner Arbeit und seinen wissenschaftlichen Rat danken.

Bei meinen Laborkollegen möchte ich mich für die gute Zusammenarbeit und das angenehme Klima bedanken. Insbesondere danke ich Jürgen Sievert, Tobias Bender und Johannes Beerlink für ihre Mithilfe bei der Korrektur meiner Arbeit. Bei Zhen Li und René Sandmann möchte ich mich für ihre herzliche Art und ihren Zuspruch bedanken.

Mein Dank gilt weiterhin der Abteilung von Prof. Dr. de Meijere für die Versorgung mit Chemikalien und Laborutensilien. Den Mitgliedern der NMR-Abteilung, insbesondere Herrn Machinek, danke ich für die Aufnahme ein- und zweidimensionalen Spektren. Zudem möchte ich mich bei Frank Hambloch für die Ausführung der Elementaranalysen bedanken. 